\title{
Dyadic Coping: A Collection of Recent Studies
}

Edited by: Bodenmann, Guy ; Falconier, Mariana K ; Randall, Ashley K

DOI: https://doi.org/10.3389/978-2-88963-031-8

Posted at the Zurich Open Repository and Archive, University of Zurich

ZORA URL: https://doi.org/10.5167/uzh-188993

Edited Scientific Work

Published Version

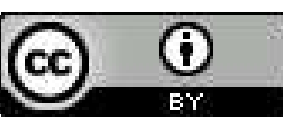

The following work is licensed under a Creative Commons: Attribution 4.0 International (CC BY 4.0) License.

Originally published at:

Dyadic Coping: A Collection of Recent Studies. Edited by: Bodenmann, Guy; Falconier, Mariana K; Randall, Ashley K (2019). Lausanne: Frontiers.

DOI: https://doi.org/10.3389/978-2-88963-031-8 


\section{DYADIC COPING: A COLLECTION OF RECENT STUDIES}

EDITED BY: Guy Bodenmann, Mariana K. Falconier and Ashley K. Randall PUBLISHED IN: Frontiers in Psychology

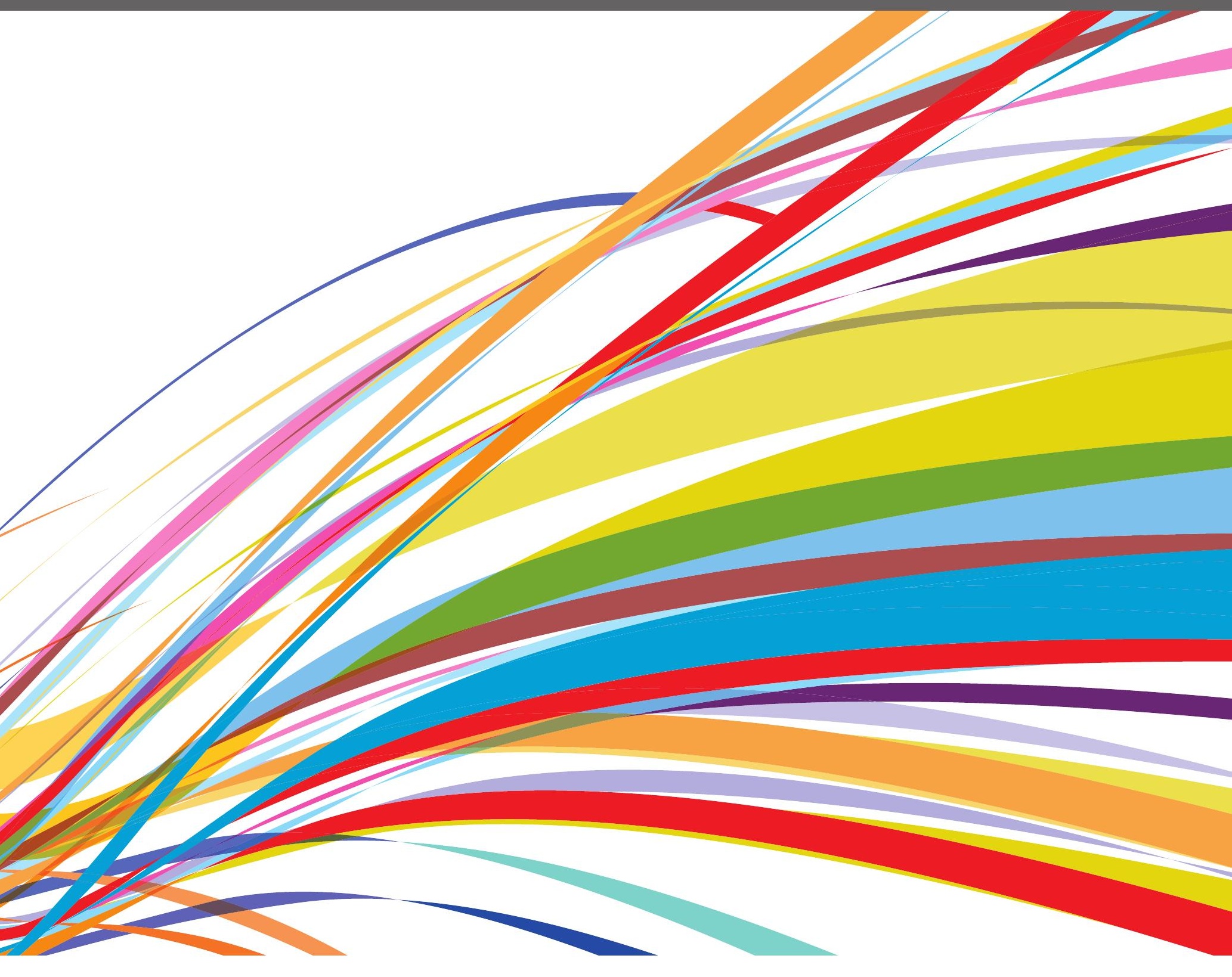

frontiers Research Topics 


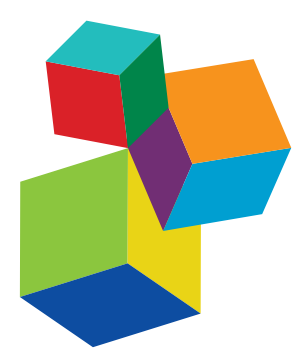

Frontiers Copyright Statement

C Copyright 2007-2019 Frontiers Media SA. All rights reserved.

All content included on this site, such as text, graphics, logos, button

icons, images, video/audio clips, downloads, data compilations and

software, is the property of or is

licensed to Frontiers Media SA

("Frontiers") or its licensees and/or subcontractors. The copyright in the text of individual articles is the property of their respective authors, subject to a license granted to Frontiers.

The compilation of articles constituting this e-book, wherever published, as well as the compilation of all other content on this site, is the exclusive property of Frontiers. For the

conditions for downloading and copying of e-books from Frontiers website, please see the Terms for Website Use. If purchasing Frontiers e-books from other websites or sources, the conditions of the website concerned apply.

Images and graphics not forming part of user-contributed materials may not be downloaded or copied without permission

Individual articles may be downloaded and reproduced in accordance with the principles of the CC-BY

licence subject to any copyright or other notices. They may not be re-sold as an e-book.

As author or other contributor you grant a CC-BY licence to others to reproduce your articles, including any graphics and third-party materials supplied by you, in accordance with the Conditions for Website Use and subject to any copyright notices which you include in connection with your articles and materials.

All copyright, and all rights therein, are protected by national and international copyright laws.

The above represents a summary only.

For the full conditions see the Conditions for Authors and the Conditions for Website Use.

ISSN 1664-8714

ISBN 978-2-88963-031-8

DOI 10.3389/978-2-88963-031-8

\section{About Frontiers}

Frontiers is more than just an open-access publisher of scholarly articles: it is a pioneering approach to the world of academia, radically improving the way scholarly research is managed. The grand vision of Frontiers is a world where all people have an equal opportunity to seek, share and generate knowledge. Frontiers provides immediate and permanent online open access to all its publications, but this alone is not enough to realize our grand goals.

\section{Frontiers Journal Series}

The Frontiers Journal Series is a multi-tier and interdisciplinary set of open-access, online journals, promising a paradigm shift from the current review, selection and dissemination processes in academic publishing. All Frontiers journals are driven by researchers for researchers; therefore, they constitute a service to the scholarly community. At the same time, the Frontiers Journal Series operates on a revolutionary invention, the tiered publishing system, initially addressing specific communities of scholars, and gradually climbing up to broader public understanding, thus serving the interests of the lay society, too.

\section{Dedication to Quality}

Each Frontiers article is a landmark of the highest quality, thanks to genuinely collaborative interactions between authors and review editors, who include some of the world's best academicians. Research must be certified by peers before entering a stream of knowledge that may eventually reach the public - and shape society; therefore, Frontiers only applies the most rigorous and unbiased reviews.

Frontiers revolutionizes research publishing by freely delivering the most outstanding research, evaluated with no bias from both the academic and social point of view. By applying the most advanced information technologies, Frontiers is catapulting scholarly publishing into a new generation.

\section{What are Frontiers Research Topics?}

Frontiers Research Topics are very popular trademarks of the Frontiers Journals Series: they are collections of at least ten articles, all centered on a particular subject. With their unique mix of varied contributions from Original Research to Review Articles, Frontiers Research Topics unify the most influential researchers, the latest key findings and historical advances in a hot research area! Find out more on how to host your own Frontiers Research Topic or contribute to one as an author by contacting the Frontiers Editorial Office: researchtopics@frontiersin.org 


\section{DYADIC COPING: A COLLECTION OF RECENT STUDIES}

Topic Editors:

Guy Bodenmann, University of Zurich, Switzerland Mariana K. Falconier, University of Maryland, United States

Ashley K. Randall, Arizona State University, United States

Dyadic coping is a concept that has reached increased attention in psychological science within the last 20 years. Dyadic coping conceptualizes the way couples cope with stress together in sharing appraisals of demands, planning together how to deal with the stressors and engage in supportive or joint dyadic coping. Among the different theories of dyadic coping, the Systemic Transactional Model (STM; Bodenmann, 1995, 1997, 2005) has been applied to many studies on couples' coping with stress. While a recent meta-analysis shows that dyadiccoping is a robust and consistent predictor of relationship satisfaction and couple's functioning in community samples, some studies also reveal the significance of dyadic coping in dealing with psychological disorders (e.g., depression, anxiety) or severe illness (e.g., cancer, diabetes, COPD, etc.).

Researchers all over the world build their research on this or other concepts of dyadic coping and many typically use the Dyadic Coping Inventory (DCl) for assessing dyadic coping. So far, research on dyadic coping has been systematically presented in two books, one written by Revenson, Kayser, \& Bodenmann in 2005, focussing on emerging perspectives on couples' coping, the other by Falconier, Randall, \& Bodenmann more recently in 2016, addressing intercultural aspects of dyadic coping in African, American, Asian and European couples.

This eBook gives an insight into recent dyadic coping research in different areas and countries.

Citation: Bodenmann, G., Falconier, M. K., Randall, A. K., eds. (2019). Dyadic Coping: A Collection of Recent Studies. Lausanne: Frontiers Media.

doi: 10.3389/978-2-88963-031-8 


\section{Table of Contents}

05

Editorial: Dyadic Coping

Guy Bodenmann, Mariana K. Falconier and Ashley K. Randall

\section{OVERVIEW ON CONCEPTS OF DYADIC COPING AND EMPIRICAL FINDINGS}

08 Dyadic Coping in Couples: A Conceptual Integration and a Review of the Empirical Literature

Mariana Karin Falconier and Rebekka Kuhn

\section{DYADIC COPING PROCESSES}

31 Examining the Effects of Couples' Real-Time Stress and Coping Processes on Interaction Quality: Language Use as a Mediator

Kevin K. H. Lau, Ashley K. Randall, Nicholas D. Duran and Chun Tao

45 Explicit Stress Communication Facilitates Perceived Responsiveness in Dyadic Coping

Ariela Francesca Pagani, Silvia Donato, Miriam Parise, Anna Bertoni, Raffaella lafrate and Dominik Schoebi

54 Dyadic Coping and its Underlying Neuroendocrine Mechanisms - Implications for Stress Regulation

Anna-Lena Zietlow, Monika Eckstein, Cristóbal Hernández, Nora Nonnenmacher, Corinna Reck, Marcel Schaer, Guy Bodenmann, Markus Heinrichs and Beate Ditzen

\section{DYADIC COPING AND ILLNESS}

64 Dyadic Coping Across the Lifespan: A Comparison Between Younger and Middle-Aged Couples With Breast Cancer

Chiara Acquati and Karen Kayser

82 Dyadic Coping in Patients Undergoing Radiotherapy for Head and Neck Cancer and Their Spouses

Hoda Badr, Krista Herbert, Mark D. Bonnen, Joshua A. Asper and Timothy Wagner

96 Dyadic Coping, Respiratory Sinus Arrhythmia, and Depressive Symptoms Among Parents of Preschool Children

Andrew Switzer, Warren Caldwell, Chelsea da Estrela, Erin T. Barker and Jean-Philippe Gouin

108 Dyadic Coping of Kidney Transplant Recipients and Their Partners: Sex and Role Differences

Daria Tkachenko, Laura Franke, Luisa Peters, Mario Schiffer and Tanja Zimmermann

121 Couples Dealing With Pediatric Blood Cancer: A Study on the Role of Dyadic Coping

Marieke Van Schoors, Tom Loeys, Liesbet Goubert, Geertrui Berghmans, Britt Ooms, Jurgen Lemiere, Koenraad Norga and Lesley Liliane Verhofstadt 
132 Links Between Communication and Relationship Satisfaction Among Patients With Cancer and Their Spouses: Results of a Fourteen-Day Smartphone-Based Ecological Momentary Assessment Study Shelby L. Langer, Joan M. Romano, Michael Todd, Timothy J. Strauman, Francis J. Keefe, Karen L. Syrjala, Jonathan B. Bricker, Neeta Ghosh, John W. Burns, Niall Bolger, Blair K. Puleo, Julie R. Gralow, Veena Shankaran, Kelly Westbrook, S. Yousuf Zafar and Laura S. Porter

147 Spousal Support for Patients With Rheumatoid Arthritis: Getting the Wrong Kind is a Pain

Jessie Pow, Ellen Stephenson, Mariët Hagedoorn and Anita DeLongis

158 Communal Coping in Couples With Health Problems

Kelly E. Rentscher

170 Strategies of Dyadic Coping and Self-Regulation in the Family Homes of Chronically III Persons: A Qualitative Research Study Using the Emotional Map of the Home Interview Method

Viola Sallay, Tamás Martos, Sheryl L. Chatfield and Andrea Dúll

\section{DYADIC COPING AND MAJOR STRESSORS}

186 The Predictive Value of Dyadic Coping in the Explanation of PTSD Symptoms and Subjective Well-Being of Work Accident Victims Susana Lameiras, Alexandra Marques-Pinto, Rita Francisco, Susana Costa-Ramalho and Maria Teresa Ribeiro

195 "What Makes us Strong?": Dyadic Coping in Italian Prospective Adoptive Couples

Elena Canzi, Silvia Donato, Laura Ferrari, Miriam Parise, Ariela Francesca Pagani, Giulia Lopez, Rosa Rosnati and Sonia Ranieri

205 Examining the Role of Dyadic Coping on the Marital Adjustment of Couples Undergoing Assisted Reproductive Technology (ART) Sara Molgora, Valentina Fenaroli, Chiara Acquati, Arianna De Donno, Maria Pia Baldini and Emanuela Saita

219 A Dyadic Approach to Understanding Associations Between Job Stress, Marital Quality, and Dyadic Coping for Dual-Career Couples in Iran Reza Fallahchai, Maryam Fallahi and Ashley K. Randall

230 Stress and Dyadic Coping in Personal Projects of Couples - A Pattern-Oriented Analysis

Tamás Martos, Viola Sallay, Marianna Nagy, Henrietta Gregus and Orsolya Filep 


\title{
Editorial: Dyadic Coping
}

\author{
Guy Bodenmann ${ }^{1 *}$, Mariana K. Falconier ${ }^{2}$ and Ashley K. Randall ${ }^{3}$ \\ ${ }^{1}$ Department of Psychology, University of Zurich, Zurich, Switzerland, ${ }^{2}$ School of Public Health, University of Maryland, \\ Virginia Tech, Blacksburg, VA, United States, ${ }^{3}$ Counseling and Counseling Psychology, Arizona State University, Tempe, AZ, \\ United States
}

Keywords: dyadic coping, couple support, spousal support, social support, emotion co-regulation

\section{Editorial on the Research Topic}

Dyadic Coping

\section{THEORETICAL BACKGROUND AND KEY ASSUMPTIONS}

Twenty years ago, dyadic coping arose from the conceptualization of stress and coping in romantic dyads, abandoning the traditional individual view of these phenomena. Since then theoretical contributions on dyadic coping (Bodenmann, 1997; Revenson et al., 2005; Revenson and Lepore, 2012; Falconier et al., 2016) and empirical studies have been published (see review article by Falconier and Kuhn), which extend work on conceptualizing dyadic coping and its beneficial association on individual and relational well-being.

Based on the individual-oriented theory of stress and coping by Lazarus and Folkman (1984), the Systemic Transactional Model (STM; Bodenmann, 1995, 2005) became an internationally recognized theory of stress and coping in couples and families. The key assumption of STM refers to the interdependence between romantic partners (Kelley et al., 1983), ascertaining that stress of one romantic partner always affects the other partner, through processes of stress spillover (Bodenmann et al., 2007) and cross-over (Neff and Karney, 2007). As such, this view of shared or mutual stress opens the possibility for common/joint dyadic coping, where either one partner supports the other in his/her own coping efforts (supportive or delegated dyadic coping) or both partners engage together in shared problem-solving or joint emotion-regulation (common dyadic coping).

Dyadic coping can be conceptualized as positive (emotion-focused, problem-focused, or delegated) or negative (e.g., superficial, ambivalent, hostile) (Bodenmann et al., 2016). From a STM perspective (Bodenmann, 1995, 2005), dyadic coping involves cognitive (individual and dyadic appraisals of stress and coping resources, individual and dyadic goals), emotional (shared emotions and co-regulation of emotions), physiological (shared arousals, impact of dyadic coping on endocrine processes) and behavioral aspects and processes (e.g., overt stress management activities, active listening to the partner's stress-related self-disclosure, verbal and non-verbal support behaviors like holding each other, hugging, giving a massage, active joint problem-solving). Dyadic coping is usually assessed by self-reports (such as e.g., the Dyadic Coping Inventory; DCI, Bodenmann, 2008), diary studies or behavioral coding (e.g., Kuhn et al., 2018; Leuchtmann et al., 2018; Lau et al.).

\section{EVOLUTION OF DYADIC COPING RESEARCH}

Entering the keyword "dyadic coping" in ISI Web of Knowledge yields an impressive figure of the development of dyadic coping research since 1992. While in 1992, four publications cited dyadic coping, in 2018, 1,347 publications were referring to couple's coping.

At the beginning dyadic coping research focused primarily on the impact of daily hassles on couples' functioning (e.g., relationship satisfaction, couple's communication, sexuality, commitment, relationship dissolution). Researchers were interested in stress spill-over and 
cross-over processes within couples exposed to daily external stress and how couples effectively cope with these stressors (Story and Bradbury, 2004; Neff and Karney, 2007; Randall and Bodenmann, 2009, 2017; Falconier et al., 2015a,b). In the last decade, the field moved further into couples dealing with critical life events or severe illness (e.g., Badr et al., 2010; Revenson and Lepore, 2012; Rottmann et al., 2015; Falconier and Kuhn) or mental disorders (Bodenmann and Randall, 2013).

More recently, dyadic coping research started to address intercultural aspects in African, American, Asian and European couples (Falconier et al., 2016; Hilpert et al., 2016).

\section{THIS SPECIAL ISSUE}

The content of the 18 contributions, forming this special issue, ranges from contributions on specific mechanisms of dyadic coping (contribution by Pagani et al.; Zietlow et al.), different types of stressors (contributions by Canzi et al.; Fallahchai et al.; Molgora et al.), health or illness issues (contribution by Acquati and Kayser; Badr et al.; Lameiras et al.; Langer et al.; Martos et al.; Pow et al.; Rentscher; Sallay et al.; Switzer et al.; Tkachenko et al.; Van Schoors et al.), and novel methods such as dyadic coping in language use (Lau et al.) up to a review article (Falconier and Kuhn), integrating the conceptual and empirical literature on dyadic coping.

\section{FUTURE DIRECTIONS}

As demonstrated by this special issue, dyadic coping represents an inspiring field of research that spans many topics and disciplines (clinical psychology, health psychology, counseling, family science, developmental and personality

\section{REFERENCES}

Badr, H., Carmack, C. L., Kashy, D. A., Cristofanilli, M., and Revenson, T. A. (2010). Dyadic coping in metastatic breast cancer. Health Psychol. 29, 169-180. doi: 10.1037/a0018165

Bodenmann, G. (1995). A systemic-transactional conceptualization of stress and coping in couples. Swiss J. Psychol. 54, 34-49.

Bodenmann, G. (1997). Dyadic coping: a systemic-transactional view of stress and coping among couples: theory and empirical findings. Eur. Rev. Appl. Psychol. 47, 137-141.

Bodenmann, G. (2005). "Dyadic coping and its significance for marital functioning," in Couples Coping with Stress: Emerging Perspectives on Dyadic Coping, eds T. A. Revenson, K. Kayser, and G. Bodenmann (Washington, DC: American Psychological Association), 33-49. doi: 10.1037/11031-002

Bodenmann, G. (2008). Dyadisches Coping Inventar: Testmanual. [Dyadic Coping Inventory. Manual]. Bern: Huber and Hogrefe Tests.

Bodenmann, G., Ledermann, T., and Bradbury, T. N. (2007). Stress, sex, and satisfaction in marriage. Pers. Relatsh. 14, 551-569. doi: 10.1111/j.1475-6811.2007.00171.x

Bodenmann, G., and Randall, A. K. (2013). Close relationships in psychiatric disorders: Curr. Opin. Psychiatry 26, 464-467. doi: 10.1097/YCO.0b013e3283642de7

Bodenmann, G., Randall, A. K., and Falconier, M. K. (2016). "The systemic transactional model (STM)," in Couples Coping with Stress: A cross-cultural Perspective, eds M. K. Falconier, A. K. Randall, and Guy Bodenmann (New York, NY: Routledge), 5-22. psychology). Future studies are encouraged to further integrate multi-methods approaches (self-report, behavior coding, physiology, voice stress, pronoun use etc.) by using longitudinal designs (collection of data over several months or years). Another promising focus is examining overt dyadic coping during everyday interactions of couples at their home, as thus far, little is known about how often and how couples really cope together in everyday life, capturing life as lived (Bolger et al., 2003).

Another focus of dyadic coping research might further address parent-child dyadic coping interactions or adults caring for aging parents, where only few studies were conducted (Zemp et al., 2016).

Furthermore, we do not know much about how dyadic coping evolves over the lifespan of couples (e.g., Johnson et al., 2016), when and it what phase of a couple's life it is particularly relevant and what are long-term effects of dyadic coping on relationship functioning and health.

Finally, more studies on clinical interventions focusing on the improvement of dyadic coping skills, such as the Couples Coping Enhancement Training (CCET) by Bodenmann and Shantinath (2004) or TOGETER by Falconier (2015) are needed. However, current research by Randall and Totenhagen shows promise in this area based on their research with sexual minority individuals experiencing stress due to their marginalized status (Randall et al., 2017a,b; Totenhagen et al., 2018).

\section{AUTHOR CONTRIBUTIONS}

All authors listed have made a substantial, direct and intellectual contribution to the work, and approved it for publication.
Bodenmann, G., and Shantinath, S. D. (2004). The Couples Coping Enhancement Training (CCET): a new approach to prevention of marital distress based upon stress and coping. Fam. Relat. 53, 477-484. doi: 10.1111/j.0197-6664.2004.00056.x

Bolger, N., Davis, A., and Rafaeli, E. (2003). Diary methods: Capturing life as it is lived. Annu. Rev. Psychol. 54, 579-616.

Falconier, M. K. (2015). Together-A couples' program to improve communication, coping, and financial management skills: development and initial pilot-testing. J. Marital Fam. Ther. 41, 236-250. doi: 10.1111/jmft.12052

Falconier, M. K., Jackson, J. B., Hilpert, P., and Bodenmann, G. (2015a). Dyadic coping and relationship satisfaction: a meta-analysis. Clin. Psychol. Rev. 42, 28-46. doi: 10.1016/j.cpr.2015.07.002

Falconier, M. K., Nussbeck, F., Bodenmann, G., Schneider, H., and Bradbury, T. (2015b). Stress from daily hassles in couples: its effects on intradyadic stress, relationship satisfaction, and physical and psychological well-being. J. Marital Fam. Ther. 41, 221-235. doi: 10.1111/jmft.12073

Falconier, M. K., Randall, A. K., and Bodenmann, G. (eds.). (2016). Couples Coping With Stress. A Cross-Cultural Perspective. New York, NY: Routledge. doi: 10.4324/9781315644394

Hilpert, P., Randall, A. K., Sorokowski, P., Atkins, D. C., Sorokowska, A., Ahmadi, K., et al. (2016). The associations of dyadic coping and relationship satisfaction vary between and within nations: a 35-nation study. Front. Psychol. 7:1106. doi: 10.3389/fpsyg.2016.01106

Johnson, M. D., Horne, R. M., and Galovan, A. M. (2016). The developmental course of supportive dyadic coping in couples. Dev. Psychol. 52, 2031-2043. doi: $10.1037 / \operatorname{dev} 0000216$ 
Kelley, H. H., Berscheid, E., Christensen, A., Harvey, J. H., Huston, T. L., Levinger, G., et al. (eds.). (1983). Close Relationships. New York, NY: W. H. Freeman.

Kuhn, R., Bradbury, T. N., Nussbeck, F. W., and Bodenmann, G. (2018). The power of listening: lending an ear to the partner during dyadic coping conversations. J. Fam. Psychol. 32, 762-772. doi: 10.1037/fam0000421

Lazarus, R. S., and Folkman, S. (1984). Stress, Appraisal, and Coping. New York, NY: Springer.

Leuchtmann, L., Zemp, M., Milek, A., Nussbeck, F. W., Brandstätter, V., and Bodenmann, G. (2018). Role of clarity of other's feelings for dyadic coping. Pers. Relatsh. 25, 38-49. doi: 10.1111/pere.12226

Neff, L. A., and Karney, B. R. (2007). Stress crossover in newlywed marriage: a longitudinal and dyadic perspective. J. Marr. Fam. 69, 594-607. doi: 10.1111/j.1741-3737.2007.00394.x

Randall, A. K., and Bodenmann, G. (2009). The role of stress on close relationships and marital satisfaction. Clin. Psychol. Rev. 29, 105-115. doi: 10.1016/j.cpr.2008.10.004

Randall, A. K., and Bodenmann, G. (2017). Stress and its associations with relationship satisfaction. Curr. Opin. Psychol. 13, 96-106. doi: 10.1016/j.copsyc.2016.05.010

Randall, A. K., Tao, C., Totenhagen, C. J., Walsh, K. J., and Cooper, A. (2017a). Associations between sexual orientation discrimination and depression among same-sex couples: moderating effects of dyadic coping. J. Couple Relationship Ther. 4, 325-345. doi: 10.1080/15332691.2016.1253520

Randall, A. K., Totenhagen, C. J., Walsh, K. J., Adams, C., and Tao, C. (2017b). Coping with workplace minority stress: associations between dyadic coping and anxiety among women in same-sex relationships. J. Lesbian Stud. 21, 70-87. doi: 10.1080/10894160.2016.1142353

Revenson, T. A., Kayser, K., and Bodenmann, G. (eds.). (2005). Couples Coping With Stress: Emerging Perspectives on Dyadic Coping. Washington, DC: American Psychological Association. doi: 10.1037/11031-000
Revenson, T. A., and Lepore, S. J. (2012). "Coping in social context," in Handbook of Health Psychology, 2nd Edn., eds A. Baum, T. A. Revenson, and J. E. Singer (New York, NY: Psychology Press), 193-217.

Rottmann, N., Hansen, D. G., Larsen, P. V., Nicolaisen, A., Flyger, H., Johansen, C., et al. (2015). Dyadic coping within couples dealing with breast cancer: a longitudinal, population-based study. Health Psychol. 34:486. doi: $10.1037 /$ hea 0000218

Story, L. B., and Bradbury, T. N. (2004). Understanding marriage and stress: essential questions and challenges. Clin. Psychol. Rev. 23, 1139-1162. doi: $10.1016 /$ j.cpr.2003.10.002

Totenhagen, C. J., Randall, A. K., and Lloyd, K. (2018). Stress and relationship functioning in same-sex couples: the vulnerability of internalized homophobia. Fam. Relat. 67, 399-413. doi: 10.1111/far e.12311

Zemp, M., Bodenmann, G., Backes, S., Sutter-Stickel, D., and Revenson, T. A. (2016). The importance of parents' dyadic coping for children. Fam. Relat. 65, 275-286. doi: 10.1111/fare.12189

Conflict of Interest Statement: The authors declare that the research was conducted in the absence of any commercial or financial relationships that could be construed as a potential conflict of interest.

Copyright $(2019$ Bodenmann, Falconier and Randall. This is an open-access article distributed under the terms of the Creative Commons Attribution License (CC BY). The use, distribution or reproduction in other forums is permitted, provided the original author(s) and the copyright owner(s) are credited and that the original publication in this journal is cited, in accordance with accepted academic practice. No use, distribution or reproduction is permitted which does not comply with these terms. 


\section{OPEN ACCESS}

Edited by:

Gianluca Castelnuovo, Catholic University of Sacred Heart,

Italy

Reviewed by:

Tanja Zimmermann,

Hannover Medical School, Germany

Noa Vilchinsky,

Bar-llan University, Israe

Lesley L. Verhofstadt,

Ghent University, Belgium

*Correspondence:

Mariana Karin Falconier

marianak@umd.edu

Specialty section:

This article was submitted to Clinical and Health Psychology,

a section of the journal

Frontiers in Psychology

Received: 03 July 2018

Accepted: 28 February 2019

Published: 26 March 2019

Citation:

Falconier MK and Kuhn R (2019)

Dyadic Coping in Couples: A Conceptual Integration and a Review

of the Empirical Literature.

Front. Psychol. 10:571.

doi: 10.3389/fpsyg.2019.00571

\section{Dyadic Coping in Couples: A Conceptual Integration and a Review of the Empirical Literature}

\author{
Mariana Karin Falconier ${ }^{1 *}$ and Rebekka Kuhn ${ }^{2}$ \\ ${ }^{1}$ Department of Family Science, School of Public Health, University of Maryland, College Park, MD, United States, \\ ${ }^{2}$ Department of Psychology, University of Zurich, Zurich, Switzerland
}

The present review on dyadic coping (DC) aims at providing a critical integration of both the conceptual and empirical DC literature and overcoming the limitations of past reviews by (a) describing, comparing, and integrating all the DC models, (b) presenting and integrating findings from studies based on DC models, and (c) suggesting directions for further research. The DC models identified and compared include: The congruence model (Revenson, 1994), the relationship-focused model (Coyne and Smith, 1991; O'Brien and DeLongis, 1996), the communal coping model (Lyons et al., 1998), the systemic-transactional model (Bodenmann, 1995, 1997), the relational-cultural model (Kayser et al., 2007), and the developmental-contextual coping model (Berg and Upchurch, 2007). After discussing each DC model, we advance a conceptual integration of all models, which serves as the framework to organize the review of the empirical literature. This integration includes the following DC dimensions: (a) Stress Communication, (b) Positive DC by One Partner (supportive DC, empathic responding, delegated DC, active engagement), (c) Positive Conjoint DC (common, collaborative, communal, mutual responsiveness); (d) Negative DC by One Partner (protective buffering, overprotection, and hostility/ambivalence), and (e) Negative Conjoint DC (common negative DC, disengaged avoidance). Developmental, relational, and contextual variables are included as factors shaping DC. To be included in the empirical review, articles had to be published in or a peer-reviewed journal in English and/or German before 2017 and include an original empirical study guided by one of the DC models. The review included 139 studies and, with the exception of the congruence model whose findings were discussed separately, findings were presented for overall DC and each of the dimensions identified in the conceptual integration. Findings were grouped also according to whether the stressor related or not to a medical or mental health condition. Demographic and cultural factors affecting DC were discussed. Overall, the empirical review suggests that in Western couples, positive individual, and conjoint DC forms, taken together or separately, have individual and relational benefits for couples coping with stress in general and/or mental health or medical stressors. Research on DC can be expanded to include other populations and stressors and use improved designs.

Keywords: coping, couples, models, review, stress 
For decades the study of stress and coping strategies focused mainly on the individual, without considering the reciprocal influential processes that are part of relational contexts (e.g., Lazarus and Folkman, 1984). The focus was limited to the stressed individual and the role that partner's support might play in reducing his or her stress. It was only in the last two decades that scholars adopted a more systemic perspective and shifted their view of stressors as affecting only one partner to affecting both, either directly when partners face the same stressful event such as a dyadic stressor (e.g., financial problems) or indirectly when the stressor may be initially related to one partner (e.g., a medical problem) but then spills into the relationship and ends up affecting the other partner as well. In other words, stress in couples was no longer conceptualized as an individual phenomenon but as a dyadic affair (e.g., Bodenmann, 1995, 1997; Lyons et al., 1998). This dyadic conceptualization of stress emphasizes not only the interdependence of partners' stress experience but it also places the coping process with external stressors (stressful situations originating outside the couple's relationship) in a relational context in which partners respond not only to their individual stress but also to each other's stress. This interpersonal view opens a new understanding of how couples deal with everyday stress as well as critical life events. Partners' coping responses to each other's stress resulting from circumstances outside the relationship is usually referred to as dyadic coping (DC). For stress that is experienced as a result of within-the-relationship stressors (e.g., conflict with partner, infidelity), partners usually use their own individual coping strategies and they do not rely on dyadic coping since they are not likely to help each other cope with that type of stress.

In line with this shift toward an interpersonal view of stress and coping in couples, various DC models have been proposed, such as the relationship-focused model (Coyne and Smith, 1991), the congruence model (Revenson, 1994), the systemic transactional model (Bodenmann, 1995, 1997), and the developmental-contextual coping model (Berg and Upchurch, 2007) among others. DC research has been typically guided by one of these conceptual models, each of which may offer some unique DC perspective. However, significant conceptual overlap also exists among those DC models (e.g., Falconier et al., 2015). A critical examination of such models and their research reveals that each model offers a partial view of the DC process and that their studies fail to integrate findings from studies guided by other DC conceptual models that focus on similar DC aspects. This fragmented approach has prevented from capturing the progress made in the field toward understanding the DC process and answering questions such as whether some aspects of DC process are more beneficial than others, whether the DC benefits vary by stressors, whether there are demographic variations in DC, or where further DC research is needed. Therefore, the present manuscript reviews and analyzes the conceptual and empirical literature with the aim of providing an integrated view of the DC process, organizing the accumulated empirical knowledge, and identifying areas for further research. In the first section of the manuscript we describe each DC model and its unique conceptual contributions in understanding the DC process while identifying its conceptual overlap with other
DC models. This comparative analysis leads to the proposal of an integrative model that includes all the different dimensions of DC identified across different models while eliminating unnecessary conceptual overlaps. The resulting integrative model allows to present a comprehensive approach to understanding the DC process and guides the organization and presentation of the accumulated empirical findings in the DC field in the second section of the present manuscript. After describing the methodology used to conduct the empirical review, this second section reports research findings in each of the DC dimensions included in the integrative model, while differentiating findings regarding medical and mental health stressors vs. other types of (non-medical) stressors. The discussion of such findings illuminates areas for further research. In other words, the goals of this paper are: (a) to describe each DC model, its contributions to understanding the DC process, and its conceptual differences and overlaps with other models; (b) to develop a model that includes all dimensions and factors identified in each DC model in order to have a comprehensive understanding of the DC process and allows to organize the empirical literature; (c) to summarize the findings from the empirical literature regarding each DC dimension and the potential effects of demographic (age, gender) and contextual factors (e.g., culture) on couples coping with medical and non-medical stressors, and (d) to discuss further research directions in the field. To the best of our knowledge, this is the first manuscript that attempts to provide such an integration and review of all the DC conceptual and empirical literature. Previous attempts to review and/or conceptually integrate the DC literature have focused exclusively on one stressor, mostly couples coping with cancer (Traa et al., 2015), have reviewed only the association between DC and relationship satisfaction (Falconier et al., 2015), or have included mostly studies applying only one DC model (Staff et al., 2017). These reviews have failed to integrate conceptually all DC existing models and/or have left out a large number of the studies that have been published in the last two decades.

\section{DYADIC COPING MODELS}

The initial DC models were formalized in the 1990s and each of them followed Lazarus and Folkman's conceptualization of stress as resulting from the perception or appraisal that the demands of a situation exceed the resources available to deal with such demands. These initial models were the congruence model (CM; Revenson, 1994), the relationship-focused model (RFM; DeLongis and O'Brien, 1990; Coyne and Smith, 1991), the communal coping model (CCM; Lyons et al., 1998), and the systemic-transactional model (STM; Bodenmann, 1995; Bodenmann et al., 2016). In the last decade these initial models were expanded to incorporate developmental and cultural aspects resulting in the relational-cultural coping model (RCCM; Kayser et al., 2007; Kayser and Revenson, 2016), and the developmentalcontextual coping model (DCCM; Berg and Upchurch, 2007). The following section includes a description of each DC model, the research areas in which each model has been applied and the instruments that have been used to measure the 
constructs. The presentation of each model seeks to uncover the unique contributions of each model to the understanding of the DC process while identifying conceptual overlaps with other DC models. This comparative analysis is necessary to create a theoretical framework that integrates all the conceptual developments about the DC process in the field.

\section{The Congruence Model (CM)}

During the 1980s different researchers became interested in the interplay between partners' individual coping styles by examining the effect of similarities and dissimilarities between those coping styles on individual and relational outcomes (e.g., Barbarin et al., 1985). Their studies marked the beginning of the DC field by considering one partner's stress and coping in relation to the other partner's and therefore acknowledging the interpersonal context of the stress and coping process in couples. For example, Cronkite and Moos (1984) studied whether similarity between partners' coping styles alleviated the effects of illness-related stress and concluded that "the personal coping resources and coping responses of each partner can alter the impact of stress and the effectiveness of coping" (p. 389). Later on, Revenson (1994) moved beyond the similarity or dissimilarity between partners' coping strategies and instead focused on the congruence, or fit, between the partners' coping styles, that is, the degree to which partners' coping responses are coordinated and mutually supporting. Revenson coined the term "congruence" coping and advanced the idea that the coordination of coping efforts or mutually reinforcing coping strategies can lead to positive psychosocial outcomes.

The CM and the dissimilarity/similarity framework has been mostly applied to the study of couples coping with stress in general (Cronkite and Moos, 1984; Giunta and Compas, 1993), cancer (e.g., Kraemer et al., 2011), and multiple sclerosis (Pakenham, 1998). Those studies usually assess each partner's individual coping style through well-known individual coping measures such as the Revised Ways of Coping Scale (Vitaliano et al., 1985) or the Coping Strategies Inventory (Carver et al., 1989).

Unlike any other DC model, the CM focuses on the interplay between partners' individual strategies to cope with their own stress rather than on partners' conjoint strategies to cope with common stressors or a partner's coping responses to the other partner's stress. In this regard it is the only DC model that examines the interpersonal effects of individual coping strategies on couple functioning.

\section{The Relationship-Focused Model (RFM)}

There were two groups of researchers, one led Coyne and Smith (1991) and another led by DeLongis and O’Brien (1990), that were the first to consider that in addition to individual emotion- and problem-focused strategies to cope with stress, individuals also responded with relationship-focused strategies "aimed at managing, regulating, or preserving relationships during stressful periods... particularly when stressors occur in interpersonal contexts" (O'Brien and DeLongis, 1996, p. 782). Each of these groups of scholars focused on different dimensions of the RFM, but both groups attended not to what each partner did to manage their own stress but to what each partner did to help the other partner cope with a stressful situation. Coyne and Smith (1991) studied the way couples responded to a partner's myocardial infarction and identified two coping mechanisms that had relationship-focused function: active engagement and protective buffering. During active engagement, an individual provides support to the sick partner by involving him or her in conversations about how she or he is thinking and feeling, or about other issues around the medical condition. As such, active engagement would be expected to represent a positive form of support and be related to positive outcomes for the stressed individual and their relationship. Protective buffering refers to the partner's efforts to hide or deny concerns and worries and yielding to the other partner to minimize conflict. Even though this form of coping may be triggered by a positive intent, most studies have shown that it usually has a negative impact on the stressed individual and the couple's relationship (for a review see Falconier et al., 2015). In collaboration with Fiske, Coyne and Smith (1991) also identified another RFM coping strategy: overprotection. This coping form is seen when a partner underestimates the sick individual's capabilities and therefore, he or she provides unnecessary support (practical or emotional) or restricts the sick partner's activities. Overprotection can be viewed conceptually as a negative form of dyadic coping and empirical evidence has provided support for its detrimental impact at the individual and relational levels (for a review see Falconier et al., 2015).

Coyne and Smith's model has been mostly applied to the study of couples coping with medical condition such as cancer (e.g., Hinnen et al., 2008), diabetes (e.g., Schokker et al., 2010), Alzheimers (Kramer, 1993), chronic-obstructive pulmonary disease (COPD; Snippe et al., 2012), or smoking (Butler et al., 2014). In order to measure RFM strategies, Coyne and Smith (1991) developed a self-report instrument known as the Relationship-Focused Coping Scales with subscales that assess active engagement and protective buffering, and in collaboration with Fiske et al. (1991) they developed a scale for overprotection.

Instead of defining three different specific dimensions, DeLongis and O'Brien (1990) distinguished between positive and negative RFM strategies. Positive strategies included empathy, providing support, and compromise, similar to the STM's supportive DC, whereas negative strategies included withdrawal and hostility, similar conceptually to the ambivalent/hostile negative DC from STM. Over time O'Brien et al. (2009) focused particularly on the use of one form of positive relationshipfocused coping: empathic responding. This DC form involves "the non-stressed partner's efforts to view the world from the other partner's perspective, experience the affective and cognitive associations that the stressful situation is evoking for the other partner, understand the partner's psychological states in his or her communication" (O'Brien et al., 2009, p. 783). Studies examining empathic responding have focused on stepfamilies (e.g., Lee-Baggley et al., 2005) and medical stressors (e.g., Marin et al., 2007) and have used daily process methods such as structured diaries and the Empathic Responding Scale (O’Brien and DeLongis, 1996). 
Unlike the CM, the RFM shifted the attention away from what partners do to cope with their own stress to identify what successful and unsuccessful strategies a partner uses to help the other partner cope with his or her own stress. In doing so, the RFM has uniquely contributed to our understanding of DC dimensions by describing protective buffering and overprotection as individual mechanisms that people tend to use to help their romantic partners cope with stressful situations, particularly medical conditions, but that they may end up having a negative impact. Similarly, another unique contribution lies in the identification of active engagement in helping a partner express his or her thoughts and feelings about a medical condition as a strategy to help a partner cope with a stressful health issue. By contrast, empathic responding, which is the other positive DC dimension described by the RFM, bears similarities with the STM construct of emotion-focused support provided by a partner to the other to help him or her cope with stress. Despite its unique contributions, the RFM does not include what partners do conjointly to cope with stress and acknowledge the role of contextual factors (e.g., culture) in shaping how couples cope with stress.

\section{The Communal Coping Model (CCM)}

In 1998 Lyons and colleagues introduced the term communal coping as occurring "when one or more individuals perceive a stressor as 'our' problem (a social appraisal) vs. 'my' or 'your' problem (an individualistic appraisal) and activate a process of shared collaborative coping" (p. 583). They viewed communal coping as a process happening in families and communities and that could have benefits for relationships and for the individual. According to the CCM there are three components involved in communal coping. First, at least one of the individuals in the relationship must have a communal coping orientation, that is, believe that conjoint coping is beneficial, necessary and/or expected to deal with a problem. Second, the process of communal coping requires communication about the stressor, that is, individuals must share the details and meaning of the situation. Third, individuals respond to the stressor with cooperative action, that is, they collaborate to develop strategies that reduce the negative impact of the situation and address the demands of the stressful situation.

Even though the CCM does not apply to couples coping only, various scholars have argued that it is a good model to understand couples coping with medical stressors. Lewis et al. (2006) have argued that couples' communal coping can lead to the adoption of risk-reducing health habits while Helgeson et al. (2017) have proposed that the CCM can be used to explain the "optimal pathway to patient adjustment among couples in which one person faces a chronic illness" (p. 1). Helgeson and colleagues emphasize that the primary goal of communal coping is to enhance not the relationship but the patient's adjustment to chronic illness. Unlike Lyons' formulation of communal coping, Helgeson and colleagues considered that shared illness appraisals may lead not only to collaboration but also to support interactions.

When communal coping has been studied in the context of couples' DC, it has focused mostly on coping with one partner's medical conditions and it was measured mostly through linguistic markers. Such studies (e.g., Rohrbaugh et al., 2012) have typically used the Linguistic Inquiry Word Count program (Pennebaker et al., 2007) to count partners'use of first-person plural pronouns in couple conversations (e.g., transcripts of marital interaction tasks or intervention sessions) such as we, us, or our, also referred to as we-talk. Some studies (e.g., Rohrbaugh et al., 2008) have also used two self-report questions, one asking the extent to which a partner views the other partner's medical condition as "our problem" and another question inquiring about the extent to which both partners work together to resolve that problem.

The CCM has emphasized the benefits of perceiving, communicating, and coping with a partner's medical condition not as an individual issue but as a couple's problem. This emphasis is also part of the other models such as the RCCM, the DCCM, and the STM that have also highlighted the benefits of such a communal approach to problems that have long been perceived and dealt with from an individual perspective. Similar to the RCCM, the CCM has to be credited for its examination of stress appraisals as communal. Unlike the DCCM and the STM, which have focused on measuring communal or collaborating coping strategies, the CCM has studied the extent to which couples appraise individual stressors such as a medical condition as a shared problem or "our" problem. Nonetheless, compared to other DC models, the CCM has had a narrower focus for its almost exclusive interest in medical problems or individual stressors. As it will be discussed later, models such as the STM include conjoint or collaborative coping as a strategy that couples may use to deal not just with individual stressors that may affect both partners but also with common or dyadic stressors. In addition, the CCM has not included other DC processes in the context of couples coping with stress such as when one partner offers emotion- or problem-focused support to a stressful partner and the stressor is not perceived as "our" problem.

\section{The Systemic-Transactional Model (STM)}

Unlike the RFM or the CM that originated in the study of couples where one partner had a serious medical condition, the STM (Bodenmann, 1995) focused on examining coping processes in couples dealing with daily hassles or minor chronic stressors. According to the STM, when partners experience stress, they resort to individual and dyadic coping strategies as well as seeking support outside the couple's relationship and "dyadic coping is used most often after individual coping efforts have been made and failed" (pp. 36-37). Similar to the CCM, the STM also includes stress communication as part of the dyadic coping process. According to the STM, each partner communicates his/her experience of stress to the other partner either verbally, non-verbally, and/or para-verbally and the other partner perceives, interprets, and decodes these signals and responds to the stressed partner with some form of coping "to maintain or restore a state of homeostasis as individuals, as a couple, and with regard to other people in the couple's social world" (Bodenmann, 2005, p. 36). The couple's coping process is seen as being affected by various factors such as context, type 
of stressor, degree of concern for both partners, attributions of causes of the stress, personal, motivational, and relational factors.

The STM is a comprehensive DC model as it involves various dimensions of positive and negative DC. Positive DC forms are viewed as benefiting both partners and their relationship and include supportive, delegated, and common DC. Supportive DC refers to one partner's attempts to assist the other partner in his/her coping efforts through problem-focused (e.g., giving advice or helping to find solutions) or emotion-focused strategies (e.g., showing understanding). Delegated DC involves efforts to help the partner reduce the stress by taking over some of his/her responsibilities. Common DC refers to coping strategies in which both partners participate more or less symmetrically or complementarily and can be either problem-focused (e.g., finding a solution together) or emotion-focused (e.g., emotionregulating together). Similar to CCM, common DC is likely to occur in situations that are affecting both partners and that are considered dyadic stressors or "we-experiences" (e.g., birth or death of a child, economic problems, child behavior problems, etc.), but unlike the CCM, it is also considered a coping strategy that may also happen in response to situations that may be initially related to one partner (e.g., job loss, disease) but is experienced as a situation affecting both partners and therefore as "we-stress" or "we-disease" (Bodenmann et al., 2016).

STM negative DC forms include hostile, ambivalent, and superficial efforts to assist the stressed partner. Hostile DC involves distancing, mocking, showing disinterest, or minimizing the seriousness of the situation. Ambivalent DC refers to offer support unwillingly or showing that support should not be necessary. Superficial DC refers to insincere efforts to support the stressed partner. Badr et al. (2010) have also added a negative form of common DC that is characterized by mutual avoidance or withdrawal.

The STM has been applied in studies of couples coping with a medical illness such as cancer (see Traa et al., 2015 for a systematic review) or COPD (Meier et al., 2012), but also with other non-medical stressors such as depression (e.g., Bodenmann et al., 2001), post-traumatic stress disorder (Witkovsky and Braakmann, 2015), immigration issues (Falconier et al., 2013a), coping with the death of a child (Bergstraesser et al., 2015), or even general stress (Rusu et al., 2016). All STM studies used the instrument developed by Bodenmann (2008) to assess DC: the Dyadic Coping Inventory (DCI). This scale was initially made up of 55 items but it has been further developed into the most common 37-item version. The DCI has been used in at least 35 countries (Hilpert et al., 2016), and validated for over 10 different cultural groups (for a review see Falconier et al., 2015). A standardized coding scheme based on the STM concepts has also been developed for observations of couples' conversations (Bodenmann, 2000).

Even though the STM did not explicitly incorporate any cultural factors in its original formulations, it did acknowledge that contextual factors could affect the DC process. Furthermore, more recently Falconier et al. (2016a) included culture into the STM as a powerful contextual factor that may affect "whether and to what extent situations are considered stressful, and whether the stressor is viewed as concerning only one partner (individual stressor) or both partners (common stressor)" (p. 28). Cultural factors "may affect the extent to which couples prefer dyadic coping over other coping strategies, the potential benefits of dyadic coping over other coping mechanisms" and shape "the specific factors that favor dyadic coping, and the preference for relying more on some dyadic coping dimensions over others" (Falconier et al., 2016b, p. 304). Falconier et al. (2016b) identified the culture's communication style and individualistic vs. collectivistic orientation as factors that may influence stress appraisal and coping responses.

The STM is the model that has guided most of the research in the DC field (Falconier et al., 2016a). This may be due to the fact that the STM is the model that includes most DC dimensions. Whereas, the RCFM has focused on what one partner does to assist the other partner cope with his or her stress and the CCM has been concerned with the appraisal of the stressor as a "we" problem and collaborative coping strategies, the STM has provided a broadened framework in which stress appraisal ("our" problem vs. "your" or "my" problem" is included and both, collaborative and individual mechanisms for assisting one partner to cope with stress or for partners coping with stress together are present. In addition to its comprehensiveness, the STM has been the only one to emphasize the stress communication process as a DC dimension.

Nonetheless, despite its comprehensiveness and broad appeal, STM studies have relied mostly on the DCI, which measures only coping strategies and not stress appraisal. In contrast to the CCM, the STM has not produced studies examining its conceptualizations on stress appraisal and its link to coping strategies. The STM does not include either particular forms of partners' negative or positive support that have been identified in other DC models such as overprotection, protective buffering, or active engagement.

\section{The Relational-Cultural Coping Model (RCCM)}

In applying the STM to the study of couples' coping with cancer, Kayser and Revenson (2016) focused not only on couples' coping strategies but also on the factors that shaped those behaviors. As a result, they developed the relational-cultural coping model (RCCM) which expanded the STM by adding relational and cultural components. First, in terms of relational aspects, Kayser and colleagues found experiencing cancer as "we-stress" or as an individual stressor affecting each partner individually determined whether the couple displayed mutual responsiveness or disengaged avoidance. Similar to communal coping and the STM common DC, mutual responsiveness, which was associated with experiencing cancer as "we-stress," referred to coping in which partners communicated about the stress and handled the situation in a coordinated way with both emotionand problem-focused responses, whereas disengaged avoidant couples, associated with experiencing cancer as an individual problem, described a response in which partners avoided talking to each other and just focused on the practical aspects of coping with the illness. They identified three key relationship factors contributing to mutual responsiveness: relationship awareness, 
authenticity, and mutuality (Kayser et al., 2007). Relationship awareness refers to "thinking about the impact of the disease on each partner and the relationship and how to sustain one's relationship given the extra demands of the illness" (p. 415). Authenticity involves "the disclosing of genuine feelings and not hiding them" (p. 416), whereas mutuality refers to "empathy as a way of relating in which each of the partners is participating as fully as possible in a shared experience" (p. 416).

Regarding the cultural component, Kayser et al. (2007) first expanded the STM by acknowledging the role of culture in shaping the way in which couples adapt to stressful situations, but later on, after conducting a study with American, Chinese, and Indian couples (2014), they proposed four specific cultural dimensions that could influence coping: family boundaries (from open to closed), gender roles (from differentiated to flexible), personal control (from acceptance to mastery), and independence (from dependence to independence). Where couples lie on the continuum of each of these dimensions is likely to affect the way in which they cope with cancer and stress in general. The RCCM was developed from qualitative studies and no instrument has been developed to measure its constructs.

The RCCM's identification of mutual responsiveness as involving both "we-ness" stress appraisals and collaborative coping responses and as beneficial in couples' coping with medical conditions is aligned with STM, and particularly, CMM conceptualizations. However, RCCM's identification of key relationship factors that contribute to either mutual responsiveness or disengaged avoidance is a unique contribution. In addition and most importantly, unlike STM and DCCM, the RCCM is the first DC model that attempted to identify through research the cultural aspects that affect stress and coping processes in the couple's context. Furthermore, it is the only model that has produced a study that did not only include a multicultural sample but it actually focused on examining cultural factors to explain differences in that diverse samples. Given these contributions, the RCCM can be characterized as a DC model that focused more on the factors that shape the stress appraisal and coping process rather than on the actual stress and coping mechanisms in couples. Nonetheless, despite its contributions, many of the RCCM concepts still need to be operationalized into measurable constructs that can be used in research. Also, considering its focus on medical conditions, it is unknown whether the cultural and relational factors identified by the RCCM as shaping the stress and coping process apply to other stressful situations.

\section{The Developmental-Contextual Coping Model (DCCM)}

The DCCM was developed by Berg and Upchurch (2007) to understand the process through which couples cope with chronic illness. Similar to the STM, the CCM, and the RCCM, the DCCM highlights the importance of the stress appraisal process that comes prior to the actual coping strategies. Appraisals can be made about the illness controllability and the illness ownership. Similar to other DC models, stress can be perceived as individual (one's own stress), indirectly (through my partner's stress experience), or shared (both partners appraise the stressor as a common one), similar to the "we-appraisal" described by the STM, the CCM, and the RCCM. However, the DCCM also acknowledges that the coping strategy also affects the appraisal processes (e.g., collaborating as one coping strategy activates the belief that the stressor is appraised as a joint stressor).

Unlike other DC models, the DCCM does not focus on stress communication but on the partner's responses, which are viewed on a continuum that spans from under-involvement to over-involvement. The DCCM also argues that DC dimensions identified in other models are one of the four coping strategies on that continuum: uninvolved, supportive, collaborative, and controlling. Uninvolved coping refers to the perception that one's partner is providing no support to help the other cope with stress, whereas supportive coping refers to the perception that the partner is providing such support either emotionally and/or instrumentally. Similar to the STM common DC, the CMM, and the RCCM mutual responsiveness, collaborative coping describes both partners' actions to cope with the stressful situation together. Controlling coping describes moments in which the non-stressed partner "dominates the actions of the other spouse by taking charge and telling the other person what to do" (Berg and Upchurch, 2007, pp. 932-933) and is associated with the protective buffering and overprotection strategies identified in the RFM.

Unlike other models, as the name suggests, the DCCM emphasizes the role of developmental and contextual factors in the appraisal of the stressor and coping responses. In terms of developmental aspects the DCCM argues that coping with an illness varies over time depending on the stage of illness and the life cycle stage. Regarding contextual factors, the DCCM views cultural differences, gender differences, the quality of the couple relationship, and the illness type as affecting stress appraisal and partners' coping responses. Interactions between different factors are taken into consideration so that, for example, different cultural groups experience different diseases at different rates.

The DCCM studies have measured coping through diaries (Berg et al., 2008), the Perceptions of Collaboration Questionnaire (PCQ; Berg et al., 2008), and structured stress and coping interviews (Berg et al., 2008). Even though the model includes developmental and contextual aspects, the DCCM studies have focused only on coping dimensions and demographic variables such as age, gender, and length of relationship.

Compared to other models and similar to the STM, the DCCM offers a more comprehensive framework by including stress appraisal, a partner's both positive and negative responses to the stressed partner, partners' collaborative coping efforts, and contextual factors that can affect stress appraisal and coping processes with medical conditions. However, the model's unique contribution is its proposal that the couple's coping strategies may vary depending on the stage of the illness. In this regard, it is the only DC model to suggest that couples may cope differently over time, even when dealing with the same stressor. This dynamic view of coping seems to be absent in other approaches or studies. Despite these contributions, the DCCM does not include all the DC dimensions identified in other models and that could 
be relevant to cope with non-medical stressors (e.g., the STM's negative DC).

\section{Model Integration}

When looking at the coping process in the context of couple's relationships, there have been two different approaches. One approach, exemplified in the $\mathrm{CM}$, continues with the tradition of examining the individual coping responses to one's stress, but it brings attention to the relational context by (a) focusing on whether partners' individual coping responses are mutually supportive and (b) whether the interplay of such strategies is beneficial for each partner and their relationship. The other approach, which is represented in the other models and could be viewed as a more dyadic conceptualization, moves beyond individual strategies for coping with one's own stress to focus on what partners do or don't do for each other and together to handle stress. These models typically assume that that when one partner experiences stress, so does the other partner due to the common nature of the stressor or to crossover effects. As discussed in the description of those DC models (the RFM, the CCM, the STM, the RCCM, and the DCCM), each of them seemed to have examined different or overlapping dimensions of the same DC phenomenon and therefore, they could be integrated into a comprehensive DC model. Some of this integration has been attempted before (e.g., Falconier et al., 2015) but, as noted earlier, it has left out some of the DC models or specific dimensions highlighted by each model. After examining conceptual overlaps and differences in the previous sections, our integrated view proposes that the DC process involves partners' communication about their stress and their responses which can be positive or negative and include individual responses to a partner's stress when the stressor is viewed as concerning one partner (individual-oriented appraisal) or conjoint responses when the stressor concerns both partners or an individual stressor is viewed as a "we" or shared problem ("we" oriented appraisal; see Figure 1). Therefore, the integration includes: (a) Stress Communication to refer to communication of the experience of stress between partners, (b) Individual Positive $D C$ to refer to one partner's positive responses to help the other cope with stress (supportive DC, empathic responding, delegated DC, active engagement), (c) Positive Conjoint DC to refer to what partners do together to cope with shared or dyadic stress (common, collaborative, communal DC, mutual responsiveness); (d) Negative Individual $D C$ to refer to one partner's negative responses to the other partner's stress (e.g., protective buffering, overprotection, hostile/ambivalent DC, and controlling DC), and (e) Negative Conjoint $D C$ to refer to partners' conjoint negative response to deal with a shared or dyadic stress (common negative DC, disengaged avoidance). Similarly to DCCM formulations, developmental, relational, and contextual variables are included in the model as factors that can shape the stress and coping process. The inclusion of the developmental factors indicate that changes in stress appraisal and use of DC strategies may change over time due to the changes or development of the stressful situation. In other words, changes in the stressor may lead to the adoption of different coping mechanisms. Similarly, DC strategies that may have been adopted to first cope with a stressor may be changed for others after some time. For example, a partner may initially respond to her husband's extended family problems with supportive DC but over time she may appraise the situation as a "we" problem and engage in positive conjoint DC. Relationship variables are those characteristics of the relationship that influence the stress appraisal and coping process. The RCCM has already proposed some relationship characteristics such as relationship awareness, authenticity, and mutuality that increase the likelihood that partners will appraise problems as shared and will engage in collaborative forms of coping. It is also possible that other relationship characteristics such as level of intimacy, satisfaction, and ability to resolve conflict constructively also affect the stress appraisal and coping process. Contextual factors refer to socio-economic conditions that may affect the availability of resources (e.g., unemployment, income level), cultural values (e.g., collectivistic vs. individualistic), and/or religious beliefs that may affect stress appraisal and coping in couples.

It is important to note that it is only when a review and analysis of all DC models is made that the uniqueness of the DC concept is fully understood and differentiated from other constructs such as partner's social support. Unlike DC, which is one partner's or both partners' response to the stress experienced by one or both partners, social support by a partner is not necessarily provided to assist the partner cope with stress. Social support, which can be informational (e.g., recommendations, advice, helpful information), instrumental (e.g., financial, material, or physical assistance), emotional (e.g., expression of affection, caring), and/or companion (availability of partner) can also occur in the absence of a stressor (Kent de Grey et al., 2018).

\section{METHODOLOGY FOR THE REVIEW OF THE EMPIRICAL LITERATURE}

\section{Literature Search}

In order to conduct the review of all the empirical literature guided by the DC models discussed above we conducted the search in the Psychology and Behavioral Sciences Collection from EBSCOhost and in Psych-INFO in 2017 and 2018. We used the following inclusion criteria for selecting studies: (a) be published in or before 2017, (b) include an original empirical study guided by one of the DC models identified in the present review, and (c) be published in a peer-reviewed journal in English and/or German in order to guarantee the scientific merit. All DC models but the STM were developed in English speaking countries, whereas the STM was developed in Switzerland. Therefore, we included journal articles both in English and German to increase the likelihood of including as many studies as possible for each DC model. In addition, the search included terms related to the models' names and constructs such as "stress," "couple," "relationship," and "intimate."

\section{Study Selection}

Studies were selected when they mentioned and based themselves explicitly on the specific model or when they used one of the model-related questionnaires developed by the authors of the models. Articles were excluded, for example, if they focused on 
Dyadic Coping Responses

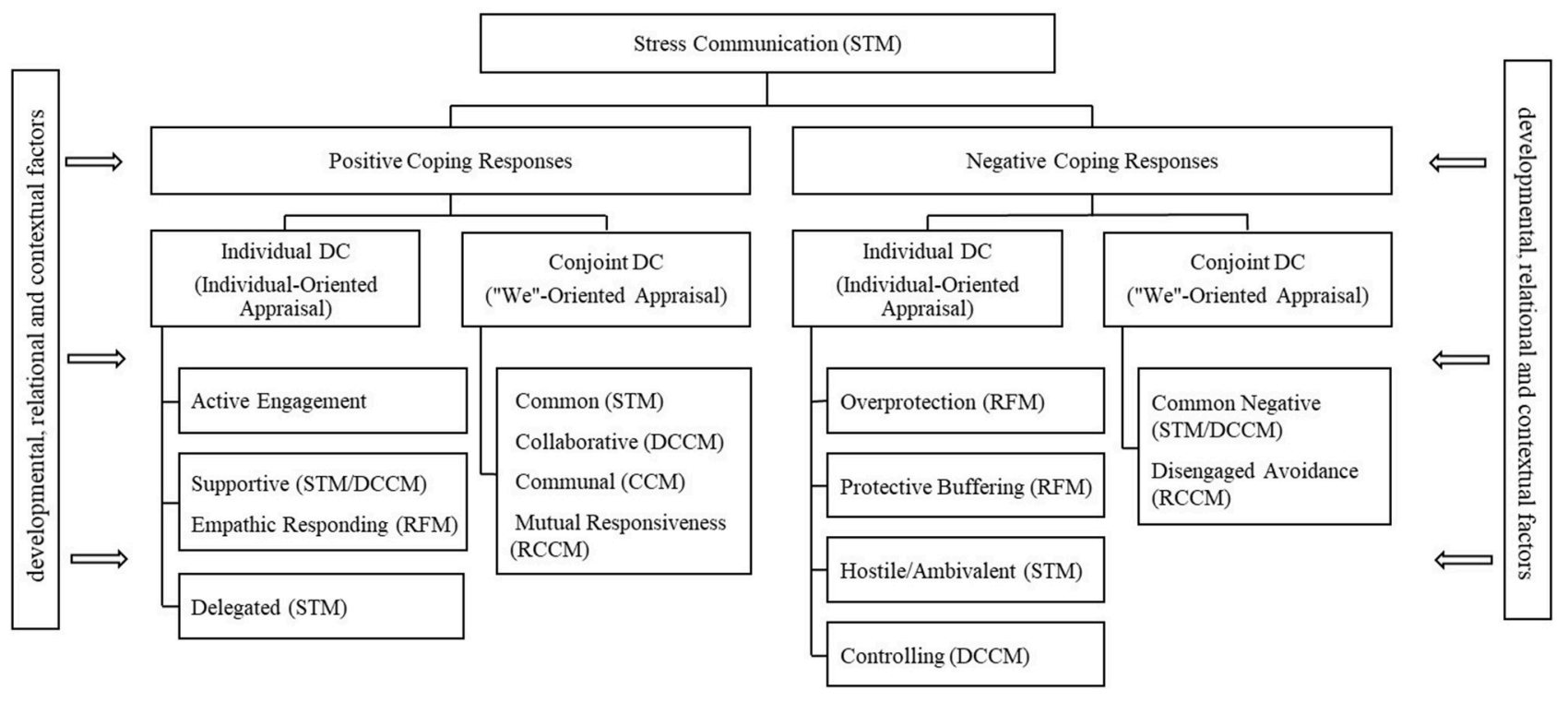

FIGURE 1 | Integration model chart.

relationship-internal conflicts instead of a relation-ship external stressor, or if they did not include any coping efforts by any partner.

The initial search through these databases yielded 1,601 results and 63 more articles were added after inspecting reference lists of included articles or because the model developers, when consulted, identified additional articles that our database search had failed to identify (see Figure 2). We removed 317 duplicate articles and screened 1,347 records in a two-step process (abstract screening, full-text screening).

The coding team included the present authors, graduate assistants, and alumni from two different universities. Coders read abstracts of all 1,347 articles and eliminated 953 records not meeting eligibility criteria. We read 394 records in full and 255 articles were further excluded for not meeting eligibility criteria (e.g., examination of only one partner's individual coping strategies, not being an empirical article, not applying a DC model, or not focusing on stress). When in doubt or disagreement, coders consulted with the rest of the coding team until an agreement was reached. To ensure accuracy, both coders read and agreed on $43 \%$ of the final articles. The current review ended up including 132 quantitative studies and 7 qualitative studies (see Figure 2 and summary of studies table available online as Supplementary Material). In total, over 37,000 couples and individuals participated in the different studies (range: 10-7,973 individual and couples). Studies were mainly cross-sectional (66\%: 92 out of 139) and 47 were longitudinal. In addition, seven studies reported an experimental design (e.g., stress tests with experimental groups).

\section{Data Extraction}

Each study was entered into a database identifying the authors, title, sample, DC model, DC measure, non-DC measures, study design, and main findings. Articles were classified into the different DC models either because they made explicit the model that guided their research or because they used DC constructs or measurements developed after the DC models. The classification resulted in the following number of articles for each model: CM: 10; RFM: 34; STM: 78; CCM: 7; RCCM: 2; DCCM: 8 (see summary of studies in Supplemental Material).

\section{FINDINGS FROM THE EMPIRICAL LITERATURE}

The review of the empirical literature has been organized into two parts. The first part presents findings from studies examining the interplay of partners' individual coping styles, and therefore mostly related to the CM. The second part discusses findings from the rest of the studies on DC that met the inclusion criteria for the present review. Findings in this second part are presented for DC as an overall construct first. Then, with the exception of negative conjoint responses and controlling DC for which no studies were found, for each of the DC dimensions outlined in the integrative model: Stress communication, individual (active engagement, supportive DC, empathic responding, and delegated DC), and conjoint (common $\mathrm{DC} /$ collaborative $\mathrm{DC} /$ communal $\mathrm{DC} /$ mutual responsiveness) positive coping responses, and individual (overprotection, protective buffering, and hostile/ambivalent DC) negative coping responses. In this way studies guided by different DC models 


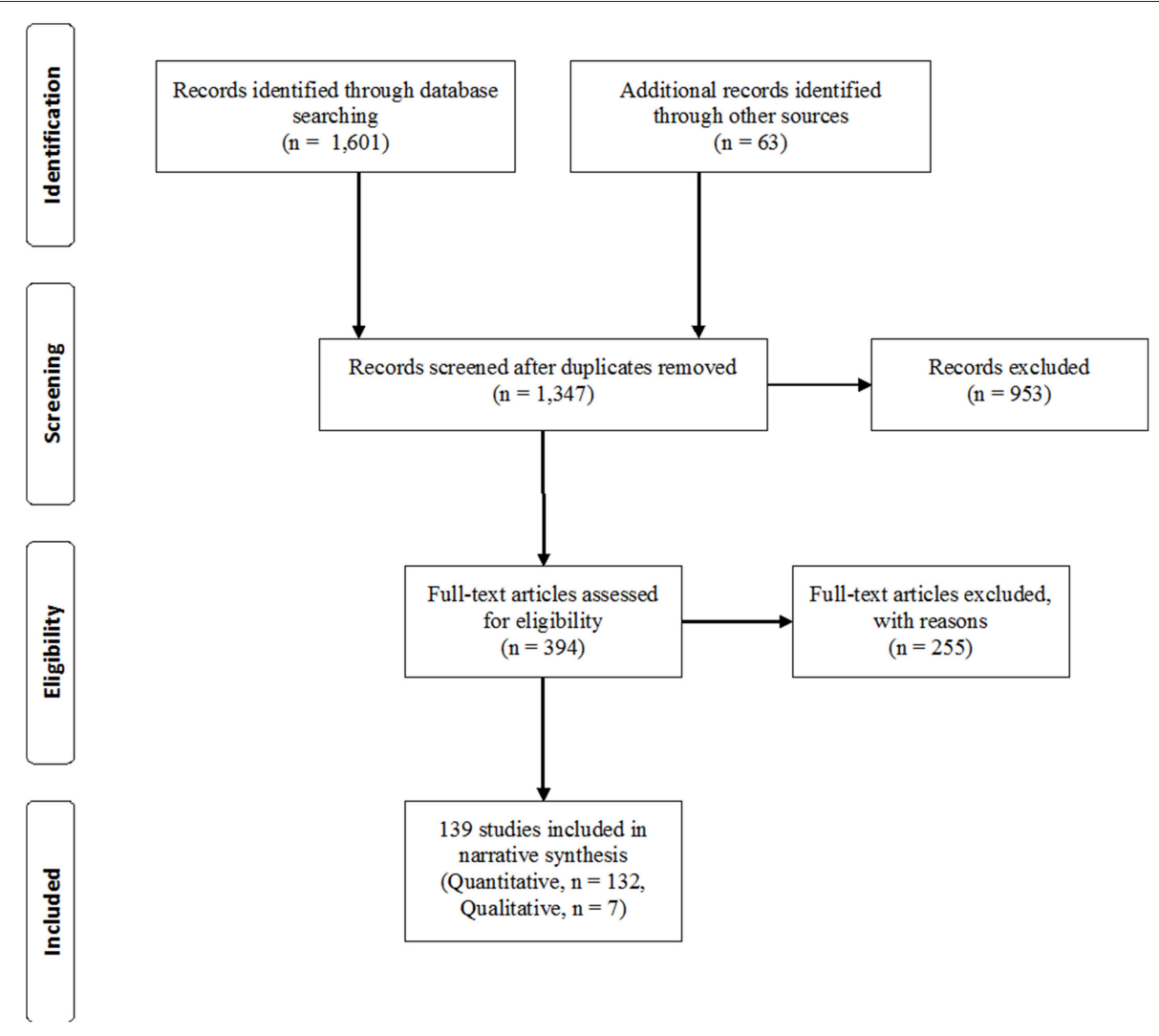

FIGURE 2 | Prisma flowchart.

but that tap on the same DC dimension can be presented together. For example, findings on STM's common DC, DCCM's collaborative DC, and CCM's communal coping can be discussed together, obtaining a full picture on the accumulated knowledge in the field regarding couples' conjoint strategies to cope with stress. In addition, findings on couples coping with medical or mental health stressors have been separated from those on couples coping with other types of stressors, referred to as nonmedical stressors. The review also includes a final section that discusses the demographic and contextual/cultural differences identified for overall DC and each dimension.

\section{CONGRUENCE BETWEEN PARTNERS' INDIVIDUAL COPING}

Except for two studies (Cronkite and Moos, 1984; Bodenmann et al., 2011), research on similarity between partners' individual coping strategies have been all related to medical stressors. Overall, findings suggest that positive individual and relational outcomes in stressful situations are not necessarily the result of similarity between partners' individual coping strategies. For example, partners' similarity in emotional and problem-focused coping helped women with non-metastatic cancer adapt 10 months later, but it was dissimilarity in emotional coping that predicted women to be happier with their couple's relationship (Kraemer et al., 2011). Israeli partners' similarity in monitoring as an information seeking style predicted better adjustment in women with cancer; however, similarity in blunting as an information seeking style predicted better adjustment in men with cancer but predicted psychological distress in their caregivers (Barnoy et al., 2006). Similarly, a study on Australian couples with MS (Pakenham, 1998) found dissimilarity in problem-focused coping to be associated with lower collective depression and better individual adjustment in both partners. In a study of parents of children diagnosed with cancer, however, similarity in emotion-focused coping helped parents be more optimistic, but it was the complementarity in problemfocused coping that predicted better marital quality and support (Barbarin et al., 1985).

Even when similar stressors are considered, results have not been consistent for similarity in coping styles. On the one hand, similarity in emotion-focused coping predicted better adjustment in women with breast cancer in Kraemer et al. study (2011) but it did not in a study by Ben-Zur et al. (2001) in which women with breast cancer reported more psychological distress and poorer functioning when both partners relied on emotion-focused coping. Nonetheless, this study also showed that complementarity in emotion-focused coping and that women's avoidance and men's preference for problem solving 
also predicted women's depression. Similarly, women's avoidance and men's problem-focused had been found in an earlier study to be associated with women's depression and men's physical symptoms in a sample of urban couples (Cronkite and Moos, 1984). However, in that study men were also more depressed when both partners used avoidance coping strategies. But again, by contrast, two American studies (Giunta and Compas, 1993; Fagundes et al., 2012) found that similarity in avoidance did not predict negative affect or psychological distress.

In short, studies on similarities between partners' individual coping styles offer inconsistent findings, even when focusing the same stressor. No socio-demographic, developmental, or cultural factors explained the different results either, all of which lends support to the idea that partners' individual coping styles should be examined in terms of the extent to which each partner's style supports the other partner's instead of blocking them or even creating another source of stress.

\section{OVERALL DC (STM)}

Several studies within the STM framework have examined DC overall as the aggregation of all or some of the DC dimensions. Furthermore, some of these studies have specifically focused on overall positive DC, excluding negative DC forms.

\section{Coping With Non-medical Stressors}

At the individual level DC has been related to positive individual forms of coping (e.g., Bodenmann et al., 2010a); less neuroticism (Merz et al., 2014), more daily physical activity in women (Reed et al., 2016), higher life satisfaction (Gabriel et al., 2016), and lower anxiety, insomnia, social dysfunction, and depression in women (Bodenmann et al., 2011). Furthermore, in experimentally induced stress conditions, DC was found to reduce stress levels (Meuwly et al., 2012) and low DC was associated with immune reactivity (Reed et al., 2016).

In terms of benefits for the relationship, DC has been associated with tenderness and togetherness (Bodenmann et al., 2006), higher sexual satisfaction, sexual behaviors, and orgasms in women (Bodenmann et al., 2010a), and relationship satisfaction and constructive communication in Western including American couples (e.g., Randall et al., 2015), Latino (e.g., Falconier et al., 2013b), and European couples (e.g., Vedes et al., 2013; Zeidner et al., 2013). Longitudinal studies in Swiss couples have found that DC predicts men's relationship satisfaction 10 years later (Ruffieux et al., 2014) and that couples maintain their relationship satisfaction over a 5 yearperiod if they are highly involved in DC, but their relationship satisfaction declines if they are not high on DC (Bodenmann, 2000). Some findings even suggest that DC is a better predictor of relationship satisfaction than individual coping (Papp and Witt, 2010) and may be beneficial above and beyond positive communication (Nussbeck et al., 2012). According to a large study across 35 different nations that included not only Western but also African and Asian countries, the extent to which one partner perceives the other as providing DC plays a more important role in predicting relationship satisfaction than the actual extent to which one partner reports engaging in DC
(Hilpert et al., 2016). Furthermore, perceived similarity in DC between partners matters more for relationship satisfaction than the actual similarity (e.g., Iafrate et al., 2012). DC can also serve as a predictor for relationship stability. After 5 years, couples could be correctly classified in $73 \%$ of the cases regarding whether they would separate or stay together according to their level of DC (Bodenmann and Cina, 2005). In addition, DC has been found to attenuate the negative impact of chronic external stress on chronic internal stress (spillover), particularly for women (Merz et al., 2014), and relationship stability (e.g., Bodenmann and Cina, 1999). Positive DC has also been found to moderate the effects of stress on verbal aggression and anger (Bodenmann et al., 2010b).

Some factors affect partners' likelihood to become involved in DC. Stressors external to the couple's relationship decreases partners' use of DC strategies (e.g., Gabriel and Bodenmann, 2006a) but dyadic empathy (Levesque et al., 2014b) and men's emotional intelligence are associated with higher DC. Additionally, men's perspective taking predicts women's DC and women's empathic concern can predict men's DC (Levesque et al., 2014a). Couples with higher relationship-focused standards (Wunderer and Schneewind, 2008), a passionate love style (Gagliardi et al., 2015), functional types of couples (validating, volatile, and conflict avoidant) (Bodenmann et al., 2004), and securely attached couples (Gagliardi et al., 2013) rely more on DC. Rational love styles predict more positive DC only in women in in Swiss and German couples (Gagliardi et al., 2015). DC has also been shown to be beneficial for other family members. Zemp et al. (2016) found that DC predicted lower internalizing and externalizing symptoms and higher prosocial behavior in children, with particularly stable effects for externalizing behavior.

\section{Coping With Medical and Mental Health Conditions}

All studies in Western populations have found an association between DC and positive individual indicators in both patients and their partners in couples coping with a medical or mental health condition. DC has been linked to physical well-being in women with breast cancer (Feldman and Broussard, 2006) and less psychological distress and higher quality of life in European couples coping with COPD (Meier et al., 2011; Vaske et al., 2015).

Similarly, studies on relational outcomes have consistently suggested benefits of DC. In Western couples DC has been associated with increased relationship satisfaction in parents raising Autistic children (Gouin et al., 2016) and better partner acceptance and relationship satisfaction in women with breast cancer (Zimmermann et al., 2010). When a partner is diagnosed with PTSD, low discrepancies between partners' DC also predict better relationship satisfaction regardless of the severity level of the PTSD (Witkovsky and Braakmann, 2015). Again, overall DC has been found to have positive effects on other family members beyond the partners. Parents' DC has been linked to better health outcomes in children with type 1 diabetes in German families (Körner et al., 2013). Factors decreasing Western couples' use of 
DC strategies include traumatic events, depression, anxiety, and COPD (e.g., Gabriel et al., 2016).

\section{STRESS COMMUNICATION (STM)}

Despite the fact that several DC models include stress communication as important aspect of the DC process, it is mostly the STM that has guided the study of this DC dimension. This may be due to the fact that, first, it is explicitly included in the STM conceptualization of DC and second, the DCI, the STM based self-report instrument, specifically includes items to assess this dimension.

\section{Coping With Non-medical Stressors}

Stress communication has consistently been found to benefit couple relationships, as it is associated with increased likelihood of both male and female partners providing support (e.g., Bodenmann et al., 2015) and better relationship satisfaction in Japanese (Yokotani and Kurosawa, 2015), Latino (Falconier et al., 2013b), and Western European and American couples (e.g., Ledermann et al., 2010; Levesque et al., 2014a). It has also been related to constructive communication in European (e.g., Ledermann et al., 2010) and Latino (Falconier et al., 2013b) couples. Additionally, stress communication is associated with positive individual coping in both men and women (e.g., Falconier et al., 2013b). A micro-analytic longitudinal study also showed that the type of stress communication is directly linked with the subsequent coping reaction even in small time frames (Kuhn et al., 2017). It has also been found that unhappy couples seem to rely more on factual stress communication and less on emotional exchanges (Bodenmann and Perrez, 1991).

\section{Coping With Medical and Mental Health Conditions}

Studies with Western couples in which one partner suffers from depression (Bodenmann et al., 2004) or cancer (e.g., Weißflog et al., 2016) have indicated that patients tend to communicate about their stress less frequently than their partners do. It is possible that depressed patients might suffer from a lack of energy, generally employ maladaptive coping strategies (Kovacs and Beck, 1978), and thus experience a decline in their communication competences (Hoffmann et al., 2016), whereas patients with cancer might consciously hold back information that would make their partner worry. Nonetheless, stress communication with medical conditions has been found to have a positive effect individually, improving COPD patients' quality of life (Vaske et al., 2015) and to trigger the provision of support by the healthy partner (e.g., Badr et al., 2010).

\section{INDIVIDUAL POSITIVE DC: DELEGATED DC (STM)}

Delegated DC, one of the positive ways to help a partner cope with stress, has been included only in STM studies as it is part of its conceptual model and its measurement instrument, the DCI.
Compared to other DC dimensions there are fewer studies that specifically focus on delegated DC.

\section{Coping With Non-medical Stressors}

Studies on couples coping with stress in general show that providing delegated DC is positively associated with individual positive coping strategies for both men and women in Latino (Falconier et al., 2013b) and Romanian couples (Rusu et al., 2016). Delegated DC is also linked to constructive conflict resolution and relationship satisfaction for Latino (e.g., Falconier et al., 2013b) and Western European couples (e.g., Vedes et al., 2013), and exclusively to relationship satisfaction for Canadian and American couples (Randall et al., 2015) and Japanese men (Yokotani and Kurosawa, 2015). Nonetheless, when compared with other DC dimensions delegated DC is less strongly linked to marital communication (Ledermann et al., 2010) and relationship satisfaction (for a review see Falconier et al., 2015).

\section{Coping With Medical and Mental Health Conditions}

Delegated coping is often studied in the context of physical or psychological conditions, probably because in the context of chronic illness it is expected that one of the ways in which partners can support the ill partner is by taking over some of their tasks. Logically, in the context of illness, it would be expected for the non-ill partner to provide more delegated DC than the sick partner. For example, COPD or cancer patients report engaging in delegated DC less frequently than their partners do (e.g., Meier et al., 2012). However, this imbalance may not be necessarily beneficial as patients with COPD report a lower quality of life when there is a higher imbalance in partners' delegated DC (Meier et al., 2011). Furthermore, another study on Danish couples coping with breast cancer found that while providing delegated DC to the patient lowers the partner's depressive symptoms, patients tend to report more depressive symptoms when they provide more delegated DC to their partner (Rottmann et al., 2015). These findings suggest that in couples coping with illness imbalance in delegated DC between partners might be beneficial but only to a certain extent.

\section{INDIVIDUAL POSITIVE DC: EMPATHIC RESPONDING (RFM)}

Empathic responding is part of the RFM and is one of the positive ways in which an individual may help a romantic partner cope with stress. Unfortunately, only a few studies have examined this DC dimension. Additionally, studies on empathic responding have measured this construct without discriminating between cognitive/affective and behavioral strategies and therefore, it is not possible to report on the effects of each set of responses.

\section{Coping With Non-medical Stressors}

Only one study examined empathic responding when coping with stress in general. This study (O'Brien et al., 2009) investigated Canadian stepfamilies using a daily dairy methodology and found that both partners perceived lower 
marital tension on the days following the use of empathic responding. However, husbands' use of empathic responding was associated with increased perception of same-day marital tension while the opposite was true for wives, suggesting gender differences in the use of empathic responding.

\section{Coping With Medical or Mental Health Conditions}

Three studies have examined empathic responding in the context of medical or mental health conditions. The first examined couples coping with the male partner's Alzheimer's disease (Kramer, 1993) and found that partners' empathic responding was related to higher satisfaction in women's caregiving. A second study was a cross-sectional examination of Canadian couples with children with disabilities (Marin et al., 2007), which found that empathic responding is not linked to psychological well-being unless the individual perceives that his or her empathic responding is not reciprocated by the partner, in which case it is associated with psychological distress. The third study (Lee-Baggley et al., 2005) indicated that the individual's conscientiousness, openness, and extraversion contributed to empathic responding in couples coping with child misbehavior while the opposite was true for agreeableness and there was no link to neuroticism.

\section{INDIVIDUAL POSITIVE DC: ACTIVE ENGAGEMENT (RFM)}

Active engagement, which is a positive way to assist a stressed partner, is an RFM concept that was developed in the context of couples coping with an illness. As a result, active engagement has been examined mostly in that context.

\section{Coping With Non-medical Stressors}

Only one study has examined active engagements as a DC strategy for couples to manage stress in general. In that study Kurosawa et al. (2015) found that in Japanese couples with pre-school children active engagement was linked with higher relationship satisfaction.

\section{Coping With Medical and Mental Health Conditions}

Most of the studies on active engagement have been conducted in relation to cancer in the Netherlands (e.g., Kuijer et al., 2000; Hinnen et al., 2009). Other medical conditions studied in relation to active engagement in couples have included TypeII diabetes in American couples (e.g., Schokker et al., 2010), heart problems in Israeli (Vilchinsky et al., 2011), and Dutch couples (Joekes et al., 2007). Across these various medical conditions several studies have found active engagement to have positive effects on the couple's relationship and either no effect or a positive effect on the individual. When partners become actively engaged, both patient and partner report better relationship satisfaction (e.g., Schokker et al., 2010), better individual coping with the illness, lower distress, higher selfefficacy, better health-related quality of life (Coyne and Smith,
1991, 1994; Kuijer et al., 2000; Joekes et al., 2007), and decreased smoking (Vilchinsky et al., 2011). Partners' active engagement has also been found to moderate the negative association between protective buffering and relationship satisfaction (Schokker et al., 2010) in patients with diabetes. Additionally, partners seem to use active engagement more than patients do (Lavery and Clarke, 1999). However, when both patients and partners use active engagement, they report better marital adjustment (Badr, 2004).

Only two studies found active engagement to be unrelated to individual outcomes. Hinnen et al. (2009) reported that partner's active engagement was not associated with cancer patients' distress, regardless of their perceptions of received support or their feelings of mastery. Similarly, Sormanti et al. (1997) found that partner's active engagement was unrelated to quality of life, depression, or health care behavior. Among factors affecting active engagement negativity about the prognosis in cancer patients was found to increase it (Kuijer et al., 2000).

\section{INDIVIDUAL POSITIVE DC: SUPPORTIVE DC (STM-DCCM)}

Supportive DC is one of the positive ways in which an individual experiencing stress can be helped by a romantic partner. It has been conceptualized within the STM and thus measured with the DCI (Bodenmann, 2008). However, DCCM includes a dimension of partner's supportiveness that is consistent with the STM's definition of supportive DC and has been mostly applied in the study of couples coping with chronic illness.

\section{Coping With Non-medical Stressors}

Studies suggest that individuals who provide emotion- and problem-focused support to a stressed partner are also more likely to use positive individual coping strategies (e.g., Randall et al., 2015) and report increased well-being (Rusu et al., 2015). In terms of couple benefits, supportive DC is linked to relationship satisfaction in Latino (Falconier et al., 2013b), European (e.g., Ledermann et al., 2010), American (Randall et al., 2015), and Canadian couples (Levesque et al., 2014a) and in Japanese husbands (Yokotani and Kurosawa, 2015). Interestingly, for one partner's relationship satisfaction, the subjective perception of how much supportive DC the partner provides seems more important than how much the supporting partners themselves indicate providing. A partner could thus provide only little support, yet, the relationship satisfaction is rather linked to what the receiving partner thinks he or she is receiving (Landis et al., 2013). Additionally, in Western couples supportive DC is related to sexuality, romance and passion, constructive conflict resolution and communication, shared meaning (e.g., Ledermann et al., 2010; Vedes et al., 2013), and relationship stability (Bodenmann and Cina, 2005). Men's supportive DC has also been found to buffer the negative effects of the female partner's immigration stress on relationship satisfaction in Latino couples living in the U.S (Falconier et al., 2013a). Regarding the developmental course of supportive DC, in a study on German couples Johnson and Horne (2016) found that supportive DC predicted significantly future commitment and willingness to 
sacrifice within 5 years, but not the other way around, indicating that supportive DC enhances relationship functioning. However, the same study (Johnson et al., 2016), found a constant decline in supportive DC over time. In young couples, however, male's more rapid decline in supportive DC was associated with a slower decline in women's supportive DC.

In terms of factors that affect providing supportive DC, a spiritual orientation favors the use of supportive DC in Latino couples (Austin and Falconier, 2013) while a traditional gender role orientation in men has the opposite effect (Falconier, 2013). Economic pressure has also been found to reduce couples' use of supportive DC over time (Johnson et al., 2016). Severe depression decreases the use of supportive DC in Swiss couples (Bodenmann et al., 2004).

\section{Coping With Medical and Mental Health Conditions}

Studies have shown both positive and negative effects of supportive DC on the individual. Supportive DC has been linked to less distress in breast cancer patients and their partners (Badr et al., 2010) and individual positive self-verbalization as well as problem-solving in couples with a currently or formerly depressed partner (Bodenmann et al., 2004). In Dutch couples with colorectal cancer, perceived spousal supportive behavior has been a negative predictor of distress over time but only for patients low in perceived personal control; couples with a high sense of personal control reported lower levels of distress 6 months later, regardless of partner support (Dagan et al., 2011). However, one study found that receiving supportive DC could increase depressive symptoms in women with breast cancer (Rottmann et al., 2015).

At a relational level, supportive DC has been associated with relationship satisfaction in Spanish couples with an autistic child (García-López et al., 2016) and in American couples coping with cancer (Checton et al., 2015). A study on American civilian women and their combat veteran partners also found that the negative association between the veteran's post-traumatic stress and their female partner's relationship satisfaction could be buffered the higher women indicated their partner's supportive DC (Lambert et al., 2015).

\section{CONJOINT DC: COLLABORATIVE/COMMON/COMMUNAL DC AND MUTUAL RESPONSIVENESS (DCCM-STM-CCM-RCCM)}

Conjoint forms of DC are responses to stress experienced by both partners and/or to problems that partners see as sharing ("our" problem) even if they originated in one partner (e.g., an illness). Compared to other DC dimensions, positive conjoint strategies, particularly STM's common DC, and DCCM's collaborative coping, has received the most attention in research. There are only a handful of studies that have looked at communal coping or mutual responsiveness in couples.

\section{Coping With Non-medical Stressors}

Studies on Latino (Falconier et al., 2013b), American (e.g., Randall et al., 2015), and Western European (e.g., Bodenmann, 2000) couples show that, similar to supportive DC, partners that engage in common DC also tend to use effective individual coping strategies. Unlike other DC dimensions, common DC has found to be associated with relationship satisfaction not only in Latino, American, and Western European couples but also in Eastern couples such as Japanese (e.g., Yokotani and Kurosawa, 2015) and Chinese (Xu et al., 2016). In European couples common DC is also linked with sexuality, romance, passion, constructive conflict resolution, shared meaning, and commitment (Ledermann et al., 2010; Vedes et al., 2013; Landis et al., 2014), and less verbal aggression and anger (e.g., Bodenmann et al., 2010b). Compared to supportive DC, common DC is a stronger predictor of relationship satisfaction (e.g., Falconier et al., 2013a) and has stronger moderating effects in the association between different love styles and relationship satisfaction in Swiss couples, particularly for the female partner (Vedes et al., 2016). Common DC also helps work through grief (Bergstraesser et al., 2015) and attenuates the negative effects of posttraumatic stress on relationship satisfaction for American female spouses of combat veterans (Lambert et al., 2015) and of immigration stress on relationship satisfaction for Latino couples (Falconier et al., 2013a). Spirituality and a non-traditional role orientation are related to more frequent common DC in Latino couples (Austin and Falconier, 2013; Falconier, 2013).

A study on communal coping (Lin et al., 2016), indirectly measured through the frequency of we-talk, found that Taiwanese wives' we-talk was linked to husbands' higher work and marital satisfaction husbands' we-talk was only related to wives' work satisfaction.

\section{Coping With Medical and Mental Health Conditions}

Common and collaborative DC have been associated with better individual problem solving and decreased negative emotional expression in currently or formerly depressed individuals and their partners (Bodenmann et al., 2004). They have also been linked to lower depression in both partners when coping with breast cancer in Danish couples (Rottmann et al., 2015) and improved physical well-being in American women with breast cancer (Feldman and Broussard, 2006) and men with prostate cancer (Berg et al., 2011). The study on men with prostate cancer (Berg et al., 2008), based on daily diary data, also reported that collaborative DC was linked with more positive and less negative emotions and individual coping effectiveness in both partners. Nonetheless, the same study also reported that for women, collaborative DC exacerbated the negative emotion covariation between the spouses. The researchers explained that "one of the potential downsides to collaborative coping for women is that one may bear the brunt of the distress that the spouse is experiencing" but that these "short-term costs of collaboration" were perhaps "associated with more long-term gains as the active management nature of collaborative coping may be associated with long-term relational benefits" (p. 513). 
However, another study (Berg et al., 2011) that also examined American couples coping with breast cancer found that even though common DC was related to better dyadic adjustment for both partners, it was associated with higher distress in patients. In line with positive findings, communal coping, as measured partners' use of we language in, has been associated with lower depression in American women with breast cancer (Robbins et al., 2013) and improved alcohol abstinence during treatment and at follow up in American couples (Hallgren and McCrady, 2016). Spouse's we-talk predicted positive change in heart failure symptoms and general health over the following 6 months (Rohrbaugh et al., 2008) and smoking abstinence 12 months after quitting in American individuals with heart or lung disease (Rohrbaugh et al., 2012).

At the relational level, common and collaborative DC have consistently been found to have positive effects in couples coping with medical conditions. It has been associated with perceptions of the partner's acceptance of appearance in German women with breast cancer (Zimmermann et al., 2010), sharing more common goals in American couples with prostate cancer (Berg et al., 2008), and increased relationship satisfaction and/or couple's cohesion in Danish couples coping with cancer (Rottmann et al., 2015) and in Australian couples in which women were at an increased risk for breast/ovarian cancer (Watts et al., 2011). We-talk as an indicator of communal coping has been associated with relationship adjustment in American couples coping with breast cancer (Robbins et al., 2013). In Kenyan couples communal coping helped HIVnegative couples try to avoid HIV acquisition and helped zero-discordant couples prevent HIV transmission and lived positively with HIV (Rogers et al., 2016). Consistent with these findings, couples coping with breast cancer that reported mutual responsiveness DC, also reported stronger relationships (Kayser et al., 2007).

\section{INDIVIDUAL NEGATIVE DC: OVERPROTECTION (RFM)}

This negative form of DC to respond to a partner's stress was introduced by the RFM. It has been studied exclusively in the context of serious medical conditions.

\section{Coping With Medical Conditions}

Except for one study that found no effect of spousal overprotectiveness on patient's adaptation to myocardial infarction and a positive association with the couple's closeness (Fiske et al., 1991), studies have reported overprotectiveness to be associated with negative outcomes, particularly individual ones. Partners' overprotection has been associated with less improvement in self-efficacy in Dutch patients with coronary disease (Berkhuysen et al., 1999), less sense of control and more psychological distress in Dutch cancer patients (Kuijer et al., 2000) and CODP patients (Snippe et al., 2012), worse physical condition in cardiac patients (Joekes et al., 2007; Vilchinsky et al., 2011), and reduced dietary adherence and more diabetes distress in American diabetic patients (Johnson et al.,
2015). Regarding relational outcomes, Hagedoorn et al. (2000) found that overprotection was associated with lower marital satisfaction only for cancer patients that were experiencing high psychological distress or physical impairment. Bertoni et al. (2015) also found that when partners in Italian couples overprotected cardiac patients, the patients engaged less in their treatment.

\section{INDIVIDUAL NEGATIVE DC: PROTECTIVE BUFFERING (RFM)}

Similar to overprotection, this form of DC was introduced by the RFM. It has been studied primarily in the context of couples coping with chronic illness.

\section{Coping With Non-medical Stressors}

Only one study has examined the role of protective buffering in couples in a non-medical context. This study examined Japanese couples with pre-school children (Kurosawa et al., 2015) and found no significant associations of protective buffering with either relationship satisfaction or well-being, suggesting the possibility that protective buffering may play a different role when coping with non-medical stressors. However, the same study found that couples with more serious stressors tended to use protective buffering as a coping strategy more often.

\section{Coping With Medical Conditions}

Protective buffering has been studied in American couples with medical conditions such as heart and/or lung problems (e.g., Butler et al., 2014), Type-II diabetes (Johnson et al., 2014), and stem cell transplantation (Langer et al., 2009), in Dutch couples with cancer (e.g., Hagedoorn et al., 2011), heart problems (Joekes et al., 2007; Vilchinsky et al., 2011), CODP (Snippe et al., 2012), and diabetes (Schokker et al., 2010), and Australian couples with cancer (Lavery and Clarke, 1999). In the context of medical stressors both the patient and his/her partner may try to help each other cope through protective buffering (e.g., Langer et al., 2009). However, findings have been inconsistent regarding who relies more on this coping strategy. Some studies have found that caregivers tend to use more protective buffering than their ill partners (e.g., Langer et al., 2009), whereas other studies reported the opposite (e.g., Manne et al., 1999).

Regardless of which partner provides protective buffering and despite seemingly positive intentions, protective buffering has negative effects on individual and relational well-being for both providers and recipients in Western couples dealing with a medical condition. Receiving protective buffering has been associated with (a) lower physical exercise and glycemic control in diabetes patients (Johnson et al., 2014), (b) poorer mental health in recipients of stem cell transplants (Langer et al., 2009), (c) depression in men with heart disease (Hagedoorn et al., 2011; Vilchinsky et al., 2011), (d) distress in cancer patients (Manne et al., 2012), and (e) lower relationship satisfaction (e.g., Langer et al., 2009), particularly when there was low partner support for cancer patients (Hagedoorn et al., 2011). Even women undergoing genetic tests for cancer reported greater distress 6 months after receiving protective buffering (Manne et al., 2004). 
Furthermore, in the presence of overprotection, which partners also tend to use when they engage in protective buffering (e.g., Kuijer et al., 2000) only protective buffering is significantly associated with distress in patients with COPD (Snippe et al., 2012). Similarly, providers of protective buffering experience lower relationship satisfaction (Hinnen et al., 2008; Schokker et al., 2010), and greater distress regardless of whether they are patients or caregivers (Suls et al., 1997; Manne et al., 2007). A study of couples' coping with lung cancer indicated that patients that engaged in protective buffering reported higher pain severity and fatigue and poorer mental health (Lyons et al., 2016).

There are only a few exceptions to this pattern of results: Badr (2004) found that when American couples are more congruent in using active engagement but more complementary in the use of avoidance coping and protective buffering, they tend to report greater marital quality. Another study found that caregivers reported higher relationship satisfaction when they provided protective buffering (Langer et al., 2009). Regarding factors affecting protective buffering, one study of couples coping with cancer found that lower life expectancy increases the use of protective buffering (Manne et al., 1999).

\section{INDIVIDUAL NEGATIVE DC: HOSTILE/AMBIVALENT DC (STM)}

In our integration of DC models, STM's form of negative DC has been addressed as hostile/ambivalent. In this way other negative DC dimensions (e.g., overprotection) can also be considered independently of the one introduced by the STM.

\section{Coping With Non-medical Stressors}

Hostile/ambivalent DC has been linked with negative individual and relational functioning. At the individual level this DC dimension is associated with higher verbal aggression, anger, insomnia, depression, men's physical symptoms, and women's social dysfunction in Swiss couples (Bodenmann et al., 2010a, 2011) and catastrophizing in Romanian couples (Rusu et al., 2016). No association has been found with individual positive forms of coping (e.g., Randall et al., 2015). At the relational level hostile/ambivalent DC is linked to lower marital quality in both partners in European couples (e.g., Vedes et al., 2013), American couples (Randall et al., 2015), Latino couples in the U.S (Falconier et al., 2013b), Canadian couples (Levesque et al., 2014a), and Japanese women (Yokotani and Kurosawa, 2015). Hostile/ambivalent DC has also been negatively associated with sexuality, romance, passion, and constructive conflict resolution in Portuguese couples (Vedes et al., 2013) and has been found to be a stronger predictor of lower relationship satisfaction than individual coping in American couples (Papp and Witt, 2010). In our integration of DC models, the STM's form of negative DC has been addressed as hostile/ambivalent so that other negative DC dimensions (e.g., overprotection) can also be considered independently of the one introduced by the STM.
In terms of factors affecting the use of use hostile/ambivalent DC, a study on Italian couples found that parents' use of this form of negative DC increases the likelihood that their children will use it similarly in their romantic relationships (Donato et al., 2012). Additionally, rational love as compared to passionate love is associated with men's hostile/ambivalent DC (Gagliardi et al., 2015) and traumatic events exacerbate the use of hostile/ambivalent DC in Swiss couples (Kramer et al., 2005). By contrast, secure attachment is linked to less frequent use of hostile/ambivalent DC (Gagliardi et al., 2013).

\section{Coping With Medical or Mental Health Conditions}

In general, hostile and ambivalent DC has been linked to negative outcomes for the individual and the relationship in couples coping with physical- and mental health-related stressors across different Western cultures. In German couples coping with COPD, use of hostile/ambivalent DC is related to lower quality of life (Vaske et al., 2015). German couples coping with a partner's hematologic malignancy reported higher unmet supportive care needs when hostile/ambivalent DC was higher (Weißflog et al., 2016). When coping with breast cancer, hostile/ambivalent DC was associated with partners' poorer emotional well-being and patients' poorer physical well-being in patients in American couples (Feldman and Broussard, 2006), and with depressive symptoms and lower relationship quality in Danish couples (Rottmann et al., 2015).

Various factors predict a more frequent use of hostile/ambivalent DC in couples. Swiss couples coping with COPD use hostile/ambivalent DC more frequently than healthy couples do (Meier et al., 2012), and an imbalance in delegated DC increases the likelihood of relying more on this negative DC form (Meier et al., 2011). Swiss couples in which one partner is depressed/was formerly depressed (Bodenmann et al., 2010a) or who have children with externalizing behaviors (Gabriel et al., 2008) also use hostile/ambivalent DC more often than couples without a depressed member or a child with externalizing behaviors. Similarly, caregiving burden predicted more frequent hostile/ambivalent DC in Canadian couples with autistic children (Gouin et al., 2016).

The only exception to this link between hostile/ambivalent DC and negative individual indicators is an Italian study conducted by Bertoni et al. (2015). In this study, when couples used hostile/ambivalent DC to cope with cardiac problems, the cardiac patient's partner was more engaged in the problem.

\section{DEMOGRAPHIC, CULTURAL, AND CONTEXTUAL FACTORS: GENDER, AGE, AND CULTURE}

Several studies on Western populations have found that both partners report women as engaging more frequently in positive forms of coping such as providing delegated DC, supportive DC, and common and collaborative DC (e.g., Bodenmann et al., 2010a; Falconier et al., 2013b; Zeidner et al., 2013). Even though results have been inconsistent regarding which partner has a 
more positive evaluation of their overall couple's coping strategies (e.g., Vedes et al., 2013), DC also plays a more important role for women's relationship satisfaction than for men's in many Western cultures (e.g., Gmelch and Bodenmann, 2007; Papp and Witt, 2010). Additionally, several studies have found that women communicate their stress more often than men in Western couples (e.g., Donato et al., 2009). Some studies have also reported men to be more likely than women to provide negative DC forms such as protective buffering (Manne et al., 1999) and hostile/ambivalent DC (e.g., Yokotani and Kurosawa, 2015). This is consistent with the finding that lesbian couples reported receiving better DC and experiencing less conflict when compared to heterosexual couples (Meuwly et al., 2013). Nonetheless, some studies have failed to find any gender differences in some DC positive forms such as delegated DC (e.g., Rusu et al., 2016) or supportive DC (García-López et al., 2016).

Studies have reported inconsistent findings regarding the effect of age or length of relationship on overall DC or any of its dimensions, regardless of whether the study focused on coping with general stress or on medical or mental health conditions. Some studies found no effect of age or relationship length on overall DC (e.g., Ruffieux et al., 2014; Reed et al., 2016), stress communication (e.g., Levesque et al., 2014a), delegated DC (e.g., Levesque et al., 2014a), active engagement (e.g., Joekes et al., 2007), protective buffering (Langer et al., 2009). By contrast, other studies have reported that younger couples engage more frequently in positive forms of $\mathrm{DC}$ than older couples including overall DC (e.g., Meyer et al., 2005), active engagement with a sick partner (e.g., Schokker et al., 2010), and supportive DC (e.g., Levesque et al., 2014a) and less frequently in negative DC forms such as overprotection (e.g., Joekes et al., 2007) and that older couples rely more frequently on factual stress communication than younger couples do (Bodenmann and Widmer, 2000). Regarding length of relationship, several studies have found a positive relation between length of relationship and DC negative forms such as protective buffering (Schokker et al., 2010) and hostile/ambivalent DC (e.g., Yokotani and Kurosawa, 2015), but one study found also a positive association for overall DC and common or collaborative DC (e.g., Feldman and Broussard, 2006).

When analyzing results from all DC studies, given that the majority of those studies have been conducted with European couples, it is not possible to reliably identify a pattern of results that could be indicative of cultural differences between Western and Eastern populations or even between Western European and non-European couples (e.g., Latin American couples). Evidence from two studies suggests that stress communication might be a DC dimension in which Western and Eastern couples manage differently. One study on Chinese couples (Xu et al., 2016) reported that men communicated stress more frequently than women did, which was in sharp contrast with the great number of Western studies that have consistently found the opposite gender pattern. A second study found that intercultural ThaiSwiss couples communicated about their stress less frequently than mono-cultural Swiss couples (Gagliardi et al., 2010).

Only a handful of studies have actually looked at the role culture in the couples' stress and coping process by examining different cultural groups in the same study. As described earlier, Kayser et al. (2014) interviewed American, Chinese, and Indian couples coping with breast cancer and concluded that compared to American couples, Asian couples viewed the illness as beyond their control and they were therefore more inclined to accept it rather than desperately trying to do something to change it. Additionally, Asian couples had more gender differentiated roles and involved their families in their coping efforts (open boundaries) more often. Asian couples also coped in ways that showed more interdependence. Another study comparing three different cultural groups found that Chinese couples reported significantly less delegated DC than Swiss and American couples (Xu et al., 2016). This set of findings suggests that Asian couples cope in ways congruent with their collectivistic orientation whereas American couples cope in ways consistent with their individualistic orientation.

A large recent, cross-sectional study across 35 different countries (Hilpert et al., 2016) found that supportive and common DC considered together predicted relationship satisfaction across all nations. It also found that couples in African countries used supportive and common DC more frequently than couples in Asian countries such as Hong Kong and South Korea. However, the study yielded two interesting findings. First, results did not support differences between Eastern and Western cultures in the association between DC and relationship satisfaction. For example, Nigeria, India, Ghana, Iran, Portugal, and Kenya were among the countries with the smallest effect of DC on relationship satisfaction and Bulgaria, Romania, Hong Kong, Slovakia, and Canada where among the countries with larger effects, indicating significant variability within a region whose countries were expected to be culturally related. Then, the size of the effect of DC on relationship satisfaction was independent from the frequency with which they used supportive and common DC. For example, couples from Bulgaria, Canada, and Greece reported using DC frequently and that coping behavior had a large impact on relationship satisfaction, whereas couples in Ghana, Kenya, and Nigeria were also high in DC behaviors but their coping had a small effect on the relationship.

\section{DISCUSSION}

\section{The DC Conceptual Integration}

The conceptual review and integration of the various DC models presented in this paper suggests that the various theoretical frameworks that have been developed can be brought together to present a more comprehensive picture of the DC process. Even though DC models differ in its origins with most of them developed to understand the couple's process to cope with a medical condition and one, the STM, to explain how couples cope with everyday stress, most models have been applied to examine DC with medical and non-medical stressors, providing support for the integration of all DC models into a larger framework that can explain all DC processes. The integration is also possible because there are no contradictions among the various DC models. To begin with, they all share a systemic perspective in which each partner's experiences of stress and 
coping with external stressors are interrelated. In addition, all DC models support the same chore principles about the DC process: (a) stress appraisals ("my" or "your" vs. "our" problem) shape DC responses; (b) partners communicate about their stress; (c) partners engage in DC individual strategies that can help the other partner cope with stress or in conjoint strategies to handle stress together; (d) DC may be positive or negative; and (e) relationship and contextual factors may affect stress appraisals and DC. DC models only differ on the attention given to each of those chore principles and the different types of individual and conjoint coping strategies.

Nonetheless, the integrative DC model advanced in this paper should continue to be expanded and refined conceptually. It is possible that new dimensions are identified beyond the ones included at present. However, new theoretical developments could consider the present integration to avoid construct overlaps and conceptual fragmentation in the field. The present integrative model should keep being expanded to also incorporate the work in related fields such as emotional co-regulation and spousal support.

\section{The Review of the DC Empirical Literature}

Following the conceptual integration of DC models, the goal of this narrative review was to present the findings from all studies on DC and each of its specific dimensions in order to provide a complete picture of the accumulated empirical knowledge and suggest areas in need of research.

This empirical review, which includes mostly studies conducted on American and European populations, suggests that taken together or separately, most dimensions of positive individual (helping partner cope with stress) and conjoint forms (partners coping together with stress) of DC are associated with better individual and relational functioning when coping with either medical or mental health conditions or other types of stressors, while the opposite is true for negative individual DC strategies In other words, when couples report using DC, empathic responding, active engagement, supportive DC, delegated DC, stress communication, or common or collaborative DC they also tend to report higher use of effective individual coping strategies, higher life satisfaction, lower psychological distress, and depression when coping with stress in general and better illness management, health related quality of life, improved physical and emotional wellbeing when coping with a mental health or medical condition. At a relational level, these couples tend to report more constructive communication, sexual and relationship satisfaction, commitment, and stability over time when coping with stress in general as well as with mental health and medical stressors. When couples use positive forms of coping they can also buffer the negative effects of stress on their individual levels of aggression and on their relationship satisfaction. In contrast to positive forms of DC, all three forms of negative DC have been related to negative individual and relational functioning. In the context of illnesses, regardless of which partner is the provider or recipient, overprotection, protective buffering, and hostile/ambivalent DC are linked to lower self-efficacy, sense of control, physical and emotional well-being, and relationship satisfaction. Similarly, when couples use hostile/ambivalent DC to cope with stress in general, they report more destructive communication and conflict resolution, and relationship dissatisfaction.

Despite this overall picture that suggests that there are individual and relationship benefits to using positive DC strategies but risks to relying on negative forms of DC, further research is necessary to better understand the DC process. Findings included in this review suggest that some DC dimensions may be more critical than others in harming or protecting individual and relational well-being. For example, common and collaborative DC tend to be more beneficial than other positive DC dimensions for both medical and non-medical stressors while delegated DC seems to be the least beneficial. Furthermore, it is possible that some DC forms might not even be beneficial across all stressful situations. For example, delegated DC does not seem to be positive for partners with medical conditions and imbalanced delegated DC between the partners is negative for patients. Similarly, a lack of reciprocation in empathic responding or supportive DC is linked to psychological distress. This set of findings indicate that not all DC strategies are equally beneficial or negative across different contexts. However, further research is necessary to examine differential effects of all DC strategies and variations across different stressors. As a illustration, it might be that controlling DC or overprotection turn out to be less negative when coping with medical conditions than when coping with other type of stressors or that active engagement has positive effects only when coping with medical conditions but not when a partner is coping with a stressful situation that he or she might want to avoid sharing with a partner or feels responsible for (e.g., conflict with a family member, job-related problem, etc.). In order to answer some these questions, more attention should be given to examine forms of DC that have been understudied such as conjoint negative DC, controlling DC, or empathic responding. In addition, DC forms should be studied across different stressful contexts. For example, most studies on medical stressors are on couples coping with cancer and therefore, further research could focus on the role of DC and each of its dimensions in couples coping with other medical conditions. Similarly, studies should also examine DC in couples coping with other non-medical stressors such as economic problems, immigration related issues, raising children with disabilities, emotional and/or behavior difficulties, caring for elderly family members, etc. Researchers should specially focus on examining DC dimensions that have only been studied in relation to only one type of stressor. As an illustration, overprotection has only be examined in the context of medical illness.

Additionally, improvement in design and measurement instruments could further advance the field and provide more reliable findings. More than 100 of the 139 articles included in the present review were reports of cross-sectional studies, precluding conclusions on causal direction. Longitudinal studies did indicate that it is DC that predicts many of the individual and relational outcomes but clearly more longitudinal studies are needed to provide further support. Also, and most importantly, except for 18 studies all studies relied on self-report questionnaires. Unfortunately, this is an important methodological constraint to 
the study of DC. First of all, some self-report measures (e.g., DCI) assess dyadic coping with stress in general and not in relation to a specific stressor, assuming that couples rely on DC to the same extent and in the same way across different contexts of stress. These measures are even more problematic when used in studies on couples coping with a particular stressor as it is not possible to know whether the responses on coping apply to the way in which the couple is managing that stressor. Then, even if the self-report measure is specific to the stressful situation, asking partners about their overall impression on DC assumes that there is stability over time and consistency in how couples cope with various types of stressors. However, the developmental perspective introduced by the DCCM in studying couples coping with illness suggests that couples' coping can change over time. This limitation might be overcome by the use of daily diaries, which would allow to see changes in DC over time and/or by stressor.

Last, and also well-known are the biases introduced by selfreports. The few studies that have been conducted in the DC field relying on observational and physiological data have allowed not only to obtain less biased data on partners' use of DC and its effects on each other but also to micro-analyze the DC process following moment-to-moment interactions instead of assessing DC at a macro level. This type of micro-analysis has helped linked, for example, differences in stress communication with particular partner's responses. For example, Kuhn et al. (2017) found that problem-oriented stress expression was strongly linked to problem-oriented dyadic coping in a time sequence of $10 \mathrm{~s}$ within a conversation, while emotion-oriented stress expression was associated with emotion-oriented dyadic coping reactions. Continuing to employ observational and physiological measurement in research may refine our understanding of DC, particularly regarding stress communication, partners $\mathrm{DC}$ responses, and the effects of DC responses on each partner and their further stress communication and use of DC.

In terms of factors affecting the use of DC strategies, the current review indicates that age, individual psychological and relationship variables, family context, and gender may play some role. Being young, empathic, emotionally intelligent, securely attached, or spiritually oriented and having relationship-focused standards all contribute to using positive forms of DC, whereas experiencing trauma, depression, and/or anxiety, and being older do not. By contrast, depression and having children with psychosocial challenges may contribute to more use of negative DC and imbalance in delegated DC. Western women tend to engage more in positive forms of $\mathrm{DC}$ whereas men use more negative forms. Furthermore, DC is more significant for relationship functioning for Western, African, and Asian women and for their male partners. These findings reinforce the need to further study the role of demographic and individual and relationship factors in the use and effects of DC in general and across different stress contexts. This research could explain why men are less likely than women to use DC strategies despite the fact that they also benefit from them or whether age and gender affect DC similarly across different stressful circumstances.

Nonetheless, the review clearly indicates that one of the areas in which the DC field seriously needs to further advance is in the examination of cross-cultural variation. Despite the fact that the cultural context has been included in various DC models (the STM, the DCCM, and the RCCM) as a factor that shapes the stress and coping process and the fact that one study indicated that DC is beneficial for the couple's relationship at least in 35 different countries (Hilpert et al., 2016), most of the research included in the present review have been conducted in Western Europe. The few studies that have examined cultural factors suggest that Asian couples cope in more collectivistic ways, whereas Western couples seem to cope in more individualistic ways. It also appears that some cultures (e.g., African) use DC more frequently than others (e.g., Asian) and that they may also differ on the extent to which their DC behaviors contribute to their relationship satisfaction regardless of DC use. Those findings call for more studies to be conducted in non-Western European populations so that differences in stress appraisal, stress communication, and use and effects of DC strategies can be understood in different cultures.

The other area of inquiry that has not received much attention in the field is DC in the context of same-sex couples. Only one study on same-sex couples was included in the present review (Meuwly et al., 2013) and it indicates that the DC quality might be better in than in heterosexual couples. Further research should be conducted to fully understand the DC process in gay and lesbian couples.

In terms of the interplay of individual coping styles and their effects on the relationship, the review of studies on similarities between partners' styles of coping with their own stress did not show consistent results, suggesting that the fit or congruence between partners' styles may matter more in terms of the impact on the individual and the relationship. However, studies still need to provide evidence for this possibility

The knowledge gained in the field about the individual and relational benefits of positive DC and the harmful effects of negative DC as well as the factors that promote positive DC have had important clinical and programmatic implications. Several interventions have been developed to help couples cope with stress together based on the concepts and empirical findings reported in the present review. A report of all interventions is beyond the scope of this review but The Couples Coping Enhancement Training (CCET; Bodenmann and Shantinath, 2004) to prevent relationship distress by teaching couples cope with stress, the Coping-Oriented Couple Therapy (Bodenmann et al., 2008) that provides a clinical intervention focused on DC, or the TOGETHER program (Falconier, 2015) to assist couples cope with financial stress are just some illustrations of the programmatic and clinical applications of DC models. Further advances in the DC field may provide critical information to design interventions and programs that can reach ethnically diverse couples, different age, religious, and socio-economic groups, same-sex couples, and both men and women.

\section{LIMITATIONS}

Despite the contributions of the present narrative review, there are also some limitations. The review only included journal articles published in English and German, which may have 
left out studies published through other outlets and in other languages. However, compared to other reviews, this review has included the largest number of studies and DC models, offering a more complete picture of the DC field.

Even though we provide supplementary material describing the sample type and size, measures, design, and the main findings of each of the studies reviewed, due to publication length limitations it is not possible to describe or integrate all studies with enough level of detail. Last, this review did not entail a critical analysis of the study designs or a statistical analysis (e.g., meta-analysis) due to the heterogeneity of variables, low number of studies for some of the variables, and need to include qualitative studies. The inherent limitations of studies using cross-sectional and self-report data, which comprise the vast majority of studies in this review, are well-known and have been addressed in our discussion as well.

\section{CONCLUSION}

Various DC models have been introduced in the last two decades. Even though each DC model has made unique contributions to the understanding of the DC process, conceptual overlap also exists across models. Given that several chore principles are shared across those models, a conceptual integration was possible. The integrative model proposed in this paper includes all the DC dimensions identified by such DC models as well as factors that affect the coping process. The review of all studies applying any of the DC models suggest that in Western couples, positive forms of coping, whether individual or conjoint and taken together or separately, are beneficial for each partner's

\section{REFERENCES}

Austin, J. L., and Falconier, M. K. (2013). Spirituality and common dyadic coping: protective factors from psychological aggression in Latino immigrant couples. J. Family Issues 34, 323-346. doi: 10.1177/0192513X124 52252

Badr, H. (2004). Coping in marital dyads: a contextual perspective on the role of gender and health. Pers. Relat. 11, 197-211. doi: 10.1111/j.1475-6811.2004.00078.x

Badr, H., Carmack, C. L., Kashy, D. A., Cristofanilli, M., and Revenson, T. A. (2010). Dyadic coping in metastatic breast cancer. Health Psychol. 29, 169-180. doi: $10.1037 / \mathrm{a} 0018165$

Barbarin, O. A., Hughes, D., and Chesler, M. A. (1985). Stress, coping, and marital functioning among partners of children with cancer. J. Marriage Family 47, 473-480. doi: $10.2307 / 352146$

Barnoy, S., Bar-Tal, Y., and Zisser, B. (2006). Correspondence in informational coping styles: How important is it for cancer patients and their spouses? Personal. Individ. Differ. 41, 105-115. doi: 10.1016/j.paid.2005. 12.013

Ben-Zur, H., Gilbar, O., and Lev, S. (2001). Coping with breast cancer: patient, spouse, and dyad models. Psychosomat. Med. 63, 32-39. doi: 10.1097/00006842-200101000-00004

Berg, C. A., and Upchurch, R. (2007). A developmental-contextual model of couples coping with chronic illness across the adult life span. Psychol. Bull. 133, 920-954. doi: 10.1037/0033-2909.133.6.920

Berg, C. A., Wiebe, D. J., \& Butner, J. (2011). Affect covariation in marital couples dealing with stressors surrounding cancer. Gerontology, 57, 167-172. doi: $10.1159 / 000318642$ individual and relational well-being when they cope with stress in general and/or mental health or medical stressors. Few studies in non-Western populations suggest similar benefits. Research on DC can be expanded to include other populations and stressors and use better designs. The accumulated knowledge in the field already has already offered enough guidance for prevention programs and clinical interventions. However, such knowledge should be taken with caution given the design and measurement limitations of the studies as well as the characteristics of the samples.

\section{AUTHOR CONTRIBUTIONS}

MF came up with idea and design of the study. She conducted a lot of the coding in English and was responsible for the majority of the conceptual review and integration. RK contributed with all the coding in German and writing about the methodology for the review of empirical studies and writing about STM and the empirical findings for STM and DCCM. MF also wrote about the empirical findings of CM, RFM, CMM, and RCCM and reworded STM findings. Both $\mathrm{MF}$ and RK worked on creating the table of the summary and findings.

\section{SUPPLEMENTARY MATERIAL}

The Supplementary Material for this article can be found online at: https://www.frontiersin.org/articles/10.3389/fpsyg. 2019.00571/full\#supplementary-material
Berg, C. A., Wiebe, D. J., Butner, J., Bloor, L., Bradstreet, C., Upchurch, R., et al. (2008). Collaborative coping and daily mood in couples dealing with prostate cancer. Psychol. Aging 23, 505-516. doi: 10.1037/a0012687

Bergstraesser, E., Inglin, S., Hornung, R., and Landolt, M. A. (2015). Dyadic coping of parents after the death of a child. Death Stud. 39, 128-138. doi: 10.1080/07481187.2014.920434

Berkhuysen, M. A., Nieuwland, W., Buunk, B. P., Sanderman, R., and Rispens, P. (1999). Change in self-efficacy during cardiac rehabilitation and the role of perceived overprotectiveness. Patient Educ. Counsel. 38, 21-32. doi: 10.1016/S0738-3991(98)00115-3

Bertoni, A., Donato, S., Graffigna, G., Barello, S., and Parise, M. (2015). Engaged patients, engaged partnerships: singles and partners dealing with an acute cardiac event. Psychol. Health Med. 20, 505-517. doi: 10.1080/13548506.2014.969746

Bodenmann, G. (1995). A systemic-transactional conceptualization of stress and coping in couples. Swiss J. Psychol. 54, 34-39.

Bodenmann, G. (1997). Dyadic coping: a systemic-transactional view of stress and coping among couples: theory and empirical findings. Eur. Rev. Appl. Psychol. 47, 137-141.

Bodenmann, G. (2000). Stress und Coping bei Paaren [Stress and Coping in Couples]. Göttingen: Hogrefe.

Bodenmann, G. (2005). "Dyadic coping and its significance for marital functioning," in Couples Coping With Stress: Emerging Perspectives on Dyadic Coping, eds T. Revenson, K. Kayser, and G. Bodenmann (Washington, DC: American Psychological Association), 33-50. doi: 10.1037/ 11031-002

Bodenmann, G. (2008). Dyadisches Coping Inventar: Testmanual [Dyadic Coping Inventory: Test manual]. Bern: Huber. 
Bodenmann, G., and Widmer, K. (2000). Stressbewältigung im alter: ein Vergleich von paaren jüngeren, mittleren und höheren Alters. [Coping in elderly couples: a comparison with young and middle-aged couples]. Zeitschrift Gerontol. Geriatr. 33, 217-228. doi: 10.1007/s003910070063

Bodenmann, G., Atkins, D. C., Schär, M., and Poffet, V. (2010a). The association between daily stress and sexual activity. J. Family Psychol. 24, 271-279. doi: 10.1037/a0019365

Bodenmann, G., Charvoz, L., Widmer, K., and Bradbury, T. N. (2004). Differences in individual and dyadic coping among low and high depressed, partially remitted, and nondepressed persons. J. Psychopathol. Behav. Assess. 26, 75-85. doi: 10.1023/B:JOBA.0000013655.45146.47

Bodenmann, G., and Cina, A. (1999). Der Einfluß von Streß, individueller Belastungsbewältigung und dyadischen Coping auf die Partnerschaftsstabilität: Eine 4-Jahres-Längschnittstudie. [The influence of stress and individual and dyadic coping on the stability of partnerships. A 4-year longitudinal study]. Zeitschr. Klin. Psychol. 28, 130-139. doi: 10.1026//0084-5345.28.2.130

Bodenmann, G., and Cina, A. (2005). Stress and coping among stablesatisfied, stable-distressed, and separated/divorced Swiss couples: a 5year prospective longitudinal study. J. Divorce Remarriage 44, 71-89. doi: 10.1300/J087v44n01_04

Bodenmann, G., Cina, A., and Schwerzmann, S. (2001). Individuelle und dyadische Copingressourcen bei Depressiven [Individual and dyadic coping resources for depressed persons]. Zeitschr. Klin. Psychol. Psychother. 30, 194-203. doi: 10.1026//1616-3443.30.3.194

Bodenmann, G., Meuwly, N., Bradbury, T. N., Gmelch, S., and Ledermann, T. (2010b). Stress, anger, and verbal aggression in intimate relationships: moderating effects of individual and dyadic coping. J. Soc. Pers. Relat. 27, 408-424. doi: 10.1177/0265407510361616

Bodenmann, G., Meuwly, N., Germann, J., Nussbeck, F. W., Heinrichs, M., and Bradbury, T. N. (2015). Effects of stress on the social support provided by men and women in intimate relationships. Psychol. Sci. 26, 1584Ü1594. doi: $10.1177 / 0956797615594616$

Bodenmann, G., Meuwly, N., and Kayser, K. (2011). Two conceptualizations of dyadic coping and their potential for predicting relationship quality and individual well-being. A comparison. Eur. Psychol. 16, 255-266. doi: 10.1027/1016-9040/a000068

Bodenmann, G., and Perrez, M. (1991). Dyadisches Coping - eine systemische Betrachtungsweise der Belastungsbewältigung in Partnerschaften [Dyadic coping - a systemic view of couples coping with stress]. J. Family Res. 3, 4-25.

Bodenmann, G., Pihet, S., and Kayser, K. (2006). The relationship between dyadic coping and marital quality: a 2-year longitudinal study. J. Family Psychol. 20, 485-493. doi: 10.1037/0893-3200.20.3.485

Bodenmann, G., Plancherel, B., Beach, S. R. H., Widmer, K., Gabriel, B., Meuwly, N., et al. (2008). Effects of coping-oriented couples therapy on depression: a randomized clinical trial. J. Consult. Clin. Psychol. 76, 944-954. doi: $10.1037 / \mathrm{a} 0013467$

Bodenmann, G., Randall, A. K., and Falconier, M. K. (2016). "Coping in couples: the systemic transactional model (STM)," in Couples Coping With Stress: A Cross-Cultural Perspective, eds M. K. Falconier, A. K., Randall, and G. Bodenmann (New York, NY: Routledge), 5-22.

Bodenmann, G., and Shantinath, S. D. (2004). The couples coping enhancement training (CCET): a new approach to prevention of marital distress based upon stress and coping. Family Relat. 53, 477-484. doi: 10.1111/j.0197-6664.2004.00056.x

Butler, E. A., Hollenstein, T., Shoham, V., and Rohrbaugh, M. J. (2014). A dynamic state-space analysis of interpersonal emotion regulation in couples who smoke. J. Soc. Pers. Relat. 31, 907-927. doi: 10.1177/0265407513508732

Carver, C. S., Scheier, M. F., and Weintraub, J. K. (1989). Assessing coping strategies: a theoretically based approach. J. Personal. Soc. Psychol. 56, 267-283. doi: 10.1037/0022-3514.56.2.267

Checton, M. G., Magsamen-Conrad, K., Venetis, M. K., and Greene, K. (2015). A dyadic approach: applying a developmental-conceptual model to couples coping with chronic illness. Health Educ. Behav. 42, 257-267. doi: 10.1177/1090198114557121

Coyne, J. C., \& Smith, D. A. F. (1994). Couples coping with a myocardial infarction: Contextual perspective on patient self-efficacy. Journal of Family Pscyhology, 8, 43-54. doi: 10.1037/0893-3200.8.1.43
Coyne, J. C., and Smith, D. A. F. (1991). Couples coping with a myocardial infarction: a contextual perspective on wives' distress. J. Personal. Soc. Psychol. 61 404-412. doi: 10.1037/0022-3514.61.3.404

Cronkite, R. C., and Moos, R. H. (1984). The role of predisposing and moderating factors in the stress-illness relationship. J. Health Soc. Behav. 25, 372-393. doi: $10.2307 / 2136377$

Dagan, M., Sanderman, R., Schokker, M. C., Wiggers, T., Baas, P. C., van Haastert, M., et al. (2011). Spousal support and changes in distress over time in couples coping with cancer: the role of personal control. J. Family Psychol. 25, 310-318. doi: $10.1037 / \mathrm{a} 0022887$

DeLongis, A., and O'Brien, T. B. (1990). "An interpersonal framework for stress and coping: an application to the families of Alzheimer's patients," in Stress and Coping in Later-Life Families, eds M. A. P. Stephens, J. H. Crowther, S. E. Hobfoll, and D. L. Tennenbaum (New York, NY: Hemisphere), 221-239.

Donato, S., Iafrate, R., Barni, D., Bertoni, A., Bodenmann, G., and Gagliardi, S. (2009). Measuring dyadic coping: the factorial structure of Bodenmann's "Dyadic Coping Questionnaire" in an Italian sample. Test. Psychometr. Methodol. Appl. Psychol. 16, 1-22.

Donato, S., Iafrate, R., Bradbury, T. N., and Scabini, E. (2012). Acquiring dyadic coping: parents and partners as models. Pers. Relat. 19, 386-400. doi: $10.1111 /$ j.1475-6811.2011.01368.x

*Donato, S., Parise, M., Iafrate, R., Bertoni, A., Finkenauer, C., and Bodenmann, G. (2015). Dyadic coping responses and partners' perceptions for couple satisfaction: an actor-partner interdependence analysis. J. Soc. Pers. Relat. 32, 580-600. doi: 10.1177/0265407514541071

Fagundes, F. C., Berg, C. A., and Wiebe, D. J. (2012). Intrusion, avoidance, and daily negative affect among couples coping with prostate cancer: a dyadic investigation. J. Family Psychol. 26, 246-253. doi: 10.1037/a0027332

Falconier, M. K. (2013). Traditional gender role orientation and dyadic coping in immigrant Latino couples: effects on couple functioning. Family Relat. 62, 269-283. doi: 10.1111/fare.12002

Falconier, M. K. (2015). Together - a couples' program to improve communication, coping, and financial management skills: development and initial pilot-testing. J. Marital Family Ther. 41, 236-250. doi: 10.1111/jmft.12052

Falconier, M. K., Jackson, J. B., Hilpert, P., and Bodenmann, G. (2015). Dyadic coping and relationship satisfaction: a meta-analysis. Clin. Psychol. Rev. 42, 28-46. doi: 10.1016/j.cpr.2015.07.002

Falconier, M. K., Nussbeck, F., and Bodenmann, G. (2013a). Immigration stress and relationship satisfaction in Latino couples: the role of dyadic coping. J. Soc. Clin. Psychol. 32, 813-843. doi: 10.1521/jscp.2013.32.8.813

Falconier, M. K., Nussbeck, F., and Bodenmann, G. (2013b). Dyadic coping in Latino couples: validity of the Spanish version of the dyadic coping inventory. Anxiety Stress Coping 26, 446-466. doi: 10.1080/10615806.2012. 699045

Falconier, M. K., Randall, A. K., and Bodenmann, G. (2016a). Couples Coping With Stress: A Cross-Cultural Perspective. New York, NY: Routledge. doi: $10.4324 / 9781315644394$

Falconier, M. K., Randall, A. K., and Bodenmann, G. (2016b). "Cultural considerations in understanding dyadic coping across cultures," in Couples Coping With Stress: A Cross-Cultural Perspective, eds M. K. Falconier, A. K., Randall, and G. Bodenmann (New York, NY: Routledge), 23-35.

Feldman, B. N., and Broussard, C. A. (2006). Men's adjustment to their partners' breast cancer: a dyadic coping perspective. Health Soc. Work 31, 117-127. doi: $10.1093 / \mathrm{hsw} / 31.2 .117$

Fiske, V., Coyne, J. C., and Smith, D. A. (1991). Couples coping with myocardial infarction. An empirical reconsideration of the role of overprotectiveness. J. Family Psychol. 5, 4-20. doi: 10.1037/0893-3200.5.1.4

Gabriel, B., and Bodenmann, G. (2006a). Elterliche Kompetenzen und Erziehungskonflikte: eine ressourcenorientierte Betrachtung von familiären Negativdynamiken [Parental competences and child raising conflicts: a resource-oriented view of familial negative cycles]. Kindheit Entwicklung 15, 9-18. doi: 10.1026/0942-5403.15.1.9

*Gabriel, B., and Bodenmann, G. (2006b). Stress und Coping bei Paaren mit einem verhaltensauffälligen Kind [Stress and coping in couples of a child with conduct problems]. Zeitschr. Klin. Psychol. Psychother. 35, 59-64. doi: 10.1026/1616-3443.35.1.59 
Gabriel, B., Untas, U., Lavner, J., Koleck, M., and Luminet, O. (2016). Gender typical patterns and the link between alexithymia, dyadic coping, and psychological symptoms. Personal. Individ. Differ. 96, 266-271. doi: 10.1016/j.paid.2016.02.029

Gabriel, B., Zeender, N., and Bodenmann, G. (2008). Stress und Coping bei Eltern von einem Kind mit einem Down-Syndrom [Stress and coping in parents of a child with Down syndrome: an examination of a theoretical model]. J. Family Res. 20, 80-95.

Gagliardi, S., Bodenmann, G., and Bregy, M. (2010). Coping bei binationalen Paaren: Ein Vergleich zwischen thailändisch-schweizerischen und mononationalen Schweizer Paaren [Coping in bi-national couples: A comparison between Thai-Swiss and mononational Swiss couples]. Zeitschrift für Klinische Psychologie Und Psychotherapie, 39(3), 141-150. doi: 10.1026/1616-3443/a000028

Gagliardi, S., Bodenmann, G., and Heinrichs, N. (2015). Dyadisches Coping und Partnerschaftszufriedenheit bei verschiedenen Liebesstilen [Dyadic coping and relationship satisfaction in different love styles]. J. Family Res. 27, 105-121. doi: 10.3224/zff.v27i1.18589

Gagliardi, S., Bodenmann, G., Heinrichs, N., Maria Bertoni, A., Iafrate, R., and Donato, S. (2013). Unterschiede in der Partnerschaftsqualität und im dyadischen Coping bei verschiedenen bindungsbezogenen Paartypen [Differences in relationship quality and dyadic coping in different attachmentrelated couple types]. Psychother. Psychosomat. Med. Psychol. 63, 185-192. doi: 10.1055/s-0032-1333291

García-López, C., Sarriá, E., Pozo, P., and Recio, P. (2016). Supportive dyadic coping and psychological adaptation in couples parenting children with Autism spectrum disorder: the role of relationship satisfaction. J. Autism Dev. Disord. 46, 3434-3447. doi: 10.1007/s10803-016-2883-5

Giunta, C. T., and Compas, B. E. (1993). Coping in marital dyads: patterns and associations with psychological symptoms. J. Marriage Family 55, 1011-1017. doi: $10.2307 / 352780$

Gmelch, S., and Bodenmann, G. (2007). Dyadisches Coping in Selbstund Fremdwahrnehmung als Prädiktor für Partnerschaftsqualität und Befinden [Dyadic coping in self- and partner perception as predictor for relationship quality and well-being]. Zeitschr. Gesundheitspsychol. 15, 177-186. doi: 10.1026/0943-8149.15.4.177

*Gmelch, S., Bodenmann, G., Meuwly, N., Ledermann, T., Steffen-Sozinova, O., and Striegl, K. (2008). Dyadisches Coping Inventar (DCI): Ein Fragebogen zur Erfassung des partnerschaftlichen Umgangs mit Stress [Dyadic Coping Inventory (DCI): a questionnaire assessing dyadic coping in couples]. J. Family Res. 20, 185-202. doi: 10.5167/uzh-7391

Gouin, J.-P., Scarcello, S., da Estrela, C., Paquin, C., and Barker, E. T. (2016). Dyadic coping and inflammation in the context of chronic stress. Health Psychol. 35, 1081-1084. doi: 10.1037/hea0000395

Hagedoorn, M., Dagan, M., Puterman, E., Hoff, C., Meijerink, W. J. H. J., Delongis, A., et al. (2011). Relationship satisfaction in couples confronted with colorectal cancer: the interplay of past and current spousal support. J. Behav. Med. 34, 288-297. doi: 10.1007/s10865-010-9311-7

Hagedoorn, M., Kuijer, R. G., Buunk, B. P., DeJong, G. M., Wobbes, T., and Sanderman, R. (2000). Marital satisfaction in patients with cancer: does support from intimate partners benefit those who need it most? Health Psychol. 19, 274-282. doi: 10.1037/0278-6133.19.3.274

Hallgren, K. A., and McCrady, B. S. (2016). We-language and sustained reductions in drinking in couple-based treatment for alcohol use disorders. Family Process 55, 62-78. doi: 10.1111/famp. 12150

Helgeson, V. S., Jakubiak, B., Van Vleet, M., and Zajdel, M. (2017), Communal coping and adjustment to chronic illness: theory update and evidence. Personal. Soc. Psychol. Rev. 22, 170-195. doi: 10.1177/10888683177 35767

*Herzberg, P. Y. (2013). Coping in relationships: the interplay between individual and dyadic coping and their effects on relationship satisfaction. Anxiety Stress Coping 26, 136-153. doi: 10.1080/10615806.2012.655726

*Hilpert, P., Bodenmann, G., Nussbeck, F. W., and Bradbury, T. N. (2013). Predicting relationship satisfaction in distressed and non-distressed couples based on a stratified sample: a matter of conflict, positivity, or support? Family Sci. 4, 110-120. doi: 10.1080/ 19424620.2013.830633

Hilpert, P., Randall, A. K., Sorokowski, P., Atkins, D. C., Sorokowska, A., Ahmadi, K., et al. (2016). The associations of dyadic coping and relationship satisfaction vary between and within nations: a 35-nation study. Front. Psychol. 7:1106 doi: 10.3389/fpsyg.2016.01106

Hinnen, C., Hagedoorn, M., Ranchor, A. V., and Sanderman, R. (2008). Relationship satisfaction in women: a longitudinal case-control study about the role of breast cancer, personal assertiveness, and partners' relationship-focused coping. Br. J. Health Psychol. 13, 737-754. doi: 10.1348/135910707X252431

Hinnen, C., Ranchor, A. V., Baas, P. C., Sanderman, R., and Hagedoorn, M. (2009). Partner support and distress in women with breast cancer: the role of patients' awareness of support and level of mastery. Psychol. Health 24, 439-455. doi: 10.1080/08870440801919513

Hoffmann, F., Banzhaf, C., Kanske, P., Gärtner, M., Bermpohl, F., and Singer, T. (2016). Empathy in depression: egocentric and altercentric biases and the role of alexithymia. J. Affect. Disord. 199(Suppl. C), 23-29. doi: 10.1016/j.jad.2016.03.007

*Holahan, C. J., Moos, R. H., Moerkbak, M. L., Cronkite, R. C., Holahan, C. K., and Kenney, B. A. (2007). Spousal similarity in coping and depressive symptoms over 10 years. J. Family Psychol. 21, 551-559. doi: 10.1037/0893-3200.21.4.551

Iafrate, R., Bertoni, A., Margola, D., Cigoli, V., and Acitelli, L. K. (2012). The link between perceptual congruence and couple relationship satisfaction in dyadic coping. Eur. Psychol. 17, 73-82. doi: 10.1027/1016-9040/a000069

Joekes, K., Maes, S., and Warrens, M. (2007). Predicting quality of life and self-management from dyadic support and overprotection after myocardial infarction. Br. J. Health Psychol. 12, 473-489. doi: 10.1348/135910706X118585

Johnson, M. D., Anderson, J. R., Walker, A., Wilcox, A., Lewis, V. L., and Robbins, D. C. (2014). Spousal protective buffering and type 2 diabetes outcomes. Health Psychol. 33, 841-844. doi: 10.1037/hea0000054

Johnson, M. D., Anderson, J. R., Walker, A., Wilcox, A., Lewis, V. L., and Robbins, D. C. (2015). Spousal overprotection is indirectly associated with poorer dietary adherence for patients with type 2 diabetes via diabetes distress when active engagement is low. Br. J. Health Psychol. 20, 360-373. doi: 10.1111/bjhp.12105

Johnson, M. D., and Horne, R. M. (2016). Temporal ordering of supportive dyadic coping, commitment, and willingness to sacrifice. Family Relat. 65, 314-326. doi: 10.1111/fare.12187

Johnson, M. D., Horne, R. M., and Galovan, A. M. (2016). The developmental course of supportive dyadic coping in couples. Dev. Psychol. 52, 2031-2043. doi: $10.1037 / \mathrm{dev} 0000216$

Kayser, K., Cheung, P. K., Rao, N., Chan,Y. C., Chan, Y., and Lo, P. H. (2014). The influence of culture on couples coping with breast cancer: a comparative analysis of couples from China, India, and the United States. J. Psychosoc. Oncol. 32, 264-288. doi: 10.1080/07347332.2014.897292

Kayser, K., and Revenson, T. A. (2016). "Including the cultural context in dyadic coping: directions for future research and practice," in Couples Coping With Stress: A Cross-Cultural Perspective, eds M. K. Falconier, A. K. Randall, and G. Bodenmann (New York, NY: Routledge/Taylor and Francis Group), 285-299.

Kayser, K., Watson, L. E., and Andrade, J. T. (2007). Cancer as a "We-disease": examining the process of coping from a relational perspective. Families Systems Health 25:404. doi: 10.1037/1091-7527.25.4.404

Kent de Grey, R., Uchino, B. N., Trettevik, R., Cronan, S., and Hogan, J. (2018). Social support. Oxford Bibliogr. Pscychol. 2018:204. doi: 10.1093/obo/9780199828340-0204

Körner, A., Würz, J., Brosseau, D. C., Brähler, E., Kapellen, T., and Kiess, W. (2013). Parental dyadic coping in families of children and adolescents with type 1 diabetes. J. Pediatr. Endocrinol. Metabol. 26, 867-875. doi: 10.1515/jpem-2012-0410

Kovacs, M., and Beck, A. T. (1978). Maladaptive cognitive structures in depression. Am. J. Psychiatry 135, 525-533. doi: 10.1176/ajp.135.5.525

Kraemer, L. M., Stanton, A. L., Meyerowitz, B. E., Rowland, J. H., and Ganz, P. A. (2011). A longitudinal examination of couples' coping strategies as predictors of adjustment to breast cancer. J. Family Psychol. 25, 963-972. doi: $10.1037 / \mathrm{a} 0025551$

Kramer, B. J. (1993). Expanding the conceptualization of caregiver coping: the importance of relationship-focused coping strategies. Family Relat. 42, 383-391. doi: 10.2307/585338

Kramer, U., Ceschi, G., Van der Linden, M., and Bodenmann, G. (2005). Individual and dyadic coping strategies in the aftermath of a traumatic experience. Swiss J. Psychol. 64, 241-248. doi: 10.1024/1421-0185.64.4.241

Kuhn, R., Milek, A., Meuwly, N., Bradbury, T. N., and Bodenmann, G. (2017). Zooming in: a microanalysis of couples' dyadic coping conversations 
after experimentally induced stress. J. Family Psychol. 31, 1063-1073. doi: 10.1037/fam0000354

Kuijer, R. G., Ybema, J. F., Buunk, B. P., De Jong, G. M., Thijs-Boer, F., and Sanderman, R. (2000). Active engagement, protective buffering, and overprotection: three ways of giving support by intimate partners of patients with cancer. J. Soc. Clin. Psychol. 19, 256-275. doi: 10.1521/jscp.2000.19.2.256

Kurosawa, T., Kato, M., and Kamiya, T. (2015). Relationship-focused coping patterns of Japanese child-rearing couples. J. Relat. Res. 6, 1-8. doi: 10.1017/jrr.2015.3

Lambert, J. E., Hasbun, A., Engh, R., and Holzer, J. (2015). Veteran PTSS and spouse relationship quality: the importance of dyadic coping. Psychol. Trauma 7, 493-499. doi: 10.1037/tra0000036

Landis, M., Bodenmann, G., Bradbury, T. N., Brandstätter, V., Peter-Wight, M., Backes, S., et al. (2014). Commitment and dyadic coping in longterm relationships. J. Gerontopsychol. Geriatr. Psychiatry 27, 139-149. doi: 10.1024/ 1662-9647/a000112

Landis, M., Peter-Wight, M., Martin, M., and Bodenmann, G. (2013). Dyadic coping and marital satisfaction of older spouses in long-term marriage. J. Gerontopsychol. Geriatr. Psychiatry 26, 39-47. doi: 10.1024/1662-9647/ a000077

Langer, S. L., Brown, J. D., and Syrjala, K. L. (2009). Intrapersonal and interpersonal consequences of protective buffering among cancer patients and caregivers. Cancer 115, 4311-4325. doi: 10.1002/cncr.24586

*Langer, S. L., Rudd, M. E., and Syrjala, K. L. (2007). Protective buffering and emotional desynchrony among spousal caregivers of cancer patients. Health Psychol. 26, 635-643. doi: 10.1037/0278-6133.26.5.635

Lavery, J. F., and Clarke, V. A. (1999). Prostate cancer: patients' and spouses' coping and marital adjustment. Psychol. Health Med. 4, 289-302. doi: 10.1080/ 135485099106225

Lazarus, R. S., and Folkman, S. (1984). Stress, Appraisal, and Coping. New York, NY: Springer Publishing.

Ledermann, T., Bodenmann, G., Gagliardi, S., Charvoz, L., Verardi, S., Rossier, J., et al. (2010). Psychometrics of the dyadic coping inventory in three language groups. Swiss J. Psychol. 69, 201-212. doi: 10.1024/1421-0185/a000024

Lee-Baggley, D., Preece, M., and DeLongis, A. (2005). Coping with interpersonal stress: the role of big five traits. J. Personal. 73, 1141-1180. doi: 10.1111/j.1467-6494.2005.00345.x

Levesque, C., Lafontaine, M.-F., Caron, A., and Fitzpatrick, J. (2014a). Validation of the English version of the dyadic coping inventory. Meas. Eval. Counsel. Dev. 47, 215-225. doi: 10.1177/0748175614522272

Levesque, C., Lafontaine, M.-F., Caron, A., Flesch, J. L., and Bjornson, S. (2014b). Dyadic empathy, dyadic coping, and relationship satisfaction: a dyadic model. Eur. J. Psychol. 10, 118-134. doi: 10.5964/ejop.v10i1.697

Lewis, M. A., McBride, C. M., Pollak, K. I., Puleo, E., Butterfield, R. M., and Emmons, K. M. (2006). Understanding health behavior change among couples: an interdependence and communal coping approach. Soc. Sci. Med. 62, 1369-1380. doi: 10.1016/j.socscimed.2005.08.006

Lin, W., Chen, L. H., and Li, T. (2016). Are "we" good? A longitudinal study of wetalk and stress coping in dual-earner couples. J. Happiness Stud. 17, 757-772. doi: 10.1007/s10902-015-9621-0

Lyons, K. S., Miller, L. M., and McCarthy, M. J. (2016). The roles of dyadic appraisal and coping in couples with lung cancer. J. Family Nurs. 22, 493-514. doi: $10.1177 / 1074840716675976$

Lyons, R. F., Mickelson, K. D., Sullivan, M. J. L., and Coyne, J. C. (1998). Coping as a communal process. J. Soc. Pers. Relat. 15, 579-605. doi: $10.1177 / 0265407598155001$

*Magsamen-Conrad, K., Checton, M. G., Venetis, M. K., and Greene, K. (2015). Communication efficacy and couples' cancer management: applying a dyadic appraisal model. Commun. Monogr. 82, 179-200. doi: 10.1080/03637751.2014.971415

Manne, S., Audrain, J., Schwartz, M., Main, D., Finch, C., and Lerman, C. (2004). Associations between relationship support and psychological reactions of participants and partners to BRCA1 and BRCA2 testing in a clinicbased sample. Ann. Behav. Med. 28, 211-225. doi: 10.1207/s15324796abm $2803 \_10$

Manne, S., Badr, H., and Kashy, D. A. (2012). A longitudinal analysis of intimacy processes and psychological distress among couples coping with head and neck or lung cancers. J. Behav. Med. 35, 334-346. doi: 10.1007/s10865-011-9349-1
Manne, S., Dougherty, J., Veach, S., and Kless, R. (1999). Hiding worries from one's spouse: protective buffering among cancer patients and their spouses. Cancer Res. Ther. Control 8, 175-188.

Manne, S. L., Norton, T. R., Ostroff, J. S., Winkel, G., Fox, K., and Grana, G. (2007). Protective buffering and psychological distress among couples coping with breast cancer: the moderating role of relationship satisfaction. J. Family Psychol. 21, 380-388. doi: 10.1037/0893-3200.21.3.380

Marin, T. J., Holtzman, S., DeLongis, A., and Robinson, L. (2007). Coping and the response of others. J. Soc. Pers. Relat. 24, 951-969. doi: $10.1177 / 0265407507084192$

Meier, C., Bodenmann, G., Moergeli, H., Peter-Wight, M., Martin, M., Buechi, S., et al. (2012). Dyadic coping among couples with COPD: a pilot study. J. Clin. Psychol. Med. Settings 19, 243-254. doi: 10.1007/s10880-011-9279-7

Meier, C., Bodenmann, G., Morgeli, H., and Jenewein, J. (2011). Dyadic coping, quality of life, and psychological distress among chronic obstructive pulmonary disease patients and their partners. Int. J. Chronic Obstruct. Pulmonary Dis. 6 , 583-595. doi: 10.2147/COPD.S24508

Merz, C. A., Meuwly, N., Randall, A. K., and Bodenmann, G. (2014). Engaging in dyadic coping: buffering the impact of everyday stress on prospective relationship satisfaction. Family Sci. 5, 30-37. doi: 10.1080/19424620.2014.927385

Meuwly, N., Bodenmann, G., Germann, J., Bradbury, T. N., Ditzen, B., and Heinrichs, M. (2012). Dyadic coping, insecure attachment, and cortisol stress recovery following experimentally induced stress. J. Family Psychol. 26, 937-947. doi: 10.1037/a0030356

Meuwly, N., Feinstein, B. A., Davila, J., Nuñez, D. G., and Bodenmann, G. (2013). Relationship quality among Swiss women in opposite-sex versus same-sex romantic relationships. Swiss J. Psychol. 72, 229-233. doi: 10.1024/1421-0185/a000115

Meyer, J., Bodenmann, G., Binz, G., and Brunner, L. (2005). Subjektiv eingeschätzte partnerschaftliche Kommunikationsqualität in fünf Alterskohorten [Subjectively rated relationship communication quality in five age cohorts]. Zeitschr. Klin. Psychol. Psychiatr. Psychother. 53, 342-355.

Nussbeck, F. W., Hilpert, P., and Bodenmann, G. (2012). Der Zusammenhang von positivem und negative Interaktionsverhalten in der Partnerschaft mit Partnerschaftszufriedenheit und Trennungsabsichten [The association between positive and negative interaction behavior with relationship satisfaction and the intention to separate in married couples]. J. Family Res. 24, 85-104.

O'Brien, T. B., and DeLongis, A. (1996). The interactional context of problem-, emotion-, and relationship-focused: the role of the big five personality factors. J. Personal. 64, 775-813. doi: 10.1111/j.1467-6494.1996.tb00944.x

O’Brien, T. B., DeLongis, A., Pomaki, G., Puterman, E., and Zwicker, A. (2009). Couples coping with stress: the role of empathic responding. Eur. Psychol. 14, 18-28. doi: 10.1027/1016-9040.14.1.18

Pakenham, K. I. (1998). Couple coping and adjustment to multiple sclerosis in care receiver-carer dyads. Family Relat. 47, 269-277. doi: 10.2307/584977

Papp, L. M., and Witt, N. L. (2010). Romantic partners' individual coping strategies and dyadic coping: implications for relationship functioning. J. Family Psychol. 24, 551-559. doi: 10.1037/a0020836

Pennebaker, J. W., Booth, R. J., and Francis, M. E. (2007). Linguistic Inquiry and Word Count: LIWC [Computer Software]. Austin, TX: LIWC.net.

Randall, A. K., Hilpert, P., Jimenez-Arista, L. E., Walsh, K. J., and Bodenmann, G. (2015). Dyadic coping in the U.S.: psychometric properties and validity for use of the English version of the dyadic coping inventory. Curr. Psychol. 35, 570-582. doi: 10.1007/ s12144-015-9323-0

Reed, R. G., O'Connor, M.-F., Pace, T. W. W., Raison, C. L., and Butler, E. A (2016). Dyadic coping and salivary interleukin-6 responses to interpersonal stress. J. Family Psychol. 31, 367-373. doi: 10.1037/fam0000249

Revenson, T. A. (1994). Social support and marital coping with chronic illness Ann. Behav. Med. 16, 122-130.

Robbins, M. L., Mehl, M. R., Simith, H. L., and Weihs, K. L. (2013). Linguistic indicators of patient, couple, and family adjustment following breast cancer. Psycho-oncology 22, 1501-1508. doi: 10.1002/pon.3161

Rogers, A. J., Achiro, L., Bukusi, E. A., Hatcher, A. M., Kwena, Z., Musoke, P. L., et al. (2016). Couple interdependence impacts HIVrelated health behaviors among pregnant couples in southwestern Kenya: a qualitative analysis. J. Int. AIDS Soc. 19, 214-224. doi: 10.7448/IAS.19.1. 21224 
Rohrbaugh, M. J., Mehl, M. R., Varda, S., Reilly, E. S., and Ewy, G. A. (2008). Prognostic significance of spouse we talk in couples coping with heart failure. J. Consult. Clin. Psychol. 78, 781-789. doi: 10.1037/a0013238

Rohrbaugh, M. J., Shoham, V., Skoyen, J. A., Jensen, M., and Mehl, M. R. (2012). We-talk, communal coping, and cessation success in a couple-focused intervention for health-compromised smokers. Family Process 51, 107-121. doi: 10.1111/j.1545-5300.2012.01388.x

Rottmann, N., Hansen, D. G., Larsen, P. V., Nicolaisen, A., Flyger, H., Johansen, C., et al. (2015). Dyadic coping within couples dealing with breast cancer: a longitudinal, population-based study. Health Psychol. 34, 486-495. doi: $10.1037 /$ hea0000218

Ruffieux, M., Nussbeck, F. W., and Bodenmann, G. (2014). Long-term prediction of relationship satisfaction and stability by stress, coping, communication, and well-Being. J. Divorce Remarriage 55, 485-501. doi: 10.1080/10502556.2014.931767

Rusu, P. P., Hilpert, P., Beach, S. R. H., Turliuc, M. N., and Bodenmann, G. (2015). Dyadic coping mediates the association of sanctification with marital satisfaction and well-being. J. Family Psychol. 29, 843-849. doi: 10.1037/fam0000108

Rusu, P. P., Hilpert, P., and Bodenmann, G. (2016). Dyadic coping in an Eastern European context: validity and measurement invariance of the Romanian version of the Dyadic Coping Inventory. Measur. Eval. Counsel. Dev. 49, 274-285. doi: 10.1177/0748175616664009

*Salazar, L. F., Stephenson, R. B., Sullivan, P. S., and Tarver, R. (2013). Development and validation of HIV-related dyadic measures for men who have sex with men. J. Sex Res. 50, 164-177. doi: 10.1080/00224499.2011.636845

*Schär, M., and Bodenmann, G. (2011). Behandlungsverlauf bei depressiven Störungen: intrapsychische und interpersonelle Prädiktoren des Patienten und dessen Partner [Changes in symptoms during treatment of depression: intrapersonal and interpersonal predictors of the patient and spouse]. Zeitschr. Klin. Psychol. Psychother. 40, 42-52. doi: 10.1026/1616-3443/a000069

*Schindler, I., Berg, C. A., Butler, J. M., Fortenberry, K. T., and Wiebe, D. J. (2010). Late-midlife and older couples' shared possible selves and psychological well-being during times of illness: the role of collaborative problem solving. J. Gerontol. Ser. B 65B, 416-424. doi: 10.1093/geronb/gbq030

Schokker, M. C., Stuive, I., Bouma, J., Keers, J. C., Links, T. P., Wolffenbuttel, B. H. R., et al. (2010). Support behavior and relationship satisfaction in couples dealing with diabetes: main and moderating effects. J. Family Psychol. 24, 578-586. doi: 10.1037/a0021009

Snippe, E., Maters, G. A., Wempe, J. B., Hagedoorn, M., and Sanderman, R. (2012). Discrepancies between patients' and partners' perceptions of unsupportive behavior in chronic obstructive pulmonary disease. J. Family Psychol. 26, 464-469. doi: 10.1037/a0028333

Sormanti, M., Kayser, K., and Strainchamps, E. (1997). A relational perspective of women coping with cancer: a preliminary study. Soc. Work Health Care 25, 89-106. doi: 10.1300/J010v25n01_10

*Stachowski, C., and Stephenson, R. (2015). Homophobia and communal coping for HIV risk management among gay men in relationships. Arch. Sex. Behav. 44, 467-474. doi: 10.1007/s10508-014-0417-9

Staff, H. R., Didymus, F. F., and Backhouse, S. H. (2017). The antecedents and outcomes of dyadic coping in close personal relationships: a systematic review and narrative synthesis. Anxiety Stress Coping 30, 498-520. doi: 10.1080/10615806.2017.1329931

Suls, J., Green, P., Rose, G., Lounsbury, P., and Gordon, E. (1997). Hiding worries from one's spouse: associations between coping via protective buffering and distress in male post-myocardial infarction patients and their wives. J. Behav. Med. 20, 333-349. doi: 10.1023/A:1025513029605

Traa, J. M., De Vries, J., Bodenmann, G., and Den Oudsten, B. L. (2015). Dyadic coping and relationship functioning in couples coping with cancer: a systematic review. Br. J. Health Psychol. 20, 85-114. doi: 10.1111/bhjp.12094

* Studies included in the Summary of Findings Table as online supplementary material but not in the manuscript
Vaske, I., Thöne, M. F., Kühl, K., Keil, D. C., Schürmann, W., Rief, W., et al. (2015). For better or for worse: a longitudinal study on dyadic coping and quality of life among couples with a partner suffering from COPD. J. Behav. Med. 38, 851-862. doi: 10.1007/s10865-015-9657-y

Vedes, A., Hilpert, P., Nussbeck, F. W., Randall, A. K., Bodenmann, G., and Lind, W. R. (2016). Love styles, coping, and relationship satisfaction: a dyadic approach: love styles and dyadic coping. Pers. Relat. 23, 84-97. doi: 10.1111 /pere. 12112

Vedes, A., Nussbeck, F. W., Bodenmann, G., Lind, W., and Ferreira, A. (2013). Psychometric properties and validity of the dyadic coping inventory in Portuguese. Swiss J. Psychol. 72, 149-157. doi: 10.1024/1421-0185/ a000108

Vilchinsky, N., Dekel, R., Leibowitz, M., Reges, O., Khaskia, A., and Mosseri, M. (2011). Dynamics of support perceptions among couples coping with cardiac illness: the effect on recovery outcomes. Health Psychol. 30, 411-419. doi: $10.1037 / \mathrm{a} 0023453$

Vitaliano, P. P., Russo, J., Carr, J. E., Maiuro, R. D., and Becker, J. (1985). The ways of coping checklist: revision and psychometric properties. Multivar. Behav. Res. 20,3-26. doi: 10.1207/s15327906mbr2001_1

Watts, K. J., Sherman, K. A., Mireskandari, S., Meiser, B., Taylor, A., and Tucker, K. (2011). Predictors of relationship adjustment among couples coping with a high risk of developing breast/ovarian cancer. Psychol. Health 26, 21-39. doi: 10.1080/08870441003592587

Weißflog, G., Hönig, K., Gündel, H., Lang, D., Niederwieser, D., Döhner, H., et al. (2016). Associations between dyadic coping and supportive care needs: findings from a study with hematologic cancer patients and their partners. Support. Care Cancer 25, 1445-1454. doi: 10.1007/s00520-0163541-3

Witkovsky, A., and Braakmann, D. (2015). Die Bedeutung partnerschaftlicher Stressbewältigung für die Beziehungsqualität und die posttraumatische Symptomausprägung [The importance of coping strategies for relationship quality and posttraumatic symptom severity]. Zeitschr. Klin. Psychol. Psychother. 4, 17-26. doi: 10.1026/1616-3443/a000285

Wunderer, E., and Schneewind, K. A. (2008). The relationship between marital standards, dyadic coping and marital satisfaction. Eur. J. Soc. Psychol. 38, 462-476. doi: 10.1002/ejsp.405

Xu, F., HIlpert, P., Randall, A. K., Li, Q., and Bodenmann, G. (2016). Validation of the dyadic coping inventory with chinese couples: factorial structure, measurement invariance, and construct validity. Psychol. Assess. 8, 127-140. doi: $10.1037 /$ pas0000329

Yokotani, K., and Kurosawa, T. (2015). A pilot examination of dyadic coping inventory among Japanese married couples. Psychologia 58, 155-164. doi: $10.2117 /$ psysoc.2015.155

Zeidner, M., Kloda, I., and Matthews, G. (2013). Does dyadic coping mediate the relationship between emotional intelligence (EI) and marital quality? J. Family Psychol. 27, 795-805. doi: 10.1037/a0034009

Zemp, M., Bodenmann, G., Backes, S., Sutter-Stickel, D., and Revenson, T. (2016). The importance of parents' dyadic coping for children. Family Relat. 65, 275-286. doi: 10.1111/fare.12189

Zimmermann, T., Scott, J. L., and Heinrichs, N. (2010). Individual and dyadic predictors of body image in women with breast cancer. Psycho-Oncology 19, 1061-1068. doi: 10.1002 /pon. 1660

Conflict of Interest Statement: The authors declare that the research was conducted in the absence of any commercial or financial relationships that could be construed as a potential conflict of interest.

Copyright (c) 2019 Falconier and Kuhn. This is an open-access article distributed under the terms of the Creative Commons Attribution License (CC BY). The use, distribution or reproduction in other forums is permitted, provided the original author(s) and the copyright owner(s) are credited and that the original publication in this journal is cited, in accordance with accepted academic practice. No use, distribution or reproduction is permitted which does not comply with these terms. 


\section{OPEN ACCESS}

Edited by:

Changiz Mohiyeddini,

Northeastern University, United States

Reviewed by:

Noa Vilchinsky,

Bar-Ilan University, Israel Kai K. Kummer,

Innsbruck Medical University, Austria

${ }^{*}$ Correspondence: Kevin K. H. Lau

khlau2@asu.edu

Specialty section:

This article was submitted to Clinical and Health Psychology, a section of the journal

Frontiers in Psychology

Received: 21 June 2018 Accepted: 03 December 2018

Published: 15 January 2019

Citation:

Lau KKH, Randall AK, Duran ND

and Tao C (2019) Examining the Effects of Couples' Real-Time Stress and Coping Processes on Interaction Quality: Language Use as a Mediator. Front. Psychol. 9:2598. doi: 10.3389/fpsyg.2018.02598

\section{Examining the Effects of Couples' Real-Time Stress and Coping Processes on Interaction Quality: Language Use as a Mediator}

\author{
Kevin K. H. Lau' ${ }^{1 *}$, Ashley K. Randall', Nicholas D. Duran ${ }^{2}$ and Chun Tao ${ }^{1}$ \\ ' Couples Coping with Stress Laboratory, Counseling and Counseling Psychology, College of Integrative Sciences and Arts, \\ Arizona State University, Tempe, AZ, United States, ${ }^{2}$ DynamiCog Laboratory, School of Social and Behavioral Sciences, \\ Arizona State University, Glendale, AZ, United States
}

Stress in romantic relationships is an all-too-common phenomenon that has detrimental effects on relationship well-being. Specifically, stress can lead to negative interactions between partners and ultimately decrease relationship functioning. The systemictransactional model of dyadic coping posits that by effectively communicating stress and coping with one's romantic partner, couples can mitigate the deleterious effects of stress. Specifically, partners can engage in positive dyadic coping, which may foster couples' sense of "we-ness," strengthen their emotional connection, and facilitate their understanding of each other's stressful experiences. However, these associations have not yet been examined during partners' real-time stress conversations. When assessing dyadic coping, a particular aspect of interest is partners' language use (i.e., pronouns, emotion words, and cognition words), as it may reflect the types of support they communicate to one another. Using real-time interaction data from 41 heterosexual couples, this study examined how couples' stress and coping processes affect perceived interaction quality following discussions of stress. Specifically, language use (i.e., pronouns, emotion words, and cognition words) was assessed as a mediator on the association between observed stress communication and perceived interaction quality. Overall, results supported our hypotheses; when one partner communicated stress, the other partner responded with language use indicative of different types of dyadic coping (i.e., more you-talk and use of emotion words, less we-talk, l-talk, and use of cognition words), which were in turn associated with interaction quality in mixed directions. Implications of these findings for romantic couples are discussed.

Keywords: systemic-transactional model, language use, interaction quality, real-time interaction data, stress, romantic relationships

\section{INTRODUCTION}

Romantic partners' experiences of stress can be detrimental to their relationship. In particular, effects of external stress (stress originating outside the relationship, such as stress from work or friends) can spill over into the relationship and create tension between partners (Bolger et al., 1989; Ledermann et al., 2010). Moreover, higher levels of external stress have been found to be associated 
with lower levels of relationship satisfaction (Bodenmann, 1997; Randall and Bodenmann, 2009; Randall and Bodenmann, 2017), as well as greater levels of relationship conflict (Bahr, 1979; Lavee et al., 1987; Neff and Karney, 2004). The systemic-transactional model of dyadic coping posits that partners can mitigate the deleterious effects of stress by effectively communicating and engaging in positive dyadic coping (Bodenmann, 1995, 1997, 2005). The benefits of positive dyadic coping have been welldocumented in the literature (see Falconier et al., 2015, for a meta-analytic review); however, there is a dearth of literature on understanding how couples' stress and coping processes may unfold in real-time conversations.

Previous studies examining the associations between couples' stress and coping mechanisms on relationship functioning have largely depended on self-report assessments, which may be unreliable as partners may provide biased responses (O'Brien et al., 1994). In addition, self-report measures may not account for the intricate communication processes that occur as partners' conversations unfold in time (e.g., explicit messages indicating how stress is impacting the partner, implicit stress communication via facial expressions). As such, more objective ways to measure partners' real-time communication, such as behavioral coding and linguistic analysis, are needed.

Behavioral coding may be helpful in assessing partners' stress communication by providing a systematic and objective set of codes that are assigned to partners based on their overt behaviors (Margolin et al., 1998). The coding scheme may also account for the various ways in which partners can communicate different types of stress (e.g., general, emotion-focused, problem-focused). Moreover, partners' language, in particular the pronouns or words (e.g., emotion- or cognition-related words) they use, may reflect engagement in dyadic coping. Given that unique patterns of language use have been associated with greater relationship functioning (e.g., Borelli et al., 2013; Rentscher et al., 2013), it is likely that when partners engage in dyadic coping, they may also use more supportive language (e.g., positive emotion words).

Existing literature illustrates positive links between dyadic coping and general relationship functioning (e.g., Bodenmann et al., 2006; Ledermann et al., 2010), but findings on the association between dyadic coping and partners' perceptions of specific conversations they have regarding their experiences of stress remain limited. As such, it is unclear how couples' stress and coping processes during momentary interactions may contribute to their overall relationship well-being. Bodenmann's (2000) stress-divorce model suggests that decreases in interaction quality may help explain the negative association between external stress and relationship satisfaction. Thus, when partners are able to effectively convey stress and support to each other in their conversations, they may view their interactions as more positive, which over time could improve their relationship satisfaction; however, this has yet to be examined.

Taken together, to our knowledge, no research to date has explored couples' real-time stress and coping processes at the conversational level, and how these interactions may contribute to partners' perceptions of interaction quality. As such, important gaps in the well-documented and empirically supported systemic transactional model of dyadic coping (Bodenmann, 1995,
1997, 2005) remain largely unexamined. To address this gap in the literature, this study examines associations between stress communication, language use, and interaction quality in discussions about stress using real-time interaction data from heterosexual couples.

\section{Systemic-Transactional Model of Dyadic Coping}

Stress can be conceptualized on a number of dimensions, which includes its origin (see Randall and Bodenmann, 2009 for a review). Stress that originates outside the relationship (i.e., external stress), such as work and finances, can negatively impact a partner's perception of stress within the relationship through stress Spillover (Bolger et al., 1989; Repetti and Wood, 1997). Stress Spillover occurs when one partner experiences external stress, and the emotions associated with that stress are carried over into the relationship, impeding positive interactions and effective stress communication between the partners. Stress communication refers to how partners convey and understand each other's stressful experiences (Bodenmann, 1995, 1997, 2005). For example, following the experience of a bad interaction with a friend, one partner may come home to express their stress to the other partner, which then draws the other partner into the coping process. Importantly, a partner may communicate their stress nonverbally (e.g., through signs of discomfort or frustration) or verbally. In this study, we focus specifically on verbal stress communication.

Verbal stress communication can be categorized into three types: (1) general or neutral explanation of stress (i.e., describing only the facts of a stressful situation without conveying emotion, offering or seeking advice), (2) emotionfocused stress communication (i.e., highlighting the emotional effect of a stressor and/or describing felt emotions), and (3) problem-focused stress communication (i.e., focusing on tangible solutions to stressors and/or soliciting practical advice from the partner). Emotion-focused stress communication can be further distinguished based on implicit or explicit emotions. Implicit emotion-focused stress communication refers to when partners discuss their stress in a vague manner, without mentioning specific emotions (e.g., "Work is making me stressed," "My friends made me feel bad"), whereas explicit emotion-focused stress communication occurs when partners identify specific emotions (e.g., "I am angry at my parents," "I am scared that I will run out of money"). Prior research has examined stress communication in the form of willingness to disclose, and results suggest that effective communication is positively associated with relationship satisfaction (e.g., Meeks et al., 1998; MacNeil and Byers, 2005; Smith et al., 2008; Montesi et al., 2011). This suggests that when partners allow themselves to be open in their conversations with each other, they may become more satisfied with their partners and in their relationships. Despite these results and the robust evidence for interpersonal communication, partners' stress communication, as conceptualized by the systemic transactional model (Bodenmann, 2005), have not yet been explicitly examined. 
Another important element to the perception of interaction quality is the response from one's interaction partner following the stress disclosure. For instance, if one partner self-discloses an upsetting experience at work and their partner responds negatively, then the stressed partner may perceive the quality of the interaction as negative (as opposed to positive). As such, it is important to also consider how partners respond to each other's stress communication and how it may contribute to the overall interaction quality.

Following the communication of one partner's stress, the other partner then responds in order to help mitigate (or exacerbate, in the case of negative dyadic coping) the stressed partner's experience (Bodenmann, 2005). Positive dyadic coping can take one of three forms: (1) emotion-focused dyadic coping (i.e., providing emotional and empathic support), (2) problem-focused dyadic coping (i.e., providing partner with new perspectives and practical solutions), and (3) delegated dyadic coping (i.e., taking on extra responsibilities so that the partner's workload is lessened). In this study, we focused on the verbal exchange between partners and therefore only examined emotion- and problem-focused dyadic coping, as they may be expressed verbally, as opposed to delegated dyadic coping, which may be more likely to be exhibited via action. Partners can also engage in negative dyadic coping (e.g., mock or invalidate partner's feelings, provide insincere support). Higher levels of perceived positive dyadic coping and lower levels of negative dyadic coping have been found to be positively associated with reduced stress (Ledermann et al., 2010), and greater relationship quality (Bodenmann et al., 2006, 2011).

An important aspect of dyadic coping is the language that partners use with each other, given that partner's words may facilitate the communication of the various types of support outlined above. Specifically, the use of pronouns may be salient to examine in partners' engagement of any form of positive dyadic coping, emotion words in emotion-focused dyadic coping, and cognition words in problem-focused dyadic coping.

\section{Language Use and Dyadic Coping}

One central aspect of the engagement in positive dyadic coping is that it can foster couples' sense of "we-ness" or cohesion between partners (Bodenmann, 2005). The extent to which partners view themselves as a close, intimate unit may be reflected in their use of pronouns, which represents the partner's attentional focus and identification (Tausczik and Pennebaker, 2010). For instance, partners who view the relationship as cohesive may be more likely to highlight the interdependence by using more plural, firstperson pronouns like "we" and "us." They may also conceptualize each other's external stress as "our" stress, an issue that they must work together in order to combat. Consistent with positive dyadic coping, it has been found that greater use of plural, personal pronouns (i.e., we-talk) is positively associated with relationship satisfaction (Borelli et al., 2013) and communication quality (Biesen et al., 2015).

Conversely, partners who do not view themselves as cohesive may highlight their individual identities by using more singular pronouns, such as "I" and "you." There is evidence suggesting that the use of singular, first-person pronouns (i.e., I-talk) is negatively correlated with relationship quality (Slatcher et al., 2008; Rentscher et al., 2013). Further, the use of singular secondperson pronouns (i.e., you-talk) negatively predicts interaction quality in couples (Biesen et al., 2015). Partners' use of youtalk may communicate distance between partners, and further, indicate blame and criticism (e.g., "You never do the dishes"). Therefore, when partners engage in any form of positive dyadic coping, they may use fewer "I" and "you" pronouns because they communicate separation between partners, which could lead to higher perceived interaction quality.

Emotion words (e.g., happy, sad, excited, and anxious) can convey individuals' emotional responses and level of immersion to certain experiences (Tausczik and Pennebaker, 2010). Individuals may use emotion words to describe whether they feel positively about an experience via words like "cheerful," and "joy," or negatively with words like "hate" and "hurt." In addition, when an individual feels burdened by a specific stressor he/she may be more willing to verbally express feelings. The use of emotion words between romantic partners has been found to be positively associated with relationship satisfaction (Slatcher and Pennebaker, 2006) as well as relationship adjustment (Baddeley and Pennebaker, 2011).

Applied to the systemic-transactional model (Bodenmann, 1995, 1997, 2005), partners' use of positive emotion words during the expression of emotion-focused dyadic coping may help partners express their feelings to one another. In the context of couples' stress-related conversations, the supporting partner's use of positive emotion words may provide encouragement and support for the stressed partner, which in turn may impact their partner's perception of the interaction. Conversely, the use of negative emotion words has not been extensively examined in the literature. Despite the lack of literature, the use of negative emotion words could be easily be argued to yield a negative perception of the interaction.

Cognition words refer to words that illustrate the processing and interpretation of information (e.g., because, know, and think). The use of cognition words has been found to benefit individuals' recounts of past stressful events, leading to more positive mental health outcomes (Cordova et al., 2001; Boals and Klein, 2005). The use of cognition words may reflect an attempt at deeper understanding of stressors, which could be considered as an effective coping mechanism. Applied to the systemic transactional model (Bodenmann, 1995, 1997, 2005), when partners engage in problem-focused dyadic coping, they may try to understand and make meaning out of each other's experiences; thus, they may use more words that indicate cognitive processing. As outlined above, communication about stress and coping could have positive effects on partners' perceptions of their interactions.

\section{Associations Between Stress Communication, Dyadic Coping, and Interaction Quality}

Communication quality is positively associated with relationship satisfaction (Litzinger and Gordon, 2005). Further, Carroll 
et al. (2013) found that, in a sample of 1,117 married individuals who were employed full-time employees, the use of constructive communication mediated the association between work-family conflict and relationship satisfaction. However, to our knowledge, no research has examined partners' perceptions of their interactions immediately following their conversations about stress.

Cross-sectional research on dyadic coping has found a robust association between perceptions of partners' dyadic coping and perceived well-being, across cultures (Falconier et al., 2016). Despite these well-documented associations, it is still unclear how partners' stress communication and coping processes unfold during real-time. Effective stress communication, and responses of positive dyadic coping, are thought to result in more satisfying interactions, resulting in greater relationship satisfaction over time (Bodenmann, 1995, 1997, 2005); however, examinations on such associations using real-time interaction data is lacking. This manuscript addresses this gap in the literature, which is pertinent to promoting the understanding of the partners' momentary experiences of stress communication and coping processes during real-time interactions.

\section{Present Study}

The present study used real-time interaction data from 41 heterosexual couples' discussions about external stress to examine the mediational effect of partners' language use on the association between observed stress communication and self-reported interaction quality (Figure 1). The use of dyadic data allowed for the examination of both actor (i.e., one partner's independent variable predicting their own outcome variable) and partner (i.e., one partner's independent variable predicting their partner's outcome variable) effects. Given our interest in partners' interactions with each other during their conversations, partner effects were predicted to be more salient than actor effects, because of the transactional nature of stress communication and dyadic coping. Further, the association between stress communication and interaction quality was expected to be more pronounced for the partner who communicates their stress (i.e., assigned to discuss their external stress topic). The following hypotheses $(\mathrm{H})$ were tested:

H1: It was hypothesized that the use of pronouns (e.g., we, I, you) would mediate the association between stress communication and perceived interaction quality. Specifically, when one partner engaged in general stress communication (i.e., neutral explanation of his/her stress), the other partner would respond with greater use of we-talk (H1a) and lower use of I-talk (H1b) and youtalk (H1c), which would then be positively associated with perceived quality of the interaction for the stressed partner.

$\mathrm{H} 2$ : It was hypothesized that the use of emotion words would mediate the association between stress communication and perceived interaction quality. Specifically, when one partner engaged in emotion-focused stress communication, the other partner would respond with more positive emotion words (e.g., happy, cheer, enjoy; $\mathrm{H} 2 \mathrm{a}$ ) and fewer negative emotion words (e.g., sad, angry; $\mathrm{H} 2 \mathrm{~b}$ ), which would then be positively associated with the first partner's perceived quality of the interaction.

H3: It was hypothesized that the use of words related to cognitive processing (e.g., think, believe, and maybe) would mediate the association between stress communication and perceived interaction quality. Specifically, when one partner engaged in problemfocused stress communication (e.g., seeking practical advice or alternative perspective), the other partner would respond with more cognition words, which would then be positively associated with interaction quality for the first partner.

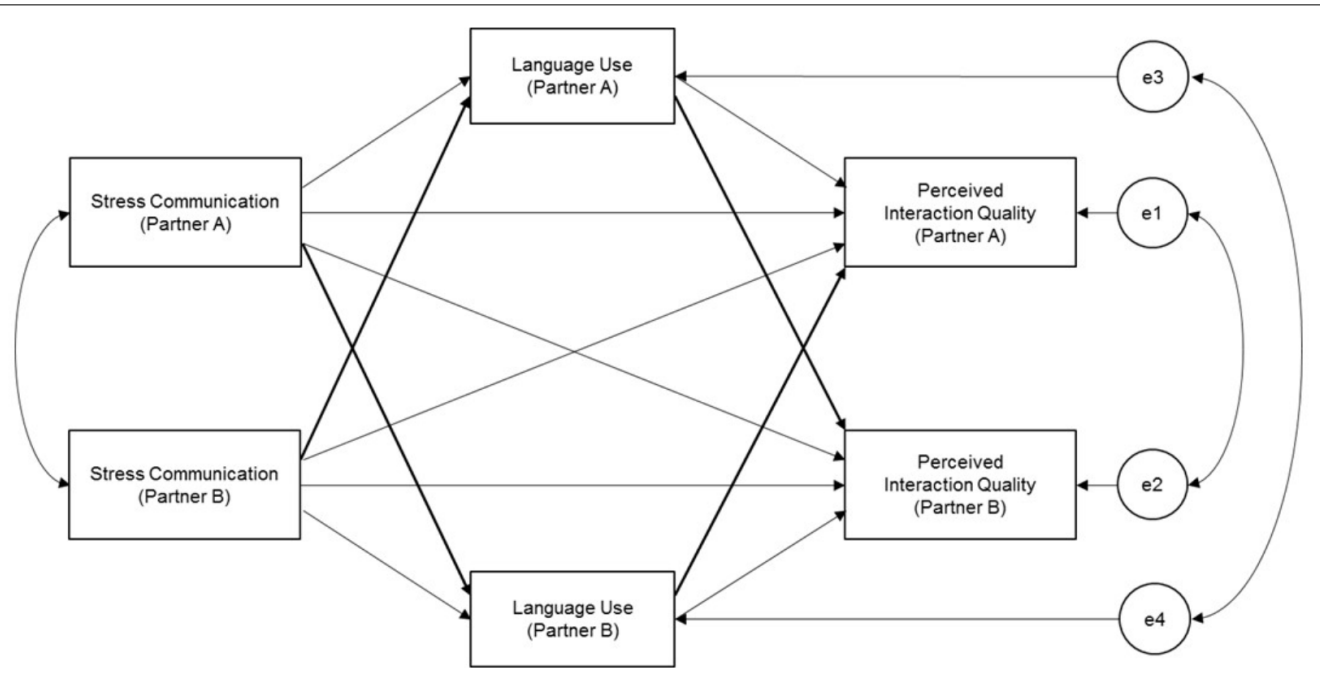

FIGURE 1 | Proposed model of language use (Time 1) mediating the association between stress communication (Time 1) and interaction quality (Time 2). 


\section{MATERIALS AND METHODS}

\section{Participants}

The initial sample for this study consisted of 54 heterosexual couples ( $N=108$ individuals) that completed the baseline questionnaire; however, only 41 couples ( $n=82$ individuals) completed both the baseline questionnaire and participated in the laboratory session; as such, results are based on the 41 couples. The mean age of women was 30.39 years $(S D=7.36)$ and the mean age of men was 30.41 years $(S D=6.87)$. The majority of participants identified as White $(n=58)$, followed by Hispanic $(n=14)$, Asian American $(n=4)$, Native Hawaiian or Pacific Islander $(n=2)$, African American $(n=1)$, and 3 individuals identified with other racial backgrounds. Most participants reported having obtained a terminal college, university, or graduate degree ( $n=38$ women, 29 men). The median range of annual income was $\$ 25,000$ to $\$ 50,000$.

Partners reported being together, on average, for 5.45 years $(S D=5.25)$. Out of the 41 couples, 4 reported that they were in committed relationships and were not cohabiting, 6 indicated that they were in committed relationships and cohabiting, 2 were engaged and not cohabiting, 10 were engaged and cohabiting, and 19 were married. Eleven of the couples reported having children.

\section{Procedure}

This study's procedures were reviewed and approved by the Institutional Review Board at Arizona State University, and all participants consented to participate. Participants were recruited via advertisements posted on Craigslist, Facebook, and electronic mailing lists belonging to various universities and professional organizations in a Southwestern region of the United States.

Data for this study were collected in three parts: (1) a screening survey to ensure couples' eligibility, (2) an initial baseline questionnaire, and (3) a laboratory session. Couples were required to fulfill the following criteria in order to participate: (1) both partners were over the age of 18 ; (2) had been in a relationship with their current partner for at least 6 weeks; and (3) both partners were willing to participate in the study. Eligible participants were electronically sent the baseline questionnaires, which contained demographic measures and measures related to relationship functioning. Participants were instructed to complete the baseline questionnaires separately. The baseline questionnaire took approximately $1 \mathrm{~h}$ to complete.

Following the completion of the baseline questionnaire, participants were scheduled for a laboratory session wherein they were asked to engage in a series of video-taped conversations. Each couple was asked to have a conversation regarding a source of external stress (i.e., originating outside of the relationship), internal stress (i.e., coming from within the relationship), and a topic of enjoyment. Each video-taped conversation lasted for $6 \mathrm{~min}$, and couples saw a message on a screen in the laboratory alerting them that they have $1 \mathrm{~min}$ left for the conversation. For the present study, only the discussions about external stress were used based on previous evidence indicating that external stress strongly predicts relationship outcomes (e.g., Story and Repetti, 2006; Bodenmann et al.,
2007; Randall and Bodenmann, 2009; Randall and Bodenmann, 2017).

Topics were determined by using the Multidimensional Stress Scale for Couples (Bodenmann, 2006), which was included in the baseline questionnaire and assessed partners' experience of various external stressors. The research team chose the external stressor that each partner rated as the most stressful. Topics were counterbalanced by partner gender (e.g., Couple 1: Female's external stress, Couple 2: Male's external stress, etc.). For each conversation, partners were instructed to discuss the chosen topic for $6 \mathrm{~min}$. Following the conversation, partners were given a brief questionnaire asking about their perception of the interaction.

Each couples' conversation was transcribed by a team of eight undergraduate research assistants using the Praat software (Boersma and Weenink, 2006). After all transcripts had been completed, a different team of three graduate research assistants checked them for accuracy. The video recordings of the stress discussions were separated into 10-s segments to prepare for the behavioral coding, resulting in a total of 36 segments for each 6min conversation. A team of three graduate research assistants, all familiar with the systemic transactional model of dyadic coping, then reviewed the videos according to the codebook (Lau et al., unpublished) and indicated whether partners exhibited signs of non-verbal and verbal stress communication and dyadic coping. After initial training, the graduate research assistants were blindly assigned videos to complete coding independently, and some of the conversations were viewed by all three of them in order to calculate inter-rater reliability (see below).

\section{Measures \\ Observed Stress Communication}

Partners' observed stress communication was measured using the English-version of Bodenmann's (2008) dyadic coping behavioral coding system (Lau et al., unpublished). The Dyadic Coping Manual outlines types of non-verbal and verbal stress communication and dyadic coping. For the purpose of this study, we utilized data from the verbal stress communication codes. The four types of verbal stress communication are: (1) general or neutral explanations of stress (e.g., "I have a work to do and my co-workers are not helping"), (2) implicit emotion-focused stress communication (e.g., "I do not like how my friend talked to me"), (3) explicit emotion-focused stress communication (e.g., "I am furious at my boss"), and (4) problem-focused stress communication (e.g., "What do you think I should do in this situation?").

The average Cohen's Kappa values, across all external stress conversations in this study for the three raters was 0.82, indicating high inter-rater reliability (Cohen, 1968). To maintain consistency with the LIWC measures (described below), percentages were calculated for each partner based on the number of times that each partner was observed engaging in stress communication during the conversations by dividing the number of segments in which one form of stress communication occurred by the total of 36 segments. 


\section{Self-Reported Interaction Quality}

Participants rated their perceived quality of the external stress interaction following their conversation. Partners responded to 19 items related to relationship satisfaction and quality on a 7-point scale, ranging from 1 (strongly disagree) to 7 (strongly agree). Examples of items included, "In the previous interaction, my partner communicated warmth rather than coldness," and, "In the previous interaction, I felt that my partner understood what I was saying." Some items were originally rated a 7 -point scale, ranging from 1 (a negative personality trait) to 7 (a positive personality trait), which were then recoded to be consistent with the scale and examples described above. For example, one item was, "In the previous interaction, my partner was 1 (untrustworthy) versus 7 (trustworthy)," and this was revised to, "In the previous interaction, my partner was trustworthy," rated between 1 (strongly disagree) and 7 (strongly agree). A total score was generated by averaging all 19 of the items, with higher scores indicating greater levels of satisfaction with the interaction. Cronbach's alphas for the 19 items were 0.93 for both females and males, demonstrating high internal reliability (Cronbach, 1951).

\section{Language Use}

To obtain data related to partners' language use, raw transcript data were analyzed using the Linguistic Inquiry and Word Count (LIWC) computer program (Pennebaker et al., 2015). LIWC calculated percentages of all pronoun types, emotion words, and cognition words in the total word count of a given text sample. Based on prior literature (Rohrbaugh et al., 2012), the transcripts were prepared in the following ways prior to running them in LIWC: (1) raw transcripts were split by speaker and all information other than the actual speech and an identifying marker was removed; and (2) filler words and expressions that contained pronouns that did not carry independent meaning (e.g., "I" in "I mean") were marked in a way that prevented LIWC from counting them toward this category. The resulting percentages of pronouns (e.g., "I"; "you”; "we"), emotion words (e.g. "happy," "sad," "scared"), and cognition words (e.g., "think," "because," "effect") in total word counts were used in our current analyses.

\section{Analytic Plan}

Dyadic data contains many sources of interdependence. Given this, prior to conducting dyadic data analysis, it is recommended to determine the distinguishability of partners' roles (Kenny et al., 2006). Typically, research with heterosexual couples has used gender as a distinguishing variable (i.e., female and male); however, in our study, we must also consider the roles of the stress communicator and the listener. Thus, we conducted two separate sets of tests-one with gender and the other with speaker-listener roles as the distinguishable variable-to determine distinguishability. Speaker-listener roles were initially determined with the study design (selecting one partner's stress as the topic of discussion), and we used the behavioral coding of stress communication to verify role assignments and made changes based on who communicated more stress during the real-time conversations. The tests of distinguishability involved comparing the -2 log likelihood goodness of fix indices of the hierarchical linear models with and without the distinguishable variables; if the difference between the goodness of fit is significant according to chi-square calculations, then it would indicate that the model with the distinguishable has greater fit and partners are assumed to be distinguishable based on the examined variable. The tests showed that neither the models with gender nor the ones with speaker-listener roles as the distinguishable variables had significantly better fit than the indistinguishable model, suggesting that partners' results did not differ based on gender or speaker-listener roles. To account for the indistinguishability, data was restructured using the "doubleentry method" suggested by Kenny et al. (2006), such that each partner's scores were entered twice, once as the actor and again as the partner, and adjustments are made to the weights of the data points and the degrees of freedom.

The Actor-Partner Interdependence Mediation Model (APIMeM; Ledermann et al., 2011) was used to analyze the restructured dataset. The method of analysis has three functions: (1) accounts for variability due to the interdependence of partners, (2) assesses the impact of one partner's predictor and mediator variables on both partners' outcomes, and (3) measures the residual covariance between the variable pairs. In this model, there are actor and partner effects between each predictor, mediator, and outcome variable, along with direct and indirect effects from the standard mediation model, resulting in a total of 12 paths (see Figure 1).

Given our hypotheses, we present the results from the partnerpartner effects (i.e., the association between one partner's stress communication, the other partner's language use, and the first partner's interaction quality) for parsimony. Data analyses were conducted using Structural Equation Modeling (SEM) with Mplus 8, which is the suggested method to test the APIMeM as it estimates all model parameters within a single equation (Cook and Kenny, 2005; Ledermann et al., 2011).

\section{RESULTS}

\section{Descriptive Analyses}

Means, standard deviations, and bivariate correlations among the study variables are presented in Table 1 . The only significant difference between female and male partners was their engagement in problem-focused stress communication, $t(40)=-2.44, p=0.01$. Specifically, male partners were observed to engage in more problem-focused stress communication, compared to their female partner. Results for the APIMeMs are described below in terms of actor and partner effects due to the indistinguishability of Partner A's and B's roles. All models showed good fit (Table 2).

\section{H1: Pronouns Mediate the Association Between General Stress Communication and Interaction Quality We-Talk}

Results revealed a significant partner effect of general stress communication on the use of we-talk $(b=-3.56, S E=0.84$, 


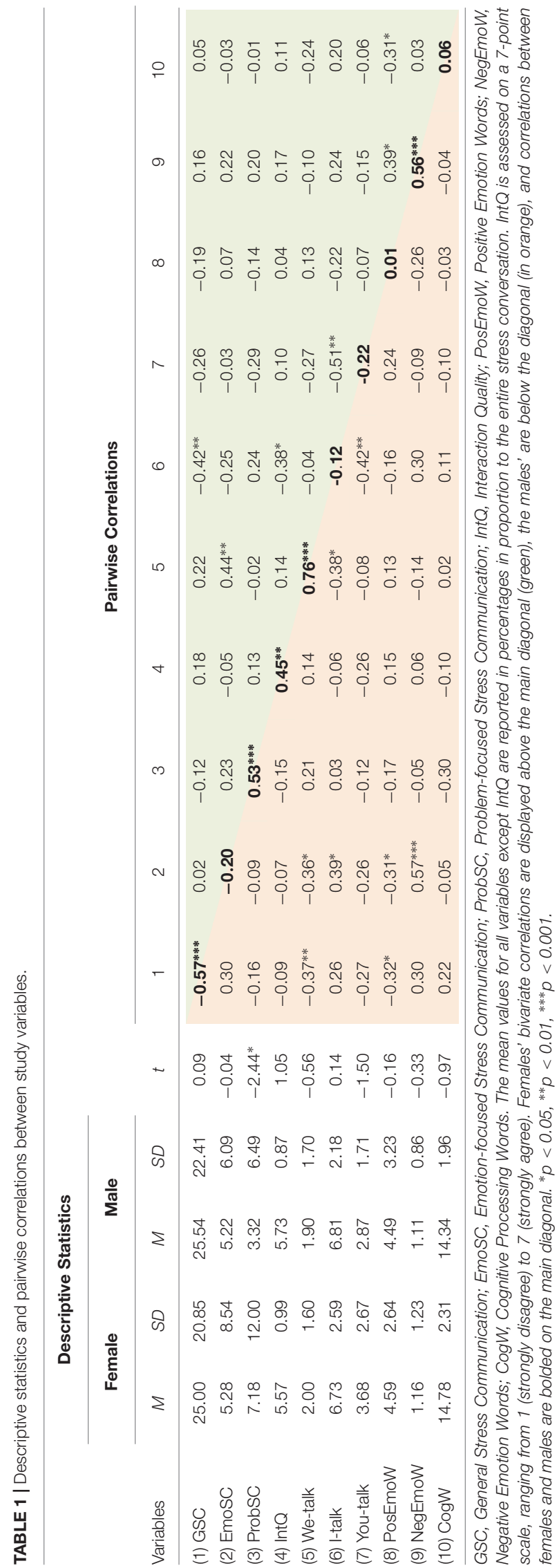

TABLE 2 | Fit indices for all models with interaction quality as dependent variable.

\begin{tabular}{lccccccc}
\hline & & \multicolumn{6}{c}{ Model fit } \\
\cline { 3 - 8 } IV & Mediator & $\chi^{2}$ & df & $\boldsymbol{p}$ & CFI & RMSEA & SRMR \\
\hline GSC & - & 0.14 & 2 & 0.93 & 1.00 & 0.00 & 0.01 \\
GSC & We-talk & 5.54 & 6 & 0.48 & 1.00 & 0.00 & 0.06 \\
GSC & I-talk & 1.01 & 6 & 0.99 & 1.00 & 0.00 & 0.02 \\
GSC & You-talk & 3.01 & 6 & 0.81 & 1.00 & 0.00 & 0.05 \\
EmoSC & - & 0.50 & 2 & 0.78 & 1.00 & 0.00 & 0.03 \\
EmoSC & PEmoW & 4.02 & 6 & 0.67 & 1.00 & 0.00 & 0.06 \\
EmoSC & NEmoW & 1.73 & 6 & 0.94 & 1.00 & 0.00 & 0.03 \\
ProbSC & - & 0.48 & 2 & 0.78 & 1.00 & 0.00 & 0.03 \\
ProbSC & CogW & 2.70 & 6 & 0.85 & 1.00 & 0.00 & 0.07 \\
\hline
\end{tabular}

IV, Independent Variable; CFI, Comparative Fit Index; RMSEA, Root Mean Square Error of Approximation; SRMR, Standardized Root Mean Square Residual; GSC, General Stress Communication; EmoSC, Emotion-focused Stress Communication; ProbSC, Problem-focused Stress Communication; PEmoW, Positive Emotion Words; NEmoW, Negative Emotion Words; CogW, Cognition Words.

$p<0.001$; Figure 2), such that one partner's general stress communication was negatively associated with the other partner's we-talk. There was also a significant partner effect of we-talk on interaction quality $(b=0.26, S E=0.05, p<0.001)$. The direct actor effect of Partner A's general stress communication on his/her own interaction quality was marginally significant, $b=0.84, S E=0.48, p=0.08$, and further, the partner-partner indirect effect was statistically significant, $b=-0.93, S E=0.31$, $p=0.002$. Thus, these findings suggest that we-talk partially mediated the association between general stress communication and perceived interaction quality, supporting H1a.

\section{I-Talk}

Results indicated a significant partner effect of general stress communication on I-talk $(b=-3.92, S E=0.99, p<0.001$; Figure 2), such that one partner's general stress communication was negatively associated with the other partner's I-talk. In addition, we found a significant partner effect of I-talk on interaction quality $(b=-0.07, S E=0.03, p=0.04)$ as well as a significant actor effect $(b=-0.08, S E=0.03, p=0.02)$. The direct actor effect was not significant, $b=-0.09, S E=0.47$, $p=0.86$; however, the partner-partner indirect effect was, $b=0.26, S E=0.13, p=0.05$. Therefore, I-talk fully mediated the association between general stress communication and interaction quality, which provided support for H1b.

\section{You-Talk}

Results showed a significant partner effect of general stress communication on you-talk $(b=2.41, S E=1.07, p=0.02$; Figure 2), such that one partner's general stress communication was positively associated with the other partner's you-talk. However, no significant partner effect was found between youtalk and interaction quality $(b=-0.05, S E=0.04, p=0.15)$. The direct actor effect was not significant, $b=0.47, S E=0.36, p=0.20$, and neither was the indirect partner-partner effect, $b=-0.13$, $S E=0.10, p=0.21$. Therefore, our hypothesis related to our focus on actor-partner-actor effects (H1c) was not supported. However, we found additional results based on the other paths that were not 


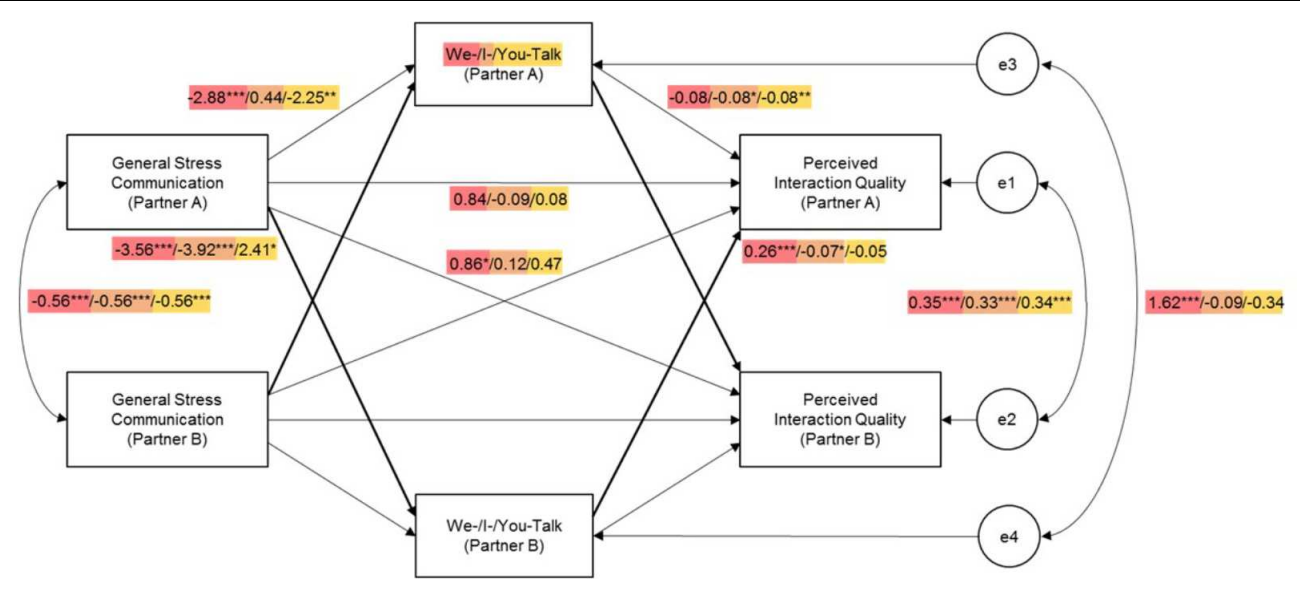

FIGURE 2 | Unstandardized model results of we-talk (red), I-talk (orange), and you-talk (yellow) mediating the association between general stress communication and perceived interaction quality. Only one set of coefficients were included due to the indistinguishability of partner roles. ${ }^{*} p<0.05,{ }^{* *} p<0.01,{ }^{* * *} p<0.001$.

hypothesized. There was a significant actor effect of general stress communication on you-talk $(b=-2.25, S E=0.76, p=0.003)$ as well as a significant actor effect of you-talk on interaction quality $(b=-0.08, S E=0.03, p=0.002)$. While the direct actor effect was not significant, $b=0.08, S E=0.46, p=0.87$, the indirect actor-actor mediational effect was, $b=0.19, S E=0.10, p=0.05$. Although not hypothesized, it was found that the actor's youtalk mediated the association between his/her own general stress communication and interaction quality.

\section{H2: Emotion Words Mediate the Association Between Emotion-Focused Stress Communication and Interaction Quality}

\section{Positive Emotion Words}

Results showed no significant partner effect of emotion-focused stress communication on the use of positive emotion words $(b=3.28, S E=2.93, p=0.26$; Figure 3) and no significant partner effect of positive emotion words on interaction quality $(b=0.01, S E=0.03, p=0.67)$. The indirect partner-partner effect was not significant, $b=0.04, S E=0.15, p=0.76$, and neither was the direct actor effect, $b=-0.57, S E=0.97, p=0.55$. The only significant association found in this model was between one partner's emotion-focused stress communication with his/her own use of positive emotion words $(b=-5.60, S E=2.61, p=0.03)$. Our findings do not support $\mathrm{H} 2 \mathrm{a}$ regarding the mediation of positive emotion words on the association between emotionfocused stress communication and interaction quality.

\section{Negative Emotion Words}

Results showed a marginally significant partner effect of emotionfocused stress communication on the use of negative emotion words $(b=1.66, S E=0.93, p=0.07$; Figure 3$)$ and a significant partner effect of negative emotion words on interaction quality $(b=0.24$, $S E=0.07, p<0.001)$. The direct actor effect was not significant, $b=-1.04, S E=0.88, p=0.24$, however, the indirect partnerpartner effect was marginally significant, $b=0.40, S E=0.25$, $p=0.10$. Based on these results, there is limited support for the mediating role of negative emotion words in the association between emotion-focused stress communication and interaction quality (H2b). In addition to these main findings, it was also found that stress communication was positively associated with one's own use of negative emotion words $(b=3.70, S E=1.03$, $p<0.001$ ), and that there was a significant indirect partnerpartner effect of stress communication on one's own use of negative words on the partner's interaction quality $(b=0.89$, $S E=0.42, p=0.04)$.

\section{H3: Cognitive Processing Words Mediate the Association Between Problem-Focused Stress Communication and Interaction Quality}

Lastly, we found a significant partner effect of problem-focused stress communication on cognition words $(b=-5.30, S E=1.15$, $p<0.001)$ and a significant partner effect of cognition words on interaction quality $(b=0.11, S E=0.03, p=0.002)$. The direct actor effect was not significant, $b=0.07, S E=0.85, p=0.94$, while indirect partner-partner effect was, $b=-0.57, S E=0.23$, $p=0.01$ (Figure 4). Overall, there is support for $\mathrm{H} 3$, in that cognitive processing words partially mediated the association between problem-focused stress communication and interaction quality. However, the association between stress communication and use of cognition words was negative, which is different from what we had hypothesized.

In sum, there was partial support for our hypotheses. Specifically, we-talk partially mediated and I-talk fully mediated the association between general stress communication and perceived interaction quality. Moreover, the use of cognitive processing words mediated the association between problemfocused stress communication and perceived interaction quality.

Although the focus of this study was on the partner effects of stress communication on language use (i.e., the association between one partner's stress communication and the other partner's language use) to highlight the interaction between 


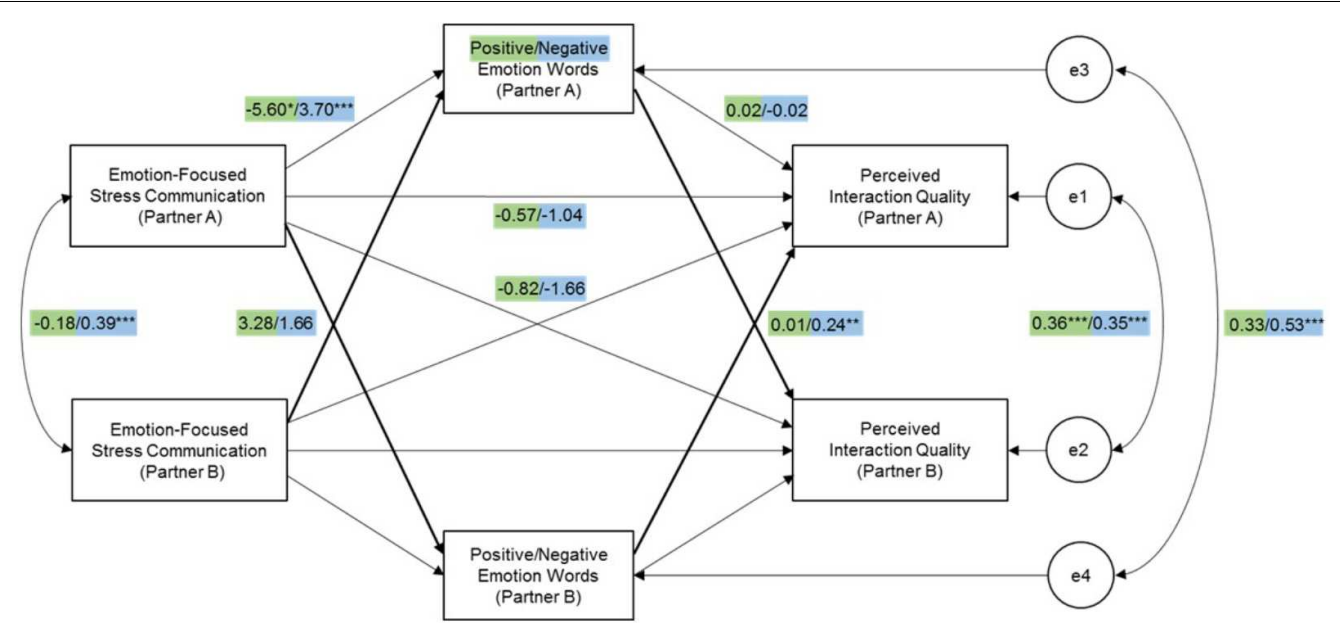

FIGURE 3 | Unstandardized model results of positive (green) and negative emotion words (blue) mediating the association between emotion-focused stress communication and perceived interaction quality. Only one set of coefficients were included due to the indistinguishability of partner roles. ${ }^{*} p<0.05,{ }^{* *} p<0.01$, $* * * p<0.001$.

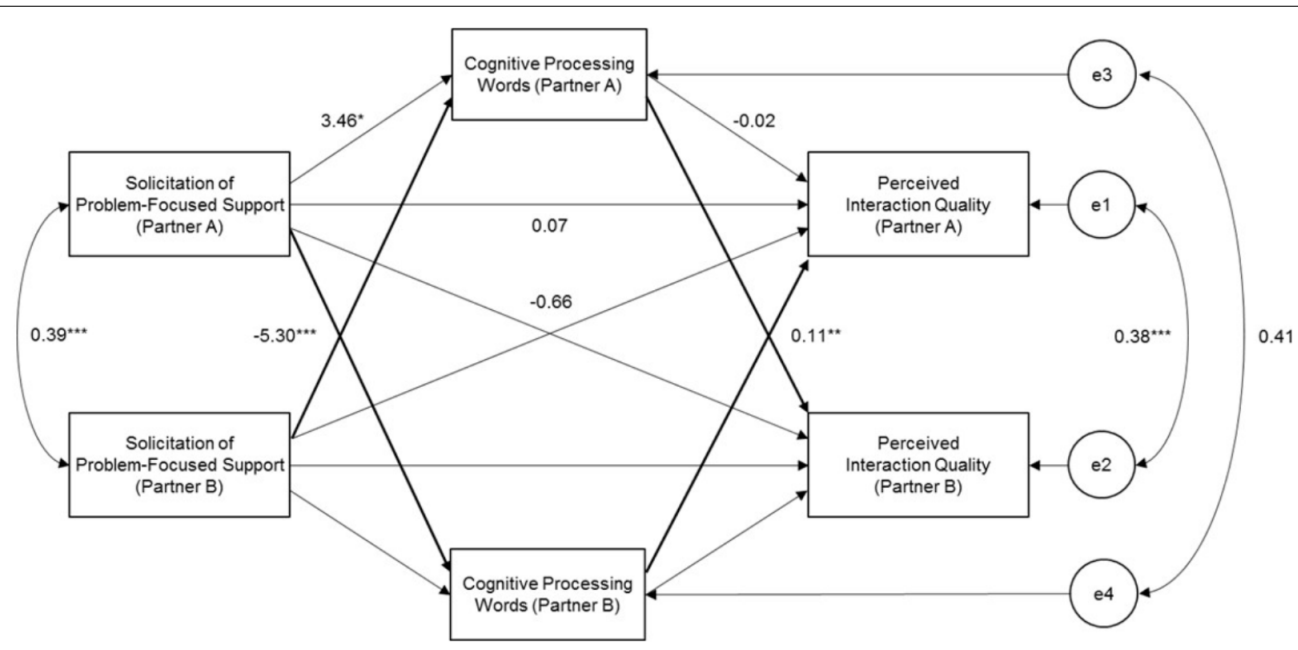

FIGURE 4 | Unstandardized model results of cognitive processing words mediating the association between problem-focused stress communication and perceived interaction quality. Only one set of coefficients were included due to the indistinguishability of partner roles. ${ }^{*} p<0.05,{ }^{* *} p<0.01,{ }^{* * *} p<0.001$.

the actor and the partner, there were other notable findings. For instance, results revealed significant negative actor effects between stress communication and we-talk, you-talk, and positive emotion words, such that when one communicates stress, he/she also uses fewer "we" and "you" pronouns and positive emotion words.

\section{DISCUSSION}

Partners' experience of external stress is a common occurrence, which can lead to significant relational concerns and even dissolution if not properly dealt with (e.g., Kiecolt-Glaser et al., 2003; Bodenmann et al., 2007; Falconier et al., 2015). In order to promote relationship well-being, it is imperative that relationship researchers understand how partners can effectively manage stress via communication and coping, particularly when discussing stressful situations. To our knowledge, this study was the first to examine the stress and coping processes described by the systemic-transactional model at the real-time conversational level. Specifically, we tested whether partners' observed engagement in stress communication and dyadic coping, more specifically in the form of language use, would contribute to their perceptions of quality of their real-time interactions about external stressors.

\section{General Stress Communication, Pronouns, and Interaction Quality}

Overall, the results supported our hypotheses regarding the mediator role of pronoun use. First, the results indicated that we-talk is a significant mediator in the association between stress 
communication and interaction quality. However, contrary to the hypothesis, the association between stress communication and we-talk was negative. That is, when one partner communicated their stress in a general or neutral manner, the other partner responded with less we-talk, which then positively predicted interaction quality. We-talk has been typically thought by researchers to communicate cohesion between couples and in fact, found to be associated with relationship satisfaction (Borelli et al., 2013). It is possible that when the supporting partner used fewer "we" words, the partner communicating stress interpreted it as the partner was not coping with him/her and therefore perceived lower quality of interaction. It could also be the case that the partner expressing their stress did not feel as though they were working together with their partner (Reid et al., 2006).

However, it seems that not only the supporting partner, but the stressed partner also engaged in lower levels of we-talk, as indicated by the negative actor association between one partner's general stress communication and his/her own we-talk. This is not unexpected given the nature of the couples' discussions about external stress, as external stress traditionally only affects one partner. As such, in this context, it would be appropriate to expect the actor (i.e., the stressed partner) to focus more on his/her own experience of stress. However, as the other partner listens, he/she may choose to emphasize what he/she could do to help alleviate the actor's stress; thus, they may be more likely to use singular pronouns (e.g., "I," "you”) than plural pronouns (e.g., "we").

Previous studies have demonstrated the negative associations between I-talk/you-talk and relationship outcomes and the positive associations between we-talk and relationship outcomes (e.g., Rentscher et al., 2015), especially when partners discuss common stressors, such as coping with cancer (Robbins et al., 2013). This research suggests that there may be instances in which the use of "we" can be more conducive to partners' perception of we-ness and joint coping efforts than others, such as in activities in which partners participate together (Aron et al., 2000). Further, Slatcher et al. (2008) suggested that the use of "we" in problemsolving discussions is unrelated to relationship quality whereas the use of "we" when describing the relationship or the future of the couple may be linked to relationship quality. These results may be used to educate couples on the importance of viewing external stress as a mutual experience or issue, which is a focus in the Couples Coping Enhancement Program (CCET; Bodenmann and Shantinath, 2004). In addition, the current findings are helpful in establishing considerations for context (e.g., the topic of discussion) and language use in future studies.

Results also showed, as expected, that one partner's general stress communication was negatively associated with the other partner's I-talk, which was in turn negatively associated with the first partner's interaction quality. This finding is consistent with the nature of the external stress conversation that the partner would engage less I-talk when attempting cope with his/her partner because the focus "should" be on their partner's stress. This finding also supports the extant literature, which shows that I-talk is negatively associated with relationship outcomes (Slatcher et al., 2008: Rentscher et al., 2013).

In addition, one partner's stress communication was found to be positively associated with the other partner's you-talk, which was not predictive of the first partner's interaction quality. This does not support our hypothesis regarding the partner-partner mediation path; however, it was interesting that we found a positive association between the actor's stress communication and the partner's you-talk. Again, individuals who engage in positive DC would be expected to use less I-talk because their focus would be directed toward their partners in order to address their partners' stress; thus, they may be using more "you” words. For instance, in attempting to cope with one's partner, one may use language like, "Have you tried doing this?" or "You must be feeling so stressed." You-talk can at times communicate blame and criticism (Biesen et al., 2015) and other times be indicative of support provision, and each use of "you" could have different effects on interaction quality. Thus, the absence of significant associations between the partner's you-talk and the actor's interaction quality may be explained by this flexible use of second-person pronouns.

\section{Emotion-Focused Stress Communication, Emotion Words, and Interaction Quality}

Contrary to our hypotheses, we did not find significant mediation for positive or negative emotion words in the association between emotion-focused stress communication and interaction quality. Findings may indicate that the use of positive emotions may be ambiguous. When the actor communicates the negative emotional effects of a stressor and the partner uses many positive emotion words, the actor may perceive that as less supportive than intended rather than supportive. The emotional burden on the actor may prevent him/her from recognizing the positive effects of the partner's use of positive emotion words and thus may feel as if the partner does not truly understand their negative emotions and is trying to avoid talking about the issue. On the other hand, what we had anticipated to be negative associations turned out to be positive associations between negative emotion words and interaction quality, possibly because the partner's use of negative emotion words may communicate validation and empathy instead. Again, when assessing language use, it is important to consider the context in which words are spoken (Tausczik and Pennebaker, 2010).

\section{Problem-Focused Stress Communication, Cognition Words, and Interaction Quality}

Despite the support for the mediational effect of cognitive processing words on the association between problem-focused stress communication and interaction quality, the association between stress communication and use of cognition words was negative. That is, when the actor communicated stress by taking a solution-focused approach, the partner used fewer cognitive processing words, which suggested that the partner might not have been engaging in problem-focused dyadic coping. This is similar to the unexpected mediational direction for the use of we-talk, in that stress communication indirectly negatively predicted interaction quality because the partner did not communicate support that the actor may have hoped for. 
This pattern may resemble demand-withdraw communication styles, in which one partner seeks discussion or resolution of an issue while the other withdraws from the interaction (Christensen and Heavey, 1990). Demand-withdraw communication patterns in couples have been found to be positively associated with relationship distress and dissolution (e.g., Eldridge and Christensen, 2002); thus, our findings appear to be consistent with extant literature.

In summary, results from this study have strong implications in understanding the stress communication and coping patterns of romantic partners. We had originally formulated our hypotheses based on the assumption that the partners in our sample would be effective at dyadic coping; however, some of our findings (i.e., the negative associations between stress communication and we-talk and cognitive processing words) suggested otherwise. The naturalistic design of this study (i.e., no assignment of speaker-listener roles) was integral because it may reveal how couples interact in reality as they switch speaker and listener roles during a stress conversation. The findings from this study could augment existing prevention programs designed to teach couples how to communicate during stress (e.g., CCET; Bodenmann and Shantinath, 2004).

\section{Limitations}

This study is not withstanding limitations. First, there may have been sample bias due to the majority of participants identifying as White and highly educated (i.e., most received at least a college degree). Membership in higher socioeconomic status may affect the way partners communicate, as well as their expectations of effective interactions (e.g., Amato and Previti, 2003). In addition, this study recruited from a population of selfselecting, heterosexual couples. Both partners had to agree to participate, so it was likely that partners were at least moderately satisfied with their relationships in order to complete a research study together. Overall, the lack of representation with respect to this sample may affect how generalizable the results are to all romantic couples facing external stress. For instance, couples who are not native to the United States may encounter immigration stress (Falconier et al., 2013; Falconier et al., 2016) and samesex couples may experience stress due to discrimination from a heteronormative environment (Randall et al., 2016; Totenhagen et al., 2017). In addition, culture may play a role in how partners communicate and cope with stress (e.g., McCubbin and McCubbin, 1988).

Another limitation of this study was related to the study variables. First, because of the number of existing parameters in our models and the final sample size, we did not include any control variables to ensure sufficient power of our analyses. Possible variables to account for include levels of stress and relationship satisfaction prior to the conversations, because they may impact how couples engage in coping processes together as well as their perceptions of the interactions. Also, this study used observed stress communication as the independent variable, as it has been shown to be associated with various aspects of relationship well-being (Falconier et al., 2015). By having trained raters code for observable behavior, it could reflect a more objective assessment of real-time dyadic coping responses of partners. Further, given that the behavioral coding and linguistic data were aggregated throughout the conversations, these scores may not be representative of the transactional nature of dyadic coping. Specifically, couples engage in discussions about stress, moment-to-moment conversational cues could occur and cause the partners to respond to each other (Mohr and Spekman, 1994; Sanders et al., 1999); thus, using cumulative variables rather than examining the variables at each time point may remove some of the meaningful information about the stress and coping processes during realtime conversations.

Another limitation to the current study is the indistinguishability of partners' roles. Partners were shown to be indistinguishable based on gender possibly because the assignment of speaker-listener roles was counterbalanced. Thus, an approximately equal number of females and males were expected to be the stress communicator and listener. Speakerlistener roles were also not explicitly assigned in our laboratory instruction, due to an attempt to preserve the naturalistic design of the study. Despite this attempt, it could be that some couples discussed areas of external stress that were salient for both partners. This may explain why speaker-listener roles were not distinguishable in our analyses. Indistinguishability could be an issue when determining the context in which the partners engaged in their word use (e.g., stress communication vs. dyadic coping). While this was one of the notable limitations of the naturalistic design, this design allowed us to examine how couples interact with one another in real-time.

\section{Future Directions}

Future research examining temporal stress communication and coping dynamics may wish to recruit a more diverse sample in terms of ethnicity, education background, and sexual orientation. Doing so could shed light on the possible variability in partners' stress levels, dyadic coping, language use, interaction quality, and overall relationship outcomes. Generally speaking, having greater variability in these measures would not only increase external validity, but it will allow for a more in-depth knowledge about how stress and coping processes occur for couples. In addition, it may be interesting to consider couples' conversations about other types of stress in terms of origin (external vs. internal), intensity (major vs. minor), and duration (acute vs. chronic; Randall and Bodenmann, 2009, 2017). Due to their differential impact on the relationship, it is possible that the coping and language processes may be different when discussing different stressors.

One of the advantages of using real-time data is the ability to assess moment-to-moment changes in affect, behavior, and cognition (Laurenceau and Bolger, 2005; Iida et al., 2012). Thus, it is important for future research to collect data and conduct analyses that allow adequate tests of these momentto-moment fluctuations. Based on this study's results, we offer several important directions for future research. First, it may be helpful to separate the dialog by speaker-listener turns rather than using the fixed 10-s intervals established by the dyadic coping behavioral coding system (Bodenmann, 2008; Randall et al., 2016). For example, Table 3 displays an example of 
TABLE 3 | Sample dataset for future studies.

\begin{tabular}{|c|c|c|c|c|c|c|c|c|c|}
\hline Couple ID & Begin & Gender & S/L & Stress_Begin & Dialog & End & Stress_End & sc & DC \\
\hline 5 & 0:00:00 & Male & & 50 & $\begin{array}{l}\text { Man umm so work start with you or me } \\
\text { I wonder if that was for both of us }\end{array}$ & 0:00:07 & 50 & 88 & 88 \\
\hline 5 & 0:00:07 & Female & & 50 & $\begin{array}{l}\text { I'm not sure which one of us it's } \\
\text { supposed to be for we can start with } \\
\text { me I guess }\end{array}$ & 0:00:16 & 50 & 88 & 88 \\
\hline 5 & $0: 00: 16$ & Male & $S$ & 50 & $\begin{array}{l}\text { I was going to start with rrlike I wonder } \\
\text { what kind of questions I should do for } \\
\text { this interview today }\end{array}$ & 0:00:20 & 50 & 88 & 88 \\
\hline 5 & 0:00:20 & Female & $L$ & 50 & $\begin{array}{l}\text { We should talk about that because it's } \\
\text { probably useful to talk about }\end{array}$ & 0:00:23 & 23 & 88 & 88 \\
\hline 5 & 0:00:22 & Male & S & 45 & $\begin{array}{l}\text { I was thinking about the only thing I can } \\
\text { think of for now since I've already kind } \\
\text { of interviewed with them was rrlike uhh } \\
\text { rrlike what's the day to day for I think it's } \\
\text { a processing assistant }\end{array}$ & $0: 00: 40$ & 23 & 2 & 88 \\
\hline 5 & $0: 00: 40$ & Female & $L$ & 23 & $\begin{array}{l}\text { So was it rrlike a different position that } \\
\text { she interviewed with for }\end{array}$ & $0: 00: 43$ & 23 & 88 & 2 \\
\hline 5 & $0: 00: 43$ & Male & $S$ & 23 & $\begin{array}{l}\text { Yes the last one was something else } \\
\text { but this one is more like what Will is } \\
\text { doing }\end{array}$ & $0: 00: 48$ & 23 & 2 & 88 \\
\hline 5 & 0:00:48 & Female & $L$ & 23 & $\begin{array}{l}\text { But I mean obviously you don't ask him } \\
\text { about his job so that would be the } \\
\text { question }\end{array}$ & 0:01:03 & 70 & 88 & 1 \\
\hline 5 & 0:01:03 & Male & S & 70 & $\begin{array}{l}\text { You typically want to leave work at work } \\
\text { unless it's rrlike something you need to } \\
\text { vent about }\end{array}$ & 0:01:06 & 70 & 2 & 88 \\
\hline 5 & $0: 01: 07$ & Female & $\mathrm{L}$ & 70 & $\begin{array}{l}\text { That's true umm yeah I don't know } \\
\text { yeah it's supposed to be rrlike you want } \\
\text { to do a really good question but }\end{array}$ & $0: 01: 26$ & 51 & 88 & 2 \\
\hline 5 & 0:01:24 & Male & S & 57 & $\begin{array}{l}\text { I don't know because I don't } \\
\text { necessarily plan on being there for the } \\
\text { next } 5 \text { years but I don't necessarily } \\
\text { don't either }\end{array}$ & $0: 01: 34$ & 63 & 1 & 88 \\
\hline 5 & 0:01:34 & Female & $L$ & 63 & $\begin{array}{l}\text { Well you don't know maybe you're } \\
\text { going to super love it and it's going to } \\
\text { be the best thing ever }\end{array}$ & $0: 01: 43$ & 39 & 88 & 2 \\
\hline 5 & $0: 01: 43$ & Male & S & 39 & $\begin{array}{l}\text { Do projects at home where it'll be like I } \\
\text { wanted and then just worry about the } \\
\text { income and we're good who knows }\end{array}$ & $0: 01: 49$ & 39 & 88 & 88 \\
\hline 5 & 0:01:49 & Female & $L$ & 39 & $\begin{array}{l}\text { You should be honest rrlike you know } \\
\text { it's not like I went to college for loan } \\
\text { processing or whatever but see you're } \\
\text { already doing good }\end{array}$ & 0:02:01 & 50 & 88 & 1 \\
\hline
\end{tabular}

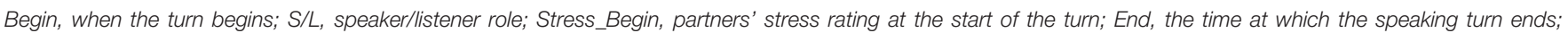
Stress_End, partners' stress rating at the end of the turn; SC, behavioral coding for stress communication; DC, behavioral coding for dyadic coping.

how data may be organized to facilitate further examination of partners' stress, coping, and micro-communication processes. Additionally, future research could also utilize statistical analyses that are appropriate in testing for fluctuations between various time points, such as the cross-lagged model (Kenny et al., 2006). The cross-lagged model could be used to examine associations between one partner's predictor variable (in the case of the present research, stress communication) at Time 1 and the other partner's outcome variable at Time 2 (i.e., language use). This analytical procedure could be especially helpful in the context of stress communication and coping process because of its transactional nature; further, it would allow researchers to more closely examine the micro-communication dynamics that occur between partners.

\section{CONCLUSION}

The findings from this study suggest language use may mediate the associations between stress communication and perceived interaction quality during real-time interactions. Relationship scholars are encouraged to further explore the interplay between couples' stress and coping using ecological momentary assessment methods (Kirchner and Shiffman, 2016). 
Additionally, mental health practitioners working with couples could benefit from implementing psychoeducation or skills training on language use during conversations about stress. Taken together, the way we express ourselves to our romantic partner during stressful interactions can have meaningful effects on how our partner perceives our stress, and how we perceive the interaction and our relationship.

\section{ETHICS STATEMENT}

This study was carried out in accordance with the recommendations of the Institutional Review Board at Arizona State University with written informed consent from all subjects. All subjects gave written informed consent in accordance with the Declaration of Helsinki. The protocol was approved by the Arizona State University Institutional Review Board.

\section{REFERENCES}

Amato, P. R., and Previti, D. (2003). People's reasons for divorcing: gender, social class, the life course, and adjustment. J. Fam. Issues 24, 602-626. doi: 10.1177/ 0192513X03024005002

Aron, A., Norman, C. C., Aron, E. N., McKenna, C., and Heyman, R. E. (2000). Couples' shared participation in novel and arousing activities and experienced relationship quality. J. Pers. Soc. Psychol. 78, 273-284. doi: 10.1037/0022-3514. 78.2 .273

Baddeley, J. L., and Pennebaker, J. W. (2011). A postdeployment expressive writing intervention for military couples: a randomized controlled trial. J. Trauma Stress 24, 581-585. doi: 10.1002/jts.20679

Bahr, S. J. (1979). The effects of welfare on marital stability and remarriage. J. Marriage Fam. 41, 553-560. doi: 10.2307/351625

Biesen, J. N., Schooler, D., and Smith, D. (2015). What a difference a pronoun makes: I/We versus you/me and worried couples' perceptions of their interaction quality. J. Lang. Soc. Psychol. 35, 1-26.

Boals, A., and Klein, K. (2005). Word use in emotional narratives about failed romantic relationships and subsequent mental health. J. Lang. Soc. Psychol. 24, 252-268. doi: 10.1177/0261927X0527 8386

Bodenmann, G. (1995). A systemic-transactional conceptualization of stress and coping in couples. J. Psychol. 54, 34-49.

Bodenmann, G. (1997). The influence of stress and coping on close relationships: a two-year longitudinal study. Swiss J. Psychol. 56, 156-164.

Bodenmann, G. (2000). Stress und Coping bei Paaren [Stress and coping in couples]. Göttingen: Hogrefe.

Bodenmann, G. (2005). "DC and its significance for marital functioning," in Couples Coping with Stress: Emerging Perspectives on DC, eds T. A. Revenson, K. Kayser, and G. Bodenmann (Washington DC: American Psychological Association), 33-49. doi: 10.1037/11031-002

Bodenmann, G. (2006). Multidimensionaler Stressfragebogen fur Paare. Fribourg: University of Fribourg.

Bodenmann, G. (2008). Kodiersystem zur Erfassung des Dyadischen Copings (SEDC). Erweiterte und Adaptierte Version [Coding System to Assess Dyadic Coping. Modified and Adapted Version]. Zurich: University of Zurich.

Bodenmann, G., Ledermann, T., and Bradbury, T. (2007). Stress, sex, and satisfaction in marriage. Pers. Relationsh. 14, 551-569. doi: 10.1111/j.1475-6811. 2007.00171.x

Bodenmann, G., Meuwly, N., and Kayser, K. (2011). Two conceptualizations of dyadic coping and their potential for predicting relationship quality and individual well-being. Eur. Psychol. 16, 255-266. doi: 10.1027/1016-9040/ a000068

\section{AUTHOR CONTRIBUTIONS}

$\mathrm{AR}$ and ND are the PIs on the study (Couples Coregulation Processes during Real-Time Interactions). $\mathrm{KL}$ and $\mathrm{AR}$ contributed to the conception and design of this study. CT led the collection of the data. KL organized the dataset, performed the statistical analysis, and wrote the first draft of the manuscript. All authors contributed to manuscript revision, read and approved the submitted version.

\section{ACKNOWLEDGMENTS}

The authors would like to acknowledge funding from ASU's Institute for Social Science Research (PIs: AR and ND), members of the Couples Coping with Stress Laboratory and the DynamiCog Laboratory for their help with data collection and processing, and the research participants.

Bodenmann, G., Pihet, S., and Kayser, K. (2006). The relationship between DC and marital quality: a 2-year longitudinal study. J. Fam. Psychol. 20, 485-493. doi: 10.1037/0893-3200.20.3.485

Bodenmann, G., and Shantinath, S. D. (2004). The Couples Coping Enhancement Training (CCET): a new approach to prevention of marital distress based upon stress and coping. Fam. Relat. 53, 477-484. doi: 10.1111/j.0197-6664.2004. 00056.x

Boersma, P., and Weenink, D. (2006). Praat (Version 4.5). [Computer software]. Amsterdam: Institute of Phoenix Sciences.

Bolger, N., DeLongis, A., Kessler, R. C., and Schilling, E. A. (1989). The effects of daily stress on negative mood. J. Pers. Soc. Psychol. 57, 808-818. doi: 10.1037/ 0022-3514.57.5.808

Borelli, J. L., Sbarra, D. A., Randall, A. K., Snavely, J. E., St. John, H. K., and Ruiz, S. K. (2013). Linguistic indicators of wives' attachment security and communal orientation during military deployment. Fam. Process. 52, 535-544. doi: $10.1111 /$ famp. 12031

Carroll, S. J., Hill, E. J., Yorgason, J. B., Larson, J. H., and Sandberg, J. G. (2013). Couple communication as a mediator between work-family conflict and marital satisfaction. Contemp. Fam. Ther. 35, 530-545. doi: 10.1007/s10591-0139237-7

Christensen, A., and Heavey, C. L. (1990). Gender and social structure in the demand/withdraw pattern of marital conflict. J. Pers. Soc. Psychol. 59, 73-81. doi: 10.1037/0022-3514.59.1.73

Cohen, J. (1968). Weighted kappa: nominal scale agreement provision for scaled disagreement or partial credit. Psychol. Bull. 70, 213-220. doi: 10.1037/ h0026256

Cook, W. L., and Kenny, D. A. (2005). The actor-partner interdependence model: a model of bidirectional effects in developmental studies. Int. J. Behav. Dev. 29, 101-109. doi: 10.1080/01650250444000405

Cordova, M. J., Cunningham, L. L. C., Carlson, C. R., and Andrykowski, M. A. (2001). Posttraumatic growth following breast cancer: a controlled comparison study. Health Psychol. 20, 176-185. doi: 10.1037/0278-6133.20.3.176

Cronbach, L. J. (1951). Coefficient alpha and the internal structure of test. Psychometrika 16, 297-334. doi: 10.1007/BF02310555

Eldridge, K. A., and Christensen, A. (2002). "Demand-withdraw communication during couple conflict: a review and analysis," in Understanding Marriage: Developments in the Study of Couple Interaction, eds P. Noller and J. Feeney (Cambridge: Cambridge University Press), 289-322.

Falconier, M. K., Jackson, J. B., Hilpert, P., and Bodenmann, G. (2015). Dyadic coping and relationship satisfaction: a meta-analysis. Clin. Psychol. Rev. 42, 28-46. doi: 10.1016/j.cpr.2015.07.002

Falconier, M. K., Nussbeck, F., and Bodenmann, G. (2013). Immigration stress and relationship satisfaction in Latino couples: the role of dyadic coping. J. Soc. Clin. Psychol. 32, 813-843. doi: 10.1521/jscp.2013.32.8.813 
Falconier, M. K., Randall, A. K., and Bodenmann, G. (eds) (2016). Couples Coping With Stress: A Cross-Cultural Perspective. Abingdon: Routledge. doi: 10.4324/ 9781315644394

Iida, M., Shrout, P. E., Laurenceau, J.-P., and Bolger, N. (2012). "Using diary methods in psychological research," in APA Handbook of Research Methods in Psychology, Vol. 1. Foundations, Planning, Measures, and Psychometrics, eds H. Cooper, P. M. Carnic, D. L. Long, A. T. Panter, D. Rindskopf, and K. J. Sher (Washington DC: American Psychological Association), 277-305. doi: 10.1037/13619-016

Kenny, D. A., Kashy, D. A., and Cook, W. L. (2006). Dyadic Data Analysis. New York, NY: The Guilford Press.

Kiecolt-Glaser, J. K., Bane, C., Glaser, R., and Malarkey, W. B. (2003). Love, marriage, and divorce: Newlyweds' stress hormones foreshadow relationship changes. J. Consult. Clin. Psychol. 71, 176-188. doi: 10.1037/0022-006X.71.1.176

Kirchner, T. R., and Shiffman, S. (2016). Spatio-temporal determinants of mental health and well-being: advances in geographically-explicit ecological momentary assessment (GEMA). Soc. Psychiatry Psychiatr. Epidemiol. 51, 1211-1223. doi: 10.1007/s00127-016-1277-5

Laurenceau, J.-P., and Bolger, N. (2005). Using diary methods to study marital and family processes. J. Fam. Psychol. 19, 86-97. doi: 10.1037/0893-3200.19.1.86

Lavee, Y., McCubbin, H. I., and Olson, D. H. (1987). The effect of stressful life events and transitions on family functioning and well-being. J. Marriage Fam. 49, 857-873. doi: 10.2307/351979

Ledermann, T., Bodenmann, G., Rudaz, M., and Bradbury, T. N. (2010). Stress, communication, and marital quality in couples. Fam. Relat. 59, 195-206. doi: 10.1111/j.1741-3729.2010.00595.x

Ledermann, T., Macho, S., and Kenny, D. A. (2011). Assessing mediation in dyadic data using the actor-partner interdependence model. Struct. Equ. Model. 18, 595-612. doi: 10.1080/10705511.2011.607099

Litzinger, S., and Gordon, K. C. (2005). Exploring relationships among communication, sexual satisfaction, and marital satisfaction. J. Sex Marital Ther. 31, 409-424. doi: 10.1080/00926230591006719

MacNeil, S. E., and Byers, S. (2005). Dyadic assessment of sexual self-disclosure and sexual satisfaction in heterosexual dating couples. J. Soc. Pers. Relationsh. 22, 169-181. doi: 10.1177/0265407505050942

Margolin, G., Oliver, P. H., Gordis, E. B., O’Hearn, H. G., Medina, A. M., Ghosh, C. M., et al. (1998). The nuts and bolts of behavioral observation of marital and family interaction. Clin. Child Fam. Psychol. Rev. 1, 195-213. doi: 10.1023/A: 1022608117322

McCubbin, H. I., and McCubbin, M. A. (1988). Typologies of resilient families: emerging roles of social class and ethnicity. Fam. Relat. 37, 247-254. doi: 10. $2307 / 584557$

Meeks, B. S., Hendrick, S. S., and Hendrick, C. (1998). Communication, love, and relationship satisfaction. J. Soc. Pers. Relationsh. 15, 755-773. doi: 10.1177/ 0265407598156003

Mohr, J., and Spekman, R. (1994). Characteristics of partnership success: partnership attributes, communication behavior, and conflict resolution techniques. Strateg. Manage. J. 15, 135-152. doi: 10.1002/smj.4250150205

Montesi, J. L., Fauber, R. L., Gordon, E. A., and Heimberg, R. G. (2011). The specific importance of communicating about sex to couples' sexual and overall relationship satisfaction. J. Soc. Pers. Relationsh. 28, 591-609. doi: 10.1177/ 0265407510386833

Neff, L. A., and Karney, B. R. (2004). How does context affect intimate relationships? Linking external stress and cognitive processes within marriage. Pers. Soc. Psychol. Bull. 30, 134-148. doi: 10.1177/0146167203255984

O’Brien, M., John, R. S., Margolin, G., and Erel, O. (1994). Reliability and diagnostic efficacy of parents' reports regarding children's exposure to marital aggression. Violence Vict. 9, 45-62. doi: 10.1891/0886-6708.9.1.45

Pennebaker, J. W., Booth, R. J., and Francis, M. E. (2015). Linguistic Inquiry and Word Count: LIWC. Austin, TX: LIWC.net.

Randall, A. K., and Bodenmann, G. (2009). The role of stress on close relationships and marital satisfaction. Clin. Psychol. Rev. 29, 105-115. doi: 10.1016/j.cpr.2008. 10.004
Randall, A. K., and Bodenmann, G. (2017). Stress and its associations with relationship satisfaction. Curr. Opin. Psychol. 13, 96-106. doi: 10.1016/j.copsyc. 2016.05.010

Randall, A. K., Tao, C., Totenhagen, C. J., Walsh, K., J., and Cooper, A. (2016). Associations between sexual orientation discrimination and depression among same-sex couples: moderating effects of dyadic coping. J. Couple Relationsh. Ther. 16, 325-345 doi: 10.1080/15332691.2016.12 53520

Reid, D. W., Dalton, E. J., Laderoute, K., Doell, F. K., and Nguyen, T. (2006). Therapeutically induced changes in couple identity: the role of we-ness and interpersonal processing in relationship satisfaction. Genet. Soc. Gen. Psychol. Monogr. 132, 241-284. doi: 10.3200/MONO.132.3.24 $1-288$

Rentscher, K. E., Rohrbaugh, M. J., Shoham, V., and Mehl, M. R. (2013). Asymmetric partner pronoun use and demand-withdraw interaction in couples coping with health problems. J. Fam. Psychol. 27, 691-701. doi: 10.1037/ a0034184

Rentscher, K. E., Soriano, E. C., Rohrbaugh, M. J., Shoham, V., and Mehl, M. R. (2015). Partner pronoun use, communal coping, and abstinence during couplefocused intervention for problematic alcohol use. Fam. Process 56, 348-363. doi: $10.1111 /$ famp. 12202

Repetti, R. L., and Wood, J. (1997). "Families accommodating to chronic stress," in Coping With Chronic Stress (Boston, MA: Springer), 191-220.

Robbins, M. L., Mehl, M. R., Smith, H. L., and Weihs, K. L. (2013). Linguistic indicators of patient, couple, and family adjustment following breast cancer. Psycho Oncol. 22, 1501-1508. doi: 10.1002/pon.3161

Rohrbaugh, M. J., Shoham, V., Skoyen, J. A., Jensen, M., and Mehl, M. R. (2012). We-talk, communal coping, and cessation success in a couple-focused intervention for health-compromised smokers. Fam. Process 51, 107-121. doi: 10.1111/j.1545-5300.2012.01388.x

Sanders, M. R., Halford, W. K., and Behrens, B. C. (1999). Parental divorce and premarital couple communication. J. Fam. Psychol. 13, 60-74. doi: 10.1037/ 0893-3200.13.1.60

Slatcher, R. B., and Pennebaker, J. W. (2006). How do I love thee? Let me count the words: the social effects of expressive writing. Psychol. Sci. 17, 660-664. doi: 10.1111/j.1467-9280.2006.01762.x

Slatcher, R. B., Vazire, S., and Pennebaker, J. W. (2008). Am "I" more important than "we"? Couples' word use in instant messages. Pers. Relationsh. 15, 407-424. doi: 10.1111/j.1475-6811.2008.00207.x

Smith, L., Heaven, P. C., and Ciarrochi, J. (2008). Trait emotional intelligence, conflict communication patterns, and relationship satisfaction. Pers. Individ. Differ. 44, 1314-1325. doi: 10.1016/j.paid.2007.11.024

Story, L. B., and Repetti, R. (2006). Daily occupational stressors and marital behavior. J. Fam. Psychol. 20, 690-700. doi: 10.1037/0893-3200.20. 4.690

Tausczik, Y. R., and Pennebaker, J. W. (2010). The psychological meaning of words: LIWC and computerized text analysis methods. J. Lang. Soc. Psychol. 20, 24-54. doi: 10.1177/0261927X09351676

Totenhagen, C. J., Randall, A. K., Cooper, A., Tao, C., and Walsh, K. J. (2017). Stress spill-over in same-sex couples: concurrent and lagged daily effects. J. GLBT Fam. Stud. 31, 236-256. doi: 10.1080/1550428X.2016.1203273

Conflict of Interest Statement: The authors declare that the research was conducted in the absence of any commercial or financial relationships that could be construed as a potential conflict of interest.

Copyright (c) 2019 Lau, Randall, Duran and Tao. This is an open-access article distributed under the terms of the Creative Commons Attribution License (CC BY). The use, distribution or reproduction in other forums is permitted, provided the original author(s) and the copyright owner(s) are credited and that the original publication in this journal is cited, in accordance with accepted academic practice. No use, distribution or reproduction is permitted which does not comply with these terms. 


\section{OPEN ACCESS}

Edited by:

Guy Bodenmann,

University of Zurich, Switzerland

Reviewed by:

Evangelos C. Karademas,

University of Crete, Greece

Carolyn E. Cutrona,

lowa State University, United States

${ }^{*}$ Correspondence: Ariela Francesca Pagani ariela.pagani@unicatt.it

Specialty section:

This article was submitted to Clinical and Health Psychology, a section of the journal

Frontiers in Psychology

Received: 18 June 2018 Accepted: 11 February 2019

Published: 27 February 2019

Citation:

Pagani AF, Donato S, Parise M,

Bertoni $A$, lafrate $R$ and Schoebi $D$ (2019) Explicit Stress Communication Facilitates Perceived Responsiveness

in Dyadic Coping.

Front. Psychol. 10:401. doi: 10.3389/fpsyg.2019.00401

\section{Explicit Stress Communication Facilitates Perceived Responsiveness in Dyadic Coping}

\author{
Ariela Francesca Pagani ${ }^{*}$, Silvia Donato², Miriam Parise ${ }^{1}$, Anna Bertoni², \\ Raffaella lafrate ${ }^{2}$ and Dominik Schoebi ${ }^{3}$
}

${ }^{1}$ Family Studies and Research University Centre, Università Cattolica del Sacro Cuore, Milan, Italy, ${ }^{2}$ Department of Psychology, Family Studies and Research University Centre, Università Cattolica del Sacro Cuore, Milan, Italy, ${ }^{3}$ Department of Psychology, University of Fribourg, Fribourg, Switzerland

The present study was aimed at examining the role of explicit stress communication in the context of dyadic coping. The general aim of the present study was to test (a) whether explicit communication of daily stressful events predicted relationship satisfaction and (b) whether the perception of responsiveness in dyadic coping mediated the association between explicit stress communication and partners' satisfaction. We analyzed daily diary data from 55 married couples and multilevel analyses suggested that, although explicit stress communication was not associated with relationship satisfaction, it predicted both partners' responsiveness in dyadic coping behaviors. Finally, responsive dyadic coping behaviors mediated the relationship between explicit stress communication and relationship satisfaction. On the whole, our findings showed that perceived responsiveness in dyadic coping with daily stressors was facilitated by explicit stress communication and that this contributed to the effectiveness of dyadic coping behaviors in fostering partners' relationship satisfaction. We discussed how the current study contributes to the understanding of the dyadic coping process and its contribution to partners' satisfaction, underscoring the importance of communication skills.

Keywords: dyadic coping, explicit stress communication, perceived responsiveness, couple relationship, daily diary

\section{INTRODUCTION}

How a couple deals with stress can have a lasting effect on the relationship, even when responding to daily stressors (Randall and Bodenmann, 2009). A couple's joint response to a stressor (i.e., dyadic coping -see par. 1.1) can protect relationships from the wear and tear of daily stress, enhance partners' intimacy and further strengthen the couple relationship (Bodenmann, 2005; Milek et al., 2015). Dyadic coping requires that both partners engage in a communication process when responding to the stressor. Key elements of such communication process include one partner disclosing relevant information about the event, and the stress and negative emotions he/she feels, and the other partner responding to this disclosure (e.g., Reis and Gable, 2015). Once a couple has engaged in such a communication sequence, the partner who initiated the sequence perceives, decodes, and evaluates the other's reactions, being sensitive to the degree to which the partner responded to their concerns and needs (i.e., to the partner's "responsiveness;" 
Reis and Shaver, 1988). Perceptions of responsiveness are an integral part of the process of dyadic coping with stress (Bodenmann, 2007). It is not uncommon, however, that partners fail to be responsive to stress expressions and related disclosures. This can be the result of a lack of motivation or skills, but it can also occur because the partner does not adequately perceive or interpret the disclosed content (Reis and Shaver, 1988; Reis and Clark, 2013; Reis and Gable, 2015). Therefore, we propose that explicit and unambiguous stress communication should inoculate couples against such maladaptive coping dynamics, enhance the partner's responsiveness in the dyadic coping process, and thereby improve adaptation to the stressor and strengthen the relationship. The current research tests these predictions. In the remainder of the introduction, we first discuss the dyadic coping process and the role of responsive reactions during this process. Next, we point out the relevance of explicit stress communication for partners' responsiveness, and specifically for responsive dyadic coping behaviors, and partners' satisfaction with their relationship.

\section{Dyadic Coping}

In everyday life, all couples encounter situations in which they have to cope with minor or major stressors, and doing so effectively helps to maintain well-being and relationship satisfaction. When partners communicate about a stressor to jointly respond to it, dyadic coping occurs (Bodenmann, 1995). Dyadic coping is associated with enhanced relationship satisfaction (for a meta-analytic review, see Falconier et al., 2015) and can protect the relationship from the negative effects of stressful events (e.g., Gasbarrini et al., 2015).

Dyadic coping is conceptualized as a dyadic process involving both partners (Bodenmann, 1995, 1997, 2005), and involving an interplay between one partner's stress signals and the other partner's coping reactions (Revenson et al., 2005). The key theoretical framework, the Systematic-Transactional Model of dyadic coping (STM; Bodenmann, 1995, 2005), is based on Lazarus and Folkman's (1984) stress and coping paradigm and conceptualizes dyadic coping as partners' participation in the stress-related regulation of emotions and behavior that directly or indirectly concern both partners. The process of dyadic coping can be thought of as circular sequence, in which one partner's communication of stress (with different levels of explicitness and through various channels) is perceived and evaluated by the other partner (cfr. "dyadic stress appraisal;" Bodenmann, 2005; Bodenmann et al., 2016), who in turn reacts with a dyadic coping response (both in terms of supporting the stressed partner or implementing joint coping strategies) ${ }^{1}$. What the other does or does not during the process is then perceived, decoded and evaluated by the partner who initiated the sequence and it is on the basis of these perceptions and evaluations that the stressed partner may feel more or less satisfied with the response and may continue with the cycle by giving his/her own feedback to the other. Partner responsiveness is a key element

\footnotetext{
${ }^{1}$ It is important to note that not only negative events, but also positive though challenging events, can trigger coping responses (e.g., Langston, 1994; Gable and Reis, 2010).
}

of these evaluations. Dyadic coping responses, in fact, have been conceptualized as either positive (i.e., providing emotional or instrumental support) or negative (i.e., unsupportive, ambivalent, or superficial forms of support; see, for a presentation of various types of dyadic coping responses: Bodenmann, 1997, 2005) and -besides their theoretical valence- they can be perceived as more or less responsive to the partners' needs, which makes them more or less beneficial for the individual and the relationship (e.g., Bodenmann, 2007; Iafrate and Donato, 2012; Donato, 2014).

Dyadic coping reactions that are highly responsive convey that one's partner is present and committed to the relationship, that he or she can be relied on, and that he or she is also a skillful support provider. It allows both partners to feel a reciprocal connection, enhancing a sense of trust, validation and support, and a sense of we-ness (Cutrona, 1996; Bodenmann, 2005). In other words, such a relationship is marked by high intimacy, with partners attending to and responding "supportively to each other's needs, wishes, concerns, and goals" (Reis and Clark, 2013, p. 400). Responsive interactions favor partners' belief that both members of the couple will take care of each other and will react supportively (Reis et al., 2004). Perceived responsiveness is important when partners decide to share personal stressors, or when they want to resolve a conflict, or share or negotiate important personal needs and goals (Reis and Clark, 2013; Reis and Gable, 2015). Specifically, perceptions of partner responsiveness were found to maximize the benefits derived from social support behaviors (e.g., Collins and Feeney, 2000; Maisel and Gable, 2009), which represent an important component of the dyadic coping process. Beyond any specific behavioral response a partner can enact in front of the other stress communication, perceptions of responsiveness are likely to affect partners' relationship satisfaction as resulting from the dyadic coping process.

Although communication is considered an integral part of the dyadic coping model, less attention has been dedicated to the way partners communicate stressful events, and whether the nature of such a communication might affect whether a partner reacts more or less responsively. While much more attention to the role of communication can be found in the support literature, and particularly in the study of support solicitation (e.g., Cunningham and Barbee, 2000), surprisingly less attention was devoted to this aspect in the dyadic coping literature. In the context of the dyadic coping process, communication actually allows the stressful event to become a relational issue: It is through the communication of the event and of the stress derived from it that partners can engage in dyadic coping reactions (Bodenmann, 2005). Moreover, in the dyadic coping process communication may not only indicate which behavioral options better match with the kind of support that is requested by the stressed partner (cfr. the "optimal matching model;" Cutrona and Russell, 1990), but also allows to make such behaviors as responsive as possible to the others' needs. The same actions of practical support (i.e., delegated dyadic coping, as define within the STM), for example, can be carried out with more or less attention to the other's need of protect his/her own sense of autonomy and competence. 


\section{The Role of Explicit Stress Communication for Responsive Dyadic Coping Behaviors}

Research on communication suggests that couples in which both partners communicate their feelings and concerns openly reported higher levels of relational satisfaction than couples who communicate without elaborating on the events or their feelings (Guerrero et al., 2011). More specifically, communication may lead to relational happiness if it is characterized by a mutual discussion of problems, by partners' expression of feelings, by partners' attempts to understand the point of view of the other, and by a negotiation to find a solution to the problem (Katriel and Philipsen, 1981; Caughlin, 2003; Chi et al., 2013). The central role of explicit communication is highlighted in a recent study on communication of positive events and daily wellbeing, showing that on days partners reported more explicit communication of positive events, they also reported better individual and relational well-being (Pagani et al., 2015). The authors defined explicit communication as referring to partners talking openly and specifically about an event, adding details about it and possibly expressing their own points of view and emotions with regard to it, while implicit communication to partners talking only indirectly or superficially about the event, without elaborating on it and/or without direct expression of their perspectives and emotional reactions to the event. While explicit communication gives the listener sufficient information to accurately understand what happened and the effects of it on the stressed partner, implicit communication can be ambiguous and lead to misunderstandings. Yet, although partners value open and explicit communication of events and emotions, and although they try to implement it, they often communicate in a closed and implicit way (Kirkman et al., 2005; Caughlin et al., 2011; Goldsmith and Domann-Scholz, 2013), raising the risk that communicated events and moods are misinterpreted or misunderstood.

For effective dyadic coping transactions more specifically, it is not only important that the stressed partner interprets the other's supportive reactions as responsive to his or her needs, but also that the stressed partner is involved in the dyadic exchange and enables the other to enact responsive behaviors. Limited attention has been paid to the role of the communication component of the dyadic coping process, even though communication is a critical part of dyadic coping trainings (CCET, Bodenmann and Shantinath, 2004; TOGETHER, Falconier, 2015). Some insights on the role of communication can be borrowed from the social support literature (for conceptual differences between dyadic coping and partner support, see Donato, 2014; Donato et al., 2015). In this literature, the contribution of the support recipient to the support process has recently gained increased attention (e.g., Lawrence et al., 2008; Verhofstadt et al., 2013). Support seekers play an active role in support transactions, contributing in important ways to how the interaction evolves (Pearlin and McCall, 1990). Moreover, research on support solicitation has shown that positive vs. negative support seeking behaviors differ between distressed and non-distressed spouses (Verhofstadt et al., 2013). In their broad definition of support seeking behaviors, the authors included explicitness of communication as a key component of positive support seeking and found that "when seeking support, distressed couples are -as compared to non-distressed ones- more inclined to make demands for help, to complain and whine and less inclined to ask for help or state their needs in an open and clear way" (Verhofstadt et al., 2013, p. 334).

Specifically, it has been theorized for the case of dyadic coping that a clear and explicit stress communication is required, so that the other could implement effective forms of coping (Bodenmann, 2005). Only recently, however, has the role of the communication mode received empirical attention. It has been found, for example, that, in couples with one depressed partner, the enhancement of mutual support and explicit communication about the personal stress through copingoriented couple therapy was positively related to partners' levels of relationship satisfaction and expressed emotions (Bodenmann et al., 2008). Clear and explicit stress communication is arguably more suited to engage the partner into a responsive interaction than implicit communication (Reis, unpublished), as it helps avoiding ambiguity about the intentions and content of the communication. More recently, additional evidence suggested that during videotaped discussions following a stress induction, partners adjusted their dyadic coping behaviors as a function of the form of stress communication used by the stressed partner (Kuhn et al., 2017). In particular, problem-oriented stress communication consistently predicted problem-oriented dyadic coping, while emotion-oriented dyadic coping was more likely to follow other forms of stress communication (emotionoriented, non-verbal, neutral; Kuhn et al., 2017). What remained unaddressed is whether explicit stress communication had implications for the perceived responsiveness of the other's dyadic coping reactions. This seems a crucial link, however, as partners' perceptions of the other's dyadic coping behaviors are considered key components of the dyadic coping process, mediating the link between actual behaviors and relationship satisfaction (Donato et al., 2015).

\section{The Current Study}

The general aim of the present study was to examine the role of explicit communication of daily stressful events for facilitating partners' responsive dyadic coping behaviors and, in turn, sustaining their relationship satisfaction. In particular, we examined whether greater communication explicitness of daily stressful events predicted higher relationship satisfaction (Hypothesis 1). Second, we tested whether perceptions of more responsive dyadic coping reactions from the partner mediated the association between the stressed partner's communication of his or her stress and his or her own relationship satisfaction (Hypothesis 2). We focused on explicitness of disclosures about the stressful event (i.e., event stress communication), rather than explicitness in the expression of the emotions connected to the event, given that explicit emotion communication was found to be rare in dyadic coping interactions (Kuhn et al., 2017). Finally, we tested whether the above associations differed by partners' gender. We did not have specific hypotheses at this regard, as research on both stress communication and dyadic coping reported relatively inconsistent findings in terms 
of gender effects. While some research found gender effects in self-disclosure (Dindia and Allen, 1992), more recent research specifically focusing on stress communication did not (Kuhn et al., 2017). Literature on dyadic coping similarly evidenced both gender differences (e.g., Bodenmann et al., 2015) and similarities (Donato et al., 2015) in partner's dyadic coping responses.

\section{MATERIALS AND METHODS}

\section{Participants}

Participants were 55 Italian heterosexual couples $(N=110$ subjects). Age range of partners was 26-64 years (women: $M=42.61, S D=7.88$; men: $M=45.45, S D=8.42$ ). Partners were together for 17 years on average $(S D=9.40)$. About half of all men $(49.1 \%)$ reported a technical school diploma or a high school diploma, while $43.6 \%$ of female partners reported a higherlevel degree. The modal net income was between 1,000 and 1,500 Euro for both women (34.6\%) and men (38.2\%). Ninetysix point four percent of participants were Catholic, while the remaining partners reported no religious affiliation. This feature is in line with the prevalence of Catholic religious affiliation in Italy. Participants were recruited partially through snowball sampling and partially through the help of their children's school. In particular, an institute (from elementary school to high school) gave us permission to handle questionnaires out to students' parents, whenever willing to participate to the study. To participate to the study partners had to be cohabiting for at least 3 years.

\section{Procedure}

Couples filled in a time-based electronic daily diary on a Personal Digital Assistant (PDA) twice a day (during the lunch break and before going to bed) for 2 weeks. Research assistants visited participants in their home to help them familiarize with the use of the PDAs and the reporting plan. Participants were instructed not to provide reports retrospectively if they had forgotten to complete the questionnaire at the expected time. The device was also programed to prevent returning to previous sets of questions. Participants were informed that participation was voluntary, that they could stop whenever they wanted without justification. Written informed consent was filled in by all couples. The study protocol was not reviewed by the ethics committee, since it was not required at the time of data collection as per University's guidelines and national regulations. However, it complied with the Ethical Guidelines of the Italian Association of Psychology (AIP) and with the Ethical Guidelines of the American Psychological Association (APA).

\section{Measures}

\section{Explicit Stress Communication}

Participants were asked to report twice a day (during the lunch break and before going to bed) whether they experienced stressful events and whether they shared them with the partner. In case they shared the event with the partner, we assessed the degree to which the event was communicated explicitly with the item "When I communicated what happened to my partner, to what extent was I clear and explicit?" Participants responded by means of a 5 -point scale (from $1=$ not at all to $5=$ very $m u c h$ ).

\section{Perceived Responsiveness}

In both daily assessments, in case participants reported they experienced a negative event, they were also asked to indicate their perception of the partner's responsiveness using the following prompt: "When I communicated this negative event, to what extent my partner...." The prompt was followed by three items based on Reis (unpublished) perceived responsiveness scale: "My partner understood me," "My partner made me feel like he/she valued my abilities and opinions," "My partner made me feel cared for." Participants reported their agreement to each item on a 5 -point scale (from $1=$ not at all to $5=$ very $m u c h$ ). The three items were combined to create average responsiveness scores. Cronbach's alpha for the scale was 0.93 .

\section{Relationship Satisfaction}

The momentary relationship satisfaction was assessed with an item starting from the stem "Today our relationship was..." and measured on a 7 -point scale $(1=$ terrible, $4=o k$, $7=$ terrific). These reports were assessed only once per day before going to bed.

\section{Data Analysis}

Due to the nested nature of our data, we used multi-level modeling for dyadic data with the software Mplus 7 (Muthén and Muthén, 1987/2017). The dataset consisted of 51 women and 51 men from 55 couples who provided 2826 reports on their daily experiences (with $9.6 \%$, or 270 missing datapoints; missing reports were taken into account by using ML estimation). Sufficient information from both partners to estimate random variation of effects, and their covariance between partners, was available for 47 couples, but estimations for the main results reported below are based on data from 102 individuals from 55 couples. A hierarchical linear model for distinguishable dyadic diary data was estimated (repeated assessments of two partners nested within couples). In this model, repeated measures of the two individuals were represented as lower level variables, while the upper level represented between-couple variability across male partners and across female partners (Bolger and Laurenceau, 2013). Communication explicitness was coded 0 when no stressful events were experienced, such that the comparison level of explicit or implicit stress communication effects were days with average explicitness or no communication.

To examine whether explicit stress communication predicted partners' responsive dyadic coping, which in turn predicted relationship satisfaction, we tested a within-subject mediation model, following a procedure proposed by Bolger and Laurenceau's (2013). We controlled for the day of the diary period. The day variable was centered at day 7 . We also included between-subject means of explicit stress communication. To make sure that our results referred to the effect of explicit stress communication rather than to the effects of the mere disclosure of the stressful event, we also controlled for the fact that the stressful event was communicated to the partner irrespective of how explicit this communication was. The inclusion of the 
disclosure variable in the model did not change the results. Disclosure, moreover, did not show any significant effect in the model. Disclosure, therefore, was not entered in the final model.

A post hoc power estimation for the parameters obtained in the current analyses, using a Monte Carlo procedure (see Bolger et al., 2012), suggested good statistical power for effects of explicit stress communication on responsiveness in dyadic coping behaviors $(1-\beta>0.84)$, and for effects of responsiveness in dyadic coping behaviors on relationship satisfaction $(1-\beta>0.97)$, and for women's $(1-\beta>0.92)$, but not for men's effects of explicit stress communication on relationships satisfaction $(1-\beta=0.17)$.

\section{RESULTS}

\section{Descriptives}

Before testing the hypotheses, we examined the means and the standard deviations of all variables (Table 1). Paired sample $t$-test showed a significant effect of gender for explicit stress communication. In particular, women reported significantly higher explicit stress communication than men, $t(46)=2.49$; $p=0.01$ (women: $M=4.06, S D=0.69$, men: $3.56, S D=0.94$ ). Moreover, paired sample $t$-test showed that the effect of gender was not significant for perceived responsiveness in partners' dyadic coping reactions, $t(46)=0.79 ; p=0.43$ (women: $M=3.39$, $S D=1.06$, men: $M=3.27, S D=1.14)$. In addition, paired sample $t$-test showed that the effect of gender was not significant for relationship satisfaction, $t(56)=-0.63 ; p=0.52$ (women: $M=4.55, S D=0.71$, men: $M=4.61, S D=0.91)$.

Finally, we examined the correlations between explicit stress communication, responsiveness, and relationship satisfaction separately for women and men. As shown in Table 2, for both partners all variables were positively correlated with each other.

\section{Associations Between Explicit Stress Communication, Responsiveness in Dyadic Coping, and Relationship Satisfaction}

In a series of preliminary analyses, we conducted model comparisons to examine whether the focal coefficients in the current model differed significantly between men and women. None of the tests suggested a significant gender effect $\left[\chi^{2}(1)<2.66 ; p>0.10\right]$, and we therefore set equality constraints, estimating a single set of coefficients for men

TABLE 1 | Values of explicit stress communication, responsiveness, and relationship satisfaction.

\begin{tabular}{|c|c|c|c|c|c|c|}
\hline & \multicolumn{2}{|c|}{ Mean } & \multicolumn{2}{|c|}{$S D$} & \multicolumn{2}{|c|}{ Range } \\
\hline & Woman & Man & Woman & Man & Woman & Man \\
\hline $\begin{array}{l}\text { Explicit stress } \\
\text { communication }\end{array}$ & 4.06 & 3.56 & 0.69 & 0.94 & $2.5-5$ & $1-5$ \\
\hline Responsiveness & 3.39 & 3.27 & 1.06 & 1.14 & $1-5$ & $1-5$ \\
\hline $\begin{array}{l}\text { Relationship } \\
\text { satisfaction }\end{array}$ & 4.55 & 4.61 & 0.71 & 0.91 & $3.20-6.27$ & $2.21-6.81$ \\
\hline
\end{tabular}

TABLE 2 | Pearson's correlations between explicit stress communication, responsiveness, and relationship satisfaction.

\begin{tabular}{lccc}
\hline & $\begin{array}{c}\text { Explicit stress } \\
\text { communication }\end{array}$ & Responsiveness & $\begin{array}{c}\text { Relationship } \\
\text { satisfaction }\end{array}$ \\
\hline $\begin{array}{l}\text { Explicit stress } \\
\text { communication }\end{array}$ & 1 & 0.34 & 0.30 \\
Responsiveness & 0.51 & $p=0.01$ & $p=0.03$ \\
Relationship & $p<0.001$ & 1 & 0.64 \\
satisfaction & 0.37 & 0.76 & 0.001 \\
\end{tabular}

Women's correlations are above the diagonal and men's correlations are below the diagonal.

and women. The results did not support the idea that more explicit communication of the stressful event was associated with relationship satisfaction, above and beyond perceptions of responsiveness $\left(\beta=-0.12, \mathrm{SE}=0.07, p=0.08, \mathrm{CI}_{95}=[-0.26\right.$, 0.02]) $(\mathrm{H} 1)^{2}$. The results showed that more explicit stress communication was positively associated with the perception of the partner's responsiveness in dyadic coping in both women and men. In particular, on days partners communicated their stressful event more explicitly they also perceived more responsive dyadic coping reactions from their partner, as compared to days when they communicated less explicitly or when no stress was reported $\left(\beta=0.37, S E=0.11, p=0.001, \mathrm{CI}_{95}=[0.15,0.58]\right)$. Overall, these results suggested that, for women and men, communicating a stressful event in an explicit way to the partner had a positive effect on the perception of partner's responsiveness in their dyadic coping behaviors. Findings also suggested that, in turn, responsiveness in dyadic coping was positively associated with partners' relationship satisfaction $(\beta=0.53, S E=0.09$, $\left.p<0.001, \mathrm{CI}_{95}=[0.35,0.71]\right)$. Finally, a test of indirect effects of explicit stress communication via perceptions of responsive dyadic coping reactions on relationship satisfaction suggested a significant mediation (H2; Figure 1; mediational path: $\beta=0.24$, $\left.S E=0.10, p=0.018, \mathrm{CI}_{95}=[0.04,0.44]\right)^{3}$.

\section{DISCUSSION}

The goal of the current work was to increase our understanding of explicit stress communication in the context of dyadic coping. In particular, we tested whether explicit communication of stressful

\footnotetext{
${ }^{2}$ Although the model popularized by Baron and Kenny (1986) proposed that establishing the relationship between $\mathrm{X}$ and $\mathrm{Y}$ is the first step in testing for a mediational effect, it is increasingly argued that step 1 (the association between $\mathrm{X}$ and $\mathrm{Y}$ ) should not be an essential condition for establishing mediation (see Kenny et al., 1998; Shrout and Bolger, 2002; Zhao et al., 2010). A number of plausible reasons exist, for which the $\mathrm{X}-\mathrm{Y}$ effect may not emerge as a statistically significant coefficient, even when mediation does exist: Expected and plausible multicollinearity between variables involved in a mediation; expected and plausible lower power to detect the X-Y effect than the mediated effect; etc. (see: Zhao et al., 2010; Kenny and Judd, 2014).

${ }^{3}$ The confidence intervals were constructed based on the standard errors of the model estimates, which are based on full maximum likelihood estimation. Bias corrected confidence intervals based on a bootstrapping procedure, commonly recommended for testing mediation, are not reported because the bootstrap procedure is not available for two-level random analyses in Mplus.
} 


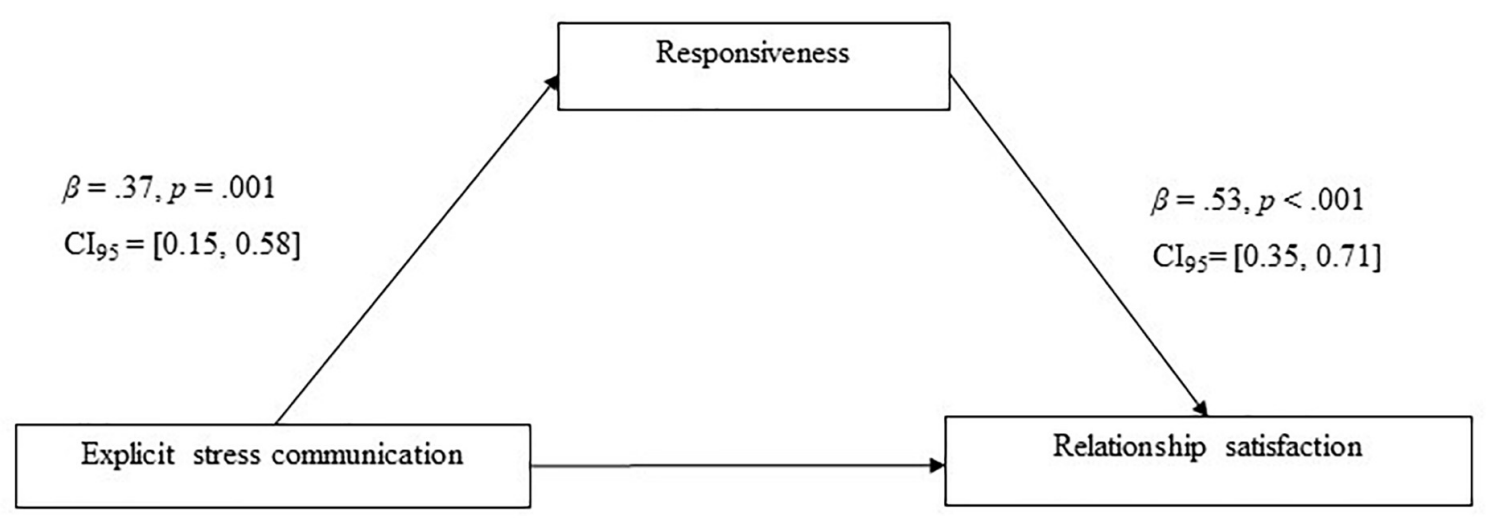

DIRECT EFFECT

$$
\begin{gathered}
\beta=-.12, p=.08, \mathrm{CI}_{95}=[-0.26,0.02] \\
\text { INDIRECT EFFECT } \\
\beta=.24, p=.018, \mathrm{CI}_{95}=[0.04,0.44]
\end{gathered}
$$

FIGURE 1 | Parameters of explicit stress communication and responsiveness for relationship satisfaction.

events predicted partners' relationship satisfaction, and whether perceptions of responsiveness in dyadic coping established an indirect link between explicitness in communication and relationship satisfaction. Overall, the findings underscore the role of explicit stress communication as a facilitator of perceived responsiveness in dyadic coping with daily stressors.

Disconfirming our first hypothesis, however, the findings did not reveal explicit stress communication as a predictor of relationship satisfaction. This finding is not in line with communication studies that suggest that self-disclosure is positively associated with partners' relationship satisfaction (e.g., Sprecher and Hendrick, 2004). This points to the possibility that, unlike in a larger relational context, explicit communication of a specific experience of stressful events on a particular day is not sufficient to bring about short-term improvements of partners' relationship satisfaction. This seems a plausible possibility, particularly since explicit communication was captured in or briefly after the stressful experience. The stressful experience itself may bear negative consequences for immediate relational well-being on its own right and therefore foreshadow the beneficial effects of explicit stress communication. Event sampling studies or longer-term momentary studies that can gather information on larger numbers of stressful events per person would allow for reliable comparisons only among different stressful episodes, which could help clarify this point. It is also possible, however, that explicit communication draws particular attention to a stressful experience, which would not only benefit the dyadic coping process, but could also lead to a more intense stress-related interaction, and therefore impede immediate improvements of satisfaction, while still facilitating improvements on a longer term. In the dyadic coping model, in fact, communication is considered as a necessary, yet not sufficient condition for the process to succeed
(Bodenmann, 2005). Thus, the stress-focus of the dyadic coping situation, and the current study, may represent a case that differs from other, more general communication contexts. A study on explicit communication of positive events predicted both women and men's relationship satisfaction (Pagani et al., 2015). Communicating about stressful circumstances involves more challenges and may therefore be riskier than communicating about positive ones. Disclosing one's negative experiences may run the risk to threaten the stressed partner's sense of efficacy and competence and therefore to elicit a negative emotional state, which could impede momentary improvements in relationship satisfaction. In negative circumstances, partners' communication may not be beneficial per se, but only when eliciting a responsive reaction by the partner, as we further discussed below.

Although more explicit stress communication did not predict higher relationship satisfaction, our findings show that both women's and men's explicit stress communication facilitated perceptions of responsiveness of the partner's dyadic coping behaviors. Notably, our focus was on within-individual effects. Partners who are prone to be explicit and open in communication could also be prone to perceive the other as more validating and caring, also irrespective of his/her actual behaviors. Our findings, nonetheless, could also reflect the possibility that explicit stress communication may facilitate the other's actual responsiveness, by signaling opportunities of support and dyadic coping to the partner and by helping him/her to avoid misunderstandings, facilitate more benevolent, external appraisals of the stressed partner's behaviors, and feel less attacked or blamed. Both of these possibilities would likely favor more responsive reactions from a helping partner during the dyadic coping process.

Finally, perceived responsiveness during dyadic coping significantly mediated the link between explicit stress communication and relationship satisfaction, confirming 
Hypothesis 2. Perceptions of responsiveness in dyadic coping behaviors may help each partner to experience the other as aware of and supportive to his/her needs and goals, and as willing to stand by his/her side with benevolent acceptance also in moments of difficulty and frailty (Rusbult et al., 2005; Gable and Reis, 2006; Reis, 2007; Reis and Gable, 2015). Such a sense of attention, support, and acceptance may well promote partners' relationship satisfaction. Indeed, one partner's perceptions about the other's dyadic coping responses were found to mediate the association between actual dyadic coping behaviors and relationship satisfaction (Donato et al., 2015). If replicated, our findings have implications for both research on dyadic coping and for preventive interventions for couples.

As for research on dyadic coping, the present study highlights how studying the communication phase of the dyadic coping process is particularly warranted. Stress communication, in fact, has revealed as an important "situational" antecedent of dyadic coping responses as they are enacted in the context of each dyadic coping interaction. While most studies focused on individual, dispositional antecedents of partners dyadic coping responses (see for a review, Donato and Pagani, 2018), the role of more proximal and situational factors facilitating or inhibiting effective dyadic coping reactions is still under-investigated. A future line of inquiry in dyadic coping research could examine each specific component of the dyadic coping process (see also Leuchtmann and Bodenmann, 2018). At this regard, in fact, only a recent study approached a micro-analytic investigation of dyadic coping conversations (Kuhn et al., 2017).

As for preventive intervention aiming at promoting partners' relationship satisfaction, our findings highlight two relevant aspects to be targeted by such interventions: Explicitness in communication of stressful events and partners' responsive dyadic coping behaviors. By increasing partners' explicitness in communication as well as fostering positive responsive reactions to the others' communication, preventive programs aimed at training partners' dyadic coping skills (CCET, Bodenmann and Shantinath, 2004) were found to enhance partners' relationship satisfaction (e.g., Bodenmann et al., 2014). In particular, our findings point to the fact that the partner's ability to make the other feel understood, validated, and cared for in stressful circumstances is of considerable importance for partners' satisfaction. Our findings also show that the stressed partner's ability to communicate clearly and explicitly can facilitate these perceptions and therefore the success of the dyadic coping process.

The present findings should be considered in light of important limitations of the study. The correlational nature of the effects allows for no strong causal interpretation. Moreover, the data were collected from a convenience sample of mostly non-distressed and relatively satisfied couples, and, therefore, further research with more representative samples is needed to confirm these findings for the broader population. For example, the same relation should be analyzed in couples seeking clinical help, and especially for those partners that show insecure attachment styles, which is related to ineffective support seeking and unresponsive caregiving (Collins and Feeney, 2000). In addition, our study focused on daily stressful events. We did not have information on whether such events represented minor or major stressors. Future research should test whether the associations examined in the present study would be different in couples dealing with minor stressful circumstances and in couples experiencing more severe, disruptive stressful conditions (see Randall and Bodenmann, 2009).

Although the present study tested important assumptions of the STM model, future research could profitably focus on also how explicit stress communication may be associated to specific dyadic coping behaviors by the partner. In the present study responsiveness was measured with regard to the partner's responses to the stressed individual, while we did not measure the partner's self-perceived responsiveness. Adding this variable in future studies could allow to test both actor and partner effects in the association examined in the present study. Moreover, cultures differ greatly with respect to communication norms and styles, in particular with regard to the emphasis on explicit communication and emotion expression (Hall, 1976; Gudykunst et al., 1996). It is therefore possible that the role of communication changes as a function of different cultural contexts. Dyadic coping, although found to be significantly associated to relationship quality across different cultural contexts, also differed in the strength of its effects across different countries (Hilpert et al., 2016). The specific cultural characteristics of the present sample may, in fact, affect the results we found. In particular, Italy presents contrasting cultural features: relatively high individualism (e.g., the value of independence and individual goals) paired with some aspects of collectivism (e.g., centrality of family of origin, widespread religious affiliation, etc.); a relatively private view of the couple relationship paired with a strong connection with familial and social ties; a traditional gender-role orientation co-existing with egalitarian expectations between partners (cfr. Donato, 2016). More specifically related to communication, Italy is a culture characterized -at least relatively to Eastern countries- by lowcontext communication (i.e., relying more on the explicit verbal code than on contextual cues). Thus, in Italy explicit stress communication may be more acceptable and expected than in high-context cultures, in which -instead- explicitly expressing the individual's needs may be avoided for the sake of relationship harmony. As a consequence, in such cultures support behaviors may be offered irrespective of explicit support seeking (cfr. Falconier et al., 2016). It is possible therefore that the association between explicit stress communication and responsiveness will be weaker in high-context cultures than in low-context ones. Future research should explore this possibility. Finally, future studies may test other possible mediators of the association between explicit stress communication and relationship satisfaction, such as for example the non-stressed partner's accuracy in understanding the stressed partner's communication. Despite the limitations of the present study, these findings confirm an important role of communication in the process of dyadic coping. The importance of explicit communication in facilitating partners' effective responses to the other's stressful events confirms the active role of both partners in dyadic coping transactions. These findings call for a more attentive examination of the communication component of the dyadic coping process in both research and intervention. 


\section{AUTHOR CONTRIBUTIONS}

$\mathrm{AP}$ and SD contributed equally to the research, development of the theoretical framework, performance of the statistical analyses, analysis of the results, and writing of the manuscript. MP

\section{REFERENCES}

Baron, R. M., and Kenny, D. A. (1986). The moderator-mediator variable distinction in social psychological research: conceptual, strategic, and statistical considerations. J. Pers. Soc. Psychol. 51, 1173-1182. doi: 10.1037/0022-3514.51. 6.1173

Bodenmann, G. (1995). A systemic-transactional conceptualization of stress and coping in couples. Swiss J. Psychol. 54, 34-39. doi: 10.1016/j.cpr.2008. 10.004

Bodenmann, G. (1997). Dyadic coping: a systemic-transactional view of stress and coping among couples. theory and empirical findings. Eur. Rev. Appl. Psychol. 47, 137-140. doi: 10.3389/fpsyg.2016.00447

Bodenmann, G. (2005). "Dyadic coping and its significance for marital functioning," in Couples Coping with Stress: Emerging Perspectives on Dyadic Coping, eds T. Revenson, K. Kayser, and G. Bodenmann (Washington, DC: American Psychological Association), 33-50. doi: 10.1037/11031-002

Bodenmann, G. (2007). "Dyadic coping and the 3-phase-method in working with couples," in Innovations in Clinical Practice: Focus on Group and Family Therapy, ed. L. VandeCreek (Sarasota, FL: Professional Resource Press), 235-252.

Bodenmann, G., Hilpert, P., Nussbeck, F. W., and Bradbury, T. N. (2014). Enhancement of couples' communication and dyadic coping by a self-directed approach: a randomized controlled trial. J. Consult. Clin. Psychol. 82, 580-591. doi: $10.1037 / \mathrm{a} 0036356$

Bodenmann, G., Meuwly, N., Germann, J., Nussbeck, F. W., Heinrichs, M., and Bradbury, T. N. (2015). Effects of stress on the social support provided by men and women in intimate relationships. Psychol. Sci. 26, 1584-1594. doi: $10.1177 / 0956797615594616$

Bodenmann, G., Plancherel, B., Beach, S. R., Widmer, K., Gabriel, B., Meuwly, N., et al. (2008). Effects of coping-oriented couples therapy on depression: a randomized clinical trial. J. Consult. Clin. Psychol. 76, 944-954. doi: 10.1037/ a 0013467

Bodenmann, G., Randall, A. K., and Falconier, M. K. (2016). "Systemictransactional model of dyadic coping," in Couples Coping with Stress: A CrossCultural Perspective, eds M. K. Falconier, A. K. Randall, and G. Bodenmann (New York, NY: Routledge), 5-22.

Bodenmann, G., and Shantinath, S. D. (2004). The couples' coping enhancement training (CCET): a new approach to prevention of marital distress based upon stress and coping. Fam. Relat. 53, 477-484. doi: 10.1111/j.0197-6664.2004. 00056.x

Bolger, N., and Laurenceau, J. P. (2013). Intensive Longitudinal Methods: An Introduction to Diary and Experience Sampling Research. New York, NY: Guilford.

Bolger, N., Stadler, G., and Laurenceau, J. P. (2012). "Power analysis for intensive longitudinal studies," in Handbook of Research Methods for Studying Daily Life, eds M. R. Mehl and T. S. Conner (New York, NY: Guilford Press), 285-301.

Caughlin, J. P. (2003). Family communication standards. Hum. Commun. Res. 29, 5-40. doi: 10.1111/j.1468-2958.2003.tb00830.x

Caughlin, J. P., Mikucki-Enyart, S. L., Middleton, A. V., Stone, A. M., and Brown, L. B. (2011). Being open without talking about it: a rhetorical/normative approach to understanding topic avoidance in families after a lung cancer diagnosis. Commun. Monogr. 78, 409-436. doi: 10.1080/03637751.2011. 618141

Chi, P., Epstein, N. B., Fang, X., Lam, D. O., and Li, X. (2013). Similarity of relationship standards, couple communication patterns, and marital satisfaction among Chinese couples. J. Fam. Psychol. 27, 806-816. doi: 10.1037/ a0034113

Collins, N. L., and Feeney, B. C. (2000). A safe haven: an attachment theory perspective on support seeking and caregiving in intimate relationships. J. Pers. Soc. Psychol. 78, 1053-1073. doi: 10.1037/0022-3514.78.6.1053 contributed to the development of the theoretical framework and writing of the manuscript. $\mathrm{AB}$ and RI supervised the writing of the manuscript. DS contributed to the performance of the statistical analyses, analysis of the results, and writing of the manuscript.

Cunningham, M. R., and Barbee, A. P. (2000). "Social support," in Close Relationships: A Sourcebook, eds C. Hendrick and S. S. Hendrick (Thousand Oaks, CA: SAGE Publications, Inc.), 274-286.

Cutrona, C. E. (1996). Social Support in Couples: Marriage as a Resource in Times of Stress. Thousand Oaks, CA: Sage. doi: 10.4135/9781483327563

Cutrona, C. E., and Russell, D. W. (1990). "Type of social support and specific stress: toward a theory of optimal matching," in Wiley Series on Personality Processes. Social Support: An Interactional View, eds B. R. Sarason I, G. Sarason, and G. R. Pierce (Oxford: John Wiley \& Sons), 319-366.

Dindia, K., and Allen, M. (1992). Sex differences in self-disclosure: a meta-analysis. Psychol. Bull. 112, 106-124. doi: 10.4236/sm.2015.52008

Donato, S. (2014). Il coping diadico, ovvero far fronte allo stress insieme: una rassegna della letteratura [Dyadic coping, that is managing stress together: a review of the literature]. Giornale Italiano Di Psicologia 3, 473-504.

Donato, S. (2016). "Dyadic coping in Italian couples," in Couples Coping with Stress: A Cross-Cultural Perspective, eds M. K. Falconier, A. K. Randall, and G. Bodenmann (New York, NY: Routledge), 137-152.

Donato, S., and Pagani, A. F. (2018). La Relazione di Coppia. Gestire Eventi Positivie Negativi [The Couple Relationship. Cope to Positive and Negative Events]. Rome: Carocci Faber.

Donato, S., Parise, M., Iafrate, R., Bertoni, A., Finkenauer, C., and Bodenmann, G. (2015). Dyadic coping responses and partners' perceptions for couple satisfaction: an actor-partner interdependence analysis. J. Soc. Pers. Relat. 32, 580-600. doi: 10.1177/0265407514541071

Falconier, M. K. (2015). TOGETHER - a couples' program to improve communication, coping, and financial management skills: development and initial pilot-testing. J. Marit. Fam. Ther. 41, 236-250. doi: 10.1111/jmft.12052

Falconier, M. K., Jackson, J. B., Hilpert, P., and Bodenmann, G. (2015). Dyadic coping and relationship satisfaction: a meta-analysis. Clin. Psychol. Rev. 42, 28-46. doi: 10.1016/j.cpr.2015.07.002

Falconier, M. K., Randall, A. K., and Bodenmann, G. (2016). Couples Coping With Stress: A Cross-Cultural Perspective. New York, NY: Routledge. doi: 10.4324/ 9781315644394

Gable, S. L., and Reis, H. T. (2006). "Intimacy and the self: an iterative model of the self and close relationships," in Close Relationships: Functions Forms, and Processes, eds P. Noller and J. Feeney (Hove: Psychology Press), 211-225.

Gable, S. L., and Reis, H. T. (2010). Good news! Capitalizing on positive events in an interpersonal context. Adv. Exp. Soc. Psychol. 42, 195-257. doi: 10.1016/ S0065-2601(10)42004-3

Gasbarrini, M. F., Snyder, D. K., Iafrate, R., Bertoni, A., Donato, S., and Margola, D. (2015). Investigating the relation between shared stressors and marital satisfaction: the moderating effects of dyadic coping and communication. Fam. Sci. 6, 143-149. doi: 10.1080/19424620.2015.1082044

Goldsmith, D. J., and Domann-Scholz, K. (2013). The meanings of "open communication" among couples coping with a cardiac event. J. Commun. 63, 266-286. doi: 10.1111/jcom.12021

Gudykunst, W. B., Matsumoto, Y., Ting-Toomey, S., Nishida, T., Kim, K., and Heyman, S. (1996). The influence of cultural individualism-collectivism, self construals, and individual values on communication styles across cultures. Hum. Commun. Res. 22, 510-543. doi: 10.1111/j.1468-2958.1996.tb00377.x

Guerrero, L. K., Anderson, P. A., and Afifi, W. A. (2011). Close Encounters: Communication in Relationships. Los Angeles, CA: Sage.

Hall, E. T. (1976). Beyond Culture. New York, NY: Doubleday.

Hilpert, P., Randall, A. K., Sorokowski, P., Atkins, D. C., Sorokowska, A., Ahmadi, K., et al. (2016). The associations of dyadic coping and relationship satisfaction vary between and within nations: a 35-nation study. Front. Psychol. 7:1106. doi: 10.3389/fpsyg.2016.01106

Iafrate, R., and Donato, S. (2012). "Coping in a relational context: the case of dyadic coping," in Handbook of the Psychology of Coping: New Research, eds B. Molinelli and V. Grimaldo (Hauppauge, NY: Nova Science Publisher), 111-132. 
Katriel, T., and Philipsen, G. (1981). What we need is communication: communication as a cultural category in some American speech. Commun. Monogr. 48, 301-317. doi: 10.1080/03637758109376064

Kenny, D. A., and Judd, C. M. (2014). Power anomalies in testing mediation. Psychol. Sci. 25, 334-339. doi: 10.1177/0956797613502676

Kenny, D. A., Kashy, D. A., and Bolger, N. (1998). "Data analysis in social psychology," in Handbook of Social Psychology, eds D. Gilbert, S. T. Fiske, and G. Lindzey (New York, NY: McGraw-Hill), 233-265.

Kirkman, M., Rosenthal, D. A., and Feldman, S. S. (2005). Being open with your mouth shut: the meaning of "openness" in family communication about sexuality. Sex Educ. 5, 49-66. doi: 10.1080/1468181042000301885

Kuhn, R., Milek, A., Meuwly, N., Bradbury, T. N., and Bodenmann, G. (2017). Zooming in: a microanalysis of couples' dyadic coping conversations after experimentally induced stress. J. Fam. Psychol. 31, 1063-1073. doi: 10.1037/ fam0000354

Langston, C. A. (1994). Capitalizing on and coping with daily-life events: expressive responses to positive events. J. Pers. Soc. Psychol. 67, 1112-1125. doi: 10.1037/ 0022-3514.67.6.1112

Lawrence, E., Bunde, M., Barry, R. A., Brock, R. L., Sullivan, K. T., Pasch, L. A., et al. (2008). Partner support and marital satisfaction: support amount, adequacy, provision, and solicitation. Pers. Relat. 15, 445-463. doi: 10.1111/j.1475-6811. 2008.00209.x

Lazarus, R. S., and Folkman, S. (1984). Stress, Appraisal, and Coping. New York City, NY: Springer.

Leuchtmann, L., and Bodenmann, G. (2018). "New perspectives on dynamics of dyadic coping," in When "We" are Stressed, eds A. Bertoni, S. Donato, and S. Molgora (Hauppauge, NY: Nova Science Publisher), 3-14.

Maisel, N. C., and Gable, S. L. (2009). The paradox of received social support: the importance of responsiveness. Psychol. Sci. 20, 928-932. doi: 10.1111/j.14679280.2009.02388.x

Milek, A., Butler, E. A., and Bodenmann, G. (2015). The interplay of couple's shared time, women's intimacy, and intradyadic stress. J. Fam. Psychol. 29, 831-842. doi: 10.1037/fam0000133

Muthén, L. K., and Muthén, B. O. (1987/2017). Mplus User's Guide, 8th Edn. Los Angeles, CA: Muthén and Muthén.

Pagani, A. F., Donato, S., Parise, M., Iafrate, R., Bertoni, A., and Schoebi, D. (2015). When good things happen: the effects of implicit and explicit capitalization attempts on intimate partners' well-being. Fam. Sci. 6, 119-128. doi: 10.1080/ 19424620.2015.1082013

Pearlin, L. I., and McCall, M. E. (1990). “Occupational stress and marital support: a description of micro-processes," in Stress Between Work and Family, eds J. Eckenrode and S. Gore (New York, NY: Plenum), 39-60. doi: 10.1007/978-14899-2097-3_3

Randall, A. K., and Bodenmann, G. (2009). The role of stress on close relationships and marital satisfaction. Clin. Psychol. Rev. 29, 105-115. doi: 10.1016/j.cpr.2008. 10.004
Reis, H. T. (2007). Steps toward the ripening of relationship science. Pers. Relat. 14, 1-23. doi: 10.1111/j.1475-6811.2006.00139.x

Reis, H. T., and Clark, M. S. (2013). "Responsiveness," in The Oxford Handbook of Close Relationships, eds J. A. Simpson and L. Campbell (New York, NY: Oxford University Press), 400-423.

Reis, H. T., Clark, M. S., and Holmes, J. G. (2004). "Perceived partner responsiveness as an organizing construct in the study of intimacy and closeness," in Handbook of Closeness and Intimacy, eds D. J. Mashek and A. Aron (Mahwah, NJ: Erlbaum), 201-225.

Reis, H. T., and Gable, S. L. (2015). Responsiveness. Curr. Opin. Psychol. 1, 67-71. doi: 10.1016/j.copsyc.2015.01.001

Reis, H. T., and Shaver, P. (1988). "Intimacy as an interpersonal process," in Handbook of Personal Relationships, ed. S. Duck (Chichester: Wiley), 367-389.

Revenson, T., Kayser, K., and Bodenmann, G. (2005). Emerging Perspectives on Couples' Coping with Stress. Washington, DC: American Psychological Association. doi: 10.1037/11031-000

Rusbult, C. E., Kumashiro, M., Stocker, S. L., and Wolf, S. T. (2005). "The michelangelo phenomenon in close relationships," in On building, Defending, and Regulating the Self: A Psychological Perspective, eds A. Tesser, J. Wood, and D. A. Stapel (New York, NY: Psychology Press), 1-29.

Shrout, P. E., and Bolger, N. (2002). Mediation in experimental and nonexperimental studies: new procedures and recommendations. Psychol. Methods 7, 422-455. doi: 10.1037/1082-989X.7.4.422

Sprecher, S., and Hendrick, S. S. (2004). Self-Disclosure in intimate relationships: associations with individual and relationship characteristics over time. J. Soc. Clin. Psychol. 23, 857-877. doi: 10.1521/jscp.23.6.857. 54803

Verhofstadt, L. L., Lemmens, G. M. D., and Buysse, A. (2013). Support-seeking, support-provision and support-perception in distressed married couples: a multi-method analysis. J. Fam. Ther. 35, 320-339. doi: 10.1111/1467-6427. 12001

Zhao, X., Lynch, J. G., and Chen, Q. (2010). Reconsidering baron and kenny: myths and truths about mediation analysis. J. Consum. Res. 37, 197-206. doi: $10.1086 / 651257$

Conflict of Interest Statement: The authors declare that the research was conducted in the absence of any commercial or financial relationships that could be construed as a potential conflict of interest.

Copyright (C) 2019 Pagani, Donato, Parise, Bertoni, Iafrate and Schoebi. This is an open-access article distributed under the terms of the Creative Commons Attribution License (CC BY). The use, distribution or reproduction in other forums is permitted, provided the original author(s) and the copyright owner(s) are credited and that the original publication in this journal is cited, in accordance with accepted academic practice. No use, distribution or reproduction is permitted which does not comply with these terms. 


\section{OPEN ACCESS}

Edited by:

Karin G. Coifman,

Kent State University, United States

Reviewed by

Reuma Gadassi-Polack

Yale University, United States

Josefina M. Grau,

Kent State University, United States

*Correspondence.

Anna-Lena Zietlow Anna-Lena.Zietlow@ med.uni-heidelberg.de

Monika Eckstein

Monika.Eckstein@

med.uni-heidelberg.de

Beate Ditzen

Beate.Ditzen@med.uni-heidelberg.de

† Shared first authorship

Specialty section:

This article was submitted to Clinical and Health Psychology,

a section of the journal

Frontiers in Psychology

Received: 05 July 2018 Accepted: 04 December 2018 Published: 09 January 2019

Citation:

Zietlow A-L, Eckstein M, Hernández C, Nonnenmacher $N$, Reck C, Schaer M, Bodenmann G, Heinrichs M and Ditzen B (2019) Dyadic Coping and Its Underlying

Neuroendocrine Mechanisms Implications for Stress Regulation.

Front. Psychol. 9:2600.

doi: 10.3389/fpsyg.2018.02600

\section{Dyadic Coping and Its Underlying Neuroendocrine Mechanisms - Implications for Stress Regulation}

\author{
Anna-Lena Zietlow ${ }^{1 * t}$, Monika Eckstein ${ }^{1 * t}$, Cristóbal Hernández ${ }^{1,2}$, \\ Nora Nonnenmacher ${ }^{3}$, Corinna Reck ${ }^{4}$, Marcel Schaer ${ }^{5}$, Guy Bodenmann ${ }^{6}$, \\ Markus Heinrichs ${ }^{7}$ and Beate Ditzen ${ }^{1 *+}$ \\ ${ }^{1}$ Center for Psychosocial Medicine, Institute of Medical Psychology, Heidelberg University Hospital, Heidelberg, Germany, \\ ${ }^{2}$ School of Psychology, Pontifical Catholic University of Chile, Santiago, Chile, ${ }^{3}$ Center for Psychosocial Medicine, General \\ Psychiatry, Heidelberg University Hospital, Heidelberg, Germany, ${ }^{4}$ Department of Psychology, Ludwig-Maximilians- \\ University, Munich, Germany, ${ }^{5}$ School of Applied Psychology, ZHAW Zurich University of Applied Sciences, Zurich, \\ Switzerland, ${ }^{6}$ Department of Clinical Psychology, Children, Youth and Family, Institute for Psychology, University of Zurich, \\ Zurich, Switzerland, ${ }^{7}$ Laboratory for Biological and Personality Psychology, Department of Psychology, \\ Albert-Ludwigs-University of Freiburg, Freiburg im Breisgau, Germany
}

Previous research suggests that neuroendocrine mechanisms underlie inter-individual stress coping in couples. The neuropeptide oxytocin (OT), while regulating stresssensitive HPA-axis activity might be crucial in this process. The purpose of this study was to examine the impact of dyadic coping abilities and OT on HPA-axis outcomes and constructive behavior during couple conflict. We conducted a secondary analysis of our previous database (Ditzen et al., 2009), assessing the modulating role of dyadic coping and intranasal OT on couple conflict behavior. The data revealed a significant interaction effect of the dyadic coping by oneself score and OT on cortisol responses during couple conflict, suggesting that particularly individuals with low a priori dyadic coping benefit from OT in terms of dampened HPA-activity. The results are in line with previous research suggesting OT's central role for stress regulation and prosocial behavior. Furthermore, an interaction with dyadic coping indicates adaptations in the sensitivity of the OT system during the individual attachment and relationship history. These data add to the evidence that the neuroendocrine attachment systems influence couple behavior. Future studies of neurobiological mechanisms underlying dyadic coping will be of high relevance for the development of prevention and intervention programs.

Keywords: dyadic coping, couple conflict, oxytocin, HPA-axis, cortisol, relationship satisfaction

\section{INTRODUCTION}

Conflicts per se are no indicator for a dysfunctional relationship, as they form part of social cohabitation. The way a couple manages to solve problems and conflicts in a constructive way and deals with negative affect are of vital importance not only for couple satisfaction but also for stress reaction and health which is also shown on neurobiological level: Research regarding couple interaction has shown that couples differ in the extent and the frequency of positive and negative affect and their psychobiological reactions toward a conflict situation (Ditzen et al., 2013; Baucom et al., 2015). The neuromodulator oxytocin (OT) seems to be involved in the rewarding and 
stimulating aspects of social interaction on a central nervous system level (Insel and Young, 2001; Zietlow et al., 2016; Feldman, 2017). Above this, OT has a regulatory effect on neuroendocrine stress responses (Neumann, 2002; Sullivan and Dufresne, 2006; Eckstein and Hurlemann, 2013), thereby modulating cognitive and endocrine regulation of stress and affect (Heatherton and Wagner, 2011).

In a secondary analysis of our database (c.f. Ditzen et al., 2009, 2013) we examined the links between OT, dyadic coping and cortisol reactivity and behavior during couple conflict interaction in a sample of healthy couples. Prior to the conflict discussion, couples self-administered intranasal OT or placebo, so that the effects of external OT in interaction with dyadic coping style could be investigated.

\section{BACKGROUND}

\section{Dyadic Coping}

The theoretical framework of the Systemic Transactional Model (Bodenmann, 1995, 2005a) proposes that in a committed close relationship the daily stress experiences of one partner concern both partners, either directly (e.g., both partners are affected by the same stressor or the couple relationship causes stress) or indirectly (one partner's stress spills over to the couple relationship) representing a dyadic phenomenon (Randall and Bodenmann, 2009). Therefore, dyadic coping which is defined as the couples' mutual, interpersonal stress regulation and the dyadic capacity to deal with couple external stressors, has become a central concept within couple research (Bodenmann, 1997, 2000, 2005b). Coping dyadically with stress always includes both stress expression and dyadic support (Bodenmann, 2005a).

Functional and flexible emotion and stress regulation capacities of the partners are of particular importance in this process (Rusu et al., 2018) which in turn lead to increasing relationship satisfaction (Bodenmann et al., 2006; Herzberg, 2013; Falconier et al., 2015) and improved stress recovery (Ditzen et al., 2008, 2011; Meuwly et al., 2012). During the transition to parenthood these processes are even more important as they affect not only the couple but also the forming family with the risk to spill over to the next generation and hence to affect child socio-emotional development.

\section{Neuroendocrine Mechanisms Involved in Coping With Stress HPA Axis Activation}

Neuroendocrine regulation of stress is a central aspect of mental and physical health with the hypothalamic-pituitaryadrenocortical (HPA) axis as one major regulating system to cope with stress on a hormonal level (Sapolsky et al., 2000). HPA axis responses are mediated through a cascade of hormones from the central nervous system (corticotrophin releasing factor, CRF) which then stimulate adrenocorticotropic hormone (ACTH) and cortisol-secretion in the periphery. Based on the dynamic negative feedback of the HPA axis at every level, the increase of cortisol will- via activation of mineralocorticoid and glucocorticoid receptors- reduce further activation and initiate recovery from stress (McEwen, 2000). Cortisol, as the endproduct of HPA activation, can be assessed from saliva, which is frequently done, in order to monitor endocrine stress-responses (Allen et al., 2017; Goodman et al., 2017).

\section{Oxytocin}

The neuropeptide OT has been known for centuries in gynecology for its role in giving birth and breastfeeding. In recent decades, the focus has changed toward the central effects on social cognition and stress regulation. Synthesized in the paraventricular nucleus of the hypothalamus, OT is released from the pituitary and reaches central and peripheral action sites. The central key nodes are situated mostly within the amygdala, insula and prefrontal areas, highly relevant for socio-emotional cognition, and top-down control of emotion, perception and behavior (Meyer-Lindenberg et al., 2011), e.g., in the context of fear (Eckstein et al., 2015). Human studies from our own group using intranasal application of OT have shown its involvement in social behavior, a.o. constructive behavior and stress-regulation during couple conflict interaction both via the HPA and sympathetic nervous system activation (Ditzen et al., 2009, 2013). On the other hand, OT release seems to be triggered by social contact and intimacy (de Jong et al., 2015), and therefore was hypothesized to build a self-reinforcing system. Importantly, OT seems to modulate these saliency and stressregulatory functions from infancy on (Doom et al., 2017).

While early studies assumed these stress dampening and anxiolytic effects are evident in general (Kirsch et al., 2005; de Oliveira et al., 2012), newer studies showed that OT effects depend on the context (Olff et al., 2013). e.g., stress dampening effects become especially evident when combined with social support by a close person (Heinrichs et al., 2003; Eckstein et al., 2014). Above this, it has been suggested that OT does not have a prosocial or stress dampening effect in general, but depending on individual traits and experiences (for an overview see Olff et al., 2013). One underlying mechanism that is discussed to define this difference is OT receptor density or sensitivity in specific brain areas (Ross et al., 2009). OT-receptors, however, cannot be investigated in the human living brain, so far. Therefore, research designs rely on intranasal administration of OT in combination with fine-grained self-report and behavioral paradigms, in order to capture specific OT-functioning during social interaction and its effects on stress responses. Such studies suggest that the activation of central OT mechanisms in a social context might influence perceptions of salience and, thereby, moderate behavioral and physiological responses to social stimuli.

Another modulatory factor is gender. There are recent studies reporting gender-dimorphic effects of OT in various domains, including attachment behavior (Scheele et al., 2012; Preckel et al., 2014) and social cognition (Hoge et al., 2014; Gao et al., 2016). These results suggest that naturally occurring higher levels of estrogens in females and testosterone in males might be involved in the sex-specific behavioral and physiological effects (Ditzen et al., 2012) in response to stressors (Taylor et al., 2000). Gao et al. (2016) hypothesized that these sex-dimorphic effects have evolved from different roles for male and female humans in parenting, raising offspring and protecting the family. 
The modulatory role of gender on couple behavior is well documented. Early studies by Gottman (1994) and Gottman et al. (1998) have suggested that men rather react to conflict with avoidance and women with persistence. Newer studies have expanded this notion to differential responses on HPA activity (Ditzen et al., 2012; Laurent et al., 2013).

\section{Neuroendocrine Mechanisms of Attachment}

Neuroendocrine theories on attachment and bonding propose a refined interplay of central nervous neuropeptides (OT), neurotransmitters (dopamine) and also steroids (e.g., cortisol) in the regulation of attachment bonds throughout the life span, both for parental attachment and pair bonding (Feldman, 2017; Frisch et al., 2017). OT seems to be involved in the rewarding and stimulating aspects of both adult dyadic interaction and parent's emotional bonding to their infant (Insel and Young, 2001).

While parent-child attachment is motivated by caregiving, and characterized by asymmetric communication, the adult pair bond is motivated by the need for symmetric communication, and emotional and physical intimacy. However, both kinds of relationships involve the need for support and emotion/stress regulation within in the dyad.

\section{The Present Study}

It seems plausible that not only individual traits and experiences, but inter-individual/ social characteristics impact the effects of OT on stress reactivity as well as interaction behavior. For the relevant social relationships in adulthood, primarily the intimate couple relationship, this would be experiences with dyadic stress coping. So far there are no investigations on the interplay of dyadic coping, as a couple's style to deal with stress, and OT's effects on physiological and behavioral stress responses.

Therefore, in a secondary analysis of our previous database (Ditzen et al., 2009) we were interested to examine the links between intranasal application of OT, reported dyadic coping, cortisol reactivity, and positive behavior during couple conflict. While we have shown before (Ditzen et al., 2009) that OT has beneficial main effects, the presented new analyses take new factors and outcomes into account in order to disentangle whether individual's dyadic coping influence these effects. Based on the above cited studies on the individual traits modulating OT effects, we expected that dyadic coping abilities would modulate the effects of OT during dyadic interaction. Precisely, we assumed that both OT and positive dyadic coping would improve behavior and reduce cortisol responses to instructed couple conflict in the laboratory. In an exploratory hypothesis, we were also interested in the interaction of these two factors, thereby investigating whether either those couples with high or low dyadic coping would particularly benefit from OT application.

\section{MATERIALS AND METHODS}

\section{Participants}

Forty-seven heterosexual couples ( $n=94$ subjects), aged 20-50 years, who were married or had been cohabiting for at least
1 year participated in the study. One couple ( $N=2$ subjects, OT group) missed to complete the Dyadic Coping Inventory and was therefore excluded from the present analysis. Exclusion criteria for participation were smoking, chronic mental or physical illness, medication intake and, for women, the intake of hormonal contraceptives, current pregnancy, and breastfeeding. All women were investigated during the luteal phase of their menstrual cycle. Subjects were informed that we were interested in hormonal influences on couple communication and that they would receive either OT or placebo before a conflict conversation in the laboratory. All couples gave written informed consent and were offered 100 Swiss Francs for participation. The Dyadic Coping Inventory (Bodenmann, 2008b), the General Health Questionnaire (GHQ; Goldberg and Williams, 1969), the Relationship Questionnaire (PFB; Hahlweg, 1996), and the Short Chronic Stress Scale (SSCS; Schulz et al., 2004) were presented to all study participants before the lab appointment, in order to assess trait characteristics. All participants provided written informed consent prior to participation. The study was approved by the ethics committee of the University of Zurich and the Canton of Zurich.

\section{Procedures}

Experiments took place in the laboratories of the Department of Psychology at the University of Zurich. To control for diurnal variation in salivary cortisol, all assessments took place between 5:00 and 7:30 pm. After baseline salivary cortisol assessment and a pregnancy test in women, subjects rated the intensity of 23 pre-determined areas of couple conflict (Hahlweg, 1996) with regard to their own relationship. Couples chose two topics (e.g., finances, educational issues, leisure time) of continuing disagreement for the later discussion (Gottman, 1994; Weiss and Heyman, 1997; Kaiser et al., 1998). After this procedure, in a double-blind design based on the randomization table prepared by the study pharmacy, couples self-administered either 40 IU ( 5 puffs in each nostril) of OT (Syntocinon Spray, Novartis, Basel, Switzerland) or placebo (containing all ingredients except the active ingredient OT) intranasally under the supervision of the study coordinator. Forty-five minutes after drug administration, couples were asked to discuss the conflict issue that they had chosen previously during the following $10 \mathrm{~min}$ (FehmWolfsdorf et al., 1999). Couples were alone in the room and were videotaped during this conflict discussion. After the conflict discussion, all subjects were asked to evaluate the discussion with a standard evaluation questionnaire (Hahlweg and Jacobson, 1984) on self-perceived aspects of the conflict (e.g., validity of the task, stressfulness of the task) and subsequently watched a nature movie for relaxation. Salivary free cortisol was repeatedly assessed with Salivette collection devices (Sarstedt, Sevelen, Switzerland) at baseline ( $-50 \mathrm{~min})$, immediately before conflict $(-1)$ and after conflict $(+10,+20,+35$, and $+55 \mathrm{~min})$. Saliva samples were stored at $-20^{\circ} \mathrm{C}$ until required for analysis with a commercially available chemiluminescence immunoassay (CLIA; IBL Hamburg, Germany) with inter- and intra-assay coefficients of variation below $10 \%$. Salivary cortisol levels were interpreted on the basis of the area under the curve with respect to ground (AUCg) after the conflict discussion, which allows a sensitive 
measure of physiological changes over time (Pruessner et al., 2003).

\section{Couple Conflict Behavior}

Conflict behavior was coded with an adapted version of the Specific Affect Coding System (SPAFF; Gottman and Krokoff, 1989; Gottman, 1994) and the Coding System for Marital and Family Interaction (KPI; Hahlweg et al., 1984) with a computeraided system of analysis [Computer Aided Observation System (CAOS); Bourquard et al., 2005]. Two trained raters who were blind with regard to the subjects' group assignment coded nonverbal (e.g., eye contact, non-verbal positive behavior, and nonverbal negative behavior) and verbal behavior (e.g., curiosity/care, emotional self-disclosure, agreement, contempt, belligerence, and defensiveness). Inter-rater reliability (Cohen's kappa) was 0.66 for non-verbal categories and $0.80-1.0$ for verbal categories. Before calculating the sum scores, all behavior categories were z-transformed to make them comparable.

To consider both positive and negative categories of interactional behavior, a ratio was calculated dividing the score of total positive behaviors by the scores of total negative behaviors. This was achieved by adding a constant of 3 to both scores before the calculation of the ratio to transform them into positive values. By doing this, when the ratio was above 1, a higher duration of positive than negative behaviors was interpreted, while when the ratio was less than 1 the opposite could be concluded. Mean values, standard deviations, and ranges of the variables can be seen in Table 1.

\section{Dyadic Coping}

Dyadic coping was assessed with the Dyadic Coping Inventory (DCI; Bodenmann, 2008b). It assesses one's own stress communication ("What I do when I am stressed?") and that enacted by the partner ("What does my partner do when he/she is stressed?"). Each partner's view of how they cope as a couple (common dyadic coping) is evaluated ("What we do when we are stressed as a couple?"). Hence, each single individual receives scores, rather than a joint score for the couple. The DCI

TABLE 1 | Descriptive statistics of dyadic coping, cortisol concentration, and behavioral ratio.

\begin{tabular}{rlrrrrr}
\hline & & Range & Minimum & Maximum & Mean & \multicolumn{1}{c}{ S.E. } \\
\hline \multirow{2}{*}{ Female } & Total DCl & 48.00 & 82.00 & 130.00 & 105.830 & 1.491 \\
& Self DCl & 24.00 & 43.00 & 70.00 & 56.700 & 0.819 \\
& Partner DCl & 26.00 & 19.00 & 45.00 & 36.511 & 0.744 \\
& Cortisol & 307.00 & 53.85 & 360.85 & 158.693 & 10.144 \\
& Behav. Ratio & 1.40 & 0.56 & 1.96 & 1.050 & 0.037 \\
Male & Total DCl & 59.00 & 68.00 & 127.00 & 102.477 & 1.631 \\
& Self DCl & 24.00 & 43.00 & 67.00 & 54.057 & 0.853 \\
& Partner DCl & 31.00 & 14.00 & 45.00 & 35.640 & 0.836 \\
& Cortisol & 552.83 & 40.50 & 593.33 & 184.254 & 14.403 \\
& Behav.Ratio & 1.17 & 0.50 & 1.67 & 1.011 & 0.339 \\
\hline
\end{tabular}

Total DCl, Dyadic Coping; Self DCl, Dyadic coping by oneself; Partner DCl, Dyadic coping by the partner; Cortisol, Cortisol AUCg; Behav. Ratio, Positive/Negative Behavioral Ratio, S.E., Standard Error. contains 37 items which are rated on a 5 -point scale (" $0=$ never" to " $4=$ very often"). In this study we used the total score "DCI total without evaluation," which is the established total score of the inventory (Bodenmann, 2008a) and the scales "Dyadic Coping by the Partner" and "Dyadic Coping by Oneself." The latter scale measures the participants self-evaluation of how they support their partners to cope with the conflict. This refers to emotional support and informational forms of support, which do not relieve the partner of the coping work, but rather support coping efforts, e.g., empathic understanding or assistance in the analysis of the problems (informational support). Higher scores indicate more supportive dyadic coping strategies. The DCI is a validated instrument in western cultures as well as in China (Xu et al., 2016).

\section{Statistical Analyses}

Statistical Package for the Social Sciences (IBM ${ }^{\mathrm{TM}}$ SPSS $^{\circledR}$ v. 24.0) was used for the descriptive analyses and plots. An Intra-Class correlation coefficient of ICC $=0.257$ for cortisol AUCg and ICC $=0.502$ for the positive/negative behavior ratio indicated non-independence of the data. Given the data is nested on dyads, and to test for group differences regarding HPA-axis reactivity after couple conflict in the treatment and placebo group, we conducted a hierarchical linear dyadic analysis following Kenny et al. (2006) recommendations for the adjustment of standard errors. For the hierarchical models, the library nlme (Pinheiro et al., 2018) of the statistical environment R ( $\mathrm{R}$ Core Team, 2017) was used. A two-level hierarchical linear model was fitted using a Restricted Maximum Likelihood (REML) estimation method, because it offers robust estimates for small samples (Peugh, 2010). The intercept was set at random to allow for an adjusted estimation of standard errors by the upper level, while no random slopes were calculated given the limitations of having only two units per couple. All predictors were grand-mean centered, while dichotomous variables were effect coded to help with the interpretation of the results (Kenny et al., 2006). Models were visually evaluated for normality and equality of variance of the residuals. Because a pattern of increased dispersion of residuals by the fitted values was found for cortisol analyses, every model was weighted by them using the "power of the covariate" function of the nlme package (Pinheiro and Bates, 2000). A likelihood ratio test was conducted to evaluate improvement in model fitness. Equal variances were assumed for dyad members in every model because no improvement in model fit was found when set as different. Aggregated cortisol levels (AUCg, for details see Pruessner et al., 2003) were added as the dependent variable and group (OT vs. placebo), dyadic coping (total score) and their interaction were computed as predictors controlling for sex, age, and body mass index. To disentangle the confounded self and partner effect of the total DCI, secondary analyses were conducted using the Dyadic Coping by Oneself and Dyadic Coping by the Partner Scale. Afterward, a fourth model was conducted to determine differences in the ratio of positive/negative behavior duration between group and Dyadic Coping interaction, including the same control variables. 


\section{RESULTS}

\section{Oxytocin, Dyadic Coping and HPA-Axis Reactivity to Couple Conflict}

For the total DCI score, the likelihood ratio test showed an improvement in model fit when adjusted for the heteroskedastic pattern of residuals [L.Ratio $(1)=11.837, p<0.001$ ]. A trend interaction effect was found between group and dyadic coping $(\beta=1.392, t(33)=1.958, p=0.059)$ on cortisol levels after the conflict discussion (see Table 2). This trend effect is depicted in Figure 1A. When the dyadic coping by oneself was entered into the equation, the likelihood ratio test also showed an improvement in model fit when adjusted for the increase on residual dispersion by predicted values [L.Ratio $(1)=10.846$, $p=0.001]$. A significant interaction effect between self-dyadic coping $[\beta=2.966, t(33)=2.190, p=0.036]$ and OT on cortisol values was found (Table 2). Finally, when dyadic coping by the partner was entered into the equation, the likelihood ratio test also showed an improvement in model fitness when adjusted [L.Ratio $(1)=8.382, p=0.004$ ], however, no interaction effect was found between the partner's coping $[\beta=2.681, t(33)=1.731$, $p=0.091]$ and oxytocin on personal cortisol values (Table 2).

To further inspect the significant interaction effect of dyadic coping by oneself with OT in cortisol levels, a median split of the DCI scores into high and low values showed that specially individuals scoring low in self-dyadic coping benefitted from OT in terms of dampened cortisol levels (see Figure 1B). The general scale showed the same substantive pattern of results.

\section{Oxytocin, Dyadic Coping and Behavior Ratio During Couple Conflict}

No significant interaction effect was found for the total DCI $[\beta=-0.003, t(32)=-1.282, p=0.209]$, dyadic coping by oneself $[\beta=-0.003, t(32)=-0.663, p=0.512]$, or by the partner $[\beta=-0.008, t(32)=-1.673, p=0.104]$. The same was the case for the main effects. Interestingly, however, on a descriptive

TABLE 2 | Dyadic modeling of Cortisol on oxytocin and dyadic coping.

\begin{tabular}{lccc}
\hline & Total DCI & Self DCI & Partner DCI \\
\hline Fixed effects & & & \\
& 168.826 & & 168.395 \\
(Intercept) & $(7.984)^{* * *}$ & $169.019(8.274)^{* * *}$ & $(8.161)^{* * *}$ \\
Group & $-6.045(7.937)$ & $-5.905(8.202)$ & $-6.058(8.201)$ \\
DCl & $-1.402(0.705)$ & $-2.018(1.347)$ & $-1.826(1.540)$ \\
Age & $-0.444(1.348)$ & $-0.337(1.424)$ & $-0.714(1.454)$ \\
BMl & $-5.406(2.619)^{*}$ & $-6.351(2.730)^{*}$ & $-4.523(2.891)$ \\
Sex & $10.538(7.689)$ & $11.007(8.181)$ & $11.252(8.140)$ \\
Group*DCl & $1.392(0.711)$ & $2.966(1.354)^{*}$ & $2.681(1.549)$ \\
Random effects & & & 0.029 \\
Intercept & 0.021 & 0.025 & 0.044 \\
Residual & 0.034 & 0.034 & \\
\hline
\end{tabular}

${ }^{* * *} p<0.001,{ }^{* *} p<0.01$, and ${ }^{*} p<0.05$; Unstandardized beta coefficients are presented with their standard errors in parentheses. Dependent variable is AUCg of cortisol after the couple's conflict. level the data showed the same pattern as for the cortisol results where participants with low DCI (median split) showed more positive than negative behaviors in the OT group, while they showed more negative than positive behaviors in the placebo condition. On the other hand, participants with high DCI showed more positive behaviors in both conditions. Analyses of the selfevaluation questionnaire with similar models did not yield any significant results.

\section{DISCUSSION}

In this presented secondary analysis (for former analyses of the dataset see also Ditzen et al., 2009, 2012) we were interested in the neuroendocrine aspects of dyadic stress coping traits and their specific influence on stress responses during couple conflict. With the administration of OT, a neuropeptide hormone associated to improved social attachment and reduced stress reactivity we aimed at imitating endogenous neural processes and tested the interactive effect of OT with couples' self-reported dyadic coping.

Results showed a significant interaction of dyadic coping and OT on cortisol responses to instructed couple conflict and indicated that particularly those participants with low a priori scores of dyadic coping benefitted from OT in terms of dampened HPA-axis. As an extension of our former analyses, we could show that improvements on the group level (Ditzen et al., 2009) where mostly driven by individuals with least coping skills.

\section{Physiological Stress Responses}

On the one hand, the cortisol results are in line with the broad range of literature showing effects of OT in down-regulating the HPA-axis, such as our previous analyses of the data (Ditzen et al., 2009). In accordance, e.g., Cardoso et al. (2013) demonstrated that OT attenuated the cortisol response elicited by physical stress. However, there are also studies that failed to find an effect of OT on cortisol levels (de Oliveira et al., 2012).

Above this, our significant interaction of OT with dyadic coping is in line with previous reports about person-dependent effects of OT (Olff et al., 2013), which suggest that personal traits moderate the effects of OT. In the present study, especially individuals who reported low levels in dyadic coping benefitted from OT treatment in terms of decreased cortisol levels. Interestingly, this effect was driven by the subscale "Dyadic Coping by Oneself." The total DCI scale is composed both by the perceived coping skills of the individual and, at the same time, the perception that the individual has on the coping skills of his/her partner. However, because the interest of this study is on the individual effects, the perception of the partners coping skill would confound both the self and partner coping skills within the interaction effect and render it uninterpretable in meaningful terms. Therefore, it's plausible that the subscale "Dyadic Coping by Oneself" turned the effect. Indeed, this adds further emphasis to the moderating role of the individual person factors. Those participants who stated that they fail to support their partners in their stress, showed most stress-dampening effects of OT. This might be due to their deficits in self-regulation which is a predisposition to regulate others (Ham and Tronick, 2009). 


\section{A}

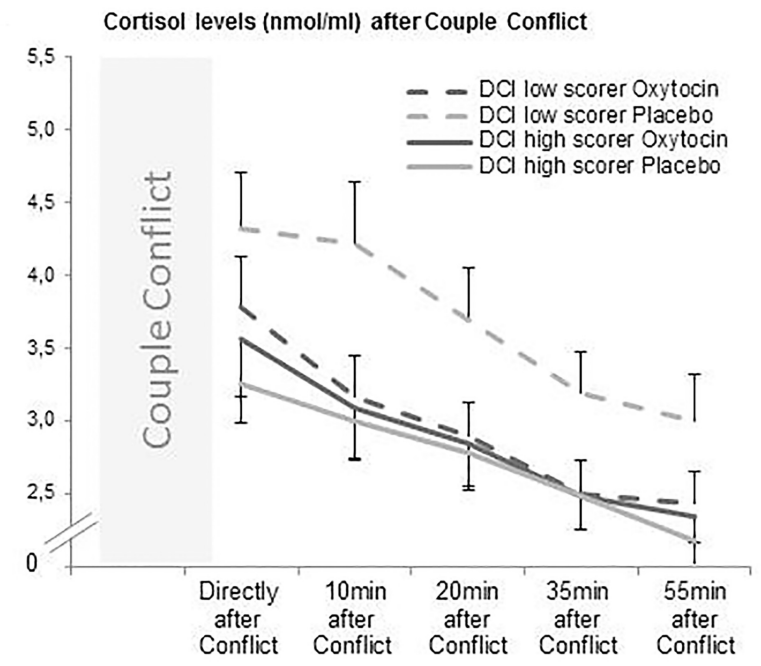

B

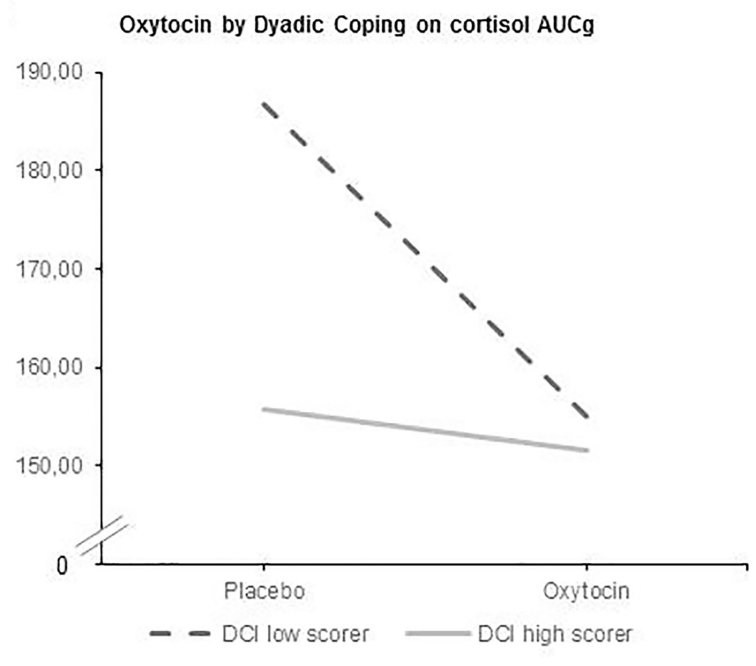

FIGURE 1 | (A,B) Oxytocin significantly decreased the level of salivary cortisol (AUCg) after the couple conflict in individuals with low scores of DCI total. DCI low scorers are marked with a continuous line, while DCl high scorers are marked with a dashed line.

In fact, a recent systematic meta-analysis (Cardoso et al., 2014) revealed that the modulatory effect of OT depends both on the specific stressor and the clinical load of the sample. It has been discussed before that individuals with clinical symptoms might benefit most from OT application in terms of reduced cortisol levels (Bartz et al., 2011; Simeon et al., 2011). Some studies showed that subjective stress response and cortisol levels were affected by OT only in those with poor individual coping and emotion regulation abilities, respectively, but not in individuals with adequate coping or emotion regulation abilities (Quirin et al., 2011; Cardoso et al., 2012). In addition, OT effects on cortisol seem to depend on the social context of the stressor (Heinrichs et al., 2003; Eckstein et al., 2014).

There is evidence from animal and human studies that stressful events themselves can trigger endogenous OT release (de Jong et al., 2015). It has also been reported that acute stress stimulates OT secretion in specific subsamples with averse childhood experiences and depending on attachment style (Pierrehumbert et al., 2012; Seltzer et al., 2014), whereas other studies failed to find OT responses to psychosocial stressor (Light et al., 2005; Ditzen et al., 2007).

\section{Couple Behavior}

The influence of OT/placebo administration or dyadic coping on couple behavior during the conflict did not reach statistical significance, which might be due to high scores of positive behavior in general in our sample of healthy couples reporting high relationship satisfaction (ceiling effect). In addition the failure to reach significance might also be due to a lack in statistical power. We would assume that the effect might become significant in a larger or more heterogeneous sample.

To our knowledge, this is the first study to investigate interindividual stress-management traits in their interaction with OT. One theoretical explanation of our findings is that an individual's history of relationships and attachment has a modulating influence on the endogenous OT system (Macdonald, 2013). This notion is supported by studies showing changes in endogenous baseline levels or OT release in response to stress after childhood trauma or early life stress (Heim et al., 2009; Opacka-Juffry and Mohiyeddini, 2012).

The involvement of OT in stress regulation and social interaction makes this system a highly relevant candidate to investigate psychobiological mechanisms of social relationships. Thus, in adult couples, dyadic stress coping is a central concept to be related to OT research. The endogenous release of OT, e.g., by intimacy or other forms of positive social interaction has probably stress regulating effects. While the present study used external given OT, these might likewise be true for endogenous released OT, e.g., after intimacy, stressors or breastfeeding (Grewen et al., 2010; de Jong et al., 2015). Indeed, we would assume better stress regulation after activities that trigger OT release in individuals with good dyadic coping skills. In previous studies by our group, intimacy between couples, operationalized as physical affection, was significantly associated with reduced salivary cortisol levels in controlled laboratory experiments and in everyday life (Ditzen et al., 2007, 2008). Clinical applications should take into account these mechanisms, e.g., in couple therapy, but also stress coping trainings might promote these behaviors by including romantic partners or the family.

The study has some limitations. The sample consisted of healthy young couples reporting high relationship satisfaction. Given opposing effects of OT in clinical samples (Bartz et al., 2010), we cannot extrapolate our findings to severe marital problems or to patient populations [see for instance a study in couple with substance abuse by Flanagan et al. (2018)]. Likewise, our sample is restricted to heterosexual couples. Since the OT system seems to react differently in homosexuals (Thienel et al., 2014) and the fact that same-sex couples experience specific 
stressors to deal with (Randall et al., 2017), it would be of great interest to investigate OT and dyadic coping in same-sex couples. Another relevant factor modulating couple behavior is gender (e.g., Gottman, 1994). In our data, however, there was no significant main or interactional effect of gender on couple behavior and cortisol. Furthermore, our findings may not be applicable for some cultures or couple circumstances, therefore generalizability is limited. Culturally based gender role expectations regarding the role of partners in supporting each other's' coping and providing emotional support could moderate how OT or DC affects stress reactivity or couple behavior. This should be investigated in future studies.

Our results in couples - with the intimate partner usually serving as the most relevant social interaction partner for adults might also have relevance for other central social relationships and types of social interactions in adult life, namely parent-child interactions. Up to $70 \%$ of the first-time parents report a decrease in relationship satisfaction after becoming parents (Gottman and Notarius, 2000) and increased occurrence of conflicts, often with child related content (Kluwer and Johnson, 2007). Thus, functional conflict strategies and problem solving capacities are of particular importance, not only for the couple, but also for the developing parent-infant-relationship (Krishnakumar and Buehler, 2000) and subsequently for infant and child development (Zemp et al., 2016). In regards to neurobiological mediators, recent studies showed an association between parental behavior and endogenous OT activity (for an overview, see Zietlow et al., 2016; Feldman, 2017) in mothers, but also in fathers (Naber et al., 2010; Weisman et al., 2014), which could indicate a joint neuroendocrine basis. Therefore, in future studies possible parallels between dyadic stress regulation in the couple and parent-infant-interaction and parenting behavior should be investigated.

\section{CONCLUSION}

Taken together the presented data suggests that the OT system in interaction with dyadic coping can improve stress regulation. In line with previous studies, we showed that rather than acting stress-regulating per se, the neuroendocrine mediator OT depend

\section{REFERENCES}

Allen, A. P., Kennedy, P. J., Dockray, S., Cryan, J. F., Dinan, T. G., and Clarke, G. (2017). The Trier social stress test: principles and practice. Neurobiol. Stress 6, 113-126. doi: 10.1016/j.ynstr.2016.11.001

Bartz, J., Simeon, D., Hamilton, H., Kim, S., Crystal, S., Braun, A., et al. (2010). Oxytocin can hinder trust and cooperation in borderline personality disorder. Soc. Cogn. Affect. Neurosci. 6, 556-563. doi: 10.1093/scan/nsq085

Bartz, J. A., Zaki, J., Bolger, N., and Ochsner, K. N. (2011). Social effects of oxytocin in humans: context and person matter. Trends Cogn. Sci. 15, 301-309. doi: 10.1016/j.tics.2011.05.002

Baucom, B. R., Dickenson, J. A., Atkins, D. C., Baucom, D. H., Fischer, M. S., Weusthoff, S., et al. (2015). The interpersonal process model of demand/withdraw behavior. J. Fam. Psychol. 29, 80-90. doi: 10.1037/ fam0000044 on individual factors such as the individual's dyadic coping mechanisms.

Both our empirical results as well as theoretical assumptions underline the importance to investigate real-time social interaction behavior in relation to psychobiological dynamics. Possible implications can be drawn for couple therapy and conflict management, but also help understanding stress coping trainings including romantic partners or the family.

\section{AUTHOR CONTRIBUTIONS}

A-LZ and ME contributed to analysis and interpretation of data, drafted the manuscript, and approved the final version. $\mathrm{CH}$ contributed to analysis and interpretation of data and approved the final version. MH, MS, and GB contributed to the study and manuscript conception and approval of the final version. NN and CR contributed to drafting of the manuscript and approval of the final version. BD contributed to study conception and design, acquisition, analysis, and interpretation of the data, drafting of the manuscript, and approval of the final version.

\section{FUNDING}

The study was supported by a Young Investigator Research Grant provided by the University of Zurich (No. 56233205) (BD), a research Fellowship for Prospective Scientists provided by the Swiss National Science Foundation PBZH1-108392 (BD), and a Grant from the Swiss National Science Foundation (PP001114788) (MH).

\section{ACKNOWLEDGMENTS}

We thank Esther Goetz, M.Sc., Mirja Hemmi, M.Sc., and Sabina Studhalter, M.Sc., for excellent research assistance in conducting the study. We acknowledge financial support by Deutsche Forschungsgemeinschaft within the funding program Open Access Publishing, by the Baden-Württemberg Ministry of Science, Research and the Arts, and by Ruprecht-KarlsUniversität Heidelberg.

Bodenmann, G. (1995). A systemic-transactional conceptualization of stress and coping in couples. Swiss J. Psychol. 54, 34-49.

Bodenmann, G. (1997). Dyadic coping-A systemic transactional view of stress and coping among couples: theory and empirical findings. Eur. Rev. Appl. Psychol. 47, 137-140.

Bodenmann, G. (2000). Stress und Coping bei Paaren [Stress and Coping in Couples]. Göttingen: Hogrefe.

Bodenmann, G. (2005a). "Dyadic coping and its significance for marital functioning," in Couples Coping with Stress: Emerging Perspectives on Dyadic Coping, eds T. Revenson, G. Bodenmann, and K. Kayser (Washington, DC: American Psychological Association), 33-49. doi: 10.1037/11031-002

Bodenmann, G. (2005b). "Dyadic coping and its significant for marital functioning," in Couples Coping with Stress: Emerging Perspectives on Dyadic Coping, eds T. Revenson and G. Bodenmann (Washington, DC: American Psychological Association), 33-50. doi: 10.1037/11031-002 
Bodenmann, G. (2008a). Dyadisches Coping Inventar [Dyadic Coping Inventory]. Göttingen: Huber \& Hogrefe.

Bodenmann, G. (2008b). Dyadisches Coping Inventar: Testmanual [Dyadic Coping Inventory: Test Manual]. Bern: Huber.

Bodenmann, G., Pihet, S., and Kayser, K. (2006). The relationship between dyadic coping and marital quality: a 2-year longitudinal study. J. Fam. Psychol. 20, 485-493. doi: 10.1037/0893-3200.20.3.485

Bourquard, E., Bodenmann, G., and Perrez, M. C. C. (2005). CAOS. Computer Aided Observation System. Fribourg: University of Fribourg.

Cardoso, C., Ellenbogen, M. A., Orlando, M. A., Bacon, S. L., and Joober, R. (2013). Intranasal oxytocin attenuates the cortisol response to physical stress: a dose-response study. Psychoneuroendocrinology 38, 399-407. doi: 10.1016/j. psyneuen.2012.07.013

Cardoso, C., Kingdon, D., and Ellenbogen, M. A. (2014). A meta-analytic review of the impact of intranasal oxytocin administration on cortisol concentrations during laboratory tasks: moderation by method and mental health. Psychoneuroendocrinology 49, 161-170. doi: 10.1016/j.psyneuen.2014. 07.014

Cardoso, C., Linnen, A.-M., Joober, R., and Ellenbogen, M. A. (2012). Coping style moderates the effect of intranasal oxytocin on the mood response to interpersonal stress. Exp. Clin. Psychopharmacol. 20, 84-91. doi: 10.1037/ a0025763

de Jong, T. R., Menon, R., Bludau, A., Grund, T., Biermeier, V., Klampfl, S. M., et al. (2015). Salivary oxytocin concentrations in response to running, sexual selfstimulation, breastfeeding and the TSST: the Regensburg Oxytocin Challenge (ROC) study. Psychoneuroendocrinology 62, 381-388. doi: 10.1016/j.psyneuen. 2015.08.027

de Oliveira, D. C., Zuardi, A. W., Graeff, F. G., Queiroz, R. H., and Crippa, J. A. (2012). Anxiolytic-like effect of oxytocin in the simulated public speaking test. J. Psychopharmacol. 26, 497-504. doi: 10.1177/0269881111 400642

Ditzen, B., Hahlweg, K., Fehm-Wolfsdorf, G., and Baucom, D. (2011). Assisting couples to develop healthy relationships: effects of couples relationship education on cortisol. Psychoneuroendocrinology 36, 597-607. doi: 10.1016/j. psyneuen.2010.07.019

Ditzen, B., Hoppmann, C., and Klumb, P. (2008). Positive couple interactions and daily cortisol: on the stress-protecting role of intimacy. Psychosom. Med. 70, 883-889. doi: 10.1097/PSY.0b013e318185c4fc

Ditzen, B., Nater, U., Schaer, M., La Marca, R., Bodenmann, G., Ehlert, U., et al. (2013). Sex-specific effects of intranasal oxytocin on autonomic nervous system and emotional responses to couple conflict. Soc. Cogn. Affect. Neurosci. 8, 897-902. doi: 10.1093/scan/nss083

Ditzen, B., Nater, U. M., Schaer, M., La Marca, R., Bodenmann, G., Ehlert, U., et al. (2012). Sex-specific effects of intranasal oxytocin on autonomic nervous system and emotional responses to couple conflict. Soc. Cogn. Affect. Neurosci. 8, 897-902. doi: 10.1093/scan/nss083

Ditzen, B., Neumann, I. D., Bodenmann, G., von Dawans, B., Turner, R. A., Ehlert, U., et al. (2007). Effects of different kinds of couple interaction on cortisol and heart rate responses to stress in women. Psychoneuroendocrinology $32, \quad 565-574$. doi: 10.1016/j.psyneuen.2007. 03.011

Ditzen, B., Schaer, M., Gabriel, B., Bodenmann, G., Ehlert, U., and Heinrichs, M. (2009). Intranasal oxytocin increases positive communication and reduces cortisol levels during couple conflict. Biol. Psychiatry 65, 728-731. doi: 10.1016/ j.biopsych.2008.10.011

Doom, J. R., Doyle, C. M., and Gunnar, M. R. (2017). Social stress buffering by friends in childhood and adolescence: effects on HPA and oxytocin activity. Soc. Neurosci. 12, 8-21. doi: 10.1080/17470919.2016.1149095

Eckstein, M., Becker, B., Scheele, D., Scholz, C., Preckel, K., Schlaepfer, T. E., et al. (2015). Oxytocin facilitates the extinction of conditioned fear in humans. Biol. Psychiatry 78, 194-202. doi: 10.1016/j.biopsych.2014. 10.015

Eckstein, M., and Hurlemann, R. (2013). [Oxytocin: evidence for a therapeutic potential of the social neuromodulator]. Nervenarzt 84, 1321-1328. doi: 10. 1007/s00115-013-3832-6

Eckstein, M., Scheele, D., Weber, K., Stoffel-Wagner, B., Maier, W., and Hurlemann, R. (2014). Oxytocin facilitates the sensation of social stress. Hum. Brain Mapp. 35, 4741-4750. doi: 10.1002/hbm.22508
Falconier, M. K., Jackson, J. B., Hilpert, P., and Bodenmann, G. (2015). Dyadic coping and relationship satisfaction: a meta-analysis. Clin. Psychol. Rev. 42, 28-46. doi: 10.1016/j.cpr.2015.07.002

Fehm-Wolfsdorf, G., Groth, T., Kaiser, A., and Hahlweg, K. (1999). Cortisol responses to marital conflict depend on marital interaction quality. Int. J. Behav. Med. 6, 207-227. doi: 10.1207/s15327558i jbm0603_1

Feldman, R. (2017). The neurobiology of human attachments. Trends Cogn. Sci. 21, 80-99. doi: 10.1016/j.tics.2016.11.007

Flanagan, J. C., Fischer, M. S., Nietert, P. J., Back, S. E., Maria, M. M.-S., Snead, A., et al. (2018). Effects of oxytocin on cortisol reactivity and conflict resolution behaviors among couples with substance misuse. Psychiatry Res. 260, 346-352. doi: 10.1016/j.psychres.2017.12.003

Frisch, J., Aguilar-Raab, C., Eckstein, M., and Ditzen, B. (2017). Einfluss von paarinteraktion auf die gesundheit. Psychotherapeut 62, 59-76. doi: 10.1007/ s00278-016-0153-9

Gao, S., Becker, B., Luo, L., Geng, Y., Zhao, W., Yin, Y., et al. (2016). Oxytocin, the peptide that bonds the sexes also divides them. Proc. Natl. Acad. Sci. U.S.A. 113, 7650-7654. doi: 10.1073/pnas.1602620113

Goldberg, D., and Williams, P. (1969). General Health Questionnaire (GHQ). Windsor: Nfer-Nelson.

Goodman, W. K., Janson, J., and Wolf, J. M. (2017). Meta-analytical assessment of the effects of protocol variations on cortisol responses to the Trier Social Stress Test. Psychoneuroendocrinology 80, 26-35. doi: 10.1016/j.psyneuen.2017. 02.030

Gottman, J. (1994). What Predicts Divorce? The Relationship Between Marital Processes and Marital Outcomes. Hillsdale, NJ: Lawrence Erlbaum Associates.

Gottman, J., and Krokoff, L. (1989). Marital interaction and satisfaction: a longitudinal view. J. Consult. Clin. Psychol. 57, 47-52. doi: 10.1037/0022-006X. 57.1.47

Gottman, J., and Notarius, C. (2000). Decade review: observing marital interaction. J. Marriage Fam. 62, 927-947. doi: 10.1111/j.1741-3737.2000. 00927.x

Gottman, J. M., Coan, J., Carrere, S., and Swanson, C. (1998). Predicting marital happiness and stability from newlywed interactions. J. Marriage Fam. 60, 5-22. doi: $10.2307 / 353438$

Grewen, K. M., Davenport, R. E., and Light, K. C. (2010). An investigation of plasma and salivary oxytocin responses in breast- and formula-feeding mothers of infants. Psychophysiology 47, 625-632. doi: 10.1111/j.1469-8986.2009. 00968.x

Hahlweg, K. (1996). Fragebogen zur Partnerschaftsdiagnostik (FPD) [Marriage Diagnostic Questionnaire]. Göttingen: Hogrefe.

Hahlweg, K., and Jacobson, N. S. (1984). Marital Interaction: Analysis and Modification. New York, NY: Guilford.

Hahlweg, K., Reisner, L., Kohli, G., Vollmer, C., Schindler, L., and Revenstorf, D. (1984). "Development and validity of a new system to analyze interpersonal communication: kategoriensystem für partnerschaftliche Interaktion [KPI]," in Marital Interaction: Analysis and Modification, eds K. Hahlweg and N. S. Jacobson (New York, NY: Guilford Press), 182-198.

Ham, J., and Tronick, E. Z. (2009). Relational psychophysiology: lessons from mother-infant physiology research on dyadically expanded states of consciousness. Psychother. Res. 19, 619-632. doi: 10.1080/1050330080260 9672

Heatherton, T. F., and Wagner, D. D. (2011). Cognitive neuroscience of selfregulation failure. Trends Cogn. Sci. 15, 132-139. doi: 10.1016/j.tics.2010. 12.005

Heim, C., Young, L. J., Newport, D. J., Mletzko, T., Miller, A. H., and Nemeroff, C. B. (2009). Lower CSF oxytocin concentrations in women with a history of childhood abuse. Mol. Psychiatry 14, 954-958. doi: 10.1038/mp. 2008.112

Heinrichs, M., Baumgartner, T., Kirschbaum, C., and Ehlert, U. (2003). Social support and oxytocin interact to suppress cortisol and subjective responses to psychosocial stress. Biol. Psychiatry 54, 1389-1398. doi: 10.1016/S00063223(03)00465-7

Herzberg, P. Y. (2013). Coping in relationships: the interplay between individual and dyadic coping and their effects on relationship satisfaction. Anxiety Stress Coping 26, 136-153. doi: 10.1080/10615806.2012. 655726 
Hoge, E. A., Anderson, E., Lawson, E. A., Bui, E., Fischer, L. E., Khadge, S. D., et al. (2014). Gender moderates the effect of oxytocin on social judgments. Hum. Psychopharmacol. Clin. Exp. 29, 299-304. doi: 10.1002/hup. 2402

Insel, T. R., and Young, L. J. (2001). The neurobiology of attachment. Nat. Rev. Neurosci. 2, 129-136. doi: 10.1038/35053579

Kaiser, A., Hahlweg, K., Fehm-Wolfsdorf, G., and Groth, T. (1998). The efficacy of a compact psychoeducational group training program for married couples. J. Consult. Clin. Psychol. 66, 753-760. doi: 10.1037/0022-006X.66. 5.753

Kenny, D. A., Kashy, D. A., and Cook, W. L. (2006). Dyadic Data Analysis. New York, NY: Guilford Press.

Kirsch, P., Esslinger, C., Chen, Q., Mier, D., Lis, S., Siddhanti, S., et al. (2005). Oxytocin modulates neural circuitry for social cognition and fear in humans. J. Neurosci. 25, 11489-11493. doi: 10.1523/JNEUROSCI.3984-05. 2005

Kluwer, E. S., and Johnson, M. D. (2007). Conflict frequency and relationship quality across the transition to parenthood. J. Marriage Fam. 69, 1089-1106. doi: 10.1111/j.1741-3737.2007.00434.x

Krishnakumar, A., and Buehler, C. (2000). Interparental conflict and parenting behaviors: a meta-analytic review. Fam. Relat. 49, 25-44. doi: 10.1111/j.17413729.2000.00025.x

Laurent, H. K., Powers, S. I., Laws, H., Gunlicks-Stoessel, M., Bent, E., and Balaban, S. (2013). HPA regulation and dating couples' behaviors during conflict: gender-specific associations and cross-partner interactions. Physiol. Behav. 118, 218-226. doi: 10.1016/j.physbeh.2013.05.037

Light, K. C., Grewen, K. M., and Amico, J. A. (2005). More frequent partner hugs and higher oxytocin levels are linked to lower blood pressure and heart rate in premenopausal women. Biol. Psychol. 69, 5-21. doi: 10.1016/j.biopsycho.2004. 11.002

Macdonald, K. (2013). Sex, receptors, and attachment: a review of individual factors influencing response to oxytocin. Front. Neurosci. 6:194. doi: 10.3389/fnins. 2012.00194

McEwen, B. S. (2000). The neurobiology of stress: from serendipity to clinical relevance. Brain Res. 886, 172-189. doi: 10.1016/S0006-8993(00)02950-4

Meuwly, N., Bodenmann, G., Germann, J., Bradbury, T. N., Ditzen, B., and Heinrichs, M. (2012). Dyadic coping, insecure attachment, and cortisol stress recovery following experimentally induced stress. J. Fam. Psychol. 26, 937-947. doi: $10.1037 / \mathrm{a} 0030356$

Meyer-Lindenberg, A., Domes, G., Kirsch, P., and Heinrichs, M. (2011). Oxytocin and vasopressin in the human brain: social neuropeptides for translational medicine. Nat. Rev. Neurosci. 12, 524-538. doi: 10.1038/nrn 3044

Naber, F., van IJzendoorn, M. H., Deschamps, P., van Engeland, H., and Bakermans-Kranenburg, M. J. (2010). Intranasal oxytocin increases fathers' observed responsiveness during play with their children: a double-blind withinsubject experiment. Psychoneuroendocrinology 35, 1583-1586. doi: 10.1016/j. psyneuen.2010.04.007

Neumann, I. D. (2002). Involvement of the brain oxytocin system in stress coping: interactions with the hypothalamo-pituitary-adrenal axis. Prog. Brain Res. 139, 147-162. doi: 10.1016/S0079-6123(02)39014-9

Olff, M., Frijling, J. L., Kubzansky, L. D., Bradley, B., Ellenbogen, M. A., Cardoso, C., et al. (2013). The role of oxytocin in social bonding, stress regulation and mental health: an update on the moderating effects of context and interindividual differences. Psychoneuroendocrinology 38, 1883-1894. doi: 10.1016/j.psyneuen.2013.06.019

Opacka-Juffry, J., and Mohiyeddini, C. (2012). Experience of stress in childhood negatively correlates with plasma oxytocin concentration in adult men. Stress 15, 1-10. doi: 10.3109/10253890.2011.560309

Peugh, J. L. (2010). A practical guide to multilevel modeling. J. Sch. Psychol. 48, 85-112. doi: 10.1016/j.jsp.2009.09.002

Pierrehumbert, B., Torrisi, R., Ansermet, F., Borghini, A., and Halfon, O. (2012). Adult attachment representations predict cortisol and oxytocin responses to stress. Attach. Hum. Dev. 14, 453-476. doi: 10.1080/14616734.2012. 706394

Pinheiro, J., Bates, D., DebRoy, S., Sarkar, D., and Team, R. C. (2018). Linear and Nonlinear Mixed Effects Models. R package version 3.1-137. Available at: https://cran.r-project.org/web/packages/nlme/index.html
Pinheiro, J. C., and Bates, D. M. (eds). (2000). "Linear mixed-effects models: basic concepts and examples," in Mixed-Effects Models in S and S-Plus, (Berlin: Springer Science \& Business Media), 3-56. doi: 10.1007/978-1-4419-0318-1_1

Preckel, K., Scheele, D., Kendrick, K., Maier, W., and Hurlemann, R. (2014). Oxytocin facilitates social approach behavior in women. Front. Behav. Neurosci. 8:191. doi: 10.3389/fnbeh.2014.00191

Pruessner, J. C., Kirschbaum, C., Meinlschmid, G., and Hellhammer, D. H. (2003). Two formulas for computation of the area under the curve represent measures of total hormone concentration versus time-dependent change. Psychoneuroendocrinology 28, 916-931. doi: 10.1016/S0306-4530(02)00108-7

Quirin, M., Kuhl, J., and Düsing, R. (2011). Oxytocin buffers cortisol responses to stress in individuals with impaired emotion regulation abilities. Psychoneuroendocrinology 36, 898-904. doi: 10.1016/j.psyneuen.2010.12.005

R Core Team (2017). R: A Language and Environment for Statistical Computing. Vienna: R Foundation for Statistical Computing.

Randall, A. K., and Bodenmann, G. (2009). The role of stress on close relationships and marital satisfaction. Clin. Psychol. Rev. 29, 105-115. doi: 10.1016/j.cpr.2008. 10.004

Randall, A. K., Totenhagen, C. J., Walsh, K. J., Adams, C., and Tao, C. (2017). Coping with workplace minority stress: associations between dyadic coping and anxiety among women in same-sex relationships. J. Lesbian stud. 21, 70-87. doi: $10.1080 / 10894160.2016 .1142353$

Ross, H. E., Freeman, S. M., Spiegel, L. L., Ren, X., Terwilliger, E. F., and Young, L. J. (2009). Variation in oxytocin receptor density in the nucleus accumbens has differential effects on affiliative behaviors in monogamous and polygamous voles. J. Neurosci. 29, 1312-1318. doi: 10.1523/JNEUROSCI.5039-08.2009

Rusu, P., Bodenmann, G., and Kayser, K. (2018). Cognitive emotion regulation and positive dyadic outcomes in married couples. J. Soc. Pers. Relationsh. 36, 359-376. doi: 10.1177/0265407517751664

Sapolsky, R. M., Romero, L. M., and Munck, A. U. (2000). How do glucocorticoids influence stress responses? Integrating permissive, suppressive, stimulatory, and preparative actions. Endocr. Rev. 21, 55-89. doi: 10.1210/edrv.21.1.0389

Scheele, D., Striepens, N., Güntürkün, O., Deutschländer, S., Maier, W., Kendrick, K. M., et al. (2012). Oxytocin modulates social distance between males and females. J. Neurosci. 32, 16074-16079. doi: 10.1523/jneurosci.2755-12.2012

Schulz, P., Schlotz, W., and Becker, P. (2004). Das Trierer Inventar zur Erfassung von chronischem Stress - Version 2 (TICS 2) [Trier Inventory for the Assessment of Chronic Stress]. Göttingen: Hogrefe.

Seltzer, L. J., Ziegler, T., Connolly, M. J., Prososki, A. R., and Pollak, S. D. (2014). Stress-induced elevation of oxytocin in maltreated children: evolution, neurodevelopment, and social behavior. Child Dev. 85, 501-512. doi: 10.1111/ cdev.12136

Simeon, D., Bartz, J., Hamilton, H., Crystal, S., Braun, A., Ketay, S., et al. (2011). Oxytocin administration attenuates stress reactivity in borderline personality disorder: a pilot study. Psychoneuroendocrinology 36, 1418-1421. doi: 10.1016/ j.psyneuen.2011.03.013

Sullivan, R. M., and Dufresne, M. M. (2006). Mesocortical dopamine and HPA axis regulation: role of laterality and early environment. Brain Res. 1076, 49-59. doi: 10.1016/j.brainres.2005.12.100

Taylor, S. E., Klein, L. C., Lewis, B. P., Gruenewald, T. L., Gurung, R. A. R., and Updegraff, J. A. (2000). Biobehavioral responses to stress in females: tend-andbefriend, not fight-or-flight. Psychol. Rev. 107, 411-429. doi: 10.1037/0033295X.107.3.411

Thienel, M., Heinrichs, M., Fischer, S., Ott, V., Born, J., and Hallschmid, M. (2014). Oxytocin's impact on social face processing is stronger in homosexual than heterosexual men. Psychoneuroendocrinology 39, 194-203. doi: 10.1016/j. psyneuen.2013.09.013

Weisman, O., Zagoory-Sharon, O., and Feldman, R. (2014). Oxytocin administration, salivary testosterone, and father-infant social behavior. Prog. Neuropsychopharmacol. Biol. Psychiatry 49, 47-52. doi: 10.1016/j.pnpbp. 2013.11.006

Weiss, R., and Heyman, R. (1997). "Couple interaction," in Clinical Handbook of Marriage and Couples Intervention, eds W. K. Halford and H. J. Markman (New York, NY: Wiley), 13-41.

Xu, F., Hilpert, P., Randall, A. K., Li, Q., and Bodenmann, G. (2016). Validation of the Dyadic Coping Inventory with Chinese couples: factorial structure, measurement invariance, and construct validity. Psychol. Assess. 28, e127-e140. doi: $10.1037 /$ pas 0000329 
Zemp, M., Milek, A., Cummings, E. M., Cina, A., and Bodenmann, G. (2016). How couple- and parenting-focused programs affect child behavioral problems: a randomized controlled trial. J. Child Fam. Stud. 25, 798-810. doi: 10.1007/ s10826-015-0260-1

Zietlow, A.-L., Heinrichs, M., and Ditzen, B. (2016). Psychobiologie sozialer Nähe. Psychother. Dialog 17, 82-84. doi: 10.1055/s-0042-109318

Conflict of Interest Statement: The authors declare that the research was conducted in the absence of any commercial or financial relationships that could be construed as a potential conflict of interest.
The reviewer JG and handling Editor declared their shared affiliation at the time of the review.

Copyright (C) 2019 Zietlow, Eckstein, Hernández, Nonnenmacher, Reck, Schaer, Bodenmann, Heinrichs and Ditzen. This is an open-access article distributed under the terms of the Creative Commons Attribution License (CC BY). The use, distribution or reproduction in other forums is permitted, provided the original author(s) and the copyright owner(s) are credited and that the original publication in this journal is cited, in accordance with accepted academic practice. No use, distribution or reproduction is permitted which does not comply with these terms. 


\section{OPEN ACCESS}

Edited by:

Guy Bodenmann,

University of Zurich, Switzerland

Reviewed by:

Nina Rottmann,

University of Southern Denmark,

Denmark

Miriam Parise,

Catholic University of Sacred Heart,

Italy

*Correspondence:

Chiara Acquati

cacquati@central.uh.edu

Specialty section

This article was submitted to Clinical and Health Psychology, a section of the journal

Frontiers in Psychology

Received: 19 June 2018 Accepted: 11 February 2019 Published: 19 March 2019

Citation: Acquati C and Kayser K (2019) Dyadic Coping Across the Lifespan: A Comparison Between Younger and Middle-Aged Couples With Breast Cancer. Front. Psychol. 10:404 doi: 10.3389/fpsyg.2019.00404

\section{Dyadic Coping Across the Lifespan: A Comparison Between Younger and Middle-Aged Couples With Breast Cancer}

\author{
Chiara Acquati $^{{ }^{*}}$ and Karen Kayser ${ }^{2}$ \\ ${ }^{1}$ Graduate College of Social Work, University of Houston, Houston, TX, United States, ${ }^{2}$ Renato LaRocca Chair of Oncology \\ Social Work, Kent School of Social Work, University of Louisville, Louisville, KY, United States
}

The association between dyadic coping and adjustment to cancer has been well-established. However, a significant gap in the literature is the understanding of how the life stage of couples may influence their dyadic coping and the accompanying quality of life. Although younger couples have been identified at higher risk for poor coping because of less collaborative behaviors and higher vulnerability to stress, only a limited number of studies have addressed younger women's coping with breast cancer in the context of close relationships. The present study addressed the differential impact of the illness on the quality of life and dyadic coping behaviors of younger and middle-aged dyads and the influence of relational mutuality on couples' coping in the two groups. A sample of 86 couples participated in a cross-sectional study; 35 younger couples were compared to 51 middle-aged dyads. Patients and partners completed measures of quality of life, dyadic coping, and mutuality. Independent-samples $t$-tests were used to examine differences in the two groups, while the Actor-Partner Interdependence Model (APIM) identified actor and partner effects of relational mutuality on dyadic coping. Younger women and their partners reported statistically significant worse quality of life and dyadic coping scores than the middle-age group. For younger couples, positive and negative coping styles were the result of both actor and partner effects of mutuality. The study highlighted the more negative impact of breast cancer on the quality of life of younger patients and partners. It also revealed a stronger influence of each partner's relational mutuality compared to the middle-age group in predicting both adaptive and maladaptive coping behavior. Future studies should continue to examine the developmental trajectory of dyadic coping across the lifespan in order to develop psychosocial interventions to promote younger dyads' coping efforts.

Keywords: dyadic coping, couples, breast cancer, lifespan, mutuality

\section{INTRODUCTION}

In the last 20 years a new attention toward interpersonal aspects of coping has emerged (Revenson et al., 2005; Kayser and Scott, 2008; Saita, 2009; Donato, 2012; Iafrate and Donato, 2012; Regan et al., 2015; Traa et al., 2015). This emphasis has resulted in the convergence of theoretical frameworks of close relationships and stress and coping with the goal to examine how coping 
develops within the context of significant relationships (Revenson et al., 2005; Iafrate and Donato, 2012). As a consequence, couples' coping has started to be conceptualized no longer referring to the separate perspectives of the two partners, but as a dyadic process involving their mutual influence (Bodenmann, 1997). Dyadic coping is conceptualized as a process shaped by the individual's close relationships (Bodenmann, 2005; Revenson et al., 2005; Peterson and Bush, 2013). It is described as "the interplay between the stress signals of one partner and the coping reactions of the other, a genuine act of shared coping" (Revenson et al., 2005; p. 4). Through a series of interactions, dyadic coping contributes to a sense of we-ness and promotes the conjoint creation of strategies to respond to the stressful event (Revenson, 1994; Bodenmann, 1997; Scott et al., 2004; Kayser et al., 2007).

The significance of dyadic coping for relationship functioning, psychological, and physical well-being has been established across several types of stressors (Bodenmann et al., 2004, 2010; Hinnen et al., 2008a,b; Randall and Bodenmann, 2009; Badr et al., 2010; Sullivan et al., 2010; Vilchinsky et al., 2010). Longitudinal studies have confirmed that dyadic coping represents a protective factor for the couple's relationship, with better relational outcomes and a reduced risk of being divorced registered among dyads reporting common coping (Bodenmann and Cina, 2005; Bodenmann et al., 2006). More recently, it has been established that dyadic coping significantly predicts relationship satisfaction and that aggregated positive forms of coping are stronger predictors of this outcome than negative ones (Falconier et al., 2015). In the context of cancer, the ability of the couple to face the illness as a unit contributes to higher relationship quality (Picard et al., 2005; Fergus and Gray, 2009; Badr et al., 2010; Traa et al., 2015). Similarly, better relationship functioning and quality of life are reported by couples that engage in relationship maintenance behaviors, social support exchanges, mutual constructive communication, and joint dyadic coping (Lavery and Clarke, 1999; Norton and Manne, 2007; Badr and Taylor, 2008; Langer et al., 2009; Hagedoorn et al., 2011a,b; Pasipanodya et al., 2012; Manne et al., 2015). However, a significant gap in the literature is the understanding of how the life stage of couples may influence their dyadic coping and the accompanying quality of life.

Previous research indicates that younger couples are more vulnerable to distress, experience poorer quality of life, and appear to be at risk for negative adjustment to the stress of the cancer because of four reasons. First, it has been documented that coping abilities increase with age, with older individuals presenting better emotion regulation and more effective collaborative coping skills (Folkman et al., 1987; Aldwin, 1994; Diehl et al., 1996; Labouvie-Vief, 2003; Revenson, 2003; Helgeson et al., 2004; Revenson and Pranikoff, 2005). Second, contextual and generational factors may impact younger couples' vulnerabilities, as younger generations report higher levels of stress, often associated with financial insecurity and unhealthy lifestyles, when compared with previous generations (American Psychological Association, 2015). Third, while there is little disagreement that marital satisfaction decreases with time (Bradbury and Karney, 2014), the literature about close relationships has provided over the last decades evidence of relational and psychological difficulties for newlyweds and younger families (Carstensen et al., 2003, 2011; Amato and Hohmann-Marriott, 2007; Luong et al., 2011; van Steenbergen et al., 2011; Scabini and Rossi, 2012; Woszidlo and Segrin, 2013; Bradbury and Karney, 2014). Researchers have recently identified different trajectories within the first years of marriage which are associated to marital dissolution. The most significant reduction in marital satisfaction is reported among couples who had low satisfaction at baseline (Lavner et al., 2012) or greatest expectations about the quality of their relationships (Lavner et al., 2013). Increased rates of divorce after 4 and 10 years of marriage have been associated to personality traits, stress, aggression, and poor communicative behaviors (Lavner and Bradbury, 2010). Investigations of the effects of personality traits and stressful events on marital satisfaction in recently married couples confirmed that partners' occupation, work interference, and family stress were negatively associated with marital satisfaction (van Steenbergen et al., 2011; Woszidlo and Segrin, 2013). Furthermore, economic hardship has been associated with higher rate of conflict (Halliday Hardie and Lucas, 2010), lower life satisfaction, higher pessimism (Haid and Seiffge-Krenke, 2012) and negative communication (Williamson et al., 2013) for newly-weds. In contrast, a study by Neff and Broady (2011) highlighted that adaptation to moderately stressful events early in the marriage is associated to reduced stress spillover effect, greater self-efficacy, and marital adjustment; suggesting that practicing stress adaptation strategies in the early years of marriage can lead to increased ability to cope with stress at a later stage of the couple's life. Finally, when dyadic coping has been investigated among different age groups and cohorts, authors have highlighted the complexity of the relational exchange within the dyad. While initial studies showed that older couples perform consistently better than younger ones (Revenson, 2003; Revenson and Pranikoff, 2005; Berg and Upchurch, 2007; Blanchard-Fields and Coats, 2008; Hoppmann et al., 2008), others have highlighted stereotypical similarity in younger couples (Iafrate et al., 2012). The authors concluded that younger partners appear to experience a heightened perceived idealization of the relationship. A recent study analyzed stress, dyadic coping, and partners' well-being in three age cohorts (Cohort 1: 20-35y.o.; Cohort 2: 40-55y.o.; Cohort 3 65-80 y.o.). Among the younger cohort, women's well-being was affected by stress and negative supportive behaviors, while males' quality of life was mostly influenced by individual coping and contextual factors (Vedes et al., 2015). Among middle-aged couples, the well-being of both partners was influenced by stress and dyadic coping. While investigators found an actor effect for female partners, the well-being of male partners appeared to be more dependent on the dyadic coping of the wife. These differences disappeared in the late-adulthood group. It is therefore possible to conclude that the relationship among stress, dyadic coping, and well-being changes across the life course experience.

If we extend our reflection on developmental differences to the experience of breast cancer, young women present higher psychological distress and poorer quality of life than older women 
(Hartl et al., 2010; Luutonen et al., 2011; So et al., 2011; Cataldo et al., 2013; Hau et al., 2013; Champion et al., 2014; BantemaJoppe et al., 2015). Young women are also more vulnerable to the disruptions of the disease on their close and intimate relationships, reporting higher concerns for their relationship with partners, difficulties disclosing the diagnosis, and higher feelings of isolation (Ruddy et al., 2013; Ahmad et al., 2015). Anxiety and depressive symptoms have been found among those who had received chemotherapy and reported low level of support from close ones and partners (Borstelmann et al., 2015; Gold et al., 2015). The literature suggests that not all couples cope effectively with the stress of cancer. In a large prospective cohort study of women diagnosed with breast cancer at age 40 or younger, $\sim 20 \%$ perceived the partner as unsupportive. For them, an increased likelihood to report anxiety symptoms existed (Borstelmann et al., 2015). Similarly, Avis et al. (2004) found that young women with higher levels of marital problems reported lower global, physical, emotional, and breast cancerspecific quality of life. This finding extends to survivorship, as younger survivors perceive less intimate or partner support than the older group, more social constraints, and lower marital satisfaction (Stava et al., 2006; Champion et al., 2014). For those experiencing difficulties in their relationship, reduced perceived benefit from the cancer experience and higher negative impact on their well-being and quality of life have been documented (Champion et al., 2014). Finally, Walsh et al. (2005) identified that although most women experienced greater closeness with their partners, 1 in 4 participants reported increased relational strain which ended in separation or end of the relationship $12 \%$ of the time. The illness also affects the quality of life of male partners (Baucom et al., 2005; Antoine et al., 2012; Duggleby et al., 2014; Hasson-Ohayon et al., 2014; Fergus et al., 2015; Borstelmann et al., 2017). Antoine et al. (2012) found that partners tended to be very close and supportive at the beginning of the cancer experience, providing high levels of mutual support, while over time they wished for the couple to resume a sense of normalcy. Other studies have highlighted (Vanlemmens et al., 2012a,b) the negative impact of the disease on psychological, physical, relational, social, sexual, domestic, professional, and economic dimensions. While couple cohesion assumed a central role for patients, caregiving concerns, and apprehension for the future became more relevant for younger partners (Christophe et al., $2015 \mathrm{a}, \mathrm{b})$. These findings are in line with a recent study conducted by Borstelmann et al. (2017) where partners of young breast cancer patients presented more maladaptive coping styles.

The present work addresses a significant gap in the literature about younger women with breast cancer by considering the impact of the diagnosis on both patients and their partners from a relational perspective. The study focuses on the concept of dyadic coping, therefore highlighting not only the impact of the illness on health-related quality of life, but extending our understanding of how young couples cope together with the illness and whether the processes are different from those of middle-aged dyads. In the present work, dyadic coping is conceptualized as a process of communicating stress and appraising coping behaviors that occur between partners (Systemic-Transactional Model; Bodenmann, 2005; Bodenmann et al., 2016). Relational mutuality, namely, the ability to be empathic with one's partner and to participate in a shared emotional experience (Jordan et al., 1991; Jordan, 2009), has emerged as an important antecedent for the enactment of coping behaviors (Kayser et al., 2007). Mutuality was found to be significantly associated to women's adjustment to cancer (Kayser et al., 1999) and it is a key relational quality that informs the coping strategies enacted by the partners according to the Relational-Cultural Model of Dyadic Coping (Kayser et al., 2007, 2010). Since we were interested in understanding how younger and middle-aged couples differ in the process of coping, the variable was included in our hypotheses and analysis.

The overall purpose of the present study was to examine differences between younger and middle-aged couples in terms of quality of life, dyadic coping, and mutuality and the influence of relational mutuality on couple's coping in the two groups. The following research questions guided this work:

(1) Do younger breast cancer patients and partners differ from middle-aged patients and partners on their quality of life, dyadic coping, and relational mutuality?

Hypothesis 1.1: Younger patients with breast cancer will report lower quality of life, relational mutuality, and higher negative dyadic coping styles compared to middle-aged breast cancer patients.

Hypothesis 1.2: Younger partners will report lower quality of life, relational mutuality, and higher negative dyadic coping styles compared to middle-aged partners of breast cancer patients.

(2) How does relational mutuality affect dyadic coping styles of breast cancer patients and their partners by age group?

Hypothesis 2.1: Patients' perceived relational mutuality will influence their own dyadic coping style and their partners' dyadic coping style.

Hypothesis 2.2: Partners' perceived relational mutuality will influence their own dyadic coping style and the patients' dyadic coping style.

Hypothesis 2.3: Differences in actor and partner effects of relational mutuality on dyadic coping will exist by age group, between younger and middle-aged dyads.

\section{MATERIALS AND METHODS}

\section{Participants and Procedure}

Participants were recruited among adult patients newly diagnosed with early-stage non-metastatic breast cancer in two medical centers in the northeast United States. ${ }^{1}$ Inclusion criteria were: (a) having received a diagnosis of primary nonmetastatic breast cancer within the last 3 months; (b) being currently involved in a close relationship with a partner; (c) being older than 18 years of age; (d) receiving routine clinical care at the participating sites; and (e) being able to understand English. The study was inclusive of heterosexual and same-sex

\footnotetext{
${ }^{1}$ This study was part of a larger intervention study and included only baseline data collected before couples were randomly assigned to the treatment arms (see Kayser et al., 2010 for further information).
} 
relationships. However, only one same-sex couple participated. Patients meeting the inclusion criteria were approached by a recruitment coordinator and questionnaires were mailed to those interested. The patients were sent letters at their home address, which included a study brochure, a decline card, and pre-stamped return envelopes. If feasible, the recruiters met with the woman and her partner in the clinic to present the study. Both the patient and partners/spouses needed to consent to be enrolled. Couples who agreed to be in the study were mailed survey questionnaires and returned their questionnaires in two separate pre-stamped envelopes. Ninety-four patients and ninety partners returned their questionnaires to the research team, with data available for 86 dyads.

In this study younger couples were identified as those where the patient was $\leq 45$ years at diagnosis. The literature about breast cancer usually refers to "younger women" as those in their reproductive years (Hulvat and Jeruss, 2009). The mean age of menopause is at 51 years for American women with a peri-menopause stage between 47 and 51 years (National Institute of Aging, 2015). In addition, when survivorship has been investigated in a younger group of breast cancer patients, researchers have usually enrolled women from the age of 50 , suggesting that they would have been $\sim 45$ years old at time of diagnosis (i.e., Champion et al., 2014). A total of 35 dyads met this criterion, and they were compared with the remaining 51 "middle-aged couples" composed by women who were between the ages of 46 and 66, with only one participant being 72 . The mean age for middle-aged cancer patients was 55 (see Table 1).

\section{Relational-Cultural Model of Dyadic Coping}

The theoretical framework of the study derives from the Relational-Cultural Model of Dyadic Coping (Kayser et al., 2007) which proposes that appraisal and responses to cancer are shaped by relational characteristics; one of these characteristics is relational mutuality. The model is influenced by the RelationalCultural Theory, a perspective that explains the individual sense of self as being in relation (Miller, 1984; Jordan et al., 1991; Jordan, 2009) and identifies the goal of human development in the acquisition of relational competence, which can be achieved by engaging in growth-fostering relationships. According to this conceptualization of dyadic coping, relationship awareness, authenticity, and mutuality determine the pattern of coping couples develop. Relationship awareness refers to the partners' awareness that the stressor is affecting both of them (Kayser et al., 2007; Kayser and Scott, 2008). Authenticity describes partners' ability to disclose genuine feelings to each other in a sensitive and appropriate way (Kayser et al., 2007; Kayser and Scott, 2008; Scott and Kayser, 2009). Finally, mutuality is defined as the ability to be empathic with the partner and to participate in a shared emotional experience (Jordan, 1997a,b; Feldman and Broussard, 2005, 2006; Kayser et al., 2007; Kayser and Scott, 2008). Depending on the presence of these characteristics, two different patterns of relational coping are enacted: mutual responsiveness or disengaged avoidance (Kayser et al., 2007; Kayser and Scott, 2008). Mutually responsive couples appraise the stress as affecting both members of the dyad, respond to each other's emotional and physical needs when coping, and grow through the stressful experience. On the contrary, disengaged avoidant couples will appraise the cancer as an individual stressor, avoid or deny the stress, and do not acknowledge benefits of the stress on their relationship (Kayser et al., 2007, 2010; Kayser and Scott, 2008). While the link between mutuality and dyadic coping can be bi-directional, based on the Relational-Cultural Theory, we hypothesize in this study that mutuality is a relational quality that is likely to influence the behaviors of positive dyadic coping.

\section{Measures \\ Quality of Life}

The quality of life of women diagnosed with breast cancer was measured by the Functional Assessment of Cancer TherapyBreast (FACT-B) Scale (Cella et al., 1993; Brady et al., 1997). The FACT-B (Version 4) is a 37-item measure that contains four general subscales assessing the physical, social/family, emotional, and functional well-being of the individual, along with the breast cancer-specific subscale that assesses concerns of particular relevance to breast cancer patients (e.g., body image, arm swelling and tenderness). Patients were invited to indicate how true each statement has been for them in the previous seven days, and items are rated on a 5 point Likert scale ranging from "Not at All" (0) to "Very Much" (4). The FACT-B consists of five subscale scores: physical well-being (PWB), social/family well-being (SWB), emotional well-being (EWB), functional wellbeing (FWB) and additional concerns (BCS), with higher scores indicating higher quality of life. From these subscale scores, two assessment total scores were calculated: the FACT-B total score, and the FACT-G score. The FACT-B total score is calculated by summing all five un-weighted subscale scores, with total scores in the range of $0-136$. The FACT-G score is calculated by summing PWB, SWB, EWB, and FWB scores, with scores in the range of $0-108$. Administration and scoring guidelines are available on the website http://www.facit.org/FACITOrg. The FACT-B has been extensively used in psychosocial oncology research and has demonstrated high validity and internal consistency (Cella et al., 1993; Brady et al., 1997; Winstead-Fry and Schultz, 1997; Webster et al., 1999, 2003; Overcash et al., 2001). In its validation study, Cronbach's alpha for the total score was 0.90 , with subscale alpha coefficients ranging from 0.63 to 0.86 (Brady et al., 1997). Evidence supported test-retest reliability, as well as convergent and divergent validity (Cella et al., 1993; Brady et al., 1997; Winstead-Fry and Schultz, 1997; Webster et al., 1999, 2003; Overcash et al., 2001). For the purpose of the present investigation, the five subscales and the FACT-G and FACT-B total scores were used when comparing quality of life between the two groups of cancer patients. Similarly to the data available in the literature, high internal consistency has been registered (FACT-G $\alpha=0.90$, FACT-B total score $\alpha=0.90$, PWB $\alpha=0.88$, $\mathrm{SWB}=0.81, \mathrm{EWB} \alpha=0.83$, FWB $\alpha=0.85, \mathrm{BCS} \alpha=0.81$ ).

Due to the unavailability of a measure of quality of life for both cancer patients and partners at time of the study, the Emotional Functioning subscale from the Quality of Life Questionnaire for Spouses (QL-SP) (Ebbesen et al., 1990) and the Illness Intrusiveness Ratings Scale (IIRS) (Binik et al., 1990) 
TABLE 1 | Socio-demographic, relational, and clinical characteristics of the sample.

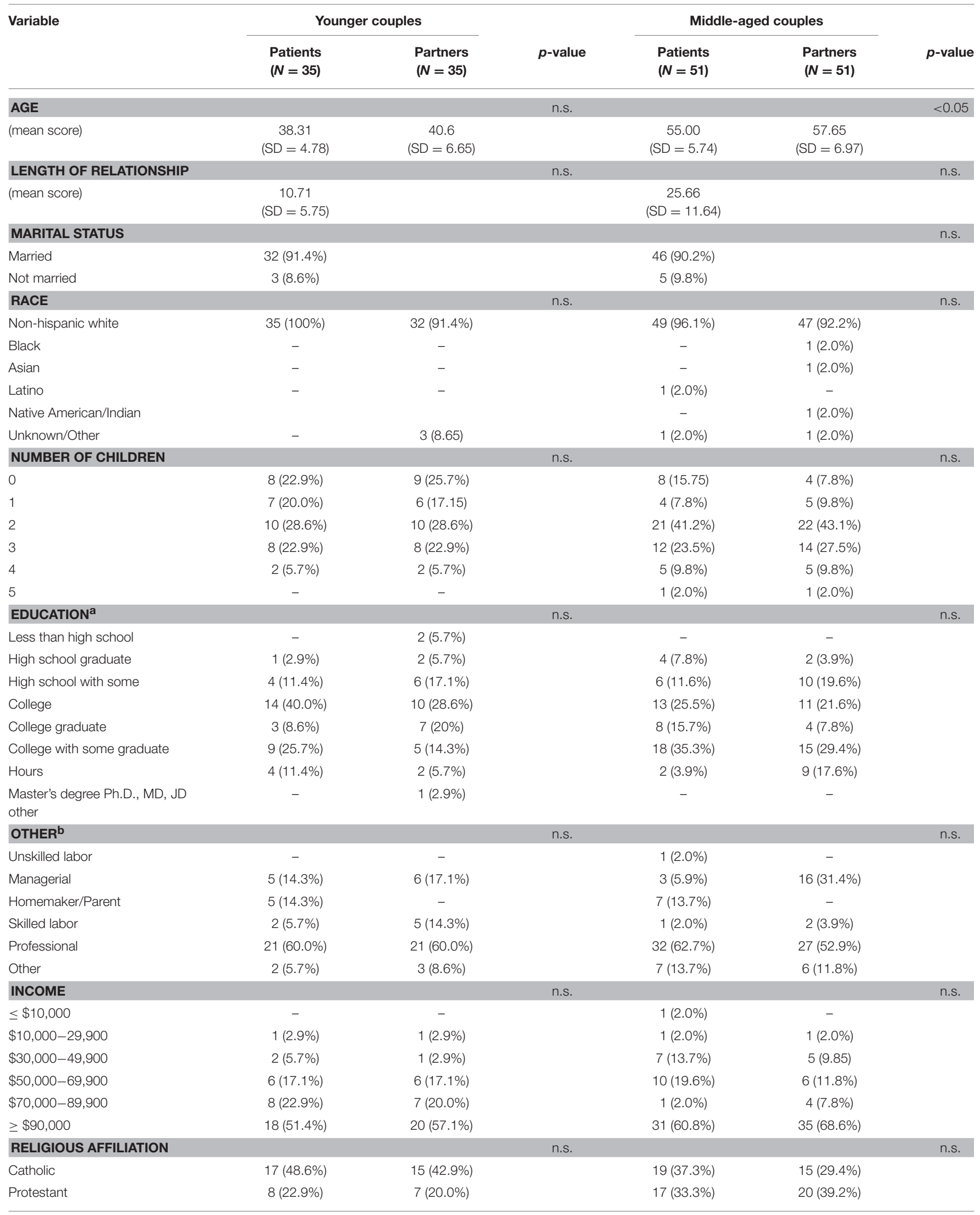


TABLE 1 | Continued

\begin{tabular}{|c|c|c|c|c|c|c|}
\hline Variable & \multicolumn{2}{|c|}{ Younger couples } & $p$-value & \multicolumn{2}{|c|}{ Middle-aged couples } & $p$-value \\
\hline Jewish & $3(8.6 \%)$ & $3(8.6 \%)$ & & 7 (13.7\%) & 10 (19.6\%) & \\
\hline Atheist/Agnostic & $1(2.9 \%)$ & $5(14.3 \%)$ & & $3(5.9 \%)$ & $4(7.8 \%)$ & \\
\hline Other & $6(17.1 \%)$ & 5 (14.3\%) & & $5(9.8 \%)$ & 2 (3.9\%) & \\
\hline No & 15 (42.9\%) & & & 18 (36.0\%) & & \\
\hline \multicolumn{7}{|l|}{ CHEMOTHERAPY } \\
\hline Yes & $10(28.6 \%)$ & & & $8(16.35)$ & & \\
\hline No & 25 (71.4\%) & & & 41 (83.7\%) & & \\
\hline \multicolumn{3}{|c|}{ PREVIOUS TREATMENT FOR DEPRESSION } & n.s. & & & n.s. \\
\hline Before cancer diagnosis & $6(17.1 \%)$ & & & 14 (28.6\%) & & \\
\hline After cancer diagnosis & - & & & $2(4.15)$ & & \\
\hline Before and after & - & & & $1(2.05)$ & & \\
\hline Not applicable & 29 (82.9\%) & & & 32 (62.7\%) & & \\
\hline
\end{tabular}

${ }^{a}$ Non-significant differences are detected also when the variable is recoded in 2 categories, $1=$ High School, and $2=$ College graduate. ${ }^{b}$ Non-significant differences are detected also when the variable is recoded in 2 categories. Unskilled labor, Homemaker, and other were recoded as 1, managerial, skilled labor and professional were recoded as 2. The Fisher's Exact Test indicates a 2-sided significance of 0.31.

TABLE 2 | Descriptives of the major study variables for younger couples.

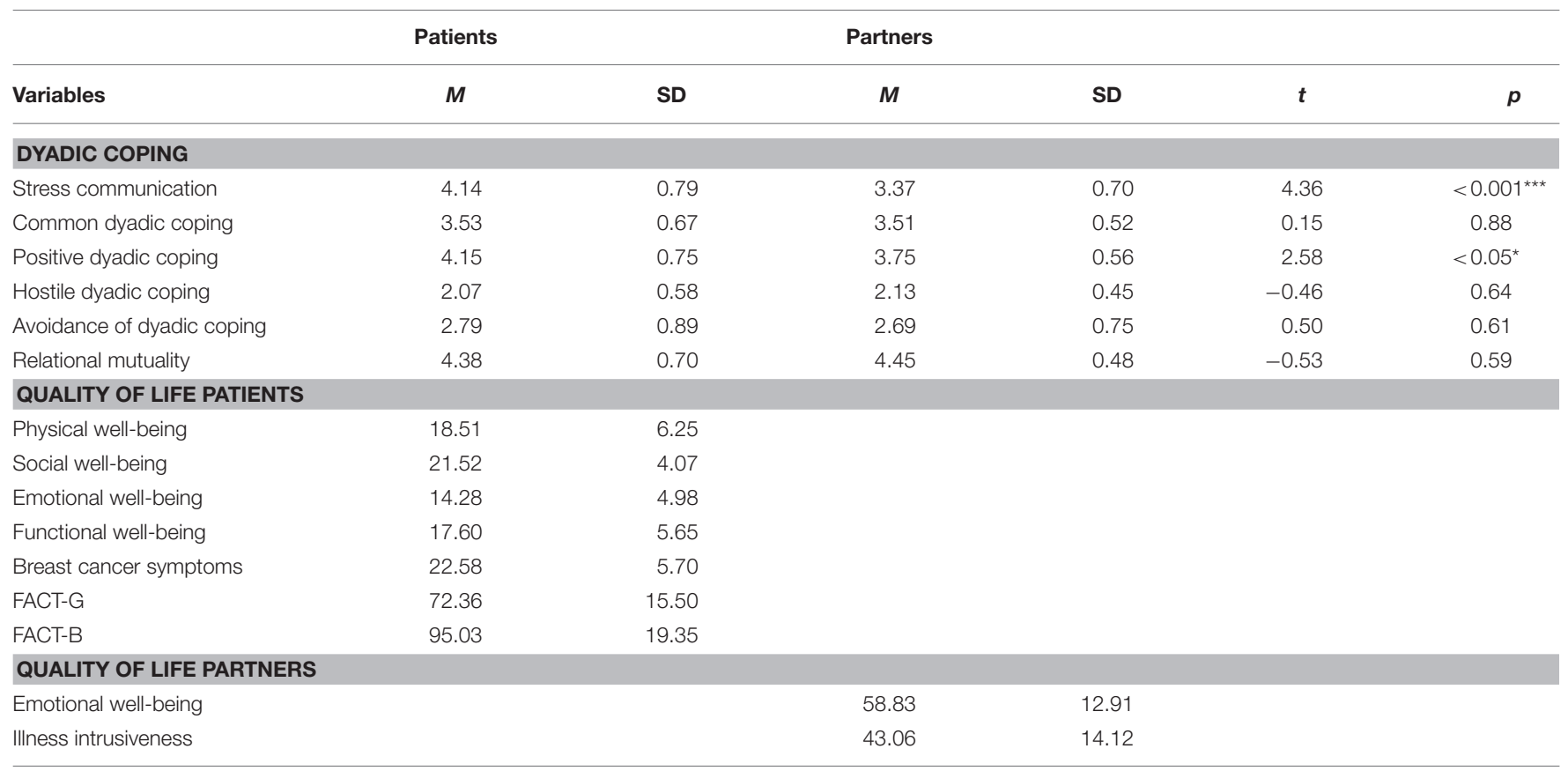

${ }^{*} p<0.05,{ }^{* *} p<0.01,{ }^{* * *} p<0.001$.

investigated quality of life among partners. The Emotional Function Dimension (14 items) examines the well-being of the individual in the previous 2 weeks by rating on a 7 point Likert scale anxiety, depression, concerns, frustration, and helplessness
(Ebbesen et al., 1990). Total scores range from 7 to 98 , with higher scores indicating better functioning. The scale demonstrated high internal consistency in previous studies (Feldman and Broussard, 2006; Iafrate et al., 2012), and Cronbach's alpha was 
TABLE 3 | Descriptives of the major study variables for Middle-Aged Couples.

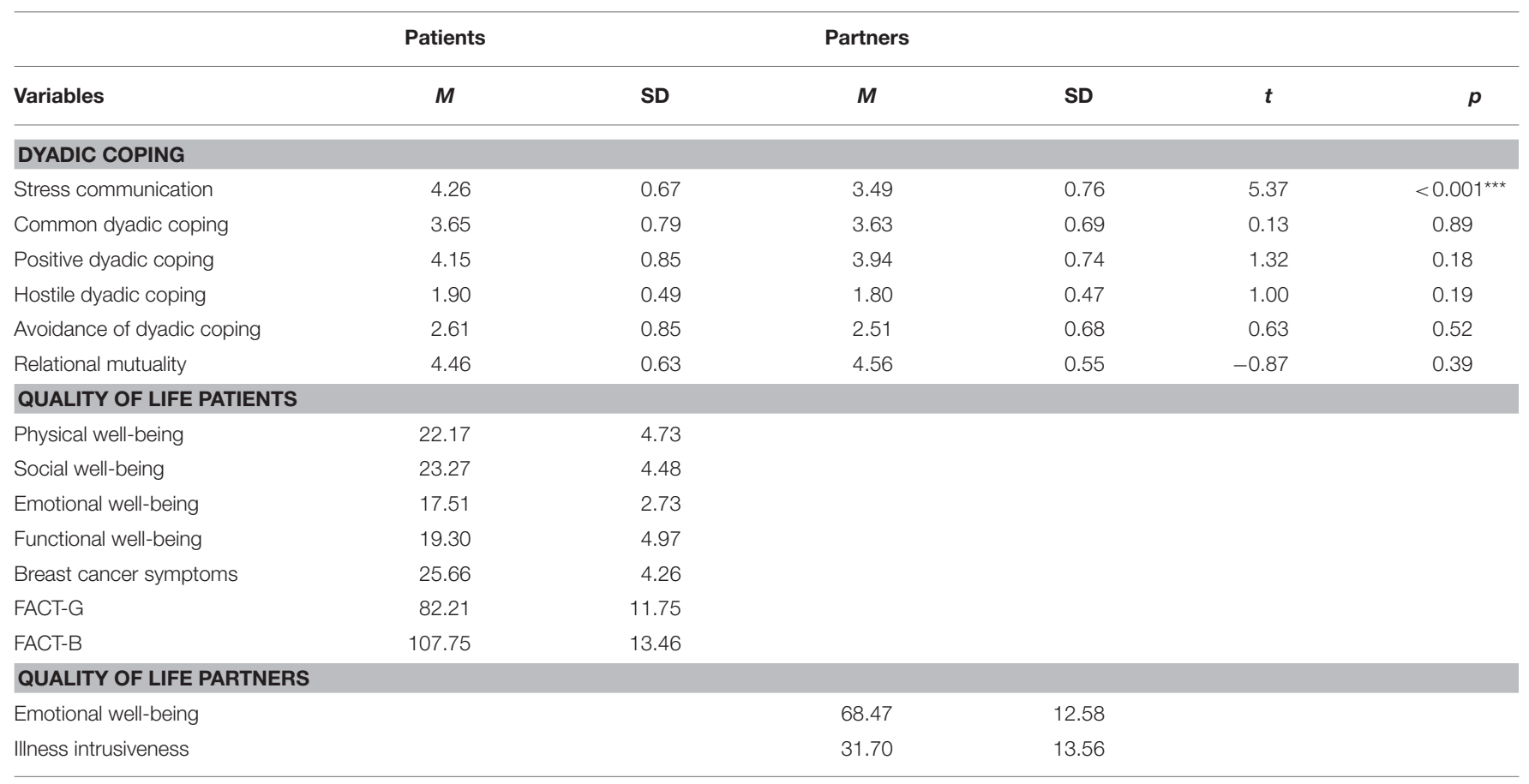

${ }^{*} p<0.05,{ }^{\star *} p<0.01,{ }^{* \star *} p<0.001$.

0.91 in the present sample. The Illness Intrusiveness Ratings Scale measures the interference of the partner's illness and treatment on 13 dimensions (Devins et al., 1983; Devins, 1994) on a 7 point Likert Scale. The total score ranges from 13 to 91, with higher scores indicating greater impact of the patient's illness on the partner. Several studies support the reliability and validity of the instrument (Binik et al., 1990; Devins et al., 1990; Devins, 1994). A systematic review highlighted that Cronbach's alpha scores ranged from the 0.80 's to the 0.90 's across studies (Devins, 2010). In the present sample the Cronbach's alpha was 0.88 .

\section{Dyadic Coping}

Dyadic coping was measured by the Dyadic Coping Scale (Bodenmann, 2000). This self-report questionnaire assesses stress communication and dyadic coping as perceived by each partner, each partner's perception of the other's coping, and each partner's view of how they cope as a couple. In this version each item (for a total of 61) is measured on a 6-point Likert scale. The Dyadic Coping Scale contains five subscales: Stress Communication (SC), Common (CDC), Positive (PDC), Hostile (HDC) and Avoidance of Dyadic Coping (ADC). Stress communication refers to the partners' ability to communicate emotion- and problem-focused stress. Examples are "I ask my partner to do things for me when I have too much to do," "I try to hide my stress from my partner so that he/she does not notice it," and "I tell my partner openly how I feel and that I would appreciate his/her support." Common dyadic coping occurs when both members of the couple experience the stressful event and they participate in the coping process in a symmetric or complementary way. They use strategies like joint problem solving, information seeking, and mutual commitment. Examples of items of this subscale are "We are supportive of each other and help one another out," "We help one another to put the problem in perspective and see it in a new light," and "We caress one another and make love." Positive dyadic coping refers to the use of supportive dyadic coping strategies like the provision of practical help, information, advice, and understanding and helping to relieve tension. Examples of items included in this subscale are: "My partner gives me the feeling that he/she understands me"; "My partner listens to me and gives me the opportunity to communicate the entire situation," and "My partner takes on things that I normally do in order to help me out." Hostile dyadic coping indicates a situation when the stress signals of one partner originate a hostile behavior by the other. Responses or behaviors that can be considered hostile include distancing, ridicule, sarcasm, clear disinterest and minimizing the emotional experience of stress of the other. Scale items that are included in this subscale are: "I make fun of my partner's stress," "I let my partner know that I do not want to be bothered with his/her problems," and "Although my partner makes time for me, his/her thoughts are somewhere else." Finally, avoidance of dyadic coping describes ambivalent and superficial coping responses, where authentic engagement is absent (Bodenmann, 1997, 2005). Examples are "When my partner is stressed, I tend to get out of his/her way," and "When my partner is stressed I tend to withdraw." Satisfactory psychometrics of the questionnaire have been reported (Feldman and Broussard, 2006). In this study, reliability scores ranged from 0.68 to 0.96 for patients, and from 0.68 to 0.95 for partners. Cronbach's alpha of each subscale for patients and partners is hereby reported for each subscale: SC: 0.68 patients and partners; CDC: 0.86 patients, 0.83 partners, PDC: 0.96 patients; 0.95 
partners; HDC: 0.80 patients; 0.70 partners; ADC: 0.68 patients, 0.68 partners. The Cronbach's alpha for the total score were 0.91 patients and 0.90 partners.

\section{Relational Mutuality}

Mutuality is defined as a "bidirectional expression of feelings, thoughts, and activity between individuals in a relationships" (Genero et al., 1992. p. 36) and involves elements of empathy, engagement, authenticity, zest, diversity and empowerment (Miller, 1986). It can be summarized as the ability to experience feelings of another person, while maintaining a sense of one's own feelings, therefore being authentic and able to empower the other member of the dyad. We used the 22-item Mutual Psychological Development Questionnaire (MPDQ) (Genero et al., 1992) that was developed to measure mutuality in adult close relationships. The MPDQ contains items from two relationship perspectivesthe self and other. It consists of items assessing the six dimensions of mutuality mentioned above. Examples of items include: "When we talk about things that matter to my spouse/partner, I am likely to be receptive/ get impatient /try to understand" and "When we talk about things that matter to me, my spouse/partner is likely to pick up on my feelings/ feel like we are not getting anywhere, share similar experiences." Hence, scores are summed in order to compute the level of mutuality reported by each person when considering the close relationship in exam, and for this reason we use the term relational mutuality in the present work. In the present sample reliability scores were high for both patients $(\alpha=0.93)$ and partners $(\alpha=0.91)$.

\section{Data Analysis}

The analysis was conducted in accordance with the recommendations of the University of Louisville Institutional Review Board. The present work was approved through the expedited review procedure because it involved materials that have been already collected and because it involved no more than minimal risk. After obtaining IRB approval, IBM SPSS Statistics 22 was used for data screening and data analysis. Descriptive statistics were obtained and mean substitution was implemented to handle missing data on the key variables. A Missing Value Analysis (MVA) was conducted on all the variables included in the dataset and revealed that missing data ranged from 0.6 to $2.3 \%$ of cases on variables of interest (from 1 to a max of 4 cases). Pearson $r$ correlations were used to assess the linear relationship between socio-demographic, clinical, and psychosocial measures. Comparisons of demographic characteristics between patients and spousal caregivers were calculated using paired sample $t$-tests for continuous variables and chi-square for ordinal and categorical variables. Differences between younger and middle-aged breast cancer patients and their partners on quality of life, dyadic coping, and mutuality have been assessed by calculating independent samples $t$-test. Then, the Actor-Partner Interdependence Model (APIM) was used to examine actor and partner effects of relational mutuality on the dyadic coping style of each member of the dyad in the two separate groups (Kenny et al., 2006).

\section{RESULTS}

\section{Sample Description}

Descriptive statistics for the demographic, relational, and clinical characteristics of the two groups are presented in Table 1. Younger patients were on average in their late thirties $(M=38.31, S D=4.78)$. The majority of them were college educated and working as professionals and reported an income higher than $\$ 90,000$ per year. Their partners were on average 40 years old $(S D=6.65)$. They were highly educated and could be considered to be middle to upper-middle class. The average length of the relationship was of 11 years $(S D=5.75)$, with most couples being married (91.4\%).

Middle-aged couples had been in a relationship for $\sim 26$ years $(S D=11.6)$ and most of them were married (90.2\%). Patients were in their mid-fifties $(M=55, S D=5.74)$, were highly educated, and working in professional settings (62.7\%). Only 16.3 $\%$ of middle-age women were currently receiving chemotherapy. Partners were in their late fifties $(M=57, S D=6.97)$, highly educated, and could be categorized as middle to upper-middle class. There were differences between patients and partners for age and occupation, with partners being significantly older $\left.\left(t_{(100)}\right)=-2.09, p<0.05\right)$ and in more managerial and professional positions (Table $\mathbf{1}$ ).

In terms of the variables of interest, younger women and their partners reported similar scores on common, hostile, and avoidance of dyadic coping. However, younger women reported higher scores than their partners on stress communication $(p<0.001)$ and positive dyadic coping behaviors $(p<0.05)$. Younger women reported affected quality of life in all the subscales of the FACT-B and partners showed levels of moderately affected emotional well-being and illness intrusiveness. Middle-aged dyads were characterized by elevated scores on dyadic coping styles like positive and common dyadic coping; indicating that the two partners were utilizing both individual and relational resources to cope with the cancer diagnosis. Middle-aged couples had low scores on hostile and avoidance dyadic coping. Women had elevated scores on the subscales that address physical, social, and overall quality of life. The areas mostly affected by the cancer diagnosis appeared to be their emotional and functional well-being. Middle-aged partners presented high scores on relational mutuality, emotional well-being, and low intrusiveness (Tables 2 and 3).

\section{Differences Between Younger and Middle-Aged Patients and Partners on Quality of Life, Relational Mutuality, and Dyadic Coping}

Independent-samples $t$-tests compared the mean scores of quality of life, relational mutuality, and dyadic coping styles of younger and middle-aged breast cancer patients and partners. Significant differences between younger and middle-age breast cancer patients were identified for physical $(p<0.01)$, emotional well-being $(p<0.01)$, and impact of breast cancer symptoms $(p<0.01)$. Younger women in this sample indicated higher physical and emotional difficulties, additional concerns related to 
TABLE 4 | Independent samples t-test comparing dyadic coping, relational mutuality, and quality of life among Younger and Middle-Age breast cancer patients.

\begin{tabular}{|c|c|c|c|c|c|}
\hline Variable & Age group & $M$ & SD & $T$ & $p$ \\
\hline \multirow[t]{2}{*}{ Stress communication } & Younger patients & 4.14 & 0.79 & -0.71 & 0.48 \\
\hline & Middle-age patients & 4.26 & 0.67 & & \\
\hline \multirow[t]{2}{*}{ Common dyadic coping } & Younger patients & 3.53 & 0.67 & -0.77 & 0.44 \\
\hline & Middle-age patients & 3.65 & 0.79 & & \\
\hline \multirow[t]{2}{*}{ Positive dyadic coping } & Younger patients & 4.15 & 0.75 & 0.04 & 0.96 \\
\hline & Middle-age patients & 4.15 & 0.85 & & \\
\hline \multirow[t]{2}{*}{ Hostile dyadic coping } & Younger patients & 2.07 & 0.57 & 1.45 & 0.15 \\
\hline & Middle-age patients & 1.90 & 0.49 & & \\
\hline \multirow[t]{2}{*}{ Avoidance of dyadic coping } & Younger patients & 2.79 & 0.89 & 0.94 & 0.35 \\
\hline & Middle-age patients & 2.61 & 0.85 & & \\
\hline \multirow[t]{2}{*}{ Relational Mutuality } & Younger patients & 4.38 & 0.70 & -0.57 & 0.57 \\
\hline & Middle-age patients & 4.46 & 0.63 & & \\
\hline \multirow[t]{2}{*}{ Physical well-being } & Younger patients & 18.51 & 6.25 & -2.94 & $0.005^{\star \star}$ \\
\hline & Middle-age patients & 22.17 & 4.73 & & \\
\hline \multirow[t]{2}{*}{ Social well-being } & Younger patients & 21.51 & 4.06 & -1.84 & 0.06 \\
\hline & Middle-age patients & 23.27 & 4.48 & & \\
\hline \multirow[t]{2}{*}{ Emotional well-being } & Younger patients & 14.28 & 4.98 & -3.48 & $0.001^{\star \star}$ \\
\hline & Middle-age patients & 17.50 & 2.73 & & \\
\hline \multirow[t]{2}{*}{ Functional well-being } & Younger patients & 17.60 & 5.65 & -1.47 & 0.14 \\
\hline & Middle-age patients & 19.30 & 4.97 & & \\
\hline \multirow[t]{2}{*}{ Breast cancer symptoms } & Younger patients & 22.59 & 5.69 & -2.86 & $0.005^{\star \star}$ \\
\hline & Middle-age patients & 25.67 & 4.26 & & \\
\hline \multirow[t]{2}{*}{ FACT-G } & Younger patients & 72.36 & 15.50 & -3.12 & $0.001^{\star *}$ \\
\hline & Middle-age patients & 82.21 & 11.75 & & \\
\hline \multirow[t]{2}{*}{ FACT-B } & Younger patients & 95.03 & 19.35 & -3.17 & $0.002^{\star \star}$ \\
\hline & Middle-age patients & 107.75 & 13.46 & & \\
\hline
\end{tabular}

${ }^{*} p<0.05,{ }^{* *} p<0.01,{ }^{* * *} p<0.001$.

breast cancer symptoms such as body appearance, social support and interaction. These results were also confirmed when the total scores of the scale were considered. No differences existed between the two groups for relational mutuality and dyadic coping style (Table 4).

When the two groups of partners were compared on the variables of interest, the results of the independent samples $t$-test indicated that the younger group scored higher on maladaptive dyadic coping styles, presented lower mean scores of stress communication, common, and positive dyadic coping, and worse quality of life than the middle-aged partners (Table 5). Significant differences were found between the two groups of partners for hostile dyadic coping $(p<0.01)$, emotional well-being $(p<0.01)$ and illness intrusiveness $(p<0.001)$. From the present analysis it is possible to affirm that younger partners experienced the illness to be more intrusive in their life, had worse emotional wellbeing, and were more likely to perceive their dyadic coping as characterized by disinterest or minimizing the seriousness of the partner's stress than those reported by middle-age partners.

\section{Actor and Partner Effects of Relational Mutuality on Dyadic Coping in Younger and Middle-Aged Couples}

Patients and partners' mean-centered scores on relational mutuality were regressed on the outcome variable in a single regression model to examine whether self-reported levels of relational mutuality of patients and partners predicted the individual's engagement in different dyadic coping styles, by conducting separate APIM analyses in the two groups for each style of coping. To facilitate a clear understanding of the analysis and avoid confusion with the partner effect, partners will be identified with the term "caregivers" in the present paragraph. Prior to the analysis, the actor and partner scores were grandmean centered and the variable role was coded as 1 for patients, and -1 for caregivers. An Intraclass Correlation Coefficient was calculated to assess non-independence between the scores of the partners. Then, an Omnibus Test of Distinguishability was conducted to assess whether treating the dyad as distinguishable improved the fit of the model. We then tested whether role acted as a moderator of actor and/or partner effects. Hence, an interaction model using REML estimation was tested first, followed by a two-intercept approach. Power estimates were obtained using $G^{*}$ Power 3.1.9.2., and the results of the power analysis were favorable. Results indicate an average effect size of 0.164 for the younger group and 0.118 for the middleaged group, with a sample of 35 and 51 dyads, p-value of 0.05 , and 0.80 power.

\section{Stress Communication}

In both groups of couples there is evidence of an actor effect of relational mutuality on stress communication $(p<0.001$ for 
TABLE 5 | Independent samples t-test comparing dyadic coping, relational mutuality, and quality of life among Younger and Middle-Age partners.

\begin{tabular}{|c|c|c|c|c|c|}
\hline Variable & Age group & $M$ & SD & $t$ & $p$ \\
\hline \multirow[t]{2}{*}{ Stress communication } & Younger partners & 3.37 & 0.70 & -0.75 & 0.45 \\
\hline & Middle-age partners & 3.49 & 0.77 & & \\
\hline \multirow[t]{2}{*}{ Common dyadic coping } & Younger partners & 3.51 & 0.52 & -0.93 & 0.33 \\
\hline & Middle-age partners & 3.63 & 0.69 & & \\
\hline \multirow[t]{2}{*}{ Positive dyadic coping } & Younger partners & 3.75 & 0.54 & -1.41 & 0.18 \\
\hline & Middle-age partners & 3.94 & 0.71 & & \\
\hline \multirow[t]{2}{*}{ Hostile dyadic coping } & Younger partners & 2.13 & 0.45 & 3.16 & $0.002^{\star \star}$ \\
\hline & Middle-age partners & 1.80 & 0.47 & & \\
\hline \multirow[t]{2}{*}{ Avoidance of dyadic coping } & Younger partners & 2.69 & 0.75 & 1.14 & 0.25 \\
\hline & Middle-age partners & 2.51 & 0.68 & & \\
\hline \multirow[t]{2}{*}{ Relational mutuality } & Younger partners & 4.45 & 0.48 & -0.97 & 0.33 \\
\hline & Middle-age partners & 4.56 & 0.55 & & \\
\hline \multirow[t]{2}{*}{ Emotional well-being } & Younger partners & 58.83 & 12.91 & -3.45 & $0.001^{\star *}$ \\
\hline & Middle-age partners & 68.47 & 12.58 & & \\
\hline \multirow[t]{2}{*}{ Illness intrusiveness } & Younger partners & 43.06 & 14.12 & 3.75 & $<0.001^{\star \star \star}$ \\
\hline & Middle-age partners & 31.71 & 13.56 & & \\
\hline
\end{tabular}

${ }^{\star} p<0.05,{ }^{* *} p<0.01,{ }^{* * *} p<0.001$.

younger couples; $p<0.05$ for middle age dyads) Individuals reporting high levels of mutuality were more likely to engage in stress communication behaviors. From our results there was no evidence that having a partner who reported higher mutuality was associated with the person's use of this coping strategy. Furthermore, mean level differences were predicted for stress communication of patients and informal caregivers, with younger and middle-age patients reporting more frequent use of this coping strategy than their respective partners/spouses.

\section{Common Dyadic Coping}

Among younger dyads, the analysis revealed the presence of actor and partner effects of relational mutuality on common dyadic coping. No significant interaction of role by actor or partner effects occurred. Younger patients and caregivers reporting high levels of mutuality were more likely to engage in common dyadic coping behaviors $(\beta=0.66, p<0.001)$. There was also evidence that having a partner who scores high on mutuality was associated with the person's use of common coping strategies $(\beta=0.24, p<0.05)$. On the contrary, among middle-aged dyads an actor effect was found $(\beta=0.63, p<0.001)$, indicating that high scores on mutuality were associated with an increase in their own dyadic coping score. The interaction role by actor effect approached significance $(p=0.055)$ (see Figure 1).

\section{Positive Dyadic Coping}

For younger dyads, an actor effect of relational mutuality on positive dyadic coping was identified ( $\beta=0.79, p<0.001)$. Younger individuals reporting high levels of mutuality were more likely to engage in positive dyadic coping behaviors. Furthermore, among younger couples, significant role differences were found, with patients predicted to report higher scores $(p<0.01)$. Among couples in the middle-age group there was evidence of an actor effect $(p<0.001)$ and that gender was a significant moderator of the actor effect for women and caregivers $(p<0.05)$. To examine the actor by role interaction for men and women separately, simple slopes were calculated. Results indicated that both patients and caregivers actor effects were significant and that the actor effect was greater for patients (patients: $\beta=0.82, p<0.001$; caregivers: $\beta=0.48, p<0.001$ ).

\section{Hostile Dyadic Coping}

Actor and partner effects were reported for the younger couples, with significant interactions of role by partner effects. Younger individuals reporting high levels of relational mutuality were less likely to report hostile dyadic coping $(\beta=-0.95, p<0.001)$. To test whether the partner effect differed by patients and caregivers, simple slopes were calculated. The caregiver partner effect was not significant $(p=0.70)$. In contrast, for patients, higher mutuality scores of the caregiver were associated with an increase in hostile dyadic coping scores $(\beta=0.56, p<0.05)$. The interaction role by actor effect approached significance $(p=0.06)$. Among middle-aged dyads evidence existed for both an actor and partner effects. Patients and caregivers presenting higher levels of mutuality were more likely to report lower levels of hostile dyadic coping $(\beta=-0.26, p<0.001)$. Similarly, having a partner reporting high scores on mutuality was associated with reduced hostile coping $(\beta=-0.18, p<0.05)$. No evidence for differences of actor and partner effects by role was identified in this group of couples (see Figure 2).

\section{Avoidance of Dyadic Coping}

Among younger couples, an actor effect was detected $(\beta=-0.72$, $p<0.001)$. Present findings indicate that as relational mutuality increases, this was associated with reduced scores on avoidance of dyadic coping in younger participants, with no interaction of actor effect by role. For middle-aged couples both actor $(\beta=-0.32, p<0.001)$ and partner effects $(\beta=-0.020, p<0.05)$ 


\section{A Younger Couples}

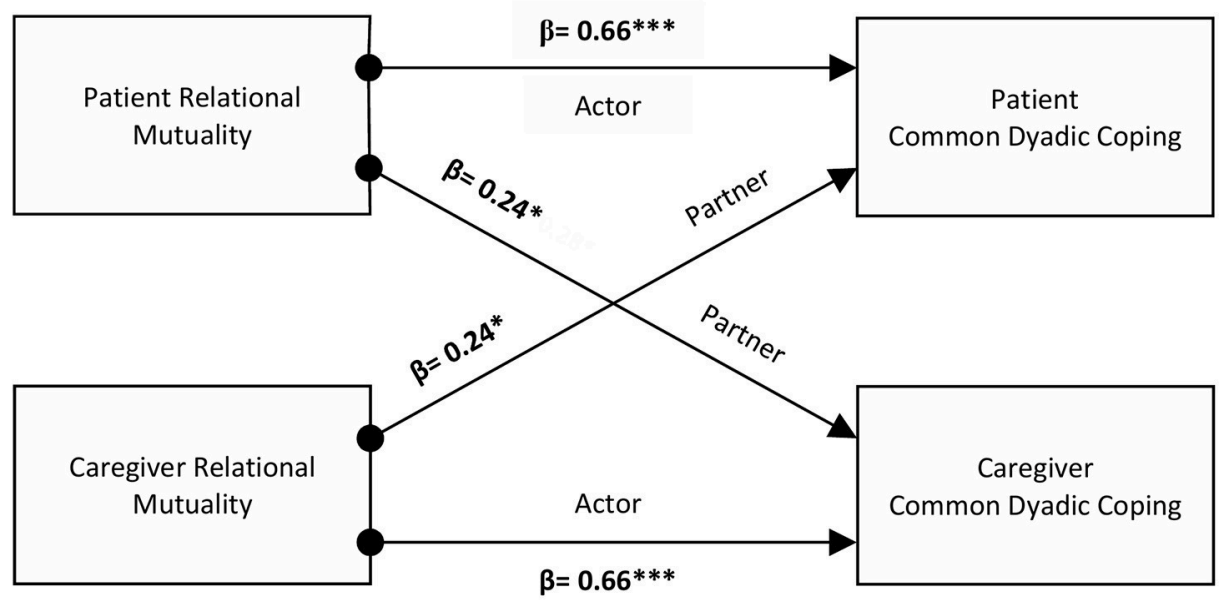

B Middle-Aged Couples

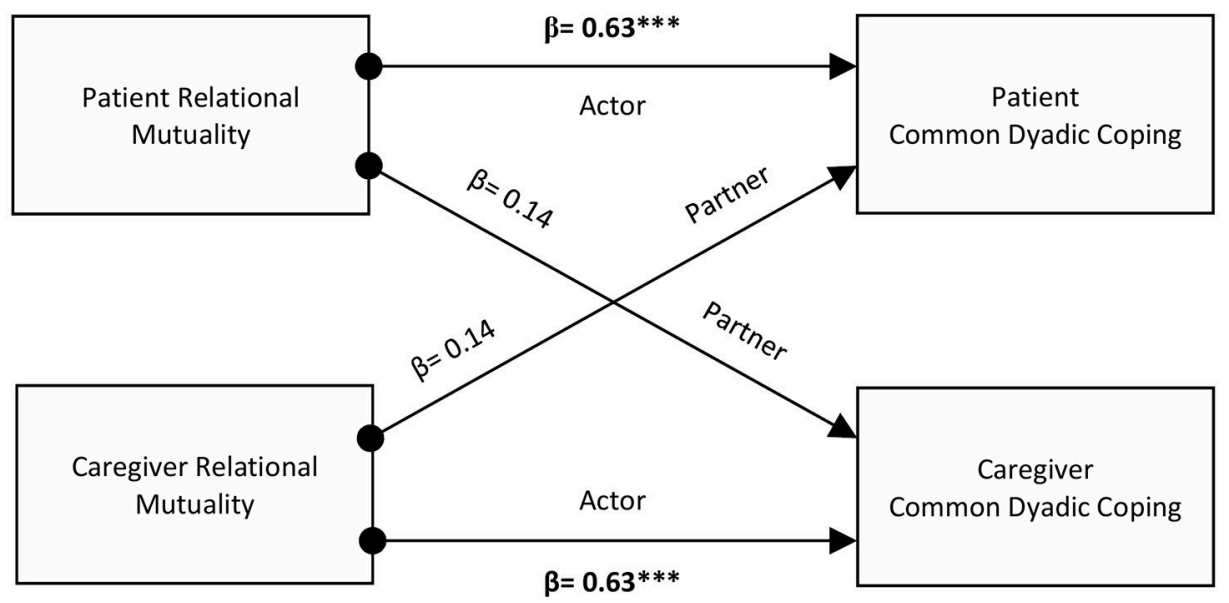

FIGURE 1 | The actor and partner effects of relational mutuality as predictors of common dyadic coping in younger and middle-aged couples. ${ }^{\star} p<0.05,{ }^{\star \star} p<0.01$, ${ }^{* * *} p<0.001$.

of relational mutuality were identified. Higher self-reported scores on mutuality were associated with reduced avoidance of dyadic coping. Similarly, having a partner scoring high on mutuality was associated to lower scores on this coping style.

\section{DISCUSSION}

In the last decade there has been an increasing recognition that younger women with breast cancer represent a separate group among all women diagnosed with the disease because of unique clinical and psychosocial issues. Among the most relevant problems reported by younger women, there is an increased concern for their relationship with the partner. However, despite the evidence that has identified more difficulties and challenges for young couples, a limited number of contributions has investigated the experience of younger women in the context of their close relationships. The present study examined younger couples' coping with breast cancer by comparing them to a group of middle-aged dyads. Results of this study confirm the differential impact of the illness on quality of life and coping responses of younger and middle-aged couples. Younger patients and their partners' adjustment to cancer appears to be significantly compromised within the first 3 months from diagnosis, with impaired functioning and worse quality of life. Younger women in this sample experienced elevated side effects of treatment and more difficult adjustment to the illness. These findings are consistent, despite the small sample size, with results from larger studies on the quality of life of younger women with breast cancer (Avis et al., 2004, 2005; Kroenke et al., 2004; Baucom et al., 2005; Luutonen et al., 2011; Howard-Anderson et al., 2012). In particular, when the same instrument (FACT-B) has been administered to younger patients, other authors have 


\section{A Younger Couples}

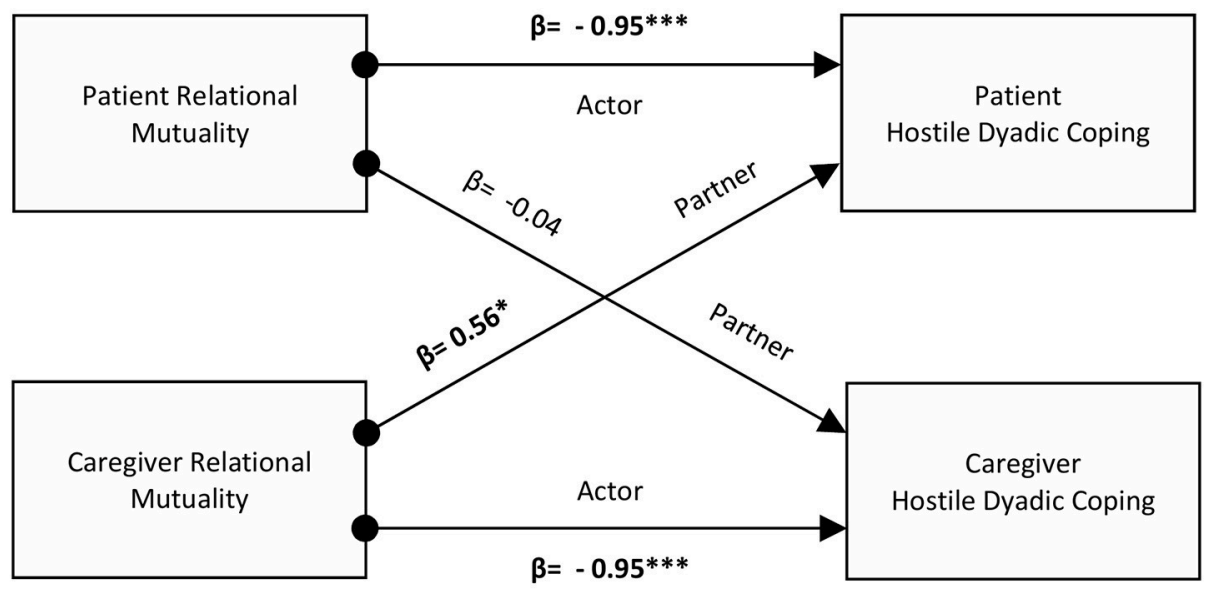

\section{B Middle-Aged Couples}

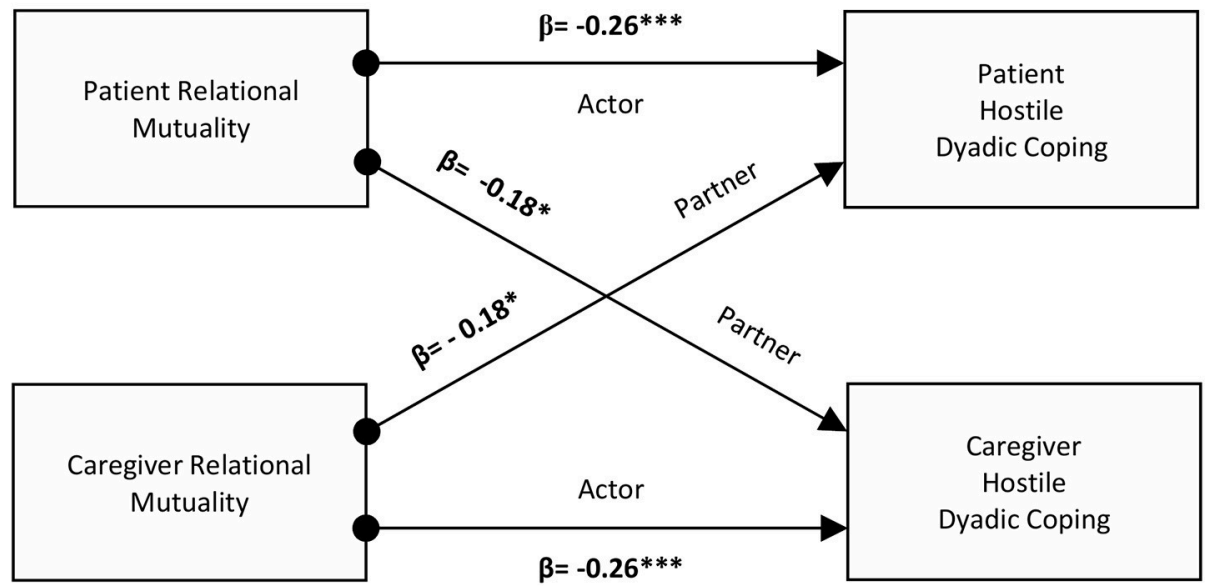

FIGURE 2 | The actor and partner effects of relational mutuality as predictors of hostile dyadic coping in younger and middle-aged couples. ${ }^{\star} p<0.05$, ${ }^{\star \star} p<0.01$, ${ }^{\star * *} p<0.001$.

identified the presence of significant differences between younger and middle-aged breast cancer patients' overall quality of life (Avis et al., 2005; DiSipio et al., 2008; Brennan et al., 2014), with more negative physical well-being, emotional well-being, and breast cancer symptoms which are consistent with our results (Park et al., 2011; So et al., 2011).

Similarly, younger partners were more negatively impacted by the diagnosis and the illness in their quality of life, with statistically significant higher intrusiveness, lower emotional well-being, and a higher use of hostile dyadic coping compared to partners of middle-aged breast cancer patients. These results are similar to those of studies that found a more detrimental effect of the diagnosis on the quality of life of younger partners, who are faced with concerns about everyday life, negative affectivity, apprehension about the future and the couple relationship (Antoine et al., 2012; Vanlemmens et al., 2012a,b; Duggleby et al.,
2014; Hasson-Ohayon et al., 2014; Christophe et al., 2015a,b; Fergus et al., 2015; Borstelmann et al., 2017). Finally, the higher score on illness intrusiveness is a finding that is consistent with the literature about cancer caregiving, which has identified higher burden, mood disturbance, and worse quality of life for those who assume this role at a younger age (Kim et al., 2012; Sjolander et al., 2012; Lee et al., 2013; Shahi et al., 2014).

The use of dyadic data analysis furthered our understanding of the role of relational mutuality among couples coping with breast cancer. Separate APIM models on younger and middleaged dyads revealed the interaction between patients' and caregivers' mutuality scores and how they are associated to different coping behaviors. Among middle-aged dyads relational mutuality was associated with reduced hostile and avoidance of dyadic coping, suggesting that these couples present mutual emotional responsiveness and this ability contributes to reduced 
coping behaviors that may compromise the relationship. At the same time, the study presents evidence about the relational exchange that characterizes young couples facing cancer. In our sample, younger dyads presented elevated interdependence as evidenced by the fact that both adaptive and maladative dyadic coping strategies were the result of patients' and partners' perceived mutuality. The most interesting differences between the two groups pertain to common and hostile dyadic coping. Higher scores for common dyadic coping existed for the younger group as a consequence of actor and partner effects, suggesting that higher scores for this coping style are the result of the individual's self-reported scores, as well as the score of the partner. The results obtained for hostile dyadic coping and mutuality seem to suggest that younger couples may be vulnerable to situations where partners are not able to equally exchange thoughts, feelings, and actions (Jordan, 1997b). In contrast, among middle-aged couples, relational mutuality was associated to lower hostile dyadic coping for both. This finding for the younger group was unexpected and it can be potentially explained as a reverse causation due to the cross-sectional nature of the study. It is also possible to hypothesize that this result indicates the need for interventions aimed at promoting more beneficial relational exchanges in younger dyads and to enhance communication strategies that facilitate the beneficial disclosure of feelings. While it is not possible to elaborate more on this finding at this time, overall our results support the need for greater attention to the adjustment of couples facing cancer earlier in their relationship.

Some implications emerge from these findings. First, our results support the role of a relationship characteristic like relational mutuality in the enactment of dyadic coping behaviors, as it has been demonstrated in previous studies (Kayser, 2005; Kayser et al., 2007, 2010; Kayser and Scott, 2008). This result echoes the literature that identifies relationship characteristics as antecedents of dyadic coping (Staff et al., 2017): stability (Bodenmann and Cina, 2005), satisfaction (Bodenmann et al., 2008; Berg et al., 2011; Landis et al., 2014; Ruffieux et al., 2014), and quality of the relationship (Bodenmann, 1997; Wise et al., 2010; Bergstraesser et al., 2015) have been found to promote coordinated forms of coping with stressors (Staff et al., 2017). Second, our study highlighted how, despite no significant differences were found between the dyadic coping scores of younger and middle-aged patients and partners (with the exception of Hostile Dyadic Coping in partners), dyadic coping responses were associated with different patterns of reciprocal influence among the two members of the dyad. Both positive and negative dyadic coping behaviors in the younger group resulted from the scores of the partners on relational mutuality. While the role of the two partners' mutuality may be more salient for younger dyads, the ability to communicate effectively their mutual empathic responsiveness may be limited in this group, while middle-aged couples implement more coordinated patterns of communication. This consideration is supported by studies where couples in longer relationships were better able to use dyadic coping styles than partners in shorter relationships (Wunderer and Schneewind, 2008; Papp and Witt, 2010; Herzberg, 2013; Staff et al., 2017). This differential ability may be also the result of developmental processes across the lifespan (Berg and Upchurch, 2007). Better competence in the ability to regulate emotions and appraisal of stress is also reported in older individuals, which tend to show greater mutuality and less maladaptive coping (Folkman et al., 1987; Aldwin, 1994; Diehl et al., 1996; Labouvie-Vief, 2003). The second developmental aspect is the temporal process of dyadic coping. Studies have highlighted the changing nature of dyadic coping over time, especially in the case of an illness (Fang et al., 2001; Martire et al., 2002; Helgeson et al., 2004; Schulz and Schwarzer, 2004). Again, age-related differences have been identified because younger individuals report greater distress and reduced ability to perform collaborative coping (Helgeson et al., 2004; Revenson and Pranikoff, 2005). In contrast, older adults become better able to cope effectively (Revenson, 2003). These findings support the need to develop interventions aimed at promoting not only the couples' ability to cope together with the illness, but also to enhance communication strategies that facilitate the beneficial disclosure of feelings, thoughts, and emotions between the two partners.

The study presents several limitations, such as small sample size, homogeneous sample composition, and cross-sectional design. The sample size affects the generalization of results to other groups, and it was not possible to elaborate on differences between participants and non-participants and potential selection biases. A more properly powered sample is needed to investigate the differential impact of breast cancer among patients and partners across the developmental trajectory. Although difficulties in the recruitment of couples for research are well-established by the literature (Kenny et al., 2006; Fredman et al., 2009; Regan et al., 2013; Hagedoorn et al., 2015), the limited sample and the use of different instruments to measure quality of life among partners impacted the possibility to test more complex models of dyadic data analysis. Furthermore, in the current study the identification of "younger" and "middle-aged" dyads was derived only by the age of the patient at diagnosis. Future studies can benefit from a more in-depth exploration of the role of developmental stages, duration of relationship, and cohort effects (Baucom et al., 2012; Revenson and Lepore, 2012). The sample was largely homogeneous in regards to race, socio-economic status, sexual orientation, and education. This project used a cross-sectional design; hence, it is not possible to elaborate whether the difference between the younger and middle-aged couples' coping persist over time. A newer version of the instrument measuring dyadic coping has been introduced (Dyadic Coping Inventory, Bodenmann, 2008) and it has been validated for the use in the US population as this study was completed (Levesque et al., 2014a; Randall et al., 2016). The inclusion of this new instrument in future research projects is recommended.

\section{IMPLICATIONS FOR PRACTICE}

Findings from the present study suggest the need for a renewed attention to the psychosocial issues of patients and partners (Institute of Medicine, 2008, 2013). Psychosocial providers and healthcare professionals need to develop greater understanding 
of how to work effectively with younger patients and their significant ones, and to promote their ability to find support within the health care system. The identification of unique needs and stressors requires the utilization of screening and assessment strategies able to capture the life experience of younger women and their well-being, in order to promote effective and timely referrals. Screening and assessment should be inclusive of the significant other in order to identify individuals who may have difficulties coping with the patient's diagnosis and its demands.

Since younger patients' and their partners' relational mutuality was associated to dyadic coping, psychosocial interventions should address the dyad as a unit of intervention. Over the last two decades, several couple-based interventions have been developed and tested in RCTs (Baik and Adams, 2011; Regan et al., 2012; Badr and Krebs, 2013). Despite these efforts, more investigation is warranted to evaluate their effectiveness and application in practice settings. Programs aimed at supporting younger dyads coping with breast cancer should assist participants identifying their relationships' qualities, positive and negative coping patterns, and their impact on both individuals' quality of life. Through this experience younger couples should be enabled to communicate effectively with each other and to establish new coping repertoires (Skerrett and Fergus, 2015). It is, however, necessary to adapt existing protocols to target topics that are relevant for couples in the early years of their relationship, marriage or for cohabitating couples (Ponzetti, 2016). Additional topics to address as part of these interventions could include: social relations with families of origin and the extensive supportive network, financial planning, intimacy and sexual functioning, fertility preservation, transition to parenthood or strategies to cope with cancer-related infertility. While preliminary data indicate beneficial changes in communication, closeness, and relationship strengths (Fergus et al., 2014, 2015), it will be important to further investigate factors associated with positive results, timing of the intervention, and the mechanism for therapeutic change (Revenson and DeLongis, 2011; Revenson and Lepore, 2012).

\section{CONCLUSION}

The present study contributes to the understanding of the experience of younger couples coping with breast cancer.
This work has highlighted the more negative effect of the illness on the quality of life of the two partners. From this investigation it emerges that patients and partners' relational mutuality scores influence the dyadic coping strategies enacted to respond to the stress of the illness. Among younger couples it appears that both positive and maladaptive outcomes in terms of couple's coping are the results of patients' and partners' mutuality. It follows that both members of the dyad have an essential role in the development of coping strategies that will promote better adjustment to the disease and the preservation of their relationship. Hence, these findings contribute to the current theoretical reflection about the process of dyadic coping and its association to individual and relational outcomes (Levesque et al., 2014b; Regan et al., 2015; Traa et al., 2015; Staff et al., 2017). Future studies, both cross-sectional and longitudinal, are needed to examine the differential impact cancer has on couples across the developmental trajectory and to provide confirmation to these results. Building on larger samples, this research will lead to better understand sources of stress and relational difficulties experienced by younger dyads, which will be critical in developing couple-based interventions to promote their coordinated coping efforts.

\section{AUTHOR CONTRIBUTIONS}

CA contributed to the study design, statistical analysis, and to the writing of the manuscript's introduction, materials and methods, results, discussion, and references sections. KK contributed to the study design, data collection, and to the writing of the introduction, results and discussion sections. All authors reviewed and approved manuscript for publication.

\section{FUNDING}

The original research study from which this secondary data analysis was conducted was supported by a research grant from the Massachusetts Department of Public Health Breast Cancer Research Program. CA was supported by an American Cancer Society Doctoral Training Grant in Oncology Social Work (DSWR-15-091-03-SW).

\section{REFERENCES}

Ahmad, S., Fergus, K., and McCarthy, M. (2015). Psychosocial issues experienced by young women with breast cancer: the minority group with the majority of need. Curr. Opin. Support Palliat. Care 9, 271-278. doi: 10.1097/SPC.0000000000000162

Aldwin, C. M. (1994). Stress, Coping, and Development: An Integrative Perspective. New York, NY: Guilford.

Amato, P. R., and Hohmann-Marriott, B. (2007). A comparison of high- and low-distress marriages that end in divorce. J. Marriage Fam. 69, 621-638. doi: 10.1111/j.1741-3737.2007.00396.x

American Psychological Association (2015). Stress in America. Paying With Our health. Available online at: https://www.apa.org/news/press/releases/stress/ 2014/stress-report.pdf

Antoine, P., Vanlemmens, L., Fournier, E., Trocme, M., and Christophe, V. (2012). Young couples' experiences of breast cancer during hormone therapy. a phenomenological interpretative dyadic analysis. Cancer Nurs. 36, 213-220. doi: 10.1097/NCC.0b013e3182 $6429 \mathrm{a} 5$

Avis, N. E., Crawford, S., and Manuel, J. (2004). Psychosocial problems among younger women with breast cancer. Psychooncology 13, 295-308. doi: 10.1002/pon.744

Avis, N. E., Crawford, S., and Manuel, J. (2005). Quality of life among younger women with breast cancer. J. Clin. Oncol. 23, 3322-3330. doi: 10.1200/JCO.2005.05.130

Badr, H., Carmack, C. L., Kashy, D. A., Cristofanilli, M., and Revenson, T.A. (2010). Dyadic coping in metastatic breast cancer. Health Psychol. 29, 169-180. doi: $10.1037 / \mathrm{a} 0018165$ 
Badr, H., and Krebs, P. (2013). A systematic review and meta-analysis of psychosocial interventions for couples coping with cancer. Psychooncology 22, 1688-1704. doi: 10.1002/pon. 3200

Badr, H., and Taylor, L. C. (2008). Effects of relationship maintenance on psychological distress and dyadic adjustment among couples coping with lung cancer. Health Psychol. 27, 616-627. doi: 10.1037/0278-6133. 27.5.616

Baik, O. M., and Adams, K. B. (2011). Improving the well-being of couples facing cancer: a review of couples based psychosocial interventions. J. Marital Fam. Ther. 37, 250-266. doi: 10.1111/j.1752-0606.2010. 00217.x

Bantema-Joppe, E. J., de Bock, G. H., Woltman-van Iersel, M., Busz, D. M., Ranchor, A. V., Langendijk, J. A., et al. (2015). The impact of age on changes in quality of life among breast cancer survivors treated with breast-conserving surgery and radiotherapy. Br. J. Cancer 112, 636-643. doi: 10.1038/bjc. 2014.632

Baucom, D. H., Kirby, J. S., Pukay-Martin, N. D., Porter, L. S., Fredman, S. J., Gremore, T. M., et al. (2012). Men's psychological functioning in the context of women's breast cancer. J. Marital Fam. Ther. 38, 317-329. doi: 10.1111/j.1752-0606.2009.00133.x

Baucom, D. H., Porter, L. S., Kirby, J. S., Gremore, T. M., and Keefe, F. J. (2005). Psychosocial issues confronting young women with breast cancer. Breast Dis. 23, 103-113. doi: 10.3233/BD-2006-23114

Berg, C. A., and Upchurch, R. (2007). A developmental-contextual model of couples coping with chronic illness across the adult life span. Psychol. Bull. 133, 920-954. doi: 10.1037/0033-2909.133.6.920

Berg, C. A., Wiebe, D. J., and Butner, J. (2011). Affect covariation in marital couples dealing with stressors surrounding prostate cancer. Gerontology 57, 167-172. doi: $10.1159 / 000318642$

Bergstraesser, E., Inglin, S., Hornung, R., and Landolt, M. A. (2015). Dyadic coping of parents after the death of a child. Death Stud. 39, 128-138. doi: 10.1080/07481187.2014.920434

Binik, Y. M., Chowanec, G. D., and Devins, G. M. (1990). Marital role strain, illness intrusiveness, and their impact on marital and individual adjustment in end-stage renal disease. Psychol. Health 4, 245-257. doi: 10.1080/08870449008400394

Blanchard-Fields, F., and Coats, A. H. (2008). The experience of anger and sadness in everyday problems impacts age differences in emotion regulation. Dev. Psychol. 44, 1547-1556. doi: 10.1037/a0013915

Bodenmann, G. (1997). Dyadic coping: a systemic-transactional view of stress and coping among couples: theory and empirical findings. Eur. Rev. Appl. Psychol. 47, 137-140.

Bodenmann, G. (2000). Stress und Coping bei Paaren [Stress and Coping in Couples]. Göttingen: Hogrefe.

Bodenmann, G. (2005). "Dyadic coping and its significance for marital functioning," in Couples Coping with Stress: Emerging Perspectives on Dyadic Coping, eds. T. A. Revenson, K. Kayser, and G. Bodenmann (Washington, DC: American Psychological Association), 33-49.

Bodenmann, G. (2008). Dyadisches Coping Inventar (DCI) [Dyadic Coping Inventory]. Bern: Huber.

Bodenmann, G., Atkins, D. C., Schär, M., and Poffet, V. (2010). The association between daily stress and sexual activity. J. Fam. Psychol. 24, 271-279. doi: $10.1037 / \mathrm{a} 0019365$

Bodenmann, G., Charvoz, L., Widmer, K., and Bradbury, T. N. (2004). Differences in individual and dyadic coping among low and high depressed, partially remitted, and nondepressed persons. J. Psychopathol. Behav. Assess. 26, 75-85. doi: 10.1023/B:JOBA.0000013655.45146.47

Bodenmann, G., and Cina, A. (2005). Stress and coping among stablesatisfied, stable-distressed and separated/divorced Swiss couples: a 5years prospective longitudinal study. J. Divorce Remarriage 44, 71-89. doi: 10.1300/J087v44n01_04

Bodenmann, G., Pihet, S., and Kayser, K. (2006). The relationship between dyadic coping and marital quality: a 2-year longitudinal study. J. Fam. Psychol. 20, 485-493. doi: 10.1037/0893-3200.20.3.485

Bodenmann, G., Plancherel, B., Beach, S. R., Widmer, K., Gabriel, B., Meuwly, N., et al. (2008). Effects of coping-oriented couples therapy on depression: a randomized clinical trial. J. Consult. Clin. Psychol. 76, 944-954. doi: $10.1037 / \mathrm{a} 0013467$
Bodenmann, G., Randall, A. K., and Falconier, M. K. (2016). "Coping in couples: the Systemic transactional Model (STM)," in Couples Coping With Stress: A Cross-Cultural Perspective, eds. M. K. Falconier, A. K. Randall, and G. Bodenmann (New York, NY: Routledge), 2-22.

Borstelmann, N., Shoshana, M., Rosenberg, S. M., Shari, I., Gelber, S. I., Meyer, M. E., et al. (2017). Partners of young breast cancer survivors: a cross-sectional evaluation of psychosocial issues and mental health. J. Clin. Oncol. 35 (Suppl. 5S):184. doi: 10.1200/JCO.2017.35.5_suppl.184

Borstelmann, N. A., Rosenberg, S. M., Ruddy, K. J., Tamimi, R. M., Gelber, S., Schapira, L., et al. (2015). Partner support and anxiety in young women with breast cancer. Psychooncology 24, 1679-1685. doi: 10.1002/ pon. 3780

Bradbury, T. N., and Karney, B. R. (2014). Intimate Relationships. New York, NY: WW Norton and Company.

Brady, M. J., Cella, D. F., Mo, F., Bonomi, A. E., Tulsky, D. S., Lloyd, S. R., et al. (1997). Reliability and validity of the functional assessment of cancer therapy-breast quality-of-life instrument. J. Clin. Oncol. 15, 974-986. doi: 10.1200/JCO.1997.15.3.974

Brennan, M., Gormally, J., Butow, P., Boyle, F., and Spillane, A. (2014). Survivorship care plans in cancer: a systematic review of care plan outcomes. Br. J. Cancer 111, 1899-1908. doi: 10.1038/bjc.2014.505

Carstensen, L. L., Fung, H., and Charles, S. (2003). Socioemotional selectivity theory and the regulation of emotion in the second half of life. Motiv. Emot. 27, 103-123. doi: 10.1023/A:1024569803230

Carstensen, L. L., Turan, B., Scheibe, S., Ram, N., Ersner-Hershfield, H., SamanezLarkin, G. R., et al. (2011). Emotional experience improves with age: evidence based on over 10 years of experience sampling. Psychol. Aging 26, 21-33. doi: $10.1037 / \mathrm{a} 0021285$

Cataldo, J. K., Paul, S., Cooper, B., Skerman, H., Alexander, K., Aouizerat, B., et al. (2013). Differences in the symptom experience of older versus younger oncology outpatients: a cross-sectional study. BMC Cancer 13:6. doi: 10.1186/1471-2407-13-6

Cella, D. F., Tulsky, D. S., Gray, G., Sarafian, B., Lloyd, S., Linn, E., et al. (1993). The Functional Assessment of Cancer Therapy (FACT) scale: development and validation of the general measure. J. Clin. Oncol. 11, 570-579. doi: 10.1200/JCO.1993.11.3.570

Champion, V. L., Wagner, L. I., Monahan, P. O., Daggy, J., Smith, L., Cohee, A., et al. (2014). Comparison of younger and older breast cancer survivors and agematched controls on specific and overall quality of life. Cancer 120, 2237-2246. doi: $10.1002 / \mathrm{cncr} .28737$

Christophe, V., Duprez, C., Congard, A., Antoine, P., Lesur, A., Fournier, E., et al. (2015a). The subjective experience of young women with non-metastatic breast cancer: the young women with breast cancer inventory. Health Qual. Life Outcomes 13:73. doi: 10.1186/s12955-015-0273-x

Christophe, V., Duprez, C., Congard, A., Fournier, E., Lesur, A., Antoine, P., et al. (2015b). Evaluate the subjective experience of the disease and its treatment in partners of young women with non-metastatic breast cancer. Eur. J. Cancer Care 25, 734-743. doi: 10.1111/ecc.12327

Devins, G. M. (1994). Illness intrusiveness and the psychosocial impact of lifestyle disruptions in chronic life-threatening disease. Adv. Ren. Replace. Ther. 1, 251-263. doi: 10.1016/S1073-4449(12)80007-0

Devins, G. M. (2010). Using the illness intrusiveness ratings scale to understand health-related quality of life in chronic disease. J. Psychosom. Res. 68, 591-602. doi: 10.1016/j.jpsychores.2009.05.006

Devins, G. M., Binik, Y. M., Hutchinson, T. A., Hollomby, D. J., Barré, P. E., and Guttmann, R. D. (1983). The emotional impact of end-stage renal disease: importance of patients' perceptions of intrusiveness and control. Int. J. Psychiatry Med. 13, 327-343. doi: 10.2190/5DCP-25BVU1G9-9G7C

Devins, G. M., Mandin, H., Hons, R. B., Burgess, E. D., Klassen, J., Taub, K., et al. (1990). Illness intrusiveness and quality-of-life in end-stage renal disease: comparison and stability across treatment modalities. Health Psychol. 9, 117-142. doi: 10.1037/0278-6133.9.2.117

Diehl, M., Coyle, N., and Labouvie-Vief, G. (1996). Age and sex differences in strategies of coping and defense across the life span. Psychol. Aging. 11, 127-139. doi: 10.1037/0882-7974.11.1.127

DiSipio, T., Hayes, S., Newman, B., and Janda, M. (2008). Health-related quality of life 18 months after breast cancer: comparison with the general 
population of Queensland, Australia. Support. Care Cancer. 16, 1141-1150. doi: 10.1007/s00520-007-0392-y

Donato, S. (2012). Il coping diadico, ovvero far fronte allo stress insieme: una rassegna della letteratura [dyadic coping, that is managing stress together: a review of the literature]. Giornale Italiano di Psicologia, 41, 473-504. doi: $10.1421 / 78499$

Duggleby, W., Doell, H., Cooper, D., Thomas, R., and Ghosh, S. (2014). The quality of life of male spouses of women with breast cancer: hope, self-efficacy, and perceptions of guilt. Cancer Nurs. 37, E28-E35. doi: 10.1097/NCC.0b013e31827ca807

Ebbesen, L. S., Guyatt, G. H., MCCartney, N., and Oldridge, N. B. (1990). Measuring quality of life in cardiac spouses. J. Clin. Epidemiol. 43, 481-487. doi: 10.1016/0895-4356(90)90137-E

Falconier, M. K., Jackson, J. B., Hilpert, P., and Bodenmann, G. (2015). Dyadic coping and relationship satisfaction: a meta-analysis. Clin. Psychol. Rev. 42, 28-46. doi: 10.1016/j.cpr.2015.07.002

Fang, C. Y., Manne, S. L., and Pape, S. J. (2001). Functional impairment, marital quality, and patient psychological distress as predictors of psychological distress among cancer patients' spouses. Health Psychol. 20, 452-457. doi: 10.1037/0278-6133.20.6.452

Feldman, B. N., and Broussard, C. A. (2005). The influence of relational factors on men's adjustment to their partners' newly diagnosed breast cancer. J. Psych. Oncol. 23, 23-43. doi: 10.1300/J077v23n02_03

Feldman, B. N., and Broussard, C. A. (2006). Men's adjustment to their partners' breast cancer: a dyadic coping perspective. Health Soc. Work 31, 117-127. doi: $10.1093 / \mathrm{hsw} / 31.2 .117$

Fergus, K., Ahmad, S., McLeod, D. L., Stephen, J., Gardner, S., Pereira, A., et al. (2015). Couplelinks- an online intervention for young women with breast cancer and their male partner: study protocol for a randomized controlled trial. Trials 16:33. doi: 10.1186/s13063-014-0534-8

Fergus, K. D., and Gray, R. E. (2009). Relationship vulnerabilities during breast cancer: patient and partner perspectives. Psychooncology 18, 1311-1322. doi: $10.1002 /$ pon. 1555

Fergus, K. D., McLeod, D., Carter, W., Warner, E., Gardner, S., Granek, L., et al. (2014). Development and pilot testing of an online intervention to support young couples' coping and adjustment to breast cancer. Eur. J. Cancer Care 23, 481-492. doi: 10.1111/ecc.12162

Folkman, S., Lazarus, R. S., Pimley, S., and Novacek, J. (1987). Age differences in stress and coping processes. Psychol. Aging 2, 171-184. doi: 10.1037/0882-7974.2.2.171

Fredman, S. J., Baucom, D. H., Gremore, T. M., Castellani, A. M., Kallman, T. A., Porter, L. S., et al. (2009). Quantifying the recruitment challenges with couple-based interventions for cancer: applications to early-stage breast cancer. Psychooncology 18,667-673. doi: 10.1002/pon.1477

Genero, N. P., Baker Miller, J., Surrey, J., and Baldwin, L. M. (1992). Measuring perceived mutuality in close relationships: validation of the mutual psychological development questionnaire. J. Fam. Psychol. 6, 36-48. doi: 10.1037/0893-3200.6.1.36

Gold, M., Dunn, L. B., Phoenix, B., Paul, S. M., Hamolsky, D., Levine, J. D., et al. (2015). Co-occurrence of anxiety and depressive symptoms following breast cancer surgery and its impact on quality of life. Eur. J. Oncol. Nurs. 20, 97-105. doi: 10.1016/j.ejon.2015.06.003

Hagedoorn, M., Dagan, M., Puterman, E., Hoff, C., Meijerink, W. J., Delongis, A., et al. (2011a). Relationship satisfaction in couples confronted with colorectal cancer: the interplay of past and current spousal support. J. Behav. Med. 34, 288-297. doi: 10.1007/s10865-010-9311-7

Hagedoorn, M., Hein, F. L., Schulz, T., van der Heide, J. J. H., Niesing, J., Westerhuis, R., et al. (2015). Are patient and relationship variables associated with participation of intimate partners in couples research? Health Psychol. 34, 270-273. doi: 10.1037/hea0000141

Hagedoorn, M., Puterman, E., Sanderman, R., Wiggers, T., Baas, P. C., van Haastert, M., et al. (2011b). Is self-disclosure in couples coping with cancer associated improvement in depressive symptoms? Health Psychol. 30, 753-762. doi: $10.1037 / \mathrm{a} 0024374$

Haid, M. L., and Seiffge-Krenke, I. (2012). Effects of (un)employment on young couples' health and life satisfaction. Psychol. Health 28, 284-301. doi: 10.1080/08870446.2012.720983
Halliday Hardie, J., and Lucas, A. (2010). Economic factors and relationship quality among young couples: comparing cohabitation and marriage. J. Marriage Fam. 72, 1141-1154. doi: 10.1111/j.1741-3737.2010.00755.x

Hartl, K., Schennach, R., Muller, M., Engel, J., Reinecker, H., Sommer, H., et al. (2010). Quality of life, anxiety, and oncological factors: a follow-up study of breast cancer patients. Psychosomatics 51, 112-123. doi: 10.1176/appi.psy.51.2.112

Hasson-Ohayon, I., Goldzweig, G., Dorfman, C., and Uziely, B. (2014). Hope and social support utilisation among different age groups of women with breast cancer and their spouses. Psychol. Health 29, 1303-1319. doi: 10.1080/08870446.2014.929686

Hau, E., Browne, L., Capp, A., Delaney, G. P., Fox, C., Kearsley, J. H., et al. (2013). The impact of breast cosmetic and functional outcomes on quality of life: long-term results from the St. George and Wollongong randomized breast boost trial. Breast Cancer Res. Treat. 139, 115-123. doi: 10.1007/s10549-0132508-z

Helgeson, V. S., Snyder, P., and Seltman, H. (2004). Psychological and physical adjustment to breast cancer over 4 years: identifying distinct trajectories of change. Health Psychol. 23, 3-15. doi: 10.1037/0278-6133.23.1.3

Herzberg, P. Y. (2013). Coping in relationships: the interplay between individual and dyadic coping and their effects on relationship satisfaction. Anxiety Stress Coping 26, 136-153. doi: 10.1080/10615806.2012.655726

Hinnen, C., Hagedoorn, M., Ranchor, A. V., and Sanderman, R. (2008a). Relationship satisfaction in women: a longitudinal case-control study abpout the role of breast cancer, personal assertiveness, and partners' relationship-focused coping. Br. J. Health Psychol. 13, 737-754. doi: 10.1348/135910707X252431

Hinnen, C., Ranchor, A. V., Sanderman, R., Snijders, T. A., Hagedoorn, M., and Coyne, J. C. (2008b). Course of distress in breast cancer patients, their partners, and matched control couples. Ann. Behav. Med. 36, 141-148. doi: 10.1007/s12160-008-9061-8

Hoppmann, C. A., Coats, A. H., and Blanchard-Fields, F. (2008). Goals and everyday problem solving: examining the link between age-related goals and problem-solving strategy use. neuropsychology, development, and cognition. Neuropsychol. Dev. Cogn. B Aging Neuropsychol. Cogn. 15, 401-423. doi: 10.1080/13825580701533777

Howard-Anderson, J., Ganz, P. A., Bower, J. E., and Stanton, A. L. (2012). Quality of life, fertility concerns, and behavioral health outcomes in younger breast cancer survivors: a systematic review. J. Natl. Cancer Inst. 104, 386-405. doi: 10.1093/jnci/djr541

Hulvat, M. C., and Jeruss, J. S. (2009). Maintaining fertility in young women with breast cancer. Curr. Treat. Options Oncol. 10, 308-317. doi: 10.1007/s11864-010-0116-2

Iafrate, R., Bertoni, A., Donato, S., and Finkenauer, C. (2012). Perceived similarity and understanding in dyadic coping among young and mature couples. Pers. Relatsh. 19, 401-419. doi: 10.1111/j.1475-6811.2011.01369.x

Iafrate, R., and Donato, S. (2012). "Coping in a relational context: the case of dyadic coping," in Handbook of the Psychology of Coping: New Research, eds. B. Molinelli and V. Grimaldo (Hauppauge, NY: Nova Science Publishers), 11-132.

Institute of Medicine (2008). Cancer Care for the Whole Patient: Meeting Psychosocial Health Needs. Washington, DC: The National Academies Press.

Institute of Medicine (2013). Delivering High Quality Cancer Care. Charting a New Course For a System in Crisis. Washington, DC: The National Academies Press.

Jordan, J. (1997a). "The relational model is a source of empowerment for women," in Women, Men, and Gender: Ongoing Debates, ed. M. R. Walsh (New Haven, CT: Yale), 373-382.

Jordan, J. (1997b). Women's Growth in Diversity. New York, NY: Gilford Press.

Jordan, J. V., Kaplan, A. G., Miller, J. B., Stiver, I. P., and Surrey, J. L. (1991). Women's Growth in Connection: Writings from the Stone Center. New York, NY: Guilford Press.

Jordan, V. J. (2009). Relational-Cultural Therapy. Washington, DC: American Psychological Association.

Kayser, K. (2005). "Enhancing dyadic coping during a time of crisis: a theory-based intervention with breast cacner patients and their partners," in Couples Coping with Stress, eds. T. A. Revenson, K. Kayser, and G. Bodenmann (Washington, DC: American Psychological Association), 175-194. 
Kayser, K., Feldman, B. N., Borstelmann, N. A., and Daniels, A. A. (2010). Effects of a randomized couple-based intervention on quality of life of breast cancer patients and their partners. Soc. Work Res. 34, 20-32. doi: 10.1093/swr/34.1.20

Kayser, K., and Scott, J. L. (2008). Helping Couples Cope With Women's Cancers. An Evidence-Based Approach for Practitioners. New York, NY: Springer.

Kayser, K., Sormanti, M., and Strainchamps, E. (1999). Women coping with cancer: the influence of relationship factors on psychosocial adjustment. Psychol. Women Q 23, 725-739. doi: 10.1111/j.1471-6402.1999. tb00394.x

Kayser, K., Watson, E., and Andrade, J. T. (2007). Cancer as a we-disease: examining the process of coping from a relational perspective. Fam. Syst. Health 25, 404-418. doi: 10.1037/1091-7527.25.4.404

Kenny, D. A., Kashy, D. A., and Cook, W. L. (2006). Dyadic Data Analysis. New York, NY: The Guilford Press.

Kim, Y., Spillers, R. L., and Hall, D. L. (2012). Quality of life of family caregivers 5 years after a relative's cancer diagnosis: follow-up of the national quality of life survey for caregivers. Psychooncology 21, 273-281. doi: 10.1002/ pon. 1888

Kroenke, C. H., Rosner, B., Chen, W. Y., Kawachi, I., Colditz, G. A., and Holmes, M. D. (2004). Functional impact of breast cancer by age at diagnosis. J. Clin. Oncol. 22, 1849-1856. doi: 10.1200/JCO.2004.04.173

Labouvie-Vief, G. (2003). Dynamic integration: affect, cognition, and the self in adulthood. Curr. Dir. Psychol. Sci. 12, 201-206. doi: 10.1046/j.0963-7214.2003.01262.x

Landis, M., Bodenmann, G., Bradbury, T. N., Brandstätter, V., Peter-Wight, M., Backes, S., et al. (2014). Commitment and dyadic coping in longterm relationships. J. Gerontopsychol. Geriatr. Psychiatry 27, 139-149. doi: $10.1024 / 1662-9647 / \mathrm{a} 000112$

Langer, S. L., Brown, J. D., and Syrjala, K. L. (2009). Intrapersonal and interpersonal consequences of protective buffering among cancer patients and caregivers. Cancer 115, 4311-4325. doi: 10.1002/cncr.24586

Lavery, J. F., and Clarke, V. A. (1999). Prostate cancer: patients' and spouses' coping and marital adjustment. Psychol. Health Med. 4, 279-302. doi: 10.1080/135485099106225

Lavner, J. A., and Bradbury, T. N. (2010). Patterns of change in newlyweds' marital satisfaction. J. Marriage Fam. 72, 1171-1187. doi: 10.1111/j.1741-3737.2010.00757.x

Lavner, J. A., Bradbury, T. N., and Karney, B. R. (2012). Incremental change or initial differences? testing two models of marital deterioration. J. Fam. Psychol. 26, 606-616. doi: 10.1037/a0029052

Lavner, J. A., Karney, B. R., and Bradbury, T. N. (2013). Newlyweds' optimistic forecasts of their marriage: for better or for worse? J. Fam. Psychol. 27, 531-540. doi: $10.1037 / \mathrm{a} 0033423$

Lee, Y. H., Liao, Y. C., Liao, W. Y., Shun, S. C., Liu, Y. C., Chan, J. C., et al. (2013). Anxiety, depression and related factors in family caregivers of newly diagnosed lung cancer patients before first treatment. Psychooncology 22, 2617-2623. doi: 10.1002/pon. 3328

Levesque, C., Lafointaine, M., Caron, A., Flesch, J. L., and Bjornson, S. (2014b). Dyadic empathy, dyadic coping, and relationship satisfaction: a dyadic model. Eur. J. Psychol. 10, 118-134. doi: 10.5964/ejop.v10i1.697

Levesque, C., Lafontaine, M.-F., Caron, A., and Fitzpatrick, J. (2014a). Validation of the english version of the dyadic coping inventory. Meas. Eval. Couns. Dev. 47, 215-225. doi: 10.1177/0748175614522272

Luong, G., Charles, S. T., and Fingerman, K. L. (2011). Better with age: Social relationships across adulthood. J. Soc. Pers. Relat. 28, 9-23. doi: $10.1177 / 0265407510391362$

Luutonen, S., Vahlberg, T., Eloranta, S., Hyvari, H., and Salminen, E. (2011). Breast cancer patients receiving postoperative radiotherapy: Distress, depressive, symptoms and unmet needs of psychosocial support. Radiother. Oncol. 100, 299-303. doi: 10.1016/j.radonc.2011.01.014

Manne, S. L., Kissane, D., Zaider, T., Kashy, D., Lee, D., Heckman, C., et al. (2015). Holding back, intimacy, and psychological and relationship outcomes among couples coping with prostate cancer. J. Fam. Psychol. 29, 708-719. doi: 10.1037/fam0000096

Martire, L. M., Stephens, M. A. P., Druley, J. A., and Wojno, W. C. (2002). Negative reactions to received spousal care: predictors and consequences of miscarried support. Health Psychol. 21, 167-176. doi: 10.1037/0278-6133. 21.2.167
Miller, J. B. (1984). The Development of Women's Sense of Self. Work in Progress No. 12. Wellesley, MA: Stone Center Working Papers Series.

Miller, J. B. (1986). Toward a New Psychology of Women. Boston, MA: Beacon Press.

National Institute of Aging (2015). Health and Aging: Menopause. Available online at: https://www.nia.nih.gov/health/publication/menopause

Neff, L. A., and Broady, E. F. (2011). Stress resilience in early marriage: can practice make perfect? J. Pers. Soc. Psychol. 101, 1050-1067. doi: 10.1037/a0023809

Norton, T. R., and Manne, S. L. (2007). Support concordance among couples coping with cancer: relationship, individual, and situational factors. J. Soc. Pers. Relat. 24, 675-692. doi: 10.1177/0265407507081454

Overcash, J., Extermann, M., Parr, J., Perry, J., and Balducci, L. (2001). Validity and reliability of the FACT-G scale for use in the older person with cancer. Am. J. Clin. Oncol. 24, 591-596. doi: 10.1097/00000421-200112000-00013

Papp, L. M., and Witt, N. L. (2010). Romantic partners' individual coping strategies and dyadic coping: implications for relationship functioning. J. Fam. Psychol. 24, 551-559. doi: 10.1037/a0020836

Park, B., Lee, S., Lee, A. R., Lee, K. H., and Hwang, S. Y. (2011). Quality of life differences between younger and older breast cancer patients. J. Breast Cancer 14, 112-118. doi: 10.4048/jbc.2011.14.2.112

Pasipanodya, E. C., Parrish, B. P., Laurenceau, J. P., Cohen, L. H., Siegel, S. D., Graber, E. C., et al. (2012). Social constraints on disclosure predict daily wellbeing in couples coping with early-stage breast cancer. J. Fam. Psychol. 26, 661-667. doi: 10.1037/a0028655

Peterson, G. W., and Bush, K. R. (2013). Handbook of Marriage and the Family, 3rd Edn. New York, NY: Springer. doi: 10.1007/978-1-4614-3987-5

Picard, L., Dumont, S., Gagnon, P., and Lessard, G. (2005). Coping strategies among couples adjusting to primary breast cancer. J. Psychosoc. Oncol. 23, 115-135. doi: 10.1300/J077v23n02_08

Ponzetti, J. J. Jr. (2016). Evidence-Based Approaches to Relationship and Marriage Education. New York, NY: Routledge.

Randall, A. K., and Bodenmann, G. (2009). The role of stress on close relationships and marital satisfaction. Clin. Psychol. Rev. 29, 105-115. doi: 10.1016/j.cpr.2008.10.004

Randall, A. K., Hilpert, P., Jimenez-Arista, L. E., Walsh, K. J., and Bodenmann, G. (2016). Dyadic coping in the U.S.: psychometric properties and validity for use of the english version of the dyadic coping inventory. Curr. Psychol. 35, 570-582. doi: 10.1007/s12144-015-9323-0

Regan, T., Lambert, S. D., and Kelly, B. (2013). Uptake and attrition in couplebased interventions for cancer: perspectives from the literature. Psychooncology 22, 2639-2647. doi: 10.1002/pon.3342

Regan, T. W., Lambert, S. D., Girgis, A., Kelly, B., Kayser, K., and Turner, J. (2012). Do couple-based interventions make a difference for couples affected by cancer? a systematic review. BMC Cancer 12:279. doi: 10.1186/1471-2407-12-279

Regan, T. W., Lambert, S. D., Kelly, B., Falconier, M., Kissane, D., and Levesque, J. V. (2015). Couples coping with cancer: exploration of theoretical framewoks from dyadic studies. Psychooncology 24, 1605-1617. doi: 10.1002/ pon. 3854

Revenson, T., and Lepore, S. J. (2012). "Coping in Social Context." in Handbook of Health Psychology, 2nd Edn, eds. A. Baum, T. A. Revenson, and J. Singer (New York, NY: Taylor and Francis Group), 193-217.

Revenson, T. A. (1994). Social support and marital coping with chronic illness. Ann. Behav. Med. 16, 122-130.

Revenson, T. A. (2003). "Scenes from a marriage: Examining supportand marital coping with chronic illness," in Social Psychological Foundations of Health and Illness, eds. J. Suls and K. Wallston (Oxford: Blackwell Publishing), 530-559.

Revenson, T. A., and DeLongis, A. (2011). "Couples coping with Chronic Illness," in The Oxford Handbook of Stress, Health and Coping, ed. S. Folkman (New York, NY: Oxford University Press), 101-123.

Revenson, T. A., Kayser, K., and Bodenmann, G. (2005). Couples Coping With Stress: Emerging Perspectives on Dyadic Coping. Washington, DC: American Psychological Association.

Revenson, T. A., and Pranikoff, J. R. (2005). A contextual approach to treatment decision making among breast cancer survivors. Health Psychol. 24, S93-S98. doi: 10.1037/0278-6133.24.4.S93

Ruddy, K. J., Greaney, M. L., Sprunck-Harrild, K., Meyer, M. E., Emmons, K. M., and Partridge, A. H. (2013). Young women with breast cancer: a focus 
group study of unmet needs. J. Adolesc. Young Adult Oncol. 2, 153-160. doi: 10.1089/jayao.2013.0014

Ruffieux, M., Nussbeck, F. W., and Bodenmann, G. (2014). Long-term prediction of relationship satisfaction and stability by stress, coping, communication, and well-being. J. Divorce Remarriage 55, 485-501. doi: 10.1080/10502556.2014.931767

Saita, E. (2009). Psico-Oncologia: Una Prospettiva Relazionale. Milano: Edizioni Unicopli.

Scabini, E., and Rossi, G. (2012). Family Transitions and Families in Transitions. Milano: VitaandPensiero.

Schulz, U., and Schwarzer, R. (2004). "Partnerschaftliche Bewältigung einer Krebserkrankung [dyadic coping with cancer]," in Stress Gemeinsam Bewältigen, eds. P. Buchwald, S. E. Hobfoll and C. Schwarzer (Göttingen: Hogrefe), 121-138.

Scott, J. L., Halford, W. K., and Ward, B. G. (2004). United we stand? the effects of a couple-coping intervention on adjustment to early stage breast or gynaecological cancer. J. Consult. Clin. Psychol. 72, 1122-1135. doi: 10.1037/0022-006X.72.6.1122

Scott, J. L., and Kayser, K. (2009). A review of couple-based interventions for enhancing women's sexual adjustment and body image after cancer. Cancer 15, 48-56. doi: 10.1097/PPO.0b013e31819585df

Shahi, V., Lapid, M. I., Kung, S., Atherton, P. J., Sloan, J. A., Clark, M. M., et al. (2014). Do age and quality of life of patients with cancer influence quality of life of the caregiver? J. Geriatr. Oncol. 5, 331-336. doi: 10.1016/j.jgo.2014.03.003

Sjolander, C., Rolander, B., Järhult, J., Mårtensson, J., and Ahlstrom, G. (2012). Health-related quality of life in family members of patients with an advanced cancer diagnosis: a one-year prospective study. Health Qual. Life Outcomes 10:89. doi: 10.1186/1477-7525-10-89

Skerrett, K., and Fergus, K. (2015). Couple Resilience. New York, NY: Springer.

So, W. K., Choi, K. C., Chan, C. W., and Chair, S. Y. (2011). Age-related differences in the quality of life of Chinese women undergoing adjuvant therapy for breast cancer. Res. Gerontol. Nurs. 4, 19-26. doi: 10.3928/1940492120101201-01

Staff, H. R., Didymus, F. F., and Backhouse, S. H. (2017): The antecedents and outcomes of dyadic coping in close personal relationships: a systematic review and narrative synthesis, Anxiety Stress Coping 30, 498-520. doi: 10.1080/10615806.2017.1329931

Stava, C. J., Lopez, A., and Vassilopoulou-Sellin, R. (2006). Health profiles of younger and older breast cancer survivors. Cancer 107, 1752-1759. doi: $10.1002 /$ cncr.22200

Sullivan, K. T., Pasch, L. A., Johnson, M. D., and Bradbury, T. N. (2010). Social support, problem solving, and the longitudinal course of newlywed marriage. J. Pers. Soc. Psychol. 98, 631-644. doi: 10.1037/a0017578

Traa, M. J., De Vries, J., Bodenmann, G., and Den Oudsten, B. L. (2015). Dyadic coping and relationship functioning in couples coping with cancer: a systematic review. Br. J. Health Psychol. 20, 85-114. doi: 10.1111/bjhp.12094

van Steenbergen, E. F., Kluwer, E. S., and Karney, B. R. (2011). Workload and the trajectory of marital satisfaction in newlyweds: job satisfaction, gender, and parental status as moderators. J. Fam. Psychol. 25, 345-355. doi: $10.1037 /$ a0023653

Vanlemmens, L., Christophe, V., Fournier, E., Dauchy, S., Boinon, D., ToudicEmily, F., et al. (2012a). The quality of life of young women with nonmetastatic braest cancer and their partners': specific needs require development of scientific questionnaires for each of them. Breast J. 18, 182-184. doi: 10.1111/j.1524-4741.2011.01218.x

Vanlemmens, L., Fournier, E., Boinon, D., Machavoine, J. L., and Christophe, V. (2012b). Quality of life of young women with early breast cancer and their partners: specific needs result in the necessity of development of specific questionnaires for the patients and the partner. Bull. Cancer 99, 685-691. doi: 10.1684/bdc.2012.1598

Vedes, A., Nussbeck, F., and Bodenmann, G. (2015). “A life cycle perspective on stress, dyadic coping and couples' well-being," in Paper presented at the $9 y \mathrm{~h}$ Workshopkongress für Klinische Psychologie und Psychoterapie/33. Symposim der Fachgruppe Klinische Psychologie und Psychotherapie der DGPs (Dresden).

Vilchinsky, N., Liat Haze-Filderman, L. H., Morton Leibowitz, M., Reges, O., Khaskia, A., and Mosseri, M. (2010). Spousal support and cardiac patients' distress: the moderating role of attachment orientation. J. Fam. Psychol. 24, 508-512. doi: 10.1037/a0020009

Walsh, S. R., Manuel, J. C., and Avis, N. E. (2005). The impact of breast cancer on younger women's relationships with their partner and children. Fam. Syst. Health 23, 80-93. doi: 10.1037/1091-7527.23.1.80

Webster, K., Cella, D., and Yost, K. (2003). The Functional Assessment of Chronic Illness Therapy (FACIT) measurement system: properties, applications, and interpretation. Health Qual. Life Outcomes 1:79. doi: 10.1186/1477-7525-1-79

Webster, K., Odom, L., Peterman, A., Lent, L., and Cella, D. (1999). The Functional Assessment of Chronic Illness Therapy (FACIT) measurement system: validation of version 4 of the core questionnaire. Qual. Life Res. 8:604. doi: 10.1186/1477-7525-1-79.

Williamson, H. C., Karney, B. R., and Bradbury, T. N. (2013). Financial strain and stressful events predict newlyweds' negative communication independent of relationship satisfaction. J. Fam. Psychol. 27, 65-75. doi: 10.1037/a0031104

Winstead-Fry, P., and Schultz, A. (1997). Psychometric assessment of the Functional Assessment of Cancer Therapy-General (FACT-G) scale in a rural sample. Cancer 79, 2446-2452.

Wise, M., Schatell, D., Klicko, K., Burdan, A., and Showers, M. (2010). Successful daily home hemodialysis patient-care partner dyads: benefits outweigh burdens. Hemodial. Int. 14, 278-288. doi: 10.1111/j.1542-4758.2010. 00443.x

Woszidlo, A., and Segrin, C. (2013). Negative affectivity and educational attainment as predictors of newlyweds' problem solving communication and marital quality. J. Psychol. 147, 49-73. doi: 10.1080/00223980.2012. 674069

Wunderer, E., and Schneewind, K. A. (2008). The relationship between marital standards, dyadic coping and marital satisfaction. Eur. J. Soc. Psychol. 38, 462-476. doi: 10.1002/ejsp.405

Conflict of Interest Statement: The authors declare that the research was conducted in absence of any commercial or financial relationships that could be construed as a potential conflict of interest.

Copyright (c) 2019 Acquati and Kayser. This is an open-access article distributed under the terms of the Creative Commons Attribution License (CC BY). The use, distribution or reproduction in other forums is permitted, provided the original author(s) and the copyright owner(s) are credited and that the original publication in this journal is cited, in accordance with accepted academic practice. No use, distribution or reproduction is permitted which does not comply with these terms. 


\section{OPEN ACCESS}

Edited by:

Guy Bodenmann,

Universität Zürich, Switzerland

Reviewed by:

Andreas Dietz,

Leipzig University, Germany

Ariela Francesca Pagani,

Università Cattolica del Sacro Cuore,

Italy

${ }^{*}$ Correspondence: Hoda Badr

hoda.badr@bcm.edu

Specialty section:

This article was submitted to Clinical and Health Psychology, a section of the journal

Frontiers in Psychology

Received: 05 June 2018 Accepted: 03 September 2018

Published: 15 October 2018

Citation:

Badr H, Herbert K, Bonnen MD,

Asper JA and Wagner T (2018)

Dyadic Coping in Patients Undergoing Radiotherapy for Head and Neck

Cancer and Their Spouses.

Front. Psychol. 9:1780

doi: 10.3389/fpsyg.2018.01780

\section{Dyadic Coping in Patients Undergoing Radiotherapy for Head and Neck Cancer and Their Spouses}

\author{
Hoda Badr ${ }^{1 *}$, Krista Herbert ${ }^{2}$, Mark D. Bonnen ${ }^{3}$, Joshua A. Asper ${ }^{3}$ and Timothy Wagner ${ }^{3}$ \\ ${ }^{1}$ Department of Medicine, Baylor College of Medicine, Houston, TX, United States, ${ }^{2}$ Department of Psychology, Rowan \\ University, Glassboro, NJ, United States, ${ }^{3}$ Department of Radiation Oncology, Baylor College of Medicine, Houston, TX, \\ United States
}

Background: Head and neck cancer (HNC) adversely affects the psychological (i.e., depression, anxiety) and marital adjustment of patients and their spouses. Dyadic coping refers to how couples cope with stress. It includes positive actions like sharing practical or emotional concerns (i.e., problem- and emotion-focused stress communication; PFSC, EFSC), and engaging in problem- or emotion-focused actions to support each other (problem- and emotion-focused dyadic coping; PFDC, EFDC). It also includes negative actions like avoidance (negative dyadic coping; NEGDC). In this secondary analysis of a randomized pilot trial of a couple-based intervention called SHARE (Spouses coping with the Head And neck Radiation Experience), we first examined associations between patients' and spouses' dyadic coping (and satisfaction with dyadic coping; SATDC) and their own/each other's psychological and marital adjustment. Next, we examined the effects of SHARE relative to usual medical care (UMC) on patients' and spouses' dyadic coping. Finally, we examined whether changes in dyadic coping were associated with changes in patients' and spouses' psychological and marital adjustment.

Methods and Measures: Thirty HNC patients ( $80 \%$ men) and their spouses ( $N=60)$ completed baseline surveys prior to initiating radiotherapy (RT) and were randomized to SHARE or UMC. One month after RT, they completed follow-up surveys.

Results: Baseline multilevel Actor-Partner Interdependence Models revealed significant actor effects of PFSC (effect size $r=-0.32)$ and PFDC $(r=-0.29)$ on depression. For marital adjustment, significant actor effects were found for PFSC, PFDC, EFDC, and SATDC ( $p<0.05, r=0.23$ to 0.38). Actor $(r=-0.35)$ and partner effects $(r=-0.27)$ for NEGDC were also significant. Moderate to large effect sizes were found in favor of SHARE on PFSC (Cohen's $d=1.14)$, PFDC $(d=0.64)$, NEGDC $(d=-0.68)$, and SATDC $(d=1.03)$. Improvements in PFDC were associated with reductions in depression and anxiety $(p<0.05)$; and, improvements in SATDC were associated with improvements in anxiety and marital adjustment $(\rho<0.05)$. 


\begin{abstract}
Conclusion: The SHARE intervention improved positive and decreased negative dyadic coping for patients and spouses. Increases in positive dyadic coping were also associated with improvements in psychological and marital adjustment. Although findings are preliminary, more research on ways to integrate dyadic coping into oncology supportive care interventions appears warranted.
\end{abstract}

Keywords: head and neck cancer, couples, caregiving, dyadic coping, radiotherapy, psychosocial intervention, depression, dyadic adjustment

\section{INTRODUCTION}

Head and neck cancers (HNCs) are malignancies of the larynx, pharynx, nasopharynx, and oral cavity. They account for approximately 560,000 new cases worldwide and $3 \%$ of cancer cases in the United States (Siegel et al., 2017). An analysis of Surveillance, Epidemiology, and End Results (SEER) data found that being married reduced the risk of dying from $\mathrm{HNC}$ by $33 \%$ (hazard ratio $[\mathrm{HR}]=0.67$; Aizer et al., 2013). Married patients also have significantly better performance status scores during HNC treatment than their unmarried counterparts (Konski et al., 2006). One possibility is that spouses (i.e., husbands/wives or significant others) often serve as primary caregivers and provide support and care to facilitate patient adherence to medical recommendations (Family Caregiver Alliance [FCA], 2012).

Radiation therapy (RT) is a common treatment modality for the management of HNC. Patients undergo RT for 5 days a week for 6-7 weeks. RT is either administered alone or it is combined with other treatments (e.g., chemotherapy or surgery; National Comprehensive Cancer Network [NCCN], 2013). Given the dosage of radiation required to successfully treat HNC tumors and the sensitivity of the location that is targeted, patients experience side effects (e.g., mucositis, xerostomia) and functional challenges (e.g., dysphagia) that make eating, drinking, and communicating extremely difficult (Epstein et al., 2001). Their quality of life (QOL) is also adversely affected due to psychological distress, rapid weight loss, dehydration, and malnutrition (Epstein et al., 2001; Massie, 2004; Duffy et al., 2006). Psychoeducational interventions can improve QOL and enhance coping with cancer (Faller et al., 2013); however, few programs have been developed specifically for HNC patients (Semple et al., 2013).

Even though being married can be beneficial for HNC patients, cancer exacts a heavy toll on spouses. Spouses serving in a caregiving role experience higher rates of distress (i.e., anxiety and depression), weakened immune responses, a greater likelihood of long-term medical problems, and higher mortality rates than their non-caregiving counterparts (Applebaum and Breitbart, 2013). In HNC, spouse distress rates are comparable to or higher than those of patients (Hodges et al., 2005; Verdonck-de Leeuw et al., 2007). Spouses also report unmet needs during the critical period when patients are undergoing RT including help with balancing competing roles/responsibilities, making time for self-care, and finding effective strategies for encouraging patient self-management (Badr et al., 2016). Addressing spouse distress is important in its own right. Moreover, distressed spouses may be unable to provide adequate caregiving and support to the patient during RT.

Cancer also challenges couples' established communication patterns, roles, and responsibilities (Manne and Badr, 2010). Whereas some individuals report that cancer improved their relationships, others experience adjustment and communication challenges that fuel interpersonal conflict and can even lead to divorce (Karraker and Latham, 2015; Badr et al., 2016). In HNC, declines in marital adjustment have been reported 1 year after treatment (Gritz et al., 1999). This is concerning because the quality of marital interaction is related to both psychological adaptation and health outcomes in cancer (Burman and Margolin, 1992).

One aspect of cancer that may be particularly challenging to negotiate for couples coping with HNC is self-care/selfmanagement during RT. Self-management refers to daily activities that minimize the impact of illness on functioning and well-being (Clark et al., 1991). HNC involves considerable self-management during and after RT. For example, patients are instructed to significantly alter their diets to prevent malnutrition, sip or spray the mouth regularly with water to prevent dehydration, use salt-soda rinses 8-10 times a day and saliva substitutes to control xerostomia, practice multiple daily repetitions of exercises to facilitate return to a normal swallow, and engage in intensive oral care routines to control mucositis and prevent dental carries (Jansma et al., 1992; Nguyen et al., 2007). Unfortunately, rates of non-adherence are high - up to $72 \%$ of patients are non-adherent with oral care recommendations and $87 \%$ are non-adherent with swallowing exercises (Epstein et al., 1995; Shinn et al., 2013). From a medical perspective, poorly managed side effects can lead to treatment interruptions, social/emotional problems, and a more complicated and costly rehabilitation process (Nitenberg and Raynard, 2000; Trotti et al., 2003). From a relationship perspective, patient non-adherence sets the stage for power struggles and conflict between the patient and caregiving spouse (McGuire, 2003). Indeed, a recent study found that $83 \%$ of HNC spouses reported increased marital conflict during RT (Badr et al., 2016). Another found that $54 \%$ of 125 HNC couples identified side effect management as a topic of considerable concern or contention (Badr et al., 2015). Thus, in order to maximize QOL and health outcomes, it is imperative to address both patient and spouse self-management as well as how the couple relates to one another and coordinates care and support during this critical period. 


\section{The SHARE Intervention}

Based on the above, we developed a 6 week telephone-based intervention called SHARE (Spouses coping with the Head And neck Radiation Experience). SHARE actively involves patients and their spouses by: (1) educating both partners about acute and long term side-effects and side-effect management; (2) teaching strategies to improve dyadic coping; and (3) teaching selfmanagement skills that are tailored to each partner's role (as patient or spouse). By teaching couples the skills to coordinate self-management and support at the start of RT, a major goal is to fortify the couple unit and mitigate the potentially adverse effects of RT on both partners' psychological and marital adjustment.

\section{Theoretical Basis}

The SHARE intervention is grounded in self-regulation theory (Cameron and Leventhal, 2012) and Bodenmann's SystemicTransactional Model (STM) of coping with stress (Bodenmann, 2005). Self-regulation theory posits that goal-setting and selfmonitoring improve self-management. However, gaps exist in our understanding of how patients and partners balance autonomy and support, and how they coordinate care while under stress. The STM posits a model of dyadic coping whereby relational partners try to mitigate the adverse impact of stress that affects either one or both partners. Dyadic coping is process consisting of (1) the communication of problem- or emotionfocused stress by partner A, (2) the awareness or perception of partner A's stress by partner B, and (3) partner B's coping reaction to partner A's behavior. Dyadic coping includes positive actions like sharing practical or emotional concerns (i.e., problem- and emotion-focused stress communication), supportive actions like helping a partner to engage in positive reframing and problemsolving (problem-focused dyadic coping), and offering empathic understanding (emotion-focused dyadic coping). It also includes negative actions like distancing, blaming, or minimizing the seriousness of a partner's stress (negative dyadic coping). Whereas positive dyadic coping is important for helping couples resolve problems and reduce emotional arousal, negative dyadic coping is considered a maladaptive couples' coping strategy. Finally, satisfaction with dyadic coping refers to each partner's view of how they cope as a couple. Despite research examining dyadic coping in a variety of illness contexts, very little is known about the dyadic coping process in HNC or how one partner's dyadic coping affects the other partner.

Our interest in dyadic coping in the context of RT for HNC stemmed from the idea that illness-specific coping efforts are often most effective at producing positive outcomes when both partners collaborate in illness management (Kuijer et al., 2000; Berg and Upchurch, 2007). Although findings regarding the association between the different forms of dyadic coping and psychological adjustment have been mixed (Badr et al., 2010; Meier et al., 2011; Rottmann et al., 2015), research has consistently demonstrated significant associations with marital adjustment (Falconier et al., 2015; Traa et al., 2015). STM interventions have also resulted in improvements in both psychological and marital adjustment in couples coping with breast and gynecological cancers (Kayser et al., 2010; Heinrichs et al., 2011). Couples who take a dyadic approach to dealing with the challenges of RT from the initiation of treatment may thus benefit in terms of better symptom control and adjustment for the patient, better psychological adjustment for the spouse, and better marital adjustment for the couple.

\section{The Current Study}

In a pilot randomized controlled trial, significant treatment effects (medium in magnitude) were observed for SHARE relative to UMC with regard to HNC-specific physical symptom burden (Cohen's $d=-0.89)$ and symptom interference $(d=-0.86)$. Medium-to-large effects favoring SHARE were also found for patient and spouse depressive symptoms $(d=-0.84)$ and cancerspecific distress $(d=-1.05)$ (Badr et al., unpublished). However, this global analysis did not allow for an examination of whether the dyadic coping skills taught in the intervention were related to improvements in patient and spouse psychological and marital adjustment. Therefore, this paper reports on a secondary analysis that was conducted to examine: (1) associations between patient and spouse reports of their own positive and negative dyadic coping efforts at baseline (prior to randomization) and their own and each other's psychological and marital adjustment; (2) effects of the SHARE intervention on patient and partner engagement in positive and negative dyadic coping relative to usual medical care (UMC); and (3) whether changes in positive and negative dyadic coping are associated with changes in psychological and marital adjustment. Based on the STM and previous research, we hypothesized that engaging in more positive and less negative dyadic coping would be associated with better psychological (i.e., fewer symptoms of depression and anxiety) and marital adjustment for both the person engaging in dyadic coping and that person's partner. We also hypothesized that patients and spouses who received the SHARE intervention would show greater improvements in positive dyadic coping (and greater reductions in negative dyadic coping) than those receiving UMC. Finally, we expected that improvements in positive dyadic coping and reductions in negative dyadic coping from baseline to 1-month follow up would be associated with improvements in psychological and marital adjustment for both HNC patients and their spouses.

\section{MATERIALS AND METHODS}

\section{Procedures}

The study was reviewed and approved by the Baylor College of Medicine Institutional Review Board. Patients were eligible if they (1) were initiating radiation treatment for HNC; (2) were spending more than $50 \%$ of the time out of bed on a daily basis, as measured by an Eastern Cooperative Oncology Group (ECOG) performance status of $<2$; and (3) had a spouse/partner who lived with them. In addition, patients and caregivers had to: (4) be >18 years old; (5) have the ability to speak/read English; and (6) be able to provide informed consent. Patients were identified through medical chart review and approached to participate during a pre-treatment clinic visit. If spouses were not present, permission was obtained to contact them by phone. All eligible couples who were approached were asked to complete a one-page anonymous survey that asked about their 
health (i.e., NCCN distress thermometer, items from the MD Anderson Symptom Inventory; MDASI) and socio-demographic characteristics regardless of whether they agreed to participate. Patients and spouses who provided written informed consent separately completed a baseline survey and either returned it by mail or at their next clinic visit. Couples who returned the questionnaire were randomly assigned to either the 6week SHARE intervention or UMC. Couples in both conditions completed follow-up paper-and-pencil surveys 1 month after RT and received gift cards upon return of each completed survey ( $\$ 10$ for baseline and $\$ 20$ for the one-month follow-up).

\section{Measures Dyadic Coping}

The 37-item Dyadic Coping Inventory (DCI; range $1=$ very rarely to $5=$ very often) assesses stress communication and dyadic coping (Bodenmann, 2008; Randall et al., 2016). Given concerns about participant burden, only 11 items from the DCI assessing stress communication, supportive and negative dyadic coping, and satisfaction with dyadic coping were used. Patients and spouses rated how they communicate when they are feeling stressed because of cancer on a 5-point Likert-type scale ( 1 = very rarely to $5=$ very often). The specific items/sub-scales are below. Mean scores for individual subscales were used in the analysis.

\section{Stress communication}

Two items assessed problem-focused stress communication (PFSC; e.g., I let my partner know that I appreciate his/her practical support, advice, or help), and two items assessed emotion-focused stress communication (EFSC; e.g., I tell my partner openly how I feel and that I would appreciate his/her support). In this study, internal consistency reliability (Cronbach's alpha) for PFSC was $\alpha_{\text {patients }}=0.62$ and $\alpha_{\text {spouses }}=0.65$, and for EFSC it was $\alpha_{\text {patients }}=0.60$ and $\alpha_{\text {spouses }}=0.58$.

\section{Supportive dyadic coping}

Two items assessed problem-focused dyadic coping (PFDC; e.g., I help my partner to see the situation in a different light) and three items assessed emotion-focused dyadic coping (EFDC; e.g., I show empathy and understanding to my partner). Internal consistency reliability (Cronbach's alpha) for PFDC was $\alpha_{\text {patients }}=0.60$ and $\alpha_{\text {spouses }}=0.66$, and for EFDC it was $\alpha_{\text {patients }}=0.75$ and $\alpha_{\text {spouses }}=0.84$.

\section{Negative dyadic coping}

Two items assessed negative dyadic coping (NEGDC; i.e., When my partner is stressed, I tend to withdraw and, I blame my partner for not coping well enough with stress). Internal consistency reliability was $\alpha_{\text {patients }}=0.66$ and $\alpha_{\text {spouses }}=0.62$.

\section{Satisfaction with dyadic coping}

A single item assessed satisfaction with dyadic coping (SATDC; i.e., I am satisfied with the support I receive from my partner and the way we deal with cancer related stress together).

\section{Psychological Adjustment}

Both depression and anxiety symptoms were assessed. The 6item PROMIS short-form depression measure assesses negative mood and views of the self over the past 7 days (Pilkonis et al., 2011). Sample items are, "I felt unhappy" and "I felt worthless." The 6-item PROMIS short-form anxiety measure assesses fear, anxious misery (e.g., worry), and hyperarousal over the same time-frame (Pilkonis et al., 2011). For both measures, responses range from 1 (never) to 5 (always) and are summed to form a raw score that can then be rescaled into a T-score (standardized) with a mean of 50 and standard deviation (SD) of 10 using tables from the PROMIS website. In this study, internal consistency reliability (Cronbach's alpha) for depression was $\alpha_{\text {patients }}=0.83$ and $\alpha_{\text {spouses }}=0.90$, and for anxiety it was $\alpha_{\text {patients }}=0.92$ and $\alpha_{\text {spouses }}=0.90$.

\section{Marital Adjustment}

The 7-item, short version of the Dyadic Adjustment Scale (DAS7) has been found to conserve, without loss of variance, the pattern of relations found between the longer, 32-item DAS and related constructs (Hunsley et al., 2001). Three items ask subjects to report on the extent of agreement/disagreement between partners on various issues (e.g., "time spent together); items are rated from $0=$ always disagree to $6=$ always agree. Three items ask how often various events occur between partners (e.g., "have a stimulating exchange of ideas); items are rated from $0=$ never to $5=$ more often than once a day. Finally, one item asks about the overall degree of happiness in the relationship ( $0=$ extremely unhappy to $6=$ perfect). Items are summed to create a total score ranging from 0 to 36; scores less than 21 indicate marital distress. Internal consistency reliability (Cronbach's alpha) was 0.85 for spouses and 0.74 for patients.

\section{Demographic and Medical Variables}

Patients and spouses reported their age, ethnicity, race, education level, employment status, marital status, and length of relationship. Patients also reported on time since initial diagnosis, disease stage, and comorbidities. Where possible, this data was verified by the patient's electronic medical record.

\section{Study Conditions \\ $U M C$}

UMC consisted of standard oncologic care for the patient (e.g., routine management of physical and psychological symptoms, and basic discussions about prognosis/treatment side effects). Partners were welcome to attend patients' routine clinic and treatment visits but were not required to do so.

\section{SHARE}

In addition to UMC, patients and spouses each received a manual with units covering: (1) self-care, (2) symptom management, (3) stress management, (4) coping with cancer as a team, (5) managing post-treatment recovery together, and (6) finding the new normal together after cancer. Units 1-3 focused on individual skills, with tailoring based on role. For example, patient-specific content included self-care, soliciting support, and balancing accepting help with autonomy. Spouse-specific content included caregiver self-care, caregiving skills (e.g., hygiene care, meal preparation, identifying red flag symptoms), and strategies for supporting patient self-management. Units 4-6 were dyadic in focus, so manual content was the same for both partners. 
In addition to the tailored manuals, patients, and spouses each received an educational CD and DVD that reinforced covered materials (e.g., relaxation and swallowing exercises), and six telephone-sessions corresponding to the units in the manual with an interventionist who had Masters'-level training in mental-health counseling (60-min each). We opted for telephone as opposed to in-person delivery due to research citing low attendance as a barrier to clinic-based program delivery (Ostroff et al., 2004), and research suggesting that telephone delivery is convenient, personal, and preferable to other home-based formats (i.e., videophone, Skype) given the body image and social withdrawal issues that are documented in HNC (Katz et al., 2002).

Intervention sessions were digitally audio-recorded to ensure fidelity. During the sessions, interventionists reviewed homework and manual content, guided participants through in-session activities, and assigned/reviewed homework to reinforce practice of skills taught. Patients and spouses each received separate calls for units 1, 2, and 3. The purpose was threefold: (1) to facilitate rapport between the interventionist and individual members of the couple before moving to the joint sessions; (2) to allow more in-depth coverage of tailored materials; and (3) to provide more time for patients/spouses to practice individual skills and receive feedback before moving to learning dyadic skills. Couples participated in joint calls (sessions 4,5 , and 6) with the interventionist via speaker-phone or three-way call.

The timeline for session delivery was based on the known symptom burden and recovery process for patients undergoing RT for HNC. Because early intervention has been shown to improve treatment tolerance and outcomes (Paccagnella et al., 2010), we delivered the first 4 sessions on a weekly basis starting the first week of RT. The goal was to teach self-care and coping skills before severe symptom onset (which usually occurs during week 4 or 5 of RT). A 4 -week break followed to allow time to apply the skills learned and for patients to recuperate. The last 2 sessions were scheduled following the 4-week break due to their focus on managing long-term side effects and the transition to survivorship.

\section{Data Analysis}

Descriptive statistics (e.g., means, standard deviations) were calculated for each of the major study variables, and paired $t$-tests were conducted to determine whether mean scores differed for patients and spouses at baseline. Associations between the study outcomes and medical (i.e., number of comorbidities, length of time since initial diagnosis, stage at diagnosis (i.e., stage 4 vs. stages 1, 2, and 3 ) and socio-demographic variables (i.e., age, length of relationship, race/ethnicity [i.e., Anglo/white vs. other], employment status [employed full/part time vs. unemployed/retired]) were examined using Pearson's correlations for continuous variables and Analyses of Variance (ANOVAs) or $t$-tests for the categorical/dichotomous variables to determine potential model covariates. Of all the medical and socio-demographic variables that we examined, only age and length of relationship were significantly associated with the study outcomes $(p<0.05)$. However, age and length of relationship were highly significantly correlated $(r=0.73)$. Given the small sample size and desire to conserve degrees of freedom, we opted to only include age as a covariate. Moreover, age was significantly correlated with all 3 study outcomes and length of relationship was only significantly correlated with anxiety.

Because data from married couples tend to be related, analyses must adjust for this non-independence so that statistical significance tests are not biased, and model the interdependence or mutual influence process itself. The ActorPartner Interdependence Model (APIM) accomplishes both goals by utilizing a multilevel modeling approach in which data from two dyad members are treated as nested scores within the same group (Kenny et al., 2006). The APIM suggests that a person's independent variable score affects his or her own dependent variable score (known as the actor effect), and his or her partner's dependent variable score (known as the partner, or cross-spouse effect). We can also determine whether these effects differ depending on role (i.e., whether the actor is a patient or spouse) or gender (i.e., whether the actor is a man or a woman). Because it is not clear whether gender or role is a stronger predictor of adjustment to cancer and because the majority of dyads in this small sample study comprised male patients and female spouses, we chose to focus on role effects.

A series of APIM analyses were conducted to examine the baseline actor and partner associations for each of the dyadic coping predictor variables of interest (i.e., problem- and emotionfocused stress communication, problem- and emotion-focused dyadic coping, negative dyadic coping, and satisfaction with dyadic coping) and the study outcomes (i.e., depression, anxiety, and marital adjustment), controlling for participant age. We also tested whether the association between a specific dyadic coping behavior and the outcome of interest differed depending on role $(1=$ patients and $-1=$ spouses $)$. The continuous predictor variables were standardized, and the error terms were allowed to differ for the two dyad members. Partial correlations (r) were used to calculate effect sizes for significant actor and partner effects.

To examine the effects of the SHARE intervention on patient and spouse engagement in dyadic coping relative to UMC, we performed a series of ANCOVAs with $\mathrm{T}_{0}$ scores as covariates and follow-up $\mathrm{T}_{1}$ outcome scores as dependent variables. The main effects tested were treatment group (SHARE or UMC) and role (patient or caregiver). We also examined the treatment group $\mathrm{X}$ role interaction.

Finally, to understand whether patient and spouse changes in dyadic coping were associated with changes in their own and each other's outcomes, a series of APIM analyses were conducted. Change scores for dyadic coping and the outcome measures were calculated by subtracting $\mathrm{T}_{0}$ from $\mathrm{T}_{1}$ values, and interactions between role and changes in dyadic coping were examined.

\section{RESULTS}

\section{Sample and Recruitment}

\section{Study Enrollment and Participation}

Sixty-four patient-caregiver dyads were screened and 16 were excluded due to one of the dyad members not being eligible. Of 
the remaining 48 eligible dyads, 34 (71\%) consented. Differences between participants and refusers on demographic and medical characteristics were examined. Results showed that refusers reported significantly greater fatigue on the MDASI $(t=2.11$, $p=0.04)$. The primary reasons for refusal were either that the patient, caregiver, or both were not interested or that they had too much going on. Four dyads dropped out before returning the baseline survey due to the patient not feeling wellenough to participate or having too much going on. Of the remaining 30 dyads, 15 were randomized to SHARE and 15 to UMC.

\section{Participant Characteristics}

Patients were mostly male (80\%), non-Hispanic White (60\%), educated with at least some college credits (73\%), middle aged $(\bar{X}=58.43, \mathrm{SD}=10.49)$, and employed full time $(60 \%) ; 77 \%$ had pharynx cancers (63\% oropharyngeal, $7 \%$ nasopharyngeal, $7 \%$ hypopharyngeal) and advanced disease (10\% - stage 1, 10\% stage $2,3 \%$ - stage $3,77 \%$ - stage $4 \mathrm{~A})$. Twenty-five $(83 \%)$ of patients were married. Average length of relationship in years was $\bar{X}=28.85$ (SD $=12.65$; Range $=3-54$ years). Spouses were mostly female $(77 \%)$, non-Hispanic white $(63 \%)$, educated with at least some college credits $(73 \%)$, middle aged $(\bar{X}=58.07, \mathrm{SD}=10.11)$, and employed full-time (59\%).

\section{Psychological adjustment}

At baseline, no patients and $30 \%$ of spouses had PROMIS Depression T-scores $>60(+1 \mathrm{SD})$, indicating high levels of depression. Also at baseline, $27 \%$ of patients and $37 \%$ of spouses had PROMIS Anxiety T-scores > 60, indicating high levels of anxiety. In $17 \%$ of dyads, both patient and spouse scored above 60. As Table 1 shows, partial correlations for patients and spouses for anxiety were significant. Spouses also reported significantly higher depression levels than patients.

\section{Marital adjustment}

At baseline, 3\% of patients and $10 \%$ of spouses scored below the DAS-7 cut-off for marital distress. At the 1-month followup, $3 \%$ of patients and $23 \%$ of spouses reported marital distress. Partial correlations for patient and marital adjustment were also significant and in the expected direction (Table 1).

\section{Baseline APIM Analyses}

No significant actor or partner interactions between any of the dyadic coping variables and role were found, so the interaction terms were removed and the models rerun. Results are below.

\section{Depression}

For PFSC, the combined actor effect across patients and spouses was significant $(b=-1.90, p=0.007)$ and yielded a medium effect size $(r=-0.32)$. However, the combined partner effect was not significant $(b=0.79, p=0.33)$. For PFDC, the combined actor effect across patients and spouses was significant and yielded a small effect size $(r=-0.29)$. The combined partner effect was also significant $(b=1.55, p=0.04)$ and yielded a small effect size $(r=0.26)$. For illustrative purposes, Figure 1 depicts the mixed models coefficients for the actor and partner associations of PFSC and PFDC with depression for patients and spouses separately. By examining the individual coefficients, we discovered that even though the overall actor effects for PFSC and PFDC were significant, the significant association between patient scores on these predictors and patient depression appeared to have been largely driving these effects. Moreover, even though the overall partner effect for PFDC was significant, the individual partner effects (i.e., the effects of patient PFDC on spouse depression and spouse PFDC on patient depression) were not significant.

\section{Anxiety}

Of all the dyadic coping predictors that we examined, only the combined actor effect of SATDC was significant $(b=-1.47$,

TABLE 1 | Baseline correlations and descriptive results ( $N=30$ patients and 30 caregivers).

\begin{tabular}{|c|c|c|c|c|c|c|c|c|c|c|c|c|c|}
\hline & 1 & 2 & 3 & 4 & 5 & 6 & 7 & 8 & 9 & 10 & $\begin{array}{l}\text { Patients Mean } \\
(S D)\end{array}$ & $\begin{array}{l}\text { Spouses } \\
\text { Mean (SD) }\end{array}$ & $t$ \\
\hline 1. PFSC & 0.16 & $0.73^{* *}$ & $0.38^{*}$ & $0.57^{* *}$ & 0.11 & $0.56^{* *}$ & $-0.45^{*}$ & -0.23 & $0.41^{*}$ & 0.12 & $3.93(0.70)$ & $3.35(0.99)$ & $2.81^{* *}$ \\
\hline 2. EFSC & $0.62^{* *}$ & $0.37^{*}$ & $0.40^{*}$ & $0.80^{* *}$ & 0.28 & $0.65^{* *}$ & -0.35 & 0.02 & 0.22 & -0.11 & 3.27 (1.00) & $2.72(0.99)$ & $2.70^{* *}$ \\
\hline 3. PFDC & 0.18 & -0.03 & $0.43^{*}$ & $0.44^{*}$ & 0.19 & 0.03 & -0.18 & 0.09 & $0.48^{* *}$ & -0.07 & $3.62(0.91)$ & 3.67 (0.95) & -0.27 \\
\hline 4. EFDC & $0.48^{* *}$ & $0.49^{* *}$ & $0.39 *$ & $0.76^{* *}$ & 0.08 & $0.72^{* *}$ & -0.36 & 0.19 & 0.40 & -0.22 & $3.93(1.12)$ & $4.10(1.05)$ & -1.27 \\
\hline 5. NegDC & $-0.48^{* *}$ & -0.14 & -0.12 & -0.11 & 0.01 & 0.20 & 0.19 & 0.11 & -0.46 & 0.07 & $1.57(0.81)$ & $1.57(0.72)$ & 0 \\
\hline 6. SatDC & $0.55^{* *}$ & $0.40^{*}$ & $0.39^{*}$ & $0.81^{* *}$ & 0.45 & $0.68^{* *}$ & $-0.40^{*}$ & -0.13 & 0.01 & 0.01 & $4.27(1.05)$ & $3.93(1.14)$ & $2.16^{*}$ \\
\hline $\begin{array}{l}\text { 7. PROMIS } \\
\text { depression raw } \\
\text { score }\end{array}$ & -0.29 & -0.10 & -0.01 & 0.36 & 0.19 & 0.05 & -0.13 & $0.41^{*}$ & -0.35 & -0.05 & $9.27(3.31)$ & $12.40(5.87)$ & $-2.40^{*}$ \\
\hline $\begin{array}{l}\text { 8. PROMIS anxiety } \\
\text { raw score }\end{array}$ & -0.12 & 0.10 & -0.11 & 0.09 & 0.15 & -0.27 & $0.53^{* *}$ & $0.49^{* *}$ & 0.11 & $-0.68^{* *}$ & $11.53(4.17)$ & $13.00(4.97)$ & -1.66 \\
\hline 9. DAS-7 & 0.30 & 0.12 & 0.22 & 0.10 & -0.32 & 0.29 & -0.10 & -0.08 & $0.38^{*}$ & -0.11 & $28.80(4.01)$ & $27.57(5.28)$ & 1.39 \\
\hline 10. Age & 0.11 & -0.05 & -0.25 & -0.20 & 0.07 & -0.04 & $-0.44^{* *}$ & -0.28 & -0.10 & $0.94^{* *}$ & $58.43(10.49)$ & 58.07 (10.11) & 0.52 \\
\hline
\end{tabular}

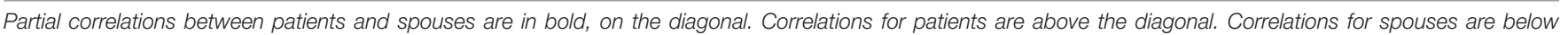

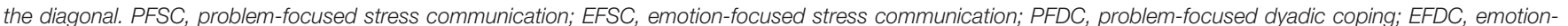

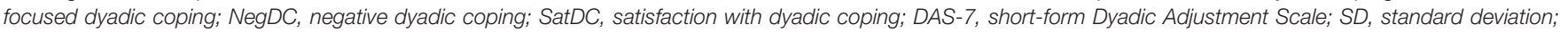
t, paired samples t-test ${ }^{*} p<0.05,{ }^{* *} p<0.01$. 

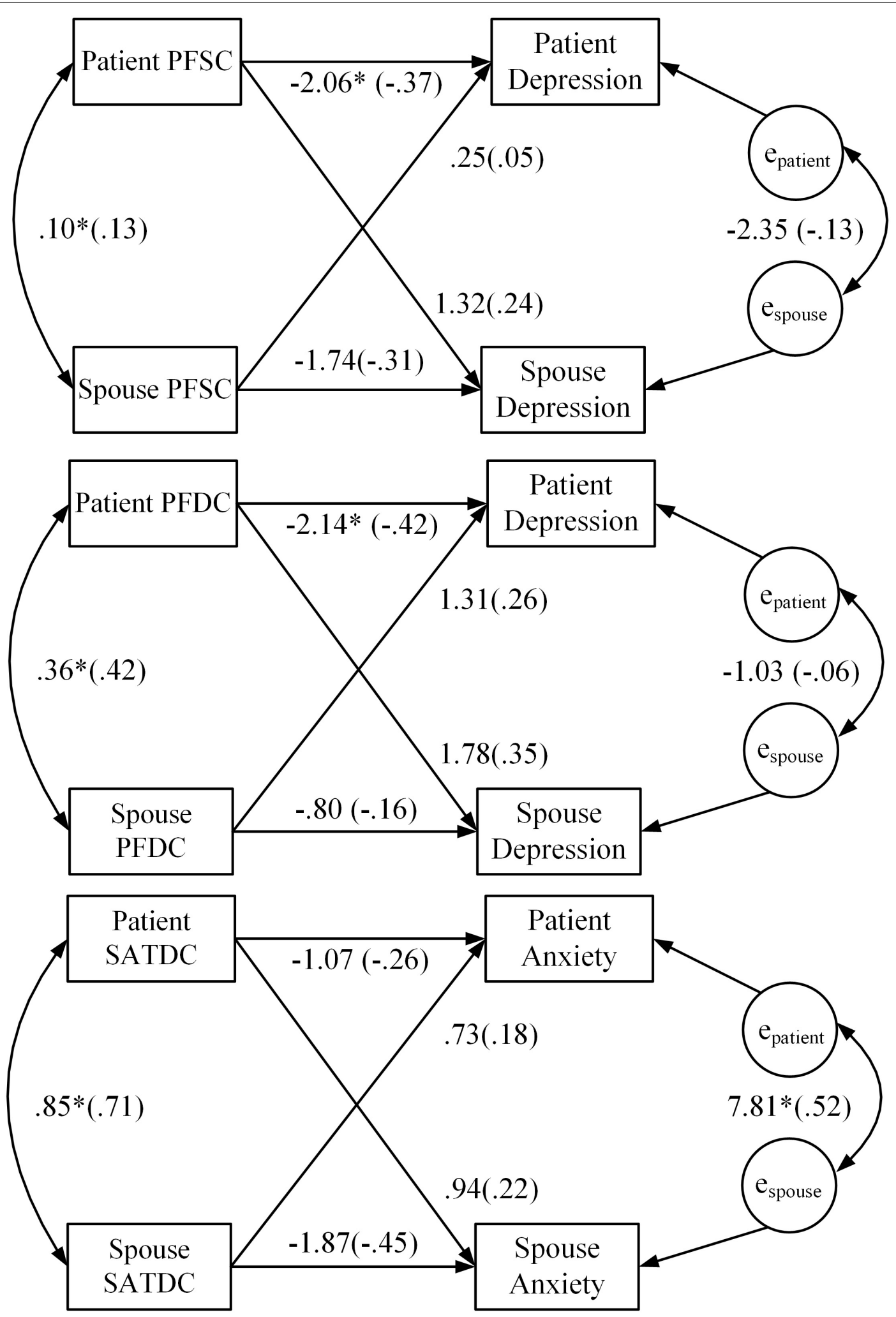

FIGURE 1 | Results of APIM baseline analysis regressing PROMIS depression and anxiety scores on patient and spouse dyadic coping. Model coefficients for the actor and partner effects for both patients and spouses are presented. Standardized coefficients are in parentheses. * $p<0.05$; PFSC, problem-focused stress communication; PFDC, problem-focused dyadic coping; SATDC, satisfaction with dyadic coping. 

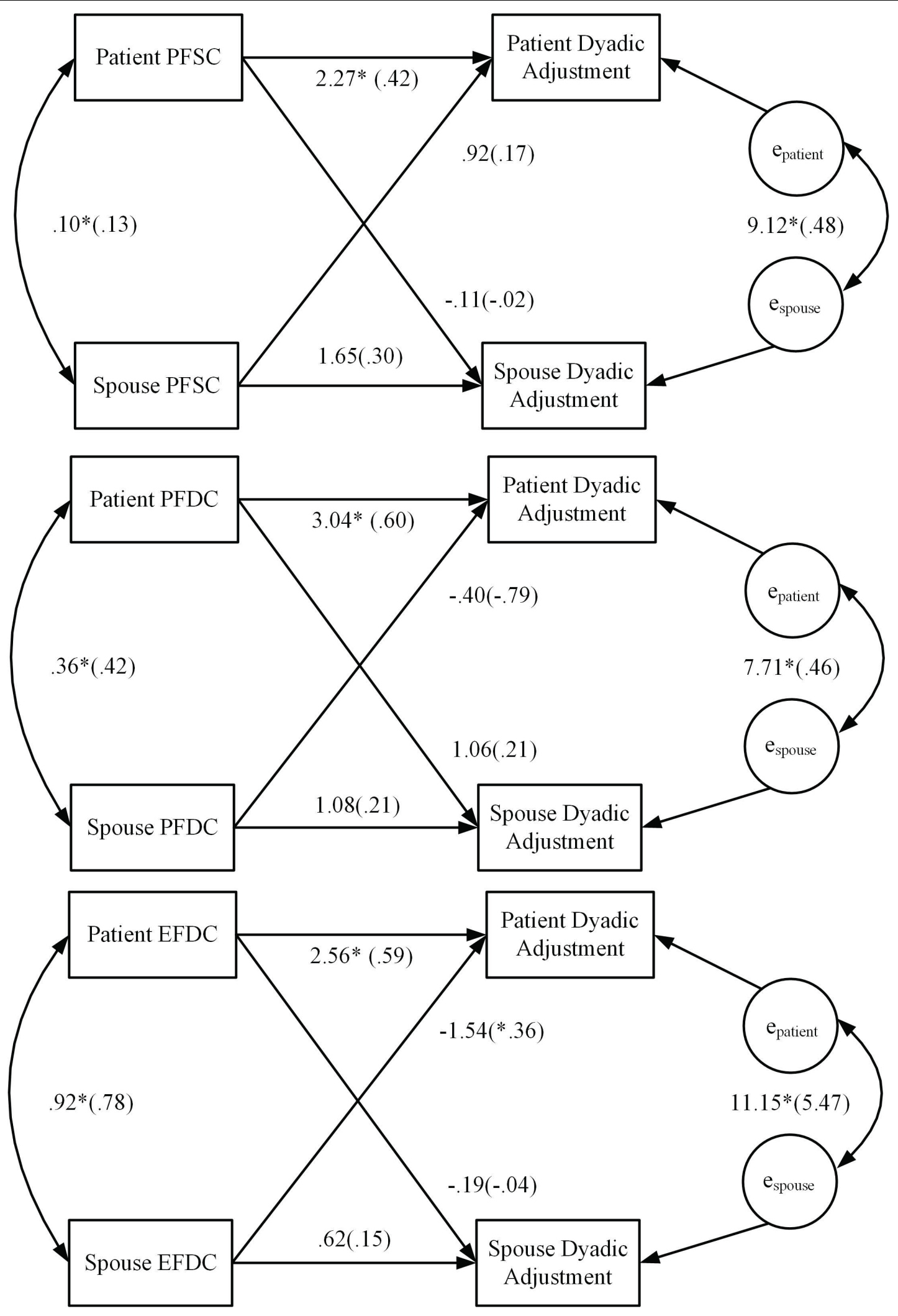

FIGURE 2 | Continued 


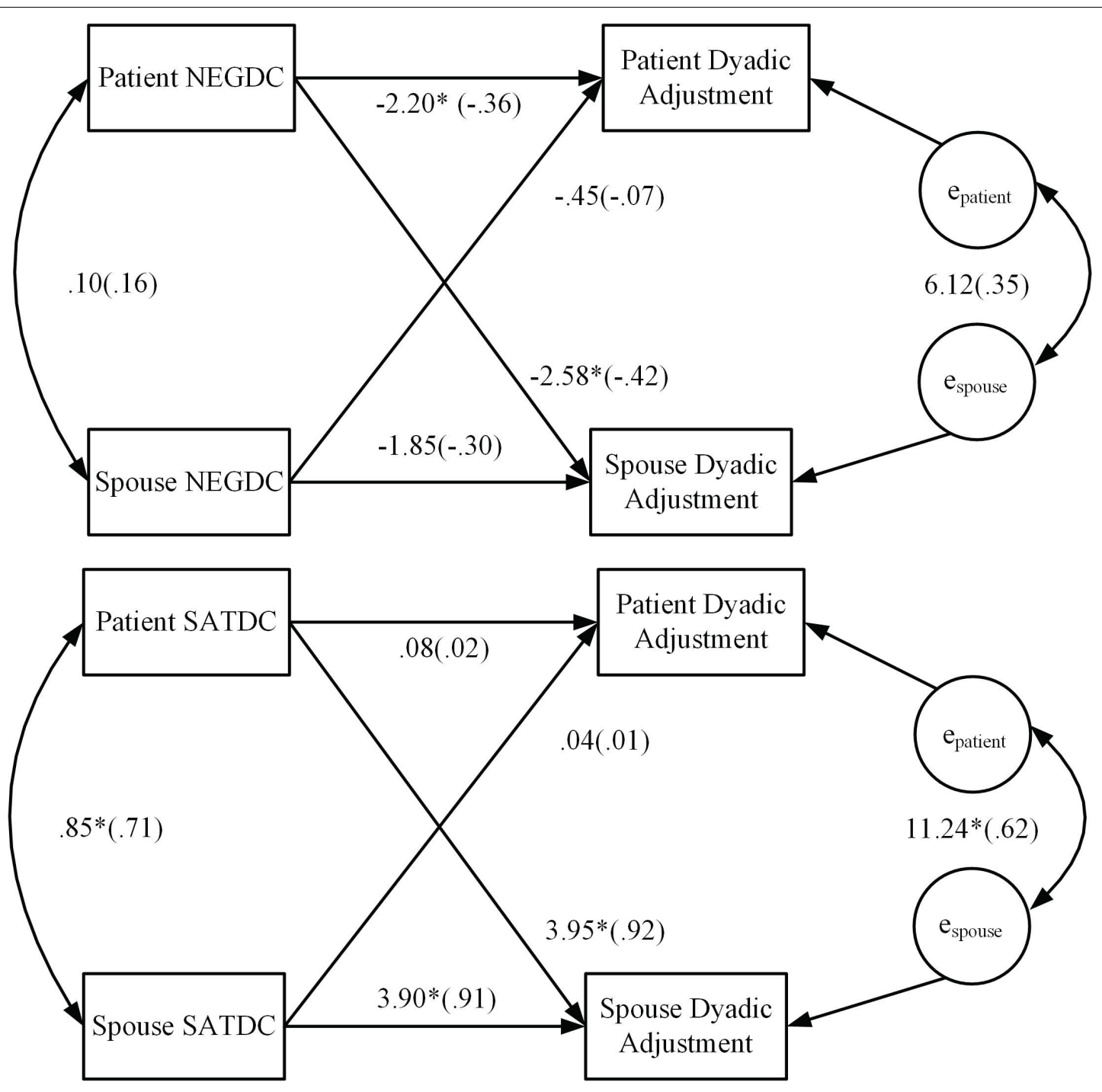

FIGURE 2 | Results of APIM baseline analysis regressing marital (dyadic) adjustment scores on patient and spouse dyadic coping. Model coefficients for the actor and partner effects for both patients and spouses are presented. Standardized coefficients are in parentheses. ${ }^{*} p<0.05$, PFSC, problem-focused stress communication; PFDC, problem-focused dyadic coping; EFDC, emotion-focused dyadic coping; NegDC, negative dyadic coping; SatDC, satisfaction with dyadic coping.

$p=0.01)$ and yielded a small effect size $(r=-0.28)$. However, the combined partner effect across patients and spouses for SATDC was not significant $(b=0.83, p=0.17)$. Figure 1 depicts the mixed models coefficients for the actor and partner associations of SATDC and anxiety for patients and spouses. As the figure shows, even though the overall actor effect was significant, the individual actor effects for patients and spouses were not significant.

\section{Marital Adjustment}

Significant combined actor effects across patients and spouses were found for PFSC $(b=1.96, p=0.01 ; r=0.34)$, PFDC $(b=2.06, p=0.001, r=0.38)$, EFDC $(b=1.59, p=0.03$, $r=0.23)$, NEGDC $(b=-2.03, p=0.01, r=-0.35)$, and SATDC $(b=1.99, p=0.001, r=0.36)$, and effect sizes were small to medium. The combined partner effect for NEGDC ( $b=-1.51, p=0.04, r=-0.27)$ was also significant and the effect size was small. Figure 2 depicts the mixed models coefficients for the actor and partner associations of PFSC, EFDC, PFDC, NEGDC, and SATDC with marital adjustment for patients and spouses separately. By examining the individual coefficients for the actor and partner effects, we discovered that even though the overall actor effects for each of these associations were significant, the coefficients for the associations between patient scores on all of the dyadic coping variables (except SATDC) and patient marital adjustment were significant, and the coefficients for the associations between all of the spouse dyadic coping variables and spouse marital adjustment were not significant (except SATDC). For NEGDC, the overall partner effect was significant but examination of the individual 
TABLE 2 | Baseline and follow-up means and SDs for stress communication and dyadic coping for patients and spouses in the SHARE intervention and UMC.

\begin{tabular}{|c|c|c|c|c|c|c|c|c|}
\hline & \multicolumn{4}{|c|}{ Patients } & \multicolumn{4}{|c|}{ Spouses } \\
\hline & \multicolumn{2}{|c|}{ SHARE } & \multicolumn{2}{|c|}{ UMC } & \multicolumn{2}{|c|}{ SHARE } & \multicolumn{2}{|c|}{ UMC } \\
\hline & $\begin{array}{l}\text { Baseline M } \\
\text { (SD) }\end{array}$ & $\begin{array}{l}\text { Follow-up M } \\
\text { (SD) }\end{array}$ & $\begin{array}{c}\text { Baseline M } \\
\text { (SD) }\end{array}$ & $\begin{array}{l}\text { Follow-up M } \\
\text { (SD) }\end{array}$ & $\begin{array}{c}\text { Baseline M } \\
\text { (SD) }\end{array}$ & $\begin{array}{l}\text { Follow-up M } \\
\text { (SD) }\end{array}$ & $\begin{array}{c}\text { Baseline M } \\
\text { (SD) }\end{array}$ & $\begin{array}{c}\text { Follow-up M } \\
\text { (SD) }\end{array}$ \\
\hline PFSC & $3.93(0.73)$ & $4.27(0.90)$ & $3.93(0.70)$ & 3.53 (1.16) & $3.30(1.18)$ & $3.83(1.04)$ & $3.40(0.81)$ & $2.93(0.90)$ \\
\hline EFSC & $3.50(0.73)$ & $3.57(0.84)$ & $3.03(1.20)$ & 3.07 (1.13) & $2.90(0.93)$ & $3.27(0.88)$ & $2.53(1.04)$ & $2.47(1.04)$ \\
\hline PFDC & $3.77(1.00)$ & $3.73(1.02)$ & $3.47(0.81)$ & $3.00(0.91)$ & $3.90(0.85)$ & $4.13(0.81)$ & $3.43(1.02)$ & $3.03(1.06)$ \\
\hline EFDC & $4.27(0.73)$ & $4.38(0.64)$ & $3.49(1.33)$ & $3.64(1.14)$ & $4.44(0.66)$ & $4.49(0.79)$ & $3.76(1.26)$ & $3.58(1.22)$ \\
\hline NEGDC & $1.73(1.02)$ & $1.80(1.05)$ & $1.40(0.51)$ & $1.97(0.77)$ & $1.60(0.83)$ & $1.47(0.64)$ & $1.53(0.61)$ & $2.17(1.21)$ \\
\hline SATDC & $4.47(0.64)$ & $4.87(0.52)$ & 4.07 (1.34) & $3.87(1.30)$ & 3.93 (1.03) & $4.27(0.88)$ & $3.93(1.28)$ & 3.60 (1.18) \\
\hline
\end{tabular}

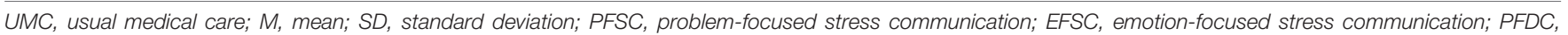

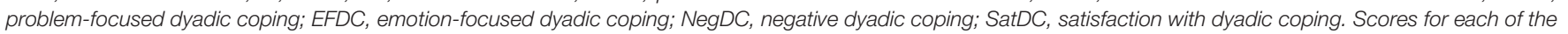
dyadic coping sub-scales range from 1 to 5 , with higher scores indicating more frequent dyadic coping.

coefficients for patients and spouses showed that only patient engagement in negative dyadic coping had an adverse effect on spouse marital adjustment. Finally, even though the overall partner effect for SATDC was not significant, patient SATDC was significantly positively associated with spouse marital adjustment.

\section{Treatment Effects}

Means for the dyadic coping variables for patients and spouses by treatment group at baseline $\left(\mathrm{T}_{0}\right)$ and follow-up $\left(\mathrm{T}_{1}\right)$ are in Table 2. Results of the ANCOVAs for dyadic coping at $\mathrm{T}_{0}$ and $\mathrm{T}_{1}$ are in Table 3. No significant main effects for role or the Group X Role interaction were found. However, at $T_{1}$ there was a significant difference on PFSC $(p<0.001)$, with the SHARE group having higher mean scores (more PFSC) than the UMC group. The effect size for this difference was Cohen's $d=1.14$ (95\% CI $=0.60$ to 1.69 ), which is a large effect (Cohen, 1988). Also at $\mathrm{T}_{1}$, significant differences were found on PFDC $(p=0.02)$ and SATDC $(p=0.001)$ with the SHARE group having higher mean scores than the UMC group. Effect sizes were $d=0.64(95 \% \mathrm{CI}=0.07$ to 1.10$)$ for PFDC, which is a medium effect, and $d=1.03(95 \% \mathrm{CI}=0.49$ to 1.57$)$ for SATDC, which is a large effect. Finally, at $\mathrm{T}_{1}$ there was a significant difference in NEGDC $(p=0.02)$, with the SHARE group having lower mean scores that the UMC group. The effect size was $d=-0.68(95 \% \mathrm{CI}=-1.21$ to -0.16$)$, which is a medium effect.

\section{APIM Change Score Analysis Depression}

As Figure 3 shows, the interaction between role and gains in a partner's PFSC was significant ( $b=1.53 ; t=2.80, p=0.01)$. Tests of the simple slopes showed that gains in spouses' PFSC did not significantly affect patients' depression $(b=0.13 ; z=0.04$, $p=$ n.s.), but gains in patients' PFSC resulted in significant reductions in spouses' depression $(b=-2.92 ; z=3.49, p=0.001)$. A significant main effect was also found for actors' PFDC $(b=-1.48 ; t=-2.10, p=0.04)$.
TABLE 3 | ANCOVA results for dyadic coping at baseline $\left(T_{0}\right)$ and at 1-month follow-up $\left(\mathrm{T}_{1}\right)$.

\begin{tabular}{lccc}
\hline & \multicolumn{3}{c}{ Treatment group } \\
\cline { 2 - 4 } Measure at $\mathbf{T}_{\mathbf{1}}$ & $\boldsymbol{F}$-value & $\boldsymbol{p}$-value & Least square means \\
\hline PFSC & 16.29 & $<0.0001$ & $\mathrm{UMC}=3.20, \mathrm{SHARE}=4.08$ \\
EFSC & n.s. & n.s. & - \\
PFDC & 6.29 & 0.02 & $\mathrm{UMC}=3.20 ;$ SHARE $=3.75$ \\
EFDC & n.s. & n.s. & - \\
NEGDC & 5.60 & 0.02 & $\mathrm{UMC}=2.16 ;$ SHARE $=1.54$ \\
SATDC & 13.38 & 0.001 & $\mathrm{UMC}=3.81 ;$ SHARE $=4.49$
\end{tabular}

UMC, usual medical care; n.s., not significant; PFSC, problem-focused stress communication; EFSC, emotion-focused stress communication; PFDC, problemfocused dyadic coping; EFDC, emotion-focused dyadic coping; NegDC, negative dyadic coping; SatDC, satisfaction with dyadic coping.

\section{Anxiety}

Although none of the role main effects or interactions were significant, significant main effects were found for gains in actors' $\operatorname{PFDC}(b=-1.77 ; t=-2.69, p=0.01)$ and SATDC $(b=-2.14$; $t=-2.80, p=0.01)$ meaning that improvements in both of these dyadic coping behaviors were associated with reductions in anxiety for both patients and spouses.

\section{Marital Adjustment}

Although none of the role main effects or interactions were significant, the main effect for gains in actors' SATDC was significant $(b=1.22 ; t=2.04, p=0.05)$. In addition, main effects for gains in partners' PFSC $(b=0.80 ; t=1.70, p<0.10)$ and partners' NEGDC $(b=-0.92 ; t=-1.94, p=0.06)$ were marginally significant. Thus, there was a trend for patients and spouses to report improvements in marital adjustment when their partners engaged in more PFSC and less NEGDC.

\section{DISCUSSION}

Even though RT for HNC can be stressful for both members of the couple, patients and spouses still find ways to support 


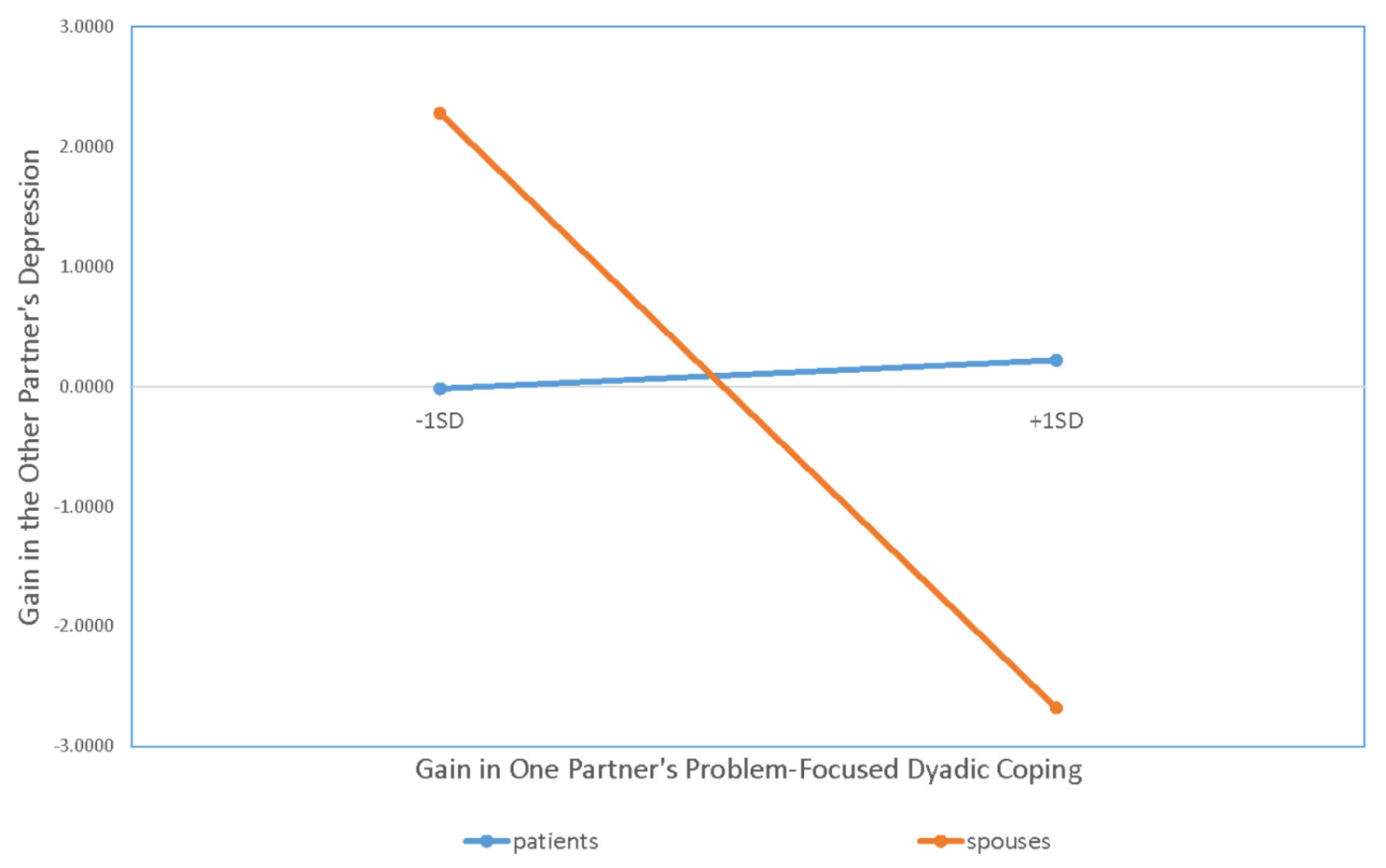

FIGURE 3 | Results of APIM analysis regressing one partner's gain scores for depression on the other partner's gain scores from problem-focused dyadic coping. Gain scores for depression were calculated by subtracting $T_{0}$ from $T_{1}$ values on PROMIS depression raw scores.

and care for each other during this emotionally and physically taxing time. Caring for and providing support to a partner who is under stress when one is already experiencing stress of his or her own can be a challenging undertaking that requires coping efforts that address each partner's well-being as well as the well-being of the relationship. With these points in mind, this study evaluated the effects of dyadic coping on both partner's psychological and marital adjustment and whether the SHARE intervention resulted in meaningful changes in patient and spouse dyadic coping relative to UMC. Providing partial support for our hypotheses, we found that engaging in some dyadic coping strategies (i.e., PFSC, PFDC, SATDC) was related to psychological and marital adjustment for both partners, whereas engagement in other dyadic coping strategies (i.e., EFDC, NEGDC) was only related to marital adjustment. We also found moderate to large effect sizes for the impact of SHARE relative to UMC on PFSC, PFDC, NEGDC, and SATDC. Finally, we found that increases in one's PFDC from baseline to the one-month follow-up were consistently associated with improvements in psychological and marital adjustment but that increases in a partner's PFSC were only associated with improvements in spouses' depression. Improvements in other dyadic coping strategies (i.e., EFSC, EFDC) were minimal and did not demonstrate significant effects on psychological adjustment, although increases in EFDC were significantly associated with increases in marital adjustment. Taken together, these findings provide important information for future couplebased interventions in HNC.
It is notable that few significant partner effects were found for the baseline APIM analyses that were conducted - and in most cases, the significant actor effects that were found were driven by significant associations for the patient only. When dealing with self-report data, partner effects are often smaller in magnitude (Ackerman et al., 2011). Thus, the small sample size in this study may have made it more difficult to detect such effects. That said, the partner effects that were significant were notable. With regard to depression, both patients and spouses reported lower depression levels when they engaged in PFDC and when their partners engaged in PFDC. Thus, PFDC may be an important target for future couple-based interventions aimed at alleviating patient and partner distress. With regard to marital adjustment, only the partner effect for NEGDC was significant. The fact that none of the partner effects for positive dyadic coping strategies were significant may be consistent with the notion that "bad is stronger than good" (Baumeister et al., 2001). Indeed, studies of marital relationships have consistently shown that the presence or absence of negative behaviors is more strongly related to the quality of couples' relationships than the presence or absence of positive behaviors (Krokoff et al., 1989; Gottman, 1991, 1994). Gottman (1994) even proposed that in order for a relationship to succeed, positive couple interactions should outnumber the negative ones by at least five to one. In this study, the ratio of positive to negative dyadic coping was approximately $2: 1$ and thus may not have been strong enough to impact partners' marital adjustment. 
With regard to the finding that more significant associations between dyadic coping and marital and psychological adjustment were found for patients, it is important to point out that tests of all of the dyadic coping by role (i.e., patient or spouse) interactions were not significant. Thus, even though examination of the individual patient and spouse coefficients showed mostly significant associations for patients, we cannot assume that role differences exist. It is possible that the small sample size made it more difficult to detect significant differences in the effects of dyadic coping based on role. Indeed, the published literature supports the idea of role differences. For example, it is possible that by virtue of the illness situation, patients are in more acute need and thus more likely to communicate their need for support to their partners (Badr et al., 2010). Given their caregiving role, spouses in turn may be more likely to provide them with support (Glasdam et al., 1996). Patients may also feel that they are contributing to the relationship and supporting their partner when they engage in PFDC, and this in turn may have positive benefits for their psychological and marital adjustment. Our future work will thus explore whether possible gender and role differences exist with regard to the effects of dyadic coping in a larger sample with sufficient power to simultaneously test for these effects. On a related note, although spouses of cancer patients may be more likely to shield their partners from their own needs and concerns, research has shown that their marital adjustment does benefit from engaging in common positive dyadic coping (Badr et al., 2010), which involves joint efforts to manage the shared stress of the couple (Bodenmann, 2005). We did not assess common dyadic coping in this study, but our future work will examine whether dyadic coping interventions that teach HNC patients to solicit spousal support and engage in PFDC and that teach patients and spouses ways to work together as a team to jointly manage their shared stress are beneficial for both members of the couple.

Another notable finding was that the actor/partner effects of EFSC and EFDC on psychological adjustment were not significant despite the fact that the disclosure of feelings and concerns is a topic that has received considerable research attention and is commonly advocated in couplebased interventions (for a review, see Badr, 2017). The idea that couples should talk about feelings is grounded in social cognitive models that posit that stressful events like cancer are a threat because they challenge existing schemas about the self and relationships (Janoff-Bulman, 1989; McLean et al., 2011). From this perspective, adaptation involves actively assimilating illness into these schemas through acceptance, reappraisal, and disclosure to a supportive partner (Lepore and Revenson, 2007; Kershaw et al., 2008). In the context of HNC, however, this can be challenging because patients (and spouses) are going to the hospital daily for RT and may become so overwhelmed and busy dealing with the day-to-day management of the illness in addition to their daily lives that they may not have the time or energy to process emotions and talk about feelings. Thus, discussions about practical support that is needed or that focus on problem-solving may be more beneficial during this acutely stressful period than discussions about emotions. Discussions about emotions may still be beneficial for HNC couples dealing with the long-term sequelae of RT and struggling to return to a normal life, or those dealing with a cancer recurrence or end-of-life, but more research is needed to follow couples for a longer period post-treatment to determine if EFSC and EFDC have any beneficial effects over time.

A related issue is that even though SHARE evidenced significant positive treatment effects for PFSC, PFDC, NEGDC, and SATDC, it did not significantly impact EFSC or EFDC. In SHARE, skills stress communication and supportive dyadic coping skills were taught through a stress reducing conversation exercise where partners took turns disclosing a topic of concern of their choice and took turns listening to and offering support to one another (Bodenmann and Shantinath, 2004). A review of the audiotapes of this session revealed that in over $50 \%$ of these discussions, the "stressor" being discussed was managing the demands of everyday life in addition to the cancer. The remainder of the topics were evenly divided between symptom management issues and wanting partners to acknowledge feelings. Given that the most of the topics of discussion centered around practical or health related topics, the topics themselves may have been more conducive for practicing PFSC and PFDC as opposed to EFSC and EFDC.

With regard to the change score analyses, we found that gains in spouses' PFSC did not significantly affect patients' depression, but gains in patients' PFSC resulted in significant reductions in spouses' depression. These findings are consistent with our previous work in HNC showing that spouses' often feel responsible for ensuring that patients adhere to self-management recommendations to control physical symptoms (Badr et al., 2016). Thus, when patients let their spouses know that they need their practical support or advice or ask for their help, spouses may feel more purposeful and in control of an otherwise difficult situation. In addition, although we found that changes in SATDC were associated with changes in marital adjustment, changes in the other dyadic coping strategies did not affect marital adjustment. One reason could be that our sample was highly martially satisfied at baseline and there was not much room for improvement. Future work should examine these associations in more martially distressed couples.

Overall, this study had several strengths. First, to our knowledge, this is the first couple-based dyadic coping intervention in HNC. Second, $40 \%$ of patients and $36 \%$ of spouses were racial/ethnic minorities, which bolsters generalizability. Other strengths include the rigorous randomized design, and data analytic approach that addressed the dependency among partners. This study also had some limitations. The sample largely comprised patients coping with advanced stage disease (stage 4A), so ability to generalize findings to patients dealing with early stage disease (stages 1-3) is limited. As initial support has now been obtained for SHARE on dyadic coping, it is important to replicate findings with a larger sample size. Given the multiple analyses performed, there was increased potential for error. Since the small sample size likely reduced the parameter estimates, a larger study would allow for examination of gender and role effects as well as the inclusion of additional covariates. A longer follow-up would allow us to examine the maintenance of effects after patients completed the acute recovery period. 
Given the dyadic nature of the study and the fragility of this patient population, retention over an extended follow-up is likely to be challenging; however, given the fact that SHARE was meant to fortify the couple against the wear and tear of HNC on adjustment, it is important to determine how long intervention effects last, whether booster sessions are needed, and whether receiving the intervention results in decreased healthcare utilization such as unnecessary hospital admissions (i.e., due to poor symptom control at home). Finally, because SHARE had multiple components, it will be important to explore what it is about the intervention that is of benefit and whether the "active ingredients" are the same for patients and spouses.

\section{CONCLUSION}

Our findings provide evidence that SHARE improved positive dyadic coping and decreased negative dyadic coping for both patients and spouses. They also showed that even during periods of great stress, positive changes in dyadic coping can occur if couples are provided with the appropriate skills training and support; and, that such changes can have a meaningful impact

\section{REFERENCES}

Ackerman, R. A., Donnellan, M. B., and Kashy, D. A. (2011). "Working with dyadic data in studies of emerging adulthood: specific recommendations, general advice, and practical tips," in Advances in Personal Relationships. Romantic Relationships in Emerging Adulthood, eds F. D. Fincham and M. Cui (New York, NY: Cambridge University Press), 67-97.

Aizer, A. A., Chen, M.-H., McCarthy, E. P., Mendu, M. L., Koo, S., Wilhite, T. J., et al. (2013). Marital status and survival in patients with cancer. J. Clin. Oncol. 31, 3869-3876. doi: 10.1200/JCO.2013.49.6489

Applebaum, A. J., and Breitbart, W. (2013). Care for the cancer caregiver: a systematic review. Palliat. Support. Care 11, 231-252. doi: 10.1017/ S1478951512000594

Badr, H. (2017). New frontiers in couple-based interventions in cancer care: refining the prescription for spousal communication. Acta Oncol. 56, 139-145. doi: 10.1080/0284186X.2016.1266079

Badr, H., Carmack, C., Kashy, D., Cristofanilli, M., and Revenson, T. (2010). Dyadic coping in metastatic breast cancer. Health Psychol. 29, 169-180. doi: 10.1037/ a0018165

Badr, H., Herbert, K., Reckson, B., Rainey, H., Sallam, A., and Gupta, V. (2016). Unmet needs and relationship challenges of head and neck cancer patients and their spouses. J. Psychosoc. Oncol. 34, 336-346. doi: 10.1080/07347332.2016. 1195901

Badr, H., Yeung, C., Lewis, M. A., Milbury, K., and Redd, W. H. (2015). An observational study of social control, mood, and self-efficacy in couples during treatment for head and neck cancer. Psychol. Health 30, 783-802. doi: 10.1080/ 08870446.2014.994633

Baumeister, R. F., Bratslavsky, E., Finkenauer, C., and Vohs, K. D. (2001). Bad is stronger than good. Rev. Gen. Psychol. 5, 323-370. doi: 10.1037/1089-2680.5. 4.323

Berg, C. A., and Upchurch, R. (2007). A developmental-contextual model of couples coping with chronic illness across the adult life span. Psychol. Bull. 133, 920-954. doi: 10.1037/0033-2909.133.6.920

Bodenmann, G. (2005). "Dyadic coping and its significance for marital functioning," in Couples Coping with Stress: Emerging Perspectives on Dyadic Coping, eds T. A. Revenson, K. Kayser, and G. Bodenmann (Washington, DC: American Psychological Association), 33-50. doi: 10.1037/110 31-002

Bodenmann, G. (2008). Dyadisches Coping Inventar (DCI). Test manual [Dyadic Coping Inventory (DCI). Test manual]. Bern: Huber. on both partners. Finally, findings suggest that interventions that target dyadic coping can result in improvements on both the individual psychological and marital adjustment levels. Although findings are preliminary and should be interpreted with caution, more research on ways to integrate dyadic coping interventions into oncology supportive care appears warranted.

\section{AUTHOR CONTRIBUTIONS}

HB contributed to study conceptualization, data collection, data analysis and interpretation, and manuscript writing. $\mathrm{KH}, \mathrm{JA}, \mathrm{MB}$, and TW contributed to data collection and manuscript writing.

\section{FUNDING}

This work was supported in part by National Cancer Institute grants R21CA 178478 (PI: Badr) and P30 CA125123 (PI: Osborne), as well as the facilities and resources at the Houston HSR\&D Center for Innovations in Quality, Effectiveness, and Safety (CIN13-413).

Bodenmann, G., and Shantinath, S. D. (2004). The couples coping enhancement training (CCET): a new approach to prevention of marital distress based upon stress and coping*. Fam. Relat. 53, 477-484. doi: 10.1111/j.0197-6664.2004. 00056.x

Burman, B., and Margolin, G. (1992). Analysis of the association between marital relationships and health problems: an interactional perspective. Psychol. Bull. 112, 39-63. doi: 10.1037/0033-2909.112.1.39

Cameron, L., and Leventhal, H. (2012). The Self-Regulation of Health and Illness Behaviour. New York, NY: Routledge. doi: 10.4324/9780203553220

Clark, N. M., Becker, M. H., Janz, N. K., Lorig, K., Rakowski, W., and Anderson, L. (1991). Self-management of chronic disease by older adults. J. Aging Health 3, 3-27. doi: 10.1177/089826439100300101

Cohen, J. (1988). Statistical Power Analysis for the Behavioral Sciences. Mahwah, NJ: Lawrence Erlbaum Associates.

Duffy, S. A., Ronis, D. L., Valenstein, M., Lambert, M. T., Fowler, K. E., Gregory, L., et al. (2006). A tailored smoking, alcohol, and depression intervention for head and neck cancer patients. Cancer Epidemiol. Biomarkers Prev. 15, 2203-2208. doi: 10.1158/1055-9965.EPI-05-0880

Epstein, J., Meij, E., Emerton, S., Le, N., and Stevenson-Moore, P. (1995). Compliance with fluoride gel use in irradiated patients. Spec. Care Dent. 15, 218-222. doi: 10.1111/j.1754-4505.1995.tb00521.x

Epstein, J., Robertson, M., Emerton, S., Phillips, N., and Stevenson-Moore, P. (2001). Quality of life and oral function in patients treated with radiation therapy for head and neck cancer. Head Neck 23, 389-398. doi: 10.1002/hed. 1049

Falconier, M. K., Jackson, J. B., Hilpert, P., and Bodenmann, G. (2015). Dyadic coping and relationship satisfaction: a meta-analysis. Clin. Psychol. Rev. 42, 28-46. doi: 10.1016/j.cpr.2015.07.002

Faller, H., Schuler, M., Richard, M., Heckl, U., Weis, J., and Küffner, R. (2013). Effects of psycho-oncologic interventions on emotional distress and quality of life in adult patients with cancer: systematic review and meta-analysis. J. Clin. Oncol. 31, 782-793. doi: 10.1200/JCO.2011.40.8922

Family Caregiver Alliance [FCA] (2012). Selected Caregiver Statistics [Online]. Available: http://www.caregiver.org/caregiver/jsp/home.jsp [Accessed August 1 2016].

Glasdam, S., Jensen, A. B., Madsen, E. L., and Rose, C. (1996). Anxiety and depression in cancer patients' spouses. Psychooncology 5, 23-29. doi: 10.1002/(SICI)1099-1611(199603)5:1<23::AID-PON206>3.0.CO;2-H

Gottman, J. (1991). Predicting the longitudinal course of marriages. J. Marital Fam. Ther. 17, 3-7. doi: 10.1111/j.1752-0606.1991.tb00856.x 
Gottman, J. (1994). What Predicts Divorce? The Relationship Between Marital Processes and Marital Outcomes. Hillsdale, NJ: Erlbaum.

Gritz, E. R., Carmack, C. L., de Moor, C. A., Coscarelli, A., Schacherer, C. W., Meyers, E. G., et al. (1999). The first year after head and neck cancer: quality of life. J. Clin. Oncol. 17, 352-360. doi: 10.1200/JCO.1999.17.1.352

Heinrichs, N., Zimmermann, T., Huber, B., Herschbach, P., Russell, D. W., and Baucom, D. H. (2011). Cancer distress reduction with a couple-based skills training: a randomized controlled trial. Ann. Behav. Med. 43, 239-252. doi: 10.1007/s12160-011-9314-9

Hodges, L., Humphris, G., and Macfarlane, G. (2005). A meta-analytic investigation of the relationship between the psychological distress of cancer patients and their carers. Soc. Sci. Med. 60, 1-12. doi: 10.1016/j.socscimed.2004. 04.018

Hunsley, J., Best, M., Lefebvre, M., and Vito, D. (2001). The seven item short form of the dyadic adjustment scale: further evidence for the construct validity. Am. J. Fam. Ther. 29, 325-335. doi: 10.1080/01926180126501

Janoff-Bulman, R. (1989). Assumptive worlds and the stress of traumatic events: applications of the schema construct. Soc. Cognit. 7, 113-136. doi: 10.1521/soco. 1989.7.2.113

Jansma, J., Vissink, A., Spijkervet, F., Roodenburg, J., Panders, A., Vermey, A., et al. (1992). Protocol for the prevention and treatment of oral sequelae resulting from head and neck radiation therapy. Cancer 70, 2171-2180. doi: 10.1002/ 1097-0142(19921015)70:8<2171::AID-CNCR2820700827>3.0.CO;2-S

Karraker, A., and Latham, K. (2015). In sickness and in health? Physical illness as a risk factor for marital dissolution in later life. J. Health Soc. Behav. 56, 420-435. doi: 10.1177/0022146515596354

Katz, M. R., Irish, J. C., Devins, G. M., Rodin, G. M., and Gullane, P. J. (2002). Psychosocial adjustment in head and neck cancer: the impact of disfigurement, gender and social support. Head Neck 25, 103-112. doi: 10.1002/hed.10174

Kayser, K., Feldman, B. N., Borstelmann, N. A., and Daniels, A. A. (2010). Effects of a randomized couple-based intervention on quality of life of breast cancer patients and their partners. Soc. Work Res. 34, 20-32. doi: 10.1002/pon.3200

Kenny, D., Kashy, D. A., and Cook, D. (2006). Dyadic Data Analysis. New York, NY: Guilford.

Kershaw, T. S., Mood, D. W., Newth, G., Ronis, D. L., Sanda, M. G., Vaishampayan, U., et al. (2008). Longitudinal analysis of a model to predict quality of life in prostate cancer patients and their spouses. Ann. Behav. Med. 36, 117-128. doi: 10.1007/s12160-008-9058-3

Konski, A. A., Pajak, T. F., Movsas, B., Coyne, J., Harris, J., Gwede, C., et al. (2006). Disadvantage of men living alone participating in radiation therapy oncology group head and neck trials. J. Clin. Oncol. 24, 4177-4183. doi: 10.1200/JCO. 2006.06.2901

Krokoff, L., Gottman, J., and Hass, S. (1989). Validation of a global rapid couples interaction scoring system. Behav. Assess. 11, 65-79.

Kuijer, R. G., Ybema, J. F., Buunk, B. P., and DeJong, G. J. (2000). Active engagement, protective buffering, and overprotection: three ways of giving support by intimate partners of patients with cancer. J. Soc. Clin. Psychol. 19, 256-275. doi: $10.1521 /$ jscp.2000.19.2.256

Lepore, S. J., and Revenson, T. A. (2007). Social constraints on disclosure and adjustment to cancer. Soc. Pers. Psychol. Compass 1, 313-333. doi: 10.1111/j. 1751-9004.2007.00013.x

Manne, S., and Badr, H. (2010). "Social relationships and cancer," in Support Processes in Intimate Relationships, eds J. Davila and K. Sullivan (New York, NY: Oxford Press), 240-264. doi: 10.1093/acprof:oso/9780195380170.003.0010

Massie, M. J. (2004). Prevalence of depression in patients with cancer. J. Natl. Cancer Inst. Monogr. 2004, 57-71. doi: 10.1093/jncimonographs/lgh014

McGuire, D. (2003). Barriers and strategies in implementation of oral care standards for cancer patients. Support. Care Cancer 11, 435-441. doi: 10.1007/ s00520-003-0466-4

McLean, L. M., Walton, T., Rodin, G., Esplen, M. J., and Jones, J. M. (2011). A couple-based intervention for patients and caregivers facing end-stage cancer: outcomes of a randomized controlled trial. Psychooncology 22, 28-38. doi: 10.1002/pon.2046

Meier, C., Bodenmann, G., Mörgeli, H., and Jenewein, J. (2011). Dyadic coping, quality of life, and psychological distress among chronic obstructive pulmonary disease patients and their partners. Int. J. Chron. Obstruct. Pulmon. Dis. 6, 583-596. doi: 10.2147/COPD.S24508
National Comprehensive Cancer Network [NCCN] (2013). Clinical Practice Guidelines in Oncology: Head and Neck Cancers Version 2.2013 [Online]. Available at: http://www.nccn.org/professionals/physician_gls/pdf/head-andneck.pdf [accessed October 20, 2015].

Nguyen, N., Smith, H., and Sallah, S. (2007). Evaluation and management of swallowing dysfunction following chemoradiation for head and neck cancer. Curr. Opin. Otolaryngol. Head Neck Surg. 15, 130-133. doi: 10.1097/MOO. 0b013e32801da0e8

Nitenberg, G., and Raynard, B. (2000). Nutritional support of the cancer patient: issues and dilemmas. Crit. Rev. Oncol. Hematol. 34, 137-168. doi: 10.1016/ S1040-8428(00)00048-2

Ostroff, J., Ross, S., Steinglass, P., Ronis-Tobin, V., and Singh, B. (2004). Interest in and barriers to participation in multiple family groups among head and neck cancer survivors and their primary family caregivers. Fam. Process 43, 195-208. doi: 10.1111/j.1545-5300.2004.04302005.x

Paccagnella, A., Morello, M., Da Mosto, M. C., Baruffi, C., Marcon, M. L., Gava, A., et al. (2010). Early nutritional intervention improves treatment tolerance and outcomes in head and neck cancer patients undergoing concurrent chemoradiotherapy. Support. Care Cancer 18, 837-845. doi: 10.1007/s00520009-0717-0

Pilkonis, P. A., Choi, S. W., Reise, S. P., Stover, A. M., Riley, W. T., and Cella, D. (2011). Item banks for measuring emotional distress from the patientreported outcomes measurement information system (PROMIS ${ }^{\circledR}$ ): depression, anxiety, and anger. Assessment 18, 263-283. doi: 10.1177/1073191111 411667

Randall, A. K., Hilpert, P., Jimenez-Arista, L. E., Walsh, K. J., and Bodenmann, G. (2016). Dyadic coping in the US: psychometric properties and validity for use of the english version of the dyadic coping inventory. Curr. Psychol. 35, 570-582. doi: 10.1007/s12144-015-9323-0

Rottmann, N., Hansen, D. G., Larsen, P. V., Nicolaisen, A., Flyger, H., Johansen, C., et al. (2015). Dyadic coping within couples dealing with breast cancer: a longitudinal, population-based study. Health Psychol. 34, 486-495. doi: 10.1037/ hea0000218

Semple, C., Parahoo, K., Norman, A., McCaughan, E., Humphris, G., and Mills, M. (2013). Psychosocial interventions for patients with head and neck cancer. Cochrane Database Syst. Rev. 7:CD009441. doi: 10.1002/14651858.CD009441. pub2

Shinn, E. H., Basen-Engquist, K., Baum, G., Steen, S., Bauman, R. F., Morrison, W., et al. (2013). Adherence to preventive exercises and self-reported swallowing outcomes in post-radiation head and neck cancer patients. Head Neck 35, 1707-1712. doi: 10.1002/hed.23255

Siegel, R., Miller, K., and Jemal, A. (2017). Cancer statistics, 2017. CA Cancer J. Clin. 67, 7-30. doi: 10.3322/caac.21387

Traa, M. J., De Vries, J., Bodenmann, G., and Den Oudsten, B. L. (2015). Dyadic coping and relationship functioning in couples coping with cancer: a systematic review. Br. J. Health Psychol. 20, 85-114. doi: 10.1111/bjhp.12094

Trotti, A., Bellm, L. A., Epstein, J. B., Frame, D., Fuchs, H. J., Gwede, C. K., et al. (2003). Mucositis incidence, severity and associated outcomes in patients with head and neck cancer receiving radiotherapy with or without chemotherapy: a systematic literature review. Radiother. Oncol. 66, 253-262. doi: 10.1016/S01678140(02)00404-8

Verdonck-de Leeuw, I. M., Eerenstein, S. E., Van der Linden, M. H., Kuik, D. J., de Bree, R., and Leemans, C. R. (2007). Distress in spouses and patients after treatment for head and neck cancer. Laryngoscope 117, 238-241. doi: 10.1097/ 01.mlg.0000250169.10241.58

Conflict of Interest Statement: The authors declare that the research was conducted in the absence of any commercial or financial relationships that could be construed as a potential conflict of interest.

Copyright $\odot 2018$ Badr, Herbert, Bonnen, Asper and Wagner. This is an open-access article distributed under the terms of the Creative Commons Attribution License (CC BY). The use, distribution or reproduction in other forums is permitted, provided the original author(s) and the copyright owner(s) are credited and that the original publication in this journal is cited, in accordance with accepted academic practice. No use, distribution or reproduction is permitted which does not comply with these terms. 


\section{OPEN ACCESS}

Edited by:

Guy Bodenmann,

Universität Zürich, Switzerland

Reviewed by:

Tanja Zimmermann,

Hannover Medical School, Germany

Steven Beach,

University of Georgia, United States

*Correspondence:

Jean-Philippe Gouin

jp.gouin@concordia.ca

Specialty section:

This article was submitted to

Clinical and Health Psychology,

a section of the journal

Frontiers in Psychology

Received: 31 May 2018 Accepted: 24 September 2018

Published: 16 October 2018

Citation:

Switzer A, Caldwell W, da Estrela C, Barker ET and Gouin J-P (2018) Dyadic Coping, Respiratory Sinus Arrhythmia, and Depressive Symptoms Among

Parents of Preschool Children.

Front. Psychol. 9:1959.

doi: 10.3389/fpsyg.2018.01959

\section{Dyadic Coping, Respiratory Sinus Arrhythmia, and Depressive Symptoms Among Parents of Preschool Children}

\author{
Andrew Switzer, Warren Caldwell, Chelsea da Estrela, Erin T. Barker and \\ Jean-Philippe Gouin*
}

Department of Psychology, Concordia University, Montreal, QC, Canada

Respiratory sinus arrhythmia (RSA) is a biomarker of cardiac vagal tone that has been linked to social functioning. Recent studies suggest that RSA moderates the impact of interpersonal processes on psychosocial adjustment. The goal of this study was to assess whether RSA would moderate the association between dyadic coping (DC) and depressive symptoms. Eighty cohabiting couples raising preschool children completed the Dyadic Coping Inventory, the Center for Epidemiological Study-Depression scale and had their RSA assessed during a laboratory session. Couples completed followup assessments of depressive symptoms 6 and 12 months later. Data were analyzed using an Actor-Partner Interdependence Model. Results indicated that RSA moderated the actor effect of negative DC on depression in men, such that men with lower RSA had a stronger association between their own ratings of negative DC within the couple relationship and their own depressive symptoms, compared to their counterparts with higher RSA. RSA also moderated the partner effect of delegated DC on depressive symptoms. Among men with higher RSA, there was a significant negative association between their partner's ratings of delegated DC within the couple relationship and the men's depressive symptoms, whereas partner-rated delegated DC was unrelated to depressive symptoms among men with lower RSA. These results suggest that men with higher RSA may possess social skills and abilities that attenuate the association between stressful marital interactions and negative mood.

Keywords: dyadic coping, respiratory sinus arrhythmia, heart rate variability, depression, stress, social support

\section{INTRODUCTION}

Stress and coping research has traditionally focused on how individuals react to and are impacted by stress from an individual perspective. The systemic-transactional model of dyadic coping (DC) highlights that for couples, coping occurs in a shared social context characterized by the interdependence of partners' responses to stress (Bodenmann, 1995). In this context, coping is often a dyadic rather than individual endeavor, whereby members of a couple work together to cope with stress. According to this model of DC, when one or both members of a dyad experience stress, both partners engage in a series of reciprocal interactions following an initial communication of stress. These interactions form a DC framework, which can take many forms. Supportive DC 
refers to strategies that assist one's partner in coping with his/her stressors (e.g., providing advice, empathy, etc.). Delegated DC refers to one partner relieving his/her partner of other responsibilities (e.g., housekeeping, shopping, etc.). Common DC refers to joint efforts of a couple to cope with a stressor directly (e.g., joint problem solving, sharing and seeking information together, etc.). These three types of DC are considered positive forms of DC. However, as with individual coping efforts, not all $\mathrm{DC}$ responses are positive. Negative DC includes reacting to a partner's stress communication with indifference, ambivalence, or hostility. The systemic-transactional model stipulates that engagement in positive DC fosters both better adjustment to stress as well as enhanced relationship satisfaction.

A large body of research has reported the benefits of positive DC for relationship functioning. A recent metaanalysis from Falconier et al. (2015) reported a moderate positive correlation $(r=0.45)$ between a couple's positive DC and relationship satisfaction. This association was observed across research methodologies (e.g., cross-sectional, longitudinal, experimental/intervention, different measurement tools), sample characteristics (e.g., culture, SES, education, age, gender), and types of stressors (e.g., chronic illness, child-related stress, etc.). Overall, more positive DC and less negative DC consistently predicted greater relationship satisfaction.

Although DC has been reliably associated with relationship satisfaction, its association with psychological adjustment is less consistent. Nevertheless, positive DC has been associated with improved psychological adjustment in different contexts. Greater positive and common DC predicted less depression in community samples of cohabiting couples (Bodenmann et al., 2011; Gana et al., 2017). Supportive DC moderated the link between discrimination stress and depression among same-sex couples (Randall et al., 2017). Positive DC also appears to promote better adjustment to chronic medical illness: in cancer patients, greater supportive and common DC have been associated with less depression (Badr et al., 2010; Regan et al., 2014; Ernst et al., 2017). Similar results have been found among couples of patients with diabetes, chronic obstructive pulmonary disease, and dementia (Meier et al., 2011; Gellert et al., 2017; Zajdel et al., 2018). Furthermore, greater supportive DC was associated with less parenting stress among parents of children with autism (García-López et al., 2016).

There are, however, some inconsistencies in this literature. The specific forms of DC associated with individual adjustment differ across studies. Rottmann et al. (2015) reported a significant association between common DC and psychological adjustment, but not with supportive DC. Conversely, Gellert et al. (2017) reported significant associations between supportive DC and delegated DC with psychological adjustment, but not with common DC. Furthermore, positive DC has been associated with increased depression and anxiety in certain contexts. For example, cancer patients who reported engaging in more delegated DC had worsened depression (Rottmann et al., 2015). Similarly, among patients with chronic obstructive pulmonary disease, greater delegated DC was associated with poorer quality of life in some (Meier et al., 2011), but not in all studies (Vaske et al., 2015). Moreover, some researchers have reported no significant association between positive DC and depression (Feldman and Broussard, 2006; Breitenstein et al., 2012).

In contrast to positive DC, negative $\mathrm{DC}$ has been more consistently related to poor psychological adjustment. When an individual feels that their communication of stress is being met with indifference, ambivalence, or hostility, they tend to report worsened psychological adjustment. Among couples from the community, greater negative DC was related to higher anxiety and depression (Bodenmann et al., 2011; Karademas and Roussi, 2016). Similarly, negative DC was associated with worsened psychological adjustment among couples facing cancer (Badr et al., 2010; Regan et al., 2014; Rottmann et al., 2015). However, some inconsistencies have also been reported. Gellert et al. (2017) reported no significant relationship between negative DC and depression.

The inconsistencies in the associations between DC and psychological adjustment suggest that individual or situational factors may moderate the impact of DC on individual adjustment. Indeed, there is evidence that not everyone benefits equally from spousal support (Vella et al., 2008; Meuwly et al., 2012; Kordahji et al., 2015). Furthermore, it is likely that not all individuals possess the social skills and abilities required to successfully enact the DC process. DC is an interactional process that involves stress communication by one partner, perception of stress by the other partner, followed by the partner's coping reaction to the stress communication (Bodenmann, 2005). These three components of the transactional cycle require specific skills for effective DC (Bodenmann and Randall, 2012). Individuals who lack the ability to effectively communicate their needs, to recognize their partner's distress, or to regulate their own negative emotions in order to provide effective support may have difficulty enacting an optimal DC process (Bodenmann et al., 2004; Gabriel et al., 2016; Levesque et al., 2017). Individual variations in social engagement capacities may thus moderate the impact of DC on psychological distress.

Respiratory sinus arrhythmia (RSA) is a measure of cardiac vagal tone that has been conceptualized as a biomarker of social engagement capacities (Porges, 2003a, 2007). RSA is calculated from the fluctuations in time intervals between consecutive heartbeats linked to the respiration cycle. At rest, the combined actions of the sympathetic and parasympathetic branches of the autonomic nervous system regulate cardiac activity. Vagaldependent parasympathetic output provides tonic and fast-acting inhibitory influences on cardiac activity that are temporarily lifted during the inspiration phase of the respiration cycle, leading to rapid fluctuations $(0.5 \mathrm{~s})$ in interbeat intervals. In contrast, sympathetic stimulation occurs on a longer timeframe over the course of 1-4 s. Accordingly, RSA, or high-frequency heart rate variability, representing fluctuations in beat-to-beat time intervals, indexes mostly vagally mediated parasympathetic output to the sino-atrial node of the heart (Berntson et al., 1997).

Two major theoretical frameworks link RSA with social functioning. Porges' polyvagal theory proposes that the 
mammalian autonomic nervous system evolved to support social engagement behavior (Porges, 2003a, 2007). The development of parasympathetic modulation of cardiac activity through the vagal nerve allowed for rapid shifts in energy mobilization that facilitated the emergence of social engagement behaviors in response to stress, instead of the more metabolically costly fight-or-flight response (Porges, 2003b). This theory also suggests that throughout vertebrate evolution, structural and functional connections emerged among brain stem nuclei involved in the neural control of cardiac activity, the striated muscles of the face, and the smooth muscles of the viscera. In more evolved mammals, the brain stem nuclei regulating heart rate activity became connected to the soft palate, pharynx, larynx, eyelid, middle ear, and other facial muscles involved in emotional expression and social communication behaviors, allowing for the coordination of physiological and behavioral states supporting social engagement responses (Porges, 2003b). The vagus nerve, linking peripheral physiology and central functions, plays a key role in quickly shifting autonomic states to modulate the repertoire of social and behavioral responses that can be expressed at a given time (Porges, 2003b). RSA is then conceptualized as a biomarker of the neurophysiological system supporting social engagement behavior (Porges, 2007). According to this theory, individuals with lower RSA are more likely to exhibit compromised spontaneous social behavior, social awareness, and emotional expressivity, and they are less physiologically regulated by positive social interactions.

The neurovisceral integration model also proposes that modulation of physiological arousal via the vagal nerve allows rapid and flexible responses to changing environmental demands. This model highlights the neural connections among the vagal nerve and cortical and subcortical brain structures that modulate the inhibitory processes regulating peripheral physiological arousal. Neuroimaging studies indicate that RSA is associated with ventromedial prefrontal cortex, anterior cingulate and amygdala activity, with greater prefrontal cortex activity being linked to higher RSA (Thayer et al., 2012). RSA is thus conceptualized as a physiological marker of topdown neural processes involved in self-regulatory capacity (Thayer and Lane, 2009). In particular, it has been argued that individuals with higher RSA may have better emotion regulation capacities that in turn allow them to maintain high relationship quality despite elevated stress (Diamond et al., 2011).

Tonic or resting RSA has been related to various markers of social functioning. Higher RSA has been associated with more prosocial behavior (Beauchaine et al., 2013), better emotion recognition (Quintana et al., 2012), better empathic accuracy (Côté et al., 2011), less self-reported alexithymia (Lischke et al., 2018), greater compassion (Stellar et al., 2015), less hostility (Sloan et al., 2001), greater attachment security (Diamond and Hicks, 2005; Maunder et al., 2012), better acculturation (Doucerain et al., 2016), and more positive marital functioning (Diamond et al., 2011; Smith et al., 2011; Donoho et al., 2015). RSA has also moderated affective responses to social interactions. Higher resting RSA was associated with a stronger association between social events and positive affect (Isgett et al., 2017).
Among dating couples, women with higher resting RSA showed a larger within-person association between their partner-reported positive couple interactions and their own positive affect, compared to women with lower RSA (Diamond et al., 2011). Individuals with higher resting RSA also exhibited a stronger association between high social support and fewer depressive symptoms over time (Hopp et al., 2013). RSA also moderated the association between maternal depression and expression of negative emotions during a mother-adolescent dyad conflict discussion (Connell et al., 2011). Collectively, these findings provide indirect evidence that RSA may influence the extent to which an individual can benefit from the DC process.

\section{The Present Study}

The primary goal of this study was to assess whether RSA would moderate the association between DC and depressive symptoms. Given that the impact of DC on psychological adjustment may be more salient during a period of increased stress (Cohen and Wills, 1985), this study was conducted among parents of young children, a normative developmental period associated increased with psychosocial stress (Umberson et al., 2010). Indeed, parents of preschool children are more likely to feel overwhelmed by the daily demands and time constraints of caring for young children, and to experience straining workfamily conflict compared to parents of older children (Scharlach, 2001; Nomaguchi and Milkie, 2003). Furthermore, the transition to parenthood is also associated with increased marital conflict and decreased marital satisfaction that may last at least until the children reach school years (Crohan, 1996; Keizer and Schenk, 2012). Given this increased exposure to psychosocial stressors, it is not surprising that a significant percentage of parents of young children experience elevated depressive symptoms that often last throughout the preschool years (Evenson and Simon, 2005; Horwitz et al., 2009; Garfield et al., 2014). This normative developmental period represents a period of time where the role of DC may be especially important in helping individuals adjust to the daily parenting challenges that the couple is facing (García-López et al., 2016; Zemp et al., 2017). In this context, the role of RSA in modulating the effect of DC may be salient. We hypothesized that individuals with higher RSA might benefit more from DC than their counterparts with lower RSA.

\section{MATERIALS AND METHODS}

\section{Participants}

Couples were invited to participate in a study of parenting stress among parents of preschool children. Participants were recruited via online advertisements as well as through schools and support groups for parents of children with developmental disabilities. Parents of children with neurodevelopmental disorders or disabilities (e.g., autism spectrum disorder, intellectual disability, cerebral palsy) were oversampled (21.3\% of dyads), because these parents tend to experience more parenting stress and greater psychological distress than parents of typically developing children (Hayes and Watson, 2013), thereby increasing the range of parenting challenges within the sample. To be included in 
the current study, cohabiting couples were required to be the legal guardian of a child under the age of 7 . Exclusion criteria included pregnancy, breastfeeding, chronic medical conditions, and regular prescribed medication use. These exclusion criteria aimed at minimizing external factors that may impact RSA.

The full sample included 84 heterosexual couples. However, one couple did not complete the DC assessment, for two other couples only one partner completed the DC assessment, and one participant did not complete the depression assessment. The final sample used for the actor-partner interdependence analyses thus included 80 couples. Participants had a mean age of 34.60 $(S D=4.70)$ years, ranging from 21 to 48 , and their children had a mean age of $36(S D=22.74)$ months, ranging from 5 to 89 . In this ethnically diverse sample, $55.36 \%$ of participants identified as Caucasians. About $36.9 \%$ of participants had completed a high school degree or lower level of education, $41.7 \%$ had completed a technical degree, and $21.5 \%$ a university degree. The average household income was $\$ 55,000$ ( $S D=\$ 8900)$ CAD. About $56.5 \%$ of the participants were employed full time, $17.9 \%$ were working part-time, and $25.6 \%$ were not currently working. Couples had been cohabiting for an average of $8.11(S D=3.42)$ years. Most couples had either one (44\%) or two (48\%) children.

\section{Procedure}

Couples first completed online self-report questionnaires assessing DC and depressive symptoms. Subsequently, they completed a 60 -min laboratory visit to assess RSA. During the laboratory visit, couples were seated side-by-side in comfortable chairs and fitted with snap electrodes in a lead II configuration for electrocardiogram (ECG) recording. They participated in several tasks: a 5-min seated and silent resting period where participants were instructed to "breathe normally and relax as much as possible without falling asleep" a 5-min questionnaire about their child's behavior problems was completed independently by each parent; a marital interaction task, in which each dyad member was instructed to take turns leading a 7-min discussion about "the most difficult aspect of raising young children and how it has impacted your relationship with your partner," as well as how they would like their partner "to change regarding the way they raise your child," and a 5-min silent and seated recovery period. Participants remained seated throughout the tasks. A retractable curtain separated the partners during the resting baseline and recovery periods in order to prevent them from interacting with each other during these time periods. An experimenter monitored the couples during the experimental tasks via a control room and prompted them to comply to the instructions when couples deviated from the protocol.

All participants were asked to refrain from consuming caffeine (France and Ditto, 1992), alcohol (Weise et al., 1986), tobacco (Hayano et al., 1990) or engaging in vigorous exercise (Houtveen et al., 2002) in the $2 \mathrm{~h}$ prior to the laboratory session. After the laboratory visit, both members of the couple independently completed a daily diary for 6 consecutive days to assess daily stress. Depressive symptoms were re-assessed using online questionnaires sent via email to participants 6- and 12-months after the laboratory visit. This study was approved by Concordia Human Research Ethics Committee. Each member of the couples provided written informed consent prior to participation. Each couple received \$100 CAD following the completion of the study.

\section{Measures}

Depressive symptoms were assessed using the Center for Epidemiological Study-Depression scale (CES-D). The CES-D assesses the frequency of various depressive symptoms in the past week (e.g., restless sleep, poor appetite, and feeling lonely). Cronbach's $\alpha$ was 0.90 in this sample. The CES-D was administered before the laboratory visit, and 6- and 12-months following the visit. Higher scores indicated more depressive symptoms.

DC was assessed using the Dyadic Coping Inventory (DCI; Bodenmann, 2008). The DCI measures DC responses to stress enacted by oneself, by one's partner, as well as the couple's joint coping efforts using a 5-point Likert scale. In the present study, supportive (Cronbach's $\alpha=0.76$ ), delegated (Cronbach's $\alpha=0.63$ ), negative (Cronbach's $\alpha=0.71$ ) and common DC (Cronbach's $\alpha=0.86$ ) were assessed. Perception of DC by oneself and one's partner were summed for the supportive, delegated, and negative DC subscales. Higher scores indicated more DC from both members of the dyad.

Daily stress was assessed in a daily diary format using three items adapted from the Perceived Stress Scale (Cohen et al., 1983). At the end of each day for 6 consecutive days after the laboratory visit, participants reported to what extent they felt: (1) "that difficulties were piling up"; (2) "overwhelmed"; (3) "that they were able to control important things in their life" (reverse coded), on a 5-point Likert scale ranging from "not at all" to "a great deal." These ratings were combined to create a daily stress measure (Cronbach's $\alpha=0.63$ ) that was subsequently averaged across days to obtain an overall measure of stress for each participant.

Respiratory sinus arrhythmia was measured as part of a 60min recording protocol. Data were collected using an ECG amplifier module within a Mindware BioNex 8-slot chassis (Mindware Technologies Ltd., Gahanna, OH, United States). Interbeat intervals were recorded continuously using a sampling rate of $1000 \mathrm{~Hz}$. The ECG recordings were analyzed using MindWare RSA Analysis software, Version 3.1 (Mindware Technologies LTD., Gahanna, OH, United States). Recording artifacts were identified using an automated algorithm, and were visually inspected and corrected when necessary. Less than $1 \%$ of beats were edited for each participant. RSA was extracted using a Fast Fourier Transform to compute the natural log of the $0.15-0.40 \mathrm{~Hz}$ frequency band in order to isolate vagal-dependent parasympathetic influences on the heart. RSA was calculated by averaging the RSA value for each 30 -s epoch across each task. The average RSA level across all tasks was used as an overall marker of vagal tone.

\section{Statistical Analyses}

First, Spearman Rho's correlations evaluated bivariate associations among actor depression, actor and partner DC, and actor RSA. An actor-partner interdependence model (APIM; Kenny et al., 2006) using multilevel modeling estimated associations between actor- and partner-rated DC and depressive 
symptoms, as well as the moderating impact of RSA and gender. The APIM model allowed for the simultaneous assessment of actor effects (e.g., the association between the wife's own ratings of DC within the couple relationship and the wife's own ratings of depressive symptoms) as well as partner effects (e.g., the association between the husband's rating of DC within the couple relationship and the wife's own rating of depression symptoms), while accounting for the within-couple dependency in the data structure. Preliminary analyses indicated that there was a significant increase in depressive symptoms for men over time, $\beta(S E)=0.18(0.07), t=2.73, p=0.007$, but not for women, $\beta(S E)=0.07$ (0.09), $t=0.79, p=0.43$. Given the lack of change in depressive symptoms over time for women, the averaged depression scores across each of the three time points for each partner were used as the dependent variable in order to model the effects of DC on depressive symptoms simultaneously for men and women. In the current sample, the dyads were distinguishable (i.e., each dyad included a male and female partner). We used the two-intercept approach in order to simultaneously calculate separate equations for men and women (Kenny et al., 2006). A heterogeneous compound symmetry $(\mathrm{CSH})$ covariance structure allowed for the estimation of unique variances of each dyad member. The moderating effect of RSA on the association between DC and depressive symptoms was tested using two-way interactions between RSA and DC. Following statistically significant interactions, simple slopes analyses were conducted by plotting the change in strength of the relationship between DC and depressive symptoms at two levels of the moderator, RSA ( 1 SD above and -1 SD below the mean).

All continuous variables were centered. RSA was normally distributed, but depressive symptoms showed a positively skewed distribution, which was corrected using a base 10 logarithmic transformation. The pattern of results did not change substantially when transformed variables were used. Analyses were run with transformed data, but untransformed analyses were plotted for greater interpretability. Given the inconsistencies in the associations between the different forms of DC and depression, separate models were run for each type of DC (Rottmann et al., 2015; Gellert et al., 2017). Having a child with a neurodevelopmental disorder was included as a covariate in each model given that these parents usually report greater psychological distress than parents of typically developing children (Olsson and Hwang, 2001). An alpha level of 0.05 was used for the present study. SAS PROC MIXED was used to perform multilevel modeling with restricted maximum likelihood estimation.

\section{RESULTS}

Correlations among the main study variables are presented in Table 1. Depressive symptoms were significantly positively correlated between cohabiting partners with a small effect size, $r=0.26, p=0.03$. Actor- and partner-ratings of DC were also positively correlated with medium effect sizes, ranging from $r=0.34$ to $r=0.57$. In contrast, there was no statistically significant correlation between partners' RSA, $r=0.08, p=0.48$.

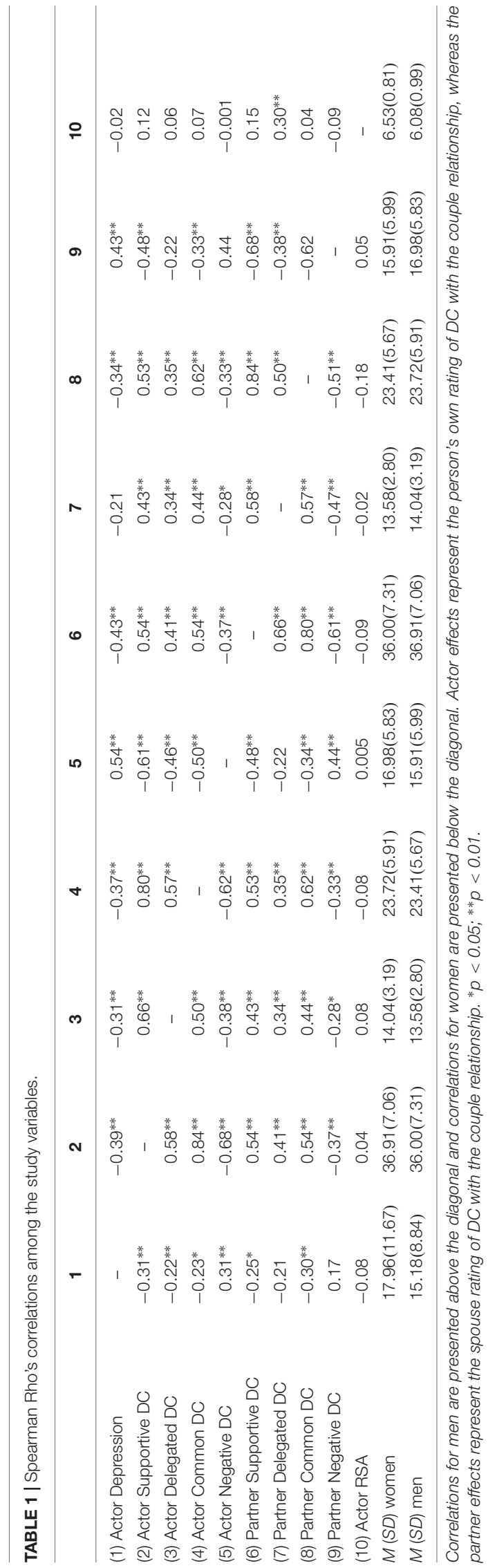


In this sample, there were no statistically significant gender differences in depressive symptoms, $t=1.54, p=0.13$, or DC, all $p$ 's $>0.25$. However, RSA was significantly higher among women than among men, $t=2.68, p=0.008$.

A series of models tested whether there were significant actor and partner effects of DC on depressive symptoms. Moderation effects were also tested using two-way interaction terms between actor- and partner-rated DC and actor RSA. In these models, being a parent of a child with a neurodevelopmental disorder and daily stress were added as covariates. Mothers of a child with a neurodevelopmental disorder reported significantly more depressive symptoms than mothers of a typically developing child, $\beta(S E)=0.22(0.07), t=3.35, p=0.001$, whereas fathers of a child with a neurodevelopmental disorder had marginally higher depressive symptoms, compared to fathers of a typically developing child, $\beta$ (SE) $=0.11(0.06), t=1.80, p=0.08$.

Table 2 presents the actor and partner DC effects as well as their interactions with actor's RSA for men. Results indicated that, for men, there were significant actor effects of delegated
DC and negative DC as well as a marginally significant actor effect of supportive DC. Significant partner effects of supportive DC and negative DC were also observed. Greater supportive and delegated DC were associated with less depressive symptoms, whereas more negative DC was related to greater depressive symptoms. There was also a marginally significant effect of daily stress, with greater daily stress being marginally associated with more depressive symptoms. Furthermore, actor's RSA significantly moderated the effect of partner-rated delegated DC on depressive symptoms. Simple slope analyses indicated that there was a significant negative association between partner-rated delegated DC and actor's depression among men with higher RSA but a non-significant positive association between the two constructs among participants with lower RSA. The interaction between partner-rated delegated DC and depressive symptoms is depicted in Figure 1. Furthermore, there was a significant interaction between actor-rated negative DC and actor RSA. Simple slope analyses indicated that the association between actor-rated negative DC and actor's depression was stronger

TABLE 2 | Actor and partner effects of DC on depression and moderation effects of RSA for men.

\begin{tabular}{|c|c|c|c|c|}
\hline & $\beta$ (SE) & $t$ & $p$ & $95 \% \mathrm{Cl}$ \\
\hline Actor DC & $-007(0.004)$ & -1.96 & $0.05^{\tau}$ & {$[-0.01,0.0001]$} \\
\hline Daily Stress & $0.01(0.007)$ & 1.94 & $0.06^{\tau}$ & {$[-0.003,0.03]$} \\
\hline RSA & $0.07(0.13)$ & 1.42 & 0.16 & {$[-0.10,0.60]$} \\
\hline Actor DC $\times$ RSA & $0.001(0.004)$ & 0.44 & 0.66 & {$[-0.007,0.01]$} \\
\hline Daily Stress $\times$ RSA & $-0.008(0.007)$ & -1.14 & 0.26 & {$[-0.02,0.006]$} \\
\hline \multicolumn{5}{|l|}{ Delegated DC } \\
\hline Actor DC & $-0.03(0.01)$ & -2.85 & $0.006^{*}$ & {$[-0.05,-0.01]$} \\
\hline Partner DC & $-0.005(0.009)$ & -0.56 & 0.57 & {$[-0.02,0.02]$} \\
\hline Daily Stress & $0.01(0.007)$ & 1.76 & $0.08^{\tau}$ & {$[-0.002,0.03]$} \\
\hline RSA & $0.38(0.17)$ & 2.16 & $0.03^{*}$ & {$[0.03,0.72]$} \\
\hline \multicolumn{5}{|l|}{ Common DC } \\
\hline Actor DC & $-0.006(0.007)$ & -0.89 & 0.38 & {$[-0.02,0.008]$} \\
\hline Partner DC & $-0.009(0.008)$ & -1.22 & 0.23 & {$[-0.02,0.006]$} \\
\hline Daily Stress & $0.01(0.007)$ & 1.83 & $0.07^{\tau}$ & {$[-0.001,0.03]$} \\
\hline RSA & $0.24(0.17)$ & 1.44 & 0.15 & {$[-0.09,0.58]$} \\
\hline Actor $\mathrm{DC} \times \mathrm{RSA}$ & $0.0001(0.009)$ & 0.006 & 0.99 & {$[-0.02,0.02]$} \\
\hline Partner DC $\times$ RSA & $-0.007(0.007)$ & -0.97 & 0.34 & {$[-0.02,0.01]$} \\
\hline Daily Stress $\times$ RSA & $-0.01(0.008)$ & -1.26 & 0.21 & {$[-0.02,0.006]$} \\
\hline \multicolumn{5}{|l|}{ Negative DC } \\
\hline Actor DC & $0.01(0.003)$ & 3.66 & $<0.001^{* *}$ & {$[0.006,0.02]$} \\
\hline Partner DC & $0.01(0.004)$ & 2.34 & $0.02^{*}$ & {$[0.001,0.02]$} \\
\hline
\end{tabular}

${ }^{\tau} p<0.10 ; * p<0.05 ; * * p<0.001$. 


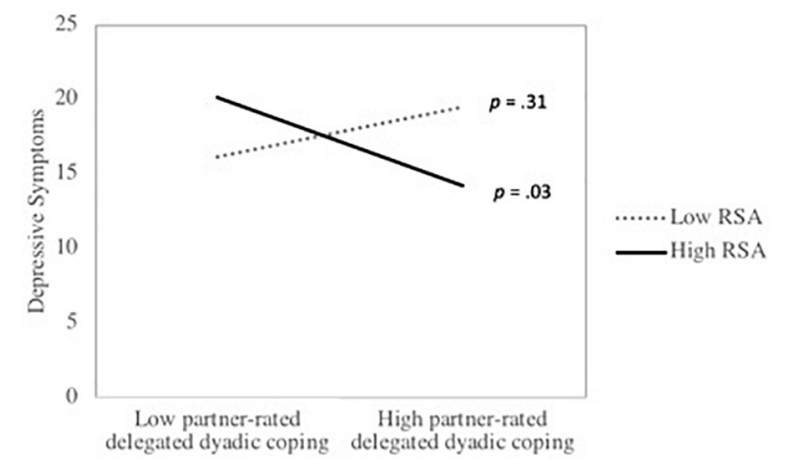

FIGURE 1 | The association between partner-rated delegated DC and depressive symptoms as a function of respiratory sinus arrhythmia (RSA) among men. $p$-values represent the significance of the simple slopes at high (1 SD above the mean) and low (1 SD below the mean) of RSA.

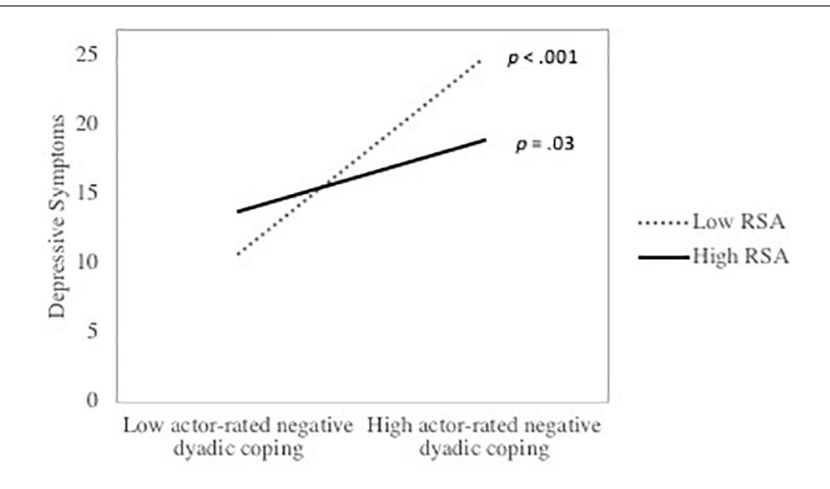

FIGURE 2 | The association between actor-rated negative DC and depressive symptoms as a function of respiratory sinus arrhythmia (RSA) among men. $p$ values represent the significance of the simple slopes at high (1 SD above the mean) and low (1 SD below the mean) of RSA.

among participants with lower RSA than among individuals with higher RSA. Figure 2 illustrates the interaction between actorrated negative DC and actor's RSA predicting actor's depressive symptoms.

Table 3 presents the actor and partner effects as well as their interactions with actor's RSA for women. Results indicated that there was a marginally significant actor effect of negative DC and a marginally significant partner effect of delegated DC on depressive symptoms. None of the moderation effects with RSA were significant. However, there was a significant or marginally significant daily stress by RSA interaction in three out of the four models. Simple slopes analysis indicated that among mothers with lower RSA there was a significant association between daily stress and depressive symptoms, and that the association was not significant among mothers with higher RSA. These results are depicted in Figure 3. To test whether the strength of the association between DC and depressive symptoms significantly differed for men and women, two-way interactions between actor and partner DC effects and gender were computed. None of the $\mathrm{DC} \times$ gender interactions were significant, all $p$ 's $>0.15$. Gender differences in the moderation effects of RSA were tested using three-way interactions among gender, DC, and RSA. None of the three-way interactions were significant, all $p$ 's $>0.24$.

\section{DISCUSSION}

The goal of this study was to evaluate whether RSA moderates the association between DC and depressive symptoms among parents of preschool children. Results indicated that actor's RSA moderated the partner effect of delegated DC as well as the actor effect of negative DC in predicting the actor's depressive symptoms. Men with higher RSA exhibited a stronger negative association between their female partner-rated delegated DC within the couple relationship and their own depressive symptoms, but a smaller association between their own rating of negative DC within the couple relationship and their own depressive symptoms compared to their counterparts with lower RSA. These findings suggest that men with higher RSA may possess social skills and abilities that support their DC skills.

In the current study, negative DC was the form of DC that showed the strongest association with depressive symptoms. This finding is consistent with results from prior studies (Bodenmann et al., 2011; Regan et al., 2014). Results indicated that RSA buffered the effect of negative DC on depressive symptoms among men, such that men with lower RSA displayed a larger association between negative DC and depression than participants with higher RSA. These results dovetail with findings from Diamond et al. (2011), who reported that men with lower RSA showed a stronger association between daily negative affect and daily negative spousal interaction. In another study, high RSA buffered the association between maternal depression and negative affect escalation during a mother-adolescent interaction task (Connell et al., 2011). These results are broadly consistent with the hypothesis that individuals with higher RSA might be better able to regulate their emotions in the face of negative social interactions (Thayer and Lane, 2009; Diamond et al., 2011), thereby diminishing the impact of negative DC interactions on their depressive symptoms.

In prior studies, delegated DC has been associated with both increased and decreased depression (Meier et al., 2011; Vaske et al., 2015). In the present study, RSA moderated the effects of partner-rated delegated DC on depressive symptoms. Among men, higher RSA was associated with a significant negative association between partner-rated delegated DC and depression, whereas there was a positive, but non-significant association between delegated DC and depressive symptoms when RSA was lower. Men with higher RSA thus benefited more from partner-rated delegated DC than men with lower RSA. These results are in line with those of Hopp et al. (2013) who reported that greater social support was associated with fewer depressive symptoms among individuals with higher RSA, whereas there was no association between social support and depression among individuals with lower RSA. Individuals with higher RSA may be more physiologically regulated by positive social interactions than their counterparts with lower RSA (Porges, 2003a), leading to a stronger stress-buffering effect of positive interpersonal relationships. However, it has also been suggested 
TABLE 3 | Actor and partner effects of DC on depression and moderation effects of RSA for women.

\begin{tabular}{|c|c|c|c|c|}
\hline & $\beta$ (SE) & $t$ & $p$ & $95 \% \mathrm{Cl}$ \\
\hline Actor DC & $-0.003(0.005)$ & -0.64 & 0.53 & {$[-0.01,0.006]$} \\
\hline Daily Stress & $0.01(0.007)$ & 1.90 & $0.06^{\tau}$ & {$[-0.001,0.03]$} \\
\hline RSA & $0.23(0.26)$ & -0.89 & 0.37 & {$[-0.28,0.75]$} \\
\hline Actor DC $\times$ RSA & $-0.004(0.005)$ & -0.79 & 0.43 & {$[-0.01,0.006]$} \\
\hline Daily Stress $\times$ RSA & $-0.02(0.009)$ & 1.90 & $0.06^{\tau}$ & {$[-0.03,0.0008]$} \\
\hline \multicolumn{5}{|l|}{ Delegated DC } \\
\hline Actor DC & $0.004(0.01)$ & -0.48 & 0.64 & {$[-0.01,0.02]$} \\
\hline Partner DC & $-0.02(0.01)$ & -1.94 & $0.06^{\tau}$ & {$[-0.04,0.001]$} \\
\hline Daily Stress & $0.01(0.007)$ & 1.86 & $0.07^{\tau}$ & {$[-0.001,0.03]$} \\
\hline RSA & $0.27(0.23)$ & -0.16 & 0.25 & {$[-0.20,0.75]$} \\
\hline \multicolumn{5}{|l|}{ Common DC } \\
\hline Actor DC & $-0.005(0.008)$ & -0.63 & 0.53 & {$[-0.02,0.01]$} \\
\hline Partner DC & $-0.006(0.009)$ & -0.71 & 0.48 & {$[-0.02,0.01]$} \\
\hline Daily Stress & $0.01(0.008)$ & 1.91 & $0.06^{\tau}$ & {$[-0.001,0.03]$} \\
\hline RSA & $0.26(0.23)$ & 1.10 & 0.28 & {$[-0.21,0.73]$} \\
\hline Actor DC $\times$ RSA & $-0.009(0.008)$ & -1.16 & 0.25 & {$[-0.02,0.006]$} \\
\hline Partner DC $\times$ RSA & $0.007(0.009)$ & -0.71 & 0.48 & {$[-0.01,0.02]$} \\
\hline Daily Stress $\times$ RSA & $-0.02(0.008)$ & -2.04 & $0.04^{*}$ & {$[-0.04,-0.0004]$} \\
\hline \multicolumn{5}{|l|}{ Negative $D C$} \\
\hline Actor DC & $0.01(0.006)$ & 1.84 & $0.07^{\tau}$ & {$[-0.001,0.02]$} \\
\hline Partner DC & $0.001(0.005)$ & 0.26 & 0.79 & {$[-0.009,0.01]$} \\
\hline
\end{tabular}

${ }^{\tau} p<0.10 ; * p<0.05$.

that delegated DC can overburden the partner in situations of high stress (Rottmann et al., 2015). Individuals with low RSA may possess less self-regulatory resources, which leaves them more easily overburdened by delegated DC, leading to an increase in depressive symptoms associated with delegated DC.

In the present study, RSA moderated the association between DC and depressive symptoms among men, but not among women. Diamond et al. (2011) reported that RSA moderated the association between negative affect and negative marital interaction among men, whereas it moderated the association between positive affect and positive interactions among women. Given that our measure of depression focused mostly on negative affect, this may explain why the findings were significant only for men. However, these gender differences should not be overinterpreted, given that the effects for men and women were not statistically different from each other. In a similar vein, RSA interacted with daily stress to predict depressive symptoms among women but not among men. The association between daily stress and depression was stronger among women with lower RSA. These results dovetail with prior results suggesting that RSA is marker of vulnerability to stress (Fabes and Eisenberg, 1997; Gouin et al., 2014).

In the DC literature, gender differences in the association between DC and psychological distress are not consistent. Although Bodenmann et al. (2011) reported that positive DC was associated with depression in women, but not in men, Chaves et al. (2018) observed that positive DC was associated with psychological distress in men but not in women. Furthermore, many studies found similar bivariate associations between DC and depression among men and women (e.g., Gabriel et al., 2016; Karademas and Roussi, 2016). In the present study, positive forms of DC were associated with depressive symptoms among men, but not among women; however, the beta coefficients between DC and depressive symptoms were in the same direction for both sexes. This suggests that a lack of statistical power may explain why these effects were not significant for women.

The association between DC and depressive symptoms is bidirectional. Greater DC can reduce depressive symptoms, but 


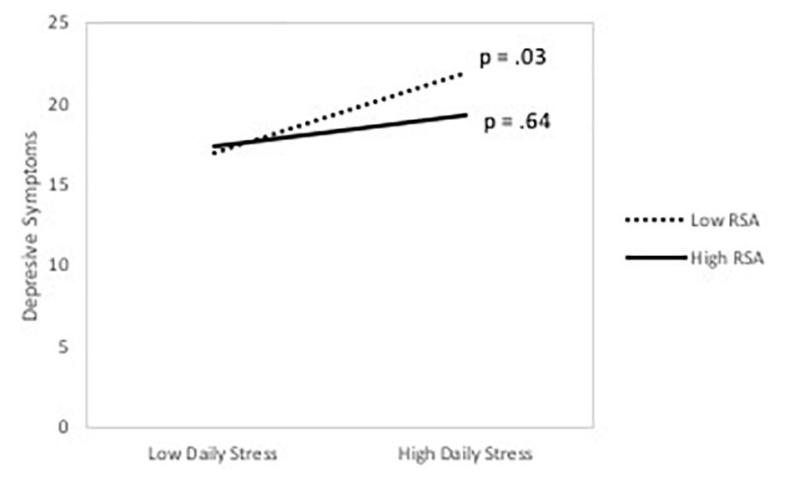

FIGURE 3 | The association between daily stress and depressive symptoms as a function of respiratory sinus arrhythmia (RSA) among women. $p$-values represent the significance of the simple slopes at high (1 SD above the mean) and low (1 SD below the mean) of RSA.

elevated depression may also erode DC (Bodenmann et al., 2004; Gana et al., 2017). Although depressive symptoms were assessed at three time points over a 12-month period in this study, there was no significant change in depressive symptoms over time for women. Therefore, the average level of depressive symptoms across the 3 times points for each partner was used in the analyses. In the context of these cross-sectional analyses, the directionality of the association between DC and depressive symptoms cannot be determined. This means that an alternative and equally plausible interpretation of these findings is that individuals with low RSA engaged in more negative DC when they experience elevated depressive symptoms compared to their counterparts with high RSA. Furthermore, individuals with high RSA may be more likely to have a partner who engaged in high delegated DC when they report low depressive symptoms. Longitudinal studies with longer follow-ups that may capture changes in both depressive symptoms and DC as they occur may help clarify the directionality of the association between these two constructs.

In this study on parenting stress of parents of preschool children, couples exhibited high levels of depressive symptoms. Indeed, the mean CES-D score was close to the clinical cutoff for risk of a major depressive disorder. Several factors may explain this high level of psychological distress. Couples rearing a child with a neurodevelopmental disorder were oversampled for this study, and these parents tend to experience higher levels of psychological distress than other parents (Olsson and Hwang, 2001). Couples who participated in this study had less education and lower household incomes than the average person in Montreal, QC, Canada (Statistics Canada, 2017). Notably, 25\% of the couples lived on or below the poverty line. Higher financial strains increase risk for depression among parents of young children (Heneghan et al., 1998). Moreover, our recruitment strategy advertising a study of parenting stress may have led to a sampling bias whereby couples who experienced higher levels of parenting stress were preferentially recruited into the study. While this sampling bias limits the generalizability of the present findings, it also highlights the role of DC in the context of elevated psychological distress. Future studies should replicate these findings in samples that are more representative of the general population. Another limitation of this study is the large number of statistical tests conducted. Given the inconsistencies in the associations between the different forms of DC and depression, and the high correlations among positive DC subscales (ranging from 0.58 - 0.84), the analyses were run in multiple models to avoid multicollinearity issues. If a family wise Bonferroni correction was applied to the findings, the moderation effect of RSA on partner-rated delegated DC would no longer be statistically significant. Furthermore, the reliability of the delegated DC subscale was relatively low in this sample. These results must therefore be interpreted cautiously, and replication of these findings is paramount.

In the current paper, an APIM framework was adopted to examine both partners' contribution to each partner's depression. The Dyadic Coping Inventory assessed the construct of DC both from an individual perspective (e.g., I show empathy and understanding to my partner) as well as from a couple perspective (e.g., We engage in serious discussion about the problem and think through what has to be done). Rather than examining how both partners' perception of DC is related to each partner's depression, one could conceptualize DC as a couple-level variable shared by both partners. Using this perspective, a Common Fate Model would be an alternative way to analyze these data (Kenny and La Voie, 1985; Lederman and Kenny, 2012). Future research should evaluate whether the association between DC and depression differ when it is conceptualized as an individual or as a dyad level variable.

These findings suggest several potential future research directions. Both the polyvagal theory and the neurovisceral integration models suggest specific mechanisms through which RSA may moderate the effect of DC (e.g., reduced emotional expressiveness, impaired emotion regulation, increased sensitivity to the social context); future studies should attempt to identify the specific pathways underlying these effects. Furthermore, interventions aimed at improving DC have been associated with improvements in depressive symptoms similar to those of cognitive-behavioral therapy and interpersonal therapy (Bodenmann et al., 2008). Future research should test whether RSA may help identify individuals with depression who are more likely to benefit from a DC-focused intervention. DC has also been related to stress-related physiological processes (Meuwly et al., 2012; Kordahji et al., 2015; Gouin et al., 2016). Future studies should test whether RSA also moderates the impact of DC on physiological stress responses.

In terms of clinical implications, these results provide further evidence that promoting DC may reduce depressive symptoms (Bodenmann et al., 2008), especially among fathers of young children. Low RSA may also help identify men who are more vulnerable to the impact of negative DC on depressive symptoms. However, in order to apply these results in clinical practice, future studies should aim at establishing norms and cut-off scores of RSA that will facilitate the identification of at risk individuals.

In summary, these findings indicate that RSA moderated the association between DC and depressive symptoms. High RSA 
buffered the association between negative DC and depression and enhanced the association between delegated DC and depressive symptoms. These results provide further evidence that RSA modulates affective responses to social interactions. More studies are needed to examine the specific pathways through which RSA influences the DC process.

\section{AUTHOR CONTRIBUTIONS}

J-PG and EB designed the study. CdE and WC collected and processed the data. J-PG analyzed the data. AS and J-PG wrote the manuscript. All authors reviewed and approved the final version of the manuscript.

\section{REFERENCES}

Badr, H., Carmack, C. L., Kashy, D. A., Cristofanilli, M., and Revenson, T. A. (2010). Dyadic coping in metastatic breast cancer. Health Psychol. 29, 169-180. doi: 10.1037/a0018165

Beauchaine, T. P., Gatzke-Kopp, L., Neuhaus, E., Chipman, J., Reid, M. J., and Webster-Stratton, C. (2013). Sympathetic- and parasympatheticlinked cardiac function and prediction of externalizing behavior, emotion regulation, and prosocial behavior among preschoolers treated for ADHD. J. Consult. Clin. Psychol. 81, 481-493. doi: 10.1037/a003 2302

Berntson, G., Thomas Bigger, J., Eckberg, D. L., Grossman, P., Kaufmann, P., Malik, M., et al. (1997). Heart rate variability: origins, methods, and interpretive caveats. Psychophysiology 34, 623-648. doi: 10.1111/j.1469-8986.1997. tb02140.x

Bodenmann, G. (1995). A systemic-transactional conceptualization of stress and coping in couples. Swiss J. Psychol. 54, 34-49.

Bodenmann, G. (2005). "Dyadic coping and its significance for marital functioning," in Couples Coping with Stress: Emerging Perspectives on DC, ed. G. Bodenmann (Washington, DC: American Psychological Association), 33-49. doi: 10.1037/11031-002

Bodenmann, G. (2008). Dyadisches Coping Inventar: Testmanual [Dyadic Coping Inventory: Test manual]. Bern: Huber.

Bodenmann, G., Charvoz, L., Widmer, K., and Bradbury, T. N. (2004). Differences in individual and dyadic coping among low and high depressed, partially remitted, and nondepressed persons. J. Psychopathol. Behav. Assess. 26, 75-85. doi: 10.1023/B:JOBA.0000013655.45146.47

Bodenmann, G., Meuwly, N., and Kayser, K. (2011). Two conceptualizations of dyadic coping and their potential for predicting relationship quality and individual well-being: a comparison. Eur. Psychol. 16, 255-266. doi: 10.1027/ 1016-9040/a000068

Bodenmann, G., Plancherel, B., Beach, S. R. H., Widmer, K., Gabriel, B., Meuwly, N., et al. (2008). Effects of coping-oriented couples therapy on depression: a randomized clinical trial. J. Consult. Clin. Psychol. 76, 944-954. doi: $10.1037 / \mathrm{a} 0013467$

Bodenmann, G., and Randall, A. K. (2012). Common factors in the enhancement of dyadic coping. Behav. Therapy 43, 88-98. doi: 10.1016/j.beth.2011. 04.003

Breitenstein, C., Milek, A., and Bodenmann, G. (2012). "Dyadic Coping, selfesteem and depressed mood in adolescents' romantic relationships," in Proceedings of the Annual congress of the European. (Association)for Behavioral and Cognitive Therapies (EABCT), Geneva.

Statistics Canada (2017). Montréal [Census metropolitan area], Quebec and Canada [Country] (table). Census Profile, 2016 - Montréal. Available at: http://www12.statcan.gc.ca/census-recensement/2016/dp-pd/prof/details/ page.cfm?Lang $=$ E $\&$ Geo $1=$ CMACA $\&$ Code $1=462 \& G e o 2=$ PR $\&$ Code $2=01 \&$ Data $=$ Count $\&$ SearchText $=$ Montreal $\&$ SearchType $=$ Begins $\&$ SearchPR $=01 \& B 1=$ Education\&TABID $=1$

\section{FUNDING}

This study was funded by a Social Sciences and Humanities Research Council of Canada Insight Development Grant and a Canada Research Chair Grant awarded to J-PG.

\section{ACKNOWLEDGMENTS}

The authors would like to thank Melanie Brouillard, Kim Desmarais, Catherine Hudon, Veronica Kost, Nadya Long, Sasha MacNeil, Cam Matamoros, Monique Riedel, and Sabrina Scarcello for assistance in data collection. The authors would also like to thank all the families who participated in this research.

Chaves, C., Canavarro, M. C., and Moura-Ramos, M. (2018). The role of dyadic coping on the marital and emotional adjustment of couples with infertility. Fam. Process. doi: 10.1111/famp.12364 [Epub ahead of print].

Cohen, S., Kamarck, T., and Mermelstein, R. (1983). A global measure of perceived stress. J. Health Soc. Behav. 24, 386-396. doi: 10.2307/2136404

Cohen, S., and Wills, T. A. (1985). Stress, social support, and the buffering hypothesis. Psychol. Bull. 98, 310-357. doi: 10.1037/0033-2909.98.2.310

Connell, A. M., Hughes-Scalise, A., Klostermann, S., and Azem, T. (2011). Maternal depression and the heart of parenting: respiratory sinus arrhythmia and affective dynamics during parent-adolescent interactions. J. Fam. Psychol. 25, 653-662. doi: 10.1037/a0025225

Côté, S., Kraus, M. W., Cheng, B. H., Oveis, C., van der Löwe, I., Lian, H., et al. (2011). Social power facilitates the effect of prosocial orientation on empathic accuracy. J. Pers. Soc. Psychol. 101, 217-232. doi: 10.1037/a0023171

Crohan, S. E. (1996). Marital quality and conflict across the transition to parenthood in African American and white couples. J. Marriage Fam. 58, 933-944. doi: 10.2307/353981

Diamond, L. M., and Hicks, A. M. (2005). Attachment style, current relationship security, and negative emotions: the mediating role of physiological regulation. J. Soc. Pers. Relationsh. 22, 499-518. doi: 10.1177/026540750505 4520

Diamond, L. M., Hicks, A. M., and Otter-Henderson, K. D. (2011). Individual differences in vagal regulation moderate associations between daily affect and daily couple interactions. Pers. Soc. Psychol. Bull. 37, 731-744. doi: 10.1177/ 0146167211400620

Donoho, C. J., Seeman, T. E., Sloan, R. P., and Crimmins, E. M. (2015). Marital status, marital quality, and heart rate variability in the MIDUS cohort. J. Fam. Psychol. 29, 290-295. doi: 10.1037/fam0000068

Doucerain, M. M., Deschênes, S. S., Aubé, K., Ryder, A. G., and Gouin, J.-P. (2016). Respiratory sinus arrhythmia is prospectively associated with early trajectories of acculturation among new international students. J. Cross Cult. Psychol. 47, 421-440. doi: 10.1177/0022022115624015

Ernst, J., Hinz, A., Niederwieser, D., Döhner, H., Hönig, K., Vogelhuber, M., et al. (2017). Dyadic coping of patients with hematologic malignancies and their partners and its relation to quality of life - a longitudinal study. Leuk. Lymphoma 58, 655-665. doi: 10.1080/10428194.2016.1194983

Evenson, R. J., and Simon, R. W. (2005). Clarifying the relationship between parenthood and depression. J. Health Soc. Behav. 46, 341-358. doi: 10.1177/ 002214650504600403

Fabes, R. A., and Eisenberg, N. (1997). Regulatory control and adults' stress-related responses to daily life events. J. Pers. Soc. Psychol. 73, 1107-1117. doi: 10.1037/ 0022-3514.73.5.1107

Falconier, M. K., Jackson, J. B., Hilpert, P., and Bodenmann, G. (2015). Dyadic coping and relationship satisfaction: a meta-analysis. Clin. Psychol. Rev. 42, 28-46. doi: 10.1016/j.cpr.2015.07.002

Feldman, B. N., and Broussard, C. A. (2006). Men's adjustment to their partners' breast cancer: a dyadic coping perspective. Health Soc. Work 31, 117-127. doi: $10.1093 / \mathrm{hsw} / 31.2 .117$ 
France, C., and Ditto, B. (1992). Cardiovascular responses to the combination of caffeine and mental arithmetic, cold pressor, and static exercise stressors. Psychophysiology 29, 272-282. doi: 10.1111/j.1469-8986.1992.tb01698.x

Gabriel, B., Untas, A., Lavner, J. A., Koleck, M., and Luminet, O. (2016). Gender typical patterns and the link between alexithymia, dyadic coping and psychological symptoms. Pers. Individ. Differ. 96, 266-271. doi: 10.1016/j.paid. 2016.02.029

Gana, K., Saada, Y., Broc, G., Koleck, M., and Untas, A. (2017). Dyadic crosssectional associations between depressive mood, relationship satisfaction, and common dyadic coping. Marriage Fam. Rev. 53, 532-555. doi: 10.1080/ 01494929.2016.1247759

García-López, C., Sarriá, E., Pozo, P., and Recio, P. (2016). Supportive dyadic coping order: the role of relationship satisfaction. J. Autism. Dev. Disord. 46, 3434-3447. doi: 10.1007/s10803-016-2883-5

Garfield, C. F., Duncan, G., Rutsohn, J., McDade, T. W., Adam, E. K., Coley, R. L., et al. (2014). A longitudinal study of paternal mental health during transition to fatherhood as young adults. Pediatrics 133 , 836-843. doi: 10.1542/peds.20133262

Gellert, P., Häusler, A., Gholami, M., Rapp, M., Kuhlmey, A., and Nordheim, J. (2017). Own and partners' dyadic coping and depressive symptoms in individuals with early-stage dementia and their caregiving partners. Aging Ment. Health. doi: 10.1080/13607863.2017.1334759 [Epub ahead of print].

Gouin, J.-P., Deschênes, S. S., and Dugas, M. J. (2014). Respiratory sinus arrhythmia during worry forecasts stress-related increases in psychological distress. Stress 17, 416-422. doi: 10.3109/10253890.2014.949666

Gouin, J.-P., Scarcello, S., da Estrela, C., Paquin, C., and Barker, E. T. (2016). Dyadic coping and inflammation in the context of chronic stress. Health Psychol. 35, 1081-1084. doi: 10.1037/hea0000395

Hayano, J., Yamada, M., Sakakibara, Y., Fujinami, T., Yokoyama, K., Watanabe, Y., et al. (1990). Short- and long-term effects of cigarette smoking on heart rate variability. Am. J. Cardiol. 65, 84-88. doi: 10.1016/0002-9149(90)90030-5

Hayes, S. A., and Watson, S. L. (2013). The impact of parenting stress: a metaanalysis of studies comparing the experience of parenting stress in parents of children with and without autism spectrum disorder. J. Autism. Dev. Disord. 43, 629-642. doi: 10.1007/s10803-012-1604-y

Heneghan, A. M., Silver, E. J., Bauman, L. J., Westbrook, L. E., and Stein, R. E. (1998). Depressive symptoms in inner-city mothers of young children: who is at risk? Pediatrics 102, 1394-1400.

Hopp, H., Shallcross, A. J., Ford, B. Q., Troy, A. S., Wilhelm, F. H., and Mauss, I. B. (2013). High cardiac vagal control protects against future depressive symptoms under conditions of high social support. Biol. Psychol. 93, 143-149. doi: 10.1016/j.biopsycho.2013.01.004

Horwitz, S. M., Briggs-Gowan, M. J., Storfer-Isser, A., and Carter, A. S. (2009). Persistence of maternal depressive symptoms throughout the early years of childhood. J. Women's Health 18, 637-645. doi: 10.1089/jwh.2008.1229

Houtveen, J. H., Rietveld, S., and Geus, E. J. C. (2002). Contribution of tonic vagal modulation of heart rate, central respiratory drive, respiratory depth, and respiratory frequency to respiratory sinus arrhythmia during mental stress and physical exercise. Psychophysiology 39, 427-436. doi: 10.1111/1469-8986. 3940427

Isgett, S. F., Kok, B. E., Baczkowski, B. M., Algoe, S. B., Grewen, K. M., and Fredrickson, B. L. (2017). Influences of oxytocin and respiratory sinus arrhythmia on emotions and social behavior in daily life. Emotion 17, 11561165. doi: 10.1037/emo0000301

Karademas, E. C., and Roussi, P. (2016). Financial strain, dyadic coping, relationship satisfaction, and psychological distress: a dyadic mediation study in Greek couples. Stress Health 33, 508-517. doi: 10.1002/smi.2735

Keizer, R., and Schenk, N. (2012). Becoming a parent and relationship satisfaction: a longitudinal dyadic perspective. J. Marriage Fam. 74, 759-773. doi: 10.1111/j. 1741-3737.2012.00991.x

Kenny, D. A., Kashy, D. A., and Cook, W. L. (2006). Dyadic Data Analysis (Methodology in the Social sciences). New York, NY: Guilford.

Kenny, D. A., and La Voie, L. (1985). Separating individual and group effects. J. Pers. Soc. Psychol. 48, 339-348. doi: 10.1037/0022-3514.48.2.339

Kordahji, H., Bar-kalifa, E., and Rafaeli, E. (2015). Attachment insecurity as a moderator of cardiovascular arousal effects following dyadic support. J. Res. Pers. 57, 89-99. doi: 10.1016/j.jrp.2015.04.004
Lederman, T., and Kenny, D. A. (2012). The common fate model for dyadic data: variations of a theoretically important but underutilized model. J. Fam. Psychol. 26, 140-148. doi: 10.1037/a0026624

Levesque, C., Lafontaine, M.-F., and Bureau, J.-F. (2017). The mediating effects of emotion regulation and dyadic coping on the relationship between romantic attachment and non-suicidal self-injury. J. Youth Adolesc. 46, 277-287. doi: 10.1007/s10964-016-0547-6

Lischke, A., Pahnke, R., Mau-Moeller, A., Behrens, M., Grabe, H. J., Freyberger, H. J., et al. (2018). Inter-individual differences in heart rate variability are associated with inter-individual differences in empathy and alexithymia. Front. Psychol. 9:229. doi: 10.3389/fpsyg.2018.00229

Maunder, R. G., Nolan, R. P., Hunter, J. J., Lancee, W. J., Steinhart, A. H., and Greenberg, G. R. (2012). Relationship between social support and autonomic function during a stress protocol in ulcerative colitis patients in remission. Inflamm. Bowel Dis. 18, 737-742. doi: 10.1002/ibd.21794

Meier, C., Bodenmann, G., Moergeli, H., and Jenewein, J. (2011). Dyadic coping, quality of life, and psychological distress among chronic obstructive pulmonary disease patients and their partners. Int. J. Chronic Obstr. Pulm. Dis. 19, 583-596. doi: 10.2147/COPD.S24508

Meuwly, N., Bodenmann, G., Germann, J., Bradbury, T., Ditzen, B., and Heinrichs, M. (2012). Dyadic coping, insecure attachment, and cortisol stress recovery following experimentally induced stress. J. Fam. Psychol. 26, 937-947. doi: $10.1037 / \mathrm{a} 0030356$

Nomaguchi, K. M., and Milkie, M. A. (2003). Costs and rewards of children: the effects of becoming a parent on adults' lives. J. Marriage Fam. 65, 356-374. doi: 10.1111/j.1741-3737.2003.00356.x

Olsson, M. B., and Hwang, C. P. (2001). Depression in mothers and fathers of children with intellectual disability. J. Intellect. Disabil. Res. 45, 535-543. doi: 10.1046/j.1365-2788.2001.00372.x

Porges, S. W. (2003a). Social engagement and attachment: a phylogenetic perspective. Ann. N. Y. Acad. Sci. 1008, 31-47. doi: 10.1196/annals.13 01.004

Porges, S. W. (2003b). The polyvagal theory: phylogenetic contributions to social behavior. Physiol. Behav. 79, 503-513. doi: 10.1016/S0031-9384(03) 00156-2

Porges, S. W. (2007). The polyvagal perspective. Biol. Psychol. 74, 116-143. doi: 10.1016/j.biopsycho.2006.06.009

Quintana, D. S., Guastella, A. J., Outhred, T., Hickie, I. B., and Kemp, A. H. (2012). Heart rate variability is associated with emotion recognition: direct evidence for a relationship between the autonomic nervous system and social cognition. Int. J. Psychophysiol. 86, 168-172. doi: 10.1016/j.ijpsycho.2012.08.012

Randall, A. K., Tao, C., Totenhagen, C. J., Walsh, K. J., and Cooper, A. N. (2017). Associations between sexual orientation discrimination and depression among same-sex couples: moderating effects of dyadic coping. J. Couple Relationsh. Therapy 16, 325-345. doi: 10.1080/15332691.2016.1253520

Regan, T. W., Lambert, S. D., Kelly, B., McElduff, P., Girgis, A., Kayser, K., et al. (2014). Cross-sectional relationships between dyadic coping and anxiety, depression, and relationship satisfaction for patients with prostate cancer and their spouses. Patient Educ. Couns. 96, 120-127. doi: 10.1016/j.pec.2014. 04.010

Rottmann, N., Hansen, D. G., Larsen, P. V., Nicolaisen, A., Flyger, H., Johansen, C., et al. (2015). Dyadic coping within couples dealing with breast cancer: a longitudinal, population-based study. Health Psychol. 34, 486-495. doi: 10.1037/ hea0000218

Scharlach, A. E. (2001). Role strain among working parents: implications for workplace and community. Community Work Fam. 4, 215-230. doi: 10.1080/ 13668800120061161

Sloan, R. P., Bagiella, E., Shapiro, P. A., Kuhl, J. P., Chernikhova, D., Berg, J., et al. (2001). Hostility, gender, and cardiac autonomic control. Psychosom. Med. 63, 434-440. doi: 10.1097/00006842-200105000-00012

Smith, T. W., Cribbet, M. R., Nealey-Moore, J. B., Uchino, B. N., Williams, P. G., MacKenzie, J., et al. (2011). Matters of the variable heart: respiratory sinus arrhythmia response to marital interaction and associations with marital quality. J. Pers. Soc. Psychol. 100, 103-119. doi: 10.1037/a0021136

Stellar, J. E., Cohen, A., Oveis, C., and Keltner, D. (2015). Affective and physiological responses to the suffering of others: compassion and vagal activity. J. Pers. Soc. Psychol. 108, 572-585. doi: 10.1037/pspi0000010 
Thayer, J. F., Åhs, F., Fredrikson, M., Sollers, J. J., and Wager, T. D. (2012). A metaanalysis of heart rate variability and neuroimaging studies: implications for heart rate variability as a marker of stress and health. Neurosci. Biobehav. Rev. 36, 747-756. doi: 10.1016/j.neubiorev.2011.11.009

Thayer, J. F., and Lane, R. D. (2009). Claude Bernard and the heart-brain connection: further elaboration of a model of neurovisceral integration. Neurosci. Biobehav. Rev. 33, 81-88. doi: 10.1016/j.neubiorev.2008.08.004

Umberson, D., Pudrovska, T., and Reczek, C. (2010). Parenthood, childlessness, and well-being: a life course perspective. J. Marriage Fam. 72, 612-629. doi: 10.1111/j.1741-3737.2010.00721.x

Vaske, I., Thöne, M. F., Kühl, K., Keil, D. C., Schürmann, W., Rief, W., et al. (2015). For better or for worse: a longitudinal study on dyadic coping and quality of life among couples with a partner suffering from COPD. J. Behav. Med. 38, 851-862. doi: 10.1007/s10865-015-9657-y

Vella, E. J., Kamarck, T. W., and Shiffman, S. (2008). Hostility moderates the effects of social support and intimacy on blood pressure in daily social interactions. Health Psychol. 27, 155-162. doi: 10.1037/0278-6133.27.2(Suppl.).S155

Weise, F., Krell, D., and Brinkhoff, N. (1986). Acute alcohol ingestion reduces heart rate variability. Drug Alcohol. Depend. 17, 89-91. doi: 10.1016/0376-8716(86) 90040-2
Zajdel, M., Helgeson, V. S., Seltman, H. J., Korytkowski, M. T., and Hausmann, L. R. M. (2018). Daily communal coping in couples with type 2 diabetes: links to mood and self-care. Ann. Behav. Med. 52, 228-238. doi: 10.1093/abm/ kax047

Zemp, M., Milek, A., Cummings, E. M., and Bodenmann, G. (2017). Longitudinal interrelations between DC and coparenting conflict in couples. J. Child Fam. Stud. 26, 2276-2290. doi: 10.1007/s10826-017-0742-4

Conflict of Interest Statement: The authors declare that the research was conducted in the absence of any commercial or financial relationships that could be construed as a potential conflict of interest.

Copyright (c) 2018 Switzer, Caldwell, da Estrela, Barker and Gouin. This is an open-access article distributed under the terms of the Creative Commons Attribution License (CC BY). The use, distribution or reproduction in other forums is permitted, provided the original author(s) and the copyright owner(s) are credited and that the original publication in this journal is cited, in accordance with accepted academic practice. No use, distribution or reproduction is permitted which does not comply with these terms. 


\section{OPEN ACCESS}

Edited by:

Mariana Karin Falconier,

Virginia Tech, United States

Reviewed by:

Simon Moss,

Charles Darwin University, Australia

Petruta Rusu,

Ştefan cel Mare University

of Suceava, Romania

Jochen Ernst,

Medizinische Fakultät,

Universitätsklinikum Leipzig, Germany

*Correspondence:

Tanja Zimmermann

zimmermann.tanja@mh-hannover.de

Specialty section

This article was submitted to Clinical and Health Psychology, a section of the journal

Frontiers in Psychology

Received: 01 June 2018 Accepted: 11 February 2019

Published: 26 February 2019

Citation:

Tkachenko D, Franke L, Peters L, Schiffer $M$ and Zimmermann T (2019) Dyadic Coping of Kidney Transplant Recipients and Their Partners: Sex and Role Differences.

Front. Psychol. 10:397. doi: 10.3389/fpsyg.2019.00397

\section{Dyadic Coping of Kidney Transplant Recipients and Their Partners: Sex and Role Differences}

\author{
Daria Tkachenko', Laura Franke ${ }^{1,2}$, Luisa Peters ${ }^{1}$, Mario Schiffer ${ }^{3}$ and \\ Tanja Zimmermann ${ }^{1 *}$
}

\begin{abstract}
' Department of Psychosomatic Medicine and Psychotherapy, Hannover Medical School, Hanover, Germany, ${ }^{2}$ Integrated Research and Treatment Center IFB-TX, Hannover Medical School, Hanover, Germany, ${ }^{3}$ Department of Nephrology, Hannover Medical School, Hanover, Germany
\end{abstract}

Background: Coping with stressful health issues - e.g., organ transplantation - can affect interpersonal relationships.

Objective: The study examines individual and dyadic coping (DC) in kidney transplant recipients and their partners under consideration of sex and role differences. The Dyadic Coping Inventory allows analyzing partners' perception of their own DC and also of their partner's behavior and investigating different perspectives with three discrepancy indexes (similarity, perceived similarity, congruence).

Methods: Fifty-six kidney transplant recipients and their partners completed self-report questionnaires ( $N=112$ ) on DC, depression, anxiety, and relationship satisfaction. The average age of the patients was 58.1 years and of the partners 57.2 years; $64.3 \%$ of the patients were male; time since transplantation was on average 9.7 years.

Results: (1) Individual and dyadic functioning: In couples with male patients female caregivers showed higher own supportive DC than the males. In couples with female patients, women reported higher own stress communication, supportive DC, total positive DC and total DC as well as depression compared to men. (2) Regarding the discrepancy indexes, in couples with male patients lower levels of similarity in DC reactions of the couple was associated with higher depression of the males as well as higher anxiety of the females. Moreover, lower comparability of the own DC with partnerperception was correlated with higher depression in males. In couples with female patients, higher comparability was associated with higher DC. Higher DC of the males was associated with lower own anxiety and better similarity in DC reactions. Lower levels of similarity of the male spouse showed correlations with higher depression and anxiety of the females. (3) Sex and role differences occurred. No significant differences between male patients and male partners occurred whereas female patients showed higher own stress communication, supportive DC, common DC, total positive DC, total DC and relationship satisfaction compared to female caregivers (role differences). The same differences were found comparing female with male patients. No differences occurred between male and female caregivers (sex differences). (4) Regarding male's relationship 
quality, male's DC total score and similarity index seem to be important predictors in couples with male patients.

Discussion: The results demonstrate the relevance of DC in couples with kidney transplantation and show differences between males and females as well as between patients and partners.

Keywords: kidney transplantation, dyadic coping, couples, relationship quality, stress communication, sex differences, depression, anxiety

\section{INTRODUCTION}

Kidney transplantation is the treatment of choice for most patients with end-stage renal disease (Reimer et al., 2002; Pinquart and Sörensen, 2007). However kidney transplantation can be considered as a stressor that impacts the psychological well-being of the patient (Goetzmann et al., 2007). The kidney transplant recipient is permanently exposed to requests in the healthcare context, such as the life-long intake of immunosuppressive medication (Behrend, 2001; Ciesek et al., 2006). Therefore increasing rates of depression, family problems and non-adherence can occur (Laederach-Hofmann et al., 2002; Butler et al., 2004; DiMatteo, 2004; Bunzel et al., 2005). The high rate of non-adherence in kidney transplant recipients can increase the risk of rejection or graft failure (Butler et al., 2004; Denhaerynck et al., 2005; Sellares et al., 2012; Pabst et al., 2015).

The support network of the patient plays an essential role regarding adherence. Relatives and caregivers are important resources providing support to the patient (Lawrence, 1974; Köllner et al., 2004). Most often, spouses are the caregivers of chronically ill patients (Rees et al., 2001). However, not only the patient but also the partner can experience distress when providing care to a chronically ill spouse (Coyne and Smith, 1991; Myaskovsky et al., 2005; Dew et al., 2007; Greif-Higer et al., 2008; Schulz and Sherwood, 2008). Moreover, the significant and often long-term emotional and physical health consequences can negatively impact the relationship quality. In addition, the perceived stress of one partner can influence the other partner's stress (Frazier et al., 1995; Kadioglu et al., 2012). Depression and anxiety symptoms can occur and negatively impact relationship satisfaction (Arapaslan et al., 2004; Eryilmaz et al., 2005; Noohi et al., 2007). Although social support, especially from the spouse, can be regarded as important for the kidney transplant recipient, also the partners themselves are affected by the disease, which can lead to distress and result in a poorer relationship functioning.

Because the kidney transplantation results in high levels of stress for both, the patient and the spouse, the transplantation can be considered as a challenge for the relationship. Partners within a dyad must be seen as an interdependent whole in which each influences the other (Fife et al., 2013). Hence, to analyze the influence of kidney transplantation on relationship quality and coping abilities seems to be important. The Dyadic Coping Inventory (DCI) is an adapted and frequently used way of assessing the coping process of couples coping with a disease (Bodenmann, 2008) and based on the Systemic Transactional Model (STM) (Bodenmann, 1997; Bodenmann et al., 2016) which describes the intercourse of a couple when one partner is confronted with a stressor and the other partner supports him/her as well as the common efforts a couple makes to cope with a shared stressor (Donato et al., 2012). The STM postulates the mutual impact of one partner's daily stress experiences, the specific behavior under stress and the wellbeing on their partner's experiences. Thus, stressors - such as a kidney transplantation and its medical treatment - affect directly or indirectly both partners of a dyad. As such, even if the kidney transplantation concerns primarily the patient, the stress reactions and coping affect the partner and could turn into dyadic issues, showing the mutuality of stress. The STM emphasizes this mutuality and interdependence between partners. The stress of one partner also affects the other person, but also the resources of one person can expand the resources of the other person. Especially joint appraisal appears as an important dyadic coping (DC) strategy and is linked to dyadic adjustment (Bodenmann et al., 2016). In couples with chronic diseases dyadic appraisals predicted higher levels of mutual self-disclosure and higher mutual responsiveness (Manne et al., 2014).

According to the STM partners can express their stress verbally and/or non-verbally as well as with implicit or explicit requests for assistance. The DC includes partner-oriented behaviors or couple-oriented behaviors and may be positive or negative (Bodenmann et al., 2016). DC is defined as an interplay between the stress signals one partner perceives, the communication of them to his/her partner and the following reactions of the other partner (Revenson et al., 2005; Donato et al., 2015). At the moment when one partner communicates the stressor in the dyadic system, the stressor becomes a dyadic concern. In this context, both, the coping efforts of one partner to support the other when he/she communicates stress and the efforts of both partners to cope together with a common stressor that affects them simultaneously (i.e., common DC), are considered as "dyadic" coping responses (Donato et al., 2015).

The DCI allows calculating sum scores of different coping levels (self-evaluation, partner evaluation, evaluation as a couple) as well as the discrepancy indexes (Gmelch and Bodenmann, 2007; Osin et al., 2018). The discrepancy indexes may help to relate the perception of the own coping and the view of the partner's coping behavior (Osin et al., 2018). In previous studies the perceived similarity, as the perception of giving and receiving support to be equilibrated in a partnership, emerged as a predictor for partnership satisfaction and the psychological wellbeing (Gmelch and Bodenmann, 2007; Bodenmann, 2008). More research is needed to define the role of the other discrepancy 
indexes, such as the similarity and the congruence index, and to describe other outcomes within the concept of DC.

Little is known so far on determining the role of the discrepancy indexes within mental outcomes as distress, depression or anxiety disorder. Nevertheless studies indicate that especially the difference between the perception of the patient and the partner is of huge importance for social support and psychological well-being when coping with a disease (Badr et al., 2010; Regan et al., 2014; Junghaenel et al., 2017; Osin et al., 2018). Studies with couples coping with breast and prostate cancer showed that negative coping of the partner and common negative coping was associated with higher distress levels and higher psychological burden of the patient, whereas positive common coping showed an association with lower distress (Badr et al., 2010; Regan et al., 2014). Comparing the evaluation of pain severity levels in patients suffering from chronically pain between patient and partner, results showed that partners who overestimated the pain severity of the patients report higher support to the spouse whereas the patients did not report more support perceived from their partners (Junghaenel et al., 2017). This finding could lead to misunderstanding and consequently to higher distress levels on both sides. Another study examined couples in which one has been diagnosed with a hemato-oncological disease in regard to social support (DC) and psychological burden (depression, anxiety disorders). Interestingly, the congruence index of the DCI showed a high agreement between the self-evaluation of the DC of the patient and the partner-evaluation. However, the consensus between the self-evaluation of the partners' DC and the partner-evaluation was lower. Apparently partners could estimate the DC of the patients better than patients estimate the DC of their partners (Osin et al., 2018). However, role differences - being patient or partner - were not investigated. Discrepancies in perception of the coping between patient and partner could help to detect the reciprocal biased perception of the DC, for example the overestimation of the own coping efforts and the underestimation of the other's coping. This aspect could also be important for analyzing DC in couples with kidney transplantation and its impacts on psychological well-being of patient and partner. To our knowledge no research on discrepancy indexes in couples in which one partner receives a graft was conducted so far.

Furthermore, sex and role (being patient or caregiver) appear to be important factors influencing coping with a kidney transplantation (Bédard et al., 2005). Little is known so far about differences in coping between males and females. For a long time differences in coping have been analyzed only in cancer samples, affecting predominantly one sex (e.g., breast cancer, prostate cancer), so that a differentiation between sex and role within the coping process was difficult. In cancer research, women reported consistently more distress than men regardless of their role (Pinquart and Sörensen, 2007; Hagedoorn et al., 2008). In transplantation research, Bédard et al. (2005) and Holtzman et al. (2011) showed that female caregivers for patients on waiting list for an organ (e.g., lung, heart, liver, and kidney) experience higher distress and higher levels of depression than male caregivers and male patients. Possible reasons for that might be the higher amount of tasks, more time provided in caregiving and less support from other family members offered to female caregivers than to male caregivers (Yee and Schulz, 2000). Additionally female caregivers report about more negative health impact when caring for male transplant recipients than male caregivers, which is associated with depression (Holtzman et al., 2011). Bédard et al. (2005) found that especially women in the caregiver role for men seem to experience higher distress levels compared with female patients or caregivers for female patients. Caregiving has traditionally been viewed as a female role, so that male caregiver may not only receive more support but also more recognition from outside of the dyad (Stoller, 1992). Another aspect to be mentioned is that women resign more frequently social activities in favor of caregiving tasks, which may contribute to feelings of isolation (Navaie-Waliser et al., 2002). Female caregivers show overprotective behaviors when caring for men, which can be seen as a reflector of caregiver's overcharge (McPherson and Addington-Hall, 2003). The sex seems to possess an important impact on individual's perception of role (patient versus caregiver) and also on psychosocial aspects.

The current study investigates (1) individual and dyadic functioning of couples after renal transplantation. (2) Moreover, the present study examines differences in coping behaviors between males and females (sex differences) and between patients and caregivers (role differences). From research so far, we expect more DC in females, especially in female caregivers. Furthermore, female caregivers are more likely to experience depression and anxiety than female patients, male caregivers, or male patients. In addition to the total score and the subscales of the DCI the discrepancy indexes that make the perception of differences in assessing the coping between patient and partner possible will be analyzed. (3) Moreover, the discrepancy indexes could shed light on the detection of the interdependence between patients and spouses as well as different perspectives on own and partner DC. Finally, DC in regard to relationship quality was analyzed (4). A positive association between DC and relationship satisfaction was expected regardless of sex or role differences.

\section{MATERIALS AND METHODS}

\section{Participants}

Kidney transplant patients and their partners were recruited as part of a cross sectional study carried out in the renal transplant clinic at Hannover Medical School. Eligibility criteria included post-mortem renal transplantation patients who underwent transplantation at least 1 year prior, in a heterosexual relationship for at least 1 year, age older than 18 years and German language competence. During a recruitment period of 10 months, from August 2016 until May 2017, patients were asked by phone approximately 1 week before their follow-up visit about their interest to participate at the study and the possibility to come accompanied by the partner. One hundred and forty-six couples fulfilled these criteria. Ninety couples did not participate at the study (most frequent reasons were no interest or time), so that the final sample consisted of $N=56$ heterosexual couples (recruitment rate of $38.4 \%$ ). The study was approved by the ethics 
committee of Hannover Medical School (No. 3003-2016) and all participants provided their written informed consent.

Patient and partner completed questionnaires separately. Sample characteristics are provided in Table 1. 64.3\% $(n=36)$ of the kidney transplant recipients were male. The sample was divided in four different groups: male kidney transplant recipients $(n=36)$ and their female partners $(n=36)$, female kidney transplant recipients $(n=20)$ and their male partners $(n=20)$.

The mean age of the male kidney recipients was 59.08 years $(S D=11.0,37-78)$, of the female transplant recipients 56.40 years $(S D=12.6,35-79), 58.85$ years $(S D=13.8,35-85)$ of the male partners and 56.33 years $(S D=10.2,35-74)$ of the female partners. Male patients were significant older than their female partners $[t(35)=4.278, p=0.000]$. Relationship length did not differ between couples with female kidney transplant recipients $(M=30.85$ years, $S D=14.59,9-56)$ and couples with male transplant recipients $[M=30.64$ years, $S D=14.0,2-54$; $t(54)=0.053, p=0.958]$. No differences were found for time since transplantation between male $(M=9.46$ years, $S D=7.04$, $1-27)$ and female kidney transplant recipients $[M=9.15$ years, $S D=6.18,2-28 ; t(53)=0.163, p=0.871]$.

\section{Measurements \\ Dyadic Coping}

The Dyadic Coping Inventory (Bodenmann, 2008), a standardized assessment of DC within couples under conditions of stress, was used to measure DC (Gmelch et al., 2008). The DCI contains 37 items rated on a 5-point Likert scale from 1 (very rarely) to 5 (very often). Patient and partner, both

TABLE 1 | Sample characteristics $(N=56)$.

\begin{tabular}{|c|c|c|}
\hline Demographic & Recipient $(n=56)$ & Caregiver $(n=56)$ \\
\hline Age mean $(S D)$ & $58.1(11.6)$ & $57.2(11.5)$ \\
\hline \multicolumn{3}{|l|}{$\operatorname{Sex} n(\%)$} \\
\hline Male & $36(64.3)$ & $20(35.7)$ \\
\hline Female & $20(35.7)$ & $36(64.3)$ \\
\hline \multicolumn{3}{|l|}{ Employment $n(\%)$} \\
\hline Employed (full-time) & $13(23.7)$ & $20(35.6)$ \\
\hline Employed (part-time) & $4(7.2)$ & $15(26.8)$ \\
\hline Unemployed & - & $1(1.8)$ \\
\hline Retired & 34 (61.9) & $16(28.6)$ \\
\hline Other & $4(7.2)$ & $4(7.2)$ \\
\hline \multicolumn{3}{|l|}{ Education $n$ (\%) } \\
\hline$<10$ years & $23(41.8)$ & $22(40.7)$ \\
\hline 10 years & $18(32.7)$ & $16(29.6)$ \\
\hline$>10$ years & $11(20.0)$ & $14(25.9)$ \\
\hline other & $3(5.5)$ & $2(3.7)$ \\
\hline $\begin{array}{l}\text { Time since transplantation } \\
\text { in years mean }(S D)\end{array}$ & $9.4(6.7)$ & \\
\hline \multicolumn{3}{|l|}{ Relationship status n (\%) } \\
\hline Married & $49(87.5)$ & \\
\hline Unmarried & $7(12.5)$ & \\
\hline $\begin{array}{l}\text { Relationship length in years } \\
\text { mean }(S D)\end{array}$ & $30.8(14.1)$ & \\
\hline
\end{tabular}

answer questions separately about the own coping perception of oneself (self-evaluation, 15 items), as well as how he/she meets with the other's coping behavior (partner evaluation, 15 items), about how he/she perceives the coping of their couple (we-evaluation, 5 items) and about the general satisfaction with DC (2 items). The DCI consists of the following nine subscales: Own stress communication (e.g., 'I let my partner know when I appreciate his/her practical support, advice, or help'), own supportive coping (e.g., 'I show empathy and understanding'), own delegated DC (e.g., 'I take on things that my partner would normally do in order to help him/her out'), own negative DC (e.g., 'I blame my partner for not coping well enough with stress'), stress communication of partner (e.g., 'My partner asks me to do things for him/her when he has too much to do'), supportive coping of partner (e.g., 'My partner expresses that he/she is on my side'), delegated DC of partner (e.g., 'When I am too busy, my partner helps me out'), negative DC of partner (e.g., 'My partner does not take my stress seriously'), common DC (e.g., 'We try to deal with the problem together and look for concrete solutions'). Stress communication tends to seek for partner's attention and interest in one's stress experience with asking for problemor emotion-oriented support. Supportive DC should reduce stress by resolving the concrete problem or reduce emotional stress arousal in assisting the others own efforts. Delegated DC diminishes stress arousal by relieving the partner. Common $D C$ helps sharing negative emotions in an attempt to regulate them jointly. Negative DC can be hostile (e.g., blaming, criticizing, sarcasm), ambivalent (support in an unwilling and unmotivated support) or superficial (support with no motivation, no authentic empathy or no real understanding) (Bodenmann et al., 2016).

Thus a total score ranging from 37 to 187 (cut off values $<111$ DC below average, values $>145$ DC above average, 111145 average DC) and two combined scales "total negative DC" (own negative DC plus negative DC of the partner) and "total positive DC” (own positive DC plus positive DC of the partner) can be assessed.

In addition to the total score and the subscales, the DCI allows combining the different perspectives of men and women (self-evaluation and partner-evaluation). These discrepancy indexes, yielding interpersonal congruence for DC strategies were calculated (see Figure 1). The similarity index shows how both partners agree on the same subscale and is a measure of similarity of the DC reactions (e.g., "What do I do when my partner is stressed?"). The perceived similarity index measures how comparable the own DC (self-evaluation) with the partner-perception is. Items like "What do I do when my partner is stressed?" and "What does my partner do when I am stressed?" are compared. The congruence index assesses how both partners consistently experience the DC of the other. Items like "What do I do when my partner is stressed?" of one partner and "What does my partner do when I am stressed?" of the other partner are compared. Lower scores of the indexes are better because they stand for high accordance within a dyad. Cronbach's alpha in the current sample is 0.92 . 


\section{Kidney Transplant Recipient}

\section{Partner}

\section{Self-evaluation}

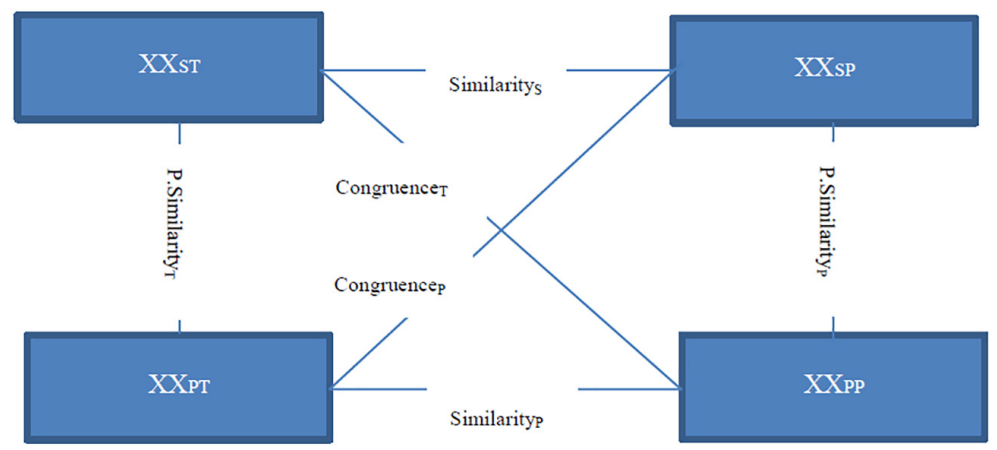

FIGURE 1 | Discrepancy indexes of the Dyadic Coping Inventory (DCI). XX, items about the stress communication, the supportive, delegated, negative dyadic coping (DC). S, self-evaluation (own DC, own supportive coping). P, partner-evaluation (supportive DC of the partner). T, kidney transplant recipient. P, partner. Modified from Bodenmann, 2008. With kind permission by Hogrefe Verlag Berne.

\section{Depression}

The German version of the nine-item Patient Health Questionnaire-Depression Scale (Kroenke et al., 2001) was used. The PHQ-9 is a well validated and widely used depression questionnaire. Participants were asked how often, during the past 2 weeks, they have been burdened, for example with insomnia, and response options were "not at all," "several days," "more than half the days," "nearly every day" scored from 0 to 3, respectively. The total score ranges from 0 to 24 . The corresponding severity levels were bordered as none (0-4), mild (5-9), moderate (10$14)$, moderately severe (15-19), and severe (20-27). Cronbach's alpha in the current sample is 0.83 .

\section{Anxiety}

The General Anxiety Disorder Screener (GAD-7) is a onedimensional, self-administered, valid and efficient tool for assessing Generalized Anxiety Disorder and measuring its severity in research and clinical practice (Spitzer et al., 2006). The participant scores the frequency of statements from 0 ("not at all") to three ("nearly every day"). The total GAD7 score is computed by addition of the answers to each item. Therefore, the total score ranges from 0 and 21 and may be categorized into four severity groups: minimal (0-4), mild (5-9), moderate (10-14) and serious (14-20). Cronbach's alpha in the current sample is 0.83 .

\section{Relationship Satisfaction}

Relationship satisfaction was assessed with the German version of the Quality of Marriage Index (QMI-D; Zimmermann et al., 2015). The QMI-D is a six-item questionnaire that uses broadly verbalized statements, such as "We have a good relationship." The participants indicate their degree of agreement on a scale ranging from one (very strong disagreement) to 7 (very strong agreement) completing five of the six items. The sixth item ranges from 1 (very strong disagreement) to 10 (very strong agreement). The total score ranges from 6 to 45 . Cut off values under 34 stand for low partnership quality. Cronbach's alpha in the current sample is 0.89 .

\section{Statistical Analysis}

Statistical analysis was carried out with the IBM SPSS Statistics 25.0 software program. Comparisons of socio-demographic and medical characteristics of the participants were presented in absolute frequencies, percentages, mean values and standard deviations. Multilevel modeling (MLM) with a pairwise dataset was performed to examine the actor and partner effects of DC, as well as of relationship quality, depression and anxiety. MLM can account for inter-dependence within the analyses of couples and is considered one of the best methods to examine effects in the Actor-Partner-Interdependence Model (APIM) (Kenny et al., 2006). T-test for independent subgroups were calculated to assess the differences in means for sex (males versus females) and role (patient versus partner). Cohen's $d$ was calculated to indicate the effect size for the comparison between two means. Pearson's correlations were calculated to assess the link between total score of the DC, discrepancy indexes, depression and anxiety of patients and partners. Multiple regression analysis of relationship quality, as the central outcome parameter of our study was conducted because of sample size only with the subgroup of male kidney transplant recipients $(n=36)$. The applied predictors were different scales of the DCI (DC total score and discrepancy indexes of males and females) and the age of male patients and female partners. Significance level for all analysis was determined to a $5 \%$ level.

\section{RESULTS}

\section{Individual and Dyadic Functioning in Couples After Renal Transplantation}

Multi-level modeling was conducted to examine differences in means between men and women within the couple. The analyses 
were conducted separately for couples with male and female kidney transplant recipients.

\section{Couples With Male Kidney Transplant Recipients}

In couples with male kidney transplant recipients the only significant difference emerged for own supportive behavior with female partners showing significant higher own supportive behavior than the male patients $\left[M_{O^{7}}=11.7, S D=3.2\right.$, $\left.M_{Q}=12.6, S D=3.1 ; t(35)=-1.26, p=0.02\right]$. No differences regarding the other subscales of the DCI and the discrepancy indexes, relationship quality, depression, and anxiety between male patients and their female partners occurred (see Tables 2, 3).

\section{Couples With Female Kidney Transplant Recipients}

In couples with female kidney transplant recipients, women showed significant higher total DC than their male spouses $[t(19)=3.27, p=0.004, d=-0.61]$ as well as higher depression scores $[t(19)=2.24, p=0.038, d=-0.65$; see Table 2]. Regarding the subscales of the DCI (see Table 1), female kidney transplant recipients compared to their male spouses showed higher own stress communication $[t(19)=3.76, p=0.001, d=-1.18]$, more own supportive DC $[t(18)=3.65, p=0.002, d=-0.98]$ as well as more total positive DC $[t(19)=3.53, p=0.002, d=0.87]$. No differences emerged for the DC discrepancy indexes (see Table 2).

\section{Relationship Between the Independent Variables}

Correlations among the independent variables were tested (see Table 4). In couples with male transplant recipients, in particular, significant positive correlations appear between DC of patient and spouse $(r=0.48, p<0.01)$ as well as between relationship satisfaction between patient and spouse $(r=0.37, p<0.05)$. $\mathrm{DC}$ of the male patient was positively associated with his relationship satisfaction $(r=0.57, p<0.01)$ as well as the relationship satisfaction of the women $(r=0.40, p<0.05)$. DC of the male patient was negatively associated with the perceived similarity index $(r=-0.36, p<0.05)$, meaning that higher DC was associated with higher comparability of own and partner DC and vice versa. DC of the female spouse showed positive correlations with own relationship satisfaction $(r=0.63, p<0.01)$ but not with the relationship satisfaction of the male patient $(r=0.16, p=0.34)$.

Regarding the discrepancy indexes of the DCI, the similarity index (self-evaluation) showed positive associations with PHQ of the male patient $(r=0.37, p<0.05)$ as well as GAD of the women $(r=0.35, p<0.05)$. Lower levels of similarity in DC reactions of the couple was associated with higher depression of the male patient as well as higher anxiety of the female spouse. Moreover, the perceived similarity of the male patient showed significant positive correlations with his depression score ( $r=0.46, p<0.01)$, meaning that lower comparability of the own $\mathrm{DC}$ with partner-perception is associated with higher depression in male patients and vice versa.

In couples with female transplant recipients, significant positive correlations appear between DC of patient and spouse $(r=0.65, p<0.01)$ as well as between relationship satisfaction between patient and spouse $(r=0.69, p<0.01)$. The DC of the female patient was negatively associated with anxiety of the male partner $(r=-0.48, p<0.05)$ as well as with own perceived similarity $(r=-0.64, p<0.01)$, meaning that higher comparability is associated with higher DC and vice versa. The DC of the male spouse showed negative correlations with own anxiety $(r=-0.51, p<0.05)$ and own similarity index $(r=-0.63, p<0.01)$. Higher DC of the males was associated with lower own anxiety and better similarity in DC reactions and vice versa. In addition, lower levels of similarity of the male spouse showed associations with higher depression of the female patient $(r=-0.52, p<0.05)$ and higher anxiety of the women $(r=-0.51, p<0.05)$ and vice versa (see Table 4).

\section{Sex and Role Differences}

Independent $t$-tests were conducted to analyze differences regarding sex and role. Comparisons were done between male patients and female patients as well as male caregivers and female caregivers (sex differences) and between male patients and male caregivers as well as female patients and female caregivers (role differences).

\section{Sex Differences}

For patients, female transplant recipients compared to male transplant recipients showed significant higher own stress communication $\left[M_{\sigma^{7}}=11.7, S D=3.2, M_{O}=16.0, S D=2.7\right.$; $t(54)=-5.02, p=0.000, d=1.39]$, higher own supportive coping $\left[M_{\sigma^{7}}=18.3, S D=2.5, M_{\text {O }}=21.3, S D=3.1 ; t(54)=-3.93\right.$, $p=0.000, d=1.10]$, more common DC $\left[M_{\sigma^{7}}=16.4, S D=3.0\right.$, $\left.M_{\varphi}=19.5, S D=3.2 ; t(51)=-3.48, p=0.001, d=1.01\right]$, as well as more total positive DC $\left[M_{\sigma^{7}}=68.9, S D=7.9\right.$, $\left.M_{\text {q }}=77.1, S D=9.5 ; t(54)=-3.47, p=0.001, d=0.97\right]$ and overall DC $\left[M_{\sigma^{7}}=125.5, S D=11.3, M_{\uparrow}=138.8, S D=17.9\right.$; $t(27.6)=-3.00, p=0.01, d=0.95]$. Moreover, differences in relationship satisfaction were found $\left[M_{\sigma^{7}}=39.7, S D=5.4\right.$, $\left.M_{\varphi}=42.7, S D=2.8 ; t(53.7)=-2.77, p=0.01\right]$. No differences occurred regarding depression $\left[M_{\sigma^{7}}=5.0, S D=3.4, M_{\odot}=5.8\right.$, $S D=4.7 ; t(54)=-0.73, p=0.47]$ or anxiety $\left[M_{\sigma^{7}}=3.8, S D=3.5\right.$, $\left.M_{\text {Q }}=3.6, S D=3.3 ; t(54)=0.30, p=0.77\right]$.

In the caregiving role, no significant differences between male and female spouses were found in terms of DC as well as for depression $\left[M_{\sigma^{7}}=3.3, S D=2.8, M_{Q}=4.4, S D=3.8\right.$; $t(54)=-1.17, p=0.25]$, anxiety $\left[M_{O^{7}}=3.3, S D=2.4, M_{O}=4.3\right.$, $S D=3.4 ; t(54)=-1.18, p=0.24]$ or relationship satisfaction $\left[M_{\sigma^{7}}=40.1, S D=7.8, M_{\text {o }}=38.7, S D=6.4 ; t(54)=0.70, p=0.49\right]$ (see Tables 2, 3).

\section{Role Differences}

For women, female transplant recipients compared to female caregivers showed significant higher own stress communication $\left[M_{\text {patients }}=16.0, S D=2.7, M_{\text {caregivers }}=12.6, S D=3.1 ; t(54)=4.05\right.$, $p=0.000, d=-1.15]$, higher common DC $\left[M_{\text {patients }}=19.5\right.$, $S D=3.2, M_{\text {caregivers }}=16.9, S D=3.5 ; t(51)=2.60, p=0.012$, $d=-0.77]$, more positive DC $\left[M_{\text {patients }}=77.1, S D=9.5\right.$, $\left.M_{\text {caregivers }}=69.7, S D=11.5 ; t(54)=2.45, p=0.018, d=-0.69\right]$ and overall DC $\left[M_{\text {patients }}=138.8, S D=17.9, M_{\text {caregivers }}=127.3\right.$, $S D=18.0 ; t(54)=2.28, p=0.05, d=-0.64]$ as well as 
TABLE 2 | Differences in the subscales of the Dyadic Coping Inventory within the couple.

\begin{tabular}{|c|c|c|c|c|c|c|c|}
\hline & \multicolumn{2}{|c|}{ Male transplant recipient $(n=36)$} & \multicolumn{2}{|c|}{ Female partner $(n=36)$} & \multicolumn{2}{|c|}{ Difference } & \multirow[b]{2}{*}{ Effect size (d) } \\
\hline & Mean (SD) & Stanine & Mean (SD) & Stanine & $t$ & $p$ & \\
\hline Own stress communication & $11.7(3.2)$ & 5 & $12.6(3.1)$ & 5 & -1.26 & 0.216 & 0.286 \\
\hline Own supportive coping & $18.3(2.5)$ & 5 & $19.6(3.3)$ & 6 & -2.38 & 0.023 & 0.444 \\
\hline Own delegated DC & $7.4(1.5)$ & 5 & $7.8(1.8)$ & 6 & -1.03 & 0.311 & 0.241 \\
\hline Own negative coping & $8.0(2.8)$ & 1 & $7.3(2.7)$ & 1 & 1.25 & 0.220 & -0.255 \\
\hline Common DC & $16.4(3.0)$ & 5 & $16.9(3.5)$ & 5 & -0.90 & 0.373 & 0.153 \\
\hline Total negative DC & $15.5(5.0)$ & & $15.2(5.7)$ & & 0.36 & 0.722 & -0.056 \\
\hline \multirow[t]{3}{*}{ Total positive DC } & $68.9(7.9)$ & & $69.7(11.5)$ & & -0.51 & 0.613 & 0.082 \\
\hline & \multicolumn{2}{|c|}{ Female transplant recipient $(n=20)$} & \multicolumn{2}{|c|}{ Male partner $(n=20)$} & \multicolumn{2}{|c|}{ Difference } & \\
\hline & Mean (SD) & Stanine & Mean (SD) & Stanine & $t$ & $p$ & Effect size $(d)$ \\
\hline Own stress communication & $16.0(2.7)$ & 7 & $11.9(4.1)$ & 5 & 3.76 & 0.001 & -1.181 \\
\hline Own supportive coping & $21.4(3.1)$ & 7 & $18.4(3.0)$ & 5 & 3.65 & 0.002 & -0.983 \\
\hline Own delegated DC & $7.4(2.2)$ & 5 & $7.4(2.2)$ & 5 & 0.00 & 1.000 & 0 \\
\hline Own negative coping & $6.9(3.1)$ & 1 & $7.1(3.0)$ & 1 & -0.21 & 0.839 & 0.066 \\
\hline Common DC & $19.5(3.2)$ & 7 & $17.7(3.9)$ & 6 & 1.84 & 0.084 & -0.505 \\
\hline Total negative DC & $13.4(5.6)$ & & $13.7(5.5)$ & & -0.37 & 0.713 & 0.054 \\
\hline Total positive DC & $77.1(9.5)$ & & $68.4(10.5)$ & & 3.53 & 0.002 & -0.869 \\
\hline
\end{tabular}

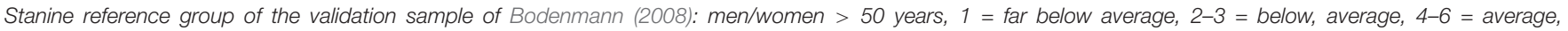
7-8 = above-average, 9 = far above-average. Significant differences in bold. $t=t$-test for dependent samples; $S D=s t a n d a r d ~ d e v i a t i o n$.

higher relationship satisfaction $\left[M_{\text {patients }}=42.7, S D=2.8\right.$, $\left.M_{\text {caregivers }}=38.8, S D=6.4 ; t(51.6)=3.20, p=0.002\right]$. No significant differences occurred for depression $\left[M_{\text {patient }}=5.8\right.$, $\left.S D=4.7, M_{\text {caregiver }}=4.4, S D=3.4 ; t(54)=1.30, p=0.20\right]$ or anxiety $\left[M_{\text {patient }}=3.6, S D=3.3, M_{\text {caregiver }}=4.3, S D=3.4\right.$; $t(54)=-0.77, p=0.45]$.

For males, no significant differences were found between male transplant recipients and male spouses in terms of DC as well as for depression $\left[M_{\text {patient }}=5.0, S D=3.4, M_{\text {caregiver }}=3.3, S D=2.8\right.$; $t(54)=1.90, p=0.06]$ or anxiety $\left(M_{\text {patient }}=3.8, S D=3.5\right.$, $\left.M_{\text {partner }}=3.3, S D=2.4 ; t(54)=0.66, p=0.51\right]($ see Tables 2, 3).

\section{Association Between DC and Age With Relationship Satisfaction in Couples With Male Kidney Transplant Recipients}

Due to the small sample size of couples with female transplant recipients $(n=20)$, the analysis was only conducted with couples with male patients $(n=36)$. A multiple regression analysis with the relationship quality of male kidney transplant recipients as dependent variable was calculated to assess the impact of DCI (total score, discrepancy indexes) and age of the patients and their partners (Table 5). A significant regression model with $67.1 \%$ of explained variance emerged $\left[F(9,25)=2.30, p=0.043, R^{2}=0.67\right]$. As significant predictors of male's relationship satisfaction occurred the DCI total score of male kidney transplant recipients $(\beta=0.75, p=0.000)$ and the similarity index of the self-evaluation of male kidney transplant recipients $(\beta=-0.49, p=0.016)$ as actor effects. No significant partner effects on male's relationship satisfaction were found.

\section{DISCUSSION}

The current study examined DC of 56 couples in which one partner is a post-mortem kidney transplant recipient and investigated the relationship between DC, relationship satisfaction and depressive and anxiety symptoms taking sex and role differences into account. The DC analysis involved calculating the discrepancy indexes (similarity index, perceived similarity index, congruence index). Finally, the association of these indexes with relationship quality, depression and anxiety were examined.

\section{Individual and Dyadic Functioning in Couples After Renal Transplantation Under Consideration of Sex and Role Differences}

Female kidney transplant recipients emerged as the group with significantly higher levels of depression compared to their male partners. In addition, female patients showed higher DC compared to their male partners, female caregivers and male patients. One possible explanation could be that women experience higher distress levels compared to men when confronted with a disease regardless of the specific diagnosis and whether they are in the patient's or the caregiver's role (Holtzman et al., 2011; Fife et al., 2013). Studies on kidney transplantation confirm these aspects: Women with end-stage renal disease react with an increase of immunologic parameters, such as interleukin1 , to the social environment, whereas no associations were found in men with end-stage renal disease (Kimmel et al., 2000; Kimmel, 2001; Kimmel and Patel, 2003). These differences between female 
TABLE 3 | Differences in dyadic coping, relationship satisfaction, depression, and anxiety within the couple.

\begin{tabular}{|c|c|c|c|c|c|c|c|}
\hline & \multirow[b]{2}{*}{ Range } & \multicolumn{2}{|c|}{ Male transplant recipient $(n=36)$} & \multicolumn{2}{|c|}{ Female partner $(n=36)$} & \multicolumn{2}{|c|}{ Difference } \\
\hline & & Mean & $S D$ & Mean & $S D$ & $t$ & $p$ \\
\hline Total dyadic coping (DCl) & $35-175$ & 125.5 & 11.3 & 127.3 & 18.0 & -0.69 & 0.494 \\
\hline Relationship quality (QMI) & $6-45$ & 39.7 & 5.4 & 38.8 & 6.4 & 0.82 & 0.417 \\
\hline Depression (PHQ-9) & $0-27$ & 5 & 3.4 & 4.4 & 3.5 & 0.89 & 0.382 \\
\hline Anxiety (GAD-7) & $0-21$ & 3.8 & 3.5 & 4.3 & 3.4 & -0.66 & 0.516 \\
\hline Congruence index (DCl) & $0-120$ & 12.3 & 4.4 & 12.3 & 4.8 & 0.03 & 0.976 \\
\hline \multirow[t]{3}{*}{ Perceived similarity (DCl) } & $0-120$ & 10.6 & 4.8 & 10.9 & 6 & 0.24 & 0.813 \\
\hline & & \multicolumn{2}{|c|}{ Self-evaluation } & \multicolumn{2}{|c|}{ Partner-evaluation } & & \\
\hline & & $M$ & $S D$ & $M$ & $S D$ & & \\
\hline \multirow[t]{3}{*}{ Actual reciprocity (DCl) } & $0-120$ & 13.3 & 4.3 & 12.9 & 5.6 & 0.33 & 0.743 \\
\hline & & \multicolumn{2}{|c|}{ Female transplant recipient $(n=20)$} & \multicolumn{2}{|c|}{ Male partner $(n=20)$} & \multicolumn{2}{|c|}{ Difference } \\
\hline & & Mean & $S D$ & Mean & $S D$ & $t$ & $p$ \\
\hline Total dyadic coping (DCI) & $35-175$ & 138.8 & 17.9 & 128.2 & 16.8 & 3.27 & 0.004 \\
\hline Relationship quality (QMI) & $6-45$ & 42.7 & 2.8 & 40.1 & 7.9 & 1.86 & 0.079 \\
\hline Depression (PHQ-9) & $0-27$ & 5.8 & 4.7 & 3.3 & 2.8 & 2.24 & 0.038 \\
\hline Anxiety (GAD-7) & $0-21$ & 3.6 & 3.3 & 3.3 & 2.4 & 0.35 & 0.073 \\
\hline Congruence index (DCl) & $0-120$ & 11.8 & 4.7 & 13.4 & 5.5 & -1.29 & 0.218 \\
\hline \multirow[t]{3}{*}{ Perceived similarity (DCl) } & $0-120$ & 11.3 & 4.6 & 11.5 & 4.9 & -0.1 & 0.923 \\
\hline & & \multicolumn{2}{|c|}{ Self-evaluation } & \multicolumn{2}{|c|}{ Partner-evaluation } & & \\
\hline & & $M$ & $S D$ & $M$ & $S D$ & & \\
\hline Actual reciprocity (DCl) & $0-120$ & 15.0 & 5.3 & 13.0 & 6.2 & 0.33 & 0.337 \\
\hline
\end{tabular}

and male patients could indicate a more intensive reaction of the female organism toward stress when being confronted with kidney diseases and accordingly the higher need of coping. The interaction between immunologic parameters, depression and DC should be investigated in more detail in future studies.

Another aspect to be considered is the different self-perception of men and women. Kimmel (2001) reported different role expectations: men are supposed to be independent, so that after kidney transplantation they regain quality of life, whereas women are characterized by being emotional, dependent and be physical beauty. Women seem to profit less from the kidney transplantation in regard to quality of life than men and experience more stress (Johnson et al., 1998; Kimmel, 2001).

To better understand the situation of a dyad dealing with kidney transplantation, the time before the transplantation should also be considered. The link between negative affect before and after kidney transplantation has been shown by Szeifert et al. (2010). Dialysis can be seen as a state of prolonged stress for the whole family (Pomaki et al., 2011). In order not to burden other family members, women tend to overcharge themselves, so that finally they might experience higher levels of distress and depression than men (Kimmel and Patel, 2003). Patients who underwent dialysis treatment frequently experience multiple losses, such as the loss of the original role within the family and the dyad, the loss of cognitive abilities and physical power (Kimmel, 2001, 2002). The loss of the original role within the family means a repositioning within the dyad, which is not automatically nullified after kidney transplantation (Pomaki et al., 2011). The higher level of depression of female kidney recipients in our sample occurred only in comparison to their partner (within the couple) and is not explainable through role (patient versus caregiver) or sex (male versus female). The high level of DC occurred in comparison to all subgroups (male partners, female patients, and male patients). That women use the dyadic system more extensively to cope than men is in line with findings of Acitelli and Antonucci (1994).

Some studies indicate that especially female caregivers caring for men are at great risk for developing high distress levels within a dyad (Yee and Schulz, 2000; Holtzman et al., 2011). Possible explanations are that women receive less support from outside of the dyad (other family members, friends), receive less recognition, provide more time to caregiving, and comply more tasks in number (Yee and Schulz, 2000; 
TABLE 4 | Correlations between dyadic coping, marital satisfaction, depression and general anxiety within the couple.

\begin{tabular}{|c|c|c|c|c|c|c|c|c|c|c|c|c|c|c|}
\hline & DC TX & DC P & QMI TX & QMI P & PHQ TX & PHQ P & GAD TX & GAD P & Sims & SimP & Cong TX & Cong $\mathrm{P}$ & PerSi TX & PerSi P \\
\hline \multicolumn{15}{|l|}{ DC TX } \\
\hline QMI TX & $0.565^{* *}$ & * $\quad 0.162$ & & & & & & & & & & & & \\
\hline QMI P & $0.402^{*}$ & $0.626^{* *}$ & $0.370^{*}$ & & & & & & & & & & & \\
\hline PHQ TX & -0.150 & 0.239 & -0.311 & 0.078 & & & & & & & & & & \\
\hline GAD TX & -0.037 & 0.088 & -0.165 & -0.029 & $0.666^{* *}$ & 0.212 & & & & & & & & \\
\hline GAD P & -0.049 & -0.178 & -0.142 & -0.128 & 0.323 & $0.802^{* *}$ & 0.313 & & & & & & & \\
\hline Sims & -0.092 & 0.081 & -0.159 & 0.166 & $0.374^{*}$ & 0.220 & 0.134 & $0.349 *$ & & & & & & \\
\hline SimP & -0.134 & -0.264 & 0.266 & -0.015 & 0.079 & -0.030 & 0.281 & 0.078 & $0.376^{*}$ & & & & & \\
\hline Cong TX & -0.113 & -0.192 & 0.117 & -0.021 & 0.212 & -0.160 & 0.191 & -0.120 & $0.492^{* *}$ & $0.650^{* *}$ & & & & \\
\hline Cong $\mathrm{P}$ & -0.278 & -0.067 & 0.092 & 0.066 & 0.125 & -0.071 & 0.13 & 0.104 & 0.367 & $0.576^{* *}$ & 0.299 & & & \\
\hline
\end{tabular}

\section{Female transplant recipient and male partner $(n=20)$}

\section{TX}

$\mathrm{DCP} \quad 0.654^{* *}$

QMI TX $\quad 0.203 \quad 0.415$

$\begin{array}{llll}\text { QMI P } & 0.108 & 0.135 & 0.691^{* *}\end{array}$

$\begin{array}{lllll}\text { PHQ TX } & 0.015 & 0.326 & 0.157 & 0.148\end{array}$

$\begin{array}{lllll}\text { PHQP } & -0.342 & -0.411 & -0.275 & -0.090\end{array}$

$\begin{array}{lllll}\text { GAD TX } & -0.075 & 0.268 & 0.157 & 0.231\end{array}$

$\begin{array}{lllll}\text { GAD P } & -0.475^{*} & -0.513^{*} & -0.043 & -0.056 \\ \text { SimS } & -0.017 & -0.158 & -0.118 & -0.144\end{array}$

$\begin{array}{llllr}\text { SimS } & -0.017 & -0.158 & -0.118 & -0.144 \\ \text { SimP } & -0.114 & -0.631^{* *} & -0.032 & 0.363\end{array}$

$\begin{array}{lllll}\text { Cong TX } & 0.088 & -0.368 & 0.039 & 0.384\end{array}$

$\begin{array}{lllll}\text { Cong P } & 0.107 & -0.353 & -0.450 & -0.261\end{array}$

$\begin{array}{ll}0.192 & \\ 0.931^{* *} & 0.298\end{array}$

$0.119 \quad 0.782^{* *} \quad 0.137$

$\begin{array}{llll}-0.182 & -0.044 & -0.382 & -0.086\end{array}$

$\begin{array}{llll}-0.516^{*} & 0.049 & -0.512^{*} & 0.084\end{array}$

$\begin{array}{llll}-0.286 & 0.226 & -0.207 & 0.223\end{array}$

$\begin{array}{llll}-0.041 & 0.127 & -0.148 & 0.123\end{array}$

$\begin{array}{llll}0.460 & 0.193 & 0.408 & 0.193\end{array}$

$-0.112$

$-0.054$

$-0.280-0.072$

0.315

$0.454 \quad 0.683^{* *}$

$0.714^{* *} \quad 0.273 \quad 0.548^{*}$

$\begin{array}{llll}0.104 & 0.065 & -0.062 & -0.113\end{array}$

$0.710^{* *} \quad 0.201 \quad 0.101 \quad 0.536^{*} \quad-0.310$

TX, transplant recipient; P, partner; DC, dyadic coping; QMI, Quality of Marriage Index; PHQ, Patient Health Questionnaire; GAD, General Anxiety Disorder; SimS/P, Similarity Index Self/Partner-evaluation; Cong, congruence index; PerSi, perceived similarity index. ${ }^{*} p<0.05 ;{ }^{* *} p<0.01$.

TABLE 5 | Summary of regression analysis for variables predicting relationship satisfaction (QMI) in male transplant recipients $(n=36)$.

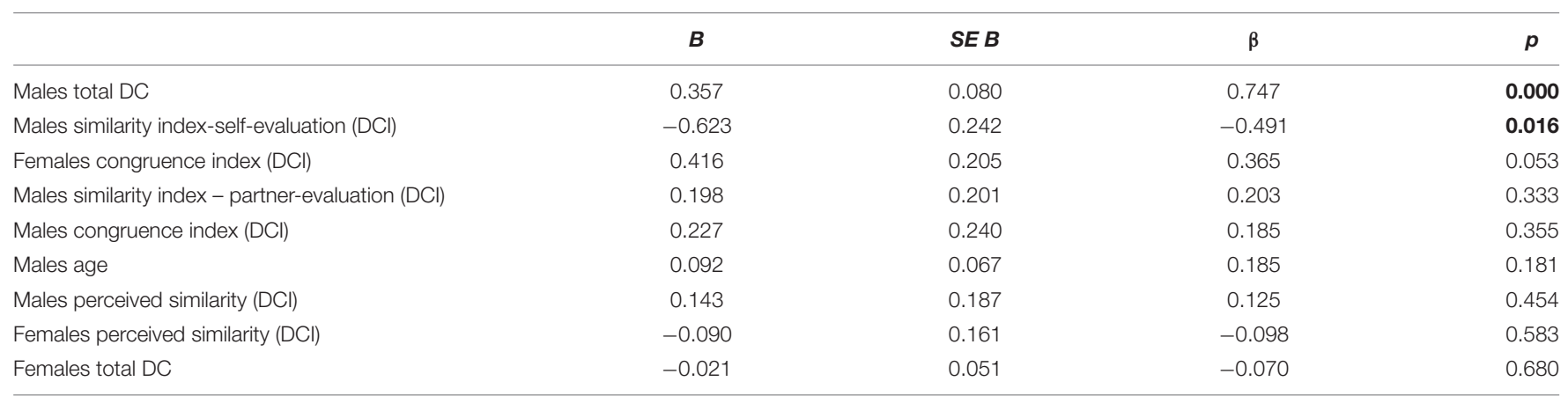

DC, dyadic coping; DCI, Dyadic Coping Inventory; QMI, Quality of Marriage Index. Significant differences in bold.

Kim et al., 2006; Fife et al., 2013). Additionally, women frequently are burdened. They are juggling house work, children and work outside of the home (Stoller, 1992; Fife et al., 2013). Our results are not in line with these findings (female caregivers did not show high scores of depression, anxiety or DC), but hints at why women in general might be more affected and feel the need to cope more when dealing with a disease.

The question why especially female kidney transplant recipients compared to their partner report higher levels 
of depression might be explained by the behavior of male caregivers: Men in the caregiver perspective of the spouse tend to use negative expressiveness and overprotectiveness as coping strategies (Thompson and Sobolew-Shubin, 1993; Pomaki et al., 2011). The negative expressiveness has been shown to be associated with mortality in female but not in men end-stage renal disease patients (Pomaki et al., 2011). Additionally, women often perceive themselves as a burden and feel overprotected as a patient (McPherson et al., 2010; Holtzman et al., 2011). Both elements, the perceived burden and overprotectiveness, are described as risk factors for developing distress and depression (McPherson et al., 2010). According to the Equity Theory of Wilson et al. (2005), a partnership has to be in balance of giving and receiving support. Chronic illnesses provide the impression of not being able to establish this equilibrium to the patient, so that consequently feelings of worthlessness and depression might develop (McPherson et al., 2010). Overprotectiveness could transmit the feeling of being able to do less than the health estate permits. Coping strategies of male caregivers might be the reason why women experience higher levels of depression and distress in the patient role compared to the partners in our sample. Whether men use negative expressiveness or overprotectiveness more often when caring for their partners within the context of kidney transplantation should be investigated in further studies.

Our results suggest that female kidney transplant recipients are at risk for developing depression with high needs for coping within the couple. Possible explanations for these circumstances could be somatic aspects of females, role and perception of women in our society, as well as male partners using unfavorable coping strategies as a caregiver. A further analysis of important risk factors for depression after kidney transplantation and the impact of DC is warranted.

\section{Impact of Dyadic Coping and Its Discrepancy Indexes on Relationship Satisfaction}

Our data reveal interesting findings regarding discrepancy indexes. In terms of differences within the couple no significant differences were found in our sample. In the study of Osin et al. (2018) including couples dealing with a hematooncological disease significant differences occurred. The partners showed significantly smaller congruence index, which indicates that the partners could estimate the coping behaviors of the patients more correctly than the other way around. Additionally, the similarity and the perceived similarity indexes suggest that patients and partners reported quite similar estimations of the own coping behavior, but underestimated the other's coping (Osin et al., 2018). Moreover, Osin et al. (2018) did not differentiate between male and female partners and patients. A possible reason for these differences not appearing in couples coping with kidney transplantation might be that a kidney transplantation does not disturb a couple in their perception as much as a hemato-oncological disease. Patients and partners perceive their coping behavior in the same degree not depending on the role (patient versus partner) when dealing with kidney transplantation.
In the sample of a healthy population of Gmelch et al. (2007) these differences did not occur either, which conforms to our assumption.

As expected, almost all discrepancy indexes correlated negatively with psychological outcomes such as DC or partnership quality in both groups, couples with male and female kidney transplant recipients. Low discrepancies were associated with positive psychological outcomes. That is in line with the study of Gmelch et al. (2007).

Our results differ in the group with female kidney transplant recipients from the one of couples with male kidney transplant recipients in regard to depression and anxiety. In couples with male kidney transplant recipients higher discrepancies were associated with negative psychological outcomes, which is in line with Osin et al. (2018). In couples with female kidney transplant recipients negative correlations between discrepancy indexes and psychological outcomes appeared. That means that more congruence correlates with higher levels of depression and anxiety of female kidney transplant recipients. The perception of female kidney transplant recipients of high partner's coping efforts might lead to feelings of guilt and evoke other negative psychological outcomes like depression and anxiety. The higher depression score of female kidney recipients within the couple supports this assumption. Similar correlation appeared in the study of Osin et al. (2018) where only the small discrepancy within the congruence index was related to high psychological burden. In our data these associations appeared in couples with female transplant recipients within all of the three discrepancy indexes. That might be explained by the issue that female kidney transplant recipients in our sample feel even guiltier because of the dependence on their partner than when patients deal with a hemato-oncological disease. Cancer is perceived as a severe and stressful life event, so that the feelings of dependence and high support from the partner are accepted (Edwards and Clarke, 2004). Contrary, kidney transplantation is seen as a step toward healing and independence (Johnson et al., 1998; Kimmel, 2001). The deception after realizing that one still feels dependent and ill (because of the immunosuppressive medication and permanent confrontation with the foreign organ) might be visible through the discrepancy indexes.

The discrepancy indexes point at female kidney transplant recipients being under higher psychological burden when estimating more correctly the coping efforts of the partner. Discrepancy indexes can help detecting feelings of guilt and might allow analysis of unconscious perception within the dyadic system. To prove all these assumptions more studies analyzing the role of discrepancy indexes are needed.

The relationship quality seems to be one of the most important aspects for the well-being and psychological outcomes of kidney transplant recipients (Frazier et al., 1995). The regression analysis of male kidney transplant recipients in regard to their relationship quality has shown actor effects, the DC total score and the similarity index of the self-evaluation, as the most predictive factors. The DCI total score as the central predictive element of partnership quality is in line with several studies (Gmelch and Bodenmann, 2007). The quality of the 
DC depends significantly and substantially on the partnership satisfaction, predicted the occurrence of divorce, psychological well-being and psychological disturbances (Kimmel et al., 2000). The second predictive factor was the similarity index of the selfevaluation. In the studies of Gmelch and Bodenmann (2007) and Rohmann and Bierhoff (2007) the perceived similarity index emerged as the most valuable predictive element of relationship quality. The Equity Theory of Walster et al. (1973) supports that the similarity between one another is positively predictive. Interestingly, partner effects of the female partner failed statistical significance. More research is needed to determine the role of several discrepancy indexes, their differences in sex and cut off values that permit the determination of a high/low index. No substantiated cut off values have been determined to our knowledge so far.

The current study has several limitations. The cross-sectional nature of our study does not permit the establishment of casual inferences about the data. Longitudinal designs are needed to prove the validity of the study. Additionally, the study relied on self-reported-perception, which also has to be taken into consideration, as a risk to receive socially desired answers deforming the reality. Nevertheless the analysis of the data showed moderate or significant correlations between patients and partners, so that the reliability of the data is given. The number of dyads with only 20 couples of female transplant recipients was small. Due to the small sample size, the generalizability of the results is limited. To investigate the role of sex larger samples are necessary. Consequently we are inapt to compare the influence of sex on kidney transplant recipients, caregivers, differences in perceived relationship quality or negative psychological outcomes. Longitudinal data are necessary.

Nevertheless, the current study reveals the importance of DC for relationship functioning within kidney transplantation.

\section{REFERENCES}

Acitelli, L. K., and Antonucci, T. C. (1994). Gender differences in the link between marital support and satisfaction in older couples. J. Pers. Soc. Psychol. 67:688.

Arapaslan, B., Soykan, A., Soykan, C., and Kumbasar, H. (2004). Cross-sectional assessment of psychiatric disorders in renal transplantation patients in Turkey: a preliminary study. Transplant. Proc. 36, 1419-1421. doi: 10.1016/j. transproceed.2004.04.087

Badr, H., Carmack, C. L., Kashy, D. A., Cristofanilli, M., and Revenson, T. A. (2010). Dyadic coping in metastatic breast cancer. Health Psychol. 29, 169-180. doi: $10.1037 / \mathrm{a} 0018165$

Bédard, M., Kuzik, R., Chambers, L., Molloy, D. W., Dubois, S., and Lever, J. A. (2005). Understanding burden differences between men and women caregivers: the contribution of care-recipient problem behaviors. Int. Psychogeriatr. 17, 99-118. doi: 10.1017/S1041610204000857

Behrend, M. (2001). Adverse gastrointestinal effects of mycophenolate mofetil. Drug Safety 24, 645-663. doi: 10.2165/00002018-200124090-00002

Bodenmann, G. (1997). Dyadic coping-a systematic-transactional view of stress and coping among couples: theory and empirical findings. Eur. Rev. Appl. Psychol. 47, 137-140.

Bodenmann, G. (2008). Dyadisches Coping Inventar - DCI. Bern: Huber.

Bodenmann, G., Randall, A. K., and Falconier, M. K. (2016). "Coping in couples: the systemic transactional model (STM)," in Couples Coping with Stress: A CrossCultural Perspective, eds M. K. Falconier, A. K. Randall, and G. Bodenmann (New York, NY: Routledge), 5-22.
Contrary to our expectation, female kidney transplant recipients and not female caregivers seem to be the group under risk for developing negative psychological consequences, such as depression. Female kidney transplant recipients seem to profit extensively from the DC. Discrepancy indexes support these assumptions. They appear as an element that could be used in future to reveal more unconscious perceptions within the dyadic interplay. Feelings of guilt or perception of imbalance of a dyad might be detected. Thus the dyadic system of a couple would be reinforced. More research on discrepancy indexes is needed. The findings of the current study could be specifically addressed in interventions for couples with kidney transplantation - especially for couples with female transplant recipients. Strengthening couples' DC could be a viable option in clinical practice. The dyadic system should be intensified in practice and used as an important way of support within kidney transplantation.

\section{AUTHOR CONTRIBUTIONS}

TZ and MS have been involved in the development of the design. DT, LF, LP, and MS did the data collection. DT, LF, LP, and TZ have been involved in data analysis and the preparation of the manuscript. All authors contributed to the interpretation of the data. All the authors have read and approved the final manuscript.

\section{FUNDING}

This work was supported by the German Federal Ministry of Education and Research (Reference Number 01EO1302) and the IFB-Tx (Grant Number 19920126).

Bunzel, B., Laederach-Hofmann, K., Wieselthaler, G. M., Roethy, W., and Drees, G. (2005). Posttraumatic stress disorder after implantation of a mechanical assist device followed by heart transplantation: evaluation of patients and partners. Transplant. Proc. 37, 1365-1368. doi: 10.1016/j.transproceed.2004. 12.248

Butler, J. A., Peveler, R. C., Roderick, P., Smith, P. W., Horne, R., and Mason, J. C. (2004). Modifiable risk factors for non-adherence to immunosuppressants in renal transplant recipients: a cross-sectional study. Nephrol. Dial. Transpl. 19, 3144-3149. doi: 10.1093/ndt/gfh505

Ciesek, S., Manns, M. P., and Strassburg, C. P. (2006). Folgeerkrankungen nach organtransplantation. Der. Intern. 47, 252-265. doi: 10.1007/s00108-0061583-1

Coyne, J. C., and Smith, D. A. (1991). Couples coping with a myocardial infarction: a contextual perspective on wives' distress. J. Pers. Soc. Psychol. 61, 404-412. doi: 10.1037/0022-3514.61.3.404

Denhaerynck, K., Dobbels, F., Cleemput, I., Desmyttere, A., Schäfer-Keller, P., Schaub, S., et al. (2005). Prevalence, consequences, and determinants of nonadherence in adult renal transplant patients: a literature review. Transpl. Int. 18, 1121-1133. doi: 10.1111/j.1432-2277.2005.00176.x

Dew, M. A., DiMartini, A. F., De Vito Dabbs, A., Myaskovsky, L., Steel, J., Unruh, M., et al. (2007). Rates and risk factors for nonadherence to the medical regimen after adult solid organ transplantation. Transplantation 83, 858-873. doi: 10.1097/01.tp.0000258599.65257.a6

DiMatteo, M. R. (2004). Social support and patient adherence to medical treatment: a meta-analysis. Health Psychol. 23, 207-218. doi: 10.1037/0278-6133.23.2.207 
Donato, S., Iafrate, R., Bradbury, T. N., and Scabini, E. (2012). Acquiring dyadic coping: partners and parents as models. Pers. Relat. 19, 386-400. doi: 10.1111/j. 1475-6811.2011.01368.x

Donato, S., Parise, M., Iafrate, R., Bertoni, A., Finkenauer, C., and Bodenmann, G. (2015). Dyadic coping responses and partners' perceptions for couples satisfaction: an actor-partner interdependence analysis. J. Soc. Pers. Relat. 32, 580-600. doi: 10.1177/0265407514541071

Edwards, B., and Clarke, V. (2004). The psychological impact of a cancer diagnosis on families: the influence of family functioning and patients' illness characteristics on depression and anxiety. Psychooncology 13, 562-576. doi: 10.1002/pon.773

Eryilmaz, M. M., Ozdemir, C., Yurtman, F., Cilli, A., and Karaman, T. (2005). Quality of sleep and quality of life in renal transplantation patients. Transplant. Proc. 37, 2072-2076. doi: 10.1016/j.transproceed.2005.03.084

Fife, B. L., Weaver, M. T., Cook, W. L., and Stump, T. T. (2013). Partner interdependence and coping with life-threatening illness: the impact on dyadic adjustment. J. Fam. Psychol. 27:702. doi: 10.1037/a0033871

Frazier, P. A., Davis-Ali, S. H., and Dahl, K. E. (1995). Stressors, social support, and adjustment in kidney transplant patients and their spouses. Soc. Work Health Care 21, 93-108. doi: 10.1300/J010v21n02_07

Gmelch, S., and Bodenmann, G. (2007). Dyadisches coping in selbst-und fremdwahrnehmung als prädiktor für partnerschaftsqualität und befinden. Zeitschrift für Gesundheitspsychologie 15, 177-186. doi: 10.1026/0943-8149. 15.4.177

Gmelch, S., Bodenmann, G., Meuwly, N., Ledermann, T., Steffen-Sozinova, O., and Striegl, K. (2008). Dyadisches coping inventar (DCI): ein fragebogen zur erfassung des partnerschaftlichen umgangs mit stress. Zeitschrift für Familienforschung 20, 185-202.

Goetzmann, L., Klaghofer, R., Wagner-Huber, R., Halter, J., Boehler, A., Muellhaupt, B., et al. (2007). Psychosocial vulnerability predicts psychosocial outcome after an organ transplant: results of a prospective study with lung, liver, and bone-marrow patients. J. Psychosom. Res. 62, 93-100. doi: 10.1016/ j.jpsychores.2006.07.023

Greif-Higer, G., Wandel, E., Otto, G., Galle, P. R., and Beutel, M. E. (2008). Psychological conflicts between relatives during the long-term course after successful living organ donation. Transplant. Proc. 40, 902-906. doi: 10.1016/ j.transproceed.2008.03.040

Hagedoorn, M., Sanderman, R., Bolks, H. N., Tuinstra, J., and Coyne, J. (2008). Distress in couples coping with cancer: a meta-analysis and critical review of role and gender effects. Psychol. Bull. 134, 1-30. doi: 10.1037/0033-2909.134.1.1

Holtzman, S., Abbey, S. E., Singer, L. G., Ross, H. J., and Stewart, D. E. (2011). Both patient and caregiver gender impact depressive symptoms among organ transplant caregivers: who is at risk and why? J. Health Psychol. 16, 843-856. doi: $10.1177 / 1359105310393542$

Johnson, C. D., Wicks, M. N., Milstead, J., Hartwig, M., and Hathaway, D. K. (1998). Racial and gender differences in quality of life following kidney transplantation. J. Nurs. Scholarsh. 30, 125-130. doi: 10.1111/j.1547-5069.1998. tb01266.x

Junghaenel, D. U., Schneider, S., and Broderick, J. E. (2017). Partners' overestimation of patients' pain severity: relationships with partners' interpersonal responses. Pain Med. 19, 1772-1781. doi: 10.1093/pm/pnx217

Kadioglu, Z. O., Kacar, S., Eroglu, A., Guven, B., and Tilif, S. (2012). Dyadic adjustment and psychological concordance of kidney transplant recipients and donors after spousal transplantation. Transplant. Proc. 44, 1608-1613. doi: 10. 1016/j.transproceed.2012.04.014

Kenny, D. A., Kashy, D. A., and Cook, W. L. (2006). Dyadic Data Analysis. New York, NY: Guilford Press.

Kim, Y., Loscalzo, M. J., Wellisch, D. K., and Spillers, R. L. (2006). Gender differences in caregiving stress among caregivers of cancer survivors. Psychooncology 15, 1086-1092. doi: 10.1002/pon.1049

Kimmel, P. L. (2001). Psychosocial factors in dialysis patients. Kidney Int. 59, 1599-1613. doi: 10.1046/j.1523-1755.2001.0590041599.x

Kimmel, P. L. (2002). Depression in patients with chronic renal disease: what we know and what we need to know. J. Psychosom. Res. 53, 951-956. doi: 10.1016/S0022-3999(02)00310-0

Kimmel, P. L., and Patel, S. S. (2003). Psychosocial issues in women with renal disease. Adv. Chron. Kidney Dis. 10, 61-70. doi: 10.1053/jarr.2003.50000
Kimmel, P. L., Peterson, R. A., Weihs, K. L., Shidler, N., Simmens, S. J., Alleyne, S., et al. (2000). Dyadic relationship conflict, gender, and mortality in urban hemodialysis patients. J. Am. Soc. Nephrol. 11, 1518-1525.

Köllner, V., Archonti, C., Schäfers, H. J., Sybrecht, G. W., and Wilkens, H. (2004). Psychische betreuung von patienten und angehörigen in der transplantationsmedizin. Psychotherapeut 49, 37-45. doi: 10.1007/s00278-0030343-0

Kroenke, K., Spitzer, R. L., and Williams, J. B. (2001). The PHQ-9: validity of a brief depression severity measure. J. Gen. Intern. Med. 16, 606-613. doi: 10.1046/j.1525-1497.2001.016009606.x

Laederach-Hofmann, K., Bunzel, B., Freundorfer, E., and Schubert, M.-T. (2002). Changes in partner relationship after organ transplantation: comparison between heart, liver, and kidney transplantation. Psychother. Psychosom. Med. Psychol. 52, 5-15. doi: 10.1055/s-2002-19663

Lawrence, P. T. (1974). Psychic consequences of loss and replacement of body parts. J. Am. Psychoanal. Assoc. 22, 170-181. doi: 10.1177/000306517402200111

Manne, S. L., Siegel, S., Kashy, D., and Heckman, C. J. (2014). Cancer-specific relationship awareness, relationship communication, and intimacy among couples coping with early stage breast cancer. J. Soc. Pers. Relat. 31, 314-334. doi: 10.1177/0265407513494950

McPherson, C. J., and Addington-Hall, J. M. (2003). Judging the quality of care at the end of life: can proxies provide reliable information? Soc. Sci. Med. 56, 95-109. doi: 10.1016/S0277-9536(02)00011-4

McPherson, C. J., Wilson, K. G., Chyurlia, L., and Leclerc, C. (2010). The balance of give and take in caregiver-partner relationships: an examination of selfperceived burden, relationship equity, and quality of life from the perspective of care recipients following stroke. Rehabil. Psychol. 55:194. doi: 10.1037/a0019359

Myaskovsky, L., Dew, M. A., Switzer, G. E., McNulty, M. L., DiMartini, A. F., and McCurry, K. R. (2005). Quality of life and coping strategies among lung transplant candidates and their family caregivers. Soc. Sci. Med. 60, 2321-2332. doi: 10.1016/j.socscimed.2004.10.001

Navaie-Waliser, M., Spriggs, A., and Feldman, P. H. (2002). Informal caregiving: differential experiences by gender. Med. Care 40, 1249-1259. doi: 10.1097/ 00005650-200212000-00012

Noohi, S., Khaghani-Zadeh, M., Javadipour, M., Assari, S., Najafi, M., Ebrahiminia, M., et al. (2007). Anxiety and depression are correlated with higher morbidity after kidney transplantation. Transplant. Proc. 39, 1074-1078. doi: 10.1016/j.transproceed.2007.04.002

Osin, R., Pankrath, A.-L., Niederwieser, D., Döhner, H., Hönig, K., Vogelhuber, M., et al. (2018). Dyadisches coping von hämatoonkologischen patienten und ihren partnern: übereinstimmungsmaße und zusammenhänge mit sozialer unterstützung und psychischer belastung. Psychother. Psych. Med. 68, 55-65. doi: 10.1055/s-0043-110137

Pabst, S., Bertram, A., Zimmermann, T., Schiffer, M., and de Zwaan, M. (2015). Physician reported adherence to immunosuppressants in renal transplant patients: prevalence, agreement, and correlates. J. Psychosom. Res. 79, 364-371. doi: 10.1016/j.jpsychores.2015.09.001

Pinquart, M., and Sörensen, S. (2007). Correlates of physical health of informal caregivers: a meta-analysis. J. Gerontol. B Psychol. Sci. Soc. Sci. 62, 126-137. doi: 10.1093/geronb/62.2.P126

Pomaki, G., DeLongis, A., Anagnostopoulou, T., and Heininger, J. (2011). Can't live with you, can't live without you: negative family exchanges and adaptation in end-stage renal disease patients. J. Health Psychol. 16, 520-529. doi: 10.1177/ 1359105310393543

Rees, J., O'Boyle, C., and MacDonagh, R. (2001). Quality of life: impact of chronic illness on the partner. J. R. Soc. Med. 94, 563-566. doi: 10.1177/ 014107680109401103

Regan, T. W., Lambert, S. D., Kelly, B., McElduff, P., Girgis, A., Kayser, K., et al. (2014). Cross-sectional relationships between dyadic coping and anxiety, depression, and relationship satisfaction for patients with prostate cancer and their spouses. Patient Educ. Couns. 96, 120-127. doi: 10.1016/j.pec.2014.04.010

Reimer, J., Franke, G. H., Lütkes, P., Kohnle, M., Gerken, G., Philipp, T., et al. (2002). Die lebensqualität von patienten vor und nach nierentransplantation. Psychother. Psych. Med. Psychol. 52, 16-23. doi: 10.1055/s-2002- 19662

Revenson, T. A., Abraido-Lanza, A. F., Majerovitz, S. D., and Jordan, C. (2005). Couples Coping With Chronic Illnes: What's Gender Got to Do With it? Washington, DC: American Psychological Association. 
Rohmann, E., and Bierhoff, H.-W. (2007). Skalen zur erfassung der equity in partnerschaften (SEEP). Zeitschrift für Sozialpsychologie 38, 217-231. doi: 10. 1024/0044-3514.38.3.217

Schulz, R., and Sherwood, P. R. (2008). Physical and mental health effects of family caregiving. J. Soc. Work Educ. 44(Suppl. 3), 105-113. doi: 10.5175/JSWE.2008. 773247702

Sellares, J., de Freitas, D. G., Mengel, M., Reeve, J., Einecke, G., Sis, B., et al. (2012). Understanding the causes of kidney transplant failure: the dominant role of antibody-mediated rejection and nonadherence. Am. J. Transplant. 12, 388-399. doi: 10.1111/j.1600-6143.2011.03840.x

Spitzer, R. L., Kroenke, K., Williams, J. B., and Lowe, B. (2006). A brief measure for assessing generalized anxiety disorder: the GAD-7. Arch. Intern. Med. 166, 1092-1097. doi: 10.1001/archinte.166.10.1092

Stoller, E. P. (1992). "Gender differences in the experiences of caregiving spouses," in Gender, Families, and Elder Care, Vol. 138, eds J. W. Dwyer and R. T. Coward (Thousand Oaks, CA: Sage Publications, Inc), 49-64.

Szeifert, L., Molnar, M. Z., Ambrus, C., Koczy, A. B., Kovacs, A. Z., Vamos, E. P., et al. (2010). Symptoms of depression in kidney transplant recipients: a cross-sectional study. Am. J. Kidney Dis. 55, 132-140. doi: 10.1053/j.ajkd.2009. 09.022

Thompson, S. C., and Sobolew-Shubin, A. (1993). Overprotective relationships: a nonsupportive side of social networks. Basic Appl. Soc. Psychol. 14, 363-383. doi: 10.1207/s15324834basp1403_8
Walster, E., Berscheid, E., and Walster, G. W. (1973). New directions in equity research. J. Pers. Soc. Psychol. 25:151. doi: 10.1037/h0033967

Wilson, K. G., Curran, D., and McPherson, C. J. (2005). A burden to others: a common source of distress for the terminally ill. Cogn. Behav. Ther. 34, 115-123. doi: 10.1080/16506070510008461

Yee, J. L., and Schulz, R. (2000). Gender differences in psychiatric morbidity among family caregivers: a review and analysis. Gerontologist 40, 147-164. doi: 10.1093/geront/40.2.147

Zimmermann, T., Lause, M., and Heinrichs, N. (2015). Fragebogen zur partnerschaftsqualität: quality of marriage index-deutsche version (QMI-D). Verhaltenstherapie 25, 51-53. doi: 10.1159/000371478

Conflict of Interest Statement: The authors declare that the research was conducted in the absence of any commercial or financial relationships that could be construed as a potential conflict of interest.

Copyright $\odot 2019$ Tkachenko, Franke, Peters, Schiffer and Zimmermann. This is an open-access article distributed under the terms of the Creative Commons Attribution License (CC BY). The use, distribution or reproduction in other forums is permitted, provided the original author(s) and the copyright owner(s) are credited and that the original publication in this journal is cited, in accordance with accepted academic practice. No use, distribution or reproduction is permitted which does not comply with these terms. 


\section{Couples Dealing With Pediatric Blood Cancer: A Study on the Role of Dyadic Coping}

Marieke Van Schoors ${ }^{1 *}$, Tom Loeys ${ }^{2}$, Liesbet Goubert ${ }^{1}$, Geertrui Berghmans ${ }^{3}$, Britt Ooms ${ }^{4}$, Jurgen Lemiere ${ }^{5,6}$, Koenraad Norga ${ }^{7,8}$ and Lesley Liliane Verhofstadt ${ }^{1}$

${ }^{1}$ Department of Experimental Clinical and Health Psychology, Ghent University, Ghent, Belgium, ${ }^{2}$ Department of Data Analysis, Ghent University, Ghent, Belgium, ${ }^{3}$ Department of Pediatric Hemato-Oncology and Stem Cell Transplantation Ghent, Ghent University Hospital, Ghent, Belgium, ${ }^{4}$ Department of Pediatric Hemato-Oncology and Immunology, University Hospital Brussels, Brussels, Belgium, ${ }^{5}$ Department of Pediatric Hemato-Oncology, University Hospital Leuven, Leuven, Belgium, ${ }^{6} \mathrm{KU}$ Leuven, Leuven, Belgium, ${ }^{7}$ Department of Pediatric Oncology, Antwerp University Hospital, Antwerp,

Belgium, ${ }^{8}$ University of Antwerp, Antwerp, Belgium

OPEN ACCESS

Edited by:

Guy Bodenmann

University of Zurich, Switzerland

Reviewed by:

Hoda Badr

Baylor College of Medicine,

United States

Sabrina Cipolletta,

University of Padua, Italy

Fridtjof W. Nussbeck,

Universität Konstanz, Germany

*Correspondence:

Marieke Van Schoors marieke.vanschoors@ugent.be

Specialty section:

This article was submitted to Clinical and Health Psychology,

a section of the journal

Frontiers in Psychology

Received: 05 June 2018 Accepted: 11 February 2019

Published: 27 February 2019

Citation:

Van Schoors M, Loeys T, Goubert L, Berghmans G, Ooms B,

Lemiere J, Norga $K$ and Verhofstadt LL (2019) Couples Dealing With Pediatric Blood Cancer:

A Study on the Role of Dyadic Coping. Front. Psychol. 10:402. doi: 10.3389/fpsyg.2019.00402
Objective: Pediatric cancer is a life-threatening disease that poses significant challenges to the ill child and his/her parents. Among the studies investigating risk and protective factors for the individual and relationship adjustment of parents being confronted with pediatric cancer, couple factors - such as dyadic coping - gained little research attention. Therefore, the aim of the current study was to explore the association between dyadic coping and individual/relationship outcomes of parents in the context of pediatric cancer.

Methods: Participants were 59 couples of children diagnosed with leukemia or NonHodgkin lymphoma. Time since diagnosis varied from diagnosis to 20 months. Both parents completed the DCl-short, DASS21, PIP, and MMQ.

Results: Positive dyadic coping (i.e., supportive and common dyadic coping) and negative dyadic coping proved to be related to individual and relational outcomes of parents facing cancer in their child. In addition, while men and women reported to be equally satisfied with their partner and their sexual relationship, women reported higher levels of individual maladjustment.

Conclusion: Our findings led to the conclusion that dyadic coping is important for both individual as well as relationship outcomes of parents when facing a diagnosis of cancer in their child. When meeting with families, both partners should be invited as a unit in order to best capture couple level experiences. Also, clinicians should be sensitive to relational and sexual issues besides individual issues, taking into account evidence-based standards for psychosocial care in pediatric oncology.

Keywords: couples, intimate relationships, pediatric cancer, dyadic coping, individual adjustment, relationship adjustment

\section{INTRODUCTION}

Pediatric cancer is an unpredictable and uncontrollable stressor that puts the diagnosed child at risk for adjustment difficulties (Alderfer and Kazak, 2006). There are a number of pediatric cancers, with blood cancer, including leukemia and lymphoma, as the most common type. Leukemia and lymphoma account for about 30 and $8 \%$ of all cancers in children, respectively 
(American Cancer Society, 2016). Due to advances in chemotherapy and stem cell transplantation, long-term survival of children with blood cancer can be achieved (Silverman and Weinstein, 1997). However, although many function well, some children with blood cancer (Rao et al., 1992; van der Does-van den Berg et al., 1995) or pediatric cancer in general (Kazak et al., 2001; Kestler and LoBiondo-Wood, 2012) experience social or emotional problems during or after treatment. In addition, the impact of a pediatric cancer diagnosis on the ill child's parents is undeniable. Every child is embedded in a broader social context, and therefore, a stressor (like pediatric cancer) influences not only the development and adaptation of that child, but also the context in which s/he lives and the subsystems with which s/he interacts (Social Ecology Model: Bronfenbrenner, 1977; Cipolletta et al., 2015). Indeed, in the context of pediatric cancer, there is abundant empirical evidence for the impact of the diagnosis and its treatment on the parents, both at the level of their individual functioning and couple functioning.

Concerning the impact of pediatric cancer on parents' individual outcomes, existing research revealed that a significant subset of parents report emotional distress, anxiety and acute or posttraumatic stress symptoms shortly after diagnosis (Grootenhuis and Last, 1997; Vrijmoet-Wiersma et al., 2008; Ljungman et al., 2014). Moreover, especially mothers seem to be impacted: they report more psychological distress than mothers of healthy children and fathers of children with cancer (Pai et al., 2007). In addition to the impact on parents' individual functioning, many studies have documented the impact of pediatric cancer on parents' intimate relationship (e.g., HoekstraWeebers et al., 1998; Patistea et al., 2000). A recently conducted systematic review (Van Schoors et al., 2017) revealed that although most couples adjust well to the crisis of a pediatric cancer diagnosis in domains such as emotional closeness, couple support and marital satisfaction, most couples do experience difficulties in the domains of sexual intimacy and conflict, both on and off treatment.

It should be noted, however, that the research described above also revealed a considerable variability -both across and within studies- in individual outcomes as well as relationship outcomes for parents facing pediatric cancer. Given this great variability, a growing number of studies has tried to explain why some parents adjust better than others. Among these studies investigating risk and protective factors for individual and relationship functioning of parents being confronted with pediatric cancer, especially individual characteristics (e.g., catastrophic thoughts in parents; Caes et al., 2014) and family characteristics (e.g., family support; Fuemmeler et al., 2003) have been the topic of investigation. In contrast, so-called couple factors -characteristics of the intimate relationship of the child's parents- that may foster or inhibit parental individual and relationship outcomes gained less research attention. The current study aimed to address this gap by focusing on a couple-level variable that could be expected to moderate the impact of pediatric cancer on parents' individual and relationship outcomes, namely, the extent to which parents deal with the stressor of pediatric cancer as a dyad ("dyadic coping;" see Bodenmann, 1995). Dyadic coping has been identified in the couple research literature as well as the stress and coping literature as playing a cardinal role in individual and relationship functioning within couples facing severe stressors (e.g., Kayser et al., 1999; Bodenmann, 2005).

"Dyadic coping" should be distinguished from other ways of coping with stress within intimate relationships, such as partners' individual coping (e.g., LaMontagne et al., 2003; Garro, 2004; Wong and Heriot, 2008) and their attempts at seeking social support from friends or relatives (e.g., Fife et al., 1987). In particular, in situations where there is the crossover of individual stress from one partner to the other (e.g., work stress) or in cases of partners' shared stress from common sources (e.g., stress related to pediatric cancer), a joint appraisal of the stressful situation is required, which triggers dyadic coping, in addition to partners' individual coping. Within the dyadic coping literature, positive as well as more negative forms of coping as a dyad are described. Positive forms of dyadic coping include supportive dyadic coping (i.e., one partner assists the other in his/her coping efforts) and common dyadic coping (i.e., both partners participate in the coping process together). Negative forms of dyadic coping include hostile (i.e., support accompanied by distancing or sarcasm), ambivalent (i.e., support that is unwillingly) or superficial (i.e., support that is insincere) dyadic coping (Bodenmann, 1995, 1997, 2005).

Both theoretical and empirical arguments speak to the need of investigating (the role of) dyadic coping in the context of pediatric cancer. First, according to the Systemic Transactional Model (STM) of Stress and Coping in Couples, stressors always affect (directly or indirectly) both partners in an intimate relationship. This is true if the situation concerns primarily one partner - then his/her stress reactions and coping affects the other and turn into dyadic issues, representing the crossover of stress and coping from one partner to the other (i.e., stressor of the self/partner) - and if the situation concerns both partners (i.e., shared stressors), both with regard to stress from daily hassles and more severe stressors (Bodenmann et al., 2016). So, stress and coping need to be understood as a systemic issue, a social process rooted in intimate relationships, with special attention to the interdependence and the mutual influence between romantic partners (Bodenmann et al., 2016). According to this theory, a pediatric cancer diagnosis needs to be considered as a shared and "dyadic stressor," as it is indeed a stressful event or encounter that concerns both partners, either directly or indirectly (Bodenmann, 1995, 1997). Both parents are directly involved in their child's illness, as shown by the finding that mostly one parent (temporarily) quits his/her job in order to accompany the diagnosed child day and night (Van Schoors et al., 2018) or by the parents' individual emotional consequences described earlier (e.g., Pai et al., 2007). Also in line with this theory is that a dyadic stressor requires dyadic coping, conceptualized as the way couples cope with stress together in sharing appraisals of demands and planning together how to deal with the stressors. The importance of studying dyadic coping within the context of pediatric cancer can be derived from studies underscoring the positive role of copingrelated activities, such as individual coping (e.g., Grootenhuis and Last, 1997) and social support (e.g., Fife et al., 1987) for the adjustment of parents and their ill child. Second, the 
importance of dyadic coping within the context of couples facing health and illness-related issues has been equally documented. For instance, the positive effect of dyadic coping on individual outcomes like health is largely documented (e.g., Berg and Upchurch, 2007; Meier et al., 2011), also in adult cancer studies (e.g., Kayser et al., 1999; Badr et al., 2008). Previous studies furthermore show robust and consistent associations between dyadic coping and relationship outcomes (Falconier et al., 2015). More specifically, a recent systematic review that focuses on couples coping with adult cancer illustrates that positive dyadic coping (i.e., supportive dyadic coping and common dyadic coping) improves relationship functioning, while negative dyadic coping impedes relationship functioning (Traa et al., 2015).

Taken together, based on theory (STM) and previous research on chronic illnesses in adulthood, we expect that dyadic coping may also be of importance in the context of pediatric cancer. More specifically, we expect that adequate dyadic coping (i.e., more supportive dyadic coping, more common dyadic coping, and less negative dyadic coping) is associated with better individual outcomes (i.e., less negative emotions: less stress, anxiety and depression, and lower levels of childhood illnessrelated parenting stress) and better relationship outcomes (i.e., higher marital and sexual adjustment) within parents being confronted with cancer in their child.

\section{MATERIALS AND METHODS}

\section{Participants}

The sample consisted of 59 heterosexual couples; all biological parents of children diagnosed with leukemia or non-Hodgkin lymphoma. They were all Caucasian and living in the Flemish part of Belgium. Mothers' mean age was 38.5 (Range 29-52); fathers' mean age was 40.5 (Range 30-56). Time since diagnosis varied from 0 to 20 months $(M=6.9, S D=6.6)$. Fortythree women and thirty-seven men had a Bachelor or Master degree. In eight families, the diagnosed child was the only child. The remaining families had either two (28 families), three (20 families) or four (3 families) children. More details on the sample are listed in Table 1. Ethical approval from the University Hospitals of Ghent, Brussels, Antwerp, and Leuven had been secured for the study and the appropriate written informed consent forms were obtained for all participants.

\section{Procedure}

The present study is part of a larger study examining the impact of pediatric cancer on families, i.e., "UGhent Families and Childhood Cancer study." For this large-scale study, children diagnosed with leukemia or non-Hodgkin lymphoma between the age of one and 18 years, their biological parents and any siblings were invited to take part in a survey study. Exclusion criteria were: (1) not speaking Dutch, (2) expression of a developmental disorder in the diagnosed child, and (3) relapse. Over a period of 3 years, 129 families participated; i.e., 65\% of the eligible families. In 65 of these families, both parents filled out the questionnaires (50\%), 59 of whom were married/co-habiting
(91\%) and 6 were divorced (9\%). As this study focuses on the intimate relationship, the final sample only included the married or co-habiting couples $(N=59)$.

\section{Measures \\ Dyadic Coping}

A short version of the Dyadic Coping Inventory (DCI; Bodenmann, 2008) was used to measure several forms of dyadic coping. The questionnaire consists of 17 items, grouped into 6 subscales: Supportive Dyadic Coping (e.g., "S/he makes me feel that s/he understands me and is committed to me"), Common Dyadic Coping (e.g., "We try to tackle the problem together and work together"), Negative Dyadic Coping (e.g., "S/he does not take my stress seriously"), Own Stress Communication (e.g., "When I feel overwrought, I show my partner that I feel bad and that I need his/her emotional support"), WE-Stress Appraisal and Individual Stress-Appraisal. In this study, only the subscales supportive dyadic coping, common dyadic coping and negative dyadic coping were included given our focus on dyadic coping. Response options for each item ranged from 1 to 5 ("very rarely" to "almost always"). Scores for each subscale were obtained by summing the relevant items. The DCI has good reliability and validity (Ledermann et al., 2010). In the present study, Cronbach's alpha coefficients were 0.53/0.83 (supportive dyadic coping), 0.67/0.95 (common dyadic coping) and 0.75/0.70 (negative dyadic coping) for men and women, respectively. The low Cronbach's alpha for the male supportive dyadic coping subscale could not be improved by dropping one or more items.

\section{Depression, Anxiety, Stress}

The Depression Anxiety Stress Scale (DASS-21; Lovibond and Lovibond, 1995) is a brief version of the 42-item DASS and consists of 21 items exploring negative emotions experienced over the last week. Participants rate the extent to which feelings of depression (e.g., "I felt that I had nothing to look forward to"), anxiety (e.g., "I experience trembling") and stress (e.g., "I found it hard to wind down") apply to them on a fourpoint scale from 0 (never) to 3 (almost always). Scores for depression, anxiety and stress were obtained by summing the relevant seven items. The DASS-21 proved to be reliable in both clinical and community samples (Antony et al., 1998). In the present study, Cronbach's alpha coefficients were (for men and women, respectively) $0.88 / 0.91$ for depression, $0.77 / 0.79$ for anxiety and $0.85 / 0.89$ for stress.

\section{Childhood Illness-Related Parenting Stress}

The Pediatric Inventory for Parents (PIP; Streisand et al., 2001) measures childhood illness-related parenting stress. The questionnaire consists of 42 items grouped into four domain scales indicating the type of stressors parents are experiencing related to caring for their ill child: (1) medical care (e.g., "helping my child with medical procedures"), (2) communication (e.g., "speaking with child about his/her illness"), (3) role functioning (e.g., "being unable to go to work/job"), and (4) emotional functioning (e.g., "feeling numb inside"). Given the overlap between the DASS-21 and the emotional functioning subscale, the latter subscale was not included. In addition, both 
TABLE 1 | Background characteristics of couples of children with Leukemia or non-Hodgkin lymphoma.

\begin{tabular}{|c|c|c|c|}
\hline & Demographic variable & & Men | Women \\
\hline \multirow[t]{5}{*}{ Parents } & $N$ (couples) & & 59 \\
\hline & Age, mean $(S D)$ & & $40.5(6.7) \mid 38.5(6.2)$ \\
\hline & Education, $n$ & Primary school & $1 \mid 0$ \\
\hline & & High school & $21 \mid 16$ \\
\hline & & Bachelor/Master & $37 \mid 43$ \\
\hline \multirow[t]{7}{*}{ III child } & $\mathrm{N}$ & & 59 \\
\hline & Sex, boys, $n$ & & 36 \\
\hline & Age, mean $(S D)$ & & $7.7(5.1)$ \\
\hline & Diagnosis, $n$ & Acute lymphoblastic leukemia (ALL) & 43 \\
\hline & & Acute myeloid leukemia (AML) & 3 \\
\hline & & Non-Hodgkin Lymphoma & 13 \\
\hline & Time since diagnosis in months (SD; Range) & & $6.9(6.6 ; 0-20)$ \\
\hline
\end{tabular}

the frequency over the last week and the level of difficulty of each item is assessed on a five-point scale (frequency: $1=$ "never" to 5 = "very often;" difficulty: 1 = "not at all" to 5 = "extremely"). Frequency and difficulty scores are summed for each of the three domain scales; these scale scores are then summed into an overall total frequency score (PIP-F) and total difficulty score (PIP-D) with higher scores indicating greater frequency and difficulty of illness-related stress. The PIP has good reliability and validity (Streisand et al., 2001). In the present study, Cronbach's alpha coefficients were 0.92/0.92 for the total frequency score and $0.91 / 0.90$ total difficulty score, men and women, respectively.

\section{Marital Adjustment}

The Maudsley Marital Questionnaire (MMQ; Arrindell et al., 1983) evaluates the marital relationship in general (e.g., "How much are you committed to this marriage?"), the sexual relationship (e.g., "Are you satisfied with the present frequency of sexual intercourse'?") and life in general (e.g., "Are you competent and successful at your job and your housework'?"). The questionnaire contains 20 items, each of which is rated on a $0-8$ scale, with 0 representing the optimum response. A cutoff score $>20$ on the marital adjustment scale can be used to identify individuals who experience marital dissatisfaction (a level of marital dissatisfaction equal to the one reported by couples referred for marital counseling; Tuinman et al., 2005). In our study, 18 men and 19 women reported a score above 20 on the marital adjustment scale. When comparing the means on the MMQ marital adjustment scale of our study with a recent, Belgian, community sample (Hellemans, 2014), the current sample reported significantly higher levels of marital dissatisfaction $(D=3.88, t=3.63, p<0.001)$. The MMQ has good reliability and validity and the psychometric qualities of the Dutch version were also found to be satisfactory (Arrindell et al., 1983; Orathinkel et al., 2007). In the present study, only the two relationship subscales were taken into account, with a Cronbach's alpha of $0.91 / 0.67$ for marital adjustment and $0.84 / 0.89$ for sexual adjustment, men and women, respectively. For both subscales, a higher score indicates more maladjustment.

\section{Data Analytic Strategy}

We first describe means (with standard deviation and range) for all study variables and assess differences between men and women using a paired $t$-test. We further present correlations between study variables for men and women separately. The correlations for each study variable between men and women illustrate the non-independence within couples. To assess the association between the perception of supportive, common and negative dyadic coping (DCI) on the one hand and the frequency and difficulty of childhood illness-related parenting stress (PIP), depression, anxiety and stress (DASS), and marital and sexual adjustment (MMQ) on the other hand, we relied on the ActorPartner Interdependence Model (APIM; Cook and Kenny, 2005). As shown in Figure 1, the APIM allows to simultaneously assess the effect of one's own perception of dyadic coping and one's

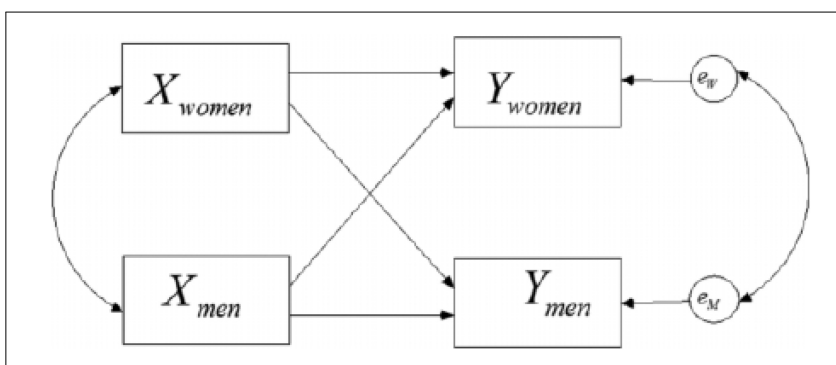

FIGURE 1 | The Actor-Partner Interdependence Model (APIM). X represents one' perception of dyadic coping (i.e., one of the dyadic coping subscales), while $Y$ represents parenting stress (PIP), depression, anxiety and stress (DASS) or marital and sexual adjustment (MMQ subscales). For supportive and negative dyadic coping: an actor effect for women (men) can be interpreted as the effect of female (male) perception of her (his) partner's supportive/negative coping efforts on the female (male) adjustment; a partner effect of women in men (of men in women) can be interpreted as the effect of female (male) perception of her (his) partner's supportive/negative coping efforts on the partner's adjustment. For common dyadic coping: an actor effect for women (men) can be interpreted as the effect of female (male) perception of the couple's common coping efforts on the female (male) adjustment; a partner effect of women in men (of men in women) can be interpreted as the effect of female (male) perception of the couple's common coping efforts on the partner's adjustment. 
partner perception of dyadic coping on one's own (actor) and one's partner outcome, while accounting for the correlation of outcomes within couples. The residuals of men and women were allowed to be correlated and to have a different variance (i.e., an unstructured residual covariance). A separate APIM was fitted for each combination of dyadic coping subscales and outcome allowing for differential effects for male and female partners. Only if the overall tests for actor and partner effects [that is, testing the goodness-of-fit of models without actor (partner) effects] turned significant, actor and partner effects were inspected. Note that in all analyses, the time since diagnosis was included as a covariate, and was allowed to have a different effect on the male and female outcomes. All analyses were performed in the Structural Equation Modeling (SEM) framework (Stas et al., 2018) using the R-package lavaan. Unstandardized regression coefficients for actor and partner effects are presented with corresponding standard error and $p$-value. To assess gender differences in actor and partner effects, the difference between the male and female actor effect (partner effect, respectively) was calculated (hereafter referred to as the difference test). All tests were performed at the 0.05 significance level. Given the exploratory nature of this study, no correction for multiple testing was performed.

\section{RESULTS}

Table 2 shows the descriptive statistics and correlations of the variables in our study. For common and negative dyadic coping, no significant gender differences were found. However, women reported experiencing more supportive behavior (supportive dyadic coping) from their partner than their male partner $\left(D^{\mathrm{M}-\mathrm{W}}=-1.00, t(56)=-2.03, p=0.047\right)$. Furthermore, higher levels of childhood illness-related stress (frequency $D^{\mathrm{M}-\mathrm{W}}=-7.72, t(57)=-2.82, p=0.007$; difficulty $\left.D^{\mathrm{M}-\mathrm{W}}=-9.04, t(57)=-4.45, p<0.001\right)$, anxiety $\left(D^{\mathrm{M}-\mathrm{W}}=-1.85, t(58)=-3.14, p<0.001\right)$, depression $\left(D^{\mathrm{M}-\mathrm{W}}=-1.61, t(58)=-2.11, \quad p=0.04\right)$ and stress $\left(D^{\mathrm{M}-\mathrm{W}}=-2.98, t(58)=-4.59, p<0.001\right)$ were found in women, as compared to men. Finally, regarding marital and sexual adjustment, no gender differences were found. Next, we discuss the results of the APIM-analyses (see Supplementary Table 1). We limit our discussion below to the gender-specific actor and partner effects for whom the global actor and partner test, respectively, were significant at 0.05 level (Table 3). Table 4 shows an overview of the significant APIM-results.

\section{Dyadic Coping and Individual Outcomes Childhood Illness-Related Parenting Stress}

More common dyadic coping reported by men was associated with lower difficulty scores of childhood illness-related parenting stress in men (actor effect; $B=-2.58, S E=0.89, p=0.004$ ). In addition, two partner effects were found: higher levels of supportive dyadic coping as perceived by men in their partner and more common dyadic coping reported by men were both associated with lower difficulty scores of parenting stress in women when facing illness in a child $(B=-3.07, S E=0.93$, $p=0.001$ and $B=-3.14, S E=1.09, p=0.004$; respectively).

\section{Negative Emotions}

When assessing the association between dyadic coping and negative emotions, one actor effect was found in men: higher levels of negative dyadic coping perceived by men were associated with higher levels of depression in men $(B=0.50, S E=0.24$, $p=0.034$ ). Furthermore, 3 partner effects were present in women. Higher levels of supporting dyadic coping as perceived by men in their partner were associated with lower levels of depression in women $(B=-0.82, S E=0.27, p=0.003)$, lower levels of anxiety in women $(B=-0.78, S E=0.19, p<0.001)$ and lower levels of stress in women $(B=-0.84, S E=0.23, p<0.001)$.

\section{Dyadic Coping and Relationship Outcomes}

Both in men and women separately, actor effects of dyadic coping emerged when considering marital adjustment as outcome. In men, we found that higher levels of supportive dyadic coping as perceived by men in their partner and more common dyadic coping reported by men $(B=-2.23, S E=0.60, p<0.001$; $B=-1.18, S E=0.60, p=0.050$, respectively) were associated with higher levels of marital adjustment reported by men. Negative dyadic coping as perceived by men in their partner was found to be associated with lower levels of marital adjustment reported by men $(B=2.50, S E=0.48, p<0.001)$. In women, we found that higher levels of supportive dyadic coping as perceived by women in their partner and more common dyadic coping reported by women $(B=-2.66, S E=0.43, p<0.001)$ were associated with higher levels of marital adjustment reported by women $(B=-1.91, S E=0.37, p<0.001)$. Negative dyadic coping as perceived by women in their partner was found to be associated with lower levels of marital adjustment reported by women $(B=2.62, S E=0.47, p<0.001)$.

One partner effect was found for coping reported by women on relationship adjustment reported by men: lower levels of negative dyadic coping as perceived by women in their partner ( $B=1.75, S E=0.47, p<0.001$ for respectively) were associated with higher levels of relationship adjustment as reported by their partner. Furthermore, negative dyadic coping as perceived by men in their partner was associated with lower levels of relationship adjustment reported by women $(B=1.37$, $S E=0.48, p=0.005$ ).

When considering sexual adjustment as an outcome, only actor effects were observed in men and women for some dyadic coping subscales. More specifically, higher levels of common dyadic coping reported by men was associated with higher levels of sexual adjustment reported by men $(B=-1.60, S E=0.53$, $p=0.003)$. Furthermore, for both men and women, higher levels of perceived negative dyadic coping in the partner were linked to lower levels of sexual adjustment (actor effects; $B=1.29$, $S E=0.47, p=0.006$ for men and $B=0.83, S E=0.41, p=0.046$ women, respectively).

\section{DISCUSSION}

Using an Actor-Partner Interdependence Model (APIM; Cook and Kenny, 2005), the present study sought to examine whether 


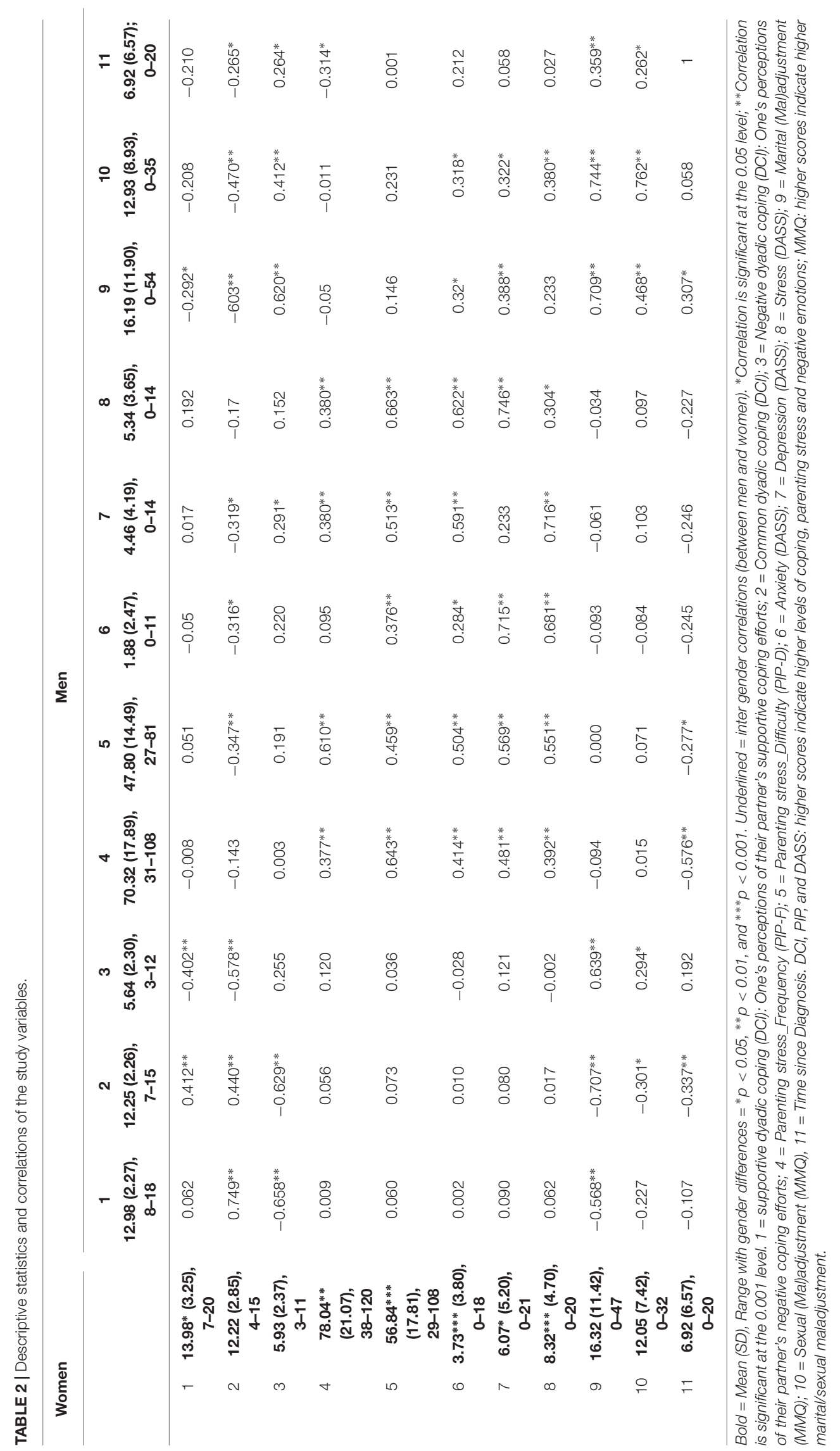


TABLE 3 | APIM analyses.

\begin{tabular}{|c|c|c|}
\hline & Overall Test & Difference Test \\
\hline Actor effect CDC on PIP-D & $X^{2}(2)=8.181, p=0.017$ & $z=-2.577, p=0.010$ \\
\hline Partner effect CDC on PIP-D & $X^{2}(2)=9.223, p=0.010$ & $z=3.120, p=0.002$ \\
\hline Partner effect SDC on PIP-D & $X^{2}(2)=10.052, p=0.007$ & $z=2.608, p=0.009$ \\
\hline Actor effect NDC on depression & $X^{2}(2)=6.220, p=0.045$ & $z=0.358, p=0.720$ \\
\hline Partner effect SDC on depression & $X^{2}(2)=8.789, p=0.012$ & $z=2.221, p=0.026$ \\
\hline Partner effect SDC on anxiety & $X^{2}(2)=18.892, p<0.001$ & $z=3.011, p=0.003$ \\
\hline Partner effect SDC on stress & $X^{2}(2)=12.092, p=0.002$ & $z=2.799, p=0.005$ \\
\hline Actor effect SDC on marital adjustment & $X^{2}(2)=25.433, p<0.001$ & $z=1.010, p=0.312$ \\
\hline Actor effect CDC on marital adjustment & $X^{2}(2)=50.539, p<0.001$ & $z=0.524, p=0.600$ \\
\hline Actor effect NDC on marital adjustment & $X^{2}(2)=49.765, p<0.001$ & $z=-0.165, p=0.869$ \\
\hline Partner effect NDC on marital adjustment & $X^{2}(2)=20.538, p<0.001$ & $z=0.393, p=0.694$ \\
\hline Actor effect CDC on sexual adjustment & $X^{2}(2)=14.308, p=0.001$ & $z=1.406, p=0.160$ \\
\hline Actor effect NDC on sexual adjustment & $X^{2}(2)=12.569, p=0.002$ & $z=0.222, p=0.824$ \\
\hline
\end{tabular}

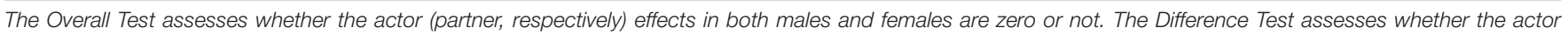

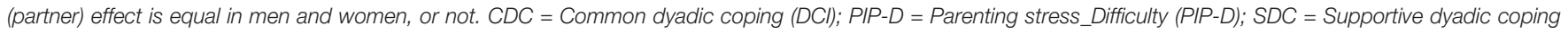
$(D C l) ; N D C=$ Negative dyadic coping (DCl); depression, anxiety and stress (DASS); marital and sexual adjustment (MMQ).

TABLE 4 | APIM-results: an overview.

\begin{tabular}{|c|c|c|c|c|c|c|c|c|c|c|c|c|c|c|c|}
\hline & & \multicolumn{7}{|c|}{ Men } & \multicolumn{7}{|c|}{ Women } \\
\hline & & 1 & 2 & 3 & 4 & 5 & 6 & 7 & 1 & 2 & 3 & 4 & 5 & 6 & 7 \\
\hline \multirow[t]{3}{*}{ Men } & SDC & & & & & & $x$ & & & $X$ & $x$ & $x$ & $X$ & & \\
\hline & CDC & & $x$ & & & & $x$ & $x$ & & $x$ & & & & & \\
\hline & NDC & & & $x$ & & & $x$ & $x$ & & & & & & X & \\
\hline \multirow[t]{3}{*}{ Women } & SDC & & & & & & & & & & & & & $X$ & \\
\hline & CDC & & & & & & & & & & & & & $X$ & \\
\hline & NDC & & & & & & $x$ & & & & & & & $x$ & X \\
\hline
\end{tabular}

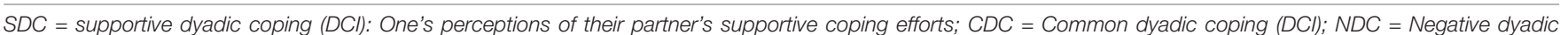

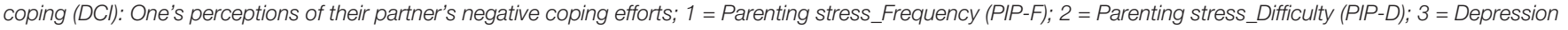
(DASS); 4 = Anxiety (DASS); 5 = Stress (DASS); 6 = Marital (Mal)adjustment (MMQ); 7 = Sexual (Mal)adjustment (MMQ). X = Statistically significant effect.

dyadic coping was related to individual outcomes (negative emotions: anxiety, depression \& stress and childhood illnessrelated parenting stress) and relationship outcomes (marital adjustment and sexual adjustment) in parents of children diagnosed with blood cancer.

\section{Summary of Results Dyadic Coping and Individual Outcomes}

Our findings indicate that both positive (i.e., supportive and common dyadic coping) and negative forms of dyadic coping matter for individual outcomes within parents being confronted with a cancer diagnosis in their child. This is in line with our prediction and with previous quantitative research on adult chronic illnesses (e.g., Meier et al., 2011; Regan et al., 2014). However, different patterns of findings emerged for supportive, common and negative dyadic coping.

More specifically, we found that the more men perceived their partner as supportive, the less depression, anxiety and stress (both general stress and difficulty scores on childhood illnessrelated stress) their partner experienced. In other words, the more men perceived their spouse as supportive, understanding and helping, the better the female partner's individual adjustment when facing pediatric cancer. These associations are in line with existing evidence that couple support is a protective and helpful factor in the individual adjustment to pediatric cancer (e.g., Morrow et al., 1982; Tarr and Pickler, 1999). However, we did not find the expected actor effects; i.e., associations between perceived supportive dyadic coping in one's partner and one's own individual adjustment. These findings seem to suggest that the benefits of support are mostly associated with support giving rather than support receiving, a finding that has also been reported by other researchers in the context of health outcomes (Brown et al., 2003). Furthermore, more common dyadic coping reported by men was associated with lower difficulty scores on illness-related parenting stress for men and for women. So, the more men had the experience that both partners participated in the coping process symmetrically or complementary, the less they and their partner struggled with the care of their ill child.

Finally, the more men perceived their partner as negative, the more depressive complaints they experienced. This is in line with previous studies investigating the association between negative dyadic coping and negative emotions in 
adult chronically ill populations (e.g., Meier et al., 2011). Looking at the differential effects of the different types of dyadic coping, negative dyadic coping seems to be of less importance for the individual well-being of parents facing pediatric cancer. This finding is not in line with the literature on adult chronic illness describing negative forms of dyadic coping to be frequently occurring (Meier et al., 2011). This contradiction can be understood in two possible ways. First, it is possible that partner effects between negative dyadic coping and individual adjustment were not found in this study due to the relative small sample size (mimicking the observed associations, the power to detect such effects with $N=59$ couples ranged from 5 to 64\%). Second, in the context of adult chronic illness, there is one partner undergoing the illness, and one experiencing the illness from a certain distance. In the context of a child's cancer diagnosis, however, the child is ill, and therefore both parents may experience the illness in a more similar way. As a consequence, it is possible that couples, after facing a cancer diagnosis in their child, tend to understand each other better than in the context of adult chronic illness, and therefore, possibly engage less in negative dyadic coping.

\section{Dyadic Coping and Relational Outcomes}

For relationship outcomes within parents being confronted with a cancer diagnosis in their child, our findings indicated that both positive (i.e., supportive and common dyadic coping) and negative forms of dyadic coping matter. This is in line with our prediction, published quantitative studies (e.g., Falconier et al., 2015) and a recent systematic review (Traa et al., 2015) in the context of adult chronic illnesses. More specifically, the present study shows that positive dyadic coping (i.e., supportive and common dyadic coping) was associated with higher marital adjustment, both in men and women (actor effects). In other words, the more a man perceives his partner as supportive and helping and the more he has the idea that both partners participate in the coping process symmetrically or complementary, the more he is satisfied with his marital relationship. The same pattern of findings was found for women. Furthermore, for negative dyadic coping, the more a man experiences distancing, mocking or sarcasm in his partner when talking about the illness, the less satisfied he is with his marital relationship. Again, this finding was replicated in women. Next to these so-called actor effects, the following partner effects were also found for marital adjustment. The more men and women perceived their partner as negative when talking about the cancer, the less satisfied their partner was in the marital relationship.

With regard to sexual adjustment, the more men experienced managing the cancer situation together, and the less negative their partner reacts, the more satisfied they were with their sexual relationship. Furthermore, the more women perceived their partner as negative, hostile or not interested, the less satisfied they were with their sexual relationship. These findings extend existing research by demonstrating that dyadic coping is not only related to marital adjustment and marital satisfaction (e.g., Falconier et al., 2015) but also to couples' sexual satisfaction.

Remarkably, for relationship adjustment, both actor effects and certain partner effects of dyadic coping were found to be important, whereas for sexual adjustment, only actor effects proved to be significant. So, how a parent describes the way in which s/he and his/her partner, as a couple, cope with the stressor together (i.e., supportive, negative or common) was at least partially related to their own and their partner's evaluation of the relationship (actor and partner effect) but only to their own evaluation of the sexual relationship (actor effect). The absence of partner effects in explaining sexual adjustment may be linked to the fact that sexuality is, in se, an intimate domain and a difficult topic to discuss. As a consequence, the assessment of one's sexual relationship may be primarily linked to one's own appraisal of dyadic coping.

\section{Gender}

Gender differences as well as important gender similarities emerged from our data. Although at the relationship level, men and women reported to be equally satisfied with their partner and their sexual relationship, men and women did differ with regard to their individual adjustment. Across all individual outcomes, women reported higher levels of maladjustment (i.e., child's illness-related stress, anxiety, depression, stress) when facing a cancer diagnosis in their child than their male partner. This is in line with previous studies in the context of pediatric cancer, showing that especially mothers are impacted by the illness of the child (Pai et al., 2007). This finding may be explained by the increased burden assumed to be experienced by mothers in the care of children with cancer, as they are for example more likely to accompany the child to medical procedures (Kazak et al., 1996) and to stay in the hospital day and night (Van Schoors et al., 2018). In terms of dyadic coping, men and women only seemed to differ in the amount of supportive coping they perceived in their partner, with women reporting higher levels of supportive coping in their partner than men. These findings are not in line with the so-called marital support gap hypothesis, assuming that women are better support providers in their relationship than men are (see Verhofstadt et al., 2007 for a critical discussion). Comparing this finding to existing research on gender differences and similarities in dyadic coping is hard, however, as previous research focused on populations in which one of the partners was ill and therefore in a more support seeking/receiving position. Furthermore, important similarities between men and women in the association between dyadic coping and the relational outcomes under study emerged, more specifically the actor effects of dyadic coping on marital adjustment. Indeed, no significant differences were found in the actor/partner effects on relational outcomes between males and females (Table 3). This means that the pattern of findings found in our male subsample was fully replicated within our female subsample and that for both parents of children with cancer, dyadic coping and relationship functioning are intertwined. However, the absence of evidence for a difference 
might also be due to the low power to detect such interactions in small samples (Gistelinck et al., 2018). For the individual outcomes, the patterns for men versus women were more heterogeneous, thus less parallels could be drawn between them. Indeed, several of the observed actor and partner effects on individual outcomes were significantly different between men and women (Table 3). Finally, gender effects also emerged in terms of effects of the predictor (i.e., the perception of dyadic coping). More specifically, men and women only differed in the partner effects of supportive dyadic coping on the individual outcomes (i.e., anxiety, depression and stress), and not in the actor effects. For common dyadic coping, however, gender differences were found in both actor and partner effects (Table 3). These tentative findings deserve further exploration in future research.

It is important to note that since no Type-I error correction was performed in this exploratory study, caution is warranted with regard to the interpretation of the above findings. All these findings should be reproduced in future studies.

\section{Strengths and Limitations}

A strength of this study is that it is the first to explore the association between dyadic coping and parental adjustment (individual and relationship outcomes), both within and between partners, after being confronted with a cancer diagnosis in their child. Furthermore, although most studies in the childhood cancer literature make use of a single-family member participant (e.g., Van Schoors et al., 2015), we included the perspectives of both partners. Discrepancies in perceptions across family members/partners (e.g., Alderfer et al., 2009) speak to the need to collect data from both members (e.g., Van Schoors et al., 2018). Additionally, by making use of the ActorPartner Interdependence Model (APIM; Cook and Kenny, 2005), we were able to model the interdependence in the dyadic relationship.

Despite the strengths of this study, some important limitations should be noted. First, we used a sample of Caucasian, heterosexual couples, thereby limiting the generalizability of our results. Future research should attempt to replicate these findings with more heterogeneous samples, e.g., also homosexual couples. Second, only Dutch speaking parents were included for participation. Therefore, with respect to the current multicultural society, this language criterion might have been a barrier for ethnic minorities. Third, we only focused on children with leukemia or non-Hodgkin lymphoma. As a consequence, it is important to highlight that parents of children with other cancer diagnoses may have different experiences. Fourth, time since diagnosis varied between the couples, ranging from 0 to 20 months. The potential biases inherent in retrospective methods like the one used in the current paper may have influenced their responses (e.g., forgetting, defensiveness). In addition, future (longitudinal) studies should also take into account the possible impact of time since diagnosis, as it is plausible to assume that the effect of dyadic coping on outcomes has a different impact depending on how long the parents face the illness of their child. Now, we simply adjusted for the effect of time since diagnosis on the outcomes, but future studies may look at the interaction of time since diagnosis and the actor and partner effects of dyadic coping. Fifth, as the associations described in this study are correlational in nature, the temporal order of the variables under investigation could not be tested with the present data. It is also possible, for instance, that better parental adjustment elicits more adaptive dyadic coping strategies, as described above.

\section{Clinical Implications}

Difficulties in the couple relationship may seem secondary to the more pressing need of ensuring adequate cancer and psychosocial care for the child. Therefore, such issues may be overlooked by psychosocial care providers in oncology or may even be downplayed by the couples themselves. However, this study shows that dyadic coping matters for individual and relational functioning in parents when facing cancer in their child. As a consequence, it is important to screen and tackle relational issues besides individual issues, taking into account evidence-based standards for psychosocial care in pediatric oncology. Interventions aimed at dealing with couple problems that get in the way of cancer care or hamper the adjustment of the child and/or family should take into account two specific recommendations. First, in working with families being confronted with a cancer diagnosis in a child, clinicians should not only focus on the adjustment of the child diagnosed with cancer or educational issues that arise post-diagnosis, but also on the impact of the illness on the parents in general and the parents' intimate relationship in particular. Moreover, clinicians should invite the couple system as a whole. Only by taking into account the perspectives of both members, couple level variables - such as dyadic coping - can be fully understood and improved when needed. Second, as previous research demonstrated that sexual relationships appear to be affected most negatively when facing a cancer diagnosis in their child (Lavee and May-Dan, 2003), clinicians should overcome their potential reluctance to discuss such topics together with the couple. Third, clinical interventions should be tailored to gender differences and specific characteristics of men and women facing pediatric cancer. For example, our findings suggest that women might be more vulnerable than men (cf. women reporting higher levels of individual maladjustment compared to men) when facing cancer in their child, and might therefore be in greater need of professional support from psycho-social workers or clinicians.

\section{CONCLUSION}

Taken together, these findings led us to the conclusion that both positive and negative dyadic coping are important for individual as well as relationship outcomes of parents when facing a diagnosis of cancer in their child. Moreover, differential associations seem to be at play between different types of coping on the one hand and individual and relationship adjustment on the other hand. In addition, while men and women reported to be 
equally satisfied with their partner and their sexual relationship, women reported higher levels of individual maladjustment.

\section{AUTHOR CONTRIBUTIONS}

All authors had been seen and reviewed the manuscript, and contributed to it in a meaningful way. MVS wrote the manuscript under the supervision of LG and LV. TL did the analyses. GB, BO,

\section{REFERENCES}

Alderfer, M. A., and Kazak, A. E. (2006). "Family issues when a child is on treatment for cancer," in Comprehensive Handbook of Childhood Cancer and Sickle Cell Disease: A Biopsychosocial Approach, ed. R. Brown (New York, NY: Oxford University Press).

Alderfer, M. A., Navsaria, N., and Kazak, A. E. (2009). Family functioning and posttraumatic stress disorder in adolescent survivors of childhood cancer. J. Fam. Psychol. 23, 717-725. doi: 10.1037/a0015996

American Cancer Society (2016). Cancer that Develop in Children. Available at: https://www.cancer.org/cancer/cancer-in-children/types-of-childhoodcancers.html

Antony, M. M., Bieling, P. J., Cox, B. J., Enns, M. W., and Swinson, R. P. (1998). Psychometric properties of the 42 -item and 21 item versions of the depression anxiety stress scales in clinical groups and a community sample. Psychol. Asses. 10, 176-181. doi: 10.1037/1040-3590.10.2.176

Arrindell, W. A., Boelens, W., and Lambert, H. (1983). On the psychometric properties of the maudsley marital questionnaire (MMQ): evaluation of selfratings in distressed and "normal" volunteer couples based on the Dutch version. Pers. Individ. Dif. 4, 293-306. doi: 10.1016/0191-8869(83)90151-4

Badr, H., Acitelli, L. K., and Carmack Taylor, C. L. (2008). Does talking about their relationship affect couples' marital and psychological adjustment to lung cancer? J. Cancer Surviv. 2, 53-64. doi: 10.1007/s11764-008-0044-3

Berg, C. A., and Upchurch, R. (2007). A developmental-contextual model of couples coping with chronic illness across the adult life span. Psychol. bull. 133, 920-954. doi: 10.1037/0033-2909.133.6.920

Bodenmann, G. (1995). A systemic-transactional conceptualization of stress and coping in couples. Swiss J. Psychol. 54, 34-49. doi: 10.1016/j.cpr.2008.10.004

Bodenmann, G. (1997). Dyadic coping - a systemic-transactional view of stress and coping among couples: theory and empirical findings. Eur. Rev. Appl. Psychol. 47, 137-140.

Bodenmann, G. (2005). "Dyadic coping and its significance for marital functioning," in Decade of Behavior. Couples Coping with Stress: Emerging Perspectives on Dyadic Coping, eds T. A. Revenson, K. Kayser, and G. Bodenmann (Washington, DC: American Psychological Association).

Bodenmann, G. (2008). Dyadisches Coping Inventar (DCI). [Dyadic Coping Inventory]. Test Manual. Bern: Huber.

Bodenmann, G., Randall, A. K., and Falconier, M. K. (2016). "Coping in couples: the systemic transactional model (STM)," in Couples Coping with Stress: A CrossCultural Perspective, eds M. K. Falconier, A. K. Randall, and G. Bodenmann (New York, NY: Routledge).

Bronfenbrenner, U. (1977). Toward an experimental ecology of human development. Am. psychol. 32, 513-531. doi: 10.1037/0003-066X.32.7.513

Brown, S. L., Nesse, R. M., Vinokur, A. D., and Smith, D. M. (2003). Providing social support may be more beneficial than receiving it. results from a prospective study of mortality. Psychol. Sci. 14, 320-327. doi: 10.1111/1467-9280. 14461

Caes, L., Goubert, L., Devos, P., Verlooy, J., Benoit, Y., and Vervoort, T. (2014). The relationship between parental catastrophizing about child pain and distress in response to medical procedures in the context of childhood cancer treatment: a longitudinal analysis. J. Pediatr. Psychol. 39, 677-686. doi: 10.1093/jpepsy/ jsu034

Cipolletta, S., Marchesin, V., and Benini, F. (2015). Family functioning as a constituent aspect of a child's chronic illness. J. Pediatr. Nurs. 30, 19-28. doi: 10.1016/j.pedn.2015.01.024
$\mathrm{JL}$, and $\mathrm{KN}$ helped in particular with the clinical implications of the manuscript.

\section{SUPPLEMENTARY MATERIAL}

The Supplementary Material for this article can be found online at: https://www.frontiersin.org/articles/10.3389/fpsyg. 2019.00402/full\#supplementary-material

Cook, W. L., and Kenny, D. A. (2005). The actor-partner interdependence model: a model of bidirectional effects in developmental studies. Int. J. Behav. Dev. 29, 101-109. doi: 10.1080/01650250444000405

Falconier, M. K., Jackson, J. B., Hilpert, P., and Bodenmann, G. (2015). Dyadic coping and relationship satisfaction: a meta-analysis. Clin. Psychol. Rev. 42, 28-46. doi: 10.1016/j.cpr.2015.07.002

Fife, B., Norton, J., and Groom, G. (1987). The family's adaptation to childhood leukemia. Soc. Sci. Med. 24, 159-168. doi: 10.1016/0277-9536(87)90248-6

Fuemmeler, B. F., Brown, R. T., Williams, L., and Barredo, J. (2003). Adjustment of children with cancer and their caregivers: moderating influences of family functioning. Fam. Syst. Health 21, 263-276. doi: 10.1037/1091-7527.21. 3.263

Garro, A. (2004). Coping patterns in mothers/caregivers of children with chronic feeding problems. J. Pediatr. Health Care 18, 138-144. doi: 10.1016/j.pedhc. 2003.08.001

Gistelinck, F., Loeys, T., Decuyper, M., and Dewitte, M. (2018). Indistinguishability tests in the actor-partner interdependence model. Br. J. Math. Stat. Psychol. 71, 472-498. doi: 10.1111/bmsp.12129

Grootenhuis, M. A., and Last, B. F. (1997). Adjustment and coping by parents of children with cancer: a review of the; literature. Support. Care Cancer 5, 466-484. doi: 10.1007/s005200050116

Hellemans, S. (2014). Intimate Partner Violence: Prevalence and Relational Dynamics. Available at: https://biblio.ugent.be/publication/4411848/file/ 4411854 .

Hoekstra-Weebers, J., Jaspers, J. P. C., Kamps, W. A., and Klip, E. C. (1998). Marital dissatisfaction, psychological distress, and the coping of parents of pediatric cancer patients. J. Marriage Fam. 60, 1012-1021. doi: 10.2307/353642

Kayser, K., Sormanti, M., and Strainchamps, E. (1999). Women coping with cancer: the influence of relationship factors on psychosocial adjustment. Psychol. Women Q. 23, 725-739. doi: 10.1111/j.1471-6402.1999.tb00394.x

Kazak, A. E., Barakat, L. P., Alderfer, M., Rourke, M. T., Meeske, K., Gallagher, P. R., et al. (2001). Posttraumatic stress in survivors of childhood cancer and mothers: development and validation of the impact of traumatic stressors interview schedule (ITSIS). J. Clin. Psychol. Med. Settings 8, 307-323. doi: 10.1023/A: 1011977031826

Kazak, A. E., Penati, B., Boyer, B., Himelstein, B., Brophy, P., Waibel, M. K., et al. (1996). A randomized controlled prospective outcome study of a psychological and pharmacological intervention protocol for procedural distress in pediatric leukemia. J. Pediatr. Psychol. 21, 615-631. doi: 10.1093/jpepsy/21.5.615

Kestler, S. A., and LoBiondo-Wood, G. (2012). Review of symptom experiences in children and adolescents with cancer. Cancer Nurs. 35, E31-E49. doi: 10.1097/ NCC.0b013e3182207a2a

LaMontagne, L., Hepworth, J. T., Salisbury, M. H., and Riley, L. P. (2003). Optimism, anxiety, and coping in parents of children hospitalized for spinal surgery. Appl. Nurs. Res. 16, 228-235. doi: 10.1053/S0897-1897(03)00051-X

Lavee, Y., and May-Dan, M. (2003). Patterns of change in marital relationships among parents of children with cancer. Health Soc. Work 28, 255-263. doi: 10.1093/hsw/28.4.255

Ledermann, T., Bodenmann, G., Gagliardi, S., Charvoz, L., Verardi, S., Rossier, J., et al. (2010). Psychometrics of the dyadic coping inventory in three language groups. Swiss J. Psychol. 69, 201-212. doi: 10.1024/1421-0185/a000024

Ljungman, L., Cernvall, M., Grönqvist, H., Ljótsson, B., Ljungman, G., and von Essen, L. (2014). Long-term positive and negative psychological late effects for parents of childhood cancer survivors: a systematic review. PLoS one 9:e103340. doi: 10.1371/journal.pone. 0103340 
Lovibond, S. H., and Lovibond, P. F. (1995). Manual for the Depression Anxiety Stress Scales, 2nd Edn. Sydney: Psychology Foundation.

Meier, C., Bodenmann, G., Morgeli, H., and Jenewein, J. (2011). Dyadic coping, quality of life, and psychological distress among chronic obstructive pulmonary disease patients and their partners. Int. J. Chronic Obstr. Pulm. Dis. 6, 583-596. doi: $10.2147 /$ copd.s24508

Morrow, G. R., Hoagland, A. C., and Morse, I. P. (1982). Sources of support perceived by parents of children with cancer: implications for counselling. Patient Couns. Health Educ. 4, 36-40. doi: 10.1016/S0738-3991(82)80033-5

Orathinkel, J., Van Steenwegen, A., and Stroobants, R. (2007). Further validation of the maudsley marital questionnaire (MMQ). Psychol. Health Med. 12, 346-352. doi: 10.1080/13548500600855481

Pai, A. L. H., Greenley, R. N., Lewandowski, A., Drotar, D., Youngstrom, E., and Peterson, C. C. (2007). A meta-analytic review of the influence of pediatric cancer on parent and family functioning. J. Fam. Psychol. 21, 407-415. doi: 10.1037/0893-3200.21.3.407

Patistea, E., Makrodimitri, P., and Panteli, V. (2000). Greek parents' reactions, difficulties and resources in childhood leukaemia at the time of diagnosis. Eur. J. Cancer Care 9, 86-96. doi: 10.1046/j.1365-2354.2000.00204.x

Rao, G. P., Malhotra, S., and Marwaha, R. K. (1992). Psychosocial study of leukemic children and their parents. Indian Pediatr. 29, 985-990.

Regan, T. W., Lambert, S. D., Kelly, B., McElduff, P., Girgis, A., Kayser, K., et al. (2014). Cross-sectional relationships between dyadic coping and anxiety, depression, and relationship satisfaction for patients with prostate cancer and their spouses. Patient Educ. Couns. 96, 120-127. doi: 10.1016/j.pec.2014.04.010

Silverman, L. B., and Weinstein, H. J. (1997). Treatment of childhood leukemia. Curr. Opin. Oncol. 9, 26-33. doi: 10.1097/00001622-199701000-00005

Stas, L., Kenny, D. A., Mayer, A., and Loeys, T. (2018). Giving dyadic data analysis away: a user friendly app for actor-partner interdependence models. Pers. Relatsh. 25, 103-119. doi: 10.1111/pere.12230

Streisand, R., Braniecki, S., Tercyak, K. P., and Kazak, A. E. (2001). Childhood illness-related parenting stress: the pediatric inventory for parents. J. Pediatr. Psychol. 26, 155-162. doi: 10.1093/jpepsy/26.3.155

Tarr, J., and Pickler, R. H. (1999). Becoming a cancer patient: a study of families of children with acute lymphocytic leukemia. J. Pediatr. Oncol. Nurs. 16, 44-50. doi: $10.1177 / 104345429901600106$

Traa, M. J., De Vries, J., Bodenmann, G., and Den Oudsten, B. L. (2015). Dyadic coping and relationship functioning in couples coping with cancer: a systematic review. Br. J. Health Psychol. 20, 85-114. doi: 10.1111/bjhp.12094

Tuinman, M. A., Fleer, J., Sleijfer, D. T., Hoekstra, H. J., and Hoekstra-Weebers, J. E. H. M. (2005). Marital and sexual satisfaction in testicular cancer survivors and their spouses. Support. Care Cancer 13, 540-548. doi: 10.1007/s00520-0040758-3

van der Does-van den Berg, A., de Vaan, G. A., van Weerde, J. F., Van WeelSipman, M., and Veerman, A. J. P. (1995). Late effects among long-term survivors of childhood acute leukemia in the Netherlands: a dutch childhood leukemia study group report. Pediatr. Res. 38, 802-807. doi: 10.1203/00006450199511000-00027

Van Schoors, M., Caes, L., Alderfer, M. A., Goubert, L., and Verhofstadt, L. (2017). Couple functioning after pediatric cancer diagnosis: a systematic review. Psycho-Oncology 26, 608-616. doi: 10.1002/pon.4204

Van Schoors, M., Caes, L., Verhofstadt, L. L., Goubert, L., and Alderfer, M. A. (2015). Systematic review: family resilience after pediatric cancer diagnosis. J. Pediatr. Psychol. 40, 856-868. doi: 10.1093/jpepsy/jsv055

Van Schoors, M., De Mol, J., Morren, H., Goubert, L., Verhofstadt, L. L., and Van Parys, H. (2018). Parents' perspectives of changes within the family functioning after a pediatric cancer diagnosis: a multi family member interview analysis. Qual. Health Res. 28, 1229-1241. doi: 10.1177/10497323177 53587

Verhofstadt, L. L., Buysse, A., and Ickes, W. (2007). Social support in couples: an examination of gender differences using self-report and observational methods. Sex Roles 57, 267-282. doi: 10.1007/s11199-0079257-6

Vrijmoet-Wiersma, C. J., van Klink, J. M., Kolk, A. M., Koopman, H. M., Ball, L. M., and Egeler, R. M. (2008). Assessment of parental psychological stress in pediatric cancer: a review. J. Pediatr. Psychol. 33, 694-706. doi: 10.1093/jpepsy/ jsn007

Wong, M. G., and Heriot, S. A. (2008). Parents of children with cystic fibrosis: how they hope, cope and despair. Child Care Health Dev. 34, 344-354. doi: $10.1111 /$ j.1365-2214.2007.00804.x

Conflict of Interest Statement: The authors declare that the research was conducted in the absence of any commercial or financial relationships that could be construed as a potential conflict of interest.

Copyright (c) 2019 Van Schoors, Loeys, Goubert, Berghmans, Ooms, Lemiere, Norga and Verhofstadt. This is an open-access article distributed under the terms of the Creative Commons Attribution License (CC BY). The use, distribution or reproduction in other forums is permitted, provided the original author(s) and the copyright owner(s) are credited and that the original publication in this journal is cited, in accordance with accepted academic practice. No use, distribution or reproduction is permitted which does not comply with these terms. 


\section{Links Between Communication and Relationship Satisfaction Among Patients With Cancer and Their Spouses: Results of a Fourteen-Day Smartphone-Based Ecological Momentary Assessment Study}

Shelby L. Langer ${ }^{1 *}$, Joan M. Romano ${ }^{2}$, Michael Todd ${ }^{1}$, Timothy J. Strauman ${ }^{3}$, Francis J. Keefe ${ }^{4}$, Karen L. Syrjala ${ }^{5}$, Jonathan B. Bricker ${ }^{6}$, Neeta Ghosh ${ }^{5}$, John W. Burns ${ }^{7}$, Niall Bolger ${ }^{8}$, Blair K. Puleo ${ }^{4}$, Julie R. Gralow ${ }^{9}$, Veena Shankaran ${ }^{9}$, Kelly Westbrook ${ }^{10}$, S. Yousuf Zafar ${ }^{10}$ and Laura S. Porter ${ }^{4}$

${ }^{1}$ College of Nursing and Health Innovation, Arizona State University, Phoenix, AZ, United States, ${ }^{2}$ Department of Psychiatry and Behavioral Sciences, University of Washington School of Medicine, Seattle, WA, United States, ${ }^{3}$ Department of Psychology, Duke University, Durham, NC, United States, ${ }^{4}$ Department of Psychiatry and Behavioral Sciences, Duke University School of Medicine, Durham, NC, United States, ${ }^{5}$ Clinical Research Division, Fred Hutchinson Cancer Research Center, Seattle, WA, United States, ${ }^{6}$ Public Health Sciences Division, Fred Hutchinson Cancer Research Center, Seattle, WA, United States, ${ }^{7}$ Department of Behavioral Sciences, Rush Medical College, Rush University, Chicago, IL, United States, ${ }^{8}$ Department of Psychology, Columbia University, New York, NY, United States, ${ }^{9}$ Medical Oncology, University of Washington School of Medicine, Seattle, WA, United States, ${ }^{10}$ Department of Medicine, Duke University School of Medicine, Durham, NC, United States

Cancer treatment poses significant challenges not just for those diagnosed with the disease but also for their intimate partners. Evidence suggests that couples' communication plays a major role in the adjustment of both individuals and in the quality of their relationship. Most descriptive studies linking communication to adjustment have relied on traditional questionnaire methodologies and cross-sectional designs, limiting external validity and discernment of temporal patterns. Using the systemic-transactional model of dyadic coping as a framework, we examined intra- and inter-personal associations between communication (both enacted and perceived) and relationship satisfaction (RS) among patients with stage II-IV breast or colorectal cancer and their spouses ( $N=107$ couples). Participants (mean age $=51,64.5 \%$ female patients, and $37.4 \%$ female spouses) independently completed twice-daily ecological momentary assessments (EMA) via smartphone for 14 consecutive days. Items assessed RS and communication (expression of feelings, holding back from expression, support and criticism of partner, and parallel ratings of partner behavior). Linear mixed models employing an Actor Partner Interdependence Model were used to examine concurrent, time-lagged, and cross-lagged associations between communication and RS. Expressing one's feelings was unassociated with RS. Holding back from doing so, in contrast, was associated with lower RS for both patients and spouses in concurrent models. These effects were both intrapersonal and interpersonal, meaning that when individuals held back from expressing their feelings, they reported lower RS and so too did their partner. Giving and receiving support were associated with 
one's own higher RS for both patients and spouses in concurrent models, and for patients in lagged models. Conversely, criticizing one's partner and feeling criticized were maladaptive, associated with lower RS (own and in some cases, partner's). Crosslagged analyses (evening RS to next-day afternoon communication) yielded virtually no effects, suggesting that communication may have a stronger influence on short-term $\mathrm{RS}$ than the reverse. Findings underscore the importance of responsive communication, more so than expression per se, in explaining both concurrent and later relationship adjustment. In addition, a focus on holding back from expressing feelings may enhance the understanding of RS for couples coping with cancer.

Keywords: dyadic coping, cancer, spouse, partner, holding back, couples, emotional expression

\section{INTRODUCTION}

A diagnosis of cancer poses great challenges for both patients and their loved ones. Patients with cancer often experience significant emotional distress including anxiety and worry, depression, and fears of disease progression and death (Syrjala and Yi, 2018). Many patients also report multiple disease and treatment-related side effects including fatigue, pain, cognitive impairment, and sexual dysfunction (Bower, 2008; Syrjala and Yi, 2018). These problems can limit patients' ability to perform many of their usual family and workplace responsibilities, thus disrupting their role functioning in important areas (Zebrack, 2000; Syrjala et al., 2004). For the many patients who are married or in committed partnerships, cancer also poses formidable challenges for their significant others and relationships (Carlson et al., 2000). Partners of individuals with cancer experience an array of psychological difficulties (Baider et al., 1996; Bishop et al., 2007). Research suggests that patients' and partners' levels of psychological distress are moderately correlated (Hagedoorn et al., 2008), and that some partners suffer more distress than do patients (Given and Given, 1992; Langer et al., 2003). In addition, patients with advanced cancer as well as their partners report more distress, role restrictions and physical difficulties than do those coping with early stage cancers (Weitzner et al., 1999; Badr et al., 2013). These impacts to both patients and partners, and the need for mutual responsiveness and support, have led to the description of cancer as a "we-disease" (Acitelli and Badr, 2005; Kayser et al., 2007).

One way in which couples can support each other is through effective communication (Kayser et al., 2007). Accumulating evidence indicates that couples' ability to communicate effectively plays a major role in the psychological adjustment of patients and partners and the quality of their relationship (Baucom et al., 2012). Specifically, communication behaviors that are associated with better patient and partner adjustment include open discussion of cancer-related concerns (often referred to as disclosure), and the ability to listen and respond supportively to one's partner. Maladaptive communication behaviors include holding back from disclosure and avoiding or responding negatively to one's partner's disclosure. A variety of questionnaires have been developed to assess adaptive and maladaptive communication behavior, including those assessing disclosure (Laurenceau et al., 2005) and holding back from disclosure (Porter et al., 2005); protective buffering, which is defined as hiding concerns or dispiriting information from one's partner, denying one's worries, or capitulating in order to avoid conflict (Hagedoorn et al., 2000); and social constraints, which are perceptions that the partner's responses to one's own disclosures are avoidant, discouraging or disapproving (Lepore and Revenson, 2007). In general, individuals who report low levels of disclosure, or high levels of holding back, protective buffering or social constraints also report greater psychological distress and poorer relationship functioning (Suls et al., 1997; Kayser et al., 1999; Hagedoorn et al., 2000; Kuijer et al., 2000; Porter et al., 2005; Manne et al., 2007, 2014b, 2015; Hinnen et al., 2009; Langer et al., 2009; Traa et al., 2015).

Even in the context of satisfying relationships, couples may experience difficulty communicating about cancer-related issues (Lichtman and Wood, 1987; Pistrang and Barker, 1995), for a number of reasons. First, both patients and partners may feel overwhelmed with the logistical, physical, and emotional challenges associated with cancer, including attending medical appointments, making medical decisions, providing emotional and physical assistance, and coping with treatment side effects and their own emotional distress. They may thus find it difficult to make time for meaningful conversation or to articulate specific concerns. Second, both patients and partners often mistakenly believe that it will be harmful or upsetting for patients to discuss their cancer, worries, or any negative aspects of the situation, and that the partners' role is to be continually cheerful and optimistic (Peters-Golden, 1982). Third, patients and partners often avoid discussing sensitive issues such as sexual functioning or disease progression and death (Porter et al., 2005; Reese et al., 2010).

Communication between patients with cancer and their intimate partners can be conceptualized in terms of the systemictransactional model of stress and coping in couples (Bodenmann, 1995). This model builds upon earlier conceptions of individuallevel stress and coping (Lazarus and Folkman, 1984) which focused on an individual's appraisal of the threat of the stressor and the extent to which s/he feels capable of coping with it. Bodenmann $(1995,2005)$ extended this model to dyads, noting that stress affects not just individual members of the dyad, but both parties, either directly or indirectly. If the stress is direct, both dyad members are affected concurrently, for example, 
if the couple's roof is leaking and needs immediate repair. If the stress is indirect, the stress experienced by one dyad member subsequently ends up impacting the other because the first is unable to adequately handle the stressor. For example, intense workplace demands might cause one dyad member to have to routinely stay late at the office, hence impeding his/her ability to pick up the couple's child after school, as was heretofore his/her usual practice. The other dyad member is thus affected. Whether or not both partners are affected directly, they can engage in dyadic coping which is conceptualized as "a systemic coping pattern, enrolling both partners in a symmetrical, complementary or occasionally asymmetric way" (Bodenmann, 1995).

Bodenmann (2005) noted that prior to the introduction of the systemic transactional model, research on stress and couples took one of three forms: (1) individual-level coping efforts in the context of a partnered relationship; (2) interactions between each dyad member's individual-level coping efforts; and (3) coping efforts on the part of one dyad member to promote better functioning for the other and the relationship as a whole. Protective buffering is mentioned as an example of the latter. One dyad member might act more positive than s/he feels so as to not upset the other. These approaches were distinguished from dyadic coping, in which both partners work conjointly to manage a stressor. Dyadic coping may be particularly relevant in the context of cancer because it is a common stressor that requires coordinated coping and management efforts, as well as mutual responsiveness (Kayser et al., 2007).

The present study focuses on two specific forms of dyadic coping: emotion-focused positive supportive dyadic coping and negative dyadic coping (Bodenmann, 2005), both particularly relevant to dyadic communication in couples dealing with cancer. According to the systemic transactional model, stress experienced by one dyad member may or may not be communicated to the other. Stress that is communicated in some way (verbally or non-verbally) is encoded and interpreted by the other dyad member, who may or may not respond. If the response conveys empathic understanding, validation and support, emotion-focused positive supportive dyadic coping is said to be occurring. If the response conveys hostility or ambivalence, negative dyadic coping is said to be occurring. Research on these specific elements of dyadic coping indicate positive associations between emotion-focused positive supportive dyadic coping and relationship satisfaction (RS), and inverse associations between negative dyadic coping and RS (Traa et al., 2015).

In addition to the elements of the systemic transactional model described above, the present study also incorporated the concept of holding back as an important element of potentially dysfunctional communication patterns in couples dealing with cancer. Holding back is seen as not simply the lack of expression or disclosure, but as an intentional or active attempt to suppress communication about difficult topics, a strategic behavior pattern that involves and affects both partners (Porter et al., 2005). Prior research has demonstrated associations between holding back and patient and partner maladjustment in the context of cancer (Porter et al., 2005; Edmond et al., 2013; Manne et al., 2014a). In addition, while the systemic transactional model focuses on stress communication specifically, we adopted a broader approach, including disclosures both related and unrelated to cancer, given the difficulty of defining a priori which communications might be stressful in the context of cancer.

In the present study, we sought to examine associations between communication (or holding back from communication), emotion-focused positive supportive dyadic coping, and negative dyadic coping at a given point in time and RS at (a) the same time and (b) a later point in time using ecological momentary assessment (EMA) methods. To date, most descriptive studies linking communication to adjustment in cancer have relied on cross-sectional designs which limit our ability to discern temporal patterns and do not allow for optimal tests of hypotheses regarding transactional causality in dyadic interactions. Few longitudinal studies exist, particularly those in which data are collected in real-time and in naturalistic settings. EMA offers a number of advantages. First, it minimizes recall biases inherent in global, retrospective measures which require participants not just to remember but also to summarize their behavior (e.g., "To what extent did you talk to your partner about cancer-related concerns during the past week?"). When considering such questions, individuals use a variety of heuristics to estimate their answers, leading to systematic biases influenced, for example, by current mood (Shiffman et al., 2008). Second, compared to both global self-report and laboratory-based assessments, EMA increases ecological validity (as participants are reporting in their real-world environment) and enables examination of within-day as well as day-to-day variations in behavior and experiences. Finally, the longitudinal nature of EMA data can be used to examine temporal sequences of behaviors and experiences (Shiffman et al., 2008). Thus, like laboratory-based observational data, EMA enables the study of both within-person and within-couple effects and directionality of such. However, it does so in the participants' natural environment and over a period of days or weeks.

At least two prior studies used a daily diary approach to explore communication among persons with breast cancer and their partners. One study (Pasipanodya et al., 2012) found that patients who reported higher perceived social constraints at baseline shared fewer positive and negative events with their partner on a daily basis, and that patients and partners who reported higher social constraints at baseline reported higher levels of negative mood and decreased relationship functioning on a daily basis. In the second study (Badr et al., 2013), women with metastatic breast cancer and their partners reported each day on the degree to which they avoided talking to each other about the patient's cancer-related concerns. Results indicated that partner but not patient avoidance varied from day to day, and that greater partner avoidance was related to increased patient negative affect the following day. Both of these studies had some important limitations including a lack of assessment of partner communication about their concerns, and samples of exclusively female patients, thereby confounding gender and role. The current study builds on these findings by assessing a wider range of patient and spouse communication behaviors among a sample that includes both male and female patients. 
The aims of the present study were to examine intraand inter-personal concurrent and lagged associations between communication, including patient and spouse reports of their own communication and perceptions of their partner's communication, and RS. Our specific hypotheses were derived primarily from the systemic transactional model but also reflected the addition of holding back as a potentially influential behavior. Actor and partner effects for both patients and spouses were hypothesized as follows: Expressing one's feelings and providing and receiving emotion-focused positive support would be positively associated with concurrent and later RS (both own and partner's). Conversely, being critical and feeling criticized would be inversely associated with concurrent and later RS (both own and partner's), as would holding back from expressing one's feelings. We also examined the cross-lagged effects of RS on nextday communication to explore the possibility of these reciprocal effects.

\section{MATERIALS AND METHODS}

\section{Participants}

All procedures, including screening and approach processes, were approved by the Institutional Review Boards of Arizona State University (Social Behavioral Committee) and Duke University School of Medicine (via expedited review; no subcommittee specified). Participants were recruited from two different cancer centers, the Duke Cancer Institute in Durham, NC and the Seattle Cancer Care Alliance in Seattle, WA (an alliance of the Fred Hutchinson Cancer Research Center and the University of Washington). The Institutional Review Board of Arizona State University served as the IRB of record for the Seattle site, with IRB authorization agreements held by the Fred Hutchinson Cancer Research Center and the University of Washington. All subjects gave written informed consent in accordance with the Declaration of Helsinki.

Inclusion criteria for patients were: age 18 or older; stage IIIV breast, colon, or rectal cancer; currently receiving or having received chemotherapy and/or hormone therapy; within 2 years of diagnosis of current cancer stage; life expectancy of at least 6 months per primary oncologist; married or in a committed, cohabiting relationship with someone of the same or opposite sex; and ability to speak and comprehend English. Inclusion criteria for spouses were: age 18 or older; married to or in a committed, cohabiting relationship with the patient; and ability to speak and comprehend English. Please note that the term "spouse" is used throughout but encompasses both spouses and non-married cohabiting partners. This is to avoid confusion with the term "partner" as used in the EMA item wording and in the statistical sense (partner effect).

Among patients screened for the protocol at the time of data extraction $(N=944), 396$ were deemed initially eligible based on medical records. Among the 396, 268 were contacted, further screened, and deemed fully eligible. Among those, 136 agreed to a face-to-face study visit with their partner, a $50.7 \%$ agreement rate. The most common reasons for refusal were lack of interest in the study or simply being too busy. Twenty of the 136 scheduled appointments were beyond the time frame of analysis for the present manuscript. Among the 116 couples seen face-to-face at the time of data extraction, 115 signed consent forms (separate forms for patients and spouses). Three of the 115 couples signed consent forms for the study in general but elected not to participate in the EMA portion of the study, due to discomfort with technology (EMA questions were posed via smartphone). Five of the 112 were excluded from the current analyses because of either insufficient data provision due to technological problems ( $n=2$ couples) or because they had not yet reached the end of the EMA phase at the time of data extraction ( $n=3$ couples), yielding an analytic sample of 107 couples.

\section{Procedures}

Patients who met initial medical inclusion criteria per medical records were sent a study brochure and letter from their primary oncologist introducing the study. This approach letter informed patients that a research team member would contact them by telephone to provide study details and gave options for patients to initiate contact themselves or to opt out. During the initial telephone contact, site research coordinators conducted further eligibility screening, including confirmation that the patient was married or in a committed and cohabiting relationship and that both patient and spouse had sufficient English comprehension and speaking ability to complete study activities.

Patients who expressed willingness to participate in the study (as a couple with their partner), were asked to identify a convenient time for a 90-min in-person visit, often scheduled around their next medical appointment to minimize additional travel to the clinic. Couples met with the site research coordinator in a private room at or near the site clinic. Written consent was obtained separately from patients and spouses following a detailed description of study procedures, risks and benefits, and assuring that any and all questions or concerns were addressed. After consent, participants completed other study activities not included in this report (e.g., a battery of psychosocial questionnaires and a cancer-related couple conversation) and then downloaded the free smartphone application.

The smartphone application was created using lifedatacorp.com, a web-based application development platform. The application is compatible with iOS and Android cell telephones. Participants used their personal phones unless they owned a different kind of phone or did not own a phone, in which case they were lent an iPod Touch device for study use. This was the case for 15 individuals. Participants who borrowed an iPod Touch completed the activity using the device as they would a smartphone. The application download was conducted in the same manner as with smartphones and application interfaces and participant views were comparable.

The lifedatacorp.com system allows users to receive questions via notification once the application has been downloaded on participants' mobile devices and they have signed up as individual users. The site research coordinators guided participants through the application download process. Upon download and required user registration, participants began receiving notifications to complete assessments twice daily for 14 days: once at 12:00 p.m. (afternoon assessment) and once at 8:00 p.m. (evening 
assessment). The response window was set for $2 \mathrm{~h}$ from the time of the first notification for both assessment periods. If participants did not begin to complete the assessment within the 2-h response window, the notification expired.

Participants received a Frequently Asked Questions user handout developed for the smartphone portion of the study and were provided with site research coordinator contact information in case of any technical difficulties. Participants were contacted 2-3 days after the application download to check in and provide assistance as needed. At the completion of the 14-day EMA, participants were sent a $\$ 75$ check or gift card if they had completed $85 \%$ or more of the notifications received. If $<85 \%$ of assessments were completed, participants were paid $\$ 3$ per notification completed.

Demographic characteristics (age, gender, race, ethnicity, education, and income) were assessed via questionnaire using Research Electronic Data Capture (REDCap), a secure web-based tracking and on-line data acquisition system (Harris et al., 2009). REDCap was also used to administer the Dyadic Adjustment Scale, a reliable and valid measure of relationship adjustment (Spanier, 1976). Medical records were extracted to screen for eligibility, using a HIPAA waiver.

\section{Measures}

Ecological momentary assessment items are described below.

\section{Communication With Partner}

Participants were asked whether or not they had talked to their partner since awakening (afternoon assessment) or since the last notification (evening assessment). What constituted "talking" was not defined and therefore left up to interpretation by participants. Accordingly, participants may have counted telephone calls or text messages with their partner as conversations, but this was not measured per se. Those responding "no" were asked why not: I didn't have any contact with my partner; I had nothing to talk about; I didn't feel well; I didn't want to bring up topics that could be upsetting; and other. Those responding "yes" were asked a series of followup questions about the conversation. The first item assessed relatedness to the cancer: To what extent was this conversation related to the cancer $(1=$ not at all; $3=$ somewhat; $5=$ a lot $)$ ? The second item assessed perceived importance: How important was this conversation to you $(1=$ not at all; $3=$ somewhat; $5=$ extremely)?

Remaining items (listed in Table 1 to illustrate mapping on to the STM) assessed the extent to which, during the conversation, participants: (1) expressed their feelings, (2) held back from expressing their feelings, (3) supported their partner, and (4) criticized their partner. The latter two items were created for the study based on face validity. The former two items were adapted from the Emotional Disclosure Scale (Pistrang and Barker, 1995). This scale asks respondents to rate the extent to which they talked to their partner about each of several cancer-related concerns (disclosure) and the extent to which they held back from doing so (holding back). For the present EMA administration, ratings were made with respect to the conversation in question and not tied to specific concerns. We also administered the full Emotional
TABLE 1 | Ecological momentary items designed to assess communication and analogous constructs per the systemic transactional model (STM).

Item

Parallel dyadic coping construct per the systemic transactional model

To what extent did you...

Express your feelings during this Stress communication conversation*

Hold back from expressing your Not addressed by STM feelings

Support your partner Emotion-focused positive supportive dyadic coping

Criticize your partner Emotion-focused negative dyadic coping

To what extent did you feel that your partner...

Expressed his/her feelings* Stress communication

Supported you Emotion-focused positive supportive dyadic coping

Criticized you Emotion-focused negative dyadic coping

Items with an asterisk assess disclosure or emotional expression, but not stress communication per se.

Disclosure Scale at baseline (pre-EMA), as part of a larger battery of questionnaires. Correlations between the two administrations were positive in sign: $r=0.54, p<0.001$ for patients and $r=0.58$, $p<0.001$ for spouses [holding back]; and $r=0.16, p=0.102$ for patients and $r=0.08, p=0.419$ for spouses [disclosure]. These associations provide some support for validity, more so for the holding back item. Note that overlap is not expected to be great given that one set of ratings is necessarily cancer-related and the other is not. Parallel EMA items assessed perceptions of partner communicative behavior (Table 1), with one exception. We did not ask participants to rate the extent to which their partner held back, assuming this would have been difficult to judge. In contrast, behaviors such as criticism are presumably more readily observable.

\section{Relationship Satisfaction (Evening Assessment Only)}

Following Auger (Auger et al., 2016), RS was assessed with a single item (item \#31) from the satisfaction subscale of the Dyadic Adjustment Scale (Spanier, 1976). Specifically, participants were asked, "All things considered, what was your degree of happiness with your relationship today: extremely unhappy (1), fairly unhappy (2), a little unhappy (3), happy (4), very happy (5), extremely happy (6), or perfectly happy (7)?” Per Goodwin (Goodwin, 1992), this item is highly correlated with total adjustment scores based on the full Dyadic Adjustment Scale excluding item \#31 ( $r=0.73$ and 0.67 from two different studies) and valid, i.e., able to discriminate persons classified as adjusted vs. distressed using the total scale score excluding item \#31.

In the present study, we administered the full DAS to patients and spouses as part of a baseline battery of questionnaires. Baseline ratings of item \#31 (general relationship happiness) were positively associated with EMA ratings of the same item (relationship happiness today), $r=0.49, p<0.001$ for patients and $r=0.57, p<0.001$ for spouses. The magnitude of these correlations suggests some degree of overlap but not complete 
overlap between the two measures. To characterize the degree of within-person non-independence (clustering) in patients' and spouses' RS responses, we computed intraclass correlation coefficients (ICCs), adjusting for temporal autocorrelation. ICC values for patients and spouses were nearly identical (0.60 and 0.61 , respectively). These values indicate that approximately $40 \%$ of the total variation in RS was at the within-person level (i.e., across days, within individuals).

\section{Analysis}

Descriptive statistics were used to characterize the sample with respect to demographic, clinical and relationship adjustment characteristics. Ecological momentary assessment response and completion rates, frequency of having talked to one's partner, whether or not a conversation was about the cancer, and perceived importance of a conversation were summarized separately by afternoon and evening. Correlational analyses examining associations among key study variables also were conducted (Supplemental Tables 1, 2).

Associations between communication variables and RS were estimated using linear mixed models within an Actor-Partner Interdependence Model (APIM) framework for dyads with distinguishable members. Three sets of APIM analyses were conducted: (1) models predicting each dyad member's RS from that member's report of his/her own communication and perceptions of his/her partner's communication on that same evening (concurrent models), (2) models predicting each dyad member's RS from that member's report of his/her own communication and perceptions of his/her partner's communication in the afternoon of the same day (lagged models), and (3) models predicting each dyad member's afternoon reports of communication and perceptions of his/her partner's communication from that member's report and his/ her partner's report of RS from the preceding evening (cross-lagged models). In concurrent and lagged models, four effects of substantive interest were estimated, depicted in the top two panels of Figure 1: (1) patient's reports of communication/perceived communication predicting his/her own RS (patient actor effects; coefficient $\mathrm{a}_{11}$ ); (2) spouse's reports of communication/perceived communication predicting his/her own RS (spouse actor effects; coefficient $\mathrm{a}_{22}$ ); (3) patient's reports of communication/ perceived communication predicting the spouse's RS (patient partner effects; coefficient $\mathrm{p}_{21}$ ); and (4) spouse's reports of communication/perceived communication predicting the patient's RS (spouse partner effects; coefficient $\mathrm{p}_{12}$ ). Parallel actor and partner effects were estimated in cross-lagged models, but with communication/ perceived communication being predicted from the preceding day's RS; see bottom panel of Figure 1.

In concurrent and lagged models, each dyad member's RS on a given evening was predicted from two dummy vectors coding for dyad member (with no overall intercept term) and four two-way interactions between these dummy vectors and person meancentered evening (or afternoon) communication/perceived communication variables, each capturing one of the four effects of substantive interest. Four parallel between-person interaction terms were included. Cross-lagged models paralleled concurrent and lagged models in their general form, but with each day's afternoon communication/perceptions of communication being predicted from the preceding evening's RS. Except where noted in tables of model coefficients, intercepts and actor and partner effects were estimated as random. An AR(1) variance/covariance structure was specified for day-level residuals. Results from analyses conducted adjusting for cancer diagnosis stage (stage II vs. stage III or IV) did not differ from those based on analyses described above. Results from the more parsimonious models (i.e., those without the stage indicator) are reported here. Descriptive analyses were conducted in SPSS 24.0 and SAS/STAT 14.1. Linear mixed models were estimated via restricted maximum likelihood using all available data in SAS/STAT 14.1 PROC MIXED. Given the large number of APIM model effects analyzed, $\alpha$ was set at 0.01 for these tests.

\section{RESULTS}

\section{Sample Characteristics}

Table 2 displays demographic characteristics of patients and spouses, and clinical characteristics of patients. Patients and spouses were, on average, 50 years old. The patient sample was comprised of $64.5 \%$ females and $35.5 \%$ males. Given that most couples were heterosexual (96\%), the reverse was true for spouses (37.4\% female). Participants were predominantly White (87\%) and non-Hispanic (96\%). Sixty-three percent had earned a 4 -year college degree or higher, and $57.5 \%$ reported a total household income of $\$ 100,000$ or higher. Most couples were married (91.6\%) and had been in their relationship for over 10 years $(72 \%)$.

With regard to clinical characteristics, $44 \%$ of patients had been diagnosed with breast cancer, $32 \%$ with colon cancer, and $24 \%$ with rectal cancer. Across diagnostic groups, $64 \%$ were coping with advanced cancer, stage III or IV. The breast cancer group was characterized by more lower stage disease (55\% stage II) whereas the colon cancer group was characterized by more higher stage disease (56\% stage IV). The rectal group was more evenly distributed in this respect (23\% stage II, $42 \%$ stage III, and $35 \%$ stage IV).

\section{Ecological Momentary Assessment Feasibility and Adherence}

Table 3 displays descriptive characteristics of the smartphonederived EMA data. Across 107 patients and spouses and both afternoon and evening periods, a total of 5,784 notifications were sent. Of these, 5,232 were responded to (90.5\%) and 5,136 were completed $(88.8 \%)$. These values were similar across patients and spouses and afternoon and evening assessment time points. Among those responding to the afternoon notification, $78.9 \%$ had conversed with their partner since awakening. Among those responding to the evening notification, $85.8 \%$ had conversed with their partner since the last assessment. The most common reason for not having conversed with one's partner was simply not having had contact with him or her $(67.9 \%$ in the afternoon and $58.8 \%$ in the evening). Conversations were deemed relatively important in nature, mean ratings $=3.17$ and 3.24 for afternoon and evening, respectively, on the 1-5 (not at all-extremely) scale, but were often not about the cancer. Seventy-one percent of 

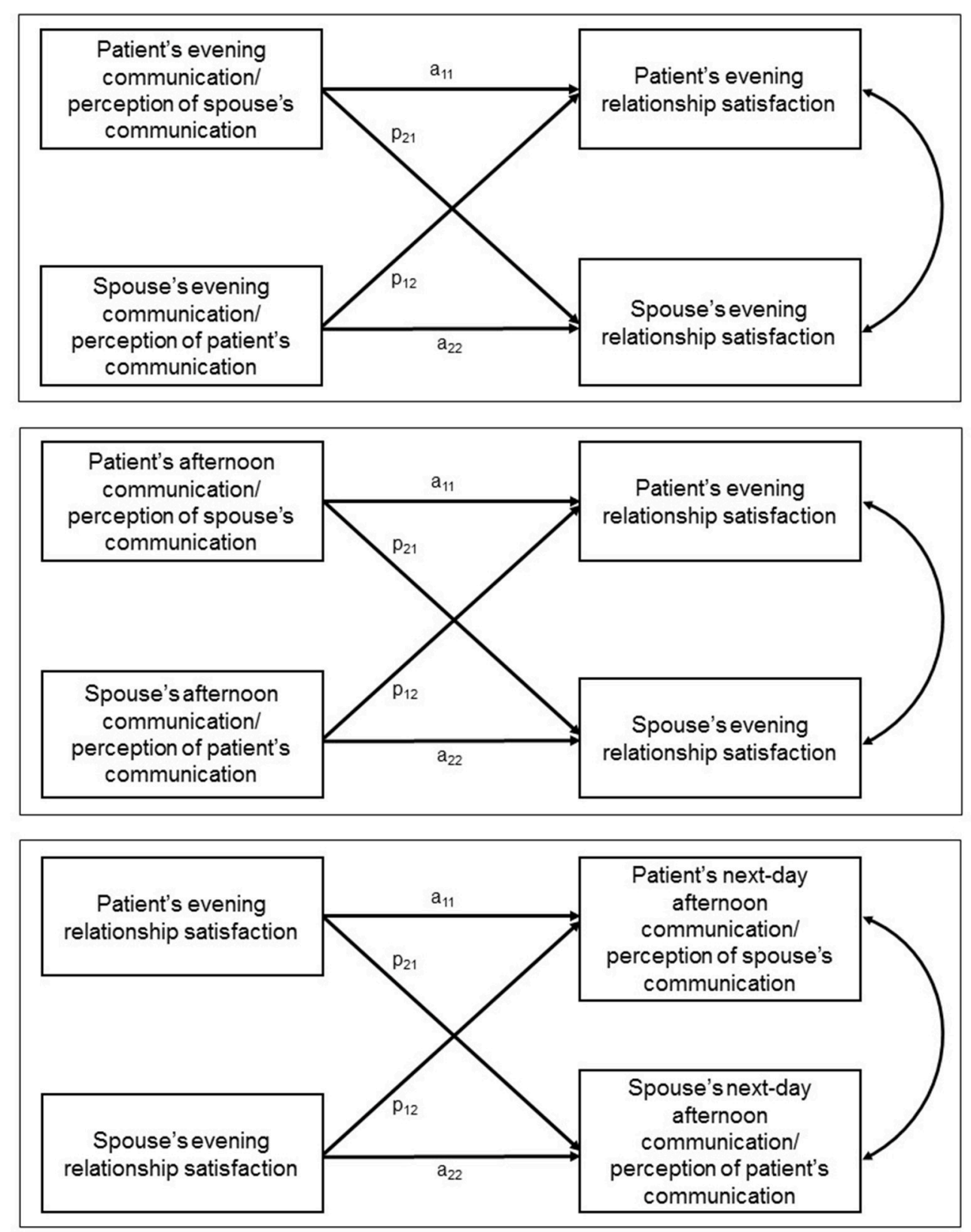

FIGURE 1 | Concurrent (top panel), lagged (middle panel), and cross-lagged (bottom panel) actor-partner interdependence models.

conversations reported in the afternoon were rated as 1 or not at all about the cancer, as were $69 \%$ of conversations reported in the evening. Subsequent (concurrent and lagged) analyses were not examined separately as a function of conversation importance or cancer-relatedness due to limited power.

\section{Concurrent Analyses}

Table 4 displays coefficients, standard errors, and p-values from linear mixed models predicting evening RS from sameday evening communication. Intercepts represent the adjusted sample means for RS for patients and spouses (second and third column, respectively). Labels for patient- and spouse-specific actor and partner effect coefficients (e.g., $\mathrm{a}_{11}$ ) correspond to those in the top panel of Figure 1. In what follows, we highlight statistically significant $(p<0.01)$ effects.

Actor effects showed associations between self-reported enacted communication and one's own RS. For both patients and spouses, providing support to one's partner was positively associated with one's own RS. Holding back from expressing one's feelings, in contrast, and criticizing one's partner, were negatively associated with one's own RS. With respect to perceived partner communication, for both patients and spouses, feeling supported 
TABLE 2 | Demographic, clinical, and relationship adjustment characteristics of the sample.

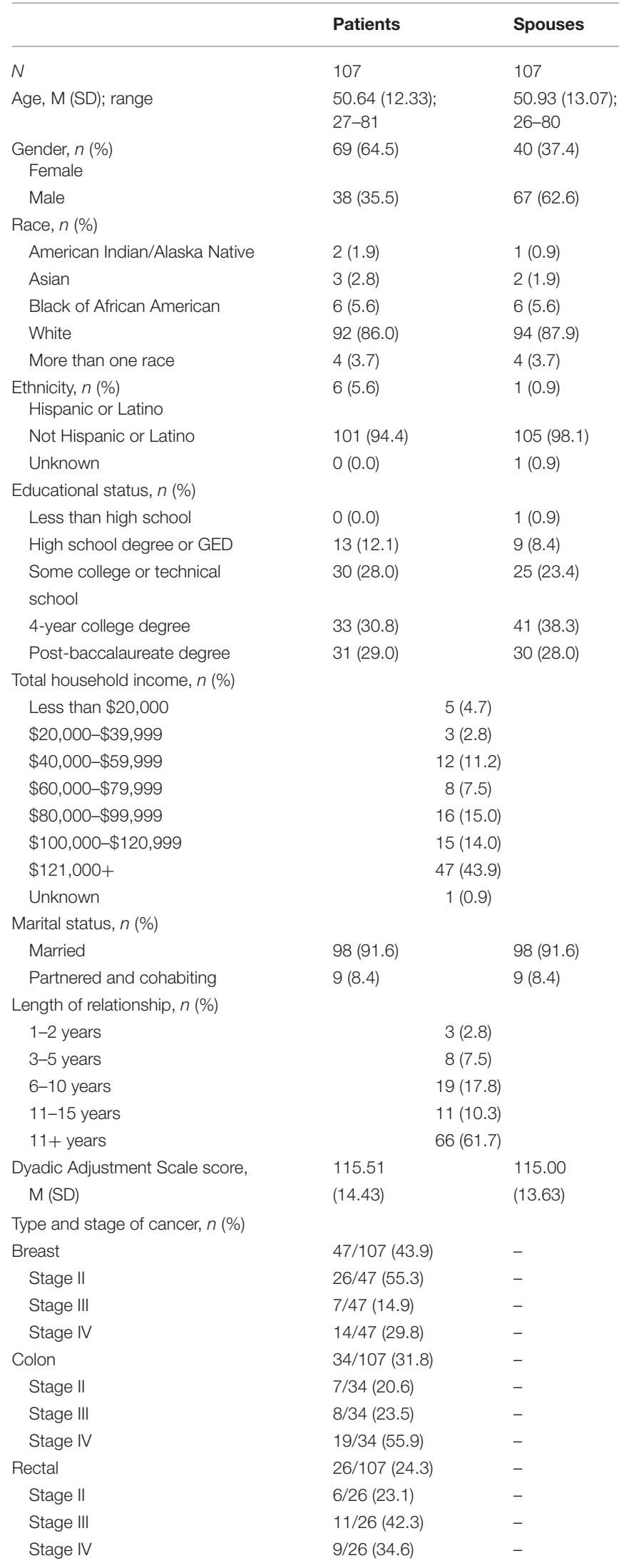

was positively associated with one's own RS, and feeling criticized was negatively associated with one's own RS.

Partner effects were also found. For both patients and spouses, their own holding back was negatively associated with their partners' RS. In addition, spouses' support of the patient was positively associated with the patients' RS, while their criticism of the patient was negatively associated with the patient's RS. Lastly, when spouses felt supported, their patient partner reported higher RS.

\section{Lagged Analyses}

Table 5 displays coefficients, standard errors, and $p$-values from linear mixed models treating afternoon communication as the predictor and same-day evening RS as the criterion. As in the concurrent models, intercepts represent the adjusted sample means of RS for patients and spouses. Significant actor effects were found only for patients. For patients, supporting one's partner in the afternoon was associated with one's own higher RS that evening. For patients, there were also intrapersonal effects of feeling supported, and feeling criticized. If patients felt supported in the afternoon, their own RS was higher that evening. Conversely, if patients felt criticized in the afternoon, their own RS was lower that evening.

Partner effects were obtained for being critical (patients only) and feeling criticized (spouses only). If patients criticized their spouse in the afternoon, the spouse reported lower RS that same evening, and if spouses felt criticized by their patient in the afternoon, their patient reported lower RS that evening.

\section{Cross-Lagged Analyses}

Table 6 displays coefficients and standard errors from linear mixed models treating evening RS as the predictor and next-day afternoon communication as the criterion. Here, each intercept represents the adjusted sample mean of the corresponding (afternoon) communication/perceived communication measure for either patients or spouses. As displayed in the upper section of Table 6, among spouses, evening RS was inversely associated with next-day afternoon holding back (actor effect). No other significant effects were found.

\section{DISCUSSION}

Results indicate that the nature and quality of communications between patients with cancer and their spouses were, as predicted, associated with concurrent ratings of RS and, to a lesser extent, later-day ratings of RS. The study used a heuristic framework based largely on the systemic transactional model, adapted to define communication broadly as not only stressrelated, but also incorporating the construct of holding back from disclosure given its relevance to this population as demonstrated in prior studies (Porter et al., 2005; Edmond et al., 2013; Manne et al., 2014a). Using an ecological twice-daily assessment over 2 weeks, we were able to examine intra- and inter-personal effects of both enacted and perceived communication in concurrent (evening to same evening) and lagged (afternoon to same-day evening) scenarios. Importantly, this innovative smartphonebased assessment was feasible, with high rates of adherence 
TABLE 3 | Descriptive statistics of smartphone-gathered ecological momentary assessment data ( $N=107$ couples).

\begin{tabular}{|c|c|c|c|}
\hline & Patient & Spouse & Total \\
\hline Total number of notifications sent, $n$ & 2,893 & 2,891 & 5,784 \\
\hline Total number of notifications responded to/ notifications sent, $n$ (\%) & $2,629(90.9)$ & $2,603(90.0)$ & $5,232(90.5)$ \\
\hline Total number of notifications completed/ notifications sent, $n(\%)$ & 2,592 (89.6) & $2,544(88.0)$ & $5,136(88.8)$ \\
\hline \multicolumn{4}{|l|}{ Afternoon notifications only } \\
\hline Number of notifications sent & 1,414 & 1,409 & 2,823 \\
\hline Number of notifications responded to/ notifications sent, n (\%) & $1,270(89.8)$ & $1,270(90.1)$ & $2,540(90.0)$ \\
\hline Number of notifications completed/ notifications sent, n (\%) & $1,251(88.5)$ & $1,246(88.4)$ & 2,497 (88.5) \\
\hline Conversed with partner since awakening, $\mathrm{n}$ & 988 & 1,016 & 2,004 \\
\hline Did not converse with partner since awakening, $\mathrm{n}$ & 277 & 246 & 523 \\
\hline \multicolumn{4}{|l|}{$\begin{array}{l}\text { Among those who did not converse with partner since awakening, reasons } \\
\text { why, } n / \text { responded to item (\%) }\end{array}$} \\
\hline I didn't have any contact with my partner & 194/277 (70.0) & $160 / 244(65.6)$ & $354 / 521$ (67.9) \\
\hline I had nothing to talk about & $18 / 277(6.5)$ & $17 / 244(7.0)$ & $35 / 521(6.7)$ \\
\hline I didn't feel well & 9/277 (3.2) & $1 / 244(0.4)$ & $10 / 521(1.9)$ \\
\hline I didn't want to bring up topics that could be upsetting & $3 / 277(1.1)$ & $1 / 244(0.4)$ & $4 / 521(0.8)$ \\
\hline Other & $53 / 277(19.1)$ & $65 / 244(26.6)$ & $118 / 521(22.6)$ \\
\hline Importance of conversation, M (SD) on 1-5 scale & $3.15(1.18)$ & $3.18(1.15)$ & $3.17(1.16)$ \\
\hline Extent to which conversation was related to cancer, $M(S D)$ on $1-5$ scale & $1.63(1.18)$ & $1.70(1.24)$ & $1.67(1.21)$ \\
\hline \multicolumn{4}{|l|}{$\begin{array}{l}\text { Extent to which conversation was related to cancer, } \mathrm{n} / \text { responded to } \\
\text { item (\%) }\end{array}$} \\
\hline Rating of 1 (not at all) & $715 / 978(73.1)$ & $705 / 1,014(69.5)$ & $1,420 / 1,992(71.3)$ \\
\hline Rating of 2 & $66 / 978(6.7)$ & 93/1,014 (9.2) & 159/1,992 (8.0) \\
\hline Rating of 3 (somewhat) & 96/978 (9.8) & $102 / 1,014(10.1)$ & 198/1,992 (9.9) \\
\hline Rating of 4 & $44 / 978(4.5)$ & $39 / 1,014(3.8)$ & $83 / 1,992(4.2)$ \\
\hline Rating of 5 (a lot) & $57 / 978(5.8)$ & $75 / 1,014(7.4)$ & 132/1,992 (6.6) \\
\hline \multicolumn{4}{|l|}{ Evening notifications only } \\
\hline Number of notifications sent & 1,479 & 1,482 & 2,961 \\
\hline Number of notifications responded to/ notifications sent, $n$ (\%) & $1,359(91.9)$ & 1,333 (89.9) & 2,692 (90.9) \\
\hline Number of notifications completed/ notifications sent, $n(\%)$ & $1,341(90.7)$ & $1,298(87.6)$ & 2,639 (89.1) \\
\hline Conversed with partner since last notification, $n$ & 1,158 & 1,153 & 2,311 \\
\hline Did not converse with partner since last notification, $n$ & 198 & 166 & 364 \\
\hline \multicolumn{4}{|l|}{$\begin{array}{l}\text { Among those who did not converse with partner since last notification, } \\
\text { reasons why, } n / \text { responded to item (\%) }\end{array}$} \\
\hline I didn't have any contact with my partner & 112/196 (57.1) & $99 / 163(60.7)$ & 211/359 (58.8) \\
\hline I had nothing to talk about & 19/196 (9.7) & 18/163 (11.0) & $37 / 359(10.3)$ \\
\hline I didn't feel well & 18/196 (9.2) & $1 / 163(0.6)$ & 19/359 (5.3) \\
\hline I didn't want to bring up topics that could be upsetting & $1 / 196(0.5)$ & 1/163 (0.6) & 2/359 (0.6) \\
\hline Other & 46/196 (23.5) & $44 / 163(27.0)$ & $90 / 359(25.1)$ \\
\hline Importance of conversation, $M(\mathrm{SD})$ on $1-5$ scale & $3.24(1.16)$ & $3.24(1.09)$ & $3.24(1.13)$ \\
\hline Extent to which conversation was related to cancer, $M(S D)$ on 1-5 scale & $1.70(1.23)$ & $1.73(1.23)$ & $1.72(1.23)$ \\
\hline \multicolumn{4}{|l|}{ Extent to which conversation was related to cancer, $n /$ responded to item (\%) } \\
\hline Rating of 1 (not at all) & $811 / 1,153(70.3)$ & $771 / 1,143(67.5)$ & $1,582 / 2,296(68.9)$ \\
\hline Rating of 2 & $83 / 1,153(7.2)$ & $110 / 1,143(9.6)$ & 193/2,296 (8.4) \\
\hline Rating of 3 (somewhat) & $123 / 1,153(10.7)$ & $136 / 1,143(11.9)$ & 259/2,296 (11.3) \\
\hline Rating of 4 & $62 / 1,153(5.4)$ & $49 / 1,143(4.3)$ & $111 / 2,296(4.8)$ \\
\hline Rating of 5 (a lot) & $74 / 1,153(6.4)$ & $77 / 1,143(6.7)$ & $151 / 2,296(6.6)$ \\
\hline
\end{tabular}

including for those without smartphone experience who were loaned iPod Touch devices.

Across both the concurrent and lagged analyses, the most consistent effects were seen for ratings of one's own and one's partner's responsiveness. In concurrent analyses, for both patients and spouses, being supportive of their partner and feeling supported by their partner were associated with higher RS. Conversely, for both patients and spouses, being critical of their 
TABLE 4 | Mixed model regression coefficients (standard errors) and p-values from concurrent analyses: evening communication predicting evening relationship satisfaction.

\begin{tabular}{|c|c|c|c|c|c|c|}
\hline \multirow[b]{2}{*}{ Predictor } & \multicolumn{2}{|c|}{ Intercept } & \multicolumn{2}{|c|}{ Actor effect } & \multicolumn{2}{|c|}{ Partner effect } \\
\hline & Patient & Spouse & $\begin{array}{c}\text { Patient } \\
\left(a_{11}\right)\end{array}$ & $\begin{array}{c}\text { Spouse } \\
\left(a_{22}\right)\end{array}$ & $\begin{array}{l}\text { Patient } \\
\left(p_{21}\right)\end{array}$ & $\begin{array}{c}\text { Spouse } \\
\left(p_{12}\right)\end{array}$ \\
\hline \multicolumn{7}{|l|}{ To what extent did you... } \\
\hline Express your feelings ${ }^{a}$ & $\begin{array}{c}5.201 \\
(0.099)\end{array}$ & $\begin{array}{l}5.126 \\
(0.096)\end{array}$ & $\begin{array}{c}0.057 \\
(0.033) \\
p=0.085\end{array}$ & $\begin{array}{c}0.045 \\
(0.030) \\
p=0.136\end{array}$ & $\begin{array}{c}-0.014 \\
(0.026) \\
p=0.593\end{array}$ & $\begin{array}{c}0.030 \\
(0.027) \\
p=0.263\end{array}$ \\
\hline $\begin{array}{l}\text { Hold back from expressing } \\
\text { your feelings }\end{array}$ & $\begin{array}{c}5.216 \\
(0.094)\end{array}$ & $\begin{array}{c}5.136 \\
(0.090)\end{array}$ & $\begin{array}{c}-\mathbf{0 . 1 4 4} \\
(0.041) \\
p<0.001\end{array}$ & $\begin{array}{c}-\mathbf{0 . 2 0 6} \\
(0.054) \\
p<0.001\end{array}$ & $\begin{array}{c}-\mathbf{0 . 1 1 7} \\
(0.039) \\
p=0.004\end{array}$ & $\begin{array}{c}-0.142 \\
(0.044) \\
p=0.002\end{array}$ \\
\hline Support your partnera & $\begin{array}{c}5.223 \\
(0.087)\end{array}$ & $\begin{array}{l}5.126 \\
(0.085)\end{array}$ & $\begin{array}{c}\mathbf{0 . 2 1 9} \\
(0.037) \\
p<0.001\end{array}$ & $\begin{array}{c}\mathbf{0 . 3 3 4} \\
(0.052) \\
p<0.001\end{array}$ & $\begin{array}{c}0.065 \\
(0.034) \\
p=0.053\end{array}$ & $\begin{array}{c}\mathbf{0 . 1 1 7} \\
(0.032) \\
p<0.001\end{array}$ \\
\hline Criticize your partner & $\begin{array}{c}5.262 \\
(0.091)\end{array}$ & $\begin{array}{c}5.171 \\
(0.087)\end{array}$ & $\begin{array}{c}-\mathbf{0 . 2 7 2} \\
(0.065) \\
p<0.001\end{array}$ & $\begin{array}{c}-\mathbf{0 . 2 2 5} \\
(0.053) \\
p<0.001\end{array}$ & $\begin{array}{c}-0.066 \\
(0.060) \\
p=0.276\end{array}$ & $\begin{array}{c}-0.187 \\
(0.066) \\
p=0.009\end{array}$ \\
\hline \multicolumn{7}{|c|}{ To what extent did you feel that your partner... } \\
\hline Expressed his/ her feelings ${ }^{a}$ & $\begin{array}{c}5.203 \\
(0.097)\end{array}$ & $\begin{array}{l}5.139 \\
(0.092)\end{array}$ & $\begin{array}{c}\mathbf{0 . 1 2 7} \\
(0.032) \\
p<0.001\end{array}$ & $\begin{array}{c}0.076 \\
(0.035) \\
p=0.035\end{array}$ & $\begin{array}{c}0.010 \\
(0.029) \\
p=0.734\end{array}$ & $\begin{array}{c}0.049 \\
(0.033) \\
p=0.130\end{array}$ \\
\hline Supported you & $\begin{array}{c}5.185 \\
(0.083)\end{array}$ & $\begin{array}{l}5.181 \\
(0.081)\end{array}$ & $\begin{array}{c}\mathbf{0 . 2 4 8} \\
(0.042) \\
p<0.001\end{array}$ & $\begin{array}{c}\mathbf{0 . 2 8 1} \\
(0.045) \\
p<0.001\end{array}$ & $\begin{array}{c}0.084 \\
(0.039) \\
p=0.033\end{array}$ & $\begin{array}{c}\mathbf{0 . 1 8 8} \\
(0.039) \\
p<0.001\end{array}$ \\
\hline Criticized you & $\begin{array}{c}5.272 \\
(0.085)\end{array}$ & $\begin{array}{c}5.166 \\
(0.084)\end{array}$ & $\begin{array}{c}-\mathbf{0 . 3 1 5} \\
(0.062) \\
p<0.001\end{array}$ & $\begin{array}{c}-\mathbf{0 . 2 9 9} \\
(0.049) \\
p<0.001\end{array}$ & $\begin{array}{c}-0.086 \\
(0.036) \\
p=0.021\end{array}$ & $\begin{array}{c}-0.087 \\
(0.058) \\
p=0.148\end{array}$ \\
\hline
\end{tabular}

a Random actor effects only. Bolded regression coefficients are significant at the $p<0.01$ level.

partner and feeling criticized by their partner were associated with lower RS. In lagged analyses, when patients reported supporting their spouse and feeling supported by their spouse, they later reported higher levels of RS. Conversely, when patients felt criticized by their spouse, they later reported lower levels of RS, and when they reported being critical, their spouse later reported lower levels of RS. These findings are commensurate with work on emotion-focused positive supportive dyadic coping and negative dyadic coping, respectively (Falconier et al., 2015), and extend this area to the realm of daily interactions of couples coping with cancer.

For both patients and spouses, and in both concurrent and lagged analyses, expressing one's own feelings was unassociated with RS. The one significant finding with regard to expression was that, in concurrent analyses, when patients perceived their partner as more expressive, their own RS was higher. This pattern of findings, combined with those above, suggests that disclosure per se may not be as crucial to RS as responses to the disclosure and how they are perceived. This is in line with results of a metaanalysis on dyadic coping and RS (Falconier et al., 2015). While disclosure may set the interaction in motion, the impact on the relationship appears to depend on the quality of the ensuing responses.

While expressiveness was largely unassociated with RS, holding back was inversely associated with RS as predicted. This finding was limited to the concurrent analyses and was the case for both actor and partner effects, and patients and spouses. In other words, when patients and spouses reported holding back from expressing their feelings, both they and their partners reported lower concurrent RS. These results are consistent with those obtained by Porter et al. (2005) using a traditional, global measure of holding back. Interestingly, the one significant finding from the cross-lagged analyses also involved holding back. When spouses reported lower levels of RS on 1 day, they were more likely to hold back from expressing feelings or concerns on the following day. Conversely, when spouses reported higher levels of RS on 1 day, they were less likely to hold back from expressing feelings or concerns on the following day. It makes sense that dissatisfaction with one's relationship on a given day might lead to reluctance to share thoughts and feelings on the following day. Clinically speaking, this suggests that attempts to bolster general satisfaction by fostering emotional closeness and intimacy and by creating opportunities for shared experiences could result in less holding back. To our knowledge, this is the first ecological study to examine temporal relationships between holding back and RS and the results suggest they may be reciprocal in nature. Taken together with previous research, these findings suggest that holding back may add explanatory power above the standard systemic transactional model, which does not address active withholding. The significant partner effects seen in the concurrent analyses and in previous research indicate that holding back is deleterious not just to the RS of the person who 
TABLE 5 | Mixed model regression coefficients (standard errors) and $p$-values from lagged analyses: afternoon communication predicting evening relationship satisfaction.

\begin{tabular}{|c|c|c|c|c|c|c|}
\hline Predictor & \multicolumn{2}{|c|}{ Intercept } & \multicolumn{2}{|c|}{ Actor effect } & \multicolumn{2}{|c|}{ Partner effect } \\
\hline \multicolumn{7}{|l|}{ To what extent did you... } \\
\hline Express your feelings & $\begin{array}{l}5.238 \\
(0.098)\end{array}$ & $\begin{array}{c}5.146 \\
(0.102)\end{array}$ & $\begin{array}{c}0.004 \\
(0.031) \\
p=0.889\end{array}$ & $\begin{array}{c}-0.004 \\
(0.038) \\
p=0.909\end{array}$ & $\begin{array}{c}-0.030 \\
(0.038) \\
p=0.432\end{array}$ & $\begin{array}{c}-0.013 \\
(0.035) \\
p=0.706\end{array}$ \\
\hline Support your partner & $\begin{array}{l}5.245 \\
(0.093)\end{array}$ & $\begin{array}{c}5.179 \\
(0.088)\end{array}$ & $\begin{array}{c}\mathbf{0 . 1 4 6} \\
(0.048) \\
p=0.003\end{array}$ & $\begin{array}{c}0.115 \\
(0.057) \\
p=0.048\end{array}$ & $\begin{array}{c}0.098 \\
(0.041) \\
p=0.026\end{array}$ & $\begin{array}{c}0.076 \\
(0.054) \\
p=0.168\end{array}$ \\
\hline Criticize your partner ${ }^{a}$ & $\begin{array}{l}5.226 \\
(0.098)\end{array}$ & $\begin{array}{l}5.147 \\
(0.090)\end{array}$ & $\begin{array}{c}-0.101 \\
(0.073) \\
p=0.179\end{array}$ & $\begin{array}{c}-0.192 \\
(0.073) \\
p=0.014\end{array}$ & $\begin{array}{c}-\mathbf{0 . 1 4 3} \\
(0.052) \\
p=0.006\end{array}$ & $\begin{array}{c}-0.104 \\
(0.053) \\
p=0.050\end{array}$ \\
\hline \multicolumn{7}{|c|}{ To what extent did you feel that your partner... } \\
\hline Supported you ${ }^{a}$ & $\begin{array}{l}5.213 \\
(0.088)\end{array}$ & $\begin{array}{l}5.184 \\
(0.084)\end{array}$ & $\begin{array}{c}\mathbf{0 . 2 0 4} \\
(0.049) \\
p<0.001\end{array}$ & $\begin{array}{c}0.083 \\
(0.047) \\
p=0.082\end{array}$ & $\begin{array}{c}-0.021 \\
(0.040) \\
p=0.603\end{array}$ & $\begin{array}{c}0.077 \\
(0.038) \\
p=0.044\end{array}$ \\
\hline Criticized you ${ }^{\mathrm{a}}$ & $\begin{array}{l}5.240 \\
(0.090)\end{array}$ & $\begin{array}{l}5.146 \\
(0.090)\end{array}$ & $\begin{array}{c}-\mathbf{0 . 1 6 9} \\
(0.050) \\
p=0.008\end{array}$ & $\begin{array}{c}-0.138 \\
(0.070) \\
p=0.055\end{array}$ & $\begin{array}{c}-0.075 \\
(0.048) \\
p=0.123\end{array}$ & $\begin{array}{c}-\mathbf{0 . 1 3 9} \\
(0.044) \\
p=0.002\end{array}$ \\
\hline
\end{tabular}

${ }^{a}$ Random actor effects only. Bolded regression coefficients are significant at the $p<0.01$ level.

does it but also that of the partner. While the phrase "holding back" suggests a lack of emotional expression, the behavior may in fact be behaviorally manifest and observable to the partner. Indeed, laboratory research on expressive suppression, defined as "the conscious inhibition of emotional expressive behavior while emotionally aroused" (Gross and Levenson, 1993) indicates that this behavior disrupts communication and increases blood pressure in both those suppressing and their partners, and results in decreased rapport (Butler et al., 2003). This construct has been examined largely in the context of laboratory-based studies involving previously unacquainted undergraduate pairs where negative emotional experience is induced, and suppression is experimentally manipulated. This construct has been understudied both in the context of cancer and in naturalistic, day-to-day settings. Our findings indicate that this may be a fruitful area for further inquiry in couples coping with cancer and perhaps other stressful experiences as well.

Overall, the lagged analyses treating afternoon communication as the predictor and same-day evening RS as the criterion yielded fewer effects than concurrent analyses, and almost exclusively effects for patients. When patients provided support, and felt supported, they reported greater RS later that day. In contrast, when patients felt criticized, they reported lower RS later that day. These findings suggest that the carry-over effect of communication behavior, be that positive or negative, may be stronger for patients, perhaps due to the vulnerabilities associated with treatment and recovery. This finding bears replication, ideally in a fully mixed-gender patient sample. Research suggests that women are more interpersonally sensitive than are men (Acitelli, 1992; Lang-Takac and Osterweil, 1992; Lambert and Hopwood, 2016). Because our sample was comprised of more female than male patients (64.5\% female), gender cannot be ruled out as a potential confound in attempts to explain any differences between patients and spouses.

While our predictions highlighted the primacy of communication in influencing RS, we also considered the possibility that there may be reciprocal relationships between these constructs such that they mutually influence each other over time. To test this, we examined RS in the evening as a predictor of next-day afternoon communication. As noted previously, these analyses yielded only one significant effect. In addition, regression coefficients were smaller in magnitude as compared to those for the lagged analyses. This suggests that, at least within the constraints of the paradigm used in this study, the influence of communication on ratings of RS may be stronger than the reverse. However, it should be noted that the cross-lagged analyses had a different time window (evening to next-day afternoon) than did the lagged (afternoon to same-day evening). Communication and RS may have been more closely connected in the lagged situation, in which the two variables were measured more proximally in time. In the cross-lagged situation, a full night and much of the following day separated ratings of RS and communication, and intervening events may have affected both variables in unknown ways.

The preponderance of findings for items designed to assess perceptions of partner behavior bear further consideration. 


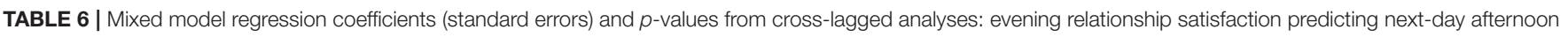
communication.

\begin{tabular}{|c|c|c|c|c|c|c|}
\hline \multirow[b]{2}{*}{ Outcome } & \multicolumn{2}{|c|}{ Intercept } & \multicolumn{2}{|c|}{ Actor effect } & \multicolumn{2}{|c|}{ Partner effect } \\
\hline & Patient & Spouse & $\begin{array}{c}\text { Patient } \\
\left(\mathrm{a}_{11}\right)\end{array}$ & $\begin{array}{c}\text { Spouse } \\
\left(\mathrm{a}_{22}\right)\end{array}$ & $\begin{array}{c}\text { Patient } \\
\left(p_{21}\right)\end{array}$ & $\begin{array}{c}\text { Spouse } \\
\left(p_{12}\right)\end{array}$ \\
\hline \multicolumn{7}{|l|}{ To what extent did you... } \\
\hline Express your feelings $^{a}$ & $\begin{array}{l}3.344 \\
(0.081)\end{array}$ & $\begin{array}{c}3.196 \\
(0.079)\end{array}$ & $\begin{array}{c}0.032 \\
(0.051) \\
p=0.535\end{array}$ & $\begin{array}{c}0.026 \\
(0.043) \\
p=0.550\end{array}$ & $\begin{array}{c}0.069 \\
(0.048) \\
p=0.151\end{array}$ & $\begin{array}{c}0.067 \\
(0.046) \\
p=0.147\end{array}$ \\
\hline $\begin{array}{l}\text { Hold back from expressing } \\
\text { your feelings }{ }^{a}\end{array}$ & $\begin{array}{c}1.460 \\
(0.048)\end{array}$ & $\begin{array}{c}1.462 \\
(0.049)\end{array}$ & $\begin{array}{c}-0.044 \\
(0.040) \\
p=0.266\end{array}$ & $\begin{array}{c}-\mathbf{0 . 0 9 1} \\
(0.032) \\
p=0.005\end{array}$ & $\begin{array}{c}-0.030 \\
(0.041) \\
p=0.470\end{array}$ & $\begin{array}{c}-0.003 \\
(0.037) \\
p=0.946\end{array}$ \\
\hline Support your partnerb & $\begin{array}{l}3.978 \\
(0.067)\end{array}$ & $\begin{array}{c}3.941 \\
(0.060)\end{array}$ & $\begin{array}{c}0.083 \\
(0.058) \\
p=0.160\end{array}$ & $\begin{array}{c}-0.007 \\
(0.040) \\
p=0.862\end{array}$ & $\begin{array}{c}0.079 \\
(0.037) \\
p=0.034\end{array}$ & $\begin{array}{c}0.038 \\
(0.039) \\
p=0.330\end{array}$ \\
\hline Criticize your partner $^{\mathrm{b}}$ & $\begin{array}{c}1.238 \\
(0.034)\end{array}$ & $\begin{array}{c}1.256 \\
(0.034)\end{array}$ & $\begin{array}{c}-0.057 \\
(0.037) \\
p=0.131\end{array}$ & $\begin{array}{c}-0.008 \\
(0.029) \\
p=0.789\end{array}$ & $\begin{array}{c}0.004 \\
(0.026) \\
p=0.890\end{array}$ & $\begin{array}{c}-0.010 \\
(0.028) \\
p=0.726\end{array}$ \\
\hline \multicolumn{7}{|c|}{ To what extent did you feel that your partner... } \\
\hline Expressed his/ her feelings ${ }^{C}$ & $\begin{array}{l}3.818 \\
(0.074)\end{array}$ & $\begin{array}{c}3.663 \\
(0.068)\end{array}$ & $\begin{array}{c}0.069 \\
(0.045) \\
p=0.128\end{array}$ & $\begin{array}{c}0.033 \\
(0.041) \\
p=0.423\end{array}$ & $\begin{array}{c}0.103 \\
(0.042) \\
p=0.015\end{array}$ & $\begin{array}{c}0.038 \\
(0.044) \\
p=0.385\end{array}$ \\
\hline Supported you ${ }^{b}$ & $\begin{array}{l}4.050 \\
(0.057)\end{array}$ & $\begin{array}{l}3.825 \\
(0.064)\end{array}$ & $\begin{array}{c}0.120 \\
(0.046) \\
p=0.012\end{array}$ & $\begin{array}{c}0.091 \\
(0.044) \\
p=0.042\end{array}$ & $\begin{array}{c}0.026 \\
(0.038) \\
p=0.493\end{array}$ & $\begin{array}{c}-0.012 \\
(0.039) \\
p=0.762\end{array}$ \\
\hline Criticized you ${ }^{b}$ & $\begin{array}{c}1.332 \\
(0.037)\end{array}$ & $\begin{array}{c}1.331 \\
(0.044)\end{array}$ & $\begin{array}{c}-0.049 \\
(0.037) \\
p=0.194\end{array}$ & $\begin{array}{c}-0.050 \\
(0.032) \\
p=0.131\end{array}$ & $\begin{array}{c}0.007 \\
(0.031) \\
p=0.821\end{array}$ & $\begin{array}{c}0.053 \\
(0.032) \\
p=0.098\end{array}$ \\
\hline
\end{tabular}

${ }^{a}$ Random partner effects only. ${ }^{b}$ Random actor effects only. ${ }^{c}$ Actor and partner effects fixed. Bolded regression coefficients are significant at the $p<0.01$ level.

Were such perceptions accurate? For example, if an individual reported being criticized by her partner, did the partner in fact report being critical? From our correlation matrices (Supplemental Tables 1, 2), we can infer that, for both patients and spouses, reports of feeling supported and feeling criticized were reflective of the way in which partners behaved. For example, patient report of feeling criticized in the afternoon was positively correlated with spouse report of having been critical at that same time $(r=0.39, p<0.01)$, and spouse report of feeling criticized in the afternoon was positively correlated with patient report of having been critical at that same time $(r=$ $0.29, p<0.01$ ). All eight relevant correlations (four for enacted and perceived support and criticism rated in the afternoon and four for enacted and perceived support and criticism rated in the evening) were positive in sign and statistically significant at the $p<0.01$ level. This suggests that appraisals were in fact attuned to partner behavior (providing support for validity) and is commensurate with previous work demonstrating the value of partner-reports in couple research (Sanford, 2010).

In interpreting the present findings, it is important to note that levels of RS (rated on a 1-7 scale) were relatively high overall, certainly above the midpoint; see intercepts in Tables 4, 5. Similarly, levels of disclosure and support (rated on a 1-5 scale) were relatively high, and levels of holding back and criticism were relatively low (also rated on a 1-5 scale); see intercepts in Table 6. These findings are consistent with those from previous studies of cancer patients and partners (Porter et al., 2005;
Manne et al., 2010). Couples who agree to be in a study on communication tend to be relatively well-adjusted. Indeed, the mean Dyadic Adjustment Scale scores for our sample fall within the non-distressed range (98-151) per standard cut-offs; see Table 2. Nonetheless, results of our regression analyses indicate important temporal patterns worthy of attention. Our findings suggest that couples' day-to-day interactions may influence their level of satisfaction with their relationship. Over time, these effects may accumulate, leading to overall declines in RS which could in turn affect mood and other important outcomes such as quality of life, pain, or physical functioning. Future work should consider more distal impacts of adaptive and maladaptive forms of communication. It would also be interesting to examine how these brief communication windows reflect patterns over a longer period of time and impact relationship stability. From the analyses reported, we cannot know whether the patterns seen reflect reactions to discrete events or represent longer term patterns of interaction among these couples.

Collectively, our findings are consistent with previous studies that point to the importance of conceptualizing cancer as a "wedisease" (Acitelli and Badr, 2005; Kayser et al., 2007) and bolster the need for joint approaches that treat both members of the couple as a source of support for the other, thereby co-facilitating adjustment (Bodenmann and Randall, 2013). Communication skills building is an essential part of this approach but, as noted by Badr (2017), simply prescribing more disclosure of emotions may not be sufficient. Rather, it may be necessary 
to address couples' motivations for avoiding cancer-related discussions, and to include training in skills for both expressing one's thoughts and feelings and listening to one's partner and responding in a supportive manner (Porter and Keefe, 2018). Our previous experience developing and testing such couplebased communication interventions suggest that they are feasible and acceptable (Porter et al., 2017; Langer et al., 2018), and that they lead to improvements in couples' relationship functioning (Porter et al., 2009, 2017). Interestingly, we have found that intervention effects are strongest among couples who report higher levels of holding back, providing further evidence of the potential importance of this variable (Porter et al., 2009). Studies using EMA methodology may provide a valuable adjunct to assessing intervention effects by examining communication and support processes as they unfold on a day-to-day basis, in the context of conversations around issues related to both cancer and other non-cancer topics.

Limitations of the present study must be considered. First, we only assessed RS once per day (in the evening), in part to minimize participant burden but also because we assumed that this variable would be relatively stable over the course of a day. Accordingly, we could not examine concurrent associations between communication and RS at the afternoon time point. Second, we did not assess conversational topic or valence of disclosures. We did, however, ask participants to rate the degree to which the topic was related to cancer and the importance of the conversation. Responses suggested that many conversations couples reported on may not have been directly cancer-related, but they were deemed relatively important. Thus, despite the rigors associated with treatment and recovery, couples frequently discuss important topics and concerns that may be unrelated to the cancer. It is unclear whether, in the context of cancer, there may be different communication patterns when discussing cancer vs. non-cancer topics, and whether these are differentially important for couples' RS. It is likely that couples infrequently engage in conversations specifically focused on the cancer in the absence of precipitating events, such as a doctor's visit or receipt of test results. Thus, studies using EMA methods may have difficulty capturing such conversations. Future studies could be designed to assess couples around the time of such events or could assess couples over multiple time periods (e.g., $1 \mathrm{week} /$ month over several months) to increase the likelihood of capturing important cancer-related conversations. Future studies might also incorporate more specific questions about the topic of conversations and valence (positivity or negativity) of disclosures. A third limitation of this study is that our analyses were limited to same and next-day effects. Examining longer time lags may yield important information regarding the degree to which communication behaviors affect RS over time. Lastly, the

\section{REFERENCES}

Acitelli, L. (1992). Gender differences in relationship awareness and marital satisfaction among young married couples. Pers. Soc. Psychol. B. 18, 102-110. doi: $10.1177 / 0146167292181015$

Acitelli, L., and Badr, H. (2005). "My illness or our illness? Attending to the relationship when one partner is ill," in Couples Coping with Stress: fact that the couples in this study were relatively well-adjusted may limit generalizability.

Despite these limitations, the present study has several notable strengths. First, the sample size was fairly large for this population and, unlike much of the literature on couple communication in cancer, includes a subset of patients coping with advanced disease (64\%). Second, our sample includes both male and female patients and was drawn from two different geographical regions across the United States. Third, our response rate was quite high $(90 \%)$, as was our completion rate $(89 \%)$, thus demonstrating that smartphone-enabled EMA is feasible, even for an arguably stressed and vulnerable population. Fourth, the inclusion of reports not just of one's own behavior but of perceptions of one's partner's behavior add significantly to our understanding of the role of appraisal in the context of dyadic coping.

In conclusion, findings from this EMA study underscore the importance of responsive communication in explaining day-today relationship adjustment among patients with cancer and their caregiving partners. These findings confirm predictions of the systemic transactional model with regard to emotionfocused positive supportive dyadic coping and negative dyadic coping (Bodenmann, 2005). They also extend the model to include the construct of holding back as potentially important in understanding RS for couples coping with cancer.

\section{AUTHOR CONTRIBUTIONS}

SL, LP, JR, FK, TS, KS, JWB, JBB, NB, JG, VS, KW, and SZ secured funding for the study. SL, LP, JR, FK, TS, KS, JWB, $\mathrm{JBB}$, and $\mathrm{NB}$ contributed to study conception and design. NG and $\mathrm{BP}$ recruited and consented participants, trained participants in the smartphone application, and tracked data collection. JG, VS, KW, and SZ supported and facilitated patient recruitment. MT performed statistical analyses. SL, MT, LP, JR, TS, and NG wrote the first draft of the manuscript. All authors reviewed and approved the submitted version.

\section{FUNDING}

This study was funded by grant R01 CA201179 from the National Cancer Institute, awarded to Multiple Principal Investigators SL and LP.

\section{SUPPLEMENTARY MATERIAL}

The Supplementary Material for this article can be found online at: https://www.frontiersin.org/articles/10.3389/fpsyg. 2018.01843/full\#supplementary-material

Emerging Perspectives on Dyadic Coping, eds T. Revenson, K. Kayser, and G. Bodenmann(Washington, DC: American Psychological Association), 121-136.

Auger, E., Menzies-Toman, D., and Lydon, J. (2016). Daily experiences and relationship well-being: the paradoxical effects of relationship identification. J. Pers. 85, 741-752. doi: 10.1111/jopy. 12283 
Badr, H. (2017). New frontiers in couple-based interventions in cancer care: refining the prescription for spousal communication. Acta Oncol. 56, 139-145. doi: 10.1080/0284186X.2016.1266079

Badr, H., Pasipanodya, E. C., and Laurenceau, J. P. (2013). An electronic diary study of the effects of patient avoidance and partner social constraints on patient momentary affect in metastatic breast cancer. Ann. Behav. Med. 45, 192-202. doi: 10.1007/s12160-012-9436-8

Baider, L., Kaufman, B., Peretz, T., Manor, O., Ever-Hadani, P., and Kaplan DeNour, A. (1996). "Mutuality of fate: adaptation and psychological distress in cancer patients and their partners," in Cancer and the Family, eds L. Baider, C. L. Cooper, and A. Kaplan De-Nour (Oxford: Wiley), 173-187.

Baucom, D. H., Porter, L. S., Kirby, J. S., and Hudepohl, J. (2012). Couple-based interventions for medical problems. Behav. Ther. 43, 61-76. doi: 10.1016/j.beth.2011.01.008

Bishop, M. M., Beaumont, J. L., Hahn, E. A., Cella, D., Andrykowski, M. A., Brady, M. J., et al. (2007). Late effects of cancer and hematopoietic stem-cell transplantation on spouses or partners compared with survivors and survivormatched controls. J. Clin. Oncol. 25, 1403-1411. doi: 10.1200/JCO.2006.07.5705

Bodenmann, G. (1995). A systemic-transactional concepqualization of stress and coping in couples. Swiss J. Psychol. 54, 34-49.

Bodenmann, G. (2005). "Dyadic coping and its significance for marital functioning," in Couples Coping with Stress: Emerging Perspectives on Dyadic Coping, eds T. A. Revenson, K. Kayser, and G. Bodenmann. (Washington, DC: American Psychological Association), 33-49. doi: 10.1037/11031-002

Bodenmann, G., and Randall, A. K. (2013). Close relationships in psychiatric disorders. Curr. Opin. Psychiatry 26, 464-467. doi: 10.1097/YCO.0b013e3283642de7

Bower, J. (2008). Behavioral symptoms in breast cancer patients and survivors: fatigue, insomnia, depression, and cognitive disturbance. J. Clin. Oncol. 26, 768-777. doi: 10.1200/JCO.2007.14.3248

Butler, E. A., Egloff, B., Wilhelm, F., Smith, N., Erickson, E., and Gross, J. (2003). The social consequences of expressive suppression. Emotion 3, 48-67. doi: 10.1037/1528-3542.3.1.48

Carlson, L. E., Bultz, B. D., Speca, M., and St. Pierre, M. (2000). Partners of cancer patients: part I. Impact, adjustment and coping across the illness trajectory. J. Psychosoc. Oncol. 18, 39-63. doi: 10.1300/J077v18n02_03

Edmond, S. N., Shelby, R., Kimmick, G., Marcom, P., Peppercorn, J., and Keefe, F. (2013). Symptom communication in breast cancer: relationships of holding back and self-efficacy for communication to symptoms and adjustment. $J$. Psychosoc. Oncol. 31, 698-711. doi: 10.1080/07347332.2013.835023

Falconier, M. K., Jackson, J., Hilpert, P., and Bodenmann, G. (2015). Dyadic coping and relationship satisfaction: a meta-analysis. Clin. Psychol. Rev. 42, 28-46. doi: 10.1016/j.cpr.2015.07.002

Given, B., and Given, C. (1992). Patient and family caregiver reaction to new and recurrent breast cancer. J. Am. Med. Women Assoc. 47, 201-206.

Goodwin, R. (1992). Overall, just how happy are you? The magical question 31 of the Spanier Dyadic Adjustment Scale. Fam. Ther. 19, 273-275.

Gross, J. J., and Levenson, R. (1993). Emotional suppression: physiology, self-report, and expressive behavior. J. Pers. Soc. Psychol. 64, 970-986. doi: 10.1037/0022-3514.64.6.970

Hagedoorn, M., Kuijer, R., Buunk, B., Dejong, G., Wobbes, T., and Sanderman, R. (2000). Marital satisfaction in patients with cancer: does support from intimate partners benefit those who need it most? Health Psychol. 19, 274-282. doi: 10.1037/0278-6133.19.3.274

Hagedoorn, M., Sanderman, R., Bolks, H. N., Tuinstra, J., and Coyne, J. C. (2008). Distress in couples coping with cancer: a meta-analysis and critical review of role and gender effects. Psychol. B. 134, 1-30. doi: 10.1037/0033-2909.134.1.1

Harris, P., Taylor, R., Thielke, R., Payne, J., Gonzalez, N., and Conde, J. (2009). Research electronic data capture (REDCap) - a metdata-drive methodology and workflow process for providing translational research informatics support. J. Biomed. Inform. 42, 377-381. doi: 10.1016/j.jbi.2008.08.010

Hinnen, C., Ranchor, A. V., Baas, P. C., Sanderman, R., and Hagedoorn, M. (2009). Partner support and distress in women with breast cancer: the role of patients' awareness of support and level of mastery. Psychol. Health 24, 439-455. doi: $10.1080 / 08870440801919513$

Kayser, K., Sormanti, M., and Strainchamps, E. (1999). The influence of relationship factors on psychosocial adjustment. Psychol. Women Quart. 23, 725-739. doi: 10.1111/j.1471-6402.1999.tb00394.x
Kayser, K., Watson, L., and Andrade, J. (2007). Cancer as a "we-disease": Examining the process of coping from a relationship perspective. Fam. Syst. Health 25, 404-418. doi: 10.1037/1091-7527.25.4.404

Kuijer, R., Ybema, J. F., Buunk, B. P., Thijs-Boer, F., and Sanderman, R. (2000). Active engagement, protective buffering, and overprotection: three ways of giving support by intimate partners of patients with cancer. J. Soc. Clin. Psychol. 19, 256-275. doi: 10.1521/jscp.2000.19.2.256

Lambert, J., and Hopwood, C. (2016). Sex differences in interpersonal sensitivities across acquaintances, friends, and romantic relationships. Pers. Indiv. Differ. 89, 162-165. doi: 10.1016/j.paid.2015.10.004

Langer, S., Abrams, J., and Syrjala, K. (2003). Caregiver and patient marital satisfaction and affect following hematopoietic stem cell transplantation: a prospective, longitudinal investigation. Psychooncology 12, 239-253. doi: $10.1002 /$ pon. 633

Langer, S., Brown, J., and Syrjala, K. (2009). Intrapersonal and interpersonal consequences of protective buffering among cancer patients and caregivers. Cancer 115, 4311-4325. doi: 10.1002/cncr.24586

Langer, S. L., Porter, L., Romano, J., Todd, M., and Lee, S. (2018). A couplebased communication intervention for hematopoietic cell transplantation survivors and their caregiving partners: feasiblity, acceptability, and change in process measures. Biol. Blood Marrow Transplant. S1083-8791(18)30265-9. doi: 10.1016/j.bbmt.2018.05.013

Lang-Takac, E., and Osterweil, Z. (1992). Separateness and connectedness: differences between the genders. Sex Roles 27, 277-289. doi: 10.1007/BF00289929

Laurenceau, J. P., Barrett, L., and Rovine, M. (2005). The interpersonal process model of intimacy in marriage: a daily-diary and multilevel modeling approach. J. Fam. Psychol. 19, 314-323. doi: 10.1037/0893-3200.19.2.314

Lazarus, R., and Folkman, S. (1984). Stress, Appraisal, and Coping. New York, NY: Springer.

Lepore, S. J., and Revenson, T. A. (2007). Social constraints on disclosure and adjustment to cancer. Soc. Pers. Psychol. Compass 1, 313-333. doi: 10.1111/j.1751-9004.2007.00013.x

Lichtman, R. R. S. E. T., and Wood, J. V. (1987). Social support and marital adjustment after breast cancer. J. Psychosoc. Oncol. 5, 47-74. doi: 10.1300/J077v05n03_03

Manne, S., Badr, H., Zaider, T., Nelson, C., and Kissane, D. (2010). Cancer-related communication, relationship intimacy, and psychological distress among couples coping with localized prostate cancer. J. Cancer Surviv. 4, 74-85. doi: 10.1007/s11764-009-0109-y

Manne, S., Kashy, D., Siegel, S., Myers Virtue, S., Heckman, C., and Ryan, D. (2014a). Unsupportive partner behaviors, social-cognitive processing, and psychological outcomes in couples coping with early stage breast cancer. J. Fam. Psychol. 28, 214-224. doi: 10.1037/a0036053

Manne, S., Kissane, D., Zaider, T., Kashy, D., Lee, D., Heckman, C., et al. (2015). Holding back, intimacy, and psychological and relationship outcomes among couples coping with prostate cancer. J. Fam. Psychol. 29, 708-719. doi: 10.1037/fam0000096

Manne, S. L., Myers, S., Ozga, M., Kissane, D., Kashy, D., Rubin, S., et al. (2014b). Holding back sharing concerns, dispositional emotional expressivity, perceived unsupportive responses and distress among women newly diagnosed with gynecological cancers. Gen. Hosp. Psychiat. 36, 81-87. doi: 10.1016/j.genhosppsych.2013.10.001

Manne, S. L., Norton, T., Ostroff, J., Winkel, G., Fox, K., and Grana, G. (2007). Protective buffering and psychological distress among couples coping with breast cancer: the moderating role of relationship satisfaction. J. Fam. Psychol. 21, 380-388. doi: 10.1037/0893-3200.21.3.380

Pasipanodya, E. C., Parrish, B. P., Laurenceau, J. P., Cohen, L. H., Siegel, S. D., Graber, E. C., et al. (2012). Social constraints on disclosure predict daily wellbeing in couples coping with early-stage breast cancer. J. Fam. Psychol. 26, 661-667. doi: $10.1037 / \mathrm{a} 0028655$

Peters-Golden, H. (1982). Breast cancer: varied perceptions of social support in the illness experience. Soc. Sci. Med. 16, 483-491. doi: 10.1016/0277-9536(82)90057-0

Pistrang, N., and Barker, C. (1995). The partner relationship in psychological response to breast cancer. Soc. Sci. Med. 40, 789-797. doi: 10.1016/0277-9536(94) 00136-H 
Porter, L. S., and Keefe, F. (2018). Couple-based communication interventions for cancer: moving beyond a 'one size fits all' approach. Acta Oncol. 57, 693-695. doi: 10.1080/0284186X.2017.1400687

Porter, L. S., Keefe, F., Baucom, D., Hurwitz, H., Moser, B., Patterson, E., et al. (2009). Partner-assisted emotional disclosure for patients with gastrointestinal cancer: results from a randomized controlled trial. Cancer 115, 4326-4338. doi: $10.1002 /$ cncr. 24578

Porter, L. S., Keefe, F., Baucom, D., Olsen, M., Zafar, S., and Uronis, H. (2017). A randomized pilot trial of a videoconference couples communication intervention for advanced GI cancer. Psycho-Oncol. 26, 1027-1035. doi: 10.1002/pon.4121

Porter, L. S., Keefe, F., Hurwitz, H., and Faber, M. (2005). Disclosure between patients with gastrointestinal cancer and their spouses. Psychooncology 14, 1030-1042. doi: 10.1002/pon.915

Reese, J. B., Shelby, R. A., Keefe, F. J., Porter, L. S., and Abernethy, A. P. (2010). Sexual concerns in cancer patients: a comparison of GI and breast cancer patients. Support. Care Cancer 18, 1179-1189. doi: 10.1007/s00520-009-0738-8

Sanford, K. (2010). Assessing conflict communication in couples: comparing the validity of self-report, partner-report, and observer ratings. J. Fam. Psychol. 24, 165-174. doi: 10.1037/a0017953

Shiffman, S., Stone, A. A., and Hufford, M. R. (2008). Ecological momentary assessment. Ann. Rev. Clin. Psychol. 4, 1-32. doi: 10.1146/annurev.clinpsy.3.022806.091415

Spanier, G. B. (1976). Measuring dyadic adjustment: new scales for assessing the quality of marriage and similar dyads. J. Marriage Fam. 38, 15-28. doi: $10.2307 / 350547$

Suls, J., Green, P., Rose, G., Lounsbury, P., and Gordon, E. (1997). Hiding worries from one's spouse: associations between coping via protective buffering and distress in male post-myocardial infarction patients and their wives. J. Behav. Med. 20, 333-349. doi: 10.1023/A:1025513029605

Syrjala, K. L., Langer, S. L., Abrams, J. R., Storer, B., Sanders, J. E., Flowers, M. E., et al. (2004). Recovery and long-term function after hematopoietic cell transplantation for leukemia or lymphoma. J. Am. Med. Assoc. 291, 2335-2343. doi: 10.1001/jama.291.19.2335

Syrjala, K. L., and Yi, J. C. (2018). "Overview of psychosocial issues in the adult cancer survivor," in UpToDate, eds P. A. Ganz, S. R. Vora (Waltham, MA: Wolters Kluwer Health). Available online at: https://www.uptodate. com/contents/overview-of-psychosocial-issues-in-the-adult-cancer-survivor? search=overview\%20of\%20psychosocial\&source=search_result\&selectedTitle $=1 \sim 150$ \&usage_type $=$ default $\&$ display_rank $=1$

Traa, M. J., De Vries, J., Bodenmann, G., and Den Oudsten, B. (2015). Dyadic coping and relationship functioning in couples coping with cancer: a systematic review. Brit. J. Health Psychol. 20, 85-114. doi: 10.1111/bjhp. 12094

Weitzner, M. A., Mcmillan, S. C., and Jacobsen, P. B. (1999). Family caregiver quality of life: differences between curative and palliative cancer treatment settings. J. Pain Symptom Manag. 17, 418-428. doi: 10.1016/S0885-3924(99)00014-7

Zebrack, B. (2000). Cancer survivor identity and quality of life. Cancer Pract. 8, 238-242. doi: 10.1046/j.1523-5394.2000. 85004.x

Conflict of Interest Statement: The authors declare that the research was conducted in the absence of any commercial or financial relationships that could be construed as a potential conflict of interest.

Copyright (c) 2018 Langer, Romano, Todd, Strauman, Keefe, Syrjala, Bricker, Ghosh, Burns, Bolger, Puleo, Gralow, Shankaran, Westbrook, Zafar and Porter. This is an open-access article distributed under the terms of the Creative Commons Attribution License (CC BY). The use, distribution or reproduction in other forums is permitted, provided the original author(s) and the copyright owner(s) are credited and that the original publication in this journal is cited, in accordance with accepted academic practice. No use, distribution or reproduction is permitted which does not comply with these terms. 


\section{OPEN ACCESS}

Edited by:

Guy Bodenmann,

Universität Zürich, Switzerland

Reviewed by:

Fridtjof W. Nussbeck

Bielefeld University, Germany

Silvia Donato,

Università Cattolica del Sacro Cuore,

${ }^{*}$ Correspondence: Anita DeLongis

anita.delongis@ubc.ca

Specialty section:

This article was submitted to Clinical and Health Psychology, a section of the journal

Frontiers in Psychology

Received: 05 June 2018

Accepted: 30 August 2018 Published: 20 September 2018

Citation:

Pow J, Stephenson E, Hagedoorn M and DeLongis $A$ (2018) Spousal Support for Patients With Rheumatoid Arthritis: Getting the Wrong Kind Is a Pain. Front. Psychol. 9:1760. doi: 10.3389/fpsyg.2018.01760

\section{Spousal Support for Patients With Rheumatoid Arthritis: Getting the Wrong Kind Is a Pain}

\author{
Jessie Pow ${ }^{1}$, Ellen Stephenson ${ }^{1}$, Mariët Hagedoorn ${ }^{2}$ and Anita DeLongis ${ }^{1 *}$ \\ ' Department of Psychology, Centre for Health and Coping Studies, The University of British Columbia, Vancouver, BC, \\ Canada, ${ }^{2}$ Department of Health Psychology, University Medical Center Groningen, University of Groningen, Groningen, \\ Netherlands
}

Research indicates that perceived support availability is beneficial, with support available from the spouse particularly important for well-being. However, actual support mobilization has shown mixed associations with recipient well-being. The primary goal of the present study was to go beyond examining the effects of global perceptions of support on recipient outcomes. Instead, we examined the effects of several specific types of support that have been found to be important in the clinical literature. In this study, we followed both members of couples in which one partner was diagnosed with rheumatoid arthritis. Patients provided reports on pain for both mornings and evenings across 1 week. Both partners also reported esteem, solicitous, and negative support mobilization received by the patient. We found that patient pain tended to increase across the day following increases in patient reports of negative support receipt and partner reports of solicitous support provision. We also found that patient pain tended to decrease across the day when partners reported increased levels of esteem support provision. Reverse causation analyses indicated higher levels of patient pain may lead partners to increase solicitous support mobilization to the patient. Findings underscore the importance of examining both partners' reports of support within a dyadic coping framework. They further suggest that not all forms of support are equally beneficial, calling for a finer grained assessment of specific support transactions.

Keywords: social support, dyadic coping, pain, rheumatoid arthritis, solicitous support, emotional support, negative support, intensive longitudinal methods

\section{INTRODUCTION}

What is the best way for the spouse to provide support? Should he express how concerned he is about the patient? Should she assure her spouse that he is loved, valued, and important? Do his avoidant or critical responses influence his partner's well-being over time? Dyadic coping theory suggests that spouse responses play a critical role in influencing well-being (Bodenmann et al., 2007), especially for those coping with chronic illness (Revenson and DeLongis, 2010). However, the literature is mixed regarding the effectiveness of support. Some studies find beneficial effects of support receipt (Pasch and Bradbury, 1998; DeLongis et al., 2004), whereas others find no effects (Barrera, 1986; Bolger et al., 1996) or even detrimental effects of support receipt (Bolger et al., 2000; Jang et al., 2003; Shrout et al., 2006; Gleason et al., 2008). Differences in findings may be attributable to limitations due to aggregating across multiple types and instances of support as well as 
limitations inherent in only examining one partner's perspective. In this paper, we addressed these issues by using an intensive longitudinal design to examine reports of several types of partner support mobilization in predicting subsequent pain among patients with rheumatoid arthritis (RA). We took a dyadic perspective by examining the perceptions of both RA patients and their partners.

Although research has emerged accounting for the role of both partners' perceptions of support in key psychosocial outcomes (Bodenmann et al., 2006; Badr and Taylor, 2009; Rosen et al., 2014, 2015), the impact of relationships on chronic illness has most often been investigated via patient reports only (Revenson and DeLongis, 2010). In couples in which one partner is coping with a chronic illness, the impact of both partners' responses on patient outcomes is not well understood. However, previous studies of RA patients and their spouses underscore the importance of examining the role of spouse variables in patient disease course. For example, in a prospective study of RA patients and their spouses, spouse reports of their own depressive symptoms predicted increased functional limitations and RArelated symptoms for patients over a one-year period, controlling for earlier patient depression and functional limitations or RArelated symptoms (Lam et al., 2009; Stephenson et al., 2014).

Outside of chronic pain contexts, studies suggest the support recipients' and support providers' perceptions both independently predict changes in recipient well-being. For example, in one study examining global levels of support, provider reports of emotional support were associated with decreases in negative affect, but recipient reports of emotional support were associated with increases in negative affect (Bolger et al., 2000). Other studies suggest that recipients and providers have unique perspectives that may jointly provide important insights into the role of support (Collins and Feeney, 2000).

Many studies on support transactions in intimate relationships have examined support as a global construct (Bolger et al., 2000; DeLongis et al., 2004). However, theoretical models outline several types of support, which are expected to have different implications for well-being (Schulz and Schwarzer, 2000; Cano et al., 2008). The focus on global levels of support mobilization is a limitation of the literature because it leads to an incomplete understanding of which specific transactions have occurred. This limited focus does not offer insights into which specific behaviors to target with interventions promoting adaptive responses to stress in couples. In the current study, we examined three types of support. First, we examined esteem/emotional support, which refers to expressions that the recipient is loved, valued, and accepted. The second type of support we examined was solicitous support, which involves conveying concern for the support recipient (Flor et al., 1989; Newton-John, 2002). Third, we examined negative support, which includes being critical of the support recipient or avoiding the support recipient (Bodenmann, 2005; Sullivan et al., 2010). Studies indicate that these forms of support are distinct constructs (Cano et al., 2008; Brock et al., 2014) and that they may be key for couples coping with chronic pain (Hemphill et al., 2016).

There are two leading models that make different predictions regarding which types of support should be effective ways of promoting well-being for those experiencing chronic pain (Cano and Williams, 2010; Hemphill et al., 2016). Pain research has traditionally relied on operant models, which indicate that pain behaviors communicate pain to others, and others' supportive responses to pain behaviors may inadvertently reinforce those behaviors (Fordyce, 1976), leading to an increase in pain. This model predicts that spousal emotional and solicitous support could reinforce pain behavior and lead to worse outcomes over time. This model also predicts that negative spouse responses extinguish pain behaviors and lead to better outcomes (Turk et al., 1992). In contrast to operant models, the interpersonal model predicts that spouse responses aimed at understanding and validating the patient's emotions and pain experiences are intimacy-building, help individuals regulate emotions, and lead to better outcomes over time (Holtzman and DeLongis, 2007; Cano et al., 2008; Cano and Williams, 2010; Hemphill et al., 2016). Interpersonal models classify negative spouse responses as unsupportive and suggest that they undermine intimacy, disrupt emotion regulation, and lead to poorer outcomes (McCracken, 2005; Cano et al., 2008; Cano and Williams, 2010; Hemphill et al., 2016).

There is partial empirical support for both the traditional operant model and the interpersonal model of pain. Findings regarding solicitous support tend to be consistent with a traditional operant model. Several cross-sectional studies of couples coping with chronic pain indicate that individuals who receive higher levels of solicitous support from their partners tend to have worse well-being than those who receive lower levels of solicitous support (Romano et al., 1995, 2000; Fillingim et al., 2003; Boothby et al., 2004; McCracken, 2005). In contrast, findings for negative and esteem support tend to support the interpersonal model. Negative responses have been linked to poorer patient outcomes, including greater emotional distress, pain, and pain catastrophizing, as well as less activity engagement and lower acceptance of pain (Kerns et al., 1990; Keefe et al., 2003; Boothby et al., 2004; Cano, 2004; McCracken, 2005). Fewer studies have examined associations between esteem/emotional support and well-being in individuals with chronic illness. However, in one study, participants with osteoarthritis who perceived higher emotional support availability tended to report poorer functional ability (Weinberger et al., 1990).

Despite several cross-sectional studies examining associations between spouse responses and patient well-being, few studies have examined within-couple or prospective associations. In addition, there has been very little research examining spouse reports. These are key limitations of the literature because this makes it difficult to know whether partners change the amount and type of support they provide in response to worsening patient symptoms. For example, is it possible that solicitous support is linked to worse patient well-being because spouses increase their provision of solicitous support when their partners are experiencing more symptoms?

In a recent study of patients with osteoarthritis, Hemphill et al. (2016) examined change in physical limitations and physical activity over 6 and 12 months as a function of spouse reports of emotional support provision, solicitous support provision, and negative support provision to patients. Emotional support 
provision as reported by spouses was a significant predictor of subsequent decreases in functional limitations and increases in physical activity over 6 months; solicitous responses were significantly associated with increases in functional limitations and decreases in physical activity over 12 months. Negative support was not significantly associated with changes in patient outcomes over time. Although this study provides evidence for a beneficial effect of emotional support and a detrimental effect of solicitous support over time, the study did not examine reports from both partners, nor did the authors examine pain as an outcome. Given this, questions remain about whether spouse reports provide complementary information beyond patient reports, and whether each type of support similarly influences different outcomes.

Intensive longitudinal studies allow for within-couple examination of time-ordered associations among spouse responses and patient well-being. There have been only a few intensive longitudinal studies examining daily associations between patient well-being and esteem/emotional, solicitous, and negative partner responses. These studies tend to find benefits of emotional support and detrimental effects of solicitous and negative support on patient well-being (Badr et al., 2013; Rosen et al., 2014; Song et al., 2015). For example, in an intensive longitudinal study examining spouse responses to vulvodynia pain, patients' sexual functioning improved on days when they reported receiving higher levels of emotional, lower solicitous, and lower negative support from their partners (Rosen et al., 2014).

A handful of studies have used intensive longitudinal methods to examine associations between spouse responses and patient pain (Holtzman and DeLongis, 2007; Burns et al., 2013; Rosen et al., 2015). In one study focusing on couples in which one partner had chronic low back pain, Burns et al. (2013), found that patient perceptions of higher spouse hostility and criticism were both associated with higher concurrent patient pain when controlling for prior pain intensity. Additionally, patient perceptions of higher spouse hostility were associated with residualized increases in patient pain over the subsequent 3 h. However, no association was found between patient perceptions of spouse criticism and subsequent changes in patient pain. Although this study provides some evidence that negative spouse responses are prospectively associated with changes in patient pain, emotional, and solicitous spouse responses were not examined. Additionally, only patient reports of spouse responses were examined.

One intensive longitudinal study examined associations between both partners' reports of spouse emotional, solicitous, and negative support and patients' reports of vulvodynia pain. In this study, pain decreased on days when patients reported receiving lower levels of solicitous and negative support (Rosen et al., 2015). Patients' pain decreased on days when their partners reported providing higher levels of esteem/emotional and lower levels of solicitous support (Rosen et al., 2015). Although this study provides initial evidence for beneficial effects of esteem/emotional and detrimental effects of solicitous and negative support on daily patient pain, prospective associations were not examined. Therefore, a viable alternative explanation of the findings is that increases in patient pain lead partners to change how they respond.

In this study, we examined the roles of both partners' perceptions of esteem/emotional, solicitous, and negative support in predicting subsequent shifts in pain. We focused on couples in which one partner had been diagnosed with RA. RA is an incurable autoimmune disease that affects up to $1 \%$ of the global population (Woolf and Pfleger, 2003). It is associated with a number of debilitating symptoms, including chronic pain, stiffness and inflammation of the joints, fatigue, and frequent shifts in mood (Smith and Wallston, 1992). Given this, the spouse can play a key role in providing support to the affected individual.

We used an intensive longitudinal design (Bolger and Laurenceau, 2013) in which patients and their partners were asked to provide reports about 6 and $12 \mathrm{~h}$ after waking to examine the influence of specific types of spousal support mobilized in the morning on subsequent changes in pain from morning to evening. We predicted that mornings when higher levels of esteem support were mobilized than typical for that couple would be associated with subsequent decreases in pain. We also predicted that times when higher levels of solicitous and negative support were mobilized than typical for that couple would be associated with subsequent increases in pain. We expected that these associations would be maintained when morning levels of potential confounding variables were controlled, including the amount of time spent with the partner and mood.

We were also interested in examining, on an exploratory basis, whether spouses change their supportive behaviors in response to within-patient fluctuations in pain. Thus, we conducted reversecausation analyses. In these analyses, we examined whether mornings when patients experienced higher levels of pain than typical for them were associated with subsequent shifts in esteem/emotional, solicitous, and negative support from the spouse.

\section{MATERIALS AND METHODS}

\section{Participants}

Couples were recruited as part of a larger study on communitydwelling patients with RA (Holtzman and DeLongis, 2007; Beggs et al., 2015). This study is the first to report findings from the spouses of these participants. Eight-hundred potential study participants were randomly selected from a database of patients registered with the Mary Pack Arthritis Society and mailed an initial contact letter describing the study and inviting participation. The Mary Pack Arthritis Society is a local organization that offers treatment and education to arthritis patients across British Columbia, Canada. One hundred eightyeight individuals contacted our research office and 160 agreed to be screened by telephone to ensure that they had been diagnosed with RA, experienced pain due to RA in the past month, and were able to read, write, and speak English. Participants in the current sample were also required to be living with a spouse or common law partner. Spouses were invited to participate following expressed interest by the patient. Of the 160 patients who agreed to participate in additional eligibility screening, 20 
(13\%) declined to participate, 17 (11\%) were excluded because they had not experienced RA pain in the past month, and 52 (33\%) were excluded because they were not married or living with a common law partner. Thus, 71 (44\%) met inclusion criteria for the larger study of patients. Forty-one (26\%) participated in the study but their spouse did not also participate and 30 patients (19\%) both met inclusion criteria and had a spouse willing to participate. One of these couples had to be dropped from analyses because the patient never saw her spouse in the morning and therefore did not report morning support mobilization. Those who contacted our research office regarding their potential participation were entered into a draw for $\$ 1000$. Additionally, all of those who met criteria and participated in the data collection phase were mailed a small gift valued at \$10 CAD.

The final sample consisted of 29 couples (29 RA patients and 29 cohabitating spouses). Patients were mostly female $(n=21$, $72 \%$ ), which is consistent with sex differences in RA prevalence rates (Public Health Agency of Canada [PHAC], 2010), and Caucasian $(n=26,90 \%)$, had children $(n=26,90 \%)$, and had a mean age of 61.1 years $(S D=10.5$, range $=42-82)$. The mean number of years since RA diagnosis was $17.7(S D=13.4$, range $=1-50)$. Of the participating RA patients, seven $(24 \%)$ were employed, twelve (41\%) were retired, five $(17 \%)$ were on sick leave, two $(7 \%)$ were on disability, and three $(11 \%)$ were homemakers at the time of the study. Patients and spouses had a relationship length averaging 31 years $(S D=15.8$, range $=6$ months -59 years). Spouses of RA patients were mostly male $(n=19,66 \%)$ and Caucasian $(n=25,86 \%)$, with a mean age of 62.9 years $(S D=9.1$, range $=46-85)$. Of the participating spouses, eleven (38\%) were employed, thirteen (45\%) were retired, one (3.7\%) was on sick leave, and one $(3.7 \%)$ was a homemaker at the time of the study. The modal family income was between $\$ 25,000$ and $\$ 50,000$ CAD.

\section{Procedure}

Participants provided informed consent over the phone and then completed brief structured telephone interviews twice a day for 1 week, which were scheduled at approximately 6 and $12 \mathrm{~h}$ after waking up. At each interview, patients were asked to report on pain and support receipt from the spouse; spouses were asked to report support provision to the patient. These reports were in reference either to their experiences so far that day (for the morning assessment) or since the last interview (for the evening assessment). The twice daily phone interviews lasted approximately 10 min per interview and were administered by a trained female research assistant. Consistent interviewers were assigned to each participant to develop and maintain rapport. Participants were asked to find a private and quiet place in which to complete the daily interviews, and interviews were conducted separately with each member of the couple. With the permission of participants, all interview sessions were tape recorded and transcribed. Telephone methods were used (as opposed to electronic methods) due to the difficulties that may have arisen with holding and operating handheld devices and/or typing, given the previously noted functional disabilities and limitations common to individuals with RA. This study was approved by the affiliated institution's Behavioral Research Ethics Board.

\section{Measures \\ Patient Pain}

Patients reported intensity of pain associated with RA during the previous half-day using a numerical rating scale (NRS) from 0 (no pain) to 10 (pain as bad as it could be). The NRS has demonstrated positive and significant associations with other measures of pain intensity (Jensen et al., 1986; Wilkie et al., 1990) and sensitivity to treatments aimed at influencing pain intensity (Paice and Cohen, 1997).

\section{Spouse Support Mobilization}

Patients and their spouses provided reports on support mobilized from the spouse to the patient using a modified version of the Berlin Social Support Scales (BSSS; Schulz and Schwarzer, 2000). Participants provided responses on a 5-point Likert scale ( 0 = does not apply, $1=$ not at all, 2 = a little, $3=$ somewhat, 4 = a lot). We collapsed across the "does not apply" and "not at all" categories such that either response received a score of one. Patient esteem/emotional support receipt was assessed with two items ("He/she showed you that he/she loves and accepts you," "He/she made you feel valued and important"; am $R_{\mathrm{c}}=0.46 ; \mathrm{pm}$ $R_{\mathrm{c}}=0.56$; all timepoints $\left.R_{\mathrm{c}}=0.53\right) .{ }^{1}$ Spouse esteem/emotional support provision was assessed with two parallel items ("You showed him/her that you love and accept him/her," "You made him/her feel valued and important"; am $R_{\mathrm{c}}=0.54 ; \mathrm{pm} R_{\mathrm{c}}=0.48$, all timepoints $R_{\mathrm{c}}=0.55$ ). Solicitous support receipt and provision were each assessed with two items (Patient receipt: "He/she comforted you when you were feeling bad," "He/she expressed concern about your condition"; am $R_{\mathrm{c}}=0.34$; pm $R_{\mathrm{c}}=0.53$; all timepoints $R_{\mathrm{c}}=0.47$; Spouse provision: "You comforted him/her when he/she was feeling bad," "You expressed concern about his/her condition"; am $R_{\mathrm{c}}=0.54 ; \mathrm{pm} R_{\mathrm{c}}=0.38$, all timepoints $=0.45$ ). Negative support receipt and provision were each assessed with two items (Patient receipt: "He/avoided you," "He/she complained about you"; am $R_{\mathrm{c}}=0.56 ; \mathrm{pm} R_{\mathrm{c}}=0.25$, all timepoints $R_{\mathrm{c}}=0.48$; Spouse provision: "You avoided him/her," "You complained about him/her"; $\mathrm{am} R_{\mathrm{c}}=0.00 ; \mathrm{pm} R_{\mathrm{c}}=0.00$, all time points $\left.R_{\mathrm{c}}=0.00\right){ }^{2}$ If participants indicated that they had not seen or spoken to their spouse since the last diary entry, spouse

${ }^{1}$ Here we report the $R_{\mathrm{c}}$, which is the internal consistency reliability of change within persons throughout the study (Cranford et al., 2006). However, it is important to note that the items we used to assess support can be considered formative rather than reflective indicators (Bollen and Lennox, 1991). That is, reflective indicators are indicators that are "caused by" an underlying latent variable and should be highly correlated (Bollen and Lennox, 1991). However, formative indicators are conceptualized as components that "cause" or "determine" the construct and are not necessarily expected to be highly correlated (Bollen and Lennox, 1991). For example, even though complaining about one's spouse and avoiding one's spouse are two components that are theorized to function together as negative support (Bodenmann, 2005), they may not occur at the same time and may not be expected to be highly correlated. Previous studies have conceptualized supportive behaviors using this measurement model (Collins and Feeney, 2000).

${ }^{2}$ An examination of the items making up spouse negative support provision indicated that the items were not correlated at the within-person level $(r=-0.01$, $n s)$. Because complaining about one's spouse and avoiding one's spouse are two components that are theorized to function together as negative support (Bodenmann, 2005), we created a variable summing across those items despite 
support questions were skipped for that timepoint and treated as missing.

\section{Control Variables}

We asked patients to report the extent to which they saw or spoke to their partner $(1=$ Not at all, $4=\mathrm{A}$ lot $)$. Positive and negative affect were assessed using the Affects Balance Scale (Derogatis, 1975). Positive affect was assessed with five items ( $\operatorname{am} R_{\mathrm{c}}=0.82$; pm $R_{\mathrm{c}}=0.78$; all timepoints $R_{\mathrm{c}}=0.80$ ). Negative affect was the combined score of the five-item depression and five-item anxiety subscales because they were highly correlated (average $r=0.69$, ranging from 0.53 to 0.89 ; am $R_{\mathrm{c}}=0.75 ; \mathrm{pm} R_{\mathrm{c}}=0.70$; all timepoints $R_{\mathrm{c}}=0.79$ ).

\section{Analytic Strategy \\ Multi-Level Modeling}

Because of the multilevel structure of the data in which days were nested within couples, we conducted multilevel analyses in R (Sarkar, 2008; Wickham, 2009, 2017; Bates et al., 2015; Bates and Maechler, 2017; Kuznetsova et al., 2017; R Core Team, 2017; Revelle, 2017; Wickham and Miller, 2017). In these analyses, within-couple variation was modeled at Level 1 and betweencouple variation was modeled at Level 2 . We began by calculating Intraclass Correlations (ICCs) for all study variables to examine the amount of variance attributable to stable differences between couples and variance attributable to fluctuations over time within couples (see Table 1). ICCs were higher than 0.18 for all variables, indicating that a multilevel approach was appropriate. We also computed within-couple Pearson correlations for study variables.

low within-person internal consistency of the items. However, we ran an additional model to supplement our main analyses that included both items as separate within-person predictors of changes in patient pain. Neither item was significantly associated with changes in pain from morning to evening ( $p s>0.100)$. Additionally, having these items in the model as separate predictors rather than as one composite predictor did not change associations we observed for the other support variables.

TABLE 1 | Descriptive statistics of study variables.

\begin{tabular}{|c|c|c|c|c|c|c|}
\hline & \multicolumn{3}{|c|}{ Patient } & \multicolumn{3}{|c|}{ Partner } \\
\hline & $\begin{array}{l}\text { Grand } \\
\text { mean }\end{array}$ & $S D$ & ICC & $\begin{array}{l}\text { Grand } \\
\text { mean }\end{array}$ & $S D$ & ICC \\
\hline \multicolumn{7}{|l|}{ AM observations } \\
\hline AM pain & 4.10 & 2.14 & 0.69 & - & - & - \\
\hline Esteem/emotional & 3.10 & 0.85 & 0.60 & 2.51 & 0.89 & 0.65 \\
\hline Solicitous & 2.22 & 1.03 & 0.64 & 2.11 & 0.95 & 0.62 \\
\hline Negative & 1.10 & 0.30 & 0.29 & 1.14 & 0.27 & 0.18 \\
\hline \multicolumn{7}{|l|}{ PM observations } \\
\hline PM pain & 3.86 & 2.17 & 0.69 & - & - & - \\
\hline Esteem/emotional & 3.18 & 0.83 & 0.60 & 2.60 & 0.89 & 0.78 \\
\hline Solicitous & 2.29 & 1.02 & 0.54 & 2.09 & 0.91 & 0.66 \\
\hline Negative & 1.09 & 0.22 & 0.18 & 1.14 & 0.24 & 0.31 \\
\hline
\end{tabular}

Grand means and standard deviations were calculated across all person-days.

\section{Hypothesis Testing}

For our main analyses, we ran a random intercept model examining the roles of morning support receipt and provision in predicting evening pain. ${ }^{3}$ Morning pain was included in all models so that we could examine effects of support on residualized change in pain from morning to evening. We included both spouses' reports of support mobilization so that we could examine the unique effects of each partner's perspective. Following this, we added quantity of time spent with the partner, negative affect, and positive affect to the model. All predictor variables were centered on the mean for each couple (i.e., groupmean centered) so that we could examine within-couple effects.

\section{Reverse Causation}

We ran a series of additional models examining whether withinpatient fluctuations in pain were significantly associated with subsequent residualized changes in each type of patient support receipt and spouse support provision. In these models, evening reports of each type of support were specified as a function of within-couple centered morning levels of that type of support and within-couple centered morning pain.

\section{RESULTS}

\section{Response Rate and Descriptive Statistics}

Response rates on the twice-daily interviews were excellent, with 404 of the possible 406 morning and evening interviews completed by patients and 404 of 406 interviews completed by spouses. Twenty-seven of the included patients and twenty-seven of the included spouses completed all 14 interviews. Two patients and two spouses each missed one interview. With few exceptions, there were no missing items within the completed interviews. A single item was missing for patient negative affect for one of the morning interviews. We addressed this by taking the average of the remaining nine items for that interview. Patients and spouses reported not seeing each other on $4 \%$ of the half-days. In these cases, participants did not complete the support items. Therefore, some participants had fewer than 14 interviews included in the analyses if they (1) missed an interview, or (2) did not see their spouse. The restricted maximum likelihood estimation of linear mixed-effects models applied here is robust to missingness and can account for unbalanced numbers of observations per group (Bates et al., 2015; Kuznetsova et al., 2017).

Table 1 displays descriptive statistics for study variables. As can be seen in the Table, the grand means for emotional support receipt and provision indicate that emotional support was mobilized "somewhat" - "a lot" across person-days. The grand means for solicitous support receipt and provision indicate that solicitous support was mobilized "a little" - "somewhat." The grand means for negative support receipt and provision were low,

\footnotetext{
${ }^{3}$ We also examined whether evening reports of support were associated with subsequent residualized change in patient pain from evening to the next morning. However, there were no significant associations of within-couple evening support receipt or provision on residualized change in well-being from evening to the next morning.
} 
corresponding to the "not at all" response option. We inspected frequencies of our study variables. These analyses revealed that frequencies of negative support were low, with values $>1$ on only $8 \%$ of the AM patient reports, $7 \%$ of the PM patient reports, $7 \%$ of the AM spouse reports, $7 \%$ of the PM spouse reports. This is a point we return to in our discussion.

Although the focus of this study is on within-couple associations among spouse responses and patient pain, for the purpose of future meta-analyses, we report within- and between-couple correlations of study variables in Table 2 . These correlations were calculated using the statsBy function in the psych package in R (Revelle, 2017), which calculates the pooled within-group correlations and the sample size weighted betweencouple correlations. Within-couple associations of each type of morning support and evening pain were in the expected directions. Mornings when patients received higher levels of esteem/emotional support and lower levels of solicitous and negative support than typical for them were nonsignificantly associated with lower levels of evening pain (for esteem/emotional support: $r=-0.10, p=0.176$; for solicitous support: $r=0.06, p=0.427$; for negative support: $r=0.12$, $p=0.108$. Additionally, mornings when partners provided higher levels of esteem/emotional support than typical for them were non-significantly associated with lower evening patient pain, $r=-0.07, p=0.230$. There were significant associations between partner reports of solicitous and negative support provision and evening patient pain: mornings when partners reported providing higher levels of solicitous and negative support than typical for them were associated with higher evening levels of patient pain (for solicitous support: $r=0.16, p=0.030$; for negative support: $r=0.17, p=0.017)$. Readers should interpret the between-couple correlations with caution given the low sample size; however, the overall pattern suggests that patients who experienced higher levels of pain tended to receive higher levels of all types of support as reported by patients and partners, although most of these correlations were not significant.

\section{Cross-Day Changes in Pain}

We conducted multilevel regression analysis predicting residualized change in patient pain as a function of each type of support mobilization to patients as reported by patients and partners. These results are displayed in Table 3. Contrary to expectations, patient reports of esteem and solicitous support were not significantly associated with subsequent changes in pain (esteem: $b=-0.26, S E=0.16, t(146)=1.63, p=0.105$; solicitous: $b=0.06, S E=0.14, t(147)=0.45, p=0.656$. However, the effects of patient negative support receipt were consistent with expectations: mornings when patients reported receiving higher levels of negative support than typical for them were associated with subsequent increases in pain, $b=0.63, S E=0.31$, $t(146)=2.00, p=0.045$. Additionally, mornings when partners reported providing higher levels of esteem support and lower levels of solicitous support than typical for them were associated with subsequent decreases in patients' pain from morning to evening (esteem: $b=-0.48, S E=0.16, t(146)=-2.93, p=0.004$; solicitous: $b=0.35, S E=0.15, t(147)=2.38, p=0.019)$. Increases in partner negative support provision were not significantly associated with subsequent residualized increases in patient pain, $b=0.74, S E=0.38, t(147)=1.94, p=0.055$. When we included time spent with the partner, negative affect, and positive affect in the model, results were unchanged. We examined statistical assumptions of linearity, homoscedasticity, and normal distribution of residuals and all three assumptions were met for both models presented in Table 3 .

\section{Reverse Causation}

Next, we examined whether morning-to-morning within-patient fluctuations in pain were associated with subsequent changes in each type of support in six multilevel regression models - one for each type of support (i.e., patient received esteem support, patient received solicitous support, patient received negative support, spouse provided esteem support, spouse provided solicitous support, and spouse provided negative support). Fluctuations in morning pain were not significantly associated with subsequent changes in levels of esteem support receipt reported by patients, $b=0.02, S E=0.03, t(156)=0.71, p=0.482$, or with subsequent changes in levels of esteem support provision reported by partners, $b=0.03, S E=0.03, t(155)=1.18, p=0.240$. However, increases in morning pain were significantly associated with subsequent increases in solicitous support receipt reported by patients, $b=0.13, S E=0.04, t(157)=2.94, p=0.004$ as well as with subsequent increases in solicitous support provision reported by partners, $b=0.09, S E=0.03, t(156)=2.68, p=0.008$. Finally, within-patient increases in pain were not significantly associated with decreases in patient negative support receipt, $b=-0.02, S E=0.01, t(158)=-1.84, p=0.067$. Changes in pain were not significantly associated with changes in negative support provision reported by partners, $b=0.001, S E=0.01$, $t(158)=-0.12, p=0.902$.

\section{DISCUSSION}

We addressed the question of how spouses might best support patients coping with chronic pain. This is one of a handful of intensive longitudinal studies to address this question and this is the first to examine effects of multiple types of spouse responses on subsequent changes in pain. We found that esteem/emotional support provision by spouses was associated with subsequent decreases in pain across the day. In contrast, solicitous support provision by spouses and negative support receipt by patients were associated with subsequent increases in pain across the day. This study provides evidence that examining only total support may mask important differences in the effects of specific types. In the past studies of social support, even when differentiating types of support, have tended to lump solicitous and esteem support together, treating both as emotional support. However, consistent with operant models of pain, our study suggests that the effects of these two types of support on patient pain outcomes may be quite different. This study also provides evidence that assessing both partners' perceptions of partner responses provides complementary information that would be missed if only one partners' perceptions were assessed. 
TABLE 2 | Within- and between-couple bivariate correlations among study variables.

\begin{tabular}{|c|c|c|c|c|c|c|c|c|c|c|c|c|c|c|}
\hline & 1 & 2 & 3 & 4 & 5 & 6 & 7 & 8 & 9 & 10 & 11 & 12 & 13 & 14 \\
\hline \multicolumn{15}{|l|}{ AM patient report } \\
\hline 1. Pain & - & 0.05 & 0.20 & 0.13 & 0.07 & $0.42^{*}$ & 0.08 & $0.89 * * *$ & 0.09 & 0.23 & 0.04 & 0.06 & $0.34^{+}$ & 0.24 \\
\hline 2. Esteem/emotional & -0.00 & - & $0.67^{* * *}$ & $-0.50^{* *}$ & $0.59^{* * *}$ & $0.41^{*}$ & $-0.50^{* *}$ & 0.16 & $0.85^{* * *}$ & $0.63^{* * *}$ & $-0.40^{*}$ & $0.62^{* * *}$ & $0.43^{*}$ & $-0.52^{* *}$ \\
\hline 3. Solicitous & 0.07 & $0.30^{* * *}$ & - & -0.30 & $0.35^{+}$ & $0.43^{*}$ & -0.30 & 0.24 & $0.59^{* * *}$ & $0.91^{* * *}$ & -0.17 & $0.38^{*}$ & $0.41^{*}$ & -0.26 \\
\hline 4. Negative & -0.06 & $-0.13^{+}$ & 0.11 & - & $-0.48^{* *}$ & $-0.36^{+}$ & $0.70^{* * *}$ & 0.12 & $-0.49^{* *}$ & $-0.36^{+}$ & $0.78^{* * *}$ & $-0.52^{* *}$ & $-0.33^{+}$ & $0.82^{* * *}$ \\
\hline \multicolumn{15}{|l|}{ AM partner report } \\
\hline 5. Esteem/emotional & 0.08 & 0.01 & 0.06 & 0.06 & - & $0.66^{* * *}$ & -0.21 & 0.04 & $0.54^{* *}$ & $0.31^{+}$ & $-0.40^{*}$ & $0.92^{* * *}$ & $0.67^{* * *}$ & $-0.47^{*}$ \\
\hline 6. Solicitous & 0.11 & 0.02 & 0.08 & 0.03 & $0.35^{* * *}$ & - & -0.12 & $0.39 *$ & $0.33^{+}$ & $0.36^{+}$ & -0.28 & $0.68^{* * *}$ & $0.95^{* * *}$ & -0.22 \\
\hline 7. Negative & $0.12^{+}$ & 0.05 & 0.01 & 0.02 & 0.05 & -0.03 & - & -0.03 & $-0.44^{*}$ & $-0.38^{*}$ & $0.49^{* *}$ & $-0.32^{+}$ & -0.16 & $0.57^{* *}$ \\
\hline \multicolumn{15}{|l|}{ PM patient report } \\
\hline 8. Pain & $0.50^{* * *}$ & -0.10 & 0.06 & 0.12 & -0.09 & $0.16^{*}$ & $0.17^{*}$ & - & 0.16 & 0.26 & 0.07 & 0.00 & 0.30 & 0.26 \\
\hline 9. Esteem/emotional & 0.05 & $0.32^{* * *}$ & 0.11 & -0.04 & 0.07 & -0.04 & -0.06 & -0.06 & - & $0.66^{* * *}$ & -0.30 & $0.54^{* *}$ & 0.27 & $-0.48^{* *}$ \\
\hline 10. Solicitous & $0.22 *$ & $0.15^{*}$ & $0.23^{* *}$ & -0.02 & -0.00 & 0.00 & 0.02 & $0.17^{*}$ & $0.16^{*}$ & - & -0.18 & $0.31^{+}$ & 0.23 & -0.26 \\
\hline 11. Negative & $-0.15^{*}$ & $-0.13^{+}$ & -0.12 & $-0.19^{* *}$ & -0.05 & 0.05 & -0.02 & -0.11 & $-0.12^{+}$ & -0.09 & - & $-0.44^{*}$ & -0.20 & $0.51^{* *}$ \\
\hline \multicolumn{15}{|l|}{ PM partner report } \\
\hline 12. Esteem/emotional & 0.10 & 0.03 & -0.03 & 0.01 & $0.23^{* *}$ & $0.13^{+}$ & -0.01 & -0.07 & 0.07 & 0.05 & 0.04 & - & $0.74^{* * *}$ & -0.44 \\
\hline 13. Solicitous & $0.22 *$ & -0.03 & 0.10 & 0.01 & 0.01 & $0.26^{* * *}$ & $-0.15^{+}$ & $0.22^{* *}$ & -0.04 & $0.22^{* *}$ & 0.02 & $0.27^{* * *}$ & - & -0.24 \\
\hline 14. Negative & -0.04 & 0.04 & 0.06 & 0.06 & 0.01 & 0.01 & -0.06 & -0.05 & 0.05 & 0.05 & 0.00 & 0.03 & 0.04 & - \\
\hline
\end{tabular}

Pooled within-couple correlations are presented below the diagonal and sample size weighted between-couple correlations are presented above the diagonal. ${ }^{+} p<0.100$, ${ }^{*} p<0.050,{ }^{* *} p<0.010,{ }^{* * *} p<0.001$

TABLE 3 | Predicting residualized change in patient pain from morning to evening as a function of morning esteem/emotional, solicitous, and negative support mobilized to patients as reported by patients and partners.

\begin{tabular}{|c|c|c|c|c|c|c|}
\hline \multirow[b]{2}{*}{ Fixed effects } & \multicolumn{3}{|c|}{ Model 1} & \multicolumn{3}{|c|}{ Model 2} \\
\hline & Estimate (SE) & $t$ (df) & $p$ & Estimate (SE) & $t(\mathrm{df})$ & $p$ \\
\hline Intercept & $3.89(0.36)$ & $10.72(28)$ & $<0.001$ & $3.89(0.37)$ & $10.69(28)$ & $<0.001$ \\
\hline AM pain & $0.50(0.07)$ & $7.56(147)$ & $<0.001$ & $0.50(0.07)$ & 7.03 (143) & $<0.001$ \\
\hline AM time spent with spouse & & & & $-0.03(0.13)$ & $-0.22(144)$ & 0.828 \\
\hline AM negative affect & & & & $-0.04(0.35)$ & $-0.12(143)$ & 0.905 \\
\hline AM positive affect & & & & $-0.03(0.19)$ & $-0.16(143)$ & 0.874 \\
\hline \multicolumn{7}{|c|}{ Patient report of support receipt } \\
\hline AM esteem/emotional & $-0.26(0.16)$ & $-1.63(146)$ & 0.105 & $-0.26(0.17)$ & $-1.52(142)$ & 0.130 \\
\hline AM solicitous & $0.06(0.14)$ & $0.45(147)$ & 0.656 & $0.05(0.14)$ & $0.36(143)$ & 0.718 \\
\hline AM negative & $0.63(0.31)$ & $2.00(146)$ & 0.048 & $0.64(0.32)$ & 1.99 (142) & 0.048 \\
\hline \multicolumn{7}{|c|}{ Partner report of support provision } \\
\hline AM esteem/emotional & $-0.48(0.16)$ & $-2.93(146)$ & 0.004 & $-0.46(0.17)$ & $-2.68(142)$ & 0.008 \\
\hline AM solicitous & $0.35(0.15)$ & $2.38(146)$ & 0.019 & $0.33(0.15)$ & 2.22 (142) & 0.028 \\
\hline AM negative & $0.74(0.38)$ & $1.94(147)$ & 0.055 & $0.56(0.42)$ & $1.34(143)$ & 0.184 \\
\hline Random effects & \multicolumn{2}{|c|}{ Standard deviation } & & \multicolumn{2}{|c|}{ Standard deviation } & \\
\hline Intercept & \multicolumn{2}{|c|}{1.907} & & \multicolumn{2}{|c|}{1.908} & \\
\hline Residual & \multicolumn{2}{|c|}{1.002} & & \multicolumn{2}{|c|}{1.009} & \\
\hline
\end{tabular}

Model based on 181-182 days from 29 couples. All predictors have been centered relative to person means that were calculated based on all available observations.

Our primary goal was to examine a model in which spouse responses are expected to lead to changes in patient adjustment. However, a competing model is that patient pain is independent of social influences, and that associations that have been observed in previous cross-sectional studies between patient pain and spouse responses are simply due to spouses reacting to patient disability. Previously, Burns et al. (2013) examined time-ordered associations between patient pain and spouse criticism and hostility. Specifically, they found that higher spouse hostility was associated with subsequent increases in patient pain, and patient pain was associated with subsequent decreases in spouse criticism and hostility. Similar to Burns et al's. (2013) study, we found some evidence for bidirectional causality between negative support receipt and pain. Higher levels of negative support receipt were associated with subsequent increases in patient pain. Although patient pain was not significantly associated with subsequent change in negative support receipt with alpha set at 0.05 , a more liberal alpha of 0.10 would lead to the conclusion that higher 
levels of patient pain were associated with subsequent decreases in negative support receipt. Future research utilizing larger samples should further examine the bidirectional association between patient pain and negative support receipt.

Our study replicates and extends the work of Burns et al. (2013), by going beyond negative spouse responses and examining esteem/emotional and solicitous spouse responses. Although we found evidence that esteem/emotional support provision by spouses may lead to decreases in patient pain, we did not find evidence that patient pain leads to shifts in esteem/emotional support mobilization to patients as reported by either the patient or the spouse. These results extend previous research indicating a general beneficial effect of esteem/emotional support to individuals with chronic illness (Weinberger et al., 1990; Rosen et al., 2014, 2015; Beggs et al., 2015; Song et al., 2015; Hemphill et al., 2016). However, this is the first study of which we are aware to examine daily time-ordered associations between esteem/emotional support and patient pain.

We found that within-couple increases in spouse reports of solicitous support provision were associated with subsequent increases in patient pain. In reverse causation analyses, we found that higher levels of patient pain were associated with subsequent increases in solicitous spouse responses as reported by both partners. These results suggest a vicious cycle of patient pain and spouse solicitousness: not only may solicitous support lead to increases in patient pain, but also increases in patient pain may lead spouses to be more solicitous.

Together, these findings point to a potential target for interventions for couples coping with chronic pain. One way that spouses could become better support providers might be to learn to change the way they respond to the patient, especially when the patient is in pain. Spouses could be taught to express love, admiration, acceptance, and confidence in the patient instead of expressing concern or worry. Changing the way that spouses react to patient pain might pave the way for better pain management. The potential vicious cycle of solicitous support and pain should be examined in future research because of potential applicability for interventions in couples coping with chronic pain.

\section{Future Directions}

In this study, most of the patients were female and most of the spouses were male. Previous studies have found gender differences in the extent to which individuals benefit from support (Neff and Karney, 2005). Although our larger proportion of female patients and male spouses reflects the distribution of RA in the population (Public Health Agency of Canada [PHAC], 2010), future studies might aim for more equal ratios of males and females in patient and spouse roles. Aiming to recruit more equal gender ratios would improve generalizability of findings to other chronic conditions and would help to disentangle the effect of patient-partner roles from husband-wife roles.

This study focused on the within-couple relations between partner responses and pain using a small sample of RA patients and their partners. We caution readers that although this study provides initial evidence on the role of support in patient outcomes, more work is needed with larger samples to replicate these results. As is often the case in samples of non-distressed couples, there were relatively few instances of negative support. This low frequency of may have limited our power to detect some of the effects of spouse negative responses. Because of the small sample and consequently limited power for between subject analyses, we were not able to test more complex models, such as models examining aggregated averages of spouse responses on average change in patient pain from morning to evening, the unique effects of morning and evening reports of spouse responses on patient pain, or interactive effects among different spouse response variables. Future research could examine these more complex and potentially more informative models. Additionally, it was not possible to examine stable factors that might influence the extent to which partner responses were associated with changes in pain. For example, patients who are more satisfied with their relationship partner in general may not be as impacted by negative support receipt compared to those who are less satisfied with their relationship partner (DeLongis et al., 2010). Additionally, there may be differences between patients in support effectiveness depending on how much pain patients tend to experience. Future research with larger samples could examine more complex models, including stable factors that might moderate associations among the variables examined here. Importantly, however, our results were unchanged when controlling for the quantity of time spent with the spouse, negative affect, and positive affect.

Future research is needed to examine how spouse responses influence well-being. Our findings here suggest that solicitous and negative spouse responses lead to increases in patient pain, and esteem/emotional spouse support leads to decreases in patient pain. We propose two potential mediators to examine in future research. The first is self-esteem. Associations have previously been found between receiving support and reduced self-esteem in recipients (Nadler et al., 1983; Nadler, 1987). Fisher et al. (1982) theorized that support includes self-threatening and supportive components. They argued that the self-threatening components lead to increased psychological distress whereas the supportive components lead to decreased psychological distress. More recently, Leary (2012) theorized that self-esteem changes as a function of the extent to which people perceive that they are relationally valued and accepted by others. We propose that whether support negatively impacts self-esteem depends on the type of support being mobilized (Pow and DeLongis, 2018). Solicitous and negative support may have detrimental effects on patient well-being across studies because these forms of support both communicate that the spouse believes that the patient is struggling and may not be able to handle things on his or her own. In contrast, esteem/emotional support communicates that the patient is loved and valued and would be expected to lead to improvements in self-esteem.

Along with self-esteem, spouse responses may also influence patient well-being by altering patient perceptions of spouse responsivity, which is the perception of understanding and validation from the spouse (Reis and Shaver, 1988). Perceived spouse responsivity has been found to fluctuate across days (Laurenceau et al., 2005) and has been associated with longterm improvements in well-being (Selcuk et al., 2015; Slatcher et al., 2015). In one cross-sectional study of couples in which 
one partner was experiencing a lupus flare-up, spouse reports of emotional support were significantly associated with higher levels of patient perceived spouse responsiveness, which was, in turn, associated with lower patient depressive symptoms (Fekete et al., 2007). Negative support had the opposite effect. Lower levels of patient perceived spouse responsiveness mediated the positive association between spouse negative support provision and patient depressive symptoms. Although this study identified perceived spouse responsiveness as a promising potential mechanism linking spouse responses and well-being for those coping with chronic illness, research is needed that examines time-ordered associations. Intensive longitudinal studies would allow for the examination of whether spouse responses are associated with subsequent shifts in perceived spouse responsiveness, and whether these shifts in spouse responsiveness account for changes in patient pain and other indicators of well-being.

\section{CONCLUSION}

Researchers typically examine coping from an individualistic perspective without examining the social context. Our findings suggest that spouse responses play a key role in promoting adaptation in individuals coping with chronic pain. Within the limitations of the current study and sample, our findings advocate for the expression of love and acceptance to individuals with chronic pain. They also advocate against expressions from the provider of worry about or criticism of the recipient.

\section{REFERENCES}

Badr, H., Pasipanodya, E. C., and Laurenceau, J.-P. (2013). An electronic diary study of the effects of patient avoidance and partner social constraints on patient momentary affect in metastatic breast cancer. Ann. Behav. Med. 45, 192-202. doi: 10.1007/s12160-012-9436-8

Badr, H., and Taylor, C. L. (2009). Sexual dysfunction and spousal communication in couples coping with prostate cancer. Psychooncology 18, 735-746. doi: 10. 1002/pon.1449

Barrera, M. (1986). Distinctions between social support concepts, measures, and models. Am. J. Commun. Psychol. 14, 413-445. doi: 10.1007/bf009 22627

Bates, D., and Maechler, M. (2017). Matrix: Sparse and Dense Matrix Classes and Methods. R package version 1.2-8. Available at: https://CRAN.R-project.org/ package $=$ Matrix

Bates, D., Maechler, M., Bolker, B., and Walker, S. (2015). Fitting linear mixedeffects models using lme4. J. Statist. Softw. 67, 1-48. doi: 10.18637/jss.v067. i01

Beggs, R., Holtzman, S., and DeLongis, A. (2015). Predicting daily satisfaction with spouse responses among people with rheumatoid arthritis. Ann. Behav. Med. 50, 24-33. doi: 10.1007/s12160-015-9728-x

Bodenmann, G. (2005). "Dyadic coping and its significance for marital functioning," in Couples Coping with Stress: Emerging Perspectives on Dyadic Coping, eds T. A. Revenson, K. Kayser, and G. Bodenmann (Washington, DC: American Psychological Association), 33-49. doi: 10.1037/110 31-002

Bodenmann, G., Charvoz, L., Bradbury, T. N., Bertoni, A., Iafrate, R., Giuliani, C., et al. (2007). The role of stress in divorce: a three-nation retrospective study. J. Soc. Pers. Relationsh. 24, 707-728. doi: 10.1177/02654075070 81456

\section{ETHICS STATEMENT}

This study was carried out in accordance with the recommendations of The University of British Columbia's Behavioural Research Ethics Board. The protocol was approved by The University of British Columbia's Behavioural Research Ethics Board. All subjects gave verbal informed consent in accordance with the Tri-Council Policy Statement (T2, 2014), the International Conference on Harmonization Good Clinical Practice Guidelines (ICH-GCP) and the requirements of the U.S. Department of Health and Human Services, as set out in the Federal Policy for the Protection of Human Subjects, 45 CFR Part 46, sub-part A.

\section{AUTHOR CONTRIBUTIONS}

$\mathrm{AD}$ designed and directed the project, developed the theoretical framework, and supervised all work. JP and ES conducted the data analyses and drafted the manuscript. $\mathrm{MH}$ contributed to the writing of the manuscript. All authors discussed the results and contributed to the final manuscript.

\section{FUNDING}

This research was supported by research grants to $\mathrm{AD}$ from the Social Science and Humanities Research Council of Canada under grants \#6568 and \#10075.

Bodenmann, G., Pihet, S., and Kayser, K. (2006). The relationship between dyadic coping and marital quality: a 2-year longitudinal study. J. Fam. Psychol. 20, 485-493. doi: 10.1037/0893-3200.20.3.485

Bolger, N., Foster, M., Vinokur, A., and Ng, R. (1996). Close relationships and adjustments to a life crisis: the case of breast cancer. J. Pers. Soc. Psychol. 70, 283-294. doi: 10.1037//0022-3514.70.2.283

Bolger, N., and Laurenceau, J.-P. (2013). Intensive Longitudinal Methods: An Introduction to Diary and Experience Sampling Research. New York, NY: Guilford.

Bolger, N., Zuckerman, A., and Kessler, R. (2000). Invisible support and adjustment to stress. J. Pers. Soc. Psychol. 79, 953-961. doi: 10.1037//0022-3514.79.6.953

Bollen, K., and Lennox, R. (1991). Conventional wisdom on measurement: a structural equation perspective. Psychol. Bull. 2, 305-314. doi: 10.1037/00332909.110.2.305

Boothby, J. L., Thorn, B. E., Overduin, L. Y., and Ward, L. C. (2004). Catastrophizing and perceived partner responses to pain. Pain 109, 500-506. doi: 10.1016/j.pain.2004.02.030

Brock, R. L., O’Hara, M. W., Hart, K. J., McCabe, J. E., Williamson, J. A., Laplante, D. P., et al. (2014). Partner support and maternal depression in the context of the Iowa floods. J. Fam. Psychol. 28, 832-843. doi: 10.1037/fam0000027

Burns, J. W., Peterson, K. M., Smith, D. A., Keefe, F. J., Porter, L. S., Schuster, E., et al. (2013). Temporal associations between spouse criticism/hostility and pain among patients with chronic pain: a within-couple daily diary study. Pain 154, 2715-2721. doi: 10.1016/j.pain.2013.07.053

Cano, A. (2004). Pain catastrophizing and social support in married individuals with chronic pain: the moderating role of pain duration. Pain 110, 656-664. doi: 10.1016/j.pain.2004.05.004

Cano, A., Barterian, J. A., and Heller, J. B. (2008). Empathic and nonempathic interaction in chronic pain couples. Clin. J. Pain 24, 678-684. doi: 10.1097/AJP. ob013e31816753d 8 
Cano, A., and Williams, A. C. (2010). Social interaction in pain: reinforcing pain behaviors or building intimacy? Pain 149, 9-11. doi: 10.1016/j.pain.2009.10.010

Collins, N., and Feeney, B. (2000). A safe haven: an attachment theory perspective on support seeking and caregiving in intimate relationships. J. Pers. Soc. Psychol. 78, 1053-1073. doi: 10.1037/0022-3514.78.6.1053

DeLongis, A., Capreol, M., Holtzman, S., O’Brien, T., and Campbell, J. (2004). Social support and social strain among husbands and wives: a multilevel analysis. J. Fam. Psychol. 18, 470-479. doi: 10.1037/0893-3200.18.3.470

DeLongis, A., Holtzman, S., Puterman, E., and Lam, M. (2010). "Spousal support and dyadic coping in times of stress," in Social Support Processes in Intimate Relationships, eds J. Davila and K. Sullivan (New York, NY: Oxford Press), 151-174.

Derogatis, L. R. (1975). The Affects Balance Scale (ABS). Baltimore, MD: Clinical Psychometric Research.

Fekete, E. M., Stephens, M. P., Mickelson, K. D., and Druley, J. A. (2007). Couples' support provision during illness: the role of perceived emotional responsiveness. Fam. Syst. Health 25, 204-217. doi: 10.1037/1090-7527.25. 2.204

Fillingim, R. B., Doleys, D. M., Edwards, R. R., and Lowery, D. (2003). Spousal responses are differentially associated with clinical variables in women and men with chronic pain. Clin. J. Pain 19, 217-224. doi: 10.1097/00002508-20030700000004

Fisher, J., Nadler, A., and Whitcher-Alagna, S. (1982). Recipient reactions to aid. Psychol. Bull. 91, 27-54. doi: 10.1037//0033-2909.91.1.27

Flor, H., Turk, D., and Rudy, T. (1989). Relationship of pain impact and significant other reinforcement of pain behaviors: the mediating role of gender, marital status and marital satisfaction. Pain 38, 45-50. doi: 10.1016/0304-3959(89) 90071-7

Fordyce, W. E. (1976). Behavioral Methods for Chronic Pain and Illness. Maryland Heights, MO: Mosby.

Gleason, M., Iida, M., Shrout, P., and Bolger, N. (2008). Receiving support as a mixed blessing: evidence for dual effects of support on psychological outcomes. J. Pers. Soc. Psychol. 94, 824-838. doi: 10.1037/0022-3514.94. 5.824

Hemphill, R. C., Martire, L. M., Polenick, C. A., and Parris Stephens, M. (2016). Spouse confidence and physical function among adults with osteoarthritis: the mediating role of spouse responses to pain. Health Psychol. 35, 1059-1068. doi: $10.1037 /$ hea0000383

Holtzman, S., and DeLongis, A. (2007). One day at a time: the impact of daily satisfaction with spouse responses on pain, negative affect and catastrophizing among individuals with rheumatoid arthritis. Pain 131, 202-213. doi: 10.1016/ j.pain.2007.04.005

Jang, Y., Haley, W. E., Mortimer, J. A., and Small, B. J. (2003). Moderating effects of psychosocial attributes on the association between risk factors and disability in later life. Aging Ment. Health 7, 163-170. doi: 10.1080/136078603100010 1111

Jensen, M. P., Karoly, P., and Braver, S. (1986). The measurement of clinical pain intensity: a comparison of six methods. Pain 27, 117-126. doi: 10.1016/03043959(86)90228-9

Keefe, F. J., Lipkus, I., Lefebvre, J. C., Hurwitz, H., Clipp, E., Smith, J., et al. (2003). The social context of gastrointestinal cancer pain: a preliminary study examining the relation of patient pain catastrophizing to patient perceptions of social support and caregiver stress. Pain 103, 151-157. doi: 10.1016/S03043959(02)00447-5

Kerns, R., Haythornthwaite, J., Southwick, S., and Giller, E. (1990). The role of marital interaction in chronic pain and depressive symptom severity. J. Psychosom. Res. 34, 401-408. doi: 10.1016/0022-3999(90)90063-a

Kuznetsova, A., Brockhoff, P. B., and Christensen, R. H. B. (2017). lmerTest package: tests in linear mixed effects models. J. Statist. Softw. 82, 1-26. doi: 10.18637/jss.v082.i13

Lam, M., Lehman, A. J., Puterman, E., and DeLongis, A. (2009). Spouse depression and disease course among persons with rheumatoid arthritis. Arthritis Rheum. 61, 1011-1017. doi: 10.1002/art.24510

Laurenceau, J.-P., Feldman Barrett, L., and Rovine, M. J. (2005). The interpersonal process model of intimacy in marriage: a daily-diary and multilevel modeling approach. J. Fam. Psychol. 19, 314-323. doi: 10.1037/0893-3200.19. 2.314
Leary, M. R. (2012). "Sociometer theory," in Handbook of Theories of Social Psychology, eds P. A. M. Van Lange, A. W. Kruglanski, and E. T. Higgins (Thousand Oaks, CA: SAGE Publications Ltd.).

McCracken, L. M. (2005). Social context and acceptance of chronic pain: the role of solicitous and punishing responses. Pain 113, 155-159. doi: 10.1016/j.pain. 2004.10.004

Nadler, A. (1987). Determinants of help seeking behavior: the effects of helper's similarity, task centrality and recipient's self-esteem. Eur. J. Soc. Psychol. 17, 57-67. doi: 10.1002/ejsp.2420170106

Nadler, A., Fisher, J., and Itzhak, S. (1983). With a little help from my friend: effect of single or multiple act aid as a function of donor and task characteristics. J. Pers. Soc. Psychol. 44, 310-321. doi: 10.1037//0022-3514.44.2.310

Neff, L. A., and Karney, B. R. (2005). Gender differences in social support: a question of skill or responsiveness? J. Pers. Soc. Psychol. 88, 79-90. doi: 10.1037/ 0022-3514.88.1.79

Newton-John, T. (2002). Solicitousness and chronic pain: a critical review. Pain Rev. 9, 7-27. doi: 10.1191/0968130202pr186ra

Paice, J. A., and Cohen, F. K. (1997). Validity of a verbally administered numeric rating scale to measure cancer pain intensity. Cancer Nurs. 20, 88-93. doi: 10.1097/00002820-199704000-00002

Pasch, L., and Bradbury, T. (1998). Social support, conflict, and the development of marital dysfunction. J. Consult. Clin. Psychol. 66, 219-230. doi: 10.1037//0022006x.66.2.219

Pow, J., and DeLongis, A. (2018). "The puzzle of support mobilization and wellbeing: potential mechanisms and methodological considerations," in When "We" are Stressed: A Dyadic Approach to Coping with Stressful Events, eds S. Donato, A. Bertoni, and S. Molgora (New York, NY: Nova Science Publishers).

Public Health Agency of Canada [PHAC] (2010). Life with Arthritis in Canada: A Personal and Public Health Challenge. Available at: http://www.phac-aspc.gc.ca/ cd-mc/arthritis-arthrite/lwaic-vaaac-10/pdf/arthritis-2010-eng.pdf

R Core Team (2017). R: A Language and Environment for Statistical Computing. Vienna: R Foundation for Statistical Computing.

Reis, H., and Shaver, P. (1988). "Intimacy as an interpersonal process," in Handbook of Personal Relationships, ed. S. Duck (Chichester: Wiley), 367-389.

Revelle, W. (2017). Psych: Procedures for Personality and Psychological Research, $R$ package version 1.7.3. Available at: https:/CRAN.R-project.org/package=psych

Revenson, T. A., and DeLongis, A. (2010). "Couples coping with chronic illness," in Oxford Handbook of Coping and Health, ed. S. Folkman (New York, NY: Oxford Press), 101-123.

Romano, J. M., Jensen, M. P., Turner, J. A., Good, A. B., and Hops, H. (2000). Chronic pain-partner interactions: further support for a behavioral model of chronic pain. Behav. Ther. 31, 415-440. doi: 10.1016/S0005-7894(00)80023-4

Romano, J. M., Turner, J. A., Jensen, M. P., Friedman, L. S., Bulcroft, R. A., Hops, H., et al. (1995). Chronic pain patient-spouse behavioral interactions predict patient disability. Pain 63, 353-360. doi: 10.1016/0304-3959(95) 00062-3

Rosen, N. O., Bergeron, S., Sadikaj, G., and Delisle, I. (2015). Daily associations among male partner responses, pain during intercourse, and anxiety in women with vulvodynia and their partners. J. Pain 16, 1312-1320. doi: 10.1016/j.jpain. 2015.09.003

Rosen, N. O., Bergeron, S., Sadikaj, G., Glowacka, M., Delisle, I., and Baxter, M. (2014). Impact of male partner responses on sexual function in women with vulvodynia and their partners: a dyadic daily experience study. Health Psychol. 33, 823-831. doi: 10.1037/a0034550

Sarkar, D. (2008). Lattice: Multivariate Data Visualization. New York, NY: Springer. doi: 10.1007/978-0-387-75969-2

Schulz, U., and Schwarzer, R. (2000). Soziale Unterstützung bei der Krankheitsbewältigung. Die Berliner Social Support Skalen (BSSS) [Social support in coping with illness: the Berlin Social Support Scales (BSSS). Diagnostica 49, 73-82. doi: 10.1026//0012-1924.49.2.73

Selcuk, E., Gunaydin, G., Ong, A. D., and Almeida, D. M. (2015). Does partner responsiveness predict hedonic and eudaimonic well-being? A 10-year longitudinal study. J. Marr. Fam. 78, 311-325. doi: 10.1111/jomf.12272

Shrout, P. E., Herman, C., and Bolger, N. (2006). The costs and benefits of practical and emotional support on adjustment: a daily diary study of couples experiencing acute stress. Pers. Relationsh. 13, 115-134. doi: 10.1111/j.14756811.2006.00108.x 
Slatcher, R. B., Selcuk, E., and Ong, A. D. (2015). Perceived partner responsiveness predicts diurnal cortisol profiles 10 years later. Psychol. Sci. 26, 972-982. doi: $10.1177 / 0956797615575022$

Smith, C. A., and Wallston, L. A. (1992). Adaptation in patients with chronic rheumatoid arthritis: application of a general model. Health Psychol. 11, 151162. doi: 10.1037/0278-6133.11.3.151

Song, S., Graham-Engeland, J. E., Mogle, J., and Martire, L. M. (2015). The effects of daily mood and couple interactions on the sleep quality of older adults with chronic pain. J. Behav. Med. 38, 944-955. doi: 10.1007/s10865-015-9651-4

Stephenson, E., DeLongis, A., Esdaile, J. M., and Lehman, A. J. (2014). Depressive symptoms and rheumatoid arthritis: spouse empathic responding as a buffer. Arthritis Care Res. 66, 532-541. doi: 10.1002/acr.22161

Sullivan, K., Pasch, L., Johnson, M., and Bradbury, T. (2010). Social support, problem solving, and the longitudinal course of newlywed marriage. J. Pers. Soc. Psychol. 98, 631-644. doi: 10.1037/a0017578

Turk, D. C., Kerns, R. D., and Rosenberg, R. (1992). Effects of marital interaction on chronic pain and disability: examining the down side of social support. Rehabil. Psychol. 37, 259-274. doi: 10.1037/h0079108

Weinberger, M., Tierney, W. M., Booher, P., and Hiner, S. L. (1990). Social support, stress and functional status in patients with osteoarthritis. Soc. Sci. Med. 30, 503-508. doi: 10.1016/0277-9536(90)90353-t

Wickham, H. (2009). Ggplot2: Elegant Graphics for Data Analysis. New York, NY: Springer-Verlag. doi: 10.1007/978-0-387-98141-3
Wickham, H. (2017). tidyverse: Easily Install and Load the 'tidyverse'. R package version 1.2.1. Available at: https://CRAN.R-project.org/package=tidyverse

Wickham, H., and Miller, E. (2017). haven: Import and Export 'SPSS', 'Stata', and 'SAS' files. $R$ package version 1.1.0. Available at: https:/CRAN.R-project.org/ package $=$ haven

Wilkie, D., Lovejoy, N., Dodd, M., and Tesler, M. (1990). Cancer pain intensity measurement: concurrent validity of three tools-finger dynamometer, pain intensity number scale, visual analogue scale. Hospice J. 6, 1-13. doi: 10.1300/ J011v06n01_01

Woolf, A. D., and Pfleger, B. (2003). Burden of major musculoskeletal conditions. Bull. World Health Organ. 81, 646-656.

Conflict of Interest Statement: The authors declare that the research was conducted in the absence of any commercial or financial relationships that could be construed as a potential conflict of interest.

Copyright (c) 2018 Pow, Stephenson, Hagedoorn and DeLongis. This is an open-access article distributed under the terms of the Creative Commons Attribution License (CC BY). The use, distribution or reproduction in other forums is permitted, provided the original author(s) and the copyright owner(s) are credited and that the original publication in this journal is cited, in accordance with accepted academic practice. No use, distribution or reproduction is permitted which does not comply with these terms. 


\section{Communal Coping in Couples With Health Problems}

\begin{abstract}
Kelly E. Rentscher*
Cousins Center for Psychoneuroimmunology, Semel Institute for Neuroscience and Human Behavior, University of California, Los Angeles, Los Angeles, CA, United States
\end{abstract}

Prior to the 1990s, the predominant view of stress and coping defined stress as occurring when an individual perceives a situation as a challenge, threat, or loss and evaluates her capacity to respond based on her available resources. As an expansion of this intrapersonal perspective, the last 20 years have seen the emergence of two prominent interpersonal perspectives on stress and coping that account for the importance of social relationships in the coping process: the Systemic Transactional Model (STM) of dyadic coping and communal coping. In this article, I outline these two perspectives and highlight their points of convergence and divergence. I propose that one difference between the models is that communal coping involves an explicit focus on a communal or shared appraisal process, in which relationship partners view a problem or stressor as "ours" rather than "yours" or "mine." I review existing methods for assessing communal coping (e.g., self-report, language use, behavioral observation) across laboratory, intervention, and real-world settings and summarize empirical evidence for the prognostic significance of communal coping for relationship and health functioning. I propose the utility of incorporating measurement of shared appraisal into future research on dyadic coping with stress, because of its potential to impact health through its influence on primary and secondary stress appraisal processes and physiological stress response systems. Finally, I outline biological and behavioral pathways through which communal coping may influence health as directions for future research.

Keywords: stress, coping, close relationships, couples, physical health, chronic illness

\section{INTRODUCTION}

Prior to the 1990s, the predominant view of stress and coping defined stress as occurring when an individual perceives a situation or an event as harmful or threatening by exceeding her available resources to address it. In their transactional theory of stress and coping, Lazarus and Folkman (1984) outlined a two-step appraisal process in which an individual first perceives a situation as a challenge or threat based on its ambiguity, controllability, and relevance to the self (primary appraisal), and then evaluates her capacity to respond to the situation based on the available resources (secondary appraisal). According to this theory, coping then involves the individual's behavioral, cognitive, and/or social response in an effort to manage, reduce, or tolerate the demands of the situation. Lazarus and colleagues further categorized these coping responses or strategies as problem-focused when they aim to manage some aspect of the problem itself, or emotion-focused when they aim to manage the individual's own emotional 
reaction to the problem. There is now an extensive literature on these appraisal and coping processes, which characterizes adaptive coping in terms of reductions in an individual's psychological distress with great benefit for individual health and well-being. Over the last 20 years, the field has seen an expansion of this intrapersonal perspective on stress and coping, with the emergence of two prominent interpersonal coping perspectives that emphasize the importance of social relationships in stress appraisal and coping processes: the Systemic Transactional Model (STM) of dyadic coping (Bodenmann, 1995, 2005; Bodenmann et al., 2016) and communal coping (Lyons et al., 1998; Helgeson et al., 2018).

The paradigmatic shift toward an interpersonal perspective on stress and coping began in the early 1990s, when Coyne and colleagues conducted a series of studies with male patients who had experienced myocardial infarction and their female spouses. Importantly, the researchers' observations while conducting this research led to findings that the wives' own distress and coping efforts in response to the coronary event were correlated with their husbands' coping responses, psychological adjustment, and health functioning following the event (Coyne and Smith, 1991, 1994; Fiske et al., 1991). Based on their findings and observations, the researchers concluded that myocardial infarction patients (and their spouses) are confronted with and manage health-related stressors in the context of their marital relationships, and that partners' coping efforts also aim to manage and maintain aspects of their relationship during stressful periods. In addition to the established problem-focused and emotion-focused coping strategies, Coyne and Smith (1991) introduced the term relationship-focused coping, which refers to two interpersonal coping processes: (1) active engagement, where partners jointly discuss the situation, inquire about the other person's feelings, and engage in collaborative problem-solving, and (2) protective buffering, where partners conceal their concerns, deny worries, or yield to each other to avoid conflict. These studies were some of the first to highlight the importance of close relationships and the role of marital partners in the coping process-not only as sources of support, but as active participants and collaborators in coping with stress and illness-that paved the way for the emergence of other interpersonal coping perspectives.

\section{The Systemic Transactional Model of Dyadic Coping}

In the mid-1990s, Guy Bodenmann developed the STM of dyadic coping as a direct extension of Lazarus and Folkman's transactional stress theory, in order to describe the processes through which romantic partners cope together with stress in the context of their relationship (Bodenmann, 1995, 2005). On a theoretical level, as reflected in the name, the STM of dyadic coping conceptualizes couples' relationships as social systems in which romantic partners mutually influence each other; and by extension, stressful events affect both partners. On account of this mutual influence, coping with stressful events includes interactive processes that occur between partners in addition to primarily intrapersonal stress appraisal and coping processes. To differentiate these intra- and interpersonal stress appraisal and coping processes, the STM first outlines three types of stress: (1) individual stress, or stress that one partner is able to cope with alone without involving the other partner or asking for assistance, (2) dyadic stress, or individual stress that is unresolved because one partner is unable to successfully cope with it alone (i.e., due to ineffective appraisals, coping efforts, or resources) and the stress becomes relevant for the couple, and (3) genuine dyadic stress, or stress that directly concerns the couple as a unit (e.g., birth of a child, search for an apartment).

With respect to stress appraisal, the STM of dyadic coping extends Lazarus and Folkman's model by proposing that individual partners engage in a primary appraisal process in which they evaluate the significance of a situation for their own well-being, their partner's well-being, and the well-being of the relationship as a unit. In addition to this primary appraisal, the STM includes three additional appraisal processes, in which the individual partners (1) assess the other partner's appraisal of the situation, (2) judge whether the other partner has realized his/her own appraisal, and (3) reevaluate and synthesize their own appraisal with their partner's appraisal. The model further specifies that, after this reevaluation process, if both partners are in agreement a "common" or dyadic appraisal may result. The STM also expands upon Lazarus and Folkman's definition of secondary appraisal by proposing that individual partners evaluate their own coping resources, their partner's resources, and the resources of the relationship as a unit. In addition to this secondary appraisal, the STM includes two additional appraisal processes, in which individual partners (1) evaluate the secondary appraisal of the other partner, and (2) evaluate and synthesize their own appraisal with their partner's appraisal.

Following individual appraisal of the stressful situation, the STM of dyadic coping outlines a stress communication process in which one partner shares his appraisal with the other partner, who interprets the partner's communication and responds with some form of dyadic coping, that could range from taking action to ignoring the communication (Bodenmann, 2005). Importantly, one assumption of the STM is that partners engage in individual efforts as their first attempts at coping, and then engage in dyadic coping when the individual efforts are unsuccessful. With respect to coping responses, the STM adopts Lazarus (1980) and Lazarus and Folkman's (1984) definitions of problem-focused and emotion-focused coping, but further defines dyadic coping as involving all efforts of one or both partners to manage stressful situations that affect one or both partners, in order to restore balance to the individual partners and to the relationship as a unit. The STM outlines three forms of dyadic coping: (1) common coping, where both partners attempt to manage a stressful situation together (e.g., through joint discussion or searching for information, mutual affection, common relaxation activities), (2) supportive coping, where one partner provides assistance to the other partner, and (3) delegated coping, where one partner requests that the other partner manage the stressful situation on account of the partner's competency, resources, or experience. 
In subsequent theory updates, Bodenmann (2005) and Bodenmann et al. (2016) expanded the STM to include both positive and negative forms of these dyadic coping processes, with positive forms comprising emotion- and problem-focused common, emotion- and problem-focused supportive, and delegated coping, and negative forms comprising hostile, ambivalent, and superficial coping. Bodenmann et al. (2016) further elaborated that common dyadic coping is expected to occur when a problem or stressful situation affects both partners, and when the partners perceive that their own personal resources may contribute to the coping process. Recently, research has also examined potential antecedents (e.g., communal goals as motivating factors) and consequences (e.g., increased sense of "we-ness" in the partners) of the dyadic coping process.

\section{Communal Coping}

The paradigmatic shift that occurred in the 1990s also influenced Lyons et al. (1998) to develop an interpersonal coping perspective called communal coping, which emphasized the embeddedness of individuals within social relationships and the importance of interpersonal processes in coping with stressful life events. Based on interpersonal systems theory, the communal coping perspective conceptualizes couples and other social units (e.g., families, communities) as dynamic systems in which any change in one partner naturally affects the other partner, and affects the relationship as a whole. Lyons et al. (1998) argued that, because of the inherent interconnectedness between relationship partners, the distinction between intrapersonal and interpersonal stress appraisal and coping processes becomes superficial, as individual partners are simultaneously influenced by and consider the effects of a situation on their partners and relationships even when they are conceivably physically "alone."

As an expansion of the relationship-focused coping-and active engagement, in particular-the communal coping perspective outlines a two-step appraisal and coping process. Specifically, communal coping occurs when one or both partners in a couple or other social unit (1) view a problem or stressful situation as "ours" (communal appraisal) rather than "yours" or "mine" (individualistic appraisal), (2) communicate about the stressful situation, including the details and meaning of the situation, and (3) engage in collaborative problem solving in which partners share responsibility for addressing the situation (communal action). The communal coping perspective further specifies that communication about the stressful situation may be verbal and/or nonverbal, and coping responses may involve conscious and/or unconscious action. As outlined above, communal coping includes two orthogonal dimensions-appraisal and action-that vary on a continuum from individualistic to communal/collaborative and form a four-quadrant model (Lyons et al., 1998, p. 586). Whereas one might locate the more traditional notion of social support in the lower-right quadrant, where partners work together to address a problem but still primarily view the problem as one person's (individualistic appraisal, communal action), one would locate communal coping in the upper-right quadrant, where partners work together to address a problem and view the problem as shared (communal appraisal, communal action). Communal coping is therefore best distinguished from social support through its shared appraisal process, regardless of whether the problem originated as one partner's problem or whether it produces similar consequences for both partners (Lyons et al., 1998). In addition, the upper left quadrant may best represent situations such as caregiving, in which partners view the problem as shared but one person assumes primary responsibility for addressing it (communal appraisal, individualistic action). For example, a woman whose partner has an illness that makes it difficult to care for himself might view a health-related problem as shared, yet assume primary responsibility for caregiving tasks. Finally, individual coping is located in the lower left quadrant, where partners view the problem as one person's and engage in solo efforts to address it.

In reference to Lazarus and Folkman's (1984) transactional theory of stress and coping, engaging in communal coping is likely to influence primary stress appraisal processes (Rentscher, 2017; Helgeson et al., 2018). Specifically, regardless of whether the problem originated as one person's, the partners appraise the problem as a challenge or threat with relevance to the couple with little distinction between the self and the partner. Communal coping is also likely to influence secondary stress appraisal processes, such that when partners assess their available coping resources they explicitly or implicitly draw on their partner's available resources in addition to their own. This "doubling" of available resources provides greater diversity of resources and a more effective set of coping strategies that may render communal coping more effective at buffering stress than social support, in which the partners' resources may be available if needed but are still provided from one person to the other rather than pooled or shared (Lyons et al., 1998). It is through these primary and secondary appraisal processes that the magnitude of a problem may be reduced and the stress response buffered. Finally, communal coping involves active coping responses characterized by collaborative problem solving and coordinated efforts to reduce the impact of the stressor.

In a recent theory update and review, Helgeson et al. (2018) proposed expanding the communal coping model in several ways based on their work with couples coping with type 2 diabetes. First, the authors proposed that among couples in which one partner has a chronic illness, partners' coping efforts are primarily aimed at improving the health and well-being of the identified patient. Second, whereas Lyons et al. (1998) posited that communal coping occurs when one or both partners view a problem as shared, the authors argued that in this context the benefits of communal coping are strongest when both partners adopt a communal or shared illness appraisal. Third, the authors proposed that supportive behaviors that one partner provides to the other that might otherwise be characterized as (unidirectional) social support may be construed as collaborative actions when partners hold a shared appraisal. Preliminary evidence for this hypothesis comes from a recent study of couples with type 2 diabetes in which shared illness appraisals moderated the association between spousal emotional support and effective patient self-management of the 
illness. Finally, the authors proposed a conceptual model that also outlines potential (1) antecedents of communal coping (e.g., relationship quality, nature of the illness) and (2) mechanisms (e.g., self-efficacy, reduced stress appraisals) through which communal coping may influence chronic illness adjustment.

\section{Comparison of Interpersonal Coping Perspectives}

The STM of dyadic coping and communal coping have several important points of convergence and divergence. With respect to points of convergence, both models (1) adopt a systemic theoretical approach and emphasize interdependence between relationship partners, (2) identify relationship quality or satisfaction as an antecedent of interpersonal coping, (3) include a dyadic form of stress appraisal in which one or both partners view a problem or stressful situation as relevant to the relationship, and (4) describe a collaborative coping response in which partners discuss the problem and engage in coping efforts together. Of the two models, the communal coping perspective is narrower in its scope. Although the model also describes individual, caretaking, and social support forms of coping, it defines communal coping as a specific process in which one or both partners view a problem as shared and engage in collaborative problem solving to address it. In comparison, the STM of dyadic coping is broader and more detailed in its description of a range of stress appraisal and coping responses that may occur within and between partners as the coping process unfolds.

In addition to these similarities, the points of divergence between the models suggest areas for development in future research. The first point concerns the type of stress and outcome of interest that have historically been the focus of each of the respective models. The STM of dyadic coping was originally developed to investigate everyday stressors (e.g., daily hassles) in more normative, non-clinical community samples of couples and the majority of research has focused on the effects of dyadic coping on relationship satisfaction (Bodenmann et al., 2016). Interestingly, more recent research has applied the STM of dyadic coping to health problems such as cancer (Badr et al., 2010; Manne et al., 2014; Regan et al., 2014; Rottmann et al., 2015), type 2 diabetes (Johnson et al., 2013), and chronic obstructive pulmonary disease (Meier et al., 2011). By contrast, although the communal coping perspective was originally developed to investigate a broad array of stressful life events and social units (e.g., couples, families), the majority of research has focused on clinical samples of couples with health problems such as congestive heart failure, type 2 diabetes, and substance use disorders (see subsequent section for review). Moreover, the outcomes of interest in these studies have ranged from chronic illness adjustment, adherence to the medical regimen, health behavior change, and disease course (e.g., symptom severity).

On a theoretical level, a second difference between the models involves the emphasis each model places on shared appraisal as the primary response to stress (Rentscher, 2017; Helgeson et al., 2018). The STM specifies that partners engage in individual coping processes as their first coping attempts and turn to dyadic coping processes if individual efforts are unsuccessful, whereas communal coping emphasizes that partners can engage in shared appraisal or collaborative action at the very initiation of a problem regardless of whether the problem originated as one person's. Although the STM outlined that the partners can arrive at a dyadic appraisal of a problem (which recent papers have also referred to as "we-stress" or "we-disease," depending on the nature of the stressor; Bodenmann et al., 2016), the model does not specify that the partners may adopt a dyadic appraisal from the initiation of an individual or dyadic stressor. In this way, the emphasis on the initial appraisal of a problem as "ours" is a unique aspect of the communal coping perspective; however, it is important to note that the primacy of shared appraisals is a theoretical concept that remains to be investigated empirically.

Emerging neuroscience research based on Social Baseline Theory provides preliminary evidence in support of the primacy of shared appraisals. Social Baseline Theory, developed by James Coan, posits that social contact and relatedness-rather than isolation and aloneness - are the natural or "baseline" conditions of the human brain, and that individuals' proximity to and interaction with others serves to regulate important aspects of the neural response to threat (Beckes and Coan, 2011; Coan and Maresh, 2014; Coan and Sbarra, 2015). To test this idea, Coan et al. (2006) conducted a functional imaging study in which women were exposed to threat of electric shock while holding either their spouse's hand or a stranger's hand. Women holding their spouse's hand showed greater attenuation in activation of brain regions associated with threat responding compared to those holding a stranger's hand, and women with higher marital quality showed larger reductions in the threat-responsive brain regions. In a recent replication and extension of this study, individuals exposed to threat of shock while holding hands with a close other (spouse, dating partner, friend) showed greater attenuations in several threat-responsive brain regions compared to those holding hands with a stranger or being alone in the scanner (Coan et al., 2017). Moreover, individuals reporting greater social support showed larger attenuations when holding hands with a close other. Adopting a similar paradigm, Beckes et al. (2013) assessed individuals' neural activation in response to threat of electric shock themselves or observing a threat of shock to a friend or stranger. Neural activation in response to threat to the self was significantly correlated with threat to a friend in several threat-response regions, but less correlated with threat to a stranger. Furthermore, individuals who reported higher scores on the Inclusion of Other in Self (IOS; Aron et al., 1992) scale, a measure of self-other overlap, showed increased activation for threat to a friend but not to a stranger. Although this study did not involve a romantic partner, findings suggest a blurred distinction between self and close others at the neural level. Together, these studies suggest that relationships marked by interdependence and closeness may influence partners' stress response at the initial appraisal of a stressor, and by extension, that partners may engage in shared appraisal and coping processes as a baseline, or primary response to stress; however, this hypothesis remains to be tested.

Finally, and related to the previous point, a third difference concerns the availability of well-developed measures to assess the stress appraisal and coping processes each of the models propose. With respect to the STM, the Dyadic Coping Inventory 
(DCI; Bodenmann, 2008) has been translated into multiple languages and is widely adopted in research on interpersonal coping. Although the DCI reliably measures the various forms of positive and negative dyadic coping responses, well-developed measures of the stress appraisal processes proposed in the model have not yet been developed. Recent advances in research on communal coping have included newly developed and validated measures for assessing both shared appraisal and collaborative action; however, some of the measures are early in their development and will benefit from additional validation in future studies. In light of recent expansions to the STM of dyadic coping that have described dyadic appraisals as "we-stress" or "we-disease" (adapted from Kayser et al., 2007), the communal coping perspective offers theoretically-consistent and validated methods for assessing shared appraisal processes that may also be of benefit to future research based on the STM and other dyadic coping perspectives.

As a more extensive review of the empirical literature on the STM of dyadic coping goes beyond the scope of this paper (see Falconier et al., 2016, for an detailed review), the remainder of this paper focuses on the contributions of the communal coping perspective to the literature with respect to advances in measurement, associated empirical findings, and an explicit emphasis on shared appraisal processes (in addition to couple collaboration) in the context of coping with health problems.

\section{APPROACHES TO MEASURING COMMUNAL COPING}

\section{Self-Report Measures}

Rohrbaugh et al. (2008) were the first to develop a self-report measure of communal coping in a study of 60 couples in which one partner had congestive heart failure. The measure is comprised of one item related to shared appraisal ("When you think about problems related to your/your partner's heart condition, to what extent do you view those as 'our problem' (shared by you and your spouse equally) or mainly your own problem?"), and one item related to collaborative action ("When a problem related to your/your partner's heart condition arises, to what extent do you and your partner work together to solve it?") that partners rated on a 5-point scale. In this sample, the two items were moderately correlated for both patients and spouses, so the authors averaged them to form a communal coping score for each partner. Patient and spouse communal coping scores were correlated with patients' use of first-person plural pronouns (we-talk; described below), providing some evidence of external validity. Surprisingly, self-reported communal coping did not relate to patient health outcomes in this study. On average, partners reported high levels of communal coping $\left(M_{\text {patients }}=4.1\right.$, $M_{\text {spouses }}=4.6$ out of 5); therefore, associations may have been constrained by a restricted range of scores on the scale.

In a recent study of 123 couples in which one partner had type 2 diabetes, Helgeson et al. (2018) expanded the Rohrbaugh et al. (2008) measure by adding three additional items. The five-item scale is comprised of two items related to shared appraisal (e.g., "When you think about problems related to your diabetes, to what extent do you view this as "our problem" [shared by you and your spouse equally] or mainly your own problem?") and three items related to collaborative action (e.g., "When a problem related to your diabetes arises, how much do you and your spouse work together to solve it?"). The authors averaged the items to form a communal coping score for each partner. Although psychometric information for the scale is not available, partner communal coping scores were significantly associated with relationship well-being, providing some evidence of external validity.

In the same sample of couples coping with diabetes, Helgeson et al. (2018) also developed a daily diary version of the scale that includes one item related to appraisal (e.g., "When you thought about diabetes today, did you view diabetes as "our problem" (shared equally by you and your partner) or mainly your own problem?") and one item related to action (e.g., "How much did you and your spouse work together to take care of diabetes?"). Partners separately completed the two items at the end of each day for 14 consecutive days. The two items were significantly correlated for both patients and spouses, so the authors averaged them to form a daily communal coping score for each partner. Patient and spouse communal coping scores were also significantly correlated on a daily basis (Zajdel et al., 2018). Psychometric information for the scale, including within-person reliability estimates, is not yet available. Finally, in the same sample of couples coping with diabetes, Helgeson et al. (2017) adapted the IOS (Aron et al., 1992) scale to create a single-item measure of communal coping. Whereas the original IOS scale was comprised of seven concentric circles that overlap to various degrees and individual partners select the pair of circles that best represents their relationship (from no overlap to complete overlap), the adapted version of the IOS asked partners to select the pair of circles that best represents how the couple has coped with the diabetes diagnosis (also ranging from no overlap to complete overlap). Patients' scores on this adapted version of the IOS correlated significantly with relationship quality, providing some evidence of external validity in this sample of couples coping with diabetes.

\section{Language Measures}

Rohrbaugh et al. (2008) were the first to investigate couples' first-person plural pronoun use (we-talk) as an unobtrusive, linguistic indicator of communal coping in the context of healthrelated communication. Of note, this study extended a sizeable body of research on we-talk in the context of couple communication as a linguistic marker of relational we-ness, that has been associated with greater positive and fewer negative interaction behaviors, more effective problem solving, and lower physiological activation during conflict (Simmons et al., 2005; Seider et al., 2009; Williams-Baucom et al., 2010). In this study, 60 couples in which one partner had congestive heart failure participated in a conjoint, coping-focused interview. To derive language measures, research assistants observed the video-recorded interviews and prepared verbatim transcripts of patient and spouse speech. Researchers submitted the transcripts to Linguistic Inquiry and Word Count (LIWC; Pennebaker et al., 2015), which extracted first-person plural (we, us, our) and first-person 
singular ( $I, m e, m y$; $I$-talk) pronoun use scores as proportions of each partner's total word count. The authors then created a we/I-ratio score to represent the proportion of total firstperson pronouns that was plural rather than singular. We-talk and $I$-talk scores were significantly inversely correlated for patients and marginally inversely correlated for spouses. Partner we/I-ratio scores were not significantly correlated. Furthermore, we/I-ratios were significantly associated with scores on the two-item communal coping scale for patients and relationship quality for both partners, providing some evidence of external validity.

In a sample of 70 couples coping with diabetes, Helgeson et al. (2017) also investigated partners' first-person plural pronoun use as a proportion of each person's total pronoun (first-, second-, and third-person pronouns) use during separate interviews with patients and spouses about how they had coped with diabetes. Patient and spouse we-talk proportion scores were significantly correlated. Somewhat surprisingly, however, partner we-talk scores were not significantly correlated with scores on the adapted IOS as a measure of communal coping.

\section{Observational Measures}

Helgeson and colleagues were the first to develop a global observational measure of communal coping in a sample of 123 couples in which one partner had type 2 diabetes. Research assistants observed videotaped interaction tasks in which the couples discussed a diabetes-related stressor and rated communal coping behavior for each partner. The measure includes a single, global item with a five-point scale ranging from 1 (low communal coping) to 5 (high communal coping). The measure instructs observers to rate the extent to which the patient or spouse views the current stressor as a joint problem based on a careful review of the whole interaction. The scale demonstrated excellent interrater reliability, with intraclass correlation coefficients (ICCs) ranging from 0.79 to 0.80 . Partner observational communal coping scores were significantly correlated with scores on the five-item communal coping self-report scale and with we-talk during individual coping-focused interviews, providing evidence of external validity. In addition, partner observational communal coping scores were significantly correlated (Van Vleet and Helgeson, 2016; Van Vleet et al., 2018).

Recently, Rentscher and colleagues developed an expanded, four-item observational measure of communal coping designed to capture therapeutic change processes in a study of 56 couples participating in couple-focused interventions for health problems. The measure is comprised of four items that assess the shared appraisal dimension (e.g., "To what degree does the patient/ spouse view the problem as one individual's ("my" or "your") problem or a shared ("our") problem?"), the collaborative action dimension of communal coping, ("To what degree does the patient/spouse deal with the problem by working alone or working together as a team?"), and a third we-ness dimension ("To what degree does the patient/spouse show a sense of independence/separateness or togetherness/we-ness as part of the couple?") designed to measure the extent to which each partner shows a sense of togetherness or interdependence as part of the couple. Trained raters observed the video-recorded therapy sessions and rated each partner in 1-min micro-segments using a nine-point bipolar scale ranging from -4 (e.g., individual problem) to +4 (e.g., shared problem). The scale demonstrated strong interrater reliability across the 1-min segments (ICCs ranged from 0.54 to 0.82 ) and excellent internal consistency (Chronbach $\alpha$ s ranged from 0.93 to 0.95 ); therefore, the four items were averaged across raters and items to form a single observational communal coping score for each partner. Partner observational communal coping scores were significantly correlated with we-talk during the therapy sessions, providing evidence of external validity. In addition, partner observational communal coping scores were significantly correlated (Rentscher, 2017; Rentscher et al., 2017, 2018).

\section{SUMMARY OF EMPIRICAL FINDINGS ON COMMUNAL COPING}

\section{Self-Report Findings}

In a sample of 60 couples in which one partner had congestive heart failure, partner scores on the two-item communal coping scale were significantly associated with relationship quality (Rohrbaugh et al., 2008); however, they did not predict changes in patient heart failure symptoms or general health functioning over a 6-month follow-up period. In a sample of 123 couples coping with diabetes, Van Vleet and Helgeson (2016) investigated associations between the five-item communal coping scale and relationship functioning within an actor-partner interdependence model (APIM). Results revealed significant actor effects, suggesting that one's own communal coping was associated with one's own reported relationship well-being and more positive perceptions of one's partner. In a separate analysis with this sample, patient reports of communal coping were also significantly associated with diabetes self-care (e.g., diet, exercise, medication adherence; Helgeson et al., 2018). The daily diary reports of communal coping were also investigated in an APIM framework, with a significant actor effect suggesting that higher levels of one's own communal coping on a given day was associated with lower depressed and angry mood and higher happy mood that day (Zajdel et al., 2018). In addition, a significant partner effect suggested that higher levels of communal coping from one's spouse on a given day is associated with one's own happy mood that day. Finally, in a smaller sample of 70 couples coping with diabetes, patient scores on the adapted IOS were positively correlated with relationship quality, and partner scores were negatively correlated with spousal psychological distress (Helgeson et al., 2017).

\section{Language Findings}

In a study of 60 couples in which one partner had congestive heart failure, patient and spouse we/I-ratio scores derived from the coping-focused interview were significantly associated with relationship quality (Rohrbaugh et al., 2008). Interestingly, spouse we/I-ratio scores predicted patient relationship quality over and above patients' own we/I-ratio scores, and vice versa. Somewhat surprisingly, patient and spouse pronoun use was 
not associated with measures of patient health status at baseline; however, spouse we-talk scores predicted positive changes in patients' heart failure symptoms and general health functioning over a subsequent 6-month follow-up period. Follow-up analyses revealed that active we-talk (we vs. us/our) accounted for the association with heart failure symptom course. In a study of 75 couples in which one partner had breast cancer, spouse we-talk during a family coping-focused interview was significantly associated with marital quality and fewer patient depressive symptoms (Robbins et al., 2013). Finally, in a recent study of 70 couples coping with diabetes, spouse we-talk was associated with less patient psychological distress and greater diabetes self-care (e.g., diet, exercise, medication adherence; Helgeson et al., 2017).

In the first study of couples' pronoun use during a clinical intervention, 20 couples in which one partner continued to smoke despite having heart or lung disease participated in a couple-focused smoking intervention (Rohrbaugh et al., 2012). Researchers derived pronoun measures from a pretreatment couple interaction task, as well as three 5-min segments sampled from two subsequent therapy sessions that were combined into one we-talk score for each partner. In this sample, we-talk over the course of the intervention was associated with relationship quality for patients but not spouses. In addition, spouse we-talk during the baseline interaction and increases in we-talk by both patients and spouses from pretreatment through the intervention predicted patients' smoking cessation success 1 year following treatment. In a combined study of four clinical trials, 188 couples in which one person had an alcohol use disorder participated in a couple-based behavioral intervention for alcohol use (Hallgren and McCrady, 2016). Patient we-talk during the first therapy session predicted the percentage of abstinence days later in the treatment, and spouse we-talk during the first session predicted patients' percentage of abstinence days 6 months following treatment. In another study, 33 couples in which one person had an alcohol use disorder participated in couple-focused interventions for alcohol use (Rentscher et al., 2015). Researchers derived pronoun measures from a pretreatment interaction task in which couples discussed the alcohol problem, as well as three 5-min segments sampled during three subsequent therapy sessions that were combined into one we-talk score for each partner. Spouse wetalk during the intervention (accounting for pretreatment wetalk) uniquely predicted successful treatment outcomes, especially when distinguishing active from passive (we vs. us/our) pronoun forms.

Interestingly, several of these studies report asymmetric (i.e., partner) effects, whereby spousal we-talk predicts patient health outcomes over and above the patient's own pronoun use (Rohrbaugh et al., 2008, 2012; Robbins et al., 2013; Rentscher et al., 2015). Considering that each of these studies involved couples in which one partner was the identified patient, changes in communal coping by the spouse (e.g., viewing the patient's health problem as their own, engaging in problem solving with the patient) may be particularly important in this context and therefore more predictive of outcomes than the patient's own communal coping. It is important to note, however, that although most of the existing research has found positive effects of spousal communal coping on patient health, one study found that asymmetric patterns in couple we/I-ratios, characterized by more we-talk relative to $I$-talk by the spouse than the patient, was associated with problematic demand-withdraw interaction patterns, suggesting a potential boundary condition of adaptive communal copingat least when partners are discrepant in their approach to (communal) coping (Rentscher et al., 2013).

Finally, in a combined sample of 56 couples participating in couple-focused interventions for health problems, Rentscher et al. $(2017,2018)$ aimed to investigate communal coping within an experimental medicine framework. Researchers derived pronoun measures from a single target session in which changes in communal coping were expected to occur. Both patients and spouses showed within-session increases in we-talk and deceases in $I$-talk following therapist implementation of a set of therapeutic techniques designed to activate communal coping, providing evidence of construct validity and suggesting successful engagement of communal coping as a therapeutic target in the couple-focused interventions. Although these studies did not directly assess the potential mechanisms that may account for increases in communal coping during the interventions, it is possible that the therapeutic techniques implemented by the therapist strengthened the partners' shared appraisal by reinforcing a sense of cohesion or togetherness and shared identity as a couple. The techniques may have also strengthened collaboration by exploring how the partners worked together to successfully resolve difficulties in the past, increasing the time spent together, and focusing on couple communication about the health problem both during and outside of the therapy sessions (Rentscher, 2017).

\section{Observational Findings}

In a sample of 123 couples in which one partner had type 2 diabetes, observational ratings of patient communal coping behavior were associated with improvements in diabetes selfcare and decreases in diabetes-related distress 6 months later (Van Vleet et al., 2018). In addition, within an APIM, a significant actor effect suggested that one's own communal coping behavior was associated with greater relationship quality (Van Vleet and Helgeson, 2016). In addition, in a combined sample of 56 couples participating in couple-focused interventions for health problems, Rentscher et al. $(2017,2018)$ also found that both partners showed within-session increases in observable communal coping behavior following therapist implementation of a set of techniques designed to activate communal coping, providing additional evidence of construct validity and successful engagement of communal coping as a therapeutic target in the couple-focused interventions.

\section{Summary}

Over the past 10 years, a growing body of research has linked communal coping-particularly by the spouse-to better relationship functioning, adjustment to chronic illness, physical health outcomes, and health behavior change in couples coping 
with health problems. In addition, researchers have developed a variety of measures for assessing communal coping across laboratory, intervention, and real-world settings. Of these measures, behavioral (i.e., language, observational) assessments of communal coping have shown particularly strong potential as they reduce social desirability concerns inherent in selfreport measures, especially for highly evaluative constructs such as communal coping. Indeed, in their recent review, Helgeson et al. (2018) reported that their observational measure of communal coping was the most predictive of psychological and behavioral outcomes in their sample of couples coping with type 2 diabetes. Language measures (e.g., we-talk) also have the advantage of serving as unobtrusive indicators of communal coping that have demonstrated strong predictive validity in couples coping with diverse health problems.

\section{FUTURE DIRECTIONS AND CONCLUSIONS}

In their recent theory update and review, Helgeson et al. (2018) proposed a conceptual model that outlined the process through which communal coping influences adjustment to chronic illness. In this model, patient adjustment is defined to include psychological well-being, self-care behavior (e.g., adherence to the medical regimen), and physical health, and partner adjustment is defined in terms of psychological well-being. As a direction for future research, I propose an expansion of this conceptual model that includes (1) a delineation of the potential biological and behavioral pathways that may link communal coping to health outcomes, (2) a differentiation of the appraisal and action dimensions of communal coping, and (3) an emphasis on relational we-ness as a unique aspect of relationship quality and antecedent of communal coping. These directions for future research are detailed below and summarized in an integrated conceptual model (Figure 1), whereby in the face of stress, a couple's sense of we-ness serves as a relational resource that translates into or activates a process of communal coping (i.e., shared appraisal, collaborative action), which in turn influences biological and behavioral pathways to affect health outcomes.
This proposed conceptual expansion suggests several new directions for future research in this area.

\section{Biobehavioral Pathways Linking Communal Coping to Health Outcomes}

Robles et al.s (2014) meta-analytic review on marital quality and health provides a useful framework for identifying the biological and behavioral pathways through which relational constructs like communal coping might impact health, and clarifying how researchers can conceptualize health outcomes with greater specificity. Broadly, the authors outlined that marital quality (both support and strain) influences several psychological (social-cognitive and affective processes, psychopathology) and behavioral (health behavior) pathways, which then impact biological mediators, surrogate endpoints, and clinical endpoints. The authors defined clinical endpoints as subjective measures of health functioning (e.g., health-related quality of life, physical symptoms, pain severity, functional impairment), objective measures of health status (e.g., occurrence of a heart attack, hospitalization), and mortality. In comparison, surrogate endpoints are biomarkers that predict clinical endpoints but represent earlier events in the disease process, such as cholesterol levels or blood pressure predicting future cardiovascular disease endpoints (e.g., coronary artery disease). Finally, biological mediators are biomarkers that are not surrogate endpoints but represent allostatic and restorative processes in response to short term demands that contribute to longer term health outcomes (Robles and Carroll, 2011). Whereas allostatic processes involve dysregulation in cardiovascular, neuroendocrine (e.g., sympathetic-adrenal-medullary and hypothalamic-pituitaryadrenal axes), and/or immune (e.g., inflammation) systems, restorative processes are complementary and help restore these biological systems to their original state prior to the demand.

In addition to the psychological mediators Helgeson et al. (2018) outlined, I propose expanding the model to include biological (e.g., cardiovascular, neuroendocrine, immune) and behavioral (e.g., health behavior) mediators, as well as surrogate and clinical endpoints based on Robles et al.s (2014) model (Figure 1). To date, the majority of research on communal coping has investigated associations with health behavior and

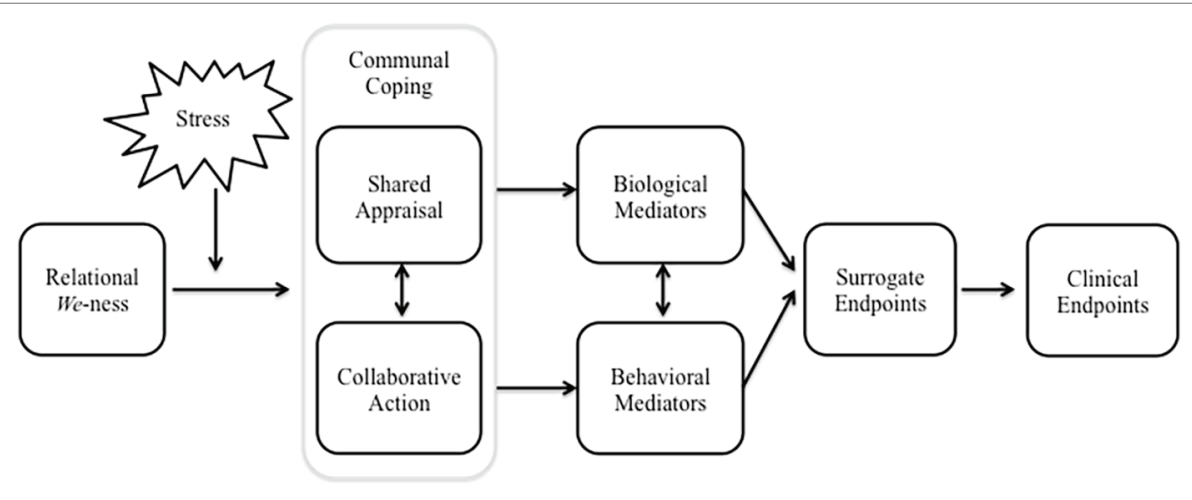

FIGURE 1 | Conceptual model depicting potential pathways through which communal coping may affect health. 
behavior change (e.g., adherence to the medical regimen, alcohol abstinence, smoking cessation) or clinical endpoints (e.g., heart failure symptom course, self-reported physical health), but the biological mechanisms remain untested. In addition, whereas most of the existing literature linking marital quality to biological mediators has focused exclusively on allostatic biological processes, less is known about how aspects of close relationships may influence restorative processes. Moreover, in a recent theoretical paper, Slatcher and Schoebi (2017) call for a greater emphasis on "marital strengths," or the positive aspects of relationships that may have unique effects on these biobehavioral mechanisms and health outcomes (e.g., over and above the more negative aspects of relationships) and serve as a protective buffer of the negative effects of stress on health; an idea that is consistent with the conceptual model proposed here.

\section{Distinguishing the Appraisal and Action Dimensions of Communal Coping}

Previous research has not been able to differentiate the appraisal and action dimensions of communal coping, as the dimensions have been highly correlated when assessed with self-report and observational scales. However, future research may benefit from further investigation in this regard (Helgeson et al., 2018; Rentscher et al., 2018), because although the two dimensions are related, they may have unique relevance to the biological and behavioral processes that may link communal coping to health outcomes.

First, the shared appraisal dimension may have a particular impact on biological mediators, because of its potential to influence primary and secondary stress appraisal processes, and therefore physiological stress response systems. Preliminary evidence in support of this hypothesis comes from the Coan et al. (2006) finding that under threat of shock, women who held their partner's hand showed reduced activation of threatrelated neural regions, and this attenuation was greater for women reporting higher marital satisfaction. Although not tested directly, differences in these neural regions may have "downstream" effects on key allostatic processes such as cardiovascular, neuroendocrine, and immune system activation. Second, in a study of non-clinical couples, Seider et al. (2009) found that we-talk by one's partner during discussion of a relationship conflict was associated with lower cardiovascular arousal for oneself. Finally, in another study of non-clinical couples, Helgeson et al. (2016) found that individual partners who participated in an acute laboratory stressor had lower blood pressure and heart rate and faster physiological recovery when researchers framed the stressor as a shared (i.e., the responsibility of both partners) rather than an individual stressor. Importantly, each of these studies suggests that communal appraisals in the face of threat or challenge may lessen the impact on physiological stress response pathways.

There is also a sizeable literature on social support that suggests that the proposed biological mediators are plausible pathways that may link communal coping to clinical endpoints. In a seminal review, Uchino (2006) summarized a body of epidemiological research establishing reliable links between social support and mortality from cardiovascular disease, cancer, and infectious diseases, and growing evidence that social support may impact morbidity and mortality through cardiovascular, neuroendocrine, and immune pathways. As outlined previously, the primary difference between social support and communal coping is that communal coping involves the presence of a communal or shared appraisal process in which relational partners view a problem or stressful situation interdependently regardless of whether the problem originated as one person's. That is, in the face of stress, social support may involve collaborative action in which partners work together to address a problem, but communal coping occurs when partners also view the problem as shared. Adopting a shared appraisal implies a degree of interdependence in the relationship that is likely to also influence both primary and secondary stress appraisals. Helgeson et al. (2018) further proposed that engaging in shared appraisals may also help partners view supportive behaviors-which are typically unidirectional, with one partner providing support to the other-as a collaborative effort to address the problem, thereby increasing the effectiveness of support behaviors. For these reasons, communal coping has the potential to confer comparable or even additional benefits for physical health through similar pathways as social support; however, this hypothesis remains to be tested in future research.

Second, the collaborative action dimension of communal coping may have a particular influence on behavioral mediators, as partners are likely to engage in health behaviors together and partner collaboration may also serve as a resource for health behavior change (Rohrbaugh, 2014). Preliminary evidence in support of this hypothesis comes from a meta-analysis of relationship factors that contribute to patient adherence to medical treatment. Although the meta-analysis did not directly examine collaboration, DiMatteo (2004) found that medical adherence was 1.7 times higher among patients with greater family cohesion and 1.5 times lower among patients with greater family conflict. In addition, individuals who reported greater closeness with their spouse were more successful in reducing their substance use over the course of individual treatment (Heinz et al., 2009). Finally, in recent studies of couple-focused intervention outlined in this review, increases in communal coping from pretreatment through the course of the intervention predicted successful patient smoking cessation and alcohol abstinence (Rohrbaugh et al., 2012; Rentscher et al., 2015). Together, these studies suggest that collaborative action to address a health problem or stressor may promote health behavior change in couples and serve as a behavioral target for interventions.

Given the potentially unique relevance of shared appraisal and collaborative action dimensions for the biological and behavioral processes that may link communal coping to health outcomes, it will be important for future research to distinguish these dimensions methodologically as well as conceptually. Although current measurement approaches have not be able to disentangle the dimensions, refinement of existing self-report, language, and observational measures may allow researchers to quantify potential differences. For example, self-report and observational instruments might be expanded to test several items for each dimension, and linguistic tools (e.g., machine 
learning) may be developed to detect patterns in the verbs that accompany instances of partner we-talk. For example, shared appraisal might be captured by phrases that include linking verbs such as "we are/seem/feel," whereas collaborative action might be captured by phrases that include action verbs such as "we do/talk/eat." Future research could also incorporate functional imaging to further investigate these dimensions at the neural level and ecological momentary assessment methods (e.g., the Electronically Activated Recorder; Mehl, 2017) to naturalistically observe these processes in daily life, extending investigations of communal coping beyond laboratory and therapy contexts and potentially increasing the range of observed behavior.

\section{Relational We-ness as an Antecedent of Communal Coping}

Buehlman et al. (1992) were the first to conceptualize we-ness (versus separateness) in married couples as the extent to which the partners identified themselves as part of a couple rather than emphasizing their individuality or independence. The authors found that observational ratings of we-ness-which were largely based on the partners' use of we-talk-were associated with greater positive and fewer negative emotional expressions during couple interactions. Since this original study, the majority of research has utilized computerized text analysis to unobtrusively measure partner we-talk as a linguistic marker of we-ness, finding that couples who engage in more we-talk also tend to engage in more positive (e.g., affectionate) and fewer negative (e.g., hostile) behaviors and solve problems more effectively (Simmons et al., 2005; Williams-Baucom et al., 2010). Conceptually, we-ness is very similar to Aron et al.'s (1992) IOS measure of self-other overlap, which consists of a set of two circles that overlap to different degrees. Indeed, one study found that individuals who used more we-talk when describing their relationship reported greater perceived overlap with their partner (Agnew et al., 1998). In addition, individuals who reported greater self-other overlap with a close friend showed a similar neural response when their friend experienced threat as when they themselves experienced threat, suggesting that we-ness with a close other may also be detected at the neural level.

Both interpersonal coping models reviewed in this paper propose some aspect of relationship functioning as an antecedent to dyadic or communal coping processes in couples (Rentscher, 2017; Helgeson et al., 2018). Specifically, Bodenmann (1995) outlined that partners may be motivated to engage in dyadic coping when they are high in marital satisfaction, a sense of togetherness, and/or goals for the future of the relationship. Likewise, Lyons et al. (1998) posited that communal coping is more likely to occur in relationships that are characterized by a high degree of closeness, and Helgeson et al. (2018) proposed relationship quality as one key antecedent to communal coping. To date, several studies have found concurrent associations between relationship quality and communal coping (Rohrbaugh et al., 2008; Robbins et al., 2013; Van Vleet and Helgeson, 2016), and one study found that higher marital satisfaction was associated with greater dyadic coping concurrently and over a five-year period (Bodenmann, 2005). Furthermore, recent studies of communal coping during couple-focused interventions for health problems have found that lower spousal pretreatment relationship distress was associated with greater patient we-talk during the first and mid-treatment therapy sessions (Hallgren and McCrady, 2016) and greater couple we-talk during a pretreatment conflict discussion was associated with larger within-session increases in we-talk among spouses following therapist implementation of techniques that aimed to promote communal coping (Rentscher, 2017). These findings suggest that relational we-ness may prime couples to engage in communal coping more readily in a therapeutic context, or be more sensitive to the effects of a communal coping intervention. In addition, these studies have employed a variety of methods to assess relational we-ness, including self-report (e.g., IOS), language, and observational measures.

In light of this emerging literature, I propose relational weness as a unique aspect of relationship quality and antecedent of communal coping in the context of health-related and other stressors. Specifically, it follows from Lyons et al's conceptualization of communal coping that a high level of we-ness, or sense of togetherness as a couple, is likely to influence partners' appraisals of stressors as shared rather than individual burdens and activate a process of collaborative action to address the problem in ways that other aspects of relationship quality such as satisfaction or intimacy may not. Future research will therefore benefit from empirical investigation of relational we-ness as a key source of relational strength and antecedent of communal and dyadic coping processes.

\section{Conclusions}

The last 20 years have seen the emergence of two prominent interpersonal perspectives on stress and coping that account for the importance of social relationships in the coping process: the STM of dyadic coping and communal coping. This article outlined these two perspectives, highlighting their points of convergence and divergence, and proposing that one difference between the models is that communal coping involves a more explicit focus on a communal or shared appraisal process. Over the last decade, researchers have developed several methods for assessing communal coping, including self-report, language use, and behavioral observation across laboratory, intervention, and real-world settings. This growing body of research has linked communal coping-particularly by the spouse-to better relationship functioning, adjustment to chronic illness, physical health outcomes, and health behavior change in couples coping with health problems. On account of this research, I proposed the utility of incorporating measurement of shared appraisal into future research on dyadic coping with stress, because of its potential to impact health through its influence on primary and secondary stress appraisal processes and physiological stress response systems. As directions for future research, I propose an integrated conceptual model of relational we-ness, communal coping, and health, whereby in the face of stress, relational we-ness translates into a communal approach to coping (i.e., shared appraisal, collaborative action) that influences biological and behavioral pathways to affect health outcomes (Figure 1). This model has the potential to advance research on communal coping process and measurement, improve our 
understanding of the pathways through which communal appraisal and collaboration may affect health, and inform intervention development by identifying couples that may be at increased risk for stress-related health declines (e.g., those low in relational we-ness) and may therefore benefit from targeted interventions.

\section{AUTHOR CONTRIBUTIONS}

$\mathrm{KR}$ is the sole author and contributor to the work in this manuscript.

\section{REFERENCES}

Agnew, C. R., Van Lange, P. A., Rusbult, C. E., and Langston, C. A. (1998). Cognitive interdependence: commitment and the mental representation of close relationships. J. Pers. Soc. Psychol. 74, 939-954. doi: 10.1037//0022-3514.74.4.939

Aron, A., Aron, E. N., and Smollan, D. (1992). Inclusion of other in the self scale and the structure of interpersonal closeness. J. Pers. Soc. Psychol. 63, 596-612. doi: 10.1037/0022-3514.63.4.596

Badr, H., Carmack, C. L., Kashy, D. A., Cristofanilli, M., and Revenson, T. A. (2010). Dyadic coping in metastatic breast cancer. Health Psychol. 29, 169-180. doi: 10.1037/a0018165

Beckes, L., and Coan, J. A. (2011). Social baseline theory: The role of social proximity in emotion and economy of action. Soc. Personal. Psychol. Compass 5, 976-988. doi: 10.1111/j.1751-9004.2011.00400.x

Beckes, L., Coan, J. A., and Hasselmo, K. (2013). Familiarity promotes the blurring of self and other in the neural representation of threat. Soc. Cogn. Affect. Neurosci. 8, 670-677. doi: 10.1093/scan/nss046

Bodenmann, G. (1995). A systemic-transactional conceptualization of stress and coping in couples. Swiss J. Psychol. 54, 34-49.

Bodenmann, G. (2005). "Dyadic coping and its significance for marital functioning" in Couples coping with stress: Emerging perspectives on dyadic coping. eds. T. Revenson, K. Kayser, and G. Bodenmann (Washington, DC: American Psychological Association), 33-50.

Bodenmann, G. (2008). Dyadisches coping inventar (DCI). Test manual [dyadic coping inventory (DCI). Test manual]. Bern, Göttingen: Huber \& Hogrefe.

Bodenmann, G., Randall, A. K., and Falconier, M. K. (2016). "Coping in couples: the systemic transactional model" in Couples coping with stress: A crosscultural perspective. eds. M. K. Falconier, A. K. Randall, and G. Bodenmann (New York: Routledge), 5-22.

Buehlman, K. T., Gottman, J. M., and Katz, L. F. (1992). How a couple views their past predicts their future: predicting divorce from an oral history interview. J. Fam. Psychol. 5, 295-318. doi: 10.1037/0893-3200.5.3-4.295

Coan, J. A., Beckes, L., Gonzalez, M. Z., Maresh, E. L., Brown, C. L., and Hasselmo, K. (2017). Relationship status and perceived support in the social regulation of neural responses to threat. Soc. Cogn. Affect. Neurosci. 12, 1574-1583. doi: 10.1093/scan/nsx091

Coan, J. A., and Maresh, E. L. (2014). "Social baseline theory and the social regulation of emotion" in The handbook of emotion regulation. 2nd edition. ed. J. Gross (New York: The Guilford Press), 221-236.

Coan, J. A., and Sbarra, D. A. (2015). Social baseline theory: the social regulation of risk and effort. Curr. Opin. Psychol. 1, 87-91. doi: 10.1016/j.copsyc.2014.12.021

Coan, J. A., Schaefer, H. S., and Davidson, R. J. (2006). Lending a hand: social regulation of the neural response to threat. Psychol. Sci. 17, 1032-1039. doi: $10.1111 /$ j.1467-9280.2006.01832.x

Coyne, J. C., and Smith, D. A. (1991). Couples coping with a myocardial infarction: a contextual perspective on wives' distress. J. Pers. Soc. Psychol. 61, 404-412. doi: 10.1037/0022-3514.61.3.404

Coyne, J. C., and Smith, D. A. (1994). Couples coping with a myocardial infarction: contextual perspective on patient self-efficacy. J. Fam. Psychol. 8, 43-54. doi: 10.1037/0893-3200.8.1.43

DiMatteo, M. R. (2004). Social support and patient adherence to medical treatment: a meta-analysis. Health Psychol. 23, 207-218. doi: 10.1037/0278-6133.23.2.207

\section{FUNDING}

This research was supported by a fellowship from the UCLA Cousins Center for Psychoneuroimmunology.

\section{ACKNOWLEDGMENTS}

This manuscript includes content that first appeared as the author's dissertation. I would like to thank Matthias Mehl, Ph.D., and Michael Rohrbaugh, Ph.D., for their invaluable feedback on previous drafts of this manuscript.

Falconier, M. K., Randall, A. K., and Bodenmann, G. (2016). Couples coping with stress: A cross-cultural perspective. New York: Routledge.

Fiske, V., Coyne, J. C., and Smith, D. A. (1991). Couples coping with myocardial infarction: an empirical reconsideration of the role of overprotectiveness. J. Fam. Psychol. 5, 4-20. doi: 10.1037/0893-3200.5.1.4

Hallgren, K. A., and McCrady, B. S. (2016). We-language and sustained reductions in drinking in couple-based treatment for alcohol use disorders. Fam. Process 55, 62-78. doi: 10.1111/famp.12150

Heinz, A. J., Wu, J., Witkiewitz, K., Epstein, D. H., and Preston, K. L. (2009). Marriage and relationship closeness as predictors of cocaine and heroin use. Addict. Behav. 34, 258-263. doi: 10.1037/0033-2909.118.1.3

Helgeson, V., Hochstedler, K., and Essien, I. (2016). Effects of manipulated communal coping on dating couples coping with a stressor. Abstract retrieved from International Association for Relationship Research database [Abstract number 344].

Helgeson, V. S., Jakubiak, B., Seltman, H., Hausmann, L., and Korytkowski, M. (2017). Implicit and explicit communal coping in couples with recently diagnosed type 2 diabetes. J. Soc. Pers. Relat. 34, 1099-1121. doi: 10.1177/0265407516669604

Helgeson, V. S., Jakubiak, B., Van Vleet, M., and Zajdel, M. (2018). Communal coping and adjustment to chronic illness: theory update and evidence. Personal. Soc. Psychol. Rev. 22, 170-195. doi: 10.1177/1088868317735767

Johnson, M. D., Anderson, J. R., Walker, A., Wilcox, A., Lewis, V. L., and Robbins, D. C. (2013). Common dyadic coping is indirectly related to dietary and exercise adherence via patient and partner diabetes efficacy. J. Fam. Psychol. 27, 722-730. doi: 10.1037/a0034006

Kayser, K., Watson, L. E., and Andrade, J. T. (2007). Cancer as a "we-disease": examining the process of coping from a relational perspective. Fam. Syst. Health 25, 404-418. doi: 10.1037/1091-7527.25.4.404

Lazarus, R. S. (1980). "The stress and coping paradigm" in Theoretical bases for psychopathology. eds. C. Eisdorfer, D. Cohen, A. Kleinman, and P. Maxim (New York: Spectrum).

Lazarus, R. S., and Folkman's, S. (1984). Stress, appraisal, and coping. New York: Springer.

Lyons, R. F., Mickelson, K. D., Sullivan, M. J. L., and Coyne, J. C. (1998). Coping as a communal process. J. Soc. Pers. Relat. 15, 579-605.

Manne, S. L., Siegel, S., Kashy, D., and Heckman, C. J. (2014). Cancer-specific relationship awareness, relationship communication, and intimacy among couples coping with early-stage breast cancer. J. Soc. Pers. Relat. 31, 314-334. doi: 10.1177/0265407513494950

Meier, C., Bodenmann, G., Mörgeli, H., and Jenewein, J. (2011). Dyadic coping, quality of life, and psychological distress among chronic obstructive pulmonary disease patients and their partners. Int. J. Chron. Obstruct. Pulmon. Dis. 6, 583-596. doi: 10.2147/COPD.S24508

Mehl, M. R. (2017). The electronically activated recorder (EAR): A method for the naturalistic observation of daily social behavior. Curr. Dir. Psychol. Sci. 26, 184-190. doi: 10.1177/0963721416680611

Pennebaker, J. W., Boyd, R. L., Jordan, K., and Blackburn, K. (2015). The development and psychometric properties of LIWC2015. Austin, TX: University of Texas at Austin.

Regan, T. W., Lambert, S. D., Kelly, B., McElduff, P., Girgis, A., Kayser, K., et al. (2014). Cross-sectional relationships between dyadic coping and anxiety, depression, and relationship satisfaction for patients with prostate cancer and their spouses. Patient Educ. Couns. 96, 120-127. doi: 10.1016/j.pec.2014.04.010 
Rentscher, K. E. (2017). Communal coping as a change process in couple-focused interventions for health problems. dissertation. Tucson (AZ): University of Arizona.

Rentscher, K. E., Rohrbaugh, M. J., and Mehl, M. R. (2017). Partner communal coping in the context of couple-focused interventions for health problems. Ann. Behav. Med. 51, S707-S708.

Rentscher, K. E., Rohrbaugh, M. J., and Mehl, M. R. (2018). Measurement considerations for the assessment of communal coping in a therapy context. Ann. Behav. Med. 52, S442-S442.

Rentscher, K. E., Rohrbaugh, M. J., Shoham, V., and Mehl, M. R. (2013). Asymmetric partner pronoun use and demand-withdraw interaction in couples coping with health problems. J. Fam. Psychol. 27, 691-701. doi: $10.1037 / \mathrm{a} 0034184$

Rentscher, K. E., Soriano, E. C., Rohrbaugh, M. J., Shoham, V., and Mehl, M. R. (2015). Partner pronoun use, communal coping, and abstinence during couple-focused intervention for problematic alcohol use. Fam. Process 56, 348-363. doi: 10.1111/famp.12202

Robbins, M. L., Mehl, M. R., Smith, H. L., and Weihs, K. L. (2013). Linguistic indicators of patient, couple, and family adjustment following breast cancer. Psycho-Oncology 22, 1501-1508. doi: 10.1002/pon.3161

Robles, T. F., and Carroll, J. E. (2011). Restorative biological processes and health. Soc. Personal. Psychol. Compass 5, 518-537. doi: 10.1111/j.1751-9004.2011.00368.x

Robles, T. F., Slatcher, R. B., Trombello, J. M., and McGinn, M. M. (2014). Marital quality and health: a meta-analytic review. Psychol. Bull. 140, 140-187. doi: $10.1037 / \mathrm{a} 0031859$

Rohrbaugh, M. J. (2014). Old wine in new bottles: decanting systemic family process research in the era of evidence-based practice. Fam. Process 53, 434-444. doi: 10.1111/famp.12079

Rohrbaugh, M. J., Mehl, M. R., Shoham, V., Reilly, E. S., and Ewy, G. A. (2008). Prognostic significance of spouse we talk in couples coping with heart failure. J. Consult. Clin. Psychol. 76, 781-789. doi: 10.1037/a0013238

Rohrbaugh, M. J., Shoham, V., Skoyen, J., Jensen, M., and Mehl, M. R. (2012). We-talk, communal coping, and cessation success in a couple-focused intervention for health-compromised smokers. Fam. Process 51, 107-121. doi: 10.1111/j.1545-5300.2012.01388.x

Rottmann, N., Hansen, D. G., Larsen, P. V., Nicolaisen, A., Flyger, H., Johansen, C., et al. (2015). Dyadic coping within couples dealing with breast cancer: a longitudinal, population-based study. Health Psychol. 34, 486-495. doi: $10.1037 /$ hea0000218
Seider, B. H., Hirschberger, G., Nelson, K. L., and Levenson, R. W. (2009). We can work it out: age differences in relational pronouns, physiology, and behavior in marital conflict. Psychol. Aging 24, 604-613. doi: 10.1037/a0016950

Simmons, R. A., Gordon, P. C., and Chambless, D. L. (2005). Pronouns in marital interaction: what do "you" and "I" say about marital health? Psychol. Sci. 16, 932-936. doi: 10.1111/j.1467-9280.2005.01639.x

Slatcher, R. B., and Schoebi, D. (2017). Protective processes underlying the links between marital quality and physical health. Curr. Opin. Psychol. 13, 148-152. doi: 10.1016/j.copsyc.2016.09.002

Uchino, B. N. (2006). Social support and health: a review of physiological processes potentially underlying links to disease outcomes. J. Behav. Med. 29, 377-387. doi: 10.1007/s10865-006-9056-5

Van Vleet, M., and Helgeson, V. S. (2016). The impact of communal coping on relationship well-being and perceptions of diabetes stressors among couples coping with type 2 diabetes. Abstract retrieved from the International Association of Relationship Research database [Abstract number 35].

Van Vleet, M., Helgeson, V. S., Seltman, H. J., Korytkowski, M. T., and Hausmann, L. R. (2018). An examination of the communal coping process in recently diagnosed diabetes. J. Soc. Pers. Relat. doi: $10.1177 / 0265407518761226$

Williams-Baucom, K. J., Atkins, D. C., Sevier, M., Eldridge, K. A., and Christensen, A. (2010). "You" and "I" need to talk about "us": linguistic patterns in marital interactions. Pers. Relat. 17, 41-56. doi: 10.1111/j.1475-6811.2010.01251.x

Zajdel, M., Helgeson, V. S., Seltman, H. J., Korytkowski, M. T., and Hausmann, L. R. (2018). Daily communal coping in couples with type 2 diabetes: links to mood and self-care. Ann. Behav. Med. 52, 228-238. doi: 10.1093/ $\mathrm{abm} / \mathrm{kax} 047$

Conflict of Interest Statement: The author declares that the research was conducted in the absence of any commercial or financial relationships that could be construed as a potential conflict of interest.

Copyright (c) 2019 Rentscher. This is an open-access article distributed under the terms of the Creative Commons Attribution License (CC BY). The use, distribution or reproduction in other forums is permitted, provided the original author(s) and the copyright owner(s) are credited and that the original publication in this journal is cited, in accordance with accepted academic practice. No use, distribution or reproduction is permitted which does not comply with these terms. 


\section{OPEN ACCESS}

Edited by:

Mariana Karin Falconier, Virginia Tech, United States

Reviewed by:

Bea Ehmann,

Hungarian Academy of Sciences,

Hungary

Benjamin W. Kelly,

Nipissing University, Canada

${ }^{*}$ Correspondence:

Viola Sallay

viola.sallay@psy.u-szeged.hu

Specialty section:

This article was submitted to Clinical and Health Psychology,

a section of the journal

Frontiers in Psychology

Received: 19 June 2018 Accepted: 11 February 2019 Published: 28 February 2019

Citation:

Sallay V, Martos T, Chatfield SL and Dúll A (2019) Strategies of Dyadic

Coping and Self-Regulation in the Family Homes of Chronically III

Persons: A Qualitative Research Study Using the Emotional Map of the

Home Interview Method.

Front. Psychol. 10:403.

doi: 10.3389/fpsyg.2019.00403

\section{Strategies of Dyadic Coping and Self-Regulation in the Family Homes of Chronically III Persons: A Qualitative Research Study Using the Emotional Map of the Home Interview Method}

\author{
Viola Sallay ${ }^{1 *}$, Tamás Martos ${ }^{1}$, Sheryl L. Chatfield ${ }^{2}$ and Andrea Dúll ${ }^{3}$ \\ ${ }^{1}$ Institute of Psychology, University of Szeged, Szeged, Hungary, ${ }^{2}$ College of Public Health, Kent State University, Kent, OH, \\ United States, ${ }^{3}$ Institute of Psychology, Eötvös Loránd University, Budapest, Hungary
}

Environmental and emotional self-regulation skills play a critical role in promoting wellbeing of individuals and in encouraging healthy relationships. However, occurrence of chronic illness in one family member complicates routine dyadic coping processes for the couple. Additionally, according to environmental psychologists, selfregulation processes are influenced by individuals' perceptions of their socio-physical environments, and during times of chronic illness, the family home is frequently the primary site of dyadic coping. To date, few researchers have investigated the complex relationship among dyadic coping, the family home, and self-regulation processes in the context of chronic illness. The purpose of this paper is to report the results of qualitative research conducted to explore these relationships by analyzing participants' emotionally significant experiences within the family home. We purposively sampled and conducted in depth semi-structured interviews with 23 adults representing 10 families with one chronically ill adult family member. Representative illnesses included epilepsy (4) and chronic back pain (6). We used the Emotional Map of the Home Interview method (EMHI), an elicitation process in which participants are initially asked to place predefined positive and negative experiences on drawn diagrams of their homes. We analyzed the data through grounded theory coding methods, including open, axial and selective coding. Results of data analysis suggest that the family home operated as a critical socio-physical environment and had a profound impact on environmental and emotional self-regulation as well as on dyadic coping when one partner experienced chronic illness. Key selective codes derived from the data that reflect the variation and nuance within this impact included: "stress communication through the home space," "coping by spatial separation" and "coping by joint striving 
for at-homeness." These results reveal formerly hidden aspects of dyadic coping with chronic illness: the role of environmental cues, represented by the family home in this study, in perceptions of stress; the coordinated use of spatial-environmental contexts to engage the appropriate self-regulatory strategies for coping with illness-related stress. These findings demonstrate the utility of $\mathrm{EMHI}$ as an assessment tool and provide meaningful theoretical and practical information about dyadic coping among couples living with chronic disease.

Keywords: chronic illness, dyadic coping, environmental self-regulation, home, qualitative study, grounded theory, Emotional Map of the Home Interview

\section{INTRODUCTION}

Environmental and emotional self-regulation skills play a critical role in promoting the well-being of individuals and in encouraging healthy relationships (Korpela, 1989). The occurrence of chronic illness in one family member not only presents additional challenge to environmental and emotional self-regulation for all family members, but also complicates routine dyadic coping processes for the couple. The purpose of this paper is to explore the dyadic coping processes in the family home in situations of chronic illness, and the impact of those processes on the couple's relationships. To this end, we followed a constructivist grounded theory methodology (Charmaz, 2014) that allowed us to systematically approach data collection and analysis with the end goal of inspiring the development of a novel theory that is not based on a priori hypotheses and does not aim to confirm existing theoretical frameworks. Although Glaser and Strauss (1967) suggested that authors working to develop grounded theory avoid extensive review of prior literature, it is necessary and desirable to be able to define the constructs of interest, and to ensure that theory building efforts are unique and warranted. This research project was informed by the components of the Systemic Transactional Model of dyadic coping (STM; Bodenmann, 2005) in combination with environmental psychological accounts of coping with chronic illness.

\section{Dyadic Coping and Chronic IIIness}

Chronic illness is a life condition that in many instances also generates chronic stress. This makes constant coping efforts necessary, both for the individual and his or her social network. Appropriate coping with illness requires a series of coordinated behaviors that are either necessary for survival (e.g., medication adherence) or contribute to improvements in health and quality of life (e.g., regular exercise). Moreover, individual efforts are embedded in social bonds. Close relationships are especially affected by the illness; close others are involved in the coping process along with other aspects of illness management. Indeed, much prior research shows that health outcomes depend considerably on the availability and quality of social support and involvement of close relationships in the coping process (Martire and Helgeson, 2017).

The Systemic Transactional Model (Bodenmann, 2005; Falconier et al., 2016) of dyadic coping posits that relational coping with stress occurs in circular chains of perceptions and reactions of the partners to each other's signs of stress and to the resulting actions. The most important elements of the process are stress appraisal and stress communication by one partner, the perception of this (verbal or non-verbal) communication by the other partner, and his or her corresponding coping reactions; by definition these coping efforts may be positive or negative. The cyclical nature of the process entails the perception of these reactions by the stressed partner, which again affects by relieving or amplifying the experienced stress. This circle may be continued until some type of resolution occurs. STM also includes joint efforts of the partners, referred to as common dyadic coping, that are engaged to handle common challenges and illustrate the systemic, mutually interdependent nature of the joint coping efforts in couples.

While STM was originally developed to model coping with daily stress and adversities in couples, it has been increasingly applied to chronic health conditions (Falconier et al., 2016). Prior research on dyadic aspects of coping with chronic illness has focused primarily on three interrelated but distinct themes. The first of these is maintenance of a high quality, functioning relationship in the context of a chronic and often life threatening stressor (i.e., a chronic illness experienced by one partner). Empirical studies have reinforced theoretical assumptions that more frequent partner use of positive and supportive, as opposed to disregarding and negative dyadic coping processes, is associated with better individual mental health (Meier et al., 2011; Regan et al., 2014; Vaske et al., 2015), and relationship functioning (Badr et al., 2010). In a systematic review of 33 articles on dyadic coping in the context of cancer in one partner where relationship functioning was an outcome, pooled results confirmed that open and constructive communication, in conjunction with positive dyadic coping of the partner, was associated with better relationship functioning (Traa et al., 2015). Alternately, dysfunctional communication (e.g., protective buffering, demand-withdraw communication), and patterns of negative dyadic coping of the partner (e.g., hostile behavior and blaming) were associated with lower relationship satisfaction.

The second prevalent theme in prior research is exploration of how dyadic coping strategies may affect both patient's and partner's adjustment to the illness itself, including aspects such as self-management of the illness, treatment related decisions and health behaviors. Previous research suggests that better dyadic coping of the partners was related to better health management 
of the illness by the patients (Helgeson et al., 2017). Interestingly, Helgeson et al. (2017) also studied implicit communal coping, measured as first-person plural pronoun usage during a diabetes discussion, and found that higher implicit communal coping of the partner predicted better self-management in the patient. They concluded that those communal coping efforts of the partners that, on face, appear less obvious might be especially beneficial for the patients.

In other research, the partner's dyadic coping also predicted his or her adjustment, although results were inconsistent. In one study with partners of patients with breast cancer, hostile dyadic coping predicted men's higher detachment and alienation from the illness experiences of the female partners, that is, they felt their partners' illness to be more intrusive (Feldman and Broussard, 2006). In another study focused on CVD patients and their partners directly after a CVD event, Bertoni et al. (2015) found that greater involvement in illness self-management was associated with higher negative coping, e.g., withdrawal, of the partner. These mixed results indicate that coping and adjustment is a complex process (c.f., Berg and Upchurch, 2007) that might have unique challenges and possibilities in certain phases.

The third prevalent theme described in prior dyadic coping in chronic illness research includes studies focused on an underlying quality of the couple's relationship that recent conceptualizations refer to as "We-disease." The term refers to a common appraisal of the illness by both partners as well as inclusion of the illness in the joint concept of the relationship. This view of the illness by the partners as inherently shared responsibility may promote both coordinated efforts and emotional sharing; these are two relational qualities that were found supportive for well-being, recovery and health maintenance in couples (e.g., Coyne et al., 2001). Empirical data support this reasoning by showing that more shared representation of the illness in the relationship is associated with better cooperation, more constructive dyadic coping and better quality of life (Kayser et al., 2007; Berg et al., 2008; Helgeson et al., 2017).

\section{Chronic Illness in the Family Home}

There has been a tendency in developed countries to strengthen the role of home care and to relocate several health services to family homes (Williams, 2002). Examples include provision of support for early mother-child relationship (Olds et al., 2002), especially in disadvantaged families (Fraser et al., 2000), home use of certain diagnostic tools (e.g., in sleep disturbances in children, Nixon and Brouillette, 2002), analgesic for chronic pain in the home (Beyer and Simmons, 2004), and home birth (Bailes and Jackson, 2000).

The home environment in chronic illness is conceptualized as the caring landscape (Williams, 2002) or therapeutic landscape (Dyck et al., 2005). The conception of therapeutic landscape refers back to the transactive nature of the relationship between a natural setting or landscape and the person, where meaning and health generating effects of the landscape are in a mutual transactive relationship with human agency. Williams suggested a holistic health paradigm that is equally concerned with physical, mental, emotional, spiritual, environmental and social factors and encompasses their interactions (c.f., Bell et al., 2018). Moreover, individuals are drawn to places that facilitate "restorative experiences" (Korpela et al., 2001, p. 573) following stress-inducing experiences. Therefore, an individual's home as a sociophysical environment might play several roles in the coping processes with chronic illness and the self-regulation challenges during this process. The home also has potential to greatly influence health in positive or negative ways, depending on the tone of associated emotions and relationship experiences (Manzo, 2003, 2005; Dyck et al., 2005) and the nature of perceived changes in the meaning of home (Donovan and Williams, 2007).

Chronic illness challenges the patient's exiting relationship with the home; this challenge extends to patients' social networks. Research with chronically ill persons repeatedly found they engaged in processes such as restructuring of routines and meanings relating to the home (Dyck et al., 2005; Donovan and Williams, 2007). Simultaneously, individuals with chronic illness make efforts to maintain their pre-illness identity through active management of the home to enable private and social activities. This requires efforts to balance conflicting priorities, for example, maintaining the privacy of home while receiving homecare, or choosing alternately to display or to hide symbols of pre-illness identity and experiences (Mærsk et al., 2018).

Researchers have identified several distinct processes by which individuals cope with the challenge of navigating this changing role of the home that results from chronic illness. Corbin and Strauss (1985) described three types of work, i.e., psychological tasks related to the restructuring of home experiences, and their interplay. These include illness work to handle illness related challenges, everyday life work to handle the tasks of living a manageable life even in the face of adversity, and biographical work for recreating the life narrative. Another process is orchestration, meaning the management of several elements of home care by spousal carers concurrently and with considerable effort and precision (Karasaki et al., 2017). It is important to note that certain aspects of the experience with the home may become especially important and at the same time fragile and vulnerable: self-expression, control, security, and restoration (Downing, 2008).

Tamm (1999) and Öhlén et al. (2014) also assessed changes in the role of the home that result when chronic disease or illness is present. Öhlén et al. (2014) categorized individuals' responses to illness at home into the following: "(i) being safe, (ii) being connected, and (iii) being centered." Tamm was additionally concerned about changes that occur when home treatment accompanies chronic disease, and questioned whether illness at home might not lead to revisualization of the home as something that more closely resembles an institution. Importantly, studies also indicate that the process of restructuring is far from being linear; instead, it is inherently stressful and followed by struggles both at individual (Riley et al., 2001) and relational levels (Downing, 2008; Moore et al., 2013; Årestedt et al., 2016; Karasaki et al., 2017). This stress almost inevitably involves coresidential relatives (Corbin and Strauss, 1985; Donovan and Williams, 2007; Årestedt et al., 2016), although influence of these individuals may

\footnotetext{
${ }^{1}$ http://dx.doi.org/10.3402/qhw.v9.23677
} 
be disproportionately more burdensome for woman caregivers (Allen and Webster, 2001; Piercy, 2007).

In summary, prior research suggests that individual and relational aspects of coping with chronic illness unfold in transaction with the family home as a complex sociophysical environment. Dyadic coping processes as described in the STM (i.e., stress appraisal and stress communication, coping responses and their perception of the partners, and common dyadic coping efforts) are specific and important types of relational coping with chronic illness. Therefore, we may assume that dyadic coping in the family home implicitly or explicitly involves not only the partners but also broader socio-physical environmental aspects. However, while the findings presented above provide helpful theoretical insight, we were unable to identify any prior published examples of research reports in which authors attempted to explain the precise interrelation of chronic illness, dyadic coping and environmental self-regulation processes in the home. This gap in the existing literature supports our exploratory and theory building approach.

\section{The Present Study}

To explore the dyadic coping processes that unfold in the context of the homes of couples living with chronic illness, we applied a constructivist-interpretative research paradigm (Lincoln and Guba, 2000; Ponterotto, 2005, 2010; Ylikoski, 2013) and the grounded theory methodology (Strauss and Corbin, 1998; Charmaz, 2014). The former assumes that interactions and constructions in a social world result in multiple realities that are equally valid, while the latter encourages intensive dialog and intersubjectivity between researcher and interviewees to explore meanings and experiences. A qualitative research strategy that fits into the constructivist-interpretive methodological paradigm has a "contextual" character that makes it especially efficient for exploring complex social phenomena. Specifically, contextual research does not rely on previously selected and defined variables; instead, research encompasses any variable that emerges through the research process.

The research described in this report reflects a focused reanalysis of data originally gathered for the first author's Ph.D. thesis (Sallay, 2014), which generally explored emotional selfregulation among family members when one partner experienced chronic illness. Analyses of these data began to reveal the critical role of dyadic coping in the home environment, which led to development of our research question for this current study: How do families with a chronically ill member use dyadic coping processes in the context of the home environment?

\section{MATERIALS AND METHODS}

The present research was designed to elicit detailed information from interviews facilitated by a visual elicitation method. Participants included adult patients with chronic illness (epilepsy and back pain) and their adult family members living in the same home. We used the "Emotional Map of the Home Interview" protocol (EMHI; Sallay, Martos, Chatfield, Dúll, in preparation), a drawing procedure followed by a semi-structured in-depth individual interview. The governing institutional review board granted approval for the procedure.

\section{Sample}

In order to find answers to the research question and subsequent questions derived from the coding process we purposively sampled 10 persons who had epilepsy or chronic back pain of any type, for at least 1 year, and who resided with at least one adult family member. The final sample consisted of 23 Hungarian adults from 10 families: four families with a person affected by epilepsy, six families with a person affected by chronic back pain. The sample included 13 women and 10 men, aged between 25 and 57, with two families residing in a rural area and the other eight living in a large urban area. See Table $\mathbf{1}$ for additional demographic details.

\section{Data Collection}

The interview guideline followed the EMHI protocol. Sallay (2014) developed the EMHI with the aim of exploring emotional and environmental self-regulation processes in the space of the home. The interview begins with the "anamnesis of the homes," that is, a process of guided recall of previous homes of the person along with the associated emotions. As a second step, we asked subjects to draw a layout of the home indicating functions and important furniture within each room. We included nine emotionally important self-regulation experiences in the interview guideline: (1) security (2) insecurity (3) wellbeing (4) tension (5) healing/change (6) suffering (7) belonging (8) withdrawal (9) illness, and we asked participants to mark the place(es) of these nine emotionally important self-regulation experiences on the layout (e.g., "Where is the place of security for you in your home?"). We also requested participants to add a tenth item that reflected an encompassing symbol of the home (see Figure 1 for an example of the layout with the places of emotionally important experiences marked on it).

We asked for stories relating to every place they marked on the map (e.g., "What stories are associated to the way you experience security in the kitchen?"). The length of the interviews varied between 43 and $82 \mathrm{~min}$. The first author completed all the interviews. Eighteen participants were interviewed in their homes, three participants were interviewed in the researcher's university office and two participants completed interviews in their respective workplaces. All interviews were tape recorded and transcribed verbatim.

Although this research was informed by constructivist grounded theory design (Charmaz, 2014), for practical and resource considerations, we did not fully employ all strategies associated with classic grounded theory methods. We focused on purposive sampling, prioritizing selection of participants who reflected the experience and situations of interest. Throughout the interviews we used the EMHI protocol and we did not extend data collection to the point where additional data analysis fails to yield new information (i.e., saturation). Nevertheless, we incorporated processes of theoretical sampling in our analysis, including reflexivity and iterative comparison between new and existing data (Charmaz, 2014). 
TABLE 1 | Characteristics of the sample.

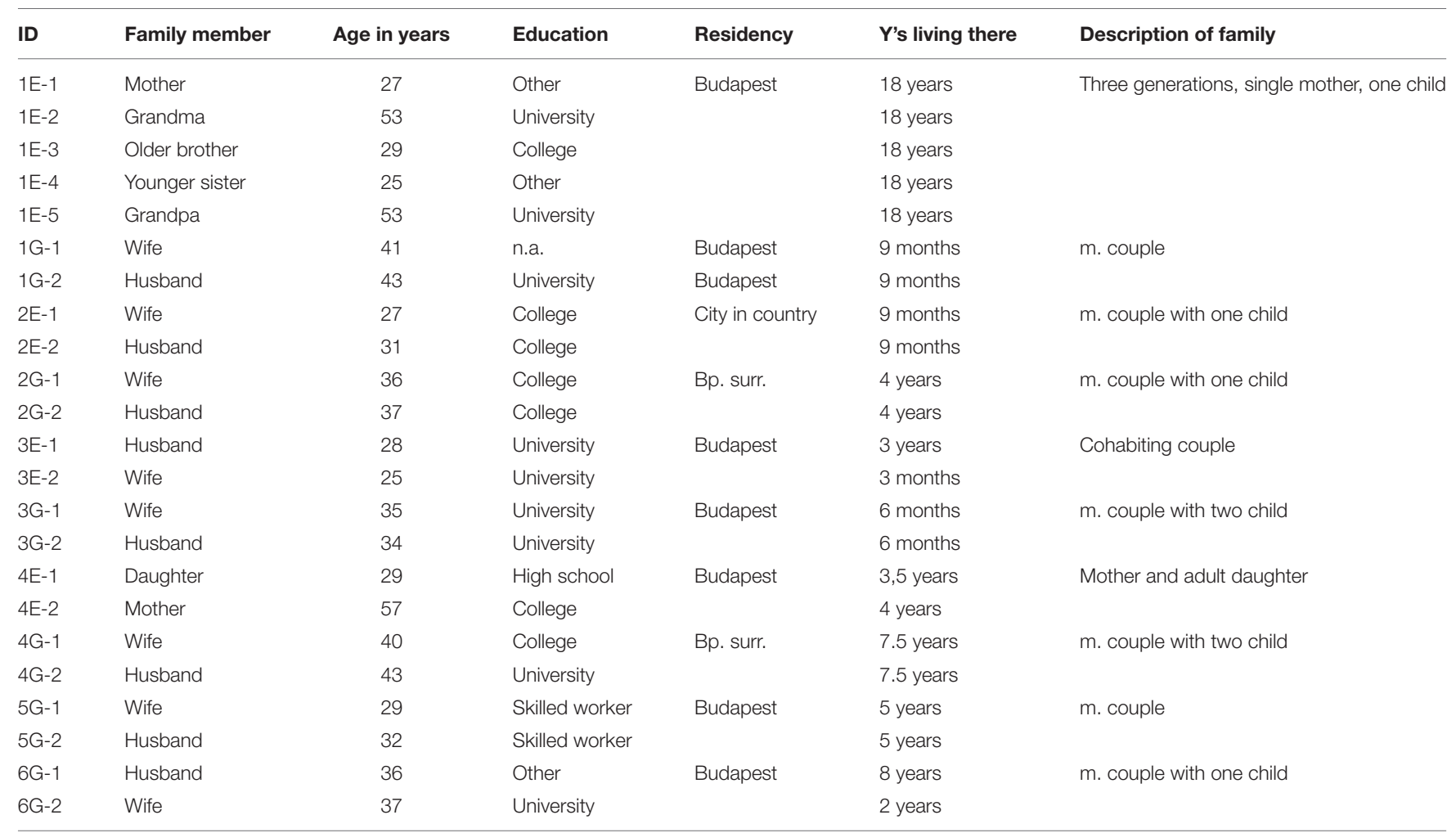

The index patient in the family has the number 1 as last digit (e.g., 3G-1); type of illness is coded as $E$ (epilepsy), or $G$ (chronic back pain); numbering of the family: first digit. fam.member, family member. In Hungarian higher education, college refers to dedicated technical or vocational training, while university education is more focused on sciences and humanities. Bp. surr., Budapest surroundings; m.couple, married couple; n.a., no answer.

Legend

1. Security

2. Insecurity

3. Well-being

4. Tension

5. Healing/change

6. Suffering

7. Belonging

8. Withdrawal

9. Illness

Sz Symbol of the home

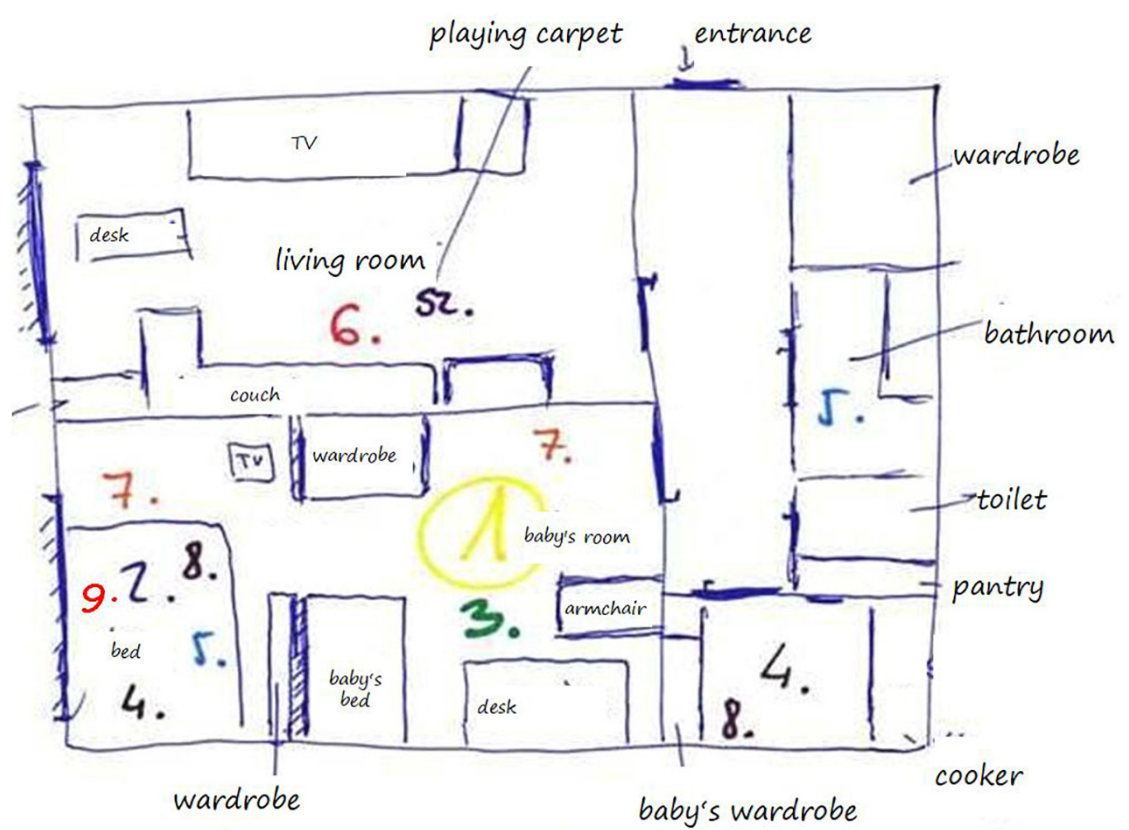

baby's wardrobe

FIGURE 1 | The Emotional Map of the Home: an example. 


\section{Data Analysis}

We followed the principles of grounded theory methodology to analyze the interview transcripts (Strauss and Corbin, 1998; Charmaz, 2014). A hallmark of grounded theory is use of theoretical sampling, which means we concurrently and iteratively engaged in processes of data analysis, comparison of new with existing data, and reflective consideration and reconsideration (Charmaz, 2014). As a result of these processes, concepts that emerged or were identified in analysis directed subsequent assessment as well. We applied a hierarchical, inductive coding process ("open coding") to each meaning unit, and developed analysis toward increased abstraction. In keeping with grounded theory tradition, we considered each line of the transcript in search for meaning units, such as sentences or paragraphs that included information referring to our research question. During the coding phase, we searched for connections, similarities and differences between meanings by means of an explicit constant comparative method (Glaser and Strauss, 1967). Raising and organizing of thematic units was made in a three level hierarchical coding process (open, axial and selective codes; Strauss and Corbin, 1998; Hallberg, 2006; Charmaz, 2014). While progressing from initial open coding to axial coding and then to selective coding, we continued to engage in theoretical sampling processes. For this analysis, theoretical sampling centered on revisiting and reflecting on data and codes, to refine axial and selective codes when categories appeared unclear or incomplete. In grounded theory, research notes are referred to as memos. We used memos in each stage of interaction with data, including the levels of coding, to record and crystallize the processes. We referred back to these memos later in the process to help describe and refine the emerging theoretical concepts. Our coding process eventually resulted in a three level "code tree" that contains the selective, axial and open codes (see Appendix).

\section{RESULTS}

Following, we provide the code tree with the selective and axial codes (see Table 2) and an expanded description of several subparts of the three primary selective codes. We validate these codes with supporting excerpts from participant interviews (translated from Hungarian).

Our analysis revealed that everyday dyadic coping processes were embedded in partners' routine movements within the home space and in the ways they used the home environment. These movements and interactions within the home environment played a prominent role in dyadic interactions, stress communication, and couples' attempts to cope with stress stemming from the symptoms of the chronic illness as well as from other areas of their relationship. In this grounded theory analysis, we elaborated three main categories, or selective codes, which describe (1) processes of stress communication through the home space, (2) dyadic coping by spatial separation, and (3) dyadic coping by joint striving for at-homeness. Just as ways of stress communication are characterized by specific person-environment interaction patterns, the dyadic coping processes also involve more or less conscious, and more or less
TABLE 2 | Code tree.

\begin{tabular}{ll}
\hline Code no. & Selective and axial codes \\
\hline 1. & Stress communication through the home space \\
1.1. & Direct stress communication is lacking - stress appears indirectly \\
through space use \\
1.2. & Open disagreement focuses on use of space \\
1.3. & Differences in partners' priorities for space use cause conflicts \\
2. & Coping by spatial separation \\
2.1. & Conscious distancing in coping with the symptoms \\
2.2. & Distancing in the coping with relationship stress \\
2.3. & Mutually reinforcing processes of distancing \\
3. & Coping by joint striving for at-homeness \\
3.1. & One partner takes care of the other in the absence of stress \\
3.2. & The risk of taking over home duties \\
3.3. & The common use of the home space supports coping with stress \\
3.4. & The joint shaping of the home space \\
\hline
\end{tabular}

ritual ways of creating spatial separation or, inversely, patterns of seeking closeness to each other in the space of the home.

\section{Stress Communication Through the Home Space}

One component of the process of dyadic coping at home is demonstrated by couples' behavioral patterns through which they involve the environment in communicating or in hiding their stressful feelings. In these experiences, stress communication either extended beyond verbal messages and was manifested by movements in space or it became apparent in the partners' discourse about the home space. The application of the EMHI protocol elicited explicit descriptions of stress communication through movements in space. Partners routinely used physical distance and specific places to express their stressful feelings. In addition, physical environment gained also symbolic significance when couples expressed relationship stress through negotiating the rules related the use of the home environment. Three variations of these phenomena emerged: lack of direct stress communication, with stress instead indirectly communicated through use of space; open disagreement focuses on use of space; difference in partners' needs for space use causing conflicts.

\section{Direct Stress Communication Is Lacking - Stress Appears Indirectly Through Space Use (Axial Code 1.1)}

In order to preserve the harmony of family life and to save children from witnessing parents' conflicts, some couples expressed the shared belief that tension cannot appear during the time spent together. In this case, partners had to find ways for dealing with their stressful feelings outside of the other partner's presence in the common home. For example, one wife (a young mother) described how she regularly relieved the tension she felt by praying alone in the living room after her husband had fallen asleep in one of the bedrooms: 
For me, the tension is connected to not sleeping. These two are related, so during the day I can mostly hold back the inner tension, usually it comes out when I either have to sleep or have the possibility to rest. So for me it's pretty much connected to the bedroom and sleeping, because during the day I can mostly hold it back somehow. So it's mostly in the evening when...I don't even have the time, by the way, to deal with this [tension] during the day.

This couple reported spending most evenings separately while dealing with daily stress. Another variation of missing stress communication occurred when chronically ill wives and husbands asserted that there was no suffering in chronic illness and their condition was not an illness but a specific "state." In their case, there was no need to take distance from each other; they managed to hide stress in the presence of their partner.

In contrast, however, some wives expressed the need to communicate their stressful feelings to their partner but believed the partner could not respond to this need, so these conversations were ineffective. A young mother described how she regularly failed to discuss the problems with their flat being too little for the family:

I usually only talk to my mom about these things. And I'm trying with daddy [her husband] too, but, but... sometimes it only makes us fight, if something like this... no... I stopped now a little bit to talk about this moving thing, but... Actually the most important would be that I'm with him. So that's the most important for me... So this idea of where to live, this shouldn't matter, but unfortunately it actually does matter.

Consequently, their shared bedroom became a place of tension for the husband: "Every night when mommy wants to talk, but daddy wants to sleep, and that's where the problems begin at the end of the day." Couples, who experienced difficulties in discussing stressful topics overtly, also described the behavioral pattern of taking turns when using home spaces. For example, some reported preferring to cook alone in the kitchen or staying in the living room when their partner was not there.

\section{Open Disagreement Appears in Use of Space (Axial Code 1.2)}

Several couples experienced that stress communication was regularly expressed in the form of disagreement. Couples, who reported having frequent disagreements on stressful issues, also described the role of the home space in these exchanges. One variation of this was that the partners were regularly using certain spaces of their home in opposite ways, and this frequently led to quarrels. In the following excerpt, a young husband explains how he was fighting with his wife to gain control over the use of the home space:

Well, there is this tension [regarding the closet] because mum [his wife] wishes to keep everything and I want to throw out everything we do not need. It usually leads to problems, but... we can manage them... I decide what I would throw out and therefore... (laughter), and that is it.

In some cases, couples explained that direct quarrels were ritually ended by one partner withdrawing to a distant or separate part of their home. One couple, for example, reported they spent most of their shared time in the living room, except for those regular moments when they entered into a disagreement and the wife withdrew to the bedroom alone. This way the bedroom often became primarily a refuge for the wife, and not a shared retreat.

Disagreements and quarrels also played an important role in the evolution of additional aspects of the relationship. This couple, who reported having regular disagreements while in bed together, also described the failure of their sexual relationship.

\section{Differences in Partners' Priorities for Space Use Cause Conflicts (Axial Code 1.3)}

Under certain circumstances the home environment itself could became a source of chronic stress for couples. For example a couple with young children had to make a consensus decision to choose a new flat, but the partners expressed different needs. Eventually one partner conceded her preferences in the final decision. But after settling in the chosen flat, the unbalanced decision continued to play a role in their everyday conflicts.

How space within the home was used could also lead to emerging accusations that caused long lasting areas of instability in the relationship. The following excerpt illustrates circularity in the flow of dissatisfaction related to space use. While drawing the layout of her home, one wife expressed her dissatisfaction with the physical environment (i.e., there was no space for her belongings after moving in) that also conveyed her dissatisfaction with the relationship as a result of feeling underappreciated:

And this is the living room. Well, these sizes, they really seem rather disproportionate but, well, here is a small wardrobe. Does it matter that my things do not fit? I could provide an excellent drawing of my things that do not fit anywhere. There are certain items that have been in the same box since I moved in many years ago. Therefore, it causes a little... Generally arguments. (...) And there is also a set of wardrobes which, of course, is not mine [it is my husband's], hence I will draw it small. Yes. (...) I have to say it is a bachelor's flat. It was designed as such and has changed a little since I arrived, and I have not been able to make any radical [changes].

Another wife described how control fight for the possession territory led to a ritual of repeated conflicts in their relationship. This wife had a computer desk in one corner of their living room where she could work and the husband had a working room on his own. Still, the husband had the habit of using his wife's computer instead of his own:

But still, the end of this was him always being here [at the wife's working corner]. And I couldn't get to my computer just to check an e-mail or look up for something online, so for me this is like... the feeling was that it compromises my liberty, that I can't do it anytime, while we made the whole thing so that there wouldn't be any problem... And then I got upset, and then he said to me not to be angry, and he would move over, and then, after this, there was something again, which is why he slowly came back to my place. And then now too, for a lot of times, I don't know why, but in the last few days he's there, in his own place. Yes. And he would come over here just a few times, which is okay. But like... the books were in heap like this and I couldn't even 
be there comfortably, so that I'm like claustrophobic too and it was tightened, the possibility to get in between the books. So for me this is the tension, when I saw that he sat down there again, I could feel the anger in me.

As with the prior example, these partners assume asymmetric roles in the debates: the husband clings to his ways of using the home space without addressing his wife, while the wife eventually respond by verbally expressing her stressful feelings resulting from this.

\section{Coping by Spatial Separation}

Coping with stress in the space of the home was strongly linked to variations of couples' spatial separation throughout the interviews. For some couples, spatial separation appeared to result from one or both partners' conscious decisions, and was a strategy used to cope with the ill person's symptoms. In other instances, spatial separation seemed to occur when partners experienced tension in the relationship, and resulted either from a conscious decision or from the couples' everyday movements. In this latter case, separation was not necessarily the explicit goal expressed by the couple, but still it occurred ritually and played an important role in preserving emotional stability in the relationship. In the third pattern of spatial separation, the need for separation stemming from a tense relationship was intertwined with the need for separation resulting from the illness experience.

\section{Conscious Distancing in Coping With the Symptoms (Axial Code 2.1)}

Couples elaborated several ways of finding distance from each other in the home space in order to deal with the ill partner's symptoms. Our analysis revealed that the phenomenon of conscious distancing occurs with varying intensity. In some cases it consisted of the ill partner regularly but briefly retiring alone in one room to alleviate his or her symptoms (for example the wife with chronic back pain taking hot shower while her husband is having dinner with their child). In another case, parents of a young adult living with epilepsy held the belief that paying extra attention to their daughter's symptoms is the wife's duty, so she remained close to their daughter; the mother and daughter slept in the same bedroom and the husband used the other bedroom.

Another wife with chronic back pain explained how sleeping separately from her husband helped her alleviate the symptoms: after sleeping in the shared bedroom:

I limp here down the stairs every morning. Then it [the pain] goes away somehow, when the day begins, but when I get out of bed and I have to go out, that's basically... that's like the death itself until I can walk down the stairs. And it's very interesting too, that we don't live together as husband and wife, we function as father and mother. We have this common agreement and actually I don't sleep here in the night (points to the bedroom), I sleep here in the guestroom with my little son and ever since we began to sleep here, the pain's gone.

In their case, both partners expressed their conscious consent to use separate bedrooms in order to ameliorate the wife's symptoms.
Separation was yet more intense for those couples when husbands stated they had "nothing to do" with coping with their wives' illnesses. In one case, the husband explained that he could not have any impact on the evolution of the wife's chronic back pain. Another husband held the belief that the wife's chronic back pain was rather a "projection of her inner state" than an illness, adding "everything starts in your head." In both cases, according to husbands' perceptions, the wife had the responsibility to manage her illness alone, at any moment during the day and at any given place in their common home.

\section{Distancing in the Coping With Relationship Stress (Axial Code 2.2)}

Coping with relationship conflicts often implied the couple ritually taking a certain distance from each other in the home space. Spatial distancing occurred on different scales from withdrawal inside the shared bedroom to creating an alternative home in another quarter of the city for one of the partners.

One couple gave the name "pouting bed" to a piece of furniture in their bedroom, as an example of a ritual of taking distance:

When we had a row, one of us moved to the pouting bed. It has begun with my wife, and then, she slept there twice, and then I also tried it once. But after an hour or so, and after that, we spoke again. We had the feeling like wanting to go back after an hour, but our pride set us back.

In case of one couple, who were parents of a girl of kindergarten age, the ritual of taking distance appeared to separate them throughout their entire house. This behavior pattern required a high degree of co-ordination between them. They had two bathrooms and two TVs in their two-story family home that they divided between each other; they additionally avoided being together in their bedroom in the evenings while the wife was awake. This is how the wife (living with a chronic back pain) explained it:

To me withdrawal is when my husband puts our child to bed and sometimes I am asleep half an hour later. But I also enjoy going to bed with my laptop to quickly browse the news in approximately $1 \mathrm{~h}$. I do that when I am on my own. Because I don't always want to watch the same TV program as my husband. In such cases, I am upstairs, he is downstairs, which is slightly bad because this way it leads to, how should I put it, separation. $\mathrm{He}$ prefers Spectrum and National, i.e., educational programs, and I may be a complete idiot, but I watch Dallas even these days, and that drives my husband crazy, and asks me if I am out of my mind because I watch Dallas. I keep saying that I watch it only as background entertainment, because I do something else at the same time but I like having approximately $1 \mathrm{~h}$ like this after the great noise has calmed down.

Their alternating use of the home space, which also extended to the bedroom for a part of the night, had evolved partly as the consequence of the husband helping his wife look after their child and do the housework (because of her chronic back pain), and partly as a way to avoid repeatedly facing the problems of intimacy between them. The wife's explanation also reflects some concern about the growing distance between them ("it is slightly bad because this way it leads to, how should I put it, 
separation"). Their separate ways, driven by their choice of TV programs, seem both reflecting their individual needs and their common way of coping with the tension in their relationship. However, this coordinated pattern of using the home space also imposed some threat according to the wife's explanation and was accompanied by the failure of their sexual life as reported by the husband.

Patterns of separation in couples' coping with relationship stress also appeared to be linked to childhood experiences. One young mother used the spatial metaphor of being in a "castle" while speaking of her feelings of security experienced in her baby's room:

I feel best here, in this room. Here can I find myself and everything I wanted the best. In this small, approximately $3 \times 3$ [meters] room. Even as a child, I always liked building a small castle and hiding there. This $3 \times 3$ room was more or less the same as a small castle.

In her fantasy, their baby's room turned into a sound fortress, which would both protect and separate her from her husband whom she resented. This way, her perception of the situation was guided by an image of her home as a child. This perception, in combination with other experiences became the basis of withdrawal from the relationship by retiring into her baby's room.

In the presence of escalating conflicts and relationship crisis, one couple's need for coping by spatial separation was so strong that they decided to rent a separate flat for the wife where she could retreat either with one of their children or alone. The wife explained that she always felt relaxed and secure there and elaborated a special attachment to this place - she called it her "snug" and described how she greeted it every time she arrived there.

\section{Mutually Reinforcing Processes of Distancing (Axial Code 2.3)}

Patterns of separation in coping with the symptoms of a chronic illness appeared to be inseparably intertwined with patterns of coping with relationship stress. For example, a couple living with the wife's chronic back pain described experiencing a growing distance between them in the course of the construction of their family house. Both partners described their respective situation as being abandoned by the other, and each felt they had to assume all responsibility for the works and had been required to engage in physically demanding duties. Their experiences were linked to the wife's symptoms in a way that restrictions of the wife's capacities became a basis for mutual blaming and consequently resulted in a growing need for distance between them.

Coping with the symptoms of the illness also implies having special environmental needs, for example a place for morning exercise or a hard mattress for sleeping. One husband's need for a hard mattress became a source of conflict in the intimate relationship of a young couple living with the husband's chronic back pain. In describing their problems with sexual intimacy, the wife explained:

But in my opinion this [sexual problems] is a thing which causes tension. And I must add that this bed, it's a very hard bed and I never agree on this with my husband. He thinks that hard bed is the good bed, but I think that the not too soft, but still softer than the one he sleeps on, because that's a hard-as-concrete bed, I have to say, I should use this expression. And on that side of the bed where I sleep I have an additional thin sponge too. So I couldn't sleep so much, I wasn't able from the beginning, that. . . we couldn't agree on this either, that what the bed should be like.

In this instance, the home environment intended to support the husband's recovery created a chronic conflict and a physical distance between partners.

\section{Coping by Joint Striving for At-Homeness}

The third component of dyadic coping process in the interviews was patterns of coping by coordinated actions aiming at creating feelings of relational and physical security accompanied by emotional and physical care - what can be called the sense of at-homeness (c.f., Seamon, 2000). Some of the partners' common actions proved to be demanding or burdensome, such as one partner taking care of the other even in the absence of stress communication, or taking over additional duties at home. Other actions of common coping, such as the common use of the home space and joint shaping of the home were associated with more positive feelings like joy, amusement, relaxation and pride.

\section{One Partner Takes Care of the Other in the Absence of Stress Communication (Axial Code 3.1)}

Several healthy partners tended to care for the other even in cases where the ill partners were hiding the stress resulting from their chronic symptoms. In case of a young couple living with the husband's epilepsy, the wife explained that she only felt safe when they were at home, because it was in the home space where she could care for her husband in the moments of his seizures. In contrast, however, in the husband's perception, their home did not have any special significance for his feelings of safety. Another husband, speaking of his wife's seizures, demonstrated greater feelings of stress and concern than his wife who was the one actually experiencing the seizures. This husband was especially worried about his wife's loss of control whereas the wife was more confident in her ability to manage the situations.

In addition to feelings of worry, one husband initiated therapeutic and healing activities for his wife who had chronic back pain but use a coping pattern based on hiding her pain. The wife explained:

For me it was an absolutely normal part of my life, I didn't even bother, it hurts, it has always been hard and now it's getting even harder and it's like part of the life, and well what, others have pain in their leg. And then he [my husband] started this, that it doesn't have to be like this, I don't have to accept this, I should start to go after this, and I should find, so I should see an expert and I should care about it. So like, he pushed it that I have to take a step forward.

The wife accepted the suggestion to see a doctor and the husband went on to initiate the home ritual of the morning therapeutic exercise, for which they get up earlier together. 
The husband was experiencing the positive outcomes of their common coping:

She is used so much to living with this thing that she doesn't say a word normally about her having a problem. This made such a difference, that we wake up earlier in the morning because of her training. . . I don't like to wake up early but I do appreciate it a lot that she wakes up.

The Risk of Taking Over Home Duties (Axial Code 3.2) While most healthy partners tended to take over household duties from the ill partners, paradoxically these actions also involved certain risks for relational or individual well-being. In some cases, the ill partners considered the quantity of duties taken over insufficient or in other cases found the partner's help inefficient. In either instance, partners reported that blaming followed the healthy partner's actions. This is how a wife living with chronic back pain described an everyday scene when she arrives home and finds dirty vessel in the sink: "Well I let the tension out, and then [the husband says], 'it's okay sweetheart, I'm coming', and then really, so it's solved, so no, there isn't any conflict because of this... Okay, I could stop myself from doing the dishes but sometimes not, so I start it and then he says 'but sweetheart, it's my turn' and, well I'm telling him 'you were at home, anyway.' Whatever, forget it." In this case, the offered help from the husband's side and a low-key blaming from the wife's side are present at the same time. The wife appreciates the way of help but is dissatisfied with the timing of the offered help.

The presence of chronic illness makes it necessary that healthy partners increasingly take over household duties for longer periods of time. This means of dyadic coping can become especially burdensome and exhausting with time. A husband of a young mother with chronic back pain explained how he struggled with this condition:

For me it's not, I don't say that it isn't hard, it is hard but... I won't become a martyr just because I have to vacuum or because I have to carry upstairs the washed clothes so she can hang them out or I have to wipe up the dust or lift up something and put it into the cabinet or get down something or just lift something. So this isn't hard, it's normal. . However in these last times my back hurts too. I don't really understand it. First the crackle and then now the pain starts to come out. But I don't think so that I'm going to be sick.

While steadily taking over duties from his wife, he seemed to be uncertain whether his feelings of being overloaded could be appreciated without having to assume the role of the "martyr."

\section{The Common Use of the Home Space Supports Coping With Stress (Axial Code 3.3)}

In contrast to couples who described patterns of coordinated avoidance and separation, others emphasized the positive effects of being close to each other, moving together in the space of the home either in presence of the children or, in the case of childless couples, the couple themselves spending their time together at home. For a young man living with epilepsy, the sense of healing was everywhere - in their flat and on the terrace - due to being close to one another during the time spent at home:
On the terrace we don't always need wine for this [healing]. The last time we just sat down and laid down and we were watching the sun and the city and we were just talking. Maybe we drank some water and cola, but that's it. And we were just joking around and we do everything on the terrace, last time I sprinkled her with water from the hose so (laughing) so it's like everything, really, we laugh from our heart.

The importance of their physical closeness is also reflected in the woman's description:

We're together in the bathroom as well, so basically always...So for example when one of us has something to do and the other doesn't then. . . like then we use the workroom/den, but except that, really, we're almost always together because if we aren't, if for example one of us is cooking and the other is in the living room, even then we're in the same space, so like a lot...

This experience of closeness, provided by the home space, also assured security for the wife who was regularly worried about her partner's seizures and the fatigue that follows the seizures.

\section{The Joint Shaping of the Home Space (Axial Code 3.4)}

Common dyadic coping activities can focus directly on shaping the home environment. Active and shared shaping of the environment not only brought positive feelings in the time spent together but also had a longer effect on the relationship as the couple had created a new environment that later on reflected their creativity and harmony. In some cases, even minor modifications could represent important changes in the environment. For example, a wife (with chronic back pain) in a childless couple reported that lately, she had opened the door of their storage room that was intended to be their child's room after they gave birth. She opened the room's door and invited her husband to eat some fruit in that room. Later she asserted that the deliberate alternative use of this space helped them with the process of conceiving a child.

The same couple described their symbols of their home very similarly: both partners expressed the feeling that the big wardrobe in their bedroom that was their common work that could be the symbol of both their home and their relationship. This is how the wife explained it:

Somehow this wardrobe too is a symbol of the flat renovation, how it went, that we decided everything together and how we were planning and how it came out. . It was like we did it together, the creative stuff; and this wardrobe was made like this.

The husband also pointed out how the wardrobe was created to support coping with the wife's symptoms:

I think that this wardrobe symbolizes pretty well how we related to this whole issue of the flat. So that for my wife it was an important thing that we could have big, well packable wardrobe, she put really big effort in the planning of the inside that what would be in which height, what would be easily accessible, how it should be divided. And I had some ideas for the appearance. So I think that it symbolizes our cooperation and this... the harmony of this common activity in our home. It's also designed to fit to her waist pain, of course, so that the important functions wouldn't be too low, as I said, because bending is hard. . . 
This process of dyadic coping with the stress of the illness and the stress stemming from furnishing a new flat led to a creative work, which reflected their cooperation every time they entered their bedroom. Thus, a glance at this wardrobe had a protective effect on their individual and relational well-being ever after its construction.

\section{DISCUSSION}

In this study, we assessed the relationships among the family home, self-regulation processes and, more closely, dyadic coping in the context of chronic illness. Reflecting back to our research question: How do families with a chronically ill member use dyadic coping processes in the context of the home environment? It was our initial assumption that the family home as sociophysical environment contributed significantly to self-regulation and relationship regulation processes. Indeed, one participant's described practice of greeting her "snug," a separate flat obtained primarily for her use, illustrates one of our essential findings: homes, and/or spaces within homes can be seen as actors in environmental self-regulation processes, thus they play significant and profound roles in dyadic coping in the context of chronic illness. In interpreting our findings, we focus on how families with a chronically ill member use the home as part of their dyadic coping processes.

\section{Summary of Main Themes}

The results of our data analysis suggested that participants' experiences could be categorized into three broad areas: processes of stress communication through the home space, dyadic coping by spatial separation, and dyadic coping by joint striving for at-homeness. These main themes and the respective subthemes were interrelated in a way that the system of these codes and their relationships described the processes through which partners communicated directly or indirectly the stress they experienced, and demonstrated variations of dyadic coping by spatial separation or by joint actions in the home space. While in some instances partners' dyadic coping by separation led to a relief from stress and pain (e.g., the wife who had no pain when sleeping apart from her husband), in other instances this pattern of coping led to an opposite outcome - escalating stress in one of the partners or both partners with consequent explicit or implicit stress communication. In a similar way, partners' dyadic coping by joint strivings resulted in stress relief in the experiences of certain couples, whereas others experienced escalating stress as a consequence of joint actions (e.g., coordinated taking over of home duties resulted in the experience of backache in the formerly healthy partner together with the failure of their sexual relationship).

The partners' perception of the outcome of their dyadic coping actions depended also on the temporal perspective: coping by spatial separation appeared to assure momentary stress relief for the wife who held the image of a castle for her baby's room but she also described her growing dissatisfaction and tension in their couple relationship partly linked to the lack of sexual intimacy between them. In addition, some couples applied a combination of dyadic coping patterns of joint actions and coordinated spatial separation related to specific places inside the home: they cooperated well in the kitchen and the living room where the husband took over household duties from his wife, but they consistently avoided each other in the bathroom and the bedroom.

\section{Spatial Aspects of Coping in STM Perspective}

While our approach and research question was informed by basic tenets of previous dyadic coping research, most prominently by Systemic Transactional Model of dyadic coping (Bodenmann, 2005; Falconier et al., 2016), we still took an interpretative constructivist stance toward qualitative data without any preformulated hypotheses. Therefore, our results are not mere demonstrations of previously described constructs but can be regarded also as potential extensions and reinterpretations of the original concepts. For example, it might be appealing to identify the three main themes (selective codes) of 'communication through the home space,' 'dyadic coping by spatial separation,' and 'dyadic coping by joint striving for at-homeness' with the three main aspects of the dyadic coping process within STM: 'stress communication,' 'negative' and 'positive (including common) dyadic coping.' Following, we consider these overlaps as well as the dissimilarities between our interpreted findings and the STM.

Based on our analysis, we suggest that the family home mediates dyadic coping activities by acting at times as a proxy of or filter for direct and explicit communication. The degree of mediation varies in association with partners' responses to one partner's chronic illness; the congruence between partners when approaching coping with illness additionally impacts use of home space. Congruence of responses occurred on a continuum. Located at one end is complete separation of response to chronic illness, as the spouses who asserted that management of illness was entirely up to the partner with the illness. A midpoint of this continuum includes empathetic responses, such as the spouse who facilitated the ill spouse in his or her disease management and self-regulatory processes. The other extreme end comprises collaborative responses, often included in the selective code coping by joint striving for at homeness, and reminiscent of the "We-disease" theme presented in the introduction of this paper (c.f., Kayser et al., 2007; Berg et al., 2008; Helgeson et al., 2017). One example of this is the couple who described co-designing their joint wardrobe.

\section{Space Use and Dyadic Coping}

Dyadic coping responses were frequently communicated through separate or shared use of space but are not necessarily constant; couples might concurrently occupy the same space, such as when sharing a meal, and adhere to a regular practice of separation in other activities, such as media use. Frequently, couples described engaging in planning or taking advantage of naturally occurring opportunities to be separate from each other. It is of interest that separation occurred related to various activities, but was described multiple times related to sleeping arrangements that at times also interfered with partners' sexual 
relationships. One couple described their use of the alternative "pouting bed" when disagreements occurred in bed; this is a particularly clear example of use of home space as a proxy for verbal communication related to nighttime intimacy. These patterns of either negatively or positively perceived individual and common acts of dyadic coping can be seen as specific manifestations of stress communication processes as described within STM. Nonetheless, our approach informed by the tenets of environmental psychology revealed non-verbal, implicit ways of stress communication by space use. Apparently, the role and significance of this kind of spatial communication - along with other types of non-verbal communication - have been seldom the explicit focus of STM-based dyadic coping research, although it is inherently present in the foundations of the model (c.f., Bodenmann, 2005). Thus, these aspects deserve further investigation and may contribute to potential extension of the original model.

\section{Control of Space}

Multiple participants described dissatisfaction over lack of control of space (c.f., Barnes, 2006), either due to storage of excessive or unwanted items, or due to a partner using the other partner's space, rather than his or her designated space, or due to perceived unequal allocation of space. In one instance, a participant who was unhappy with having less space for her personal possessions than her partner did, drew an explicit parallel between this perceived lower priority placed on her space needs with less appreciation of her in general. For two participants, including the spouse who procured her own "snug," and another mother who described her baby's room as analogous to her childhood imagined "castle," there was security in having a distinct space under one's own control. These findings echo but expand upon previous findings, which posited that dyadic coping varied through the lifespan (Berg and Upchurch, 2007), and was vulnerable to contextual factors (Bodenmann et al., 2015). Specifically, we found that available space, and perceptions of control related to available space, comprised explicit factors that might appear to be contextual but played a more significant role by being used as means of communication of coping responses.

Obviously, control aspects of space use are interwoven with the social-ecological context of actual relationships as well. Financial power of a family may significantly influence the potential availability of private spaces and equipments. This way, financial problems and limitations as a context may be partly represented in spatial-territorial stress in everyday relational behaviors. Moreover, we need to consider that ownership of a house or flat plays a key role in the financial strategies of Hungarian families (Toussaint et al., 2012) and families often take extra burdens to achieve this long-term goal. Experiences of the respondents, for example that of the woman who struggled for control in her husband's flat, stem from this socialeconomical background where upward residential mobility is difficult for lower middle class parents. Personal and relational stress and coping behaviors in the family home - as reflected in the interviews and the codes - cannot be exclusively tied to intrapersonal and interpersonal processes but broader socialecological contexts have to be considered as well.

\section{Control of Closeness and Distance}

The selective codes 'spatial separation' and 'joint striving for at-homeness' as spatially embedded forms of dyadic coping represent two characteristically distinct ways of responding to the challenges of chronic illness and the resulting relationship tensions. Strategies under the code 'spatial separation' often involve actions of distancing, withdrawal and even lack of sexual encounters while 'joint striving for at-homeness' entails coordinated and mutually reinforcing rituals. It is well known that themes of closeness vs. distance are often associated with varying levels of well being and functionality in relationships. As a general trend it can be stated that closeness brings benefits for the relationships and distance is rather detrimental (Arriago et al., 2004). As noted earlier, these aspects may have their parallels in negative and positive forms of dyadic coping as well. Withdrawal, for example in form of alternating use of the common spaces, was a coping response to relationship stress in couples; from an STM perspective, these behaviors may be regarded as lack of support for the stressed partner, or even neglecting her needs. In contrast, joint shaping of the home (under the code 'striving for at-homeness') can be easily acknowledged as a positive, common dyadic coping act.

However, closer inspection of the variations of these main themes shows that opposite tendencies may be also found in both main themes. Conscious distancing may be an adequate and coordinated response to illness symptoms, although it still can cause adverse relationship experiences too. Couples also experienced alternating use of the spaces as sign of their wellcoordinated coping with everyday challenges. In a similar way, joint strivings for at-homeness had their complex, sometimes even ambivalent character too. Interviewees spontaneously gave account of the risks of overprotection and delegated dyadic coping on behalf of the healthy partner. These ambivalent aspects of both 'distancing' and 'joint strivings' can be better understood when we consider that distancing may help overview, clarity and autonomy while closeness may eventually involve control and coercion (Kanat-Maymon et al., 2016). Therefore, our data suggest that there are complex, multifaceted interrelations between strategies of spatial behavior and dyadic coping processes. The inclusion of spatial aspects of dyadic coping seems especially important if we consider the results of Helgeson et al. (2017) who found that implicit ways of communal coping in the partner (e.g., in forms of we-talk) were especially beneficial for diabetes patients. Since much of spatial behavior is implicit in nature, we may assume that it conveys powerful messages about the relational meaning of the actual dyadic coping efforts.

\section{Theoretical Outlook on Dyadic Coping Research}

In sum, this research expands prior work on STM in the context of chronic disease by illustrating the profound role of the family home as a mediator. Recent research and commentary on the role of place in health (e.g., Corburn, 2017; Lovell et al., 2017) has tended to focus on higher, macro and meso levels of 
place, including health promoting support offered within one's community of residence, or the impact of local and national policies on physical or mental health. Our results suggest that for many individuals, aspects of the family home, including features, distribution of space, and temporarily or long-term sharing with coresidential relatives, are simultaneously the background for and an aspect of dyadic coping in illness. When a family member is chronically ill, family functioning is challenged (Ryan et al., 2012) and the family's satisfaction with the home environment can be linked to the fact if family members participate or are considered in the home design (Coulombe et al., 2016). Developing or worsening illness might be accompanied by increasing importance of the home environment with decreasing impact of macro and meso environments.

Bodenmann (2008) suggested disease specific patterns in coping exist. While distinguishing between coping for epilepsy versus chronic back pain was not a stated goal of this research, we respectfully suggest based on these findings, that use of the home space as a mediator of coping and self-regulatory activities was demonstrated throughout the sample. It might be that the explicit intent of home space shared or separate use varies based on condition, as suggested by one partner of a participant with epilepsy who perceived the home as a safer environment in the instance of a seizure, but it is possible there are factors at a higher level of abstraction, including safety or security, that are perceived consistently across various illness or disease states. Clearly, further research is needed to focus on the role of the home in dyadic coping within specific illness or disease states. While we did not formulate a comprehensive theoretical account on these phenomena, the results presented here may inspire a new line of investigation in order to develop a novel theory.

\section{Limitations}

As with any individual interview research, data are subject to deliberate or inadvertent inaccuracies, although within the constructivist-interpretative research paradigm, we embrace data that reflect how individuals experience, interpret and chose to communicate reflect the reality(ies) of interest. Another limitation is presented by our use of the Emotional Map of the Home method, which incorporated an exploratory dimension to the research design over and above the exploration related to the research question. This framework had clear advantages in allowing us to gather data that were particularly thoughtful and nuanced because the process encouraged participants to provide a layer of interpretive reflection that enhanced and increased authenticity of simple descriptive examples. However, there were associated disadvantages in that we did not capture details of the illness experience that were not elicited through this method. Further research in areas related to environmental psychology such as proxemics and home safety perceptions and practices, and investigation of other types of chronic illness, are indicated to improve understanding of dyadic coping in chronic illness. That said, we believe our sample for this study was large and diverse enough to provide ample rich data to address the research purpose and facilitate initial theory development.

\section{Implications}

These findings related to chronic illness, dyadic coping, and the family home have implications that researchers should explore in other contexts. Our findings related to the role of the family home in dyadic coping in the context of chronic illness are of potential importance for other domains of investigation. The role of home environment in the development of dyadic coping patterns can be studied in non-clinical samples like in relationships of emerging adults who seek to establish their adult life both in terms of the environmental circumstances and the basic rules ("relationship contract"; Sager, 1976; or "couple's pact," Cigoli and Scabini, 2006) of their long-term bond. Moreover, family home may be an important part of dyadic coping processes of families with special life situation other than chronic illness, such as families struggling with financial strains and challenges. Increases are anticipated in the proportion of older adults throughout the world, and many will experience some type of age-related disability or disease (United Nations Department of Economic and Social Affairs Population Division, 2015). Given population age trends, the previously discussed trend toward provision of an increasing number and scope of medical services in by carers in homes rather than institutions, and many individuals' expressed preference for aging in place, improved understanding of the role the home plays in relationships, dyadic coping, and illness is an issue of ongoing importance. Consequently, our environmental psychologically informed approach toward relationships may be applied in professional trainings, patient education programs (c.f., Riley et al., 2001; Glasgow and Emmons, 2007) and may broaden the scope of health promotion in general. As an example, couple therapy and couple relationship enhancement programs focusing on dyadic coping strategies of the couples (e.g., CCET, Bodenmann and Shantinath, 2004; and TOGETHER, Falconier, 2015) may benefit from the spatial aspects of dyadic coping described here by making couples more aware of the spatial aspects of their behavior and from the qualitative data assessment methodology that we used here, that is, the Emotional Map of the Home Interview protocol that was intended not only for research but also for counseling purposes.

\section{CONCLUSION}

In our analysis, we have demonstrated several key processes of how individuals living with chronic illness and close others implicitly or explicitly use their home environment in the process of coping with life conditions related to illness. Relationship science researchers and practitioners should address whether families living with a chronic illness understand how homeenvironment transactions can and do bear significance for their coping capacities, and, finally, to their emotional, relational and physical health. Our results may also contribute to more elaborated and complex theoretical considerations on selfregulation and dyadic coping processes. Living with chronic illness in the family home - whether as patient or a close relative challenges dyadic coping skills and strategies at the highest level but may also discover hidden resources and possibilities. Our descriptions of transactive relationships between the partners, 
their relationship processes including dyadic coping efforts and the spatial-temporal context around them provide rich examples for both challenging and empowering aspects of the life situation of chronic illness.

\section{DATA AVAILABILITY}

The raw data for this study (interview transcripts in Hungarian) supporting the conclusions of this manuscript will be made available by the authors, without undue reservation, to any qualified researcher.

\section{ETHICS STATEMENT}

This study was carried out in accordance with the recommendations of the Codex of Ethics of Scientific

\section{REFERENCES}

Allen, S., and Webster, P. (2001). When wives get sick: gender role attitudes, marital happiness, and husbands' contributions to household labor. Gender Soc. 15, 898-916. doi: 10.1177/089124301015006007

Årestedt, L., Benzein, E., Persson, C., and Rämgård, M. (2016). A shared respiteThe meaning of place for family well-being in families living with chronic illness. Int. J. Qual. Stud. Health Well Being 11:30308. doi: 10.3402/qhw.v11. 30308

Arriago, X. B., Goodfrien, W., and Lohmann, A. (2004). "Beyond the individual: concomitants of closeness in the social and physical environment," in Handbook of Closeness and Intimacy, eds D. J. Mashek and A. Aron (Mahwah, NJ: Lawrence Erlbaum Assoc., Inc), 287-304.

Badr, H., Carmack, C. L., Kashy, D. A., Cristofanilli, M., and Revenson, T. A. (2010). Dyadic coping in metastatic breast cancer. Health Psychol. 29, 169-180. doi: $10.1037 / \mathrm{a} 0018165$

Bailes, A., and Jackson, M. E. (2000). Shared responsibility in home birth practice: collaborating with clients. J. Midwifery Womens Health 45, 537-543. doi: 10. 1016/S1526-9523(00)00073-8

Barnes, S. (2006). Space, choice and control, and quality of life in care settings for older people. Environ. Behav. 38, 589-604. doi: 10.1177/0013916505281578

Bell, S. L., Foley, R., Houghton, F., Maddrell, A., and Williams, A. M. (2018). From therapeutic landscapes to healthy spaces, places and practices: a scoping review. Soc. Sci. Med. 196, 123-130. doi: 10.1016/j.socscimed.2017.11.035

Berg, C. A., and Upchurch, R. (2007). A developmental-contextual model of couples coping with chronic illness across the adult life span. Psychol. Bull. 133:920. doi: 10.1037/0033-2909.133.6.920

Berg, C. A., Wiebe, D. J., Butner, J., Bloor, L., Bradstreet, C., Upchurch, R., et al. (2008). Collaborative coping and daily mood in couples dealing with prostate cancer. Psychol. Aging 23, 505-516. doi: 10.1037/a0012687

Bertoni, A., Donato, S., Graffigna, G., Barello, S., and Parise, M. (2015). Engaged patients, engaged partnerships: singles and partners dealing with an acute cardiac event. Psychol. Health Med. 20, 505-517. doi: 10.1080/13548506.2014. 969746

Beyer, L. E., and Simmons, L. E. (2004). Home treatment of pain for children and adolescents with sickle cell disease. Pain Manag. Nurs. 5, 126-135. doi: 10.1016/j.pmn.2004.03.001

Bodenmann, G. (2005). "Dyadic coding and its significance on marital functioning," in Couples Coping with Stress: Emerging Perspectives on Dyadic Coding, eds T. Revenson, K. Kayser, and G. Bodenmann (Washington, DC: American Psychological Association), 33-49. doi: 10.1037/110 31-002

Bodenmann, G. (2008). Dyadic coping and the significance of this concept for prevention and therapy. Z. Gesundheitspsychol. 16, 108-111. doi: 10.1026/09438149.16.3.108
Knowledge of the Hungarian Academy of Sciences, approved by the Medical Research Council (a board of the Hungarian Ministry of Human Capacities) with written informed consent from all subjects. All subjects gave written informed consent in accordance with the Declaration of Helsinki. The protocol was approved by the Medical Research Council.

\section{AUTHOR CONTRIBUTIONS}

VS and $\mathrm{AD}$ designed the study. VS carried out the research. VS and TM conducted data analysis. All authors contributed to the presented interpretation of findings. VS, TM, and SC wrote sections of the manuscript and read and all authors approved the final version.
Bodenmann, G., Meuwly, N., Germann, J., Nussbeck, F. W., Heinrichs, M., and Bradbury, T. N. (2015). Effects of stress on the social support provided by men and women in intimate relationships. Psychol. Sci. 26, 1584-1594. doi: $10.1177 / 0956797615594616$

Bodenmann, G., and Shantinath, S. D. (2004). The couples coping enhancement training (CCET): a new approach to prevention of marital distress based upon stress and coping. Fam. Relat. 53, 477-484. doi: 10.1111/j.0197-6664.2004. 00056.x

Charmaz, K. (2014). Constructing Grounded Theory. Thousand Oaks, CA: Sage.

Cigoli, V., and Scabini, E. (2006). Family Identity. Ties. Symbols, and Transitions. Mahwah, NJ: Lawrence Erlbaum Associates.

Corbin, J., and Strauss, A. (1985). Managing chronic illness at home: three lines of work. Qual. Sociol. 8, 224-247. doi: 10.1007/BF00989485

Corburn, J. (2017). Urban place and health equity: critical issues and practices. Int. J. Environ. Res. Public Health 14:117. doi: 10.3390/ijerph14020117

Coulombe, S., Jutras, S., Labbé, D., and Jutras, D. (2016). Residential experience of people with disabilities: a positive psychology perspective. J. Environ. Psychol. 46, 42-54. doi: 10.1258/jtt.2010.090902

Coyne, J. C., Rohrbaugh, M. J., Shoham, V., Sonnega, J. S., Nickla, J. M., and Cranford, J. A. (2001). Prognostic importance of marital quality for survival of congestive heart failure. Am. J. Cardiol. 88, 526-529. doi: 10.1016/S00029149(01)01731-3

Donovan, R., and Williams, A. (2007). "Home as therapeutic landscape: family caregivers providing palliative care at home," in Therapeutic Landscapes, ed. A. Williams (Farnham: Ashgate Publishing), 199-220.

Downing, M. J. Jr. (2008). The role of home in HIV/AIDS: a visual approach to understanding human-environment interactions in the context of long-term illness. Health Place 14, 313-322. doi: 10.1016/j.healthplace.2007.08.001

Dyck, I., Kontos, P., Angus, J., and McKeever, P. (2005). The home as a site for long-term care: meanings and management of bodies and spaces. Health Place 11, 173-185. doi: 10.1016/j.healthplace.2004.06.001

Falconier, M. K. (2015). TOGETHER - a couples' program to improve communication, coping, and financial management skills: development and initial pilot-testing. J. Marital Fam. Ther. 41, 1-15. doi: 10.1111/jmft.12052

Falconier, M. K., Randall, A. K., and Bodenmann, G. (2016). "Coping in Couples: The Systemic Transactional Model (STM)," in Couples Coping with Stress: A Cross Cultural Perspective, eds M. K. Falconier, A. K. Randall, and G. Bodenmann (New York, NY: Routledge), 31-48. doi: 10.4324/9781315644394

Feldman, B. N., and Broussard, C. A. (2006). Men's adjustment to their partners' breast cancer: a dyadic coping perspective. Health Soc. Work 31, 117-127. doi: $10.1093 / \mathrm{hsw} / 31.2 .117$

Fraser, J. A., Armstrong, K. L., Morris, J. P., and Dadds, M. R. (2000). Home visiting intervention for vulnerable families with newborns: follow-up results of a randomized controlled trial. Child Abuse Neglect. 24, 1399-1429. doi: 10. 1016/S0145-2134(00)00193-9 
Glaser, B., and Strauss, A. (1967). The Discovery of Grounded Theory: Strategies for Qualitative Research. New Brunswick, NJ: Aldine Transaction.

Glasgow, R. E., and Emmons, K. M. (2007). How can we increase translation of research into practice? Types of evidence needed. Annu. Rev. Public Health 28, 413-433. doi: 10.1146/annurev.publhealth.28.021406.144145

Hallberg, L. R.-M. (2006). The "core category" of grounded theory: making constant comparisons. Int. J. Qual. Stud. Health Well-being 1, 141-148. doi: $10.1080 / 17482620600858399$

Helgeson, V. S., Jakubiak, B., Seltman, H., Hausmann, L., and Korytkowski, M. (2017). Implicit and explicit communal coping in couples with recently diagnosed type 2 diabetes. J. Soc. Pers. Relat. 34, 1099-1121. doi: 10.1177/ 0265407516669604

Kanat-Maymon, Y., Roth, G., Assor, A., and Raizer, A. (2016). Controlled by love: the harmful relational consequences of perceived conditional positive regard. J. Pers. 84, 446-460. doi: 10.1111/jopy.12171

Karasaki, M., Warren, N., and Manderson, L. (2017). Orchestrating home. Experiences with spousal stroke care. Med. Anthropol. Theory 4, 79-104. doi: 10.17157/mat.4.1.366

Kayser, K., Watson, L. E., and Andrade, J. T. (2007). Cancer as a "we-disease": examining the process of coping from a relational perspective. Fam. Syst. Health 25, 404-418. doi: 10.1037/1091-7527.25.4.404

Korpela, K. M. (1989). Place-identity as a product of environmental self-regulation. J. Environ. Psychol. 9, 241-256. doi: 10.1016/S0272-4944(89)80038-6

Korpela, K. M., Hartig, T., Kaiser, F. G., and Fuhrer, U. (2001). Restorative experience and self-regulation in favorite places. Environ. Behav. 33, 572-589. doi: 10.1016/j.amepre.2009.01.022

Lincoln, Y. S., and Guba, E. G. (2000). "Paradigmatic controversies, contradictions, and emerging confluences," in Handbook of Qualitative Research, 2nd Edn, eds N. K. Denzin and Y. S. Lincoln (London: Sage), 163-188.

Lovell, S. A., Gray, A. R., and Boucher, S. E. (2017). Place, health and community attachment: Is community capacity associated with self-rated health at the individual level? SSM Populat. Health 3, 153-161. doi: 10.1016/j.ssmph.2016. 12.002

Martire, L. M., and Helgeson, V. S. (2017). Close relationships and the management of chronic illness: associations and interventions. Am. Psychol. 72, 601-612. doi: $10.1037 / \mathrm{amp} 0000066$

Manzo, L. C. (2003). Beyond house and haven: toward a revisioning of emotional relationships with places. J. Environ. Psychol. 23, 47-61. doi: 10.1016/S02724944(02)00074-9

Manzo, L. C. (2005). For better or worse: exploring multiple dimensions of place meaning. J. Environ. Psychol. 25, 67-86. doi: 10.1016/j.jenvp.2005.01.002

Meier, C., Bodenmann, G., Mörgeli, H., and Jenewein, J. (2011). Dyadic coping, quality of life, and psychological distress among chronic obstructive pulmonary disease patients and their partners. Int. J. Chron. Obstruct. Pulmon. Dis. 6, 583-596. doi: 10.2147/COPD.S24508

Moore, A., Carter, B., Hunt, A., and Sheikh, K. (2013). 'I am closer to this place'Space, place and notions of home in lived experiences of hospice day care. Health Place 19, 151-158. doi: 10.1016/j.healthplace.2012.11.002

Mærsk, J. L., Cutchin, M. P., and la Cour, K. (2018). Identity and home: understanding the experience of people with advanced cancer. Health Place 51, 11-18. doi: 10.1016/j.healthplace.2018.02.003

Nixon, G. M., and Brouillette, R. T. (2002). Scoring arousals in the home environment. Sleep Med. 3, S21-S27. doi: 10.1016/S1389-9457(02)00159-4

Öhlén, J., Ekman, I., Zingmark, K., Bolmsjö, I., and Benzein, E. (2014). Conceptual development of "at-homeness" despite illness and disease: a review. In. J. Qual. Stud. Health Well Being 9:23677. doi: 10.3402/qhw.v9.23677

Olds, D. L., Robinson, J., and O’Brien, R. (2002). Home visiting by paraprofessionals and by nurses: a randomized, controlled trial. Pediatrics 110, 486-496. doi: 10.1542/peds.110.3.486

Piercy, K. W. (2007). Characteristics of strong commitments to intergenerational family care of older adults. J. Gerontol. Ser. B Psychol. Sci. Soc. Sci. 62, S381S387. doi: 10.1093/geronb/62.6.S381
Ponterotto, J. G. (2005). Qualitative research in counseling psychology: a primer on research paradigms and philosophy of science. J. Couns. Psychol. 52, 126-136. doi: 10.1037/0022-0167.52.2.126

Ponterotto, J. G. (2010). Qualitative research in multicultural psychology: philosophical underpinnings, popular approaches, and ethical considerations. Cultur. Divers. Ethnic Minor. Psychol. 16, 581-589. doi: 10.1037/a0012051

Regan, T. W., Lambert, S. D., Kelly, B., McElduff, P., Girgis, A., Kayser, K., et al. (2014). Cross-sectional relationships between dyadic coping and anxiety, depression, and relationship satisfaction for patients with prostate cancer and their spouses. Patient Educ. Couns. 96, 120-127. doi: 10.1016/j.pec.2014.04.010

Riley, K. M., Glasgow, R. E., and Eakin, E. G. (2001). Resources for health: a socialecological intervention for supporting self-management of chronic conditions. J. Health Psychol. 6, 693-705. doi: 10.1177/135910530100600607

Ryan, C. E., Epstein, N. B., Keitner, G. I., Miller, I. W., and Bishop, D. S. (2012). Evaluating and Treating Families: The McMaster approach. Abingdon: Routledge. doi: 10.4324/9780203843840

Sager, C. J. (1976). Marriage Contracts and Couple Therapy: Hidden Forces in Intimate Relationships. New York, NY: Bruner/Mazel.

Sallay, V. (2014). Környezeti-Érzelmi Önszabályozási Folyamatok a Családi Otthon Terében. [Environmental-emotional processes of self-regulation in the family home.]. Doctoral dessertation, ELTE, Budapest.

Seamon, D. (2000). "Phenomenology in environment-behavior research," in Theoretical Perspectives in Environment-Behavior Research, eds S. Wapner, J. Demick, T. Yamamoto, and H. Minami (New York, NY: Plenum), 157-178. doi: 10.1007/978-1-4615-4701-3_13

Strauss, A., and Corbin, J. (1998). Basics of Qualitative Research: Grounded Theory Procedures and Techniques. Thousand Oaks, CA: Sage.

Tamm, M. (1999). What does a home mean and when does it cease to be a home? Home as a setting for rehabilitation and care. Disabil. Rehabil. 21, 49-55. doi: 10.1080/096382899297963

Toussaint, J., Szemzo, H., Elsinga, M., Hegedüs, J., and Teller, N. (2012). Owneroccupation, mortgages and intergenerational transfers: the extreme cases of Hungary and the Netherlands. Int. J. Housing Policy 12, 69-90. doi: 10.1080/ 14616718.2012.651327

Traa, M. J., De Vries, J., Bodenmann, G., and Den Oudsten, B. L. (2015). Dyadic coping and relationship functioning in couples coping with cancer: a systematic review. Br. J. Health Psychol. 20, 85-114. doi: 10.1111/bjhp. 12094

United Nations Department of Economic and Social Affairs Population Division (2015). World Population Ageing 2015 (ST/ESA/SER.A/390). Available at: http://www.un.org/en/development/desa/population/publications/pdf/ageing/ WPA2015_Report.pdf

Vaske, I., Thöne, M. F., Kühl, K., Keil, D. C., Schürmann, W., Rief, W., et al. (2015). For better or for worse: a longitudinal study on dyadic coping and quality of life among couples with a partner suffering from COPD. J. Behav. Med. 38, 851-862. doi: $10.1007 /$ s10865-015-9657-y

Williams, A. (2002). Changing geographies of care: employing the concept of therapeutic landscapes as a framework in examining home space. Soc. Sci. Med. 55, 141-154. doi: 10.1016/S0277-9536(01)00209-X

Ylikoski, P. (2013). Causal and constitutive explanation compared. Erkenntnis 78, 277-297. doi: 10.1007/s10670-013-9513-9

Conflict of Interest Statement: The authors declare that the research was conducted in the absence of any commercial or financial relationships that could be construed as a potential conflict of interest.

Copyright (®) 2019 Sallay, Martos, Chatfield and Dúll. This is an open-access article distributed under the terms of the Creative Commons Attribution License (CC BY). The use, distribution or reproduction in other forums is permitted, provided the original author(s) and the copyright owner(s) are credited and that the original publication in this journal is cited, in accordance with accepted academic practice. No use, distribution or reproduction is permitted which does not comply with these terms. 


\section{APPENDIX: FULL CODE TREE}

\section{Code no. Selective, axial and open codes}

1. Stress communication through the home space

1.1. Direct stress communication is lacking - stress appears indirectly through space use

1.1.1 Tension cannot appear during the time spent together

1.1.2 There is no suffering in chronic illness

1.1.3 Only one of the partners needs conversation - thus it fails

1.1.4 Partners take turns when using home spaces

1.2. Open disagreement appears in use of space

1.2.1 Disagreements on oppositely used spaces

1.2.2 Direct quarreling followed by withdrawal

1.2.3 Quarrels occur while in bed together, sexual relationship fails

1.3. Differences in partners' priorities for space use cause conflicts

1.3.1 Continuing conflict when one partner defers to the other's priorities

1.3.2 Distribution of space as a proxy for feelings of value

1.3.3 Control fight for the possession of the territories

2. Coping by spatial separation

2.1. Conscious distancing in coping with the symptoms

2.1.1 Alleviation of symptoms through daily rituals of retiring

2.1.2 Wife's duty to care for sick child distances partners

2.1.3 Sleeping separately helps alleviate symptoms

2.1.4 The healthy partner has "nothing to do" with the coping with the other's illness

2.2 .

2.2.1

2.2 .2

2.2 .3

2.2 .4

2.2 .5

2.3 .

Distancing in the coping with relationship stress

Rituals of withdrawal inside the shared room

Partners avoid each other in the bedroom - lack of sexual life

Rituals of withdrawal to the next room

Childhood experiences informing rituals of withdrawal

The ritual of creating an alternative home

Mutually reinforcing processes of distancing

Growing distance in the pursuit of a home related goal

A recovery supporting home environment creates distance in the relationship

The way of parental care creates distance

Coping by joint striving for at-homeness

One partner takes care of the other in the absence of stress communication

The healthy partner provides security for the ill partner in the space of the home

The ill partner's loss of control more profoundly affects her partner

The healthy partner initiates a shared ritual to alleviate the other's symptoms

The risk of taking over home duties

The ill partner considers insufficient the duties taken over

The partner's help is inefficient/other help would be needed

The takeover of duties is followed by blaming

The healthy partner gets tired of taking over duties and shows symptoms

The common use of the home space supports coping with stress

The common actions around the children's needs provide a pleasant feeling

The couple's common actions in the home space provide security and healing

Being together at home compensates for the changes in relationships

The joint shaping of the home space

Changing use of the home space can provide hope

The furniture collaboratively created by the couple can support coping with the symptoms 


\section{OPEN ACCESS}

Edited by:

Guy Bodenmann,

Universität Zürich, Switzerland

Reviewed by:

Noa Vilchinsky,

Bar-llan University, Israel

Serge Brand

Universität Basel, Switzerland

*Correspondence:

Susana Lameiras

susana.lameiras@gmail.com

Specialty section:

This article was submitted to Clinical and Health Psychology,

a section of the journal

Frontiers in Psychology

Received: 05 June 2018

Accepted: 20 August 2018 Published: 07 September 2018

Citation:

Lameiras S, Marques-Pinto A, Francisco $R$, Costa-Ramalho $S$ and Ribeiro MT (2018) The Predictive

Value of Dyadic Coping in the Explanation of PTSD Symptoms and Subjective Well-Being of Work

Accident Victims.

Front. Psychol. 9:1664. doi: 10.3389/fpsyg.2018.01664

\section{The Predictive Value of Dyadic Coping in the Explanation of PTSD Symptoms and Subjective Well-Being of Work Accident Victims}

\author{
Susana Lameiras ${ }^{1,2 *}$, Alexandra Marques-Pinto ${ }^{1}$, Rita Francisco ${ }^{3,4}$, \\ Susana Costa-Ramalho ${ }^{3,4}$ and Maria Teresa Ribeiro' ${ }^{1}$ \\ 1 Faculdade de Psicologia, Centro de Investigação em Ciências Psicológicas, Universidade de Lisboa, Lisbon, Portugal, \\ ${ }^{2}$ Faculdade de Psicologia e de Ciências da Educação, Universidade de Coimbra, Coimbra, Portugal, ${ }^{3}$ Faculdade \\ de Ciências Humanas, Universidade Católica Portuguesa, Lisbon, Portugal, ${ }^{4}$ Católica Research Centre for Psychological, \\ Family and Social Wellbeing (CRC-W), Universidade Católica Portuguesa, Lisbon, Portugal
}

Objective: Work accidents may be considered dyadic stressors in so far as they not only affect the worker, but also the couple's relationship. Dyadic coping, as the process by which couples manage the stress experienced by each partner, can strengthen individual health and well-being as well as couple relationship functioning. Accidents at work have progressively been studied from a perspective that focuses on their negative effects on PTSS, anxiety, and depression. However, to a large extent, the dyadic coping processes and results following a work accident are still to be identified and clarified. In this study, we examined the predictive value of dyadic coping in the explanation of PTSS and subjective well-being of work accident victims.

Method: This study comprised a sample of 62 individuals involved in work accidents within the last 24 months (61.3\% males) and their partners ( $N=124 ; M=46.25$ years, $S D=11.18)$. All participants responded to the Dyadic Coping Inventory and the work accident victims also answered the PTSD Checklist - Civilian (PCL-C) and the Mental Health Continuum - Short Form (MHC-SF). Two hierarchical multiple regression analyses were performed using two different variable set models: Model 1 comprised the control variables gender and age, and Model 2 included the workers' and the partners' dyadic coping variables.

Results: Results showed that dyadic coping reported by both workers and their respective partners (Model 2) was a significant predictor of workers' PTSS $(p<0.01$ ) and subjective well-being $(p<0.001)$, explaining $31.2 \%$ of the variance in PCL-C and $68.7 \%$ in MHC-SF results. More specifically, the partners' supportive dyadic coping (by the self) and delegated dyadic coping (by the partner) were significant predictors of the workers' lower PTSS and virtually all the dyadic copying strategies of both the workers' and their partners' were significant predictors of the workers' higher subjective well-being. 


\begin{abstract}
Conclusion: Dyadic coping of both the workers and their partners predicts the workers' PTSS and subjective well-being. These findings point to the need to work with couples who have experienced a work accident, with a view to improving the workers' mental health outcomes.
\end{abstract}

Keywords: work accidents, dyadic coping, PTSS, subjective well-being, couples

\section{INTRODUCTION}

Work accidents experienced by a member of a couple may be thought of as dyadic stressors since they also affect the partner and the couple relationship functioning. The present research focused on the dyadic coping processes that sustain adaptive efforts following a work accident, with a view to understanding the predictive value of dyadic coping in the explanation of work accident victims' post-traumatic stress disorder symptoms (PTSSs) and subjective well-being.

A work accident is defined in the European Statistics on Accident at Work as "a discrete occurrence in the course of work which leads to physical or mental harm" (Eurostat, 2013, p. 5). A work accident is considered to occur in the course of work when it takes place during the time spent at work or while performing an occupational activity. Accidents at work occur quite frequently. It is estimated that 3,211,706 accidents at work occurred in the European Union in 2015, causing at least 1 day of absenteeism (Eurostat, 2018), and that in the same year 208,457 work accidents occurred in Portugal (Pordata, 2018).

Empirical evidence suggests that, in specific circumstances, accidents at work may be traumatic events and possibly lead to the development of PTSD symptoms, involving an involuntary re-experience of the accident and avoidance of related memories (Gustafsson and Ahlström, 2004). The development of PTSD symptoms may emerge in the aftermath of work accidents involving injuries such as burns or amputation, and also following accidents at work in which there is no actual physical harm such as an armed robbery, attack, or hijack (MacDonald et al., 2003; Schaefer et al., 2012).

When work accidents happen to a married or cohabiting individual, it can be a stressful and challenging experience not only for the worker but also for his/her partner and the relationship itself. According to the systemic-transactional model of Bodenmann (1995), stressors are dyadic when they are directly related to the couple, namely when both members are dealing with the same stressor, and indirectly related when the stress of one of the partners affects both of them (Kramer et al., 2005).

In the same vein, married (or cohabiting) victims of work accidents do not cope with the situation alone, and the relationship with their partner may be their primary coping resource. Dyadic coping is a construct, based on Lazarus and Folkman's (1984) Transactional Stress Theory, which defines the efforts on the part of one or both partners to deal with stress and to create or restore the psychological, physical, and social homeostasis of the couple relationship (Bergstraesser et al., 2015). According to this perspective, dyadic coping may be regarded as a form of interaction between partners when they are dealing with a stressful situation, and both individuals and their respective partners are independently and mutually influenced by each other in their adjustment processes (Bodenmann, 1995). The two main goals of dyadic coping are to reduce the stress for each member of the couple, and to maintain the quality of the relationship. Research over recent decades has confirmed the significant value of dyadic coping in predicting couples' relationship satisfaction and subjective well-being (e.g., Bodenmann et al., 2011).

The dyadic coping process is activated when one of the partners communicates the stressor to the other, whether verbally or non-verbally. The other member of the couple receives and interprets the signs of stress and responds with some type of dyadic coping (Hansen et al., 2015). Bodenmann proposes three types of dyadic coping: supportive dyadic coping, which is related to the stress response behaviors of one partner toward the other, such as assistance, encouragement and emotional support; delegated dyadic coping in which one partner is primarily affected by the stressful event and asks the other to take over several tasks to reduce his or her levels of stress; and common dyadic coping, which is defined as couple implemented behaviors to actively collaborate in problem solving, joint decision making and seeking ways of reducing the stress together (e.g., CostaRamalho et al., 2017). Some types of dyadic coping, like hostile, ambivalent and superficial, can also be negative (Bodenmann et al., 2006). Studies and reviews regarding the impacts of different types of dyadic coping are presented in the following paragraphs.

Traditional approaches toward coping and adjustment to adversity have tended to focus on the individual, however in recent decades an increasing number of studies have pointed to the profound impacts of traumatic events on both members of the couple and their relationship, and have sought to examine partners' dyadic coping efforts. In the case of chronic illness, for instance, research has shown that couples are challenged to re-establish communication patterns, roles and responsibilities (Bodenmann et al., 2004; Gabriel et al., 2016). Within the scope of serious illness, more positive dyadic coping styles correlate with higher relationship satisfaction, higher relationship quality, and higher quality of life (Bodenmann, 2000; Revenson et al., 2005). Several other reviews have highlighted the importance of couples' intervention in order to boost dyadic coping strategies while dealing with cancer, and have revealed the positive repercussions of this approach in terms of psychosocial adjustment and relationship functioning not only in patients but also in their partners (Badr and Krebs, 2013). Traa et al. (2015) also showed the importance of adequate dyadic coping efforts, such as stress communication, supportive behaviors, and 
positive dyadic coping, for the maintenance and improvement of relationship functioning in couples coping with cancer. Other reviews have shown that traumatic events, such as the loss of a child (Albuquerque et al., 2015), terror attacks (Gilber et al., 2011) and natural catastrophes like earthquakes (Marshall et al., 2017), can bring some couples closer while others demonstrate significant adjustment problems that are linked to conflict and may result in divorce (e.g., Badr and Acitelli, 2017). Adaptive processes mediate the effects of personal characteristics and stressful events on marital adjustment. Some behaviors promote marital resilience, such as communication, cooperation, and mutuality and the absence of these factors may undermine the couple's adjustment when facing a stressful event (Karney and Crown, 2007).

Moreover, Bodenmann (2005) suggested that different types of dyadic coping strategies may have positive or negative effects on marital functioning. In the case of the former, these strategies may include problem-focused or emotion-focused supportive dyadic coping, common dyadic coping and delegated dyadic coping, while negative effects may result from hostile, ambivalent, and superficial dyadic coping. Nevertheless, the effectiveness of different forms of coping may be hard to establish a priori, since it depends on a host of variables such as the nature of the stressors, the pattern of coping strategies used to confront them or the outcome variables selected to evaluate their effectiveness (Folkman and Moskowitz, 2004). For instance, as regards mental health outcomes, Lafaye et al. (2014) showed that when patients or spouses used emotion-focused coping strategies they experienced higher levels of anxiety and depressive symptoms. Likewise, Manne and Badr (2008) found that in couples in which one partner is chronically ill, some forms of positively intended dyadic coping such as excessive kindness, concern, and support may be dysfunctional as they paralyze the patient's own coping efforts.

Accidents at work have progressively been studied from a trauma perspective, focusing on the negative psychopathological consequences for the worker, with particular emphasis on PTSD, anxiety, and depression (MacDonald et al., 2003). However, dyadic coping processes, which sustain adaptive effort in the aftermath of an accident, and their relationship with the mental health and subjective well-being of work accident victims, are yet to be investigated.

Gender and age often function as demographic variables in trauma research, and, to date, the results of a number of epidemiological studies have pinpointed both gender and age differences in PTSD, noting different developmental distributions of PTSD for men and women across their life courses. In a more detailed way, some studies point to the fact that women present a higher level of PTSD throughout life depending on the exposure to stress factors (i.e., poverty, lack of social support, partner violence). Overall, research has demonstrated that PTSD is more dependent on social, economic, cultural, and historical factors than dependent on age (Ditlevsen and Elklit, 2010).

Therefore, the aim of this study is to understand the predictive value of dyadic coping, reported by workers who have suffered a work accident and their partners in the explanation of the worker's PTSS and subjective well-being, while controlling for the effects of the worker's gender and age. Inasmuch no empirical studies were found on dyadic coping processes following a work accident and their relationships with mental health and well-being and it is hard to establish a priori the effectiveness of different forms of dyadic coping, the present study set out to analyze these relationships in an exploratory manner.

This study may contribute to further the knowledge on workers who have suffered a work accident and their partners, a population the scientific literature has largely forgotten. More specifically, it may contribute to detail the consequences of work accidents with regard to post-traumatic stress symptoms and subjective well-being, as well as the role played by the couple dyadic coping efforts in the prediction of those consequences. Moreover this study may provide empirical evidence with a view to stimulating interventions geared toward getting the best out of the workers and their partners' dyadic coping when facing a work accident.

\section{MATERIALS AND METHODS}

\section{Participants}

The sample comprised 62 individuals involved in a work accident within the last 24 months $(61.3 \%$ males $)$ and their partners $(N=124)$ aged between 19 and 68 years $(M=46.25 ; S D=11.18)$. Participants had been married or cohabiting from 1 to 41 years $(M=16.60 ; S D=11.50)$. The majority of participants had children $(82.3 \%)$ and lived in Portugal, mostly in the central region $(60.5 \%)$. In terms of schooling, $42.8 \%$ had not completed secondary education, $26.6 \%$ had completed secondary education, and $25.8 \%$ held a university degree. The majority of the respondents were Portuguese (96\%).

\section{Procedure}

Data were collected between January 2016 and December 2017 in the medical clinics of Insurance Companies in Lisbon and Oporto 1 month or more after the work accident had occurred. Paper and pencil questionnaires were given by nurses and physiotherapists to individuals who had had a work accident and who were in a marital relationship (marriage or cohabitation) and, at the same point in time, to their partners. The workers' participation rate is unknown but the partners' participation rate was of $100 \%$. Workers answered the Dyadic Coping Inventory (DCI), the Mental Health Continuum - Short Form (MHC-SF), and the PTSD Checklist - Civilian (PCL-C) and their partners answered the DCI. The questionnaires of both workers and their partners received a similar code number to enable their pairing while preserving the anonymity of the answers. The participants were volunteers and no financial remuneration was provided for their participation. The time taken to complete the questionnaires varied between $30 \mathrm{~min}$ for the workers and $10 \mathrm{~min}$ for their partners. Anonymity and confidentiality were guaranteed to the participants, as was the data being used strictly for research purposes. Both the workers and their partners were informed of the main objectives of the research and signed an informed 
consent form. The study was approved by the Ethics Committee of the University where the study is in course.

\section{Measures Dyadic Coping Inventory}

The Portuguese adaptation by Vedes et al. (2013) of the DCI by Bodenmann (2008) was used. The DCI is a self-report inventory of dyadic coping with 37 items (e.g., My partner shows empathy and understanding to me), scored using a five point Likert scale ranging from 1 (very rarely) to 5 (very often). The original DCI has nine subscales: Stress Communication by Self, Stress Communication by Partner (or other), Supportive DC by Self, Supportive DC by Partner, Negative DC by Self, Negative DC by Partner, Delegated DC by Self, Delegated DC by Partner and Joint DC in addition to a global score. The Joint DC subscale describes the coping behavior shown by both partners, while the first eight subscales refer to the coping behavior of the self and partner separately. In the present study, all sub-scales presented acceptable to good alphas in both the workers' sample and the partners' sample, except in the sub-scales Stress Communication by Self and Negative DC by Self in the workers' sample, and Delegated DC by Self in the partners' sample which presented low alphas and were therefore excluded from the analysis.

\section{PTSD Checklist - Civilian}

The PCL-C (Weathers et al., 1993; Portuguese version by Marcelino and Gonçalves, 2012) is a standardized self-report scale for PTSD symptoms comprising 17 items (e.g., Repeated, disturbing memories, thoughts, or images of a stressful experience from the past?) to be answered on a five point Likert scale from 1 (not at all) to 5 (extremely). In the current study, the PCL-C instructions asked the participants to give their answers bearing in mind specifically the actual work accident they had suffered. The PCL-C is usually used as a continuous measure of PTSD symptom severity, whereby a global score may be obtained simply by adding up the scores for all 17 items, with values ranging from 17 to 85, and higher scores indicating more severe PTSD symptoms. Although the PCL-C Portuguese version is still not in conformity with DSM-V, which has add a new dimension to the criteria for diagnosis, in this study only the global score was used (American Psychiatric Association, 2000). The PCL-C has demonstrated strong psychometric properties namely in the Portuguese validation by Marcelino and Gonçalves (2012; e.g., alpha value of 0.94 ), and an excellent alpha value of 0.95 was obtained in the workers' sample in this study.

\section{Mental Health Continuum - Short Form}

The MHC-SF (Keyes, 1998; Portuguese version by Carvalho et al., 2016), consists of 14 items (e.g., During the past month how often have you felt happy?) ranging from 1 (never) to 6 (every day), reflecting emotional, psychological and social wellbeing. The emotional well-being subscale is defined in terms of positive affect or satisfaction with life, psychological wellbeing refers to self-acceptance, environmental mastery, positive relations with others, personal growth, autonomy and purpose in life and social well-being is related to social contribution, social integration, social actualization, social acceptance, and social coherence. Three specific scores may be estimated, one for each well-being dimension, as well as a global score (ranging from 14 to 84), reflecting subjective well-being as a whole. The Portuguese version of the MHC-SF used in the present study demonstrated strong psychometric properties (e.g., alpha value of 0.90 for the total questionnaire; Carvalho et al., 2016). In this study, the global score was used and an excellent alpha value of 0.90 was attained.

\section{Data Analysis}

The data were analyzed using SPSS, version 25. Descriptive statistics (means and standard deviations) and Pearson correlations were computed for the study variables. Regression analyses were used to assess the predictive value of dyadic coping in the explanation of workers' PTSS and subjective well-being, while controlling for the effects of their gender and age. More specifically, two hierarchical multiple regression analyses (enter method) were performed using two different variable set models: to control for the effects of demographic variables, the gender and age of the workers were introduced in Model 1. Model 2 included the workers' and partners' dyadic coping variables. Prior to conducting the hierarchical multiple regressions, the relevant assumptions of this statistical analysis were verified through the graphical analysis of the studentized residuals, the DurbinWatson statistic and the VIF statistic. The Durbin-Watson test statistic value found $(d=1.77)$ falls inside the range of 1.5 to 2.5 , therefore indicating that the data are not autocorrelated (Field, 2009). One predictor (i.e., workers' Supportive DC by Partner) with VIF ( $=22.62$ ) above the acceptable limits (Field, 2009) was removed from the model to avoid the effects of multicollinearity. Those with $p<0.05$ were considered to be significant effects.

\section{RESULTS}

Table 1 presents the means, standard deviations, intercorrelations, and internal consistencies (Cronbach's alpha) for the measures used in this study (PCL-C, MHC-SF, and DCI for the workers' sample and DCI for the partners' sample).

In the sample of work accident victims, the global scores for PTSS and for subjective well-being attained mean values above the respective midpoint of each of the distribution scores (minimum of 17 and maximum of 85 ; minimum of 14 and maximum of 84 , respectively). ${ }^{1}$

The workers' PTSS presented a significant strong negative correlation with the workers' subjective well-being, significant weak to moderate negative correlations with the workers' Stress Communication (partner), Supportive DC (both self and partner) and Delegated DC (self), and also significant moderate to strong negative correlations with the partners' Stress Communication (self) and Delegated DC (partner). On the other hand, the workers' subjective well-being presented significant moderate to

\footnotetext{
${ }^{1}$ There are no studies regarding the cutoff points for the Portuguese population, but using the criteria provided by the National Center for PTSD (National Center for Posttraumatic Stress Disorder [NCPTSD], 2018) for the general population (3035 ) and for a specialized medical clinic population (36-44), 36 and 28 participants in this research, respectively, would be above the cutoff point.
} 


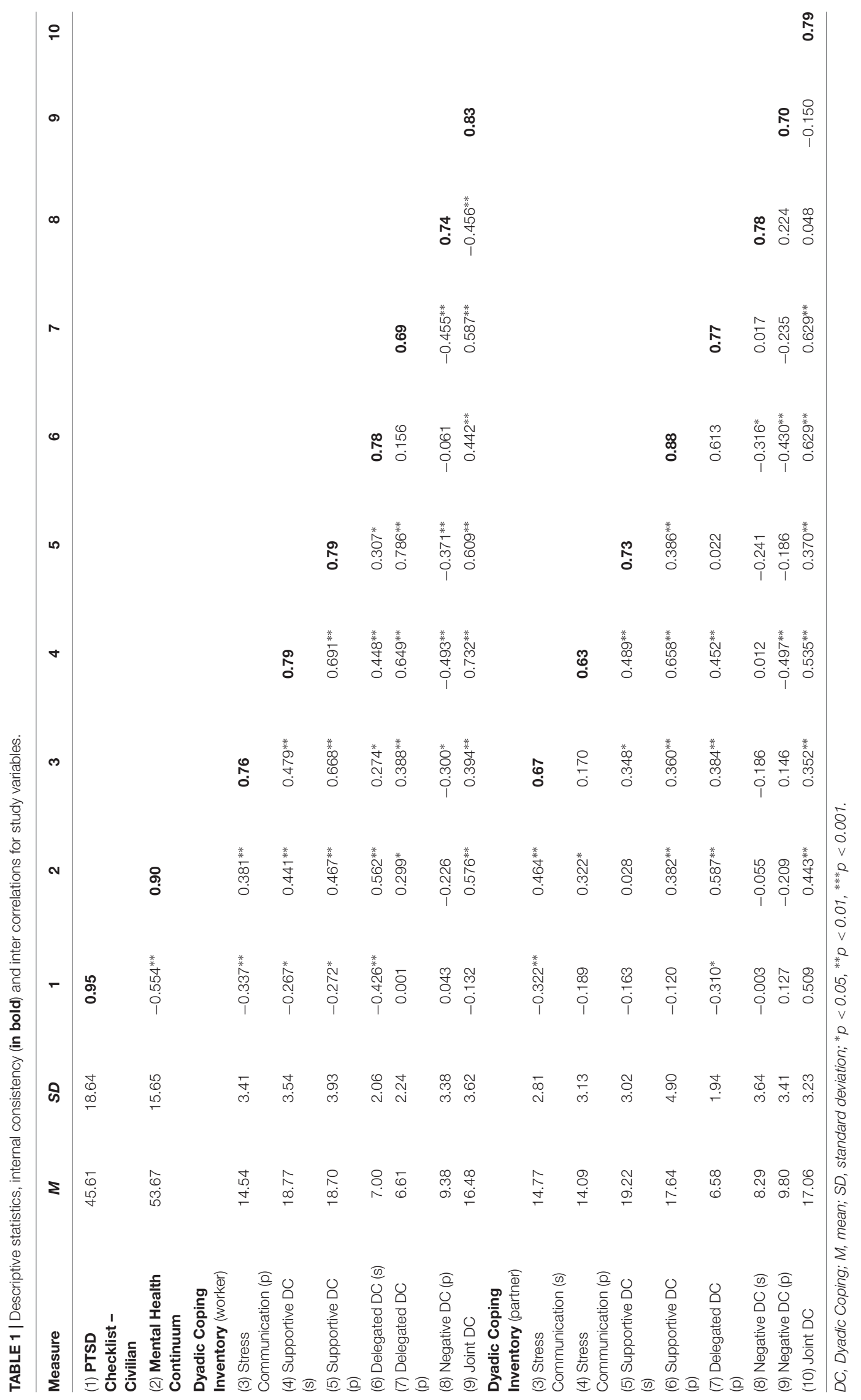


strong positive correlations with all the workers' DCI subscales, except Negative DC (partner), and also with the partners' Stress Communication (both self and partner), Supportive DC (partner), Delegated DC (partner), and Joint DC.

In the workers' sample, the DCI subscales presented significant moderate to strong positive inter-correlations, with the exception of the correlations between Delegated DC (self) and Delegated DC (partner) and Negative DC (partner), which were weak and non-significant, and the correlations between Negative DC (partner) and the other subscales which were also negative. In the partners' sample Stress Communication (both self and partner) presented significant moderate to strong positive correlations with Supportive DC (both self and partner), Delegated DC (partner), and Joint DC. This last subscale also revealed significant moderate to strong positive correlations with Supportive DC (both self and partner) and Delegated DC (partner). Supportive DC (self) presented significant moderate positive correlations with Supportive DC (partner). Negative DC (partner) presented significant moderate to strong but negative correlations with Stress Communication (partner) and Supportive DC (partner); similarly, Negative DC (self) showed a significant moderate negative correlation with Supportive DC (partner).

Two two-step hierarchical multiple regression analyses were conducted with PTSS and subjective well-being as the dependent variables. The gender and age of the victims were entered at step one of the regression, to control for demographic effects (Model 1 ), and the workers' and partners' dyadic coping were entered at step two (Model 2). The regression statistics are presented in Table 2.

The hierarchical multiple regressions results revealed that, at step one, the demographic variables (Model 1) did not contribute significantly to the regression models of either the workers' PTSS, $F(2,59)=0.443, p>0.05$ or subjective well-being $F(2,59)=2.672$, $p>0.05$.

Entering the workers' and partners' dyadic coping variables explained $31.2 \%$ of the variance in the workers' PTSS, and this model (Model 2) was significant, $F(16,45)=2,726, p<0.01$. When all the independent variables were included in this final step of the regression model, none of the demographic variables or the workers' dyadic coping variables were significant predictors of the workers' PTSS. In contrast, the partners' dyadic coping variables, Supportive DC by Self and Delegated DC by Partner, were significant negative predictors of the workers' PTSS, and the partners' dyadic coping variables Supportive DC by Partner and Joint DC were significant positive predictors of the workers' PTSS.

Finally, the introduction of the workers' and partners' dyadic coping variables explained $68.7 \%$ of the variance in the workers' subjective well-being, and this model (Model 2) was significant, $F(16,45)=9,365, p<0.001$. When all the independent variables were included in Model 2, the age of the workers, the workers' Delegated DC by Partner and Joint DC and the partners' Delegated DC by Partner were significant positive predictors of the workers' subjective well-being, while the partners' Supportive DC by Self was a significant negative predictor of the workers' subjective well-being.

\section{DISCUSSION}

The main aim of the present study was to understand the predictive value of dyadic coping, of both workers and their partners, following a work accident, in the explanation of the workers' PTSS and subjective well-being.

The workers' PTSS and subjective well-being were negatively correlated, as expected, in accordance with the current literature (Berle et al., 2018). As for the relations between dyadic coping and PTSS, results showed that the higher the use of some types of dyadic coping by the workers who had suffered a work accident, such as Stress Communication (partner), Supportive DC (both self and partner) and Delegated DC (self), and of Stress Communication (self) and Delegated DC (partner) by the partners, the lower the PTSS levels. Regarding the relations of dyadic coping with the workers' subjective wellbeing, results also revealed that the higher the workers' and their partners' own dyadic coping strategies, with the exception of Negative DC, the higher their subjective well-being. Taken together, these results support the notion that in a dyadic stress situation, both members of the couple unleash coping efforts to maintain the functioning of the relationship (Bodenmann, 2000; Revenson et al., 2005; Badr and Krebs, 2013; Traa et al., 2015). Furthermore, they are consistent with studies of the last decade, confirming that dyadic coping is significantly related to relationship satisfaction and subjective well-being (Bodenmann et al., 2011). Positive dyadic coping is actually regarded as a central dimension to relationship quality and the partner's wellbeing, as it enhances mutual trust, respect, commitment, and a sense of the relationship being comforting and supportive (Bodenmann, 2000).

Contrary to previous epidemiological studies pinpointing both gender and age differences in PTSS (Ditlevsen and Elklit, 2010), with women usually having a higher risk than men of developing PTSS after a traumatic event (Olff, 2017), in this study these demographic variables did not predict significantly the workers' PTSS, although age proved to be a positive significant predictor of their subjective well-being. Results of prior research are inconsistent with regard to the relationships between subjective well-being and age or gender, and these relations appear to be dependent on various psychological and cultural features (Lucas and Gohm, 2000).

The dyadic coping variables, of both the workers and their partners, explained $31.2 \%$ of the workers' PTSS, and $68.7 \%$ of their subjective well-being. These results are in line with previous literature pointing to the positive repercussions of dyadic coping strategies used by couples to deal with cancer, in terms of the patients' psychosocial adjustment and relationship functioning (e.g., Badr and Krebs, 2013). Similarly, recent research, for instance in the context of severe illness of one partner, has highlighted that, in general, more positive dyadic coping styles correlate with higher relationship quality and satisfaction, but also with higher quality of life (Bodenmann, 2000; Revenson et al., 2005; Meier et al., 2011).

Furthermore, although the workers' demographic and dyadic coping variables were not significant predictors of their PTSS, the partner's Supportive DC (self) and Delegated DC (partner) 
TABLE 2 | Results of the hierarchical multiple regression analyses in the predictors of the workers' PTSD and subjective well-being.

\begin{tabular}{|c|c|c|c|c|c|c|c|c|c|c|c|c|}
\hline & \multicolumn{6}{|c|}{ PTSD } & \multicolumn{6}{|c|}{ Well-Being } \\
\hline & \multicolumn{3}{|c|}{ Model 1} & \multicolumn{3}{|c|}{ Model 2} & \multicolumn{3}{|c|}{ Model 1} & \multicolumn{3}{|c|}{ Model 2} \\
\hline & $B$ & SE B & $\beta$ & $B$ & SE B & $\beta$ & $B$ & SE B & $\beta$ & $B$ & SE B & $B$ \\
\hline Workers' Gender & 3.962 & 4.908 & 0.104 & -5.837 & 5.942 & -0.154 & -4.399 & 3.976 & -0.138 & 1.752 & 3.364 & 0.055 \\
\hline Workers' Age & -0.100 & 0.219 & -0.059 & -0.182 & 0.263 & -0.107 & 0.353 & 0.177 & $0.249^{*}$ & 0.588 & 0.149 & $0.414^{* * *}$ \\
\hline $\begin{array}{l}\text { Workers' Stress } \\
\text { Communication by P. }\end{array}$ & & & & 1.128 & 1.362 & 0.207 & & & & -0.044 & 0.771 & -0.010 \\
\hline $\begin{array}{l}\text { Workers' Supportive } \\
\text { DC by S. }\end{array}$ & & & & -2.631 & 1.445 & -0.500 & & & & -0.006 & 0.818 & -0.001 \\
\hline $\begin{array}{l}\text { Workers' Delegated DC } \\
\text { by S. }\end{array}$ & & & & -2.197 & 1.712 & -0.243 & & & & 0.623 & 0.969 & 0.082 \\
\hline $\begin{array}{l}\text { Workers' Delegated DC } \\
\text { by P. }\end{array}$ & & & & 2.697 & 1.457 & 0.324 & & & & 1.813 & 0.825 & $0.260^{*}$ \\
\hline $\begin{array}{l}\text { Workers' Negative DC } \\
\text { by P. }\end{array}$ & & & & -0.247 & 0.981 & -0.045 & & & & 0.641 & 0.555 & 0.139 \\
\hline Workers' Joint DC & & & & -0.427 & 1.276 & -0.083 & & & & 1.692 & 0.723 & $0.392^{*}$ \\
\hline $\begin{array}{l}\text { Partners' Stress } \\
\text { Communication by S. }\end{array}$ & & & & -1.607 & 1.361 & -0.243 & & & & 1.182 & 0.771 & 0.212 \\
\hline $\begin{array}{l}\text { Partners' Stress } \\
\text { Communication by P. }\end{array}$ & & & & -1.207 & 1.517 & -0.203 & & & & 0.706 & 0.859 & 0.142 \\
\hline $\begin{array}{l}\text { Partners' Supportive } \\
\text { DC by S. }\end{array}$ & & & & -3.409 & 1.414 & $-0.553^{*}$ & & & & -1.591 & 0.800 & $-0.308^{*}$ \\
\hline $\begin{array}{l}\text { Partners' Supportive } \\
\text { DC by P. }\end{array}$ & & & & 3.149 & 1.120 & $0.828^{* *}$ & & & & -0.740 & 0.634 & -0.232 \\
\hline $\begin{array}{l}\text { Partners' Delegated DC } \\
\text { by P. }\end{array}$ & & & & -8.481 & 2.901 & $-0.885^{* *}$ & & & & 4.577 & 1.643 & $0.569 * *$ \\
\hline $\begin{array}{l}\text { Partners' Negative DC } \\
\text { by S. }\end{array}$ & & & & 0.712 & 0.809 & 0.139 & & & & -0.693 & 0.458 & -0.161 \\
\hline $\begin{array}{l}\text { Partners' Negative DC } \\
\text { by P. }\end{array}$ & & & & 0.051 & 0.874 & 0.009 & & & & -0.954 & 0.495 & -0.208 \\
\hline Partners' Joint DC & & & & 4.136 & 1.319 & $0.718^{* *}$ & & & & -0.828 & 0.747 & -0.171 \\
\hline$R^{2}$ & & 0.015 & & & 0.492 & & & 0.083 & & & 0.769 & \\
\hline$F$ for change in $R^{2}$ & & 0.443 & & & $3.021^{* *}$ & & & 2.672 & & & $9.547^{* * *}$ & \\
\hline Adjusted $R^{2}$ & & -0.019 & & & 0.312 & & & 0.052 & & & 0.687 & \\
\hline$F$ & & 0.443 & & & $2.726^{* *}$ & & & 2.672 & & & $9.365^{* * *}$ & \\
\hline
\end{tabular}

P., Partner; S., Self; DC, Dyadic Coping; ${ }^{*} p<0.05,{ }^{* *} p<0.01,{ }^{* * *} p<0.001$.

were negative predictors, and Supportive DC (partner) and Joint DC were positive predictors of the workers' PTSS. Additionally, all dyadic coping strategies were significant positive predictors of the workers' subjective well-being, with the exception of the partners' Supportive DC (self) that appeared to be a negative predictor. Globally, these results reveal the partners' dyadic coping strategies as a set of efforts to deal with the work accident stressor of the workers (Bodenmann, 1995) and are consistent with a recent review on severe diseases (Traa et al., 2015), which showed the importance of stress communication, supportive behaviors, and positive dyadic coping for the maintenance or enhancement of relationship functioning in couples coping with cancer. On the other hand, although Bodenmann (2005) suggested that supportive and common dyadic coping are positive forms of coping, the present results of the predictive value of the partners' supportive and joint dyadic coping are more in line with previous studies describing the negative effects of positively intended dyadic coping strategies (e.g.,
Manne and Badr, 2008). Thus, they reinforce the claim that coping functions do not reveal anything $a$ priori as to what the effects of a specific type of coping will be (Folkman and Moskowitz, 2004).

\section{CONCLUSION}

A work accident can be a stressor event not only for the worker but also for his/her partner, and the strategies used by both members of the couple may prove to be inefficient when they connect with higher levels of PTSS and lower levels of workers' subjective well-being. On the other hand, couples manage to use dyadic coping strategies that allow the workers to experience lower levels of PTSS and higher subjective well-being. These results may inform future intervention efforts following a work accident and highlight the importance of couple intervention with a view to promoting the use of dyadic coping strategies with positive effects on workers' mental health. 


\section{LIMITATIONS}

Despite the relevance of the results for theory and intervention in dyadic coping and trauma, this study presents several limitations. The first limitation regards the sample's small size and heterogeneity in terms of length of relationship and of gender distribution. Research has shown differences related to gender (and age) in PTSS levels (Ditlevsen and Elklit, 2010; Olff, 2017), which were not found in this study, possibly due to the fact that the majority of participants were men. Thus, in future research, it is important to ensure a greater balance between the gender distribution of participants. Secondly, dyadic coping following a traumatic event has been studied using other outcome variables related to marital functioning, such as conjugal satisfaction (Bodenmann, 1995, 2000), which were not considered in the present study on the trauma of work accidents and deserve to be studied in future research. Thirdly, although a convenience sample was used, not knowing the participation rate of the workers is another limitation of the study, raising issues regarding the sample's representativeness. Fourthly, the workers' and partners' perceptions of the severity of the work accident were not taken into account in the present study and future studies should control for the effect of this variable when assessing the predictive value of different types of dyadic coping strategies in the mental health of the victims of a work accident. Similarly, future research on this topic should also control for the effect of post-trauma problems with insurance companies on the mental health and subjective well-being of the victims. Additionally, as the current study is cross-sectional, reverse prediction relations between the variables can't be ruled out and PTSS and subjective well-being may have contributed to the increased/decreased use of the different dyadic coping strategies.

\section{REFERENCES}

Albuquerque, S., Pereira, M., and Narciso, I. (2015). Couple's relationship after the death of a child: a systematic review. J. Child. Fam. Stud. 25, 30-53. doi: 10.1007/s10826-015-0219-2

American Psychiatric Association (2000). Diagnostic and Statistical Manual of Mental Disorders, 4th Edn. Washington, DC: American Psychiatric Association.

Badr, H., and Acitelli, L. (2017). Re-thinking dyadic coping in the context of chronic illness. Curr. Opin. Psychol. 13, 44-48. doi: 10.1016/j.copsyc.2016. 03.001

Badr, H., and Krebs, P. (2013). A systematic review and meta-analysis of psychosocial interventions for couples coping with cancer: a systematic review and meta-analysis of psychosocial interventions for couples coping with cancer. Psycho Oncol. 22, 1688-1704. doi: 10.1002/pon. 3200

Bergstraesser, E., Inglin, S., Hornung, R., and Landolt, M. (2015). Dyadic coping of parents after death of a child. Death Stud. 39, 128-138. doi: 10.1080/07481187. 2014.920434

Berle, D., Hilbrink, D., Russel-Williams, C., Kiely, R., Hardaker, N., Garwood, N., et al. (2018). Personal wellbeing in posttraumatic stress disorder (PTSD): association with PTSD symptoms during and following treatment. BMC Psychol. 6:7. doi: 10.1186/s40359-0180219-2

Bodenmann, G. (1995). A systemic-transactional conceptualization of stress and coping in couples. Swiss J. Psychol. 54, 34-49.

Bodenmann, G. (2000). Stress und Coping bei Paaren [Stress and coping in couples]. Göttingen: Hogrefe.
Finally, the one-time evaluation design used in this study also entails a high risk of not controlling for some variables, and consequently it would be of great relevance to investigate the dyadic coping efforts after a work accident using a longitudinal study design. Therefore, evaluation across time of subjective wellbeing, PTSS, and dyadic coping strategies should be considered in future studies, with the further aim of designing well-grounded therapeutic guidelines.

\section{ETHICS STATEMENT}

This study was carried out in accordance with the recommendations of the Ethics Committee of the Faculty of Psychology, University of Lisbon. All participants gave written consent in accordance with the Declaration of Helsinki.

\section{AUTHOR CONTRIBUTIONS}

SL prepared the study design, organized the sample recruitment, collected data, and contributed to the writing of the manuscript's introduction, methods, results, discussion, prepared the tables and references sections. AM-P prepared the study design, performed statistical analyses and prepared the tables, contributed to the writing of the manuscript's introduction, results, and discussion. RF prepared the study design, performed statistical analyses and contribute to the writing of the introduction, methods, results, and discussion sections. SC-R contributed to write all sections of the manuscript. MR contributed to write all sections of the manuscript. All authors reviewed and approved manuscript for publication.

Bodenmann, G. (2005). "Dyadic coping and its significance for mrital functioning," in Couples Coping with Stress: Emerging Perspectives on Dyadic Coping, eds T. Revenson, K. Kayser, and G. Bodenmann (Washington, DC: American Psychological Association), 33-49. doi: 10.1037/110 31-002

Bodenmann, G. (2008). Dyadisches Coping Inventar (DCI). Test manual [Dyadic Coping Inventory (DCI). Test manual]. Bern: Huber.

Bodenmann, G., Charvoz, L., Widmar, K., and Bradbury, T. (2004). Differences in individual and dyadic coping among low and high depressed partially remitted, and nondepressed persons. J. Psychopathol. Behav. Assess 26, $75-85$.

Bodenmann, G., Meuwly, N., and Kayser, K. (2011). Two conceptualizations of dyadic coping and their potential for predicting relationship quality and individual well-being: A comparison. Eur. Psychol. 16, 255-266. doi: 10.1027/ 1016-9040/a000068

Bodenmann, G., Pihet, S., and Kayser, K. (2006). The relationship between dyadic coping and marital quality: a 2-year longitudinal study. J. Fam. Psychol. 20, 485-493. doi: 10.1037/0893-3200. 20.3.485

Carvalho, J., Pereira, N., Pinto, A., and Maroco, J. (2016). Psychometric properties of the mental health continuum-short form: a study of portuguese speaking children/youths. J. Child. Fam. Stud. 25, 2141-2154. doi: 10.1007/s10826-0160396-7

Costa-Ramalho, S., Marques-Pinto, A., and Ribeiro, M. (2017). The retrospective experience of climate in the family-of-origin and dyadic coping in couple relaionships: pathways to dyadic adjustment. J. Fam. Stud. 23, 3. doi: 10.1080/ 13229400.2015.1131732 
Ditlevsen, D. N., and Elklit, A. (2010). The combined effect of gender and age on posttraumatic stress disorder: do men and women show differences in the lifespan distribution of the disorder? Ann. Gen. Psychiatry 9:32. doi: 10.1186/ 1744-859X-9-32

Eurostat (2018). Accidents at Work by Economic Activity and Size of Enterprise. Availableat: http://ec.europa.eu/eurostat/web/products-datasets/-/hsw_n2_05

Eurostat (2013). European Statistics on Accidents at Work. Available at: http://ec.europa.eu/eurostat/documents/3859598/5926181/KS-RA-12-102EN.PDF/56cd35ba-1e8a-4af3-9f9a-b3c47611ff1c

Field, A. P. (2009). Discovering Statistics Using SPSS: and Sex and Drugs and Rock ' $n$ ' roll, 3rd Edn. London: Sage.

Folkman, S., and Moskowitz, J. T. (2004). Coping: pitfalls and Promise. Annu. Rev. Psychol. 55, 745-774. doi: 10.1146/annurev.psych.55.090902.141456

Gabriel, B., Bodenmann, G., and Beach, S. (2016). Gender differences in observed and perceived stress and coping in couples with a depressed partner. Open J. Depress. 5, 7-20. doi: 10.4236/ojd.2016.52002

Gilber, O., Weinberg, M., and Gil, S. (2011). The effects of coping strategies on PTSD in victims of a terror attack and their spouses. J. Soc. Pers. Relat. 29, 246-261. doi: 10.1177/0265407511426939

Gustafsson, M., and Ahlström, G. (2004). Problems experienced during the first year of an acute traumatic hand injury: a prospective study. J. Clin. Nurs. 13, 986-995. doi: 10.1111/j.1365-2702.2004.01019.x

Hansen, N., Larsen, P., Nicolaisen, A., Flyger, H., Johansen, C., and Hagedorn, M. (2015). Dyadic coping within couples dealing with breast cancer: a longitudinal, population-based study. Health Psychol. 34, 486-495. doi: 10.1037/hea000 0218

Karney, B., and Crown, J. (2007). Families Under Stress: An Assessment of Data, Theory, and Research on Marriage and Divorce in the Military. Washington, DC: National Defense Research Institute.

Keyes, C. L. M. (1998). Social well-being. Soc. Psychol. Q. 61, 121-140. doi: 10.2307/ 2787065

Kramer, U., Ceschi, G., Van der Linden, M., and Bodenmann, G. (2005). Individual and dyadic coping strategies in the aftermath of a traumatic experience. Swiss J. Psychol. 64, 241-248. doi: 10.1024/1421-0185.64.4.241

Lafaye, A., Petit, S., Richaud, P., Hovédé, N., Baguet, F., and Cousson-Gélie, F. (2014). Dyadic effects of coping strategies on emotional state and quality of life in prostate cancer patients and their spouses. Psych. Onco. 23. doi: 10.1002/pon. 3483

Lazarus, R., and Folkman, S. (1984). Stress, Appraisal and Coping. Springer Publishing Company.

Lucas, R., and Gohm, C. (2000). Culture and Subjective Well-being, eds E. Diener and E. M. Suh Cambridge, MA: MIT Press.

MacDonald, H., Colotla, V., Flamer, S., and Karlinsky, H. (2003). Post-traumatic stress disorder (PTSD) in the workplace: a descriptive study of workers experiencing PTSD resulting from work injury. J. Occup. Rehabil. 13, 63-77. doi: 10.1023/A:1022563930482

Manne, S., and Badr, H. (2008). Intimacy and relationship processes in couples' psychosocial adaptation to cancer. Cancer 112, 2541-2555. doi: 10.1002/ cncr. 23450

Marcelino, D., and Gonçalves, S. (2012). Perturbação pós-stress traumático: características psicométricas da versão portuguesa da posttraumatic stress disorder checklist - civilian (PCL-C) [Posttraumatic stress disorder: psychometric characteristics of the Portuguese version of Posttraumatic Stress Disorder Checklist - Civilian Version (PCL-C)]. Rev. Port. Saúde Pública 30, 71-75. doi: 10.1016/j.rpsp.2012.03.003

Marshall, E., Kuijer, R., Simpson, J., and Szepsenwol, O. (2017). Standing on shaky ground? Dyadic and longitudinal associations between posttraumatic stress and relationship quality post-earthquake. J. Fam. Psychol. 31, 721-733. doi: $10.1037 /$ fam0000305

Meier, C., Bodenmann, G., Mörgeli, H., and Jeneweinm, J. (2011). Dyadic coping, quality of life, and psychological distress among chronic obstructive pulmonary disease patients and their partners. Int. J. Chron. Obstruct. Pulm. Dis. 6, 583-595. doi: 10.2147/COPD.S24508

National Center for Posttraumatic Stress Disorder [NCPTSD] (2018). Using the PTSD Checklist for DSM-VI (PCL). Available at: https://www.ptsd.va.gov/ professional/assessment/documents/PCL_handoutDSM4.pdf

Olff, M. (2017). Sex and gender differences in post-traumatic stress disorder: an update. Eur. J. Psychotraumatol. 8:1351204. doi: 10.1080/20008198.2017. 1351204

Pordata (2018). Acidentes de trabalho: Total e mortais (Accidents at work: Total and mortal). Available at: http://www.pordata.pt/Portugal/Acidentes+de+trabalho+ total+e+mortais- 72

Revenson, T., Kayser, K., and Bodenmann, G. (eds) (2005). Couples Coping with Stress: Emerging Perspectives on Dyadic Coping. Washington, DC: American Psychological Association. doi: 10.1037/11031-000

Schaefer, L., Lobo, B., and Kristensen, C. (2012). Transtorno de estresse póstraumático decorrente de acidentes de trabalho: implicações psicológicas, socioeconómicas e jurídicas. [Post-traumatic stress disorder resulting from accidents at work: psychological, socio-economic and legal implications]. Estud. Psicol. 17, 329-336. doi: 10.1590/S1413-294X2012000200018

Traa, M., Vries, J., Bodenmann, G., and Den Oudsten, B. (2015). Dyadic coping and relationship functioning in couples coping with cancer: a systematic review. Br. J. Health Psychol. 20, 85-114. doi: 10.1111/bjhp. 12094

Vedes, A., Nussbeck, F., Bodenmann, G., Lind, W., and Ferreira, A. (2013). Psychometric properties and validity of the dyadic coping inventory in Portuguese. Swiss J. Psychol. 72, 149-157. doi: 10.1024/1421-0185/a0 00108

Weathers, F., Litz, B., Herman, D., Huska, J., and Keane, T. (1993). "The PTSD Checklist (PCL): Reliability, validity and diagnostic utility," in Proceedings of the 9th Annual Meeting of the International Society for Traumatic Stress Studies, San Antonio, TX.

Conflict of Interest Statement: The authors declare that the research was conducted in the absence of any commercial or financial relationships that could be construed as a potential conflict of interest.

Copyright (C) 2018 Lameiras, Marques-Pinto, Francisco, Costa-Ramalho and Ribeiro. This is an open-access article distributed under the terms of the Creative Commons Attribution License (CC BY). The use, distribution or reproduction in other forums is permitted, provided the original author(s) and the copyright owner(s) are credited and that the original publication in this journal is cited, in accordance with accepted academic practice. No use, distribution or reproduction is permitted which does not comply with these terms. 


\section{OPEN ACCESS}

Edited by:

Guy Bodenmann,

University of Zurich, Switzerland

Reviewed by:

Nathalie Meuwly,

Université de Fribourg, Switzerland

Reza Fallahchai,

University of Hormozgan, Iran

${ }^{*}$ Correspondence:

Elena Canzi

elena.canzi@unicatt.it

Specialty section:

This article was submitted to

Clinical and Health Psychology, a section of the journal

Frontiers in Psychology

Received: 18 June 2018

Accepted: 11 February 2019

Published: 06 March 2019

Citation:

Canzi E, Donato S, Ferrari L,

Parise M, Pagani AF, Lopez G, Rosnati $R$ and Ranieri S (2019) "What Makes Us Strong?": Dyadic Coping

in Italian Prospective Adoptive Couples. Front. Psychol. 10:399. doi: 10.3389/fpsyg.2019.00399

\section{"What Makes Us Strong?": Dyadic Coping in Italian Prospective Adoptive Couples}

\author{
Elena Canzi ${ }^{*}$, Silvia Donato², Laura Ferrari², Miriam Parise ${ }^{1}$, Ariela Francesca Pagani ${ }^{1}$, \\ Giulia Lopez' ${ }^{1}$, Rosa Rosnati ${ }^{2}$ and Sonia Ranieri ${ }^{3}$
}

${ }^{1}$ Family Studies and Research University Centre, Università Cattolica del Sacro Cuore, Milan, Italy, ${ }^{2}$ Department of Psychology, Family Studies and Research University Centre, Università Cattolica del Sacro Cuore, Milan, Italy, ${ }^{3}$ Department of Psychology, Family Studies and Research University Centre, Università Cattolica del Sacro Cuore, Piacenza, Italy

Becoming an adoptive parent is a particularly stressful transition, given the additional challenges couples have to face. Dyadic coping, an under-investigated dimension in the adoption literature, may play a relevant role for prospective adoptive couples' ability to better cope with the adoptive process. The general aim of the present study was to investigate the association between dyadic coping and relationship functioning, in terms of relationship satisfaction and couple generativity, among prospective adoptive couples. Participants were 103 prospective adoptive couples pursuing international adoption in Italy. Couples were asked to fill in a self-report questionnaire. Results of the Actor-Partner Interdependence Model showed that prospective adoptive partners reported high levels of positive and common dyadic coping and low levels of negative dyadic coping - suggesting partners' ability to successfully cope together with a common stressor - a high level of relationship satisfaction, and an average level of couple generativity. Moreover, analyses showed significant actor effects of one's own perception of the partner's dyadic coping (positive, negative, and common) on one's own relationship satisfaction and on couple generativity for both wives and husbands. With regard to partner effects, we found that both partners' perceptions of the other's dyadic coping responses (positive, negative, and common) were associated with the other's relationship satisfaction, with the only exception of wives' perceptions of common dyadic coping, which were not associated with their husbands' relationship satisfaction. As for couple generativity, the only significant partner effect referred to negative dyadic coping responses for both wives and husbands.

\footnotetext{
Keywords: prospective adoptive couples, dyadic coping, relationship satisfaction, couple generativity, actor partner interdependence model
}

\section{INTRODUCTION}

\section{Prospective Adoptive Couples: Stressors and Resources}

Becoming parents is a crucial family transition associated with significant relational, psychological, and social changes. Prospective adoptive couples have to face specific challenges and tasks that make them particularly vulnerable to stress (Canzi et al., 2017b). Most prospective adoptive couples, for example, faced infertility (Cohen et al., 1993; Daniluk and Hurtig-Mitchell, 2003), struggling with the related elaboration process. In addition, they are going through the assessment procedures to 
obtain adoption suitability, interfacing with bureaucratic systems, which are very demanding and stressful (Palacios and Sánchez-Sandoval, 2006). On the psychological side, moreover, during the pre-adoption phase, they could experience the anticipatory stress related to concerns about the child and the first encounter with him/her; they also have to prepare to legitimize each other as parents of a child "born by others," the so-called entitlement process (Cohen et al., 1996). Prospective adoptive partners often become parents late in life (in Italy in 2015, the average age for the husband was 45.8 years and for the wife was 44.1 years), after a long waiting period (3 years and 7 months on average in Italy in 2015) (Commission for Intercountry Adoption, 2014/2015), and they expect to cope with children who are likely to be emotionally and behaviorally compromised at arrival, due to their past experiences (i.e., abandonment, neglect, institutionalization; Canzi et al., 2018). All these stressors are likely to impact on couples' psychological well-being (Goldberg et al., 2010) as well as on their future adjustment to parenthood (Salcuni et al., 2015). Indeed, the pre-adoption phase is so demanding that, between 2006 and 2015 , only the $68.6 \%$ of Italian couples obtaining the decree of suitability have given a mandate to the adoption agency; among them, only the $66.4 \%$ has completed the entire adoption procedure applying for an authorization allowing foreign children to enter Italy (Commission for Intercountry Adoption, 2014/2015). Therefore, more than one-third of the decrees of approval that are issued became ineffective with a great loss of money, resources, and opportunities for these couples as well as for children awaiting adoption. These data suggest that to successfully cope with this demanding pre-adoption phase, couples are required to pool all their individual and relational resources, especially marital ones.

Despite the centrality of this phase of preparation and adaptation for the adoption process, relatively few studies have been conducted on prospective adoptive couples. Most of these studies especially focused on parents' socio-demographic characteristics, motivations, expectations, ethnic prejudice, and personal well-being. Generally, prospective adoptive couples resulted to have personal resources, in terms of psychological well-being, emotional stability, low levels of ethnic prejudice, and high positive intergroup contacts (Deater-Deckard and Petrill, 2004; Bausch, 2006; Salcuni et al., 2006; Welsh et al., 2008; Zhang and Lee, 2010; Park and Hill, 2014; Canzi et al., 2017a). Much less investigated were their relational and marital resources (e.g., Levy-Shiff et al., 1990). Although research conducted on adoptive parents during later stages of the adoption transition evidenced a global positive quality of adoptive couples' relationship (Lansford et al., 2001; Ceballo et al., 2004; Rosnati et al., 2013; Canzi et al., 2017b), we do not know much about prospective adoptive couples' relationship more generally and about their coping ability more specifically. Nonetheless, a study examining adoptive couples relationship quality across the transition to adoption shows that pre-adoptive coping resources represent a protective factor against a pre to post-adoption decrease in satisfaction (Goldberg et al., 2010). Given the peculiar stressors faced by prospective adoptive couples, in fact, partners' ability to cope jointly against stressful events (i.e., dyadic coping) may be considered as a functional skill that can help to overcome these challenging situations as well as strengthen their relationship (Bodenmann, 1997, 2005).

\section{Dyadic Coping: A Resource for Couples' Functioning}

Dyadic coping refers to a dyadic process in which partners cope together with stressful circumstances. Among the different conceptualizations of dyadic coping (cfr. Iafrate and Donato, 2012; Acquati and Saita, 2017), we focused here on the Systemic Transactional Model (STM) developed by Bodenmann (1995; 1997; 2000). Within the STM, stress can be conceptualized not only as an individual phenomenon, but also as a dyadic event: Dyadic stress refers to those circumstances that affect (either directly or indirectly through the other partner's stress) both members of the couple and elicit joint appraisal of the situation as well as common coping responses to it (Bodenmann, 1995; Lyons et al., 1998). Specifically, dyadic coping is a process in which one partner's communication of stress is perceived, decoded and evaluated by the other, who then responds with his/her coping reactions. Such responses can be either (emotion-oriented or problem-oriented) supportive behaviors one partner enacts toward the other (e.g., one partner showing understanding or offering solutions) or common responses both partners engage in to cope with stress together (e.g., joint problem solving, relaxing together, etc.). The aim of dyadic coping is twofold: It is intended to restore or maintain both partners' individual wellbeing, by reducing the partners' levels of stress, and to promote couple functioning, by strengthening partners' sense of we-ness and reciprocal trust (Cutrona, 1996; Bodenmann, 2005). Dyadic coping styles, however, can also be ineffective or unskillful (i.e., the so-called negative dyadic coping). When this is the case, the coping process risks to be unsuccessful and the relationship undermined (e.g., Donato and Parise, 2012; Falconier et al., 2015). Differently from the other conceptualizations of dyadic coping, that were elaborated mostly within the context of chronic illness, Bodenmann's theory was first developed to define coping with daily hassles (minor stressors; Donato et al., 2015). Only later it was extended to critical life events (major stressors), such as acute and chronic illness (e.g., Revenson and DeLongis, 2010; Bertoni et al., 2015; Traa et al., 2015), couples' coping with normative transitions (e.g., transition to marriage, Donato et al., 2012; couples' aging, Landis et al., 2013) as well as non-normative ones (e.g., couples' facing the death of a child, Bergstraesser et al., 2015; couples dealing with a child with autism, García-López et al., 2016; step-family formation, Lee-Baggley et al., 2005).

Only one study, to our knowledge, analyzed dyadic coping in the context of adoption (Hock and Mooradian, 2012). This study examined the contributions of individual and relational characteristics (dyadic coping, dyadic adjustment, and conflict resolution styles) to the quality of adoptive mothers' co-parenting and found that higher levels of positive dyadic coping were associated with better co-parenting. Moreover, dyadic coping was a stronger predictor of co-parenting quality than conflict resolution and marital quality. This study, however, could be 
usefully extended in three respects. First, the study focused on the post-adoption phase rather than on the pre-adoption one. Second, Hock and Mooradian's (2012) study tested the effects of dyadic coping on adoptive mothers, rather than focusing on the couple as a whole. In dyadic coping, however, both partners are engaged and influential on one another. In addition, within the transition to adoptive parenthood, both partners are strongly involved and engaged from the very beginning of the process. In particular, adoptive fathers seem to represent a crucial resource for the adjustment to adoption and for children lifespan development (Ferrari et al., 2015; Ranieri et al., 2017), so that some authors have spoken of an "adoptive-enhanced fatherhood" (Levy-Shiff et al., 1997). A dyadic approach to studying dyadic coping within these couples is therefore particularly warranted. Third, Hock and Mooradian's (2012) study focused on the role of dyadic coping for adoptive mothers' parenting skills. The role that dyadic coping plays for prospective adoptive couples' marital quality is as important as its effects on their parenting skills for at least two reasons. On the one hand, given that marital functioning prior to adoption is related to adoptive parents' risk of relationship dissolution (e.g., Goldberg and Garcia, 2015), it is worth investigating those factors that can foster these couples' relationship. On the other, given the links between marital quality and parenting skills, well-documented in the general population (Erel and Burman, 1995; Margolin et al., 2001; Stone et al., 2002; Bradford et al., 2003; Stright and Bales, 2003), examining predictors of prospective adoptive couples' marital functioning would also suggest a potential way to indirectly promote their parental competences.

\section{The Present Study}

The goal of this study was to investigate the association between dyadic coping and relationship functioning among Italian prospective adoptive couples. Specifically, we focused on two different aspects of relationship functioning: Relationship satisfaction and couple generativity. While research generally identifies as one of the main outcomes of couple relationship with relational satisfaction, couple generativity is relatively underinvestigated. A well-known psychological theory by Erikson (1963) postulates that for the adult individual the most important developmental outcome is not the achievement of a mere wellbeing, rather it refers to the unfolding of his/her capacity of being "generative." Generativity is the ability to move away from a narcissistic self-concern to take care of those who are to follow (Erikson, 1963; McAdams et al., 1993). Generativity does not refer exclusively to procreation (i.e., the biological level), but may be expressed also at the social level, by taking care of future generations through teaching, mentoring, political engagement as well as engagement with youth protection and health. Inspired by this theory, some scholars (Cigoli and Scabini, 2006; Parise et al., 2017) have started to argue that the good functioning of a relationship cannot be measured only in terms of relationship satisfaction, but it may involve also the ability to go beyond one's boundaries as a couple and to take care of social bonds. Couple generativity seems a relevant component of prospective adoptive couples' functioning, as adoption in itself can be considered a form of social generativity (Cigoli and
Scabini, 2006; Scabini and Rossi, 2014). Research on community couples has found that couple generativity is related to partners' trust, intimacy, commitment, and romantic affect (Bertoni et al., 2012), but no studies investigated generativity (nor the role of dyadic coping for it) in prospective adoptive couples.

\section{MATERIALS AND METHODS}

\section{Participants and Procedure}

Participants were 103 heterosexual prospective adoptive couples living in the North of Italy. Couples were contacted in the process of completing international adoptions, before the actual arrival of the child. All partners ( $N=206$ individuals) were married. The exclusion criterion was having already one or more children at the time of the study. Wives' average age was $40.2(S D=4.1)$, ranging from 29 to 46 years, and husbands' average age was 41.8 $(S D=4.4)$, ranging from 29 to 57 years. Sixty-two point one percent of couples have resorted to assisted reproductive technology, on average $3.44(S D=2)$ times (range $1-8)$. The average duration of marriage was 8.4 years $(S D=4.2)$ and ranged from 1 to 19 years. All couples had attained a medium-high level of education: $54 \%$ of wives and $57 \%$ of husbands had up to 13 years of education, while the remainder had studied for 16 years or more. All participants were recruited through advertisements placed in different venues and contexts (e.g., schools, family associations, and adoption agencies) and through snowball sampling. Participants were given two self-report questionnaires, one for the wife and one for the husband, and were asked to complete their respective questionnaire independently from their partner. Anonymity and data confidentiality were guaranteed. All participants took part in the study voluntarily and gave informed and written consent. The study protocol was not reviewed by the ethics committee, since it was not required at the time of data collection, according to the local and national guidelines. However, it followed the standard ethical guidelines of the Italian Association of Psychology (AIP) and the standard ethical guidelines of the American Psychological Association (APA).

\section{Measures}

The instrument used was a self-report questionnaire composed of the following scales.

\section{Dyadic Coping}

To measure dyadic coping we used the Dyadic Coping Questionnaire (Fragebogen zur Erfassung des Dyadischen Copings als stabile Tendenz; FDCT-N, Bodenmann, 1997; Donato et al., 2009). This scale is composed of 41 items on a 5 -point scale (from $1=$ never to $5=$ very often) and measures the processes involved in dyadic coping, including stress communication, dyadic coping responses, and satisfaction with dyadic coping (Bodenmann, 1995, 1997, 2005). In this study, we considered the subset of items referring to the perceptions of the other's dyadic coping responses. First, we assessed perceptions of the other's positive dyadic coping (seven items), that is the extent to which the responses of the partner to one's own stress are supportive. Sample item is: "When I am stressed, my 
partner shows me his/her interest and understanding." Second, we assessed perceptions of the other's negative dyadic coping (five items), that is, the extent to which the partner's responses to one's own stress are perceived as negative. Sample item is: "My partner makes fun of my stress and mocks me." Third, we assessed perceptions of common dyadic coping (seven items), that is how both partners respond to communicated stress. Sample item is: "We try to cope with the problem together and search for practical solutions." We created a global index of positive dyadic coping responses ( $\alpha=0.74$ wives and $\alpha=0.72$ for husbands), a global index of negative dyadic coping responses ( $\alpha=0.53$ wives and $\alpha=0.58$ for husbands), and a global index of common dyadic coping responses ( $\alpha=0.76$ wives and $\alpha=0.72$ for husbands) by averaging the corresponding items. A higher score indicated a higher level of the corresponding dyadic coping response.

\section{Relationship Satisfaction}

To measure relationship satisfaction we used the Quality of Marriage Index (Norton, 1983). The scale is a six-item inventory: The first five items (e.g., "The relationship with my partner makes me happy") are on a 7 -point scale $(1=$ completely disagree, 7 = completely agree), whereas the last item, measuring a global perception of relationship satisfaction, is on a 10 -point scale ( $1=$ very unhappy, $10=$ very happy). We used the first five items and averaged them to a global index of satisfaction $(\alpha=0.91$ for wives and $\alpha=0.90$ for husbands). A higher score indicated a higher level of relationship satisfaction.

\section{Couple Generativity}

Couple generativity was assessed through the Couple Generativity Scale (Parise et al., 2017), which is composed of four items on a 9-point scale (from 1 = completely disagree to 9 = completely agree). Items are: "We are committed as a couple to our community"; "We are a reference point for our friends"; "We think that our experience as a couple can be made available to other people"; "Our friends often asks for advice to us as a couple." The items were averaged to form a global index of couple generativity and showed good internal consistency ( $\alpha=0.77$ wives and $\alpha=0.76$ for husbands). A higher score indicated a higher level of couple generativity.

\section{Data Analyses}

To deal with data interdependence, we used the actor-partner interdependence model (APIM, Kenny, 1996; Kenny and Cook, 1999) for testing the association of dyadic coping responses (positive, negative, and common) with relationship satisfaction and couple generativity. The APIM is a dyadic data analytic approach that treats the couple as the unit of analysis. That is, the APIM estimates effects for both members of the couple simultaneously, while controlling for their interdependence (Kenny et al., 2006), and tests the interpersonal effects of one couple member's report on one's own (i.e., actor effect) and on the other member's (i.e., partner effect) outcome. We tested both actor effects and partner effects using the software AMOS 22. Finally, to examine gender differences, women's and men's paths of interest in the model were constrained to be equal and the $\chi^{2}$ difference test was performed. In case the constrained model showed no significantly different fit from the unconstrained one, the constrained, more parsimonious model was retained. In the figures, whenever no gender differences emerged, we presented pooled coefficients across genders as final estimates of the empirical models.

\section{RESULTS}

\section{Descriptives}

The sample was composed of partners that generally reported to perceive the other as highly supportive (i.e., providing a high level of positive dyadic coping and a low level of negative dyadic coping), to successfully cope together with a common stressor, showing similar or slightly better dyadic coping abilities than reported in other Italian samples (Donato et al., 2015, 2018; Parise et al., 2018). Moreover, they reported high levels of relationship satisfaction, and average levels of couple generativity (see Table 1). As for gender differences, wives reported higher levels of common dyadic coping $[t(102)=2.86, p=0.005]$ and couple generativity $[t(102)=2.94, p=0.004]$ than husbands. No other significant gender differences emerged. Correlations between dyadic coping indexes as well as between dyadic coping and outcomes were as expected (see Table 1). In particular, in both wives and husbands, positive and common dyadic coping were positively correlated with each other and negatively with negative dyadic coping. Again in both wives and husbands, positive and common dyadic coping were positively associated with both relationship satisfaction and couple generativity, while negative dyadic coping was negatively correlated with the above outcomes. Relationship satisfaction and couple generativity were positively correlated with each other, but correlations were low to moderate in size, thereby suggesting that the two constructs were not overlapping.

\section{Associations Between Perceptions of Dyadic Coping Responses and Relationship Satisfaction}

As for the association between perceptions of dyadic coping responses (positive, negative, and common) and relationship satisfaction, all models showed significant actor effects of one's own perception of the partner's dyadic coping on one's own relationship satisfaction for both wives and husbands (Figures 1-3). That is, one's perceptions of the other as supportive (i.e., positive DC) as well as the couple as a good team in coping with stress (i.e., common DC) were positively associated with one's own relationship satisfaction. One's perceptions of the other as unsupportive (i.e., negative DC) were negatively related to one's own relationship satisfaction. No gender differences were found in actor effects. With regard to partner effects, we found that both partners' perceptions of dyadic coping responses also predicted the other partner's relationship satisfaction, with the only exception of wives' perceptions of common dyadic coping which were not associated with their husbands' relationship satisfaction. Specifically, partners' perceptions of the other as supportive 
TABLE 1 | Correlations, means, and SD of the variables of the study.

\begin{tabular}{|c|c|c|c|c|c|c|c|}
\hline Variable & 1 & 2 & 3 & 4 & 5 & $M$ & $S D$ \\
\hline 1. Positive dyadic coping & $0.31^{* * *}$ & $-0.43^{* * *}$ & $0.51^{* * *}$ & $0.48^{* * *}$ & $0.39^{* * *}$ & 3.93 & 0.59 \\
\hline 2. Negative dyadic coping & $-0.46^{* * *}$ & $0.35^{* * *}$ & $-0.27^{* *}$ & $-0.42^{* * *}$ & $-0.27^{* *}$ & 1.27 & 0.32 \\
\hline 3. Common dyadic coping & $0.44^{* * *}$ & $-0.25^{*}$ & $0.50^{* * *}$ & $0.54^{* * *}$ & $0.40^{* * *}$ & 3.91 & 0.54 \\
\hline 4. Relationship satisfaction & $0.35^{* * *}$ & $-0.37^{* * *}$ & $0.41^{* * *}$ & $0.56^{* * *}$ & $0.41^{* * *}$ & 6.51 & 0.70 \\
\hline 5. Couple generativity & $0.21^{*}$ & $-0.21^{*}$ & $0.26^{* *}$ & $0.24^{*}$ & $0.70^{* * *}$ & 5.01 & 2.00 \\
\hline M & 3.91 & 1.22 & 3.74 & 6.53 & 4.57 & & \\
\hline$S D$ & 0.51 & 0.33 & 0.63 & 0.62 & 1.87 & & \\
\hline
\end{tabular}

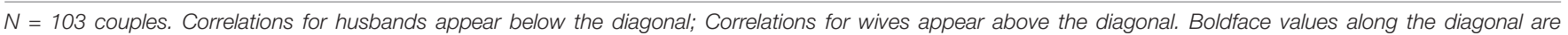

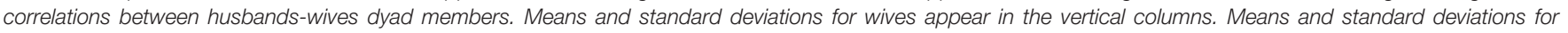
husbands appear in the horizontal columns; ${ }^{*} p<0.05,{ }^{* *} p<0.01,{ }^{* * *} p \leq 0.001$.

were positively related to the other's relationship satisfaction, while -on the contrary- partners' perceptions of the other as unsupportive were negatively related to the other's relationship satisfaction (Figures 1, 2). No gender differences were found in the above partner effects, while partner effects related to the common dyadic coping model were significantly different between husbands and wives (Figure 3). Specifically, while husbands' perceptions of common dyadic coping were positively associated with their wives' relationship satisfaction, wives' perceptions were not.

\section{Associations Between Perceptions of Dyadic Coping Responses and Couple Generativity}

As for the association between perceptions of dyadic coping responses (positive, negative, and common) and couple generativity, all the APIM models showed significant actor effects for both husbands and wives (Figures 4-6). Specifically, partners' perceptions of the other as supportive and of the couple's positive common efforts to cope with stress were positively related to their own couple generativity, while partners' perceptions of the other as unsupportive were negatively related to their own couple generativity. No gender differences were found in actor effects. With regard to partner effects, we found that partners' perceptions of the other's negative dyadic coping responses predicted the other's couple generativity (Figure 5). Specifically, partners' perceptions of negative dyadic coping from their partner negatively predicted their partner's couple generativity. No gender differences were found in the above association and no other partner effects were detected.

\section{DISCUSSION}

The present study aimed at investigating the perceptions of dyadic coping responses (positive, negative, and common) among Italian prospective adoptive couples, as well as exploring the associations with relationship functioning in terms of relationship satisfaction and couple generativity. Results showed that prospective adoptive couples reported high levels of positive dyadic coping and low levels of negative dyadic coping, suggesting partners' ability to successfully cope together with a common stressor, a high level of relationship satisfaction, and an average level of couple generativity. In line with the literature (Lansford et al., 2001; Ceballo et al., 2004; Rosnati et al., 2013; Canzi et al., 2017b), adoptive couples resulted to be well-equipped and to have relational resources, especially in terms of couple relationship functioning. It could be that couples choosing adoption are those who can count on a wide range of resources. Such resources may derive from partners' personal skills or from their good relational adjustment as well as from the experiences related to the adoption transition. Most of these couples, in fact, struggled with many critical events. Several, for example, failed assisted reproductive treatments. We can speculate that, even for couples who were not facing these experiences prior to adoption, going through the difficulties related to the decision to adopt strengthened their bond, encouraged their investment in the couple relationship, and increased their resilience. We can therefore hypothesize that the pre-adoptive period and its challenges can function somehow as a "training" process, that could enhance and promote partners' competences and resources to face the challenges related to the adjustment to adoption. This possibility is in line with models showing that challenging and stressful life experiences can benefit some couples by improving their resources and contribute to positive growth (Meichenbaum, 1985; Riley, 2013). Future research should test this possibility by evaluating the links between the level of stress experienced in the pre-adoption process, dyadic coping competences, and relationship quality in this type of couples.

Moreover, analyses evidenced that one's perceptions of the other as supportive (i.e., positive DC) as well as the couple as a good team in coping with stress (i.e., common DC) were positively associated with one's own relationship satisfaction and couple generativity. On the contrary, one's perceptions of the other as unsupportive (i.e., negative DC) were negatively related to one's own relationship satisfaction and couple generativity. Relationship satisfaction resulted to be sensitive to partner effects as well and therefore be associated to the other's perceptions of one's own dyadic coping responses, with the only exception of wives' perceptions of common dyadic coping that were not related to husbands' relationship satisfaction. Couple generativity was also predicted by the other's perceptions of one's own negative dyadic coping, while no partner effects were found for positive and common dyadic coping responses. 


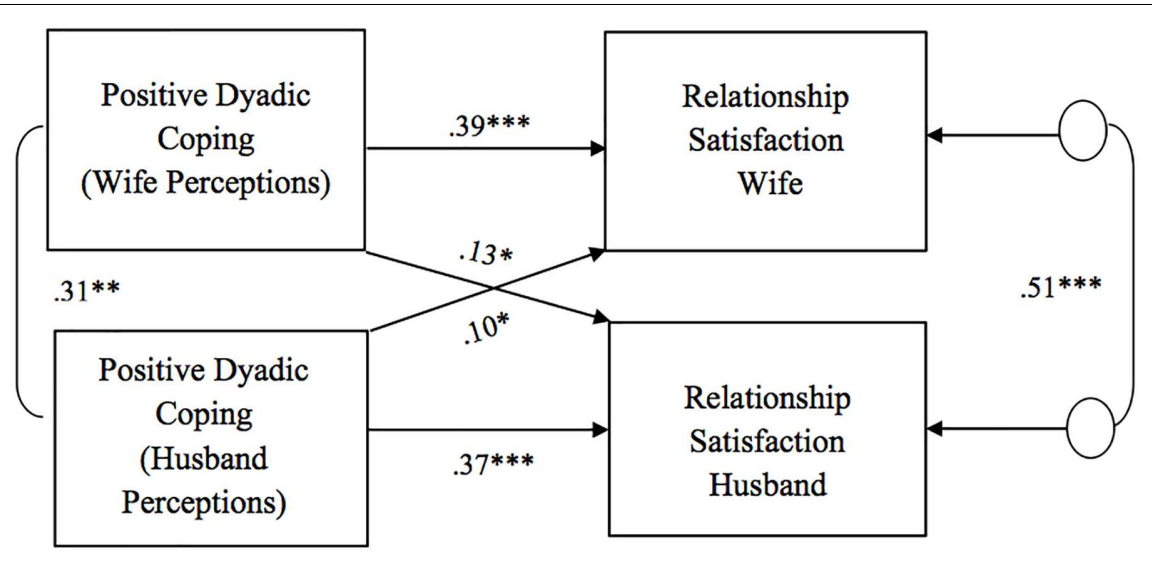

FIGURE 1 Associations between perceptions of positive dyadic coping responses and relationship satisfaction. Path coefficients are standardized estimates; ${ }^{*} p=0.05 ;{ }^{* *} p<0.01 ;{ }^{* * *} p \leq 0.001$.

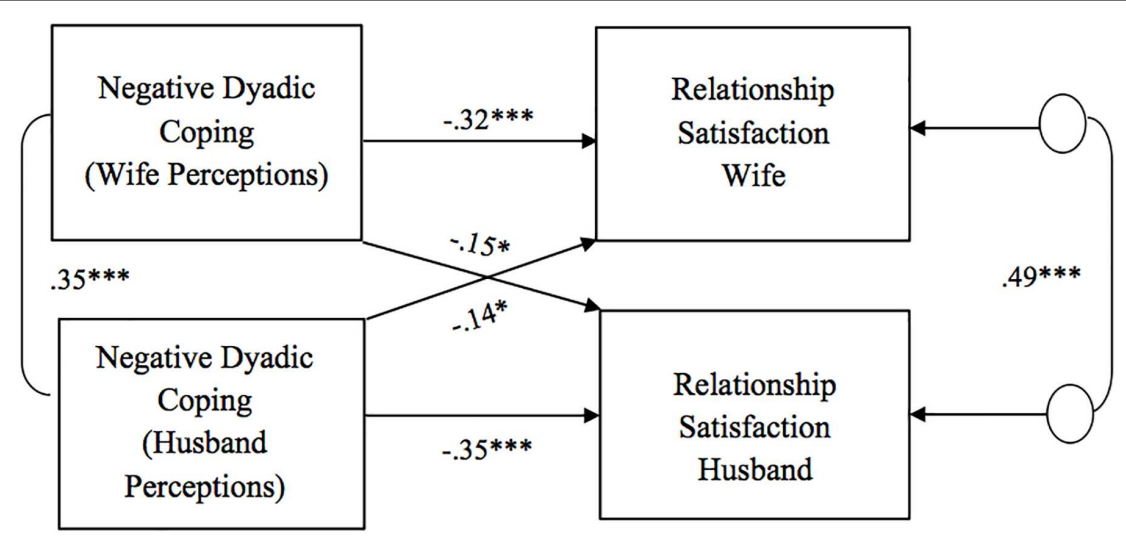

FIGURE 2 | Associations between perceptions of negative dyadic coping responses and relationship satisfaction. Path coefficients are standardized estimates; $* p<0.05 ; * * * p \leq 0.001$.

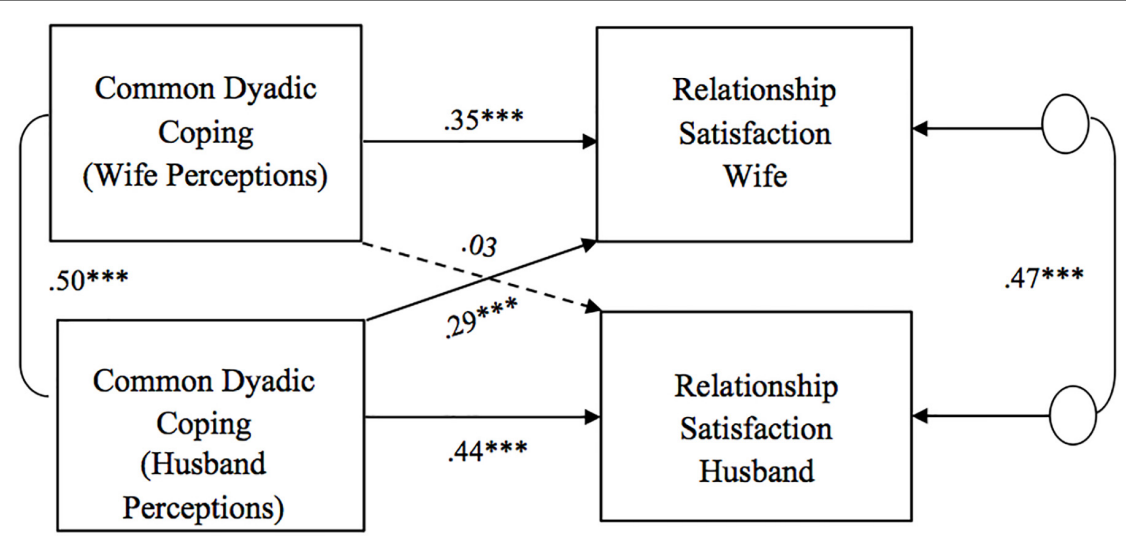

FIGURE 3 | Associations between perceptions of common dyadic coping responses and relationship satisfaction. Path coefficients are standardized estimates; $* * * p \leq 0.001$.

These findings reveal in both wives and husbands the presence of significant actor effects on relationship satisfaction and couple generativity. With regard to relationship satisfaction, these results are in line with the literature on dyadic coping in other populations (e.g., Donato et al., 2015; Hilpert et al., 2016) and suggest that, when positive, dyadic coping is a 


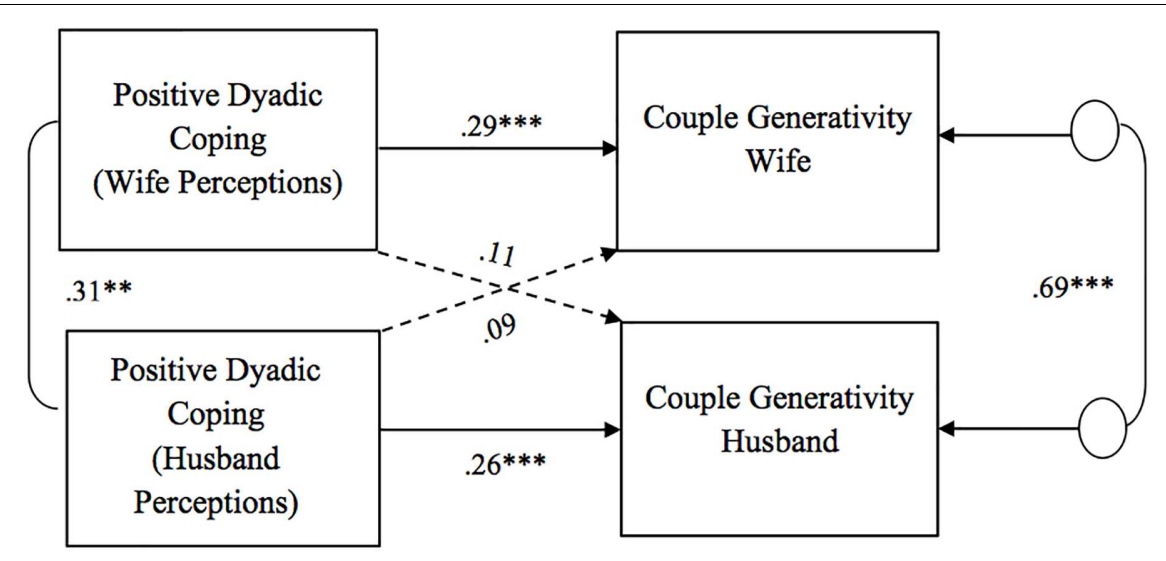

FIGURE 4 | Associations between perceptions of positive dyadic coping responses and couple generativity. Path coefficients are standardized estimates; ${ }^{* *} p<0.01 ;{ }^{* * *} p \leq 0.001$.

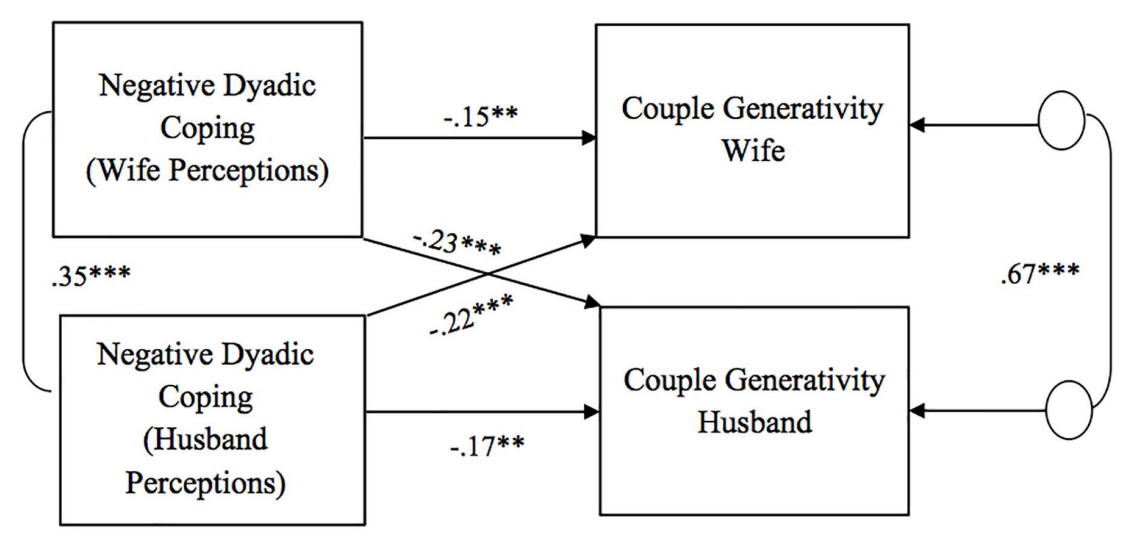

FIGURE 5 | Associations between perceptions of negative dyadic coping responses and couple generativity. Path coefficients are standardized estimates; ${ }^{* *} p<0.01 ; * * * p \leq 0.001$.

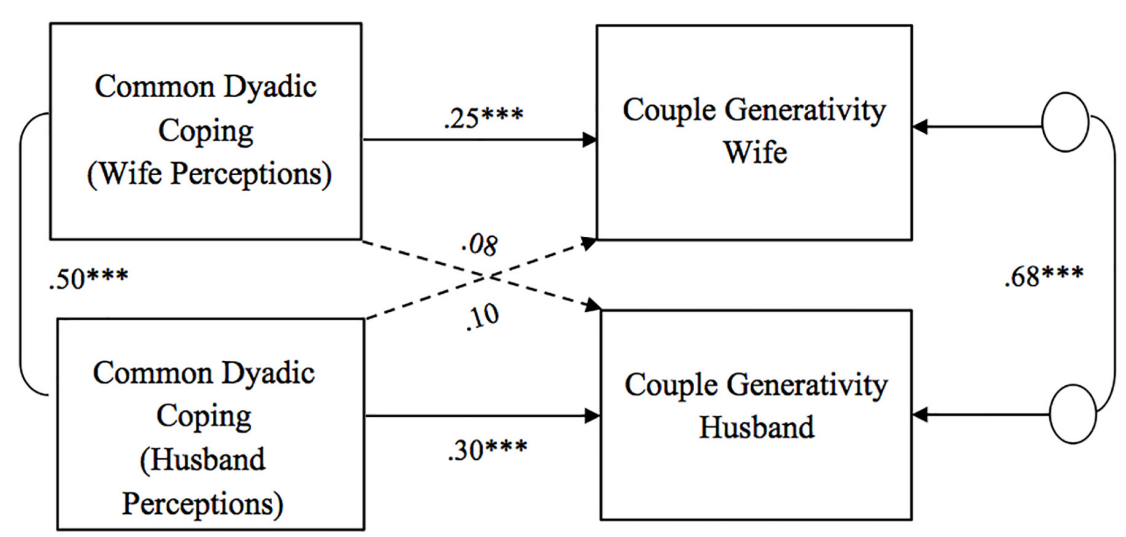

FIGURE 6 | Associations between perceptions of common dyadic coping responses and couple generativity. Path coefficients are standardized estimates; $* * * p \leq 0.001$

relevant resource for this kind of couples. Feeling supported by the other in times of stress, and feeling that both are engaged in dealing with the problem, promotes prospective adoptive partners' relationship satisfaction, while perceiving the other as hostile or ambivalent in stress management may undermine their relationship satisfaction. These findings extend 
the literature on the consequences of dyadic coping in two respects. First, they confirm the role of dyadic coping also for prospective adoptive couples. Second, findings related to couple generativity show that resources that are internal to the couple, such as dyadic coping, allow partners to go beyond themselves and their couple relationship. The way partners are able (or not able) to take care of each other in times of stress seems to spill over to their ability to care for others beyond the couple. This spillover effect seems especially important when partners become parents (Zemp et al., 2016, 2017) and may be crucial for adoptive partners.

The present findings also show significant partner effects. In particular, the perceptions the other holds about one's own dyadic coping responses are associated with one's relationship satisfaction, thereby confirming the interdependent and dyadic nature of the dyadic coping process. Dyadic coping, in fact, is a process in which both partners are involved and in which both partners' individual and relational well-being is at stake (Bodenmann, 1995, 2005). For adoptive couples, moreover, both partners' involvement is especially required in the transition: assessment procedures put both partners in the spotlight and when the child arrives both partners are involved to the same extent from the very beginning at his/her arrival and along his/her development (Levy-Shiff et al., 1997; Ferrari et al., 2015; Ranieri et al., 2017). With regard to common dyadic coping, only wives' relationship satisfaction is subject to a partner effect. While wives' satisfaction is sustained by both their own and their husbands' perceptions of common dyadic coping, that is their perceptions of common, couple-level efforts to deal with stress, husbands' satisfaction is promoted by their own perceptions of common dyadic coping only. This finding could be explained by women's relational orientation (Cross and Madson, 1997): especially when referring to partners' perceptions of the couple as a whole, women seem affected by their own and their partners' feelings. Men, on the contrary, being more independence-oriented (Cross and Madson, 1997), may rely more on their own perceptions. On couple generativity the only significant partner effect refers to negative dyadic coping responses. The other's perceptions about one's own dyadic coping responses as hostile, distant, or ambivalent, that is the other's perceptions about one not investing in the relationship and being destructive in times of stress, undermine one's ability to be generative. It seems that, to be generative as a couple, it is important that partners refrain from destructive responses and actually invest their resources in favor of the other and of the couple relationship. Noticeably, if one partner is perceived as destructive for the relationship, this also impedes him/her to use the relationship as a resource for others. The literature on dyadic coping has testified the detrimental consequences

\section{REFERENCES}

Acquati, C., and Saita, E. (2017). Affrontare Insieme la Malattia. Il Coping Diadico fra Teoria e Pratica [Dealing with Illness Together. Dyadic Coping in Theory and Practice]. Roma: Carocci Editore. of perceived negative dyadic coping on community couples' relationship satisfaction (e.g., Donato and Parise, 2012). This study extends previous findings to prospective adoptive couples and to couple generativity, especially showing that not only actor effects of perceived negative dyadic coping, but also partner effects are relevant for both relationship satisfaction and couple generativity.

The present findings bear also practical relevance for preventive and supportive interventions with prospective adoptive parents. In particular, this study highlights the importance of sustaining prospective adoptive parents' ability to be a good team in coping and facing together with a common stressor, in order to improve their relationship quality as well as their willingness to take care of others and to promote the well-being of young generations and of the society. This could, in turn, contribute to enhance parental competencies and to create a positive and collaborative family climate.

The validity and implications of the present findings should be considered in light of some limitations. First, our sample size is small, so caution is needed when generalizing our findings to the whole population of prospective adoptive parents. Second, we are unable to draw causal inferences, due to our correlational design. Future longitudinal studies could help confirm the direction of effects as well as explore the role of dyadic coping in the postadoption phase. A final limitation has to do with the exclusive reliance on self-reports. Further research could rely on daily and observational measures in order to deeply capture the complexity of marital functioning.

Despite these limitations, our study, highlights the relevance of extending the investigation of dyadic coping to couples in the pre-adoption phase. Our results, in fact, clearly show that dyadic coping is a crucial resource for prospective adoptive couples' relationship, which may help them better face the challenges related to adoption.

\section{AUTHOR CONTRIBUTIONS}

All authors equally contributed to the development of the theoretical framework, to the performance of the statistical analyses, to the analysis of the results, and to the writing of the manuscript.

\section{ACKNOWLEDGMENTS}

We would like to thank the adoption agencies and the professionals who collaborated with this research project and all the families who took part to the research.

Bausch, R. S. (2006). Predicting willingness to adopt a child: a consideration of demographic and attitudinal factors. Sociol. Perspect. 49, 47-65. doi: 10.1525/ sop.2006.49.1.47

Bergstraesser, E., Inglin, S., Hornung, R., and Landolt, M. A. (2015). Dyadic coping of parents after the death of a child. Death Stud. 39, 128-138. doi: 10.1080/ 07481187.2014 .920434 
Bertoni, A., Donato, S., Graffigna, G., Barello, S., and Parise, M. (2015). Engaged patients, engaged partnerships: singles and partners dealing with an acute cardiac event. Psychol. Health Med. 20, 505-517. doi: 10.1080/13548506.2014. 969746

Bertoni, A., Parise, M., and Iafrate, I. (2012). "Beyond satisfaction: generativity as a new outcome of couple functioning," in Marriage Psychological Implications, Social Expectations, and Role of Sexuality, eds P. E. Esposito and C. I. Lombardi (Hauppauge, NY: Nova Science Publisher), 115-132.

Bodenmann, G. (1995). Dyadisches coping und partnerschaftszufriedenheit [Dyadic coping and marital satisfaction]. Psychol. Beitr. 37, 72-89.

Bodenmann, G. (1997). Dyadic coping - a systemic-transactional view of stress and coping among couples: theory and empirical findings. Eur. Rev. Appl. Psychol. 47, 137-140.

Bodenmann, G. (2000). Stress und Coping bei Paaren [Stress and Coping in Couples]. Göttingen: Hogrefe.

Bodenmann, G. (2005). "Dyadic coping and its significance for marital functioning," in Couples Coping with Stress: Emerging Perspectives on Dyadic Coping, eds T. Revenson, K. Kayser, and G. Bodenmann (Washington, DC: American Psychological Association), 33-50. doi: 10.1037/11031-002

Bradford, K., Barber, B. K., Olsen, J. A., Maughan, S. L., Erickson, L. D., Ward, D., et al. (2003). A multi-national study of interparental conflict, parenting, and adolescent functioning: South Africa, Bangladesh, China, India, Bosnia, Germany, Palestine, Colombia, and the United States. Marriage Fam. Rev. 35, 107-137. doi: 10.1300/J002v35n03_07

Canzi, E., Ferrari, L., Ranieri, S., and Rosnati, R. (2017a). Ethnic prejudice and intergroup contact in prospective adoptive parents. Adopt. Quart. 20, 181-194. doi: 10.1080/10926755.2017.1291460

Canzi, E., Ranieri, S., Barni, D., and Rosnati, R. (2017b). Predictors of parenting stress during early adoptive parenthood. Curr. Psychol. 1-10. doi: 10.1007/ s12144-017-9657-x

Canzi, E., Rosnati, R., Palacios, J., and Román, M. (2018). Internationally adopted children's cognitive and social-emotional development during the first postadoption year: a longitudinal study. Eur. J. Dev. Psychol. 15, 517-530. doi: 10.1080/17405629.2017.1316257

Ceballo, R., Lansford, J., Abbey, A., and Stewart, A. (2004). Gaining a child: comparing the experiences of biological parents, adoptive parents and stepparents. Fam. Relat. 53, 38-48. doi: 10.1111/j.1741-3729.2004. 00007.x

Cigoli, V., and Scabini, E. (2006). "Relazione familiare: la prospettiva psicologica," in Le Parole Della Famiglia, eds E. Scabini and G. Rossi (Milano: Vita e Pensiero), 13-46.

Cohen, N. J., Coyne, J., and Duvall, J. (1993). Adopted and biological children in the clinic: family, parental and child characteristics. J. Child Psychol. Psychiatry 34, 545-562. doi: 10.1111/j.1469-7610.1993.tb01035.x

Cohen, N. J., Coyne, J. C., and Duvall, J. D. (1996). Parents' sense of “entitlement" in adoptive and nonadoptive families. Fam. Process 35, 441-456. doi: 10.1111/j. 1545-5300.1996.00441.x

Commission for Intercountry Adoption (2014/2015). Commission for Intercountry Adoption Statistical Report. Available at: http://www.commissioneadozioni.it/ media/153043/report_statistico_2014-2015.pdf

Cross, S. E., and Madson, L. (1997). Models of the self: self-construals and gender. Psychol. Bull. 122, 5-37. doi: 10.1037/0033-2909.122.1.5

Cutrona, C. E. (1996). Social Support in Couples: Marriage as a Resource in Times of Stress. Thousand Oaks, CA: Sage Pubblications. doi: 10.4135/9781483327563

Daniluk, J. C., and Hurtig-Mitchell, J. (2003). Themes of hope and healing: infertile couples' experiences of adoption. J. Counsel. Dev. 81, 389-399. doi: 10.1002/j. 1556-6678.2003.tb00265.x

Deater-Deckard, K., and Petrill, S. A. (2004). Parent-child dyadic mutuality and child behavior problems: an investigation of gene-environment processes. J. Child Psychol. Psychiatry 45, 1171-1179. doi: 10.1111/j.1469-7610.2004. 00309. $\mathrm{x}$

Donato, S., Iafrate, R., Barni, D., Bertoni, A., Bodenmann, G., and Gagliardi, S. (2009). Measuring dyadic coping: the factorial structure of bodenmann's "dyadic coping questionnaire" in an Italian sample. Test. Psych. Methodol. 16, 25-47.

Donato, S., Iafrate, R., Bradbury, T. N., and Scabini, E. (2012). Acquiring dyadic coping: parents and partners as models. Pers. Relat. 19, 386-400. doi: 10.1111/j. 1475-6811.2011.01368.x
Donato, S., Pagani, A. F., Parise, M., Bertoni, A., and Iafrate, R. (2018). “Through thick and thin: perceived partner responses to negative and positive events," in When "We" are Stressed: A Dyadic Approach to Coping with Stressful Events, eds A. Bertoni, S. Donato, and S. Molgora (Hauppauge, NY: Nova Science Publisher), 41-64.

Donato, S., and Parise, M. (2012). "The role of enacted and perceived dyadic coping for young couples' satisfaction," in Handbook of the Psychology of Coping: New Research, eds B. Molinelli and V. Grimaldo (Hauppauge, NY: Nova Science Publisher), 261-278.

Donato, S., Parise, M., Iafrate, R., Bertoni, A., Finkenauer, C., and Bodenmann, G. (2015). Dyadic coping responses and partners' perceptions for couple satisfaction: an actor-partner interdependence analysis. J. Soc. Pers. Relat. 32, 580-600. doi: 10.1177/0265407514541071

Erel, O., and Burman, B. (1995). Interrelatedness of marital relations and parentchild relations: a meta-analytic review. Psychol. Bull. 118, 108-132. doi: 10.1037/ 0033-2909.118.1.108

Erikson, E. H. (1963). Childhood and Society. New York, NY: W.W. Norton \& Company.

Falconier, M. K., Jackson, J. B., Hilpert, P., and Bodenmann, G. (2015). Dyadic coping and relationship satisfaction: a meta-analysis. Clin. Psychol. Rev. 42, 28-46. doi: 10.1016/j.cpr.2015.07.002

Ferrari, L., Ranieri, S., Barni, D., and Rosnati, R. (2015). Parent-child relationship and adoptees' psychological well-being in adolescence and emerging adulthood: disentangling maternal and paternal contribution. Fam. Sci. 6, 77-86. doi: 10. 1080/19424620.2015.1081005

García-López, C., Sarriá, E., Pozo, P., and Recio, P. (2016). Supportive dyadic coping and psychological adaptation in couples parenting children with autism spectrum disorder: the role of relationship satisfaction. J. Aut. Dev. Disord. 46, 3434-3447. doi: 10.1007/s1080

Goldberg, A. E., and Garcia, R. (2015). Predictors of relationship dissolution in lesbian, gay, and heterosexual adoptive parents. J. Fam. Psychol. 29, 394-404. doi: $10.1037 /$ fam 0000095

Goldberg, A. E., Smith, J. Z., and Kashy, D. A. (2010). Preadoptive factors predicting lesbian, gay, and heterosexual couples' relationship quality across the transition to adoptive parenthood. J. Fam. Psychol. 24, 221-232. doi: 10.1037/ a0019615

Hilpert, P., Randall, A. K., Sorokowski, P., Atkins, D. C., Sorokowska, A., Ahmadi, K., et al. (2016). The associations of dyadic coping and relationship satisfaction vary between and within nations: a 35-nation study. Front. Psychol. 7:1106. doi: 10.3389/fpsyg.2016.01404

Hock, R. M., and Mooradian, J. K. (2012). Co-parenting quality among adoptive mothers: contributions of socioeconomic status, child demands and adult relationship characteristics. Child Fam. Soc. Work 17, 85-95. doi: 10.1111/j. 1365-2206.2011.00775.x

Iafrate, I., and Donato, S. (2012). "Coping in a relational context: the case of dyadic coping," in Handbook of the Psychology of Coping, eds B. Molinelli and V. Grimaldo (Hauppauge, NY: Nova Science Publisher), 111-132.

Kenny, D. A. (1996). Models of non-independence in dyadic research. J. Soc. Pers. Relat. 13, 279-294. doi: 10.1177/0265407596132007

Kenny, D. A., and Cook, W. (1999). Partner effects in relationship research: conceptual issues, analytic difficulties, and illustration. Pers. Relat. 6, 433-448. doi: 10.1111/j.1475-6811.1999.tb00202.x

Kenny, D. A., Kashy, D. A., and Cook, W. L. (2006). The Analysis of Dyadic Data. New York, NY: Guilford Press.

Landis, M., Peter-Wight, M., Martin, M., and Bodenmann, G. (2013). Dyadic coping and marital satisfaction of older spouses in long-term marriage. J. Gerontopsychol. Geriatr. Psychiatry 26, 39-47. doi: 10.1024/1662-9647/ a000077

Lansford, J. E., Ceballo, R., Abbey, A., and Stewart, A. J. (2001). Does family structure matter? A comparison of adoptive, two-parent biological, singlemother, stepfather, and stepmother households. J. Marriage Fam. 63, 840-851. doi: 10.1111/j.1741-3737.2001.00840.x

Lee-Baggley, D., Preece, M., and DeLongis, A. (2005). Coping with interpersonal stress: role of big five traits. J. Pers. 73, 1141-1180. doi: 10.1111/j.1467-6494. 2005.00345. $\mathrm{x}$

Levy-Shiff, R., Bar, O., and Har-Even, D. (1990). Psychological adjustment of adoptive parents-to-be. Am. J. Orthopsychiatry 60, 258-267. doi: 10.1037/ h0079165 
Levy-Shiff, R., Zoran, N., and Shulman, S. (1997). International and domestic adoption: child, parents, and family adjustment. Int. J. Behav. Dev. 20, 109-129. doi: 10.1080/016502597385478

Lyons, R. F., Mickelson, K. D., Sullivan, M. J. L., and Coyne, J. C. (1998). Coping as a communal process. J. Soc. Pers. Relat. 15, 579-605. doi: 10.1177/ 0265407598155001

Margolin, G., Gordis, E. B., and John, R. S. (2001). Coparenting: a link between marital conflict and parenting in two parent families. J. Fam. Psychol. 15, 3-21. doi: 10.1037/0893-3200.15.1.3

McAdams, D. P., de St. Aubin, E., and Logan, R. L. (1993). Generativity among young, midlife, and older adults. J. Pers. Soc. Psychol. 8, 221-230. doi: 10.1037/ 0882-7974.8.2.221

Meichenbaum, D. A. (1985). Stress Inoculation Training. New York, NY: Pergamon Press.

Norton, R. (1983). Measuring marital quality: a critical look at the dependent variable. J. Marriage Fam. 45, 141-151. doi: 10.2307/351302

Palacios, J., and Sánchez-Sandoval, Y. (2006). Stress in parents of adopted children. Int. J. Behav. Dev. 30, 481-487. doi: 10.1177/0165025406071492

Parise, M., Gatti, F., and Iafrate, R. (2017). Religiosity, marital quality and couple generativity in Italian couples belonging to a Catholic spiritual association: a quali-quantitative study. J. Religion Health 56, 1856-1869. doi: 10.1007/s1094

Parise, M., Pagani, A. F., Donato, S., and Sedikides, C. (2018). Self-Concept Clarity and Relationship Quality at the Dyadic Level: The Roles of Couple Identity and Dyadic Coping. Available at: https://eprints.soton.ac.uk/id/eprint/427522.

Park, N. K., and Hill, P. W. (2014). Is adoption an option? The role of importance of motherhood and fertility help-seeking in considering adoption. J. Fam. Issues 35, 601-626. doi: 10.1177/0192513X13493277

Ranieri, S., Ferrari, L., Barni, D., and Rosnati, R. (2017). Launching adoptees into adulthood: mothers' and fathers' promotion of volitional functioning. J. Prevent. Intervent. Commun. 45, 168-179. doi: 10.1080/10852352.2016.1198124

Revenson, T. A., and DeLongis, A. (2010). "Couples coping with chronic illness," in Handbook of Coping and Health, ed. S. Folkman (New York, NY: Oxford Press), $101-123$.

Riley, K. (2013). “Benefit finding," in Encyclopedia of Behavioral Medicine, eds M. D. Gellman and J. R. Turner (New York, NY: Springer), 208-210.

Rosnati, R., Ranieri, S., and Barni, D. (2013). Family and social relationships and psychosocial well-being in Italian families with internationally adopted and non-adopted children. Adopt. Quart. 16, 1-16. doi: 10.1080/10926755.2012. 731030

Salcuni, S., Ceccato, P., Di Riso, P., and Lis, A. (2006). Diagnosi multi-prospettica di genitori in attesa di adozione. Rassegna di Psicologia 23, 49-68.
Salcuni, S., Miconi, D., Altoè, G., and Moscardino, U. (2015). Dyadic adjustment and parenting stress in internationally adoptive mothers and fathers: the mediating role of adult attachment dimensions. Front. Psychol. 6:1279. doi: 10.3389/fpsyg.2015.01279

Scabini, E., and Rossi, G. (2014). Allargare(lo )Spazio Familiare: Adozione e Affido. Studi Interdisciplinari Sulla Famiglia. Milano: Vita e Pensiero.

Stone, G., Buehler, C., and Barber, B. K. (2002). "Interparental conflict, parental psychological control, and youth problem behavior," in Intrusive Parenting: How Psychological Control Affects Children and Adolescents, ed. B. K. Barber (Washington, DC: American Psychological Association), 53-95. doi: 10.1037/ 10422-003

Stright, A. D., and Bales, S. S. (2003). Coparenting quality: contributions of child and parent characteristics. Fam. Relat. 52, 232-240. doi: 10.1111/j.1741-3729. 2003.00232.x

Traa, M. J., De Vries, J., Bodenmann, G., and Den Oudsten, B. L. (2015). Dyadic coping and relationship functioning in couples coping with cancer: a systematic review. Br. J. Health Psychol. 20, 85-114. doi: 10.1111/bjhp.12094

Welsh, J. A., Viana, A. G., Petrill, S. A., and Mathias, M. D. (2008). Ready to adopt: characteristics and expectations of pre-adoptive families. Adopt. Quart. 11, 176-203. doi: 10.1080/10926750802421982

Zemp, M., Bodenmann, G., Backes, S., Sutter-Stickel, D., and Revenson, T. A. (2016). The importance of parents' dyadic coping for children. Fam. Relat. 65, 275-286. doi: 10.1111/fare.12189

Zemp, M., Milek, A., Cummings, E. M., and Bodenmann, G. (2017). Longitudinal interrelations between dyadic coping and coparenting conflict in couples. J. Child Fam. Stud. 26, 2276-2290. doi: 10.1007/s10826-017-0742-4

Zhang, Y., and Lee, G. (2010). Intercountry versus transnational adoption: analysis of adoptive parents' motivations and preferences in adoption. J. Fam. Issues 32, 75-98. doi: 10.1177/0192513X10375410

Conflict of Interest Statement: The authors declare that the research was conducted in the absence of any commercial or financial relationships that could be construed as a potential conflict of interest.

Copyright (c) 2019 Canzi, Donato, Ferrari, Parise, Pagani, Lopez, Rosnati and Ranieri. This is an open-access article distributed under the terms of the Creative Commons Attribution License (CC BY). The use, distribution or reproduction in other forums is permitted, provided the original author(s) and the copyright owner(s) are credited and that the original publication in this journal is cited, in accordance with accepted academic practice. No use, distribution or reproduction is permitted which does not comply with these terms. 


\section{OPEN ACCESS}

Edited by:

Guy Bodenmann,

University of Zurich, Switzerland

Reviewed by:

Sarah Holley,

San Francisco State University,

United States

Mariana Moura-Ramos,

University of Coimbra, Portugal

*Correspondence:

Sara Molgora

sara.molgora@unicatt.it

Specialty section:

This article was submitted to Clinical and Health Psychology, a section of the journal

Frontiers in Psychology

Received: 19 June 2018 Accepted: 12 February 2019 Published: 08 March 2019

Citation:

Molgora S, Fenaroli V, Acquati C, De

Donno A, Baldini MP and Saita E (2019) Examining the Role of Dyadic Coping on the Marital Adjustment of

Couples Undergoing Assisted Reproductive Technology (ART).

Front. Psychol. 10:415 doi: 10.3389/fpsyg.2019.00415

\section{Examining the Role of Dyadic Coping on the Marital Adjustment of Couples Undergoing Assisted Reproductive Technology (ART)}

\author{
Sara Molgora ${ }^{1 *}$, Valentina Fenaroli ${ }^{1}$, Chiara Acquati ${ }^{2}$, Arianna De Donno ${ }^{1}$, \\ Maria Pia Baldini ${ }^{3}$ and Emanuela Saita ${ }^{1}$ \\ ${ }^{1}$ Department of Psychology, Catholic University of Sacred Hearth, Milan, Italy, ${ }^{2}$ Graduate College of Social Work, University \\ of Houston, Houston, TX. United States, ${ }^{3}$ IRCCS Ca 'Granda Foundation Maggiore Policlinico Hospital, Milan, Italy
}

A couple is considered to be infertile if unable to conceive after 12 months of unprotected sexual intercourse. An extended body of literature supports that infertility and infertility treatments contribute to emotional, social, sexual, and relational issues that can have a negative impact on each partner's well-being and on the couple relationship. Recent findings suggest that a dyadic approach should be used when working with couples coping with these stressors. However, most research to date has focused on the association between infertility and individual's psychological outcomes, rather than on the experience of infertility-related stress and coping from a relational perspective. Consequently, assuming that infertility is a dyadic stressor and that the ability of the partners to cope with this experience is the result of both individual and relational coping strategies, this study aimed to investigate dyadic coping and marital adjustment among couples at the beginning of an Assisted Reproductive Technology (ART) treatment. A sample of 167 heterosexual couples $(N=334)$ undergoing ART treatment at the fertility clinic of a large hospital in Milan from January to December 2017 was recruited. Each participant completed self-reported questionnaires examining marital adjustment (Dyadic Adjustment Scale) and dyadic coping (Dyadic Coping Questionnaire). Demographics and clinical variables were also collected. Data were analyzed using the Actor Partner Interdependence Model (APIM), testing the effect of each partner's dyadic coping style on their own and their partner's marital adjustment. Results revealed that both women and partners' scores on positive dyadic coping styles (common, emotion-focused, problem-focused, and delegated dyadic coping) contributed to higher marital adjustment. This result suggests that couples unable to engage in this type of reciprocal supportive behaviors and those unsatisfied with their coping efforts may be more vulnerable while undergoing ART treatments. Furthermore, findings highlighted some gender differences for stress communication and negative dyadic coping suggesting the presence of specific dynamics within couples facing an ART treatment. Implications for clinical practice and future research are discussed.

Keywords: dyadic coping, marital adjustment, assisted reproduction (ART), infertile couple, APIM (Actor-Partner Interdependence Model) 


\section{INTRODUCTION}

Starting from the historical definition of the World Health Organization that defined infertility as the inability for a couple to conceive after a year of regular, unprotected sexual intercourse (World Health Organization, 1992), its current definition has been expanded to cover a wider spectrum of conditions that affect individuals' and couples' capacity to reproduce (Zegers-Hochschild et al., 2017). In particular, although infertility still represents a disease of the reproductive system-which can be categorized as organic (i.e., linked to organic causes) or functional (i.e., linked to non-organic causes) (Vitale et al., 2017), it is acknowledged that the failure to conceive does not always depend on a disease; thus, the concept of an impairment of function which can lead to a disability has been introduced (ZegersHochschild et al., 2017). Worldwide the estimated prevalence of infertility is about $8-12 \%$ (Tao et al., 2012), a percentage that increases significantly in Italy, reaching approximately $30 \%$ according to data from the Ministry of Health (www. salute.gov.it/portale/fertility). This datum can be explained by considering the phenomenon of the progressive postponement of births in our country, so that, currently, the average age of first childbirth is 32.4 years for women and 35.3 for men, placing Italy as the second country in the European context for delayed maternity (Istat, 2017; Loghi and Crialesi, 2017). This situation supports the relevance of the topic for investigators interested in the study of couples coping with stress in the context of health and family issues (Vitale et al., 2017; Stanhiser and Steiner, 2018).

After a diagnosis of infertility, many couples undergo assisted reproductive technology (ART) treatments in order to become parents. This is a term that includes a wide spectrum of techniques developed to help couples achieve a viable pregnancy. These techniques can be divided in first and second level techniques, with different levels of medicalization. Specifically, for the first levels techniques nowadays couples have the following techniques available: ovulation induction (OI), that involves taking a hormone medication (by tablet or injection) in order to stimulate the production of follicle-stimulating hormone, and artificial insemination (AI) (or intrauterine insemination, IUI), that involves insertion of a male partner's semen through the woman's cervix and into the uterus at or just before the time of ovulation. With reference to the second level techniques, the following procedure are available: in vitro fertilization (IVF), that means that the woman's eggs and the man's sperm are left in a culture dish in the laboratory to allow the egg to be fertilized before placing the embryo into the woman's uterus; gamete intrafallopian transfer (GIFT), currently little used, that is considered as a more natural version of IVF because the woman's eggs are retrieved from her ovaries and the egg and sperm are left to fertilize naturally; intracytoplasmic sperm injection (ICSI), that follows the same process as IVF, except involving the direct injection of a single sperm into each egg to achieve fertilization. Furthermore, there are also some procedures that involve the use of donor eggs, donor sperm, or previously frozen embryos. ART treatments have been steadily increasing in recent years (European IVFmonitoring Consortium (EIM) et al., 2017), and, in the same way, the proportion of ART babies among the total number of babies born has increased over the years, now reaching $2.4 \%$ (Ferraretti et al., 2017).

Overall, infertility represents a stressful condition, if not a traumatic one, for those who want to have a child because it is associated with the loss and grief connected with not being able to conceive naturally (Koert and Daniluk, 2018). Previous studies reported that the condition of infertility affects the psychological well-being of both women and men, which can feel like depression, guilt, anxiety, and isolation (Schmidt, 2006; El Kissi et al., 2013; Péloquin et al., 2018). If infertilityrelated distress impacts the quality of life of both partners (Maroufizadeh et al., 2015; Martins et al., 2016), some gender differences are reported (e.g., Ying et al., 2015): infertile women seem to feel more stress about their condition and to experience more depressive symptoms than infertile men (e.g., Berghuis and Stanton, 2002; Kroemeke and Kubicka, 2018). At the same time, gender differences were specifically found also in dealing with ART treatments (Bayley et al., 2009; Davidovà and Pechovà, 2014): indeed, women overall report higher levels of anxiety than men, although over the course of several cycles the average score of anxiety increases for both partners (Schaller et al., 2016). In particular, women's main anxiety seems to be the possible failure to achieve a pregnancy, while men's main anxiety is related with their concern for their partner's health risks (Schaller et al., 2016).

Furthermore, infertile women report lower levels of quality of life than their partners during all phases of the treatment cycle and this difference is greater if the couple has experienced more than one failure of the ART cycles (Agostini et al., 2017). Finally, women are found to implement emotion-focused coping strategies, while men prefer problem-focused coping strategies (Shapiro, 2009). These differences could be partially explained considering that for women the central aspect of infertility is the desire for a child that reinforces their decision to undergo to an ART procedure, while for men the transition to fatherhood often perceived as a more socially defined transition to fulfill the male role (Davidovà and Pechovà, 2014).

Some studies reported that the distress experienced by the partners does not depend on ART techniques (the type of the ART treatment or the number of previous treatments) (Lowyck et al., 2009; Sina et al., 2010; Van Der Merwe and Greeff, 2015); however, according to some authors (Brandes et al., 2009; Gameiro et al., 2012), the distress can impact patients' decisions to discontinue treatment prematurely.

Moreover, distressing feeling and thoughts related to infertility as well as to ART techniques can affect not only each partnerwith a specific pattern of adjustment (Moura-Ramos et al., 2016), but also the couple itself as a unit (Cigoli and Scabini, 2006; Schwerdtfeger and Shreffler, 2009; Reis et al., 2013; Turner et al., 2013; Maroufizadeh et al., 2015; Moura-Ramos et al., 2016; Greil et al., 2017). Some authors have analyzed the association between stress related to infertility and ARTs and marital relationship, reporting contrasting results (Van Der Merwe and Greeff, 2015; Chaves et al., 2018). Some authors found that infertility does not reduce marital satisfaction (Amiri 
et al., 2016), and facing infertility-related stress can contribute to strengthening marital satisfaction and communication among partners, with couples experiencing greater closeness as a consequence of their ability to face the fertility problem as a shared experience (Monga et al., 2004; Schmidt et al., 2005). Others have found that higher levels of stress associated with infertility predicted lower couple satisfaction and an overall worse marital quality (Van Der Merwe and Greeff, 2015; Gana and Jakubowska, 2016). In particular, Van Der Merwe and Greeff (2015) considered four different dimensions of marital quality: quality of communication, intimacy, sexual satisfaction and an overall couple adjustment, and found that the level of infertilityrelated stress was associated with all four dimensions of marital relationship. This deterioration in the marital relationship following an infertility diagnosis can lead to separation and repartnering (Martins et al., 2014a). Peterson et al. (2003) reported that couples in which men and women perceived similar levels of infertility-related distress reported higher levels of marital adjustment compared with couples in which partners perceived this stress differently. These differences in research findings could be partially explained by considering that several factors (e.g., demographic, economic, social, etc.) may play a role in determining marital satisfaction also in infertile couples (Samadaee-Gelehkolaee et al., 2016). Furthermore, some other variables (e.g., socio-demographic variables, coping strategies, social support, etc.) have been found to mediate the relation between infertility-related stress and the couple relationship, explaining these contrasting results (Ghafouri et al., 2016; Pasha et al., 2017; Greil et al., 2018). Finally, a gender effect for the impact of infertility-related stress on the couple relationship has been found, so that dissimilar results could be partially due to differences between males and females. Indeed, although some authors did not find any difference in marital satisfaction and adjustment between wives and husbands (Yazdani et al., 2016), other authors reported gender differences that move in contrasting directions. For example, Lee and Sun (2000) found that wives were less satisfied than their husbands with their relationship. On the contrary, Peterson et al. (2011) found that a greater percentage of women, compared with men, reported high levels of marital benefit as a positive consequence of the infertility experience. In the same way, differences between males and females emerged when considering the variables that predicted marital satisfaction in infertile couples. For example, Greil et al. (2018) reported that only women, and not men, were significantly more satisfied with their couple relationship when neither partner self-identified as having a fertility problem.

Since infertility represents an unplanned and unexpected stressor, partners usually have considerable difficulty adequately managing this infertility-related stress and activate a variety of coping strategies in order to maintain or regain control over their lives (Peterson et al., 2008). Strategies that partners activate to cope with infertility and following ART can affect both their personal well-being (Rooney and Domar, 2016; Zurlo et al., 2018) as well as their marital well-being (Peterson et al., 2006a, 2008). Several studies have analyzed the coping strategies that partners use to face infertility, distinguishing between more functional or dysfunctional ones (e.g., Bayley et al., 2009). For example, Rockliff et al. (2014) found that the use of escapist coping strategies was associated with increased emotional distress. And, again, Peterson et al. (2006a) reported that avoidance coping strategies are the strongest predictors of decrease in marital adjustment. However, most studies examined these coping strategies from an individual perspective, using the individual as the unit of analysis (Peterson et al., 2008) and did not analyze the reciprocal impact of one partner's coping strategies on his or her partner's wellbeing or take into account partner interdependence (Pasch and Sullivan, 2017). Using the couple as the unit of analysis, instead, it becomes possible to better investigate the reciprocal influence between partners.

From this dyadic perspective some studies reported gender differences, with men more influenced by their partner than vice versa (Bodenmann et al., 2006). A recent study reported that women's relationship satisfaction more strongly influences their partners' relationship satisfaction (Greil et al., 2018). A similar result was obtained in another study, finding that men's infertility stress was associated with their partners' level of perceived support, but not vice versa (Martins et al., 2014b).

Since infertility can be considered a couple-level (i.e., dyadic) stressor because both partners are affected by this problem and both have to face it, partners are required to cope with these critical experiences together. According to the systemic transactional model of dyadic coping (Bodenmann, 2005; Leuchtmann and Bodenmann, 2018), dyadic coping can be defined as an interpersonal and circular process of managing stressful events shared by both partners within a couple. It is a multidimensional construct depending on several factors (e.g., the situation, individual and dyadic appraisal and goals, partners' competencies), so that partners can engage in positive as well as negative strategies to manage the stressful situation they have to cope with. In particular, different forms of positive dyadic coping can be distinguished: supportive dyadic coping, delegated dyadic coping, and common dyadic coping (Bodenmann, 1995, 2005). Supportive and delegated dyadic coping refer to the efforts of one partner to express solidarity with the other partner (i.e., the stressed partner), providing, respectively, information and practical advice and taking over his or her daily tasks. Specifically, in delegated dyadic coping, the partner is explicitly asked to provide his or her help to the other partner. Both supportive and delegated dyadic coping can be emotion-focused (i.e., focused on partners' emotional distress) or problem-focused (focused on the problem itself). Common dyadic coping refers to the efforts that both partners make together to overcome a direct dyadic stress (Donato et al., 2009). Overall, positive dyadic coping allows partners to maintain or restore their individual well-being as well as to enhance the quality of the couple's relationship, strengthening their sense of we-ness and their reciprocal trust (Bodenmann, 2005; Donato et al., 2009). On the contrary, negative dyadic coping refers to activities following the partner's expression of stress characterized by a negative connotation (e.g., ambivalent or insincere behaviors, superficial interest, hostile comments, etc.). Some studies revealed gender differences in the use of dyadic coping strategies in couples (Staff et al., 2017). For example, women perceive themselves more able to communicate their stress than men (Bodenmann and Cina, 
2005; Molgora et al., 2018). Furthermore, women report higher levels of negative dyadic coping (Ledermann et al., 2007), while men perceive dyadic coping to be more efficient than women do (Molgora et al., 2018). Bodenmann et al. (2015) investigated gender differences in support provided to the partner, finding that the support is moderated by the level of stress: in lowstressed conditions men and women provide similar support to the stressed partner, while in a high-stressed situation men provide lower-quality support than women, but only in response to women's emotionally oriented expression of stress.

Over the years, the literature has clearly highlighted how dyadic coping is strongly associated with marital quality, despite cultural and gender differences (Hilpert et al., 2016). Indeed, dyadic coping has been widely investigated in different types of couples and considering several critical events and/or transitions that the couple may face. For example, there are a lot of studies that considered couples facing illnesses or health-related sources of stress (e.g., cancer, cardiac disease, respiratory disease, etc.) (e.g., Hagedoorn et al., 2008; Badr et al., 2010, 2018; Regan et al., 2014; Rottmann et al., 2015; Traa et al., 2015; Switzer et al., 2018; Vilchinsky and Dekel, 2018; Zimmermann and Rauch, 2018). Furthermore, many studies investigated the association between dyadic coping and marital quality in non-clinical couples, in different stage of the life course (late adolescent couples, newly married couples, couples during pregnancy, older couples, etc.) (Landis et al., 2013; Donato et al., 2014; Alves et al., 2018; Breitenstein et al., 2018; Molgora et al., 2018). Specifically, positive dyadic coping was found to predict couple satisfaction and adjustment over time, whereas negative dyadic coping was associated with couple distress (Bodenmann et al., 2006; Falconier et al., 2015; Rusu et al., 2018). Furthermore, the association between dyadic coping and marital quality can be mediated by the partners' ability to communicate their stress (Ledermann et al., 2010). However, to the authors' knowledge, only one other recent study (Chaves et al., 2018) has specifically analyzed the relationship between dyadic coping and marital adjustment in relation to infertility, and in particular in couples undergoing ART, highlighting the central role of men's dyadic coping strategies for the marital adjustment of both partners: indeed, while males' marital adjustment is influenced by the perception of their own coping, females' marital adjustment is influenced by their partners' perception. However, this study, although it considered both women and men, did not use a properly dyadic approach to investigate the reciprocal influence between partners.

The present study is aimed at investigating the differences between men's and women's perception of dyadic coping strategies as well as marital adjustment in a sample of Italian couples undergoing ART. Furthermore, we tested the relationship between dyadic coping and marital adjustment. Because of the shared nature of the infertility experience, we used the Actor-Partner Interdependence Model (APIM) to analyze the effect of each partner's perception of dyadic coping strategies on its own and partners' marital adjustment. Since, to our knowledge, no previous study has investigated the association between dyadic coping and marital adjustment in infertile couples using this methodology, we adopted an explorative approach, testing the relation between all the different dyadic coping strategies and marital adjustment. However, following the results of a previous study reporting a stronger association between positive dyadic coping and marital adjustment (Falconier et al., 2015), and considering that the infertility experience has a common (i.e., dyadic) dimension, beyond individual gender-related specificities, we will expect that positive dyadic coping strategies, and in particular common dyadic coping, would have been associated with higher individual's (i.e., actor effect) and partner's (i.e., partner effect) perceptions of marital adjustment.

\section{METHODS}

\section{Participants}

From January to December 2017, a total of 230 couples, which represent all the couples entering an ART program at a public hospital in Milan, were contacted regarding participation in this study. Of these, 30 did not consent to participate, while 200 agreed to take part in this cross-sectional study. Of this number, 33 couples were excluded because of incomplete questionnaires, with the final sample comprised of 167 childless couples. Chisquare and independent samples $t$-test analyses showed no differences between couples who completed the questionnaires and those who did not complete all measures as regards sociodemographic and infertility-related variables as well as the other study variables (dyadic coping and couple adjustment).

Mean age of participants was $36.13(S D=3.92$; range $=22-$ 44) for women and 38.9 (DS = 5.08; range: $22-58$ ) for men. $35.8 \%$ of women and $28.3 \%$ of men in the sample had a high-school diploma; $15.1 \%$ of women and $11.0 \%$ of men had a junior highschool diploma; $33.5 \%$ of women and $37.6 \%$ of men were college graduates. Almost all the participants $(91.1 \%$ of women and $96 \%$ of men) were employed. Most of the women (59\%) are office workers; among men, $18.6 \%$ are blue collar workers, $35.9 \%$ are office workers, and $13.9 \%$ are managers.

As for characteristics related to infertility, $45.1 \%$ of diagnoses were female factor, $27.8 \%$ male factor, $11.3 \%$ both partners' factor; $15.8 \%$ of infertility diagnoses were idiopathic. More than half of the couples (57.1\%) never underwent an ART cycle; $42.9 \%$ had already undergone an average of 2 (mean $=2.29$; $S D=$ 1.58) ART treatment. In preparation for our analysis, couples who had completed previous ART attempts were compared to couples with no previous history of ART. No differences were found regarding the study variables of interest for women. On the contrary, men who had undergone previous ART reported lower levels of dyadic coping $\left[F_{(1,165)}=5.31 ; p<0.05\right]$ and marital adjustment $\left[F_{(1,165)}=4.83 ; p<0.05\right]$ than men who had never undergone treatments. $64.5 \%$ of couples were enrolled in IVF and $32 \%$ in ICSI treatments.

\section{Measures \\ Dyadic Adjustment Scale (DAS) (Spanier, 1976;}

Gentili et al., 2002)

This scale measures couple's adjustment through 32 items: 31 items are related to specific aspects of a couple's interactions and one item assesses the overall happiness with the relationship. 
The higher the total score, obtained by summing the 32 items, the greater is the perceived couple adjustment. The instrument shows good internal consistency both for men (Cronbach's alpha $=0.90$ ) and women (Cronbach's alpha $=0.90$ ).

\section{Dyadic Coping Questionnaire (DCQ) ${ }^{1}$ (Bodenmann, 2000; Donato et al., 2009)}

This scale measures dyadic coping behaviors through 41 items on a Likert-type 5-point scale ranging from "never" to "very often." In particular, 39 items are related to six different styles of dyadic coping (stress communication-8 items, emotionfocused -6 items, problem-focused -4 items, delegated -4 items, negative -10 items, and common -7 items) while the last two items evaluate satisfaction and efficacy for dyadic coping. The scores of the negative coping items have to be reversed, so the higher the score of this subscale, the lower is the use of hostile, ambivalent or superficial strategies by the partner. The mean of all positive and reversely coded negative items reveals the partners' perceived total dyadic coping skills. The higher the score, the more the partners feel they are jointly managing the stressful situation. The subscales related to stress communication, emotion-focused, problem-focused, delegated and negative dyadic coping consider both self-perception (i.e., one's own dyadic coping) and other perception (i.e., partner's dyadic coping). Considering both self- and other-perception allows a comparison between partners' perceptions and an investigation of the balance between the support provided and received by partners, both at an intra-individual and an interpersonal level (Donato et al., 2009). For this reason, we analyzed both self-perception and other perception in our models. Examples of items are the following: "I tell my partner openly how I feel and that I would appreciate his/her emotional support" (stress communication, self-perception); "My partner shows me that he/she is stressed and is not feeling well" (stress communication, other-perception); "I listen to my partner, give him/her the opportunity to express his/her stress, comfort and encourage him/her" (emotion-focused, self-perception); "My partner listens to me, gives me the opportunity to express my stress, comforts and encourages me" (emotion-focused, otherperception); "I try to analyze the situation together with my partner and help him/her to understand and deal with the problem" (problem-focused, self-perception); "My partner helps me to see the stressful situation in a different light and to put the problem in perspective" (problem-focused, other-perception); "I take on things that my partner would normally do in order to help him/her out" (delegated, self-perception); "When I am too busy, my partner helps me out" (delegated, other-perception); "When my partner is stressed, I withdraw from him/her" (negative, selfperception); "My partner makes fun of my stress and mocks me" (negative, other-perception); "We help each other to put the

\footnotetext{
${ }^{1}$ DCQ is the only scale on dyadic coping validated for the Italian population (Donato et al., 2009). It is somewhat longer than the more recent Dyadic Coping Inventory (DCI; Bodenmann, 2008) that measures dyadic coping behaviors through 37 items. DCQ and DCI measure the same subscales and consider both self-perception (coping by oneself) and other perception (coping by partner). However, the DCQ includes two single items, respectively, for dyadic coping satisfaction and efficacy, while the DCI has only one item for the perceived quality of dyadic coping.
}

problem in perspective and see it in a new light" (common). The reliability of each subscale and total score on dyadic coping was satisfactory, with scores ranging from 0.78 to 0.90 for men and from 0.74 to 0.93 for women.

A socio-demographic and clinical form was included, including information about age, educational level, job situation and clinical variables about infertility, i.e., the diagnosis (when known), the number of previous ART treatments and the type of ART treatment couples are undergoing.

\section{Procedure}

The research project was approved by the Institutional Review Board of the Catholic University of the Sacred Heart. All participants were informed about the research aim and methodology and signed a written informed consent form. Data were collected at the beginning of the assisted reproductive technology procedure. Specifically, both partners were recruited when they were in a day hospital for some preliminary exams before they entering treatment (e.g., hormonal stimulation). The beginning of treatment took place few days later they have completed the questionnaire. Each partner was asked to complete an on-site questionnaire independently from the other partner. Anonymity and data confidentiality were guaranteed.

\section{Data Analysis}

Descriptive statistics were conducted to illustrate the sample characteristics for demographics, clinical factors, and variables of interest. Differences between the men and women on dyadic coping and couple adjustment were investigated with pairedsamples $t$-test, by comparing the score of the two partners on each of the Dyadic Coping Questionnaire subscale and on the total score of the Dyadic Adjustment Scale. Pearson $r$ correlations were used to assess the association between dyadic coping and dyadic adjustment.

To determine the impact of women and men's dyadic coping on their own as well as their partners' scores on marital adjustment, data were analyzed with the Actor Partner Interdependence Model (APIM), because this model accounts for the non-independence of dyadic data and because this approach treats data from each member of the dyad as nested within the same group (Kenny et al., 2006). The APIM model has been extensively used in the study of close relationships, attachment, caregiving, and couples coping with stress and allows the investigators to consider the reciprocal influence of each partner on their own and their partner's outcome measure simultaneously (see Figure 1). The model states that the person's score on an independent variable can influence their own, as well as their partner's score, on the dependent variable. For this study, the actor effect was the impact of a person's dyadic coping on his or her own marital adjustment. The partner effect was the impact of each person's dyadic coping on the marital adjustment of the other member of the dyad. In the present study, we examined couple adjustment using both actor and partner dyadic coping strategies scores-both selfperceived and other perceived-as predictor variables. Women and men mean-centered predictor variables were regressed on their outcome variables in a single regression model. We also investigated gender interactions to test whether gender 


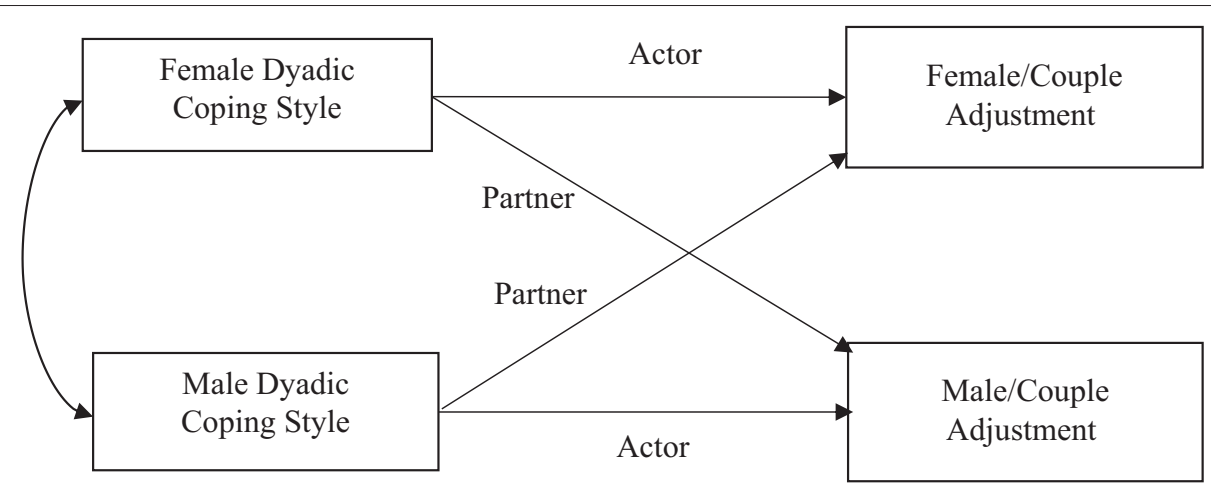

FIGURE 1 | Actor and partner effects of dyadic coping predicting couple adjustment using the APIM model.

differences were present; in case of a significant actor or partner effect interaction, separate regression analyses for females and males were conducted. Within our results, standardized coefficients indicate that an increase in the predictor variable resulted in an increase of the dependent variable. To conduct dyadic data analysis on the present database, the Intra Class Correlation Coefficient (ICC) was calculated between the outcome variables of women and men to examine the amount of non-independence within the couple. Then, an Onmibus Test of Distinguishability was conducted to assess whether treating the dyad as distinguishable improved the fit of the model. We then tested whether gender acted as a moderator of actor and/or partner effects. Hence, an interaction model using REML estimation was tested first, followed by a twointercept approach. If no significant interaction was found between role and actor or partner effect, the standardized coefficient of the average effect is reported to remind the reader of its significance. Analyses were completed with SPSS package, version 24, using the mixed models procedure, with an alpha level of 0.05 .

\section{RESULTS}

\section{Descriptive Information of the Variables of Interest}

Table 1 provides an overview of the study's variables of interest, with reported means and variance indicators of dyadic coping (total score and subscales) and marital adjustment.

Table 2 presents the bivariate associations between dyadic coping and marital adjustment for the two genders.

\section{Differences Between Men and Women}

Paired-sample $t$-test analyses showed no differences between men and women either regarding the DAS score or the DCQ score, with the exception of stress communication and efficacy of dyadic coping. Women reported significantly higher stress communication scores, $\left[t_{(166)}=4.270 ; p<0.001\right)$ and were perceived as more able to communicate their stress to $[t(166)=$ $-8.115 ; p<0.001)$. Moreover, men reported significantly higher efficacy related to dyadic coping than women $\left[t_{(159)}=-32.674\right.$; $p<0.001]$.

\section{APIM of Dyadic Coping Strategies on Couple's Adjustment}

The analysis examined actor and partner effects of each dimension of dyadic coping included in the Dyadic Coping Questionnaire (both self-perceived and other-perceived) in predicting marital adjustment among the dyads involved in the current study.

First, self-perceived dyadic coping strategies were examined, with separate analysis conducted for each subscale (Table 3).

For self-perceived stress communication overall actor and partner effects on marital adjustment were detected: both men and women reporting high levels of stress communication were more likely to report high scores on couples' adjustment. Furthermore, individuals whose partners reported high scores on stress communication were also predicted to report high scores on couple's marital adjustment scores. Gender was a significant moderator of the actor effect $(p<0.05)$, while the interaction between gender and partner effect approached significance $(p=$ 0.06). Thus, after calculating simple slopes, our results indicated that only the female actor effect was statistically significant $(p<0.001)$. For self-perceived emotion-focused, problem-focused and delegated dyadic coping, each analysis revealed both actor and partner effects of the dyadic coping strategy on the score reported on the DAS. Individuals reporting high levels of these coping strategies were more likely to report higher marital adjustment scores. Similarly, men and women whose partner reported higher levels of these dyadic coping strategies were predicted to report high levels of couple adjustment. In our analysis about self-perceived negative dyadic coping, there is evidence of only an actor effect of negative dyadic coping on couple's adjustment: individuals reporting high levels of this coping strategy were more likely to report higher scores on couple's adjustment.

Other-perceived subscales were then examined (Table 4). Actor and partner effects were identified for all the subscales investigated: higher marital adjustment scores were predicted for 
TABLE 1 | Descriptive statistics of relationship satisfaction and dyadic coping scores for men and women.

\begin{tabular}{|c|c|c|c|c|}
\hline & $\begin{array}{r}\text { Wo } \\
\text { Mea }\end{array}$ & & & \\
\hline \multirow[t]{2}{*}{ DAS total score } & \multicolumn{2}{|c|}{$124.37(12.25)$} & \multicolumn{2}{|c|}{125.59 (11.73) } \\
\hline & Self-perception & Other-perception & Self-perception & Other-perception \\
\hline DCQ total score & \multicolumn{2}{|c|}{120.79 (16.48) } & \multicolumn{2}{|c|}{$117.40(16.13)$} \\
\hline Problem-focused supportive DC & $5.88(1.43)$ & $6.05(1.40)$ & $5.90(1.49)$ & $5.90(1.59)$ \\
\hline Delegated DC & $5.24(1.34)$ & $5.10(1.73)$ & $5.52(1.49)$ & $5.15(1.60)$ \\
\hline Negative DC & $11.71(1.75)$ & $17.41(3.17)$ & $11.36(1.83)$ & $17.60(3.54)$ \\
\hline Common DC & \multicolumn{2}{|c|}{$21.92(4.12)$} & \multicolumn{2}{|c|}{$22.55(8.38)$} \\
\hline DC satisfaction & \multicolumn{2}{|c|}{$3.30(0.73$} & \multicolumn{2}{|c|}{$3.23(0.84)$} \\
\hline
\end{tabular}

Statistically significant values are shown in bold. ${ }^{* \star *} p<0.001$.

those who scored high on all these dyadic coping dimensions as well as for participants whose partners had elevated scores. Specifically, for other-perceived stress communication, emotionfocused dyadic coping, problem-focused dyadic coping, and delegated dyadic coping, higher scores on the outcome measure were predicted for men and women who scored high on these subscales, and high marital adjustment was predicted also for those whose partner presented elevated scores on these coping behaviors. Additionally, no mean level differences in couple functioning score were identified for the two partners. For other-perceived negative dyadic coping, gender was a significant moderator of both actor and partner effects. Results indicate that only the partner effect for the score reported by men was significant $(p<0.01)$, while both male and female actor effects were statistically significant.

Finally, we examined the influence of common dyadic coping, satisfaction and efficacy of couples' dyadic coping behaviors in predicting the couples' adjustment (Table 5). From our analysis, there is evidence that gender was a significant moderator of both actor and partner effects. Only the female actor effect was significant $(p<0.001)$, while both male and female partner effects were statistically significant $(p<0.001)$. Finally, both actor and partner effects of satisfaction with dyadic coping behaviors existed in our sample $(p<0.001)$. Gender moderated the actor effect $(p<0.05)$, which was significant for both men and women $(p<0.001)$ and greater for women. Finally, mean-level differences were identified for efficacy of dyadic coping between the two partners $(p<0.05)$, with greater scores on marital adjustment reported by women. Actor $(p<0.001)$ and partner effects $(p<0.001)$ were identified.

\section{DISCUSSION}

\section{Gender Differences in Dyadic Coping and Marital Adjustment}

Since infertility and ART treatments represent stressful experiences for each partner as well as for the couple relationship, independently of the specific type of treatment (Van Der Merwe and Greeff, 2015; Koert and Daniluk, 2018), the present study examined the relationship between dyadic coping and marital adjustment in a sample of Italian infertile couples at the beginning of ART treatment. In particular, we explored whether specific dyadic coping strategies, both self-perceived and otherperceived, have an impact on marital adjustment, considering direct (actor) as well as indirect (partner) effect. Furthermore, we were interested in exploring gender differences, since the literature highlights the presence of differences between males and females both for coping strategies as well as for the impact that infertility has on individual and relational well-being (Ying et al., 2015; Staff et al., 2017).

An initial result is that partners overall have similar representations of their relationship quality in terms of adjustment as well as of their ability to jointly cope with the critical experience of ART. These findings are partially congruent with previous studies on couples' adjustment in non-clinical samples, indicating no significant gender difference (Gager and Sanchez, 2003; Jackson et al., 2014), although other studies have found wives generally reporting a lower marital adjustment than husbands (Amato et al., 2007; Kamp Dush et al., 2008). Considering the specific condition of infertility, the findings reported in literature are controversial: indeed, while some authors did not find any significant difference in marital adjustment between infertile women and infertile men (Yazdani et al., 2016), other researchers found gender differences going in contrasting directions (Lee and Sun, 2000; Peterson et al., 2011). Hence, it is possible to assert that the difference between wives and husbands as regards marital adjustment is partially connected to the different strategies female and male partners adopt in order to cope with the infertility experience and, therefore, to the different impact that infertility has on the individual well-being of women and men. Indeed, although some studies underscored that the infertility condition affects the psychological well-being of both women and men (Schmidt, 2006; El Kissi et al., 2013; Péloquin et al., 2018), the majority 


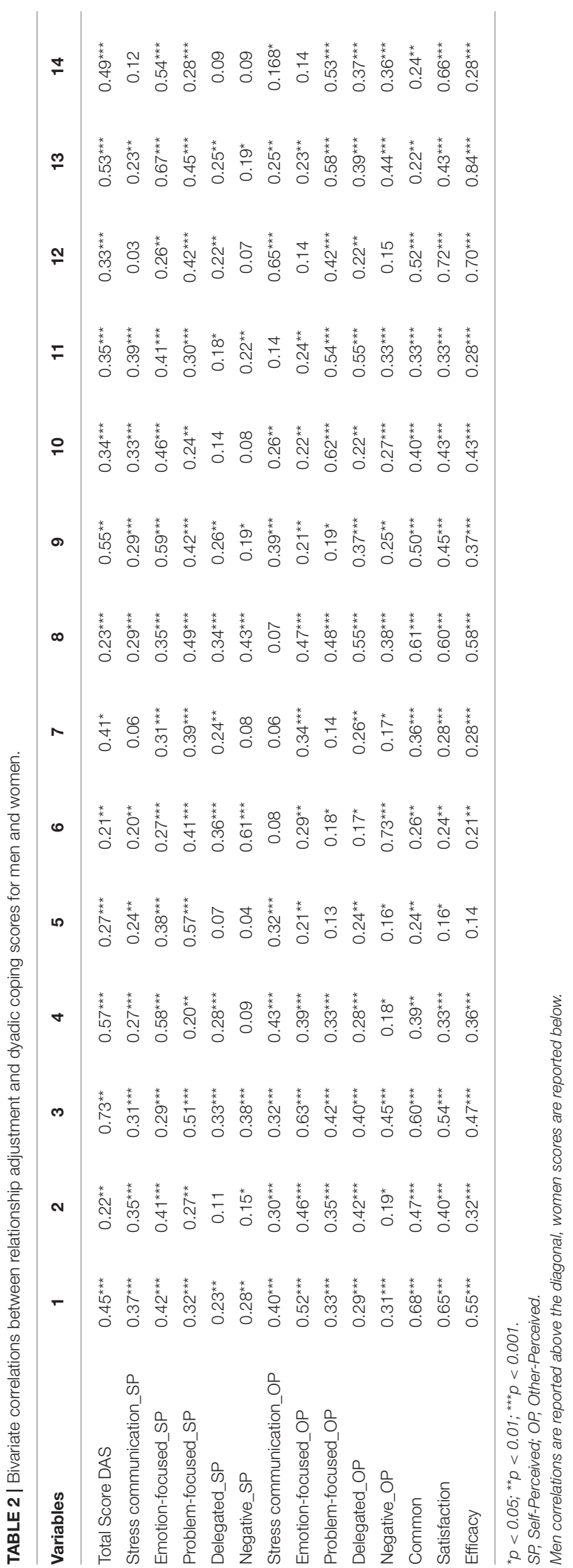

TABLE 3 | Predicting self-reported marital adjustment from self-perceived dyadic coping scores.

\begin{tabular}{|c|c|c|c|}
\hline & \multirow[b]{2}{*}{$\beta$} & \multicolumn{2}{|c|}{$95 \%$ confidence interval } \\
\hline & & Lower & Upper \\
\hline \multicolumn{4}{|c|}{ PREDICTING FEMALE MARITAL ADJUSTMENT } \\
\hline \multicolumn{4}{|c|}{ Own marital adjustment (actor effects) } \\
\hline F Stress Communication & $3.82^{* \star \star}$ & 1.79 & 5.84 \\
\hline F Emotion-Focused DC & $4.39^{\star \star \star}$ & 2.59 & 6.18 \\
\hline F Problem-Focused DC & $2.94^{\star \star \star}$ & 1.77 & 4.12 \\
\hline F Delegated DC & $2.37^{\star}$ & 0.44 & 4.30 \\
\hline F Negative DC & $3.17^{\star \star}$ & 0.86 & 5.49 \\
\hline \multicolumn{4}{|c|}{ Partner's marital adjustment (partner effects) } \\
\hline M Stress Communication & $2.00^{\star \star}$ & 0.79 & 3.21 \\
\hline M Emotion-Focused DC & $3.23^{* \star \star}$ & 1.57 & 4.89 \\
\hline M Problem-Focused DC & $2.36^{* \star \star}$ & 1.21 & 3.53 \\
\hline M Delegated DC & $1.95^{\star}$ & 0.18 & 3.72 \\
\hline M Negative DC & 0.94 & -1.29 & 3.18 \\
\hline \multicolumn{4}{|c|}{ PREDICTING MALE MARITAL ADJUSTMENT } \\
\hline \multicolumn{4}{|c|}{ Own marital adjustment (actor effects) } \\
\hline M Stress Communication & 0.72 & -1.08 & 2.53 \\
\hline M Emotion-Focused DC & $5.40^{\star \star \star}$ & 3.91 & 6.88 \\
\hline M Problem-Focused DC & $2.94^{\star \star \star}$ & 1.77 & 4.12 \\
\hline M Delegated DC & $1.96^{\star}$ & 0.26 & 3.66 \\
\hline M Negative DC & $3.78^{\star \star \star}$ & 1.73 & 5.83 \\
\hline \multicolumn{4}{|c|}{ Partner's marital adjustment (partner effects) } \\
\hline F Stress Communication & $2.00^{\star \star}$ & 0.79 & 3.21 \\
\hline F Emotion-Focused DC & $2.83^{\star \star}$ & 1.22 & 4.45 \\
\hline F Problem-Focused DC & $2.36^{\star \star \star}$ & 1.20 & 3.53 \\
\hline F Delegated DC & $2.06^{\star}$ & 0.21 & 3.92 \\
\hline F Negative DC & 0.91 & -1.19 & 3.02 \\
\hline
\end{tabular}

of studies today are in agreement that the infertility experience is more stressful for women than for men, with infertile women generally reporting more stress regarding their condition (Berghuis and Stanton, 2002; Peterson et al., 2008; Ying et al., 2015; Greil et al., 2018; Kroemeke and Kubicka, 2018). At the same time, according to researchers who do not find differences between the partners, it is possible that the stress and emotional hardship connected to the experience of infertility are shared by the partners through a spill-over effect from one partner to the other, and that, for this reason, couple adjustment also presents similar levels. It should also be emphasized that, according to some authors, the congruence between the partners as regards couple adjustment representation is a positive factor for the couple itself and a protective factor, both in infertile couples as well as in the context of dyadic coping (Peterson et al., 2003; Iafrate et al., 2012).

Since dyadic coping impacts on marital adjustment, as several studies have extensively reported (e.g., Bodenmann et al., 2006; Falconier et al., 2015; Rusu et al., 2018), we can suppose that the presence in our study of similar levels of marital adjustment in males and females may be explained considering the specific role 
TABLE 4 | Predicting self-reported marital adjustment from other-perceived dyadic coping scores.

\begin{tabular}{|c|c|c|c|}
\hline & \multirow[b]{2}{*}{$\beta$} & \multicolumn{2}{|c|}{$95 \%$ confidence interval } \\
\hline & & Lower & Upper \\
\hline \multicolumn{4}{|c|}{ PREDICTING FEMALE MARITAL ADJUSTMENT } \\
\hline \multicolumn{4}{|c|}{ Own marital adjustment (actor effects) } \\
\hline F Stress Communication & $3.42^{\star \star \star}$ & 2.08 & 4.75 \\
\hline F Emotion-Focused DC & $4.57^{\star \star \star}$ & 2.71 & 6.43 \\
\hline F Problem-Focused DC & $3.03^{\star \star}$ & 1.08 & 4.98 \\
\hline F Delegated DC & $3.03^{\star *}$ & 1.29 & 4.77 \\
\hline F Negative DC & $4.74^{\star \star \star}$ & 2.83 & 6.64 \\
\hline \multicolumn{4}{|c|}{ Partner's marital adjustment (partner effects) } \\
\hline M Stress Communication & $2.39^{\star \star \star}$ & 1.07 & 3.73 \\
\hline M Emotion-Focused DC & $2.85^{\star \star}$ & 1.04 & 4.65 \\
\hline M Problem-Focused DC & $2.00^{*}$ & 0.29 & 3.71 \\
\hline M Delegated DC & $2.18^{*}$ & 0.27 & 4.09 \\
\hline M Negative DC & -0.96 & -2.71 & 0.79 \\
\hline \multicolumn{4}{|c|}{ PREDICTING MALE MARITAL ADJUSTMENT } \\
\hline \multicolumn{4}{|c|}{ Own marital adjustment (actor effects) } \\
\hline M Stress Communication & $3.42^{\star \star \star}$ & 2.08 & 4.75 \\
\hline M Emotion-Focused DC & $4.69^{\star \star \star}$ & 3.01 & 6.36 \\
\hline M Problem-Focused DC & $2.82^{\star \star}$ & 1.25 & 4.40 \\
\hline M Delegated DC & $4.15^{\star \star \star}$ & 2.38 & 5.92 \\
\hline M Negative DC & $1.68^{*}$ & 0.02 & 3.36 \\
\hline \multicolumn{4}{|c|}{ Partner's marital adjustment (partner effects) } \\
\hline F Stress Communication & $2.39^{\star \star \star}$ & 1.07 & 3.73 \\
\hline F Emotion-Focused DC & $2.94^{\star \star}$ & 1.21 & 4.67 \\
\hline F Problem-Focused DC & $3.72^{\star \star \star}$ & 1.92 & 5.52 \\
\hline F Delegated DC & $2.14^{\star \star}$ & 0.53 & 3.75 \\
\hline F Negative DC & $3.22^{\star \star}$ & 1.41 & 5.05 \\
\hline
\end{tabular}

of coping strategies and the several effects that these strategies have not only at an intra-individual level, but also at interpersonal level (Donato et al., 2009). Indeed, dyadic coping strategies, that represent the efforts of both partners to face together with a critical and stressful experience, can contribute to a greater sharing of the feelings and thoughts related to infertility and, in this way, to a perceived more similar distress related to this experience.

In our study, significant differences existed between women and their male partners only for stress communication, both when self- and other-perceived communication were assessed, as well as efficacy. Specifically, women perceived themselves more able to communicate stress to their partner than men and, at the same time, men perceived their partner more able to communicate stress than themselves. Overall, this finding confirms previous studies that reported some gender specific patterns within the couple relationship (Helgeson, 2011) and, specifically, the greater ability of women to communicate their distress to the partner and the greater use of avoidant behaviors among men, which could be related to a major difficulty in expressing and communicating their emotions (Jackson et al.,
TABLE 5 | Predicting self-reported marital adjustment from common dyadic coping, satisfaction with dyadic coping behaviors and efficacy of dyadic coping behaviors.

\begin{tabular}{|c|c|c|c|}
\hline & \multirow[b]{2}{*}{$\beta$} & \multicolumn{2}{|c|}{$95 \%$ confidence interval } \\
\hline & & Lower & Upper \\
\hline \multicolumn{4}{|c|}{ PREDICTING FEMALE MARITAL ADJUSTMENT } \\
\hline \multicolumn{4}{|c|}{ Own marital adjustment (actor effects) } \\
\hline F Common DC & $12.35^{\star \star \star}$ & 10.03 & 14.67 \\
\hline F Satisfaction with DC & $7.03^{\star \star \star}$ & 5.36 & 8.69 \\
\hline F Efficacy of DC & $6.94^{\star \star \star}$ & 4.85 & 9.02 \\
\hline \multicolumn{4}{|c|}{ Partner's marital adjustment (partner effects) } \\
\hline M Common DC & $1.27^{\star}$ & 0.14 & 2.41 \\
\hline M Satisfaction with DC & $2.72^{\star \star \star}$ & 1.26 & 4.17 \\
\hline M Efficacy of DC & $2.30^{\star}$ & 0.43 & 4.16 \\
\hline \multicolumn{4}{|c|}{ PREDICTING MALE MARITAL ADJUSTMENT } \\
\hline \multicolumn{4}{|c|}{ Own marital adjustment (actor effects) } \\
\hline M Common DC & 0.06 & -0.58 & 1.85 \\
\hline M Satisfaction with DC & $4.45^{\star \star \star}$ & 2.99 & 5.92 \\
\hline M Efficacy of DC & $5.25^{\star \star \star}$ & 3.37 & 7.13 \\
\hline \multicolumn{4}{|c|}{ Partner's marital adjustment (partner effects) } \\
\hline F Common DC & $9.77^{\star \star \star}$ & 7.28 & 12.27 \\
\hline F Satisfaction with DC & $3.84^{\star \star \star}$ & 2.16 & 5.51 \\
\hline F Efficacy of DC & $2.82^{\star \star}$ & 0.84 & 4.81 \\
\hline
\end{tabular}

2014). Furthermore, it is coherent with other studies that highlight how women's infertility stress is greater than that of men, despite being related to partner behaviors (Martins et al., 2014b). Moreover, our result confirms other studies on dyadic coping that revealed gender differences in the use of dyadic coping strategies in couples (Staff et al., 2017), with women perceiving themselves more able to communicate their stress than men (Bodenmann and Cina, 2005; Molgora et al., 2018). We can hypothesize that, even in our sample, gender differences in communication, now widely recognized in the literature, become evident in a situation-the ART experience-that sees women particularly involved both on the physical level (think of all the exams to which women are subjected, to the hormonal stimulation, to the pick-up to which they are prepared, etc.) and the psychological one. Indeed, it is likely that women experience a very intense stress, higher than what men experience in the same moment, but that they also can better express and communicate it to the partner. Finally, this result highlights again a perceptual congruence between the two partners within the couple (Iafrate et al., 2012); this congruence could be connected to the overall good couple relationship found in our sample. Indeed, although the Dyadic Adjustment Scale did not have cut-off values, the mean of our samples denotes high scores, in line with normative data on the general population, indicating a satisfactory couple relationship. From a clinical perspective, we could wonder whether this good quality represents a defense on the part of the partners who are facing the pain of infertility. Certainly, we can argue that in this specific moment the couple seems to be 
working to cope with some individual struggles connected to this experience which have some gender-specific dimensions.

As for efficacy, men perceived their dyadic coping to be more efficient than women, confirming findings of a previous study on couple transitioning to parenthood in which men were found to perceive dyadic coping too be more efficient than women did (Molgora et al., 2018). We can speculate that this perception can be associated with differential stress-related consequences of the infertility as well as the ART experience between men and women (Bayley et al., 2009; Davidovà and Pechovà, 2014; Ying et al., 2015).

Considering the association between dyadic coping and marital adjustment, as the literature has widely recognized on non-clinical couples in different stages of the life course (Donato et al., 2014; Falconier et al., 2015; Molgora et al., 2018), results confirm that in our sample of infertile couples undergoing an ART treatment the ability to manage the infertility-related stress as a "common problem" is linked to a better couple adjustment and increases marital adjustment. Although some studies have reported that men and women manage the stress for infertility by activating gender-specific individual coping strategies (Peterson et al., 2006b), our findings show that partners are able to contemporaneously face this critical experience together, living their problem as a "dyadic problem."

\section{Association Between Dyadic Coping and Marital Adjustment}

The use of the APIM promoted a meaningful examination of the association between dyadic coping and marital adjustment, and of the experience of infertile couples as they approach assisted reproduction. As outlined by the theoretical framework and the available evidence to date, some positive dyadic coping dimensions were found to be associated with higher levels of relationship adjustment both in women and their partners, without gender differences. We might suppose that these findings explain the common dimension of the infertility experience, which, as we reported in the introduction, involved both partner beyond the type of diagnosis and requires the activation of functional coping strategies both in women and in their partners. In particular, both an actor and a partner effect were found for emotion-focused, problem-focused and delegated dyadic coping, both self-perceived and other-perceived, as well as for common coping, meaning that women and their partners reporting high levels of these strategies were more likely to report higher adjustment scores and, at the same time, men and women whose partners reported higher levels of these dyadic coping strategies were predicted to report high levels of couple adjustment. These results are consistent with the literature on dyadic coping, as more effective coping styles have been associated with better relational outcomes across samples and over time (Bodenmann and Cina, 2005; Falconier et al., 2015; Bodenmann et al., 2016).

Although for most of the coping subscales actor and/or partner effects were similar in both men and women, in some specific dimensions gender specificities emerged. Specifically, we found only a female actor effect of stress communication (selfperceived) on couple adjustment, partially supporting what has been already found in studies showing that women assign greater importance than men to communication within the couple (Matud, 2004).

Moreover, although for both partners the actor effect of dyadic coping satisfaction on couple adjustment is significant, the results show that this effect is stronger for women. Therefore, although for both partners the satisfaction of being able to face a stressful event together contributes to their marital relationship, we can assume that this connection acquires a specific centrality for the woman, more directly involved in the ART process than the partner and more sensitive to the partner's dyadic support. At this crucial moment in a couple's life, it is possible that the woman's marital adjustment is more strongly linked to the feeling that the partner is engaged in these "common" efforts.

Some gender specificities have also been found for men. Specifically, the results show a stronger partner effect for men in common dyadic coping. In our study men's perception of couple adjustment is predicted by the partners' common dyadic coping perception. As already mentioned above, the ART process mainly involves the woman, who is subjected to a great physical and mental stress. We can hypothesize that in this phase men are particularly focused on the well-being of their partner and that their couple's adjustment is strongly influenced by the woman's perception that the dyad is coordinating their strategies. At the same time, the fact that women feel they are jointly coping with the ART process can make the partner feel more involved, and therefore more satisfied with the couple relationship. Moreover, other studies using this dyadic approach reported an overall gender difference, with men more influenced by their partner than vice versa (Bodenmann et al., 2006; Greil et al., 2018).

Finally, the findings about negative coping indicate that men are more satisfied with their relationship if their partners feel empathetically (without superficial or ambivalent behaviors) supported by them. Once again, we might suppose that the male perception of the relationship is in this phase very focused on the needs of the woman, who is especially involved in the process of ART, not only at a psychological level but also at a physical one.

Although our study was among the first to examine dyadic coping in the context of infertility, results confirmed what the literature has presented in other groups of couples facing other stressors: a relationship exists between dyadic coping and marital adjustment. The partners' ability to jointly cope with the stressful ART process makes the couple more adjusted. The results also show how these effects are reciprocal within the couple: one's partner's perception about dyadic coping affects one's own couple adjustment, and vice versa. However, some gender specificities emerged: women and men show some typical differences in their relationship and in the way they cope with stress. Women are strongly involved in the ART process, and this can activate some specific couple dynamics, with a particular focus on the needs of the woman.

In conclusion, the intrinsically dyadic nature of the infertility experience can contribute to explaining the presence of a high congruence in our sample between men and women with respect to the dyadic coping strategies put into action and to the impact that these strategies have on marital adjustment, as well as to the effects of reciprocal influence between one's perception and that of one's partner (cross-partner effects). Nevertheless, some specific aspects emerge connected to gender that are 
expressed in differences in some dimensions of dyadic coping, and which in part reflect differences also found in the general population, which speak to a gender specific functioning. These differences can also be connected to specific thoughts and feelings linked to this experience that see men and women involved in different ways. The differing involvement of men and women in the infertility experience and subsequent treatments can be understood in terms of a socio-cultural dimension, that is connected to the different meanings and expectations that society nurtures with respect to motherhood and fatherhood, as well as in terms of more structural aspects, which we could say pertain to identity and are connected to the deep significance that motherhood has for women, as distinct from what fatherhood means for men.

\section{Limitations}

Some limitations affect this study. First, some medical variables related to infertility (i.e., partner with the diagnosis of infertility, number of treatment, type of ART) were not controlled. If for methodological reasons mostly connected to the sample size, we did not include in our models the medical variables in our possession (in particular, the number of IVF treatments), we can assume that the actor and partner effects of dyadic coping strategies on marital adjustment are impacted by these variables. Furthermore, this is a crosssectional study that involves couples undergoing ART, so the direction of the association we tested is theory driven. A longitudinal design is needed to better understand the association between dyadic coping and marital adjustment and to find trajectories of change over time. Finally, we have considered the impact of dyadic coping styles on marital adjustment. Future research could investigate the role of individual well-being (e.g., anxiety and depressive symptoms) as a moderator of this relation. Indeed, some studies have reported how dyadic coping impacts individual mental health (e.g., Bodenmann et al., 2008).

Despite these limitations, the present study underscored the importance of considering the decision to undergo assisted

\section{REFERENCES}

Agostini, F., Monti, F., Andrei, F., Paterlini, M., Palomba, S., and La Sala, G. B. (2017). Assisted reproductive technology treatments and quality of life: a longitudinal study among subfertile women and men. J. Assist. Reprod. Genet. 34, 1307-1315. doi: 10.1007/s10815-017-1000-9

Alves, S., Fonseca, A., Canavarro, M. C., and Pereira, M. (2018). Dyadic coping and dyadic adjustment in couples with women with high depressive symptoms during pregnancy. J. Reprod. Infant Psychol. 36, 504-518. doi: 10.1080/02646838.2018.1490496

Amato, P. R., Booth, A., Johnson, D. R., Johnson, D. R., and Rogers, S. J. (2007). Alone Together: How Marriage in America is Changing. Cambridge, MA: Harvard University Press.

Amiri, M., Sadeqi, Z., Hoseinpoor, M. H., and Khosravi, A. (2016). Marital satisfaction and its influencing factors in fertile and infertile women. J. Fam. Reproduc. Health 10, 139-145.

Badr, H., Carmack, C. L., Kashy, D. A., Cristofanilli, M., and Revenson, T. A. (2010). Dyadic coping in metastatic breast cancer. Health Psychol. 29, 169-180. doi: $10.1037 / \mathrm{a} 0018165$ reproductive technology treatments as a shared experience, i.e., a dyadic stressor, which requires dyadic strategies.

\section{Clinical Implications}

These research findings have important clinical implications and may assist in developing interventions to promote individual and dyadic coping with infertility. The presence of good levels of couple adjustment, similar between partners, and the fact that adaptive coping strategies impact on couple adjustment, suggest that in this crucial moment (start of an ART procedure), the couple can represent an important resource for partners. This could be a useful element also for medical staff (doctors, nurses, etc.), who can rely on a good "couple's alliance" managing the stressful ART process. From a clinical point of view, the results obtained lead us to think that the couple could be positively considered during the ART process as a useful "common container" of the individual efforts, often connected to gender-specific components, of each partner in the ART process. In other words, the results lead us to ask ourselves if the couple relationship could represent not the specific focus (i.e., "the object") of psychological interventions, but rather an effective resource through which to enhance the well-being of the individual partners, so differently involved in the ART process. Indeed, in this phase of life partners show to be, for different reasons, perhaps also defensively, satisfied with their relationship and able to jointly manage the common ART experience.

\section{AUTHOR CONTRIBUTIONS}

SM contributed to developing the study design and to writing the entire manuscript. VF contributed to developing the study design, the data set for individual, and dyadic data analysis, and to writing the method, and discussion sections. CA performed dyadic data analysis and contributed to writing the method, results and discussion sections. ADD contributed to developing the study design and to reviewing the manuscript. MB contributed to the data collection. ES contributed to developing the study design, and to writing the discussion section. All the authors reviewed and approved the manuscript for publication.

Badr, H., Herbert, K., Bonnen, M. D., Asper, J. A., and Wagner, T. (2018). Dyadic coping in patients undergoing radiotherapy for head and neck cancer and their spouses. Front. Psychol. 9:1780. doi: 10.3389/fpsyg.2018.01780

Bayley, T. M., Slade, P., and Lashen, H. (2009). Relationships between attachment, appraisal, coping and adjustment in men and women experiencing infertility concerns. Hum. Reproduc. 24, 2827-2837. doi: 10.1093/humrep/dep235

Berghuis, J. P., and Stanton, A. L. (2002). Adjustment to a dyadic stressor: a longitudinal study of coping and depressive symptoms in infertile couples over an insemination attempt. J. Consult. Clin. Psychol. 70, 433-438. doi: 10.1037/0022-006X.70.2.433

Bodenmann, G. (1995). A systemic-transactional view of stress and coping in couples. Swiss J. Psychol. 54, 34-49.

Bodenmann, G. (2000). Stress Und Coping Bei Paaren [Stress and Coping in Couples]. Göttingen: Hogrefe.

Bodenmann, G. (2005). "Dyadic coping and its significance for marital functioning," in Couples Coping With Stress: Emerging Perspectives on Dyadic Coping, eds T. A. Revenson, K. Kayser, and G. Bodenmann (Washington, DC: American Psychological Association), 33-49. doi: 10.1037/ 11031-002 
Bodenmann, G. (2008). Dyadisches Coping Inventar: Testmanual [Dyadic Coping Inventory: Test manual]. Bern: Huber.

Bodenmann, G., and Cina, A. (2005). Stress and coping among stablesatisfied, stable-distressed and separated/divorced Swiss couples: a 5-years prospective longitudinal study. J. Divorce Remarr. 44, 71-89. doi: 10.1300/ J087v44n01_04

Bodenmann, G., Meuwly, N., Germann, J., Nussbeck, F. W., Heinrichs, M., and Bradbury, T. N. (2015). Effects of stress on the social support provided by men and women in intimate relationships. Psychol. Sci. 26, 1584-1594. doi: $10.1177 / 0956797615594616$

Bodenmann, G., Pihet, S., and Kayser, K. (2006). The relationship between dyadic coping and marital quality: a 2-year longitudinal study. J. Fam. Psychol. 20, 485-493. doi: 10.1037/0893-3200.20.3.485

Bodenmann, G., Plancherel, B., Beach, S. R., Widmer, K., Gabriel, B., Meuwly, N., et al. (2008). Effects of coping-oriented couples therapy on depression: a randomized clinical trial. J. Consult. Clin. Psychol. 76:944. doi: $10.1037 / \mathrm{a} 0013467$

Bodenmann, G., Randall, A. K., and Falconier, M. K. (eds.). (2016). "Coping in couples: The Systemic Transactional Model (STM)," in Couples Coping With Stress: A Cross-Cultural Perspective, (New York, NY: Routledge), 2-22.

Brandes, M., Van Der Steen, J. O. M., Bokdam, S. B., Hamilton, C. J. C. M., De Bruin, J. P., Nelen, W. L. D. M., et al. (2009). When and why do subfertile couples discontinue their fertility care? A longitudinal cohort study in a secondary care subfertility population. Hum. Reproduc. 24, 3127-3135. doi: 10.1093/humrep/dep340

Breitenstein, C. J., Milek, A., Nussbeck, F. W., Davila, L., and Bodenmann, G. (2018). Stress, dyadic coping, and relationship satisfaction in late adolescent couples. J. Soc. Pers. Relat. 35, 770-790. doi: 10.1177/0265407517698049

Chaves, C., Canavarro, M. C., and Moura-Ramos, M. (2018). The role of dyadic coping on the marital and emotional adjustment of couples with infertility. Fam. Process. doi: 10.1111/famp. 12364. [Epub ahead of print]

Cigoli, V., and Scabini, E. (2006). Family Identity: Ties, Symbols and Transitions. New York, NY: Taylor.

Davidovà, K., and Pechovà, O. (2014). Infertility and assisted reproduction technologies through a gender lens. Hum. Affairs 24, 363-375. doi: 10.2478/s13374-014-0234-9

Donato, S., Iafrate, R., Barni, D., Bertoni, A., Bodenmann, G., and Gagliardi, S. (2009). Measuring dyadic coping: The factorial structure of Bodenmann's "Dyadic Coping Questionnaire" in an Italian sample. TPM 16, 25-47.

Donato, S., Parise, M., Iafrate, R., Bertoni, A., Finkenauer, C., and Bodenmann, G. (2014). Dyadic coping responses and partners' perceptions for couple satisfaction. An actor-partner interdependence analysis. J. Soc. Pers. Relation. 32, 580-600. doi: 10.1177/0265407514541071

El Kissi, Y., Romdhane, A. B., Hidar, S., Bannour, S., Ayoubi Idrissi, K., Khairi, H., et al. (2013). General psychopathology, anxiety, depression and self-esteem in couples undergoing infertility treatment: a comparative study between men and women. Eur. J. Obstetr. Gynecol. Reproduc. Biol. 167, 185-189. doi: 10.1016/j.ejogrb.2012.12.014

European IVF-monitoring Consortium (EIM), European Society of Human Reproduction and Embryology (ESHRE), Calhaz-Jorge, C., De Geyter, C., Kupka, M. S., de Mouzon, J., et al. (2017). Assisted reproductive technology in Europe, 2013: results generated from European registers by ESHRE. Hum. Reproduc. 32, 1957-1973. doi: 10.1093/humrep/dex264

Falconier, M. K., Jackson, J. B., Hilpert, P., and Bodenmann, G. (2015). Dyadic coping and relationship satisfaction: a meta-analysis. Clin. Psychol. Rev. 42, 28-46. doi: 10.1016/j.cpr.2015.07.002

Ferraretti, A. P., Nygren, K., Nyboe Andersen, A., de Mouzon, J., Kupka, M., Calhaz-Jorge, C., et al. (2017). Trends over 15 years in ART in Europe: an analysis of 6 million cycles. Hum. Reproduc. Open 2. doi: 10.1093/hropen/hox012

Gager, C. T., and Sanchez, L. (2003). Two as one? Couples' perceptions of time spent together, marital quality, and the risk of divorce. J. Fam. Issues 24, 21-50. doi: 10.1177/0192513X02238519

Gameiro, S., Boivin, J., Peronace, L., and Verhaak, C. M. (2012). Why do patients discontinue fertility treatment? A systematic review of reasons and predictors of discontinuation in fertility treatment. Hum. Reproduc. Update 18, 652-669. doi: 10.1093/humupd/dms031
Gana, K., and Jakubowska, S. (2016). Relationship between infertility-related stress and emotional distress and marital satisfaction. J. Health Psychol. 21, 1043-1054. doi: 10.1177/1359105314544990

Gentili, P., Contreras, L., Cassaniti, M., and D'Arista, F. (2002). La dyadic adjustment scale: una misura dell'adattamento di coppia [The dyadic adjustment scale: A measure of couple adjustment]. Minerva Psichiatr. $43,107-116$.

Ghafouri, S. F., Ghanbari, S., Fallahzadeh, H., and Shokri, O. (2016). The relation between marital adjustment and posttraumatic growth in infertile couples: the mediatory role of religious coping strategies. J. Reproduc. Infertil. 17, 221-229.

Greil, A. L., Slauson-Blevins, K. S., McQuillan, J., Lowry, M. H., Burch, A. R., and Shreffler, K. M. (2018). Relationship satisfaction among infertile couples: implications of gender and self-identification. J. Fam. Issues 39, 1304-1325. doi: 10.1177/0192513X17699027

Greil, A. L., Slauson-Blevins, K. S., Shreffler, K. M., Johnson, K. M., Lowry, M. H., Burch, A. R., and McQuillan, J. (2017). Decline in ethical concerns about reproductive technologies among a representative sample of US women. Public Understand. Sci. 26, 789-805. doi: 10.1177/0963662515625402

Hagedoorn, M., Sanderman, R., Bolks, H. N., Tuinstra, J., and Coyne, J. C. (2008). Distress in couples coping with cancer: a meta-analysis and critical review of role and gender effects. Psychol. Bull. 134, 1-30. doi: 10.1037/0033-2909.134.1.1

Helgeson, V. S. (2011). "Gender, stress and coping," in The Oxford Handbook of Stress, Health and Coping, ed S. Folkman (New York, NY: Oxford University Press), 63-85.

Hilpert, P., Randall, A. K., Sorokowski, P., Atkins, D. D., Sorokowska, A., Ahmadi, K., et al. (2016). The associations of dyadic coping and relationship satisfaction vary between and within nations: A 35-nation study. Front. Psychol. 7:1106. doi: 10.3389/fpsyg.2016.01106

Iafrate, R., Bertoni, A., Margola, D., Cigoli, V., and Acitelli, L. K. (2012). The link between perceptual congruence and couple relationship satisfaction in dyadic coping. Eur. Psychol. 17, 73-82. doi: 10.1027/1016-9040/a000069

Istat (2017). Natalità e Fecondità Della Popolazione Residente [Birth and Fertility of the Resident Population]. Statistiche report. Roma: Istituto Nazionale di Statistica.

Jackson, J. B., Miller, R. B., Oka, M., and Henry, R. G. (2014). Gender differences in marital satisfaction: a meta-analysis. J. Marriage Fam. 76, 105-129. doi: 10.1111/jomf.12077

Kamp Dush, C. M., Taylor, M. G., and Kroeger, R. A. (2008). Marital happiness and psychological well-being across the life course. Fam. Relations 57, 211-226. doi: 10.1111/j.1741-3729.2008.00495.x

Kenny, D. A., Kashy, D. A., and Cook, W. L. (2006). Dyadic Data analysis. New York, NY: Cambridge University Press.

Koert, E., and Daniluk, J. C. (2018). When time runs out: reconciling permanent childlessness after delayed childbearing. J. Reprod. Infant Psychol. 35, 342-352. doi: 10.1080/02646838.2017.1320363

Kroemeke, A., and Kubicka, E. (2018). Positive and negative adjustment in couples undergoing infertility treatment: the impact of support exchange. PLOS ONE 13:e200124. doi: 10.1371/journal.pone.0200124

Landis, M., Peter-Wight, M., Martin, M., and Bodenmann, G. (2013). Dyadic coping and marital satisfaction of older spouses in long-term marriage. GeroPsych 26, 39-47. doi: 10.1024/1662-9647/a000077

Ledermann, T., Bodenmann, G., and Cina, A. (2007). The efficacy of the Couples Coping Enhancement Training (CCET) in improving relationship quality. J. Soc. Clin. Psychol. 26, 940-959. doi: 10.1521/jscp.2007.26.8.940

Ledermann, T., Bodenmann, G., Rudaz, M., and Bradbury, T. N. (2010). Stress, communication, and marital quality in couples. Fam. Relat. 59, 195-206. doi: 10.1111/j.1741-3729.2010.00595.x

Lee, T. Y., and Sun, G. H. (2000). Psychological response of Chinese infertile husbands and wives. Arch. Androl. 45, 143-148. doi: 10.1080/01485010050193913

Leuchtmann, L., and Bodenmann, G. (2018). "New perspectives on dynamics of dyadic coping", in When "We" are Stressed. A Dyadic Approach to Coping With Stressful Events, eds. A. Bertoni, S. Donato, and S. Molgora (New York, NY: Nova Science Publisher), 3-14.

Loghi, M., and Crialesi, R. (2017). La Salute Riproduttiva Della Donna [The Reproductive Health of the Woman]. Roma: Istituto Nazionale di Statistica.

Lowyck, B., Luyten, P., Corveleyn, J., D'Hooghe, T., Buyse, E., and Demyttenaere, K. (2009). Well-being and relationship satisfaction of couples dealing with an 
in vitro fertilization/ intracytoplasmic sperm injection procedure: a multilevel approach on the role of self-criticism, dependency, and romantic attachment. Fertil. Steril. 91, 387-394. doi: 10.1016/j.fertnstert.2007.11.052

Maroufizadeh, S., Karimi, E., Vesali, S., and Omani Samani, R. (2015). Anxiety and depression after failure of assisted reproductive treatment among patients experiencing infertility. Int. J. Gynaecol. Obstetr. 130, 253-256. doi: 10.1016/j.ijgo.2015.03.044

Martins, M. V., Basto-Pereira, M., Pedro, J., Peterson, B. D., Almeida, V., Schmidt, L., et al. (2016). Male psychological adaptation to unsuccessful medically assisted reproduction treatments: a systematic review. Hum. Reprod. Update 22, 466-478. doi: 10.1093/humupd/dmw009

Martins, M. V., Costa, P., Peterson, B. D., Costa, M. E., and Schmidt, L. (2014a). Marital stability and repartnering: infertility-related stress trajectories of unsuccessful fertility treatment. Fertil. Steril. 102, 1716-1722. doi: 10.1016/j.fertnstert.2014.09.007

Martins, M. V., Peterson, B. D., Almeida, V., Mesquita-Guimarães, J., and Costa, M. E. (2014b). Dyadic dynamics of perceived social support in couples facing infertility. Hum. Reproduc. 29, 83-89. doi: 10.1093/humrep/det403

Matud, M. P. (2004). Gender differences in stress and coping styles. Pers. Individ. Dif. 37, 1401-1415. doi: 10.1016/j.paid.2004.01.010

Molgora, S., Acquati, C., Fenaroli, V., and Saita, E. (2018). Dyadic coping and marital adjustment during pregnancy: a cross-sectional study on Italian couples expecting their first child. Int. J. Psychol. doi: 10.1002/ijop.12476. [Epub ahead of print]

Monga, M., Alexandrescu, B., Katz, S. E., Stein, M., and Ganiats, T. (2004). Impact of infertility on quality of life, marital adjustment, and sexual function. Urology 63, 126-130. doi: 10.1016/j.urology.2003.09.015

Moura-Ramos, M., Gameiro, S., Canavarro, M. C., Soares, I., and Almeida-Santos, T. (2016). Does infertility history affect the emotional adjustment of couples undergoing assisted reproduction? The mediating role of the importance of parenthood. Br. J. Health Psychol. 21, 302-317. doi: 10.1111/bjhp.12169

Pasch, L. A., and Sullivan, K. T. (2017). Stress and coping in couples facing infertility. Curr. Opin. Psychol. 13, 131-135. doi: 10.1016/j.copsyc.2016.07.004

Pasha, H., Basirat, Z., Esmailzadeh, S., Faramarzi, M., and Adibrad, H. (2017). Marital intimacy and predictive factors among infertile women in northern Iran. J. Clin. Diagn. Res. 11, QC13-QC17. doi: 10.7860/JCDR/2017/24972.9935

Péloquin, K., Brassard, A., Arpin, V., Sabourin, S., and Wright, J. (2018). Whose fault is it? Blame predicting psychological adjustment and couple satisfaction in couples seeking fertility treatment. J. Psychosomat. Obstetr. Gynecol. 39, 64-72. doi: 10.1080/0167482X.2017.1289369

Peterson, B. D., Newton, C. R., and Rosen, K. H. (2003). Examining congruence between partners' perceived infertility-related stress and its relationship to marital adjustment and depression in infertile couples. Fam. Process 42, 59-70. doi: 10.1111/j.1545-5300.2003.00059.x

Peterson, B. D., Newton, C. R., Rosen, K. H., and Schulman, R. S. (2006a). Coping processes of couples experiencing infertility. Fam. Relat. 55, 227-239. doi: 10.1111/j.1741-3729.2006.00372.x

Peterson, B. D., Newton, C. R., Rosen, K. H., and Skaggs, G. E. (2006b). Gender differences in how men and women who are referred for IVF cope with infertility stress. Hum. Reproduct. 21, 2443-2449. doi: 10.1093/humrep/del145

Peterson, B. D., Pirritano, M., Block, J. M., and Schmidt, L. (2011). Marital benefit and coping strategies in men and women undergoing unsuccessful fertility treatments over a 5-year period. Fertil. Steril. 95, 1759-1763. doi: 10.1016/j.fertnstert.2011.01.125

Peterson, B. D., Pirritano, M., Christensen, U., and Schmidt, L. (2008). The impact of partner coping in couples experiencing infertility. Hum. Reproduc. 23, 1128-1137. doi: 10.1093/humrep/den067

Regan, T. W., Lambert, S. D., Kelly, B., McElduff, P., Girgis, A., Kayser, K., et al. (2014). Cross-sectional relationships between dyadic coping and anxiety, depression, and relationship satisfaction for patients with prostate cancer and their spouses. Patient Educ. Couns. 96, 120-127. doi: 10.1016/ j.pec.2014.04.010

Reis, S., Xavier, M. R., Coelho, R., and Montenegro, N. (2013). Psychological impact of single and multiple courses of assisted reproductive treatments in couples: a comparative study. Eur. J. Obstetr. Gynecol. Reproduc. Biol. 171, 61-66. doi: 10.1016/j.ejogrb.2013.07.034

Rockliff, H. E., Lightman, S. L., Rhidian, E., Buchanan, H., Gordon, U., and Vedhara, K. (2014). A systematic review of psychosocial factors associated with emotional adjustment in in vitro fertilization patients. Hum. Reprod. Update 20, 594-613. doi: 10.1093/humupd/dmu010

Rooney, K. L., and Domar, A. D. (2016). The impact of stress on fertility treatment. Curr. Opin. Obstetr. Gynecol. 28, 198-201. doi: 10.1097/GCO.0000000000000261

Rottmann, N., Hansen, D. G., Larsen, P. V., Nicolaisen, A., Flyger, H., Johansen, C., et al. (2015). Dyadic coping within couples dealing with breast cancer: a longitudinal, population-based study. Health Psychol. 34, 486-495. doi: $10.1037 /$ hea0000218

Rusu, P. P., Bodenmann, G., and Kayser, K. (2018). Cognitive emotion regulation and positive dyadic outcomes in married couples. J. Soc. Personal Relation. 36, 359-376. doi: 10.1177/0265407517751664

Samadaee-Gelehkolaee, K., McCarty, B. W., Khalilian, A., Hamzehgardeshi, Z., Peyvandi, S., Elyasi, F., et al. (2016). Factors associated with marital satisfaction in infertile couple: a comprehensive literature review. Glob. J. Health Sci. 8, 96-109. doi: 10.5539/gjhs.v8n5p96

Schaller, M. A., Griesinger, G., and Banz-Jansen, C. (2016). Women show a higher level of anxiety during IVF treatment than men and hold different concerns: a cohort study. Arch. Gynecol. Obstet. 293, 1137-1145. doi: 10.1007/s00404-016-4033-x

Schmidt, L. (2006). Psychosocial burden of infertility and assisted reproduction. Lancet 367, 379-380. doi: 10.1016/S0140-6736(06)68117-8

Schmidt, L., Holstein, B., Christensen, U., and Boivin, J. (2005). Does infertility cause marital benefit? An epidemiological study of 2,250 women and men in fertility treatment. Patient Educ. Counsel. 59, 244-251. doi: 10.1016/j.pec.2005.07.015

Schwerdtfeger, K. L., and Shreffler, K. M. (2009). Trauma of pregnancy loss and infertility among mothers and involuntarily childless women in the United States. J. Loss Trauma 14, 211-227. doi: 10.1080/15325020802537468

Shapiro, C. (2009). Therapy with infertile heterosexual couples: It's not about gender - or is it? Clin. Soc. Work J. 37, 140-149. doi: 10.1007/s10615-008-0149-1

Sina, M., TerMeulen, R., and Carrasco de Paula, I. (2010). Human infertility: is medical treatment enough? A cross-sectional study of a sample of Italian couples. J. Psychosomat. Obstetr. Gynecol. 31, 158-167. doi: 10.3109/0167482X.2010.487952

Spanier, G. (1976). Measuring dyadic adjustment: new scales for assessing the quality of marriage and similar dyads. J. Marriage Fam. 38, 15-28. doi: $10.2307 / 350547$

Staff, H. R., Didymus, F. F., and Backhouse, S. H. (2017). The antecedents and outcomes of dyadic coping in close personal relationships: a systematic review and narrative synthesis. Anxiety Stress Coping 30, 498-520. doi: 10.1080/10615806.2017.1329931

Stanhiser, J., and Steiner, A. Z. (2018). Psychosocial aspects of fertility and assisted reproductive technology. Obstet. Gynecol. Clin. North Am. 45, 563-574. doi: 10.1016/j.ogc.2018.04.006

Switzer, A., Caldwell, W., da Estrela, C., Barker, E. T., and Gouin, J.P. (2018). Dyadic coping, respiratory sinus arrhythmia, and depressive symptoms among parents of preschool children. Front. Psychol. 9:1959. doi: 10.3389/fpsyg.2018.01959

Tao, P., Coates, R., and Maycock, B. (2012). Investigating marital relationship in infertility: a systematic review of quantitative studies. J. Reproduc. Infertil. 13, 71-80.

Traa, M. J., De Vries, J., Bodenmann, G., and Den Oudsten, B. L. (2015). Dyadic coping and relationship functioning in couples coping with cancer: a systematic review. Br. J. Health Psychol. 20, 85-114. doi: 10.1111/bjhp.12094

Turner, K., Reynolds-May, M. F., Zitek, E. M., Tisdale, R. L., Carlisle, A. B., and Westphal, L. M. (2013). Stress and anxiety scores in first and repeat IVF cycles: a pilot study. PLOS ONE 8:e63743. doi: 10.1371/journal.pone.0063743

Van Der Merwe, E., and Greeff, A. P. (2015). Infertility related-stress within the marital relationship. Int. J. Sex. Health 27, 522-531. doi: 10.1080/19317611.2015.1067275

Vilchinsky, N., and Dekel, R. (2018). "Cardiac disease-induced PTSD: The need for a dyadic perspective," in When "We" are Stressed. A Dyadic Approach to Coping With Stressful Events, eds A. Bertoni, S. Donato, and S. Molgora (New York, NY: Nova Science Publisher), 109-124.

Vitale, S. G., La Rosa, V. L., Rapisarda, A. M. C., and Lagan,à, A. S. (2017). Psychology of infertility and assisted reproductive treatment. 
The Italian situation. J. Psychosomat. Obstetr. Gynecol. 38, 1-3. doi: 10.1080/0167482X.2016.1244184

World Health Organization (1992). Recent Advances in Medically Assisted Conception. Geneva: World Health Organization.

Yazdani, F., Kazemi, A., Fooladi, M. M., and Samani, H. R. O. (2016). The relations between marital quality, social support, social acceptance and coping strategies among the infertile Iranian couples. Eur. J. Obstetr. Gynecol. Reproduct. Biol. 200, 58-62. doi: 10.1016/j.ejogrb.2016.02.034

Ying, L. Y., Wu, L. H., and Loke, A. Y. (2015). Gender differences in experiences with and adjustments to infertility: a literature review. Int. J. Nurs. Stud. 52, 1640-1652. doi: 10.1016/j.ijnurstu.2015. 05.004

Zegers-Hochschild, F., Adamson, G. D., Dyer, S., Racowsky, C., de Mouzon, J., Sokol, R., et al. (2017). The international glossary on infertility and fertility care, 2017. Fertil. Steril. 108, 393-406. doi: 10.1016/j.fertnstert. 2017.06.005

Zimmermann, T., and Rauch, S.-L. (2018). "Dyadic coping in patients with prostate and laryngeal cancer and their partners," in When "we" are stressed.
A Dyadic Approach to Coping With Stressful Events, eds A. Bertoni, S. Donato, and S. Molgora (New York, NY: Nova Science Publisher), 125-138.

Zurlo, M. C., Cattaneo Della Volta, M. F., and Vallone, F. (2018). Predictors of quality of life and psychological health in infertile couples: the moderating role of duration of infertility. Qual. Life Res. 27, 945-954. doi: 10.1007/s11136-017-1781-4

Conflict of Interest Statement: The authors declare that the research was conducted in the absence of any commercial or financial relationships that could be construed as a potential conflict of interest.

Copyright $\odot 2019$ Molgora, Fenaroli, Acquati, De Donno, Baldini and Saita. This is an open-access article distributed under the terms of the Creative Commons Attribution License (CC BY). The use, distribution or reproduction in other forums is permitted, provided the original author(s) and the copyright owner(s) are credited and that the original publication in this journal is cited, in accordance with accepted academic practice. No use, distribution or reproduction is permitted which does not comply with these terms. 


\section{OPEN ACCESS}

Edited by:

Gianluca Castelnuovo,

Catholic University of the Sacred

Heart, Italy

Reviewed by:

Tanja Zimmermann

Hannover Medical School, Germany

Sara Molgora,

Catholic University of the Sacred

Heart, Italy

*Correspondence:

Reza Fallahchai

r.fallahchai@hormozgan.ac.ir

Specialty section:

This article was submitted to

Clinical and Health Psychology,

a section of the journal

Frontiers in Psychology

Received: 21 June 2018 Accepted: 19 February 2019

Published: 18 April 2019

Citation:

Fallahchai $R$, Fallahi $M$ and Randall AK (2019) A Dyadic Approach

to Understanding Associations Between Job Stress, Marital Quality, and Dyadic Coping for Dual-Career

Couples in Iran

Front. Psychol. 10:487.

doi: 10.3389/fpsyg.2019.00487

\section{A Dyadic Approach to Understanding Associations Between Job Stress, Marital Quality, and Dyadic Coping for Dual-Career Couples in Iran}

\author{
Reza Fallahchai ${ }^{1 *}$, Maryam Fallahi ${ }^{1}$ and Ashley K. Randall ${ }^{2}$ \\ 'Department of Psychology, University of Hormozgan, Bandar Abbas, Iran, ${ }^{2}$ Counseling and Counseling Psychology, \\ Arizona State University, Tempe, AZ, United States
}

In Iran, dual-career couples face many stressors due to their demands of balancing work and family. Moreover, the experience of this stress can negatively affect partners' martial quality. Recent studies have shown the positive impact of dyadic coping on well-being; however, a majority of this research has been conducted with Western cultures. As such, there is a dearth of literature on understanding how supportive and common dyadic coping may have a positive association with work-family stress for couples in Iran. Using a sample of 206 heterosexual dual-career couples from Iran, this study examines the associations between job stress and marital quality, and possible moderating effects of common and perceived partner supportive dyadic coping. As predicted, job stress was negatively associated with marital quality, and this association with further moderated by gender, such that women who experienced greater job stress also reported lower marital quality. Additionally, dyadic coping moderated the association between job stress and marital quality. Common dyadic coping attenuated the negative association between job stress and marital quality. The findings shed light on the possible beneficial effects of teaching supportive and common dyadic coping techniques to dual-career couples in Iran.

Keywords: job stress, Iranian dual-career couples, dyadic coping, marital quality, work-family conflict

\section{INTRODUCTION}

Iran is in transition from a society that once focused on agricultural economics to one that is now focused on industrial economy, urbanization, mass media development, and public education (Askari-Nodoushan et al., 2009). In recent decades, family values, structures, and norms have undergone wide-ranging changes due to the shifts in the structure of the Irian society, because of industrialization, urbanization and the expansion of mass media, as well as cultural and value changes, individualism from the dissemination of Western ideas and values (Azadarmaki et al., 2012). These changes have led to shifts in the structure of societies, which can be best observed in changes in the cultural ideals of individualism (Askari-Nodoushan et al., 2009). Examples of this change include the increased age of marriage in 2016 (women: from 18.4 to 23.4; men: from 25 to 27.4), decreasing fertility from 6.3 in 1986 to 1.75 in 2016 (Shojaei and Yazdkhasti, 2017), and increased divorce rates from 8.6 in 1991 to 34.1 in 2018 (Statistics Center of Iran, 2018). 
Changes in the Iranian society have also had an impact on the formation and expansion of a nuclear family (Abbasi-Shavazi and McDonald, 2008), wherein husbands were once thought to have the authority in the household due to their economic responsibility, and wives were thought to be responsible for child-rearing (Richter et al., 2014). Due to the modernization of society, women's increase in educational attainment and rates of employment (Saraie and Tajdari, 2011), women are now thought to have an equal role in all the decision-making of issues related to family (Askari-Nodoushan et al., 2009). The participation of women in the workforce has caused fundamental changes in family and occupational structures, including the increase in dual-career couples (Schaer et al., 2008), so that it has gradually become the dominant model of marital life in most countries (Haddock et al., 2006).

Despite the increase of women in the workforce, which comes along with managing the demands of work-related stress, women in Iran are still expected to attend to their family roles as wives and mothers (Rafatjah, 2011). Consequently, in dual-career families, both partners must perform multiple tasks as well as maintain efforts to create a balance between these roles (Atta et al., 2013). Research on 155 dual-career couples in Bangladesh has shown that childcare, work-family conflict, family-work conflict, and marital relations are the most important challenges for dual-career couples (Sultana et al., 2014). Consequently, the balance of work and family roles can be stressful (Rafatjah, 2011), and may lead to conflicts between partners (Soleimanian and Nazari, 2007; Nazari and Goli, 2008; Oreizi et al., 2011), which over time can lead to decreased marital quality.

Given the overwhelming number of dual-career couples in Iran (Khosravi et al., 2010; Motahari et al., 2012; Fallahchai and Khaluee, 2016; Mazhari et al., 2016), investigating the unique stressors these couples may face is an important concern for mental health practitioners working with these couples (Saginak and Saginak, 2005). Few studies have investigated marital quality in dual-career couples in a collectivist context (Quek et al., 2011), which leaves a dearth of understanding on factors can affect partners' marital quality. Given the changing cultural climate in Iran, it is necessary for relational scholars to examine ways in which dual-career couples can cope with stress in order to possible reduce marital dissatisfaction (Soleimanian and Nazari, 2007). Additionally, dyadic coping has been found to moderate the associations between work-family conflicts in Canada (Lapierre and Allen, 2006), as well as preventing the harmful effects of stress on relational functioning and physical and mental health (Levesque et al., 2014; Merz et al., 2014).

\section{Associations Between Job Stress and Marital Quality}

Job stress is defined as a reaction to the experience of stressors related to work domains (Wierda-Boer et al., 2009), which can be accompanied by role-overload due to occupational and family responsibilities. Not surprisingly, job stress can also affect within the family due to stress spillover and crossover (Neff and Karney, 2005), ultimately leading to a decrease in marital quality in both partners. Stress spillover refers to how the stress experienced from an aspect of life (e.g., occupation) spills over causing stress to another aspect (e.g., family) (Geurts and Demerouti, 2003). For example, when a person has a stressful day at work this may affect the way they interact with their partner (e.g., shutting down), causing stress at home. Workfamily spillover is defined as the transfer of the effects of work and family on one another that generate similarity between work and family (Edwards and Rothbard, 2000) and workfamily spillover transfers from one domain (e.g., occupation) to another domain (family) (Haines et al., 2006; Schaer et al., 2008). Stress spillover in marital relationships can lead to negative behaviors, such as anger toward the partners (Schulz et al., 2004), which, can negatively affects marital satisfaction (Randall and Bodenmann, 2017). Stress crossover refers to the interpersonal transfer of stress from one partner to another (Haines et al., 2006). For example, one partner's experience of stress can affect their partner's experience as well (Randall et al., 2017). Bodenmann et al. (2007) demonstrated that stress outside the relationship (external stress; Randall and Bodenmann, 2009) significantly triggers stress within the relationship (internal stress), which is commonly found to be associated with marital quality.

Not surprisingly, the experience of job stress has been found to reduce marital quality in both partners (Obradovic and CudinaObradovic, 2009). A majority of research in this domain has been conducted in the United States or with Western samples and has found that men were affected by work-family conflict as much as women, however, women were more likely to be affected by family-work conflict than men (e.g., Tatman et al., 2006). However, recent research is starting to examine these associations with non-Western samples. For example, Sandberg et al. (2012) examined the association between family-to-work spillover job satisfaction and health using a sample of 1026 married workers in Singapore. Results of this study showed that marital distress was a significant predictor of job satisfaction and health. Taken together, given the negative associations between job-stress and marital quality (Neff and Karney, 2007; van Steenbergen et al., 2011) and increased rates of divorce in Iran (National Organization for Civil Registration, 2017), it is important to consider ways in which couples could cope with stress that may prevent the harmful effects of stress on relational well-being (Randall and Bodenmann, 2009, 2017; Merz et al., 2014).

\section{Moderating Associations of Dyadic Coping}

The conceptualization of stress as a dyadic construct, one that affects both partners in a romantic relationship (Randall and Bodenmann, 2009). Given this conceptualization, partners can attempt to cope with stress by engaging in (positive) dyadic coping. Specifically, dyadic coping refers to the ways in which partners cope with stress in the context of their relationship (Bodenmann et al., 2011). Although positive and negative forms of dyadic coping exist (see Bodenmann, 2005), here we focus specifically on positive forms of dyadic coping given its strong association with relational well-being for couples around the world (Falconier et al., 2016). Additionally, a recent meta-analysis 
by Falconier et al. (2015) found that supportive and common dyadic coping were found to be powerful (positive) predictors of relationship satisfaction (e.g., Bodenmann and Cina, 2006; Ruffieux et al., 2014).

Positive forms of dyadic coping can be classified into three categories: supportive dyadic coping, delegated dyadic coping, and common dyadic coping (Bodenmann, 2005). Supportive dyadic coping refers to the efforts that one couple makes to express empathic understanding, solidarity with his/her partner, and providing practical. For example, if a partner is under stress, his/her partner may respond by expressing empathy and then providing practical advice on how to help cope with the stress. Delegated dyadic coping refers to a new division of tasks in which one partner asks for practical support. For example, a partner takes over certain tasks of the partner when his/her partner asks for help. Lastly, common dyadic coping represents the joint efforts of couples to deal with stress. For example, both partners engage in joint problem solving when they face a stressful situation (e.g., work-family conflict). Furthermore, research suggests that common dyadic coping plays an important role in reducing negative daily stress (e.g., Bodenmann et al., 2011; Falconier et al., 2015), increasing the quality of the relationship, reducing symptoms of depression and distress in both couples (Rottmann et al., 2015).

Prior research has focused on the direct association between dyadic coping and marital quality (e.g., Iafrate et al., 2012; Falconier et al., 2015; Gasbarrini et al., 2015), as well as on the indirect association (i.e., moderation) between variables (e.g., Falconier et al., 2013; Levesque et al., 2014; Herzberg and Sierau, 2016).

\section{Direct Associations}

Bodenmann et al. (2006a) who examined the association between dyadic coping and marital quality among 90 Swiss couples over a 2 year period found that dyadic coping was positively correlated with relationship quality for couples. Additionally, using a sample of 187 heterosexual couples from Switzerland, Levesque et al. (2014) investigated dyadic empathy, dyadic coping, and relationship satisfaction. Results from this study showed that, among men, perspective-taking significantly increased their partner's desire to use positive dyadic coping strategies. For female, empathy increased their partners' coping strategies.

\section{Indirect Associations}

Dyadic coping has also been shown to have moderating effects on the association between stress and individual and relational wellbeing. For example, supportive and common dyadic coping were found to reduce the negative associations between immigration stress on relationship satisfaction for 104 Latino immigrant couples in the United States, especially for women (Falconier et al., 2013). The results of the study by Merz et al. (2014), examined the moderation role of dyadic coping in association between internal stress and relationship satisfaction on 131 couples, showed that dyadic coping reduced the effects of chronic stress on relationship satisfaction especially in women. Most recently, Hilpert et al. (2018) studied stress and coping processes at both between- and within-person levels in 84 dual-earning couples in China. The results of this study indicated that at the between persons level, both in men and women, the association between stress and relationship outcomes was decreased if the partner provided more support, but at the level of within persons, the results indicated that partner support had only a significant buffer effect in women. Taken together, supportive dyadic coping has been shown to be effective in reducing stress and improving the quality of relationships (Vedes et al., 2013); however, this has yet to be examined in dual- career couples, especially those from Iran, which is the goal of the present study.

\section{THE PRESENT STUDY}

The goal of the present study is to investigate the association between job stress and marital quality in dual-career couples from Iran. Additionally, given the robust positive associations between dyadic coping and relational outcomes found across cultures (Falconier et al., 2016), we also examine how supportive and common dyadic coping may moderate the association between job stress and marital quality. To do so, we collected dyadic data from both partners in a romantic relationship, which allows us to examine both actor and partner effects (Kenny et al., 2006). Actor effects refer to the associations of partner's reports of their independent variable (job stress) on their dependent variable (marital quality), whereas partner effects refer to the associations between how one partner's reports job stress are associated with their partner's marital quality.

In sum, we tested the following hypotheses $(\mathrm{H})$ :

$\mathrm{H1}$ : In line with research that has found a positive association between job stress and marital conflict in dualcareer couples from the United States and Western Europe (Michel et al., 2009; Allen and Finkelstein, 2014; Fellows et al., 2016; Yucel, 2017), it is hypothesized that a partner's job stress will be negatively associated with one's own (actor effect) and their partner's marital quality (partner effect).

$\mathrm{H} 2 \mathrm{a}$ : Based upon prior studies suggesting that supportive dyadic coping moderates the negative association between stress and relationship quality (Bodenmann, 2005; Bodenmann et al., 2010; Vedes et al., 2013; Rottmann et al., 2015; Breitenstein et al., 2018) it is hypothesized that perception of partner's of supportive dyadic coping will moderate the association between job stress and his/her own marital quality. Moreover, it is hypothesized that actual reports of supportive dyadic coping will be associated with partner's reports of marital quality (partner effect).

$\mathrm{H} 2 \mathrm{~b}$ : Based on prior studies that have found a positive association between common dyadic coping and relationship quality (Bodenmann, 2005; Bodenmann et al., 2010; Papp and Witt, 2010), it is hypothesized that common dyadic coping will moderate the association between job stress and marital quality, such that for individuals who perceive their partner as engaging in common dyadic coping, they will also show a positive association between their coping with job stress and their marital quality (actor effects). Additionally, we hypothesize that we will also find a positive association between self-reported job stress and perception of marital quality. 
TABLE 1 | Descriptive of study variables.

\begin{tabular}{|c|c|c|c|c|c|c|c|c|c|}
\hline \multirow[t]{2}{*}{ Variable } & \multicolumn{3}{|c|}{ Husbands $(N=203)$} & \multicolumn{3}{|c|}{ Husbands $(N=203)$} & \multirow[t]{2}{*}{ Paired $t$} & \multirow[t]{2}{*}{$p$} & \multirow[t]{2}{*}{ Cohen's d } \\
\hline & $\mathbf{M}$ & SD & Range & $\mathbf{M}$ & SD & Range & & & \\
\hline Age & 32.19 & 4.19 & $26-41$ & 27.42 & 3.76 & 23-39 & 0.56 & 0.60 & 0.02 \\
\hline Relationship duration & 11.60 & 9.10 & $2-23$ & 12.10 & 10.20 & $3-25$ & 0.51 & 0.58 & 0.02 \\
\hline Number of children & 2.00 & 1.10 & $1-3$ & 2.20 & 1.20 & $1-4$ & 0.49 & 0.71 & 0.012 \\
\hline Job stress & 90.00 & 12.45 & $35-175$ & 86.07 & 11.03 & $35-175$ & $-2.04^{\star}$ & 0.03 & 0.31 \\
\hline Marital quality & 117.16 & 17.24 & $0-151$ & 109.00 & 13.70 & $0-151$ & $1.90^{*}$ & 0.02 & 0.28 \\
\hline $\begin{array}{l}\text { Perceived partner } \\
\text { supportive dyadic } \\
\text { coping }\end{array}$ & 19.46 & 3.58 & $5-25$ & 19.23 & 2.94 & $10-50$ & 1.16 & 0.21 & 0.08 \\
\hline $\begin{array}{l}\text { Common dyadic } \\
\text { coping }\end{array}$ & 19.01 & 3.22 & $5-25$ & 19.55 & 3.23 & $5-25$ & 1.30 & 0.38 & 0.15 \\
\hline
\end{tabular}

$* p<0.05$.

\section{Gender Differences}

Although studies have found men and women report the same levels of stress in work-family conflict (Barnett and Gareis, 2006; Martinengo et al., 2010), men and women show different behavioral patterns in response to stress; women showed a higher level of negative spillover than men (Mennino et al., 2005). Related to job-stress in particular, Barling et al. (2004) have shown that there are important gender differences in the degree to which job and family stress is transmitted to negative family processes, including cognitions, behaviors, and interactions within the family that lead to negative outcomes. Given this, we also examine whether gender will moderate the association between job stress and marital quality.

\section{MATERIALS AND METHODS}

\section{Participants and Procedure}

This research was reviewed and approved by the ethics and research committee of Hormozgan University prior to the start of data collection. All participants provided written and informed consent. They were recruited in person from civil institutions, local police, education and social services in Shiraz, Iran. Participants had to meet the following criteria in order to participate (a) married for at least 2 years, (b) both of the partners had to have been working at least 2 years, and (c) have full-time employment status. Eligible couples were given two packages of research questionnaire in separate envelopes with a unique ID. Participants were asked to fill in their questionnaires independently from their spouse and send back the questionnaires upon completion.

Data were collected from 238 couples; however, 32 couples were removed from the current analysis for having incomplete data. The final sample consisted of 206 heterosexual couples ( $n=412$ individuals). On average, the men were 35.7 years old ( $S D=9.1$ years; range: 25-62 years) and women were 31.1 years old ( $S D=9.3$ years; range: $21-51$ years). The sample was highly education with $62.6 \%$ of participants reporting having B.A. degrees, $26.2 \%$ had M.A. and Ph.D. degrees, and $11.2 \%$ had high school diplomas. Participants reported being married for an average of 11 years $(S D=7.2)$. The average number of children was 2 ranging from 1 to 3 .

\section{Measures}

\section{Demographic Information}

Standard demographic information relating to age, gender, level of education, length of relationship, number of children was collected. See Table 1 for descriptive information.

\section{Job Stress}

Participant's perception of job stress was measured using the Persian version of the Health and Safety (HSE) Management Standards Indicator Tool (HSE-MS IT; Cousins et al., 2004). The HSE-MS IT is a 35 -item on a 5 -point Likert type scale $(1=$ never to 5 = always) developed to measure work-related stress risk factors at an organizational level (Marcatto et al., 2014). This measure showed good reliability in the current study for men and women ( $\alpha=0.82$ and 0.85 , respectively).

\section{Marital Quality}

Participants' reports of marital quality were measured using the Persian version of Dyadic Adjustment Scale (Sanai Zakir, 2000; DAS; Spanier, 2001). The DAS includes 32 items used to assess partners' marital quality (e.g., Fișiloğlu and Demir, 2000; Chiara et al., 2014; Bachem et al., 2018). Twenty-seven items are rated on a 6- point Likert scale (15 items: $0=$ always disagree to $5=$ always agree; 12 items $0=$ never to $5=$ all the time); two items are on a 5 -point Likert scale $(0=$ never to $4=$ everyday $)$; two items are yes/no type questions $(0=$ no $1=y e s)$; and one is on a 7 -point Likert scale $(0=$ extremely unhappy to $7=$ perfect $)$. Items are summed, wherein higher scores are reflective of greater marital quality. Results of current study showed good reliability for men and women ( $\alpha=0.86$ and 0.87 , respectively).

\section{Dyadic Coping}

The Persian version of the Dyadic Coping Inventory (DCI; Fallahchai et al., 2017) was used to measure participant's reports of dyadic coping. The DCI is a self-report instrument consisting 
of 37 items, with responses arranged on a 5-point likerttype scale $(1=$ never to $5=$ always $)$. The DCI contains six subscales to measure each partner's stress communication and specific dyadic coping; however, for the purpose of our study, we examined the following: emotion-focused dyadic coping, problem-focused dyadic coping, and common dyadic coping. To create a composite score of perceived partner supportive dyadic coping, we took the average of emotion-focused and problemfocused supportive dyadic coping. For each area assessed, participants reported on their own and their perceived partner behaviors; reports of perceived partner dyadic coping were used in the present analysis. This measure showed good reliability for perceived partner supportive dyadic coping for men and women ( $\alpha=0.83$ and 0.84 , respectively), and common dyadic coping for men and women ( $\alpha=0.88$ and 0.89 , respectively).

\section{Control Variables}

Age (e.g., Michel et al., 2009; Spell et al., 2009), relationship length, and the number of children (e.g., Hassan et al., 2010; Wang et al., 2010; Mache et al., 2015) have been previously found to be negatively associated with work-family conflicts. As such, we controlled for these variables in our analysis.

\section{Statistical Analyses}

Dyadic data - data collected from two partners - contains sources of interdependence between partners' reports (Kenny et al., 2006). Analyses were run with Actor-Partner InterdependenceModel (APIMs) (Cook and Kenny, 2005) which allows researchers to control for the interdependence between reporting partners' scores, and also examine both actor and partner effects. To analyze both actor and partner effects, we used Structural Equation Modeling (SEM) for distinguishable dyads (e.g., men and women) because SEM allows for the estimation the association between variables free from measurement error, while also including the examination of the goodness of fit of the base models and the measurement structure of all study variables simultaneously (Ledermann and Kenny, 2017).

In order to evaluate model fit, we used the Root Mean Square Error of Approximation (RMSEA; $0.01=$ excellent fit; 0.05 = good fit; 0.08 = mediocre fit; MacCallum et al., 1996), and Comparative Fit Index (CFI; $0.95=$ excellent fit; $0.90=$ adequate fit; Hu and Bentler, 1999). Each model contained the control variables noted above. All analyses were conducted using AMOS 21 (Arbuckle, 2006).

\section{RESULTS}

\section{Descriptive Statistics}

Descriptive statistics for the study variables are presented in Table 1. Results showed significant gender differences in selfreported job stress; wives reported significantly higher scores in job stress $(t=-2.04, p=0.03)$. Interestingly, compared to wives, husbands reported higher marital quality $(t=1.90, p=0.02)$. We did not find differences between husbands and wives reports of engaging in partner supportive dyadic coping.
TABLE 2 | Correlations between study variables.

\begin{tabular}{lccccccc}
\hline & $\mathbf{1}$ & $\mathbf{2}$ & $\mathbf{3}$ & $\mathbf{4}$ & $\mathbf{5}$ & $\mathbf{6}$ & $\mathbf{7}$ \\
\hline 1. Age & 0.15 & 0.08 & 0.09 & -0.18 & 0.09 & 0.14 & 0.07 \\
2. RD & 0.04 & 1.00 & -0.13 & 0.14 & -0.10 & -0.11 & -0.14 \\
3. NC & 0.08 & -0.13 & 1.00 & 0.07 & $-0.21^{*}$ & -0.17 & -0.15 \\
4. JS & 0.08 & 0.12 & 0.17 & $0.79^{* *}$ & $-0.42^{* *}$ & $-0.41^{* *}$ & $-0.36^{* *}$ \\
5. MQ & 0.08 & 0.08 & 0.10 & $-0.34^{* *}$ & $0.77^{* *}$ & $0.75^{* *}$ & $0.62^{* *}$ \\
6. PSDC & 0.10 & -0.12 & 0.90 & $-0.42^{* *}$ & $0.70^{* *}$ & $0.78^{* *}$ & $0.67^{* *}$ \\
7. CDC & 0.11 & -0.14 & -0.15 & $-0.30^{* *}$ & $0.63^{* *}$ & $0.72^{* *}$ & $0.74^{* *}$ \\
\hline
\end{tabular}

RD, Relationship Duration; NC, Number of children, JS, Job stress, MQ, Marital Quality, SDC, Perceived Partner Supportive Dyadic Coping, CDC, Common Dyadic Coping. Husbands' correlations are presented above the diagonal and wives' correlations are presented below the diagonal. Between-partner correlations are presented across the diagonal. **, $0.01, *, 0.05$.

Significant correlations among the scales ranged from $(-0.43<r>0.77)$ for both husbands and wives. Table 2 shows correlations among measured variables for husbands (above the diagonal), for wives (below the diagonal).

\section{Associations Between Job Stress and Marital Quality}

A model with the direct actor and partner effects of job stress predicting change in spouses' marital quality was examined. Gender was included in the models to test whether the associations between job stress and marital quality differed between husbands and wives. The model fit well: $\chi^{2}=8.789$, $p<0.45$, CFI $=0.95$, RMSEA $=0.02$.

It was hypothesized that job stress would have a main effect on marital quality after controlling for age, marital duration, and number of child. Results indicated that there was a significant negative association between one's own job stress and marital quality for both husbands and wives (actor effect; husbands: $\beta=-0.32, p<0.001$; wives: $\beta=-0.42, p<0.001$ ). Additionally, we found partner effects for job stress and marital quality for both husbands $(\beta=-0.41, p<0.001)$ and wives $(\beta=-0.34, p<0.001)$; one's reports of job stress was negatively associated with their partner's reports of marital quality (see Figure 1).

Moreover, results indicated the actor association differed by gender. For example, significant gender differences were found in associations between job stress and marital quality $(\beta=-0.39$, $p<0.01$ ), for wives. Specifically, when wives reported greater job stress they also reported lower marital quality. However, gender did not moderate the partner effect.

\section{Moderating Associations of Dyadic Coping}

\section{Perceived Partner Supportive Dyadic Coping (H2a)}

The Goodness-of-fit for the model with perceived partner supportive dyadic coping as the moderator was very good: $\chi^{2}=6.45 ; p=0.451$; with $\mathrm{CFI}=0.92$ and $\mathrm{RMSEA}=0.04$. All the actor effects were significant and in the expected direction, but the partner effects were not significant. 

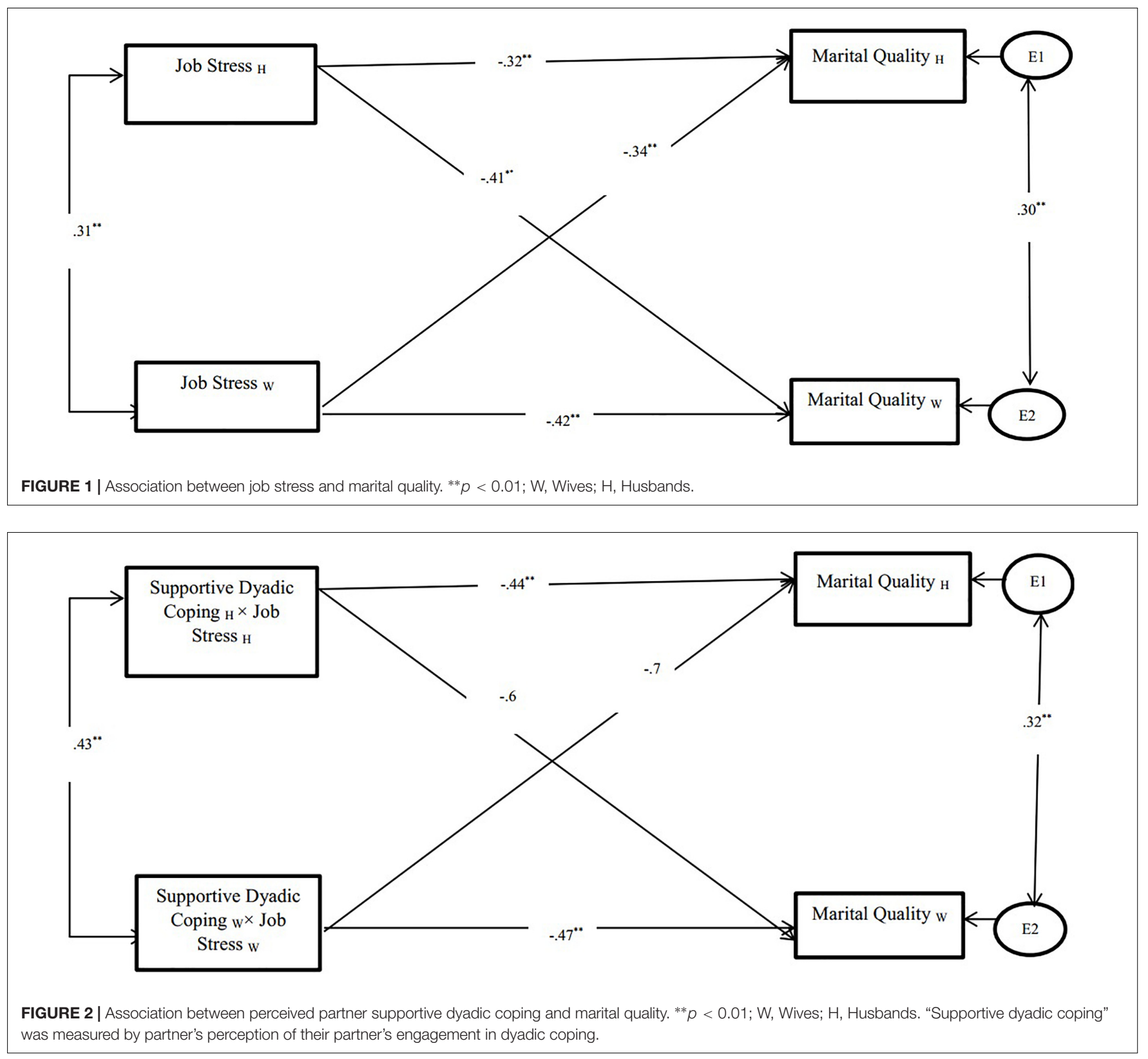

\section{Actor Effects}

The structural path from the interaction between husbands' perceived partner supportive dyadic coping and husbands' job stress to husbands' marital quality was significant $(\beta=-0.44$, $p<0.001)$, which suggests that when husbands perceive their wife as engaging in supportive dyadic coping they report greater marital quality. Additionally, results found that perceived partner supportive dyadic coping moderated the association between job stress and marital quality, this effect was significant for wives $(\beta=-0.47, p<0.001)$.

\section{Partner Effects}

Results showed that the interaction between husbands' perceived partner supportive dyadic coping and husbands' job stress to wives' marital quality was not significant $(\beta=-0.6, p>0.05)$, and the interaction between wives' perceived partner supportive dyadic coping and wives' job stress to husbands' marital quality was not significant $(\beta=-0.7, p>0.05)$. Therefore, the partner effects were not significant both for husbands and wives (see Figure 2).

\section{Common Dyadic Coping (H2b)}

Results of estimating the APIM revealed very good Goodnessof-fit for the model $\left(\chi^{2}=5.23, p=0.32\right.$ with $\mathrm{CFI}=0.96$ and RMSEA $=0.031)$.

\section{Actor Effects}

The structural path from the interaction between common dyadic coping and job stress to marital quality was significant. This effect 


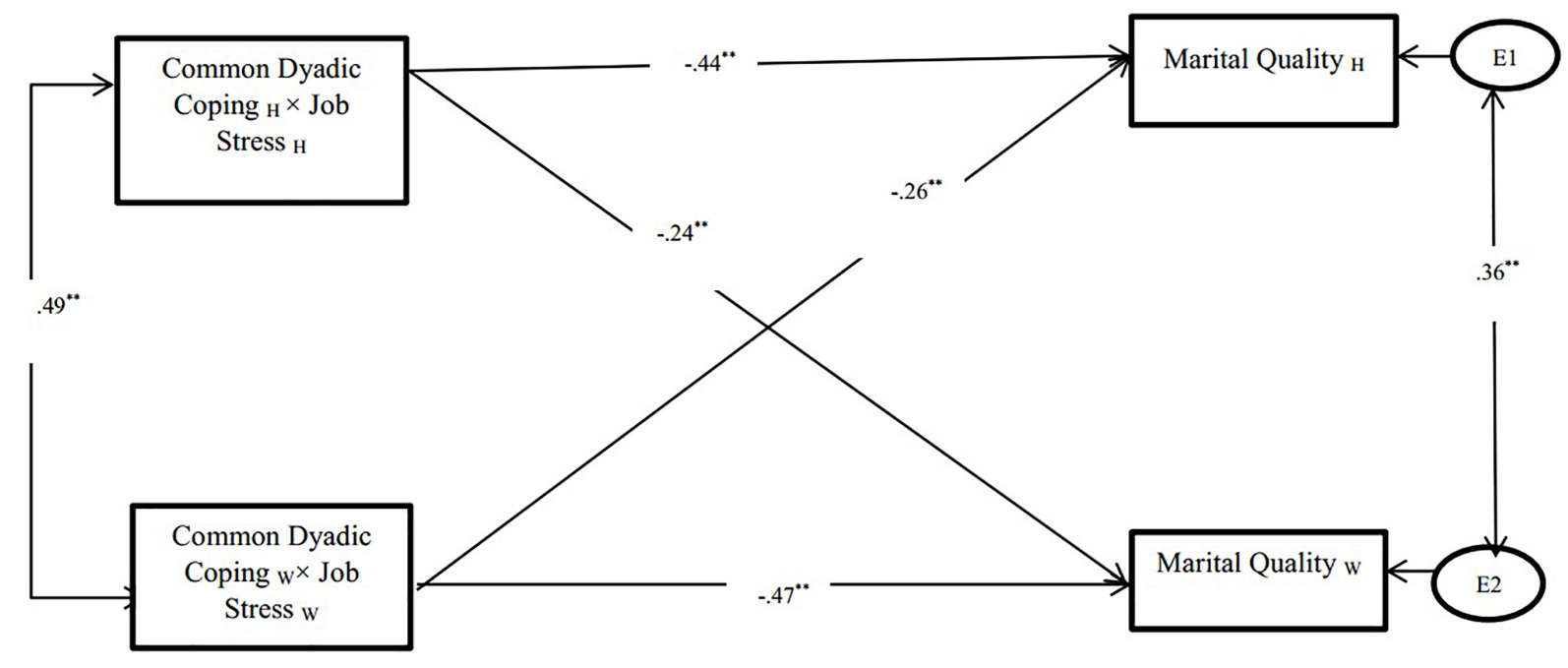

FIGURE 3 | Association between common dyadic coping and marital quality. ${ }^{* *} p<0.01$; W, Wives; H, Husbands.

was found for both husbands $(\beta=-0.44, p<0.001)$ and wives $(\beta=-0.47, p<0.001)$.

\section{Partner Effects}

Results showed that the interaction between husbands' common dyadic coping and husbands' job stress to wives' marital quality was significant $(\beta=-0.24, p<0.05)$. Moreover, the interaction between wives' common dyadic coping and wives' job stress to husbands' marital quality was significant $(\beta=-0.26, p<0.05)$ (see Figure 3).

Taken together, results revealed that perceived partner supportive dyadic coping and common dyadic coping moderated the negative association between job stress and marital quality in expected directions.

\section{DISCUSSION}

Given the change of social-cultural structure in Iran (AskariNodoushan et al., 2009; Azadarmaki et al., 2012), and the increase of dual-career couples (Soleimanian and Nazari, 2007; Ghodrati, 2015), the aim of this study was to investigate the association between job stress and marital quality for dual- career couples, and assess possible moderating associations of supportive and common dyadic coping. Results from this study largely support our hypotheses, however, interesting gender differences emerged, which are explained below.

\section{Associations Between Job Stress and Marital Quality}

We hypothesized that a partner's job stress would be negatively associated with one's own marital quality (actor effect) and their partner's marital quality (partner effect). Findings of this study found that both wives and husbands in a dual- career marriage report similar levels of job stress, and these reports were similarly associated with marital quality (both actor and partner effects). This finding is in line with the results of previous studies (Anafarta, 2011; Šimunic and Gregov, 2012; Efeoğlu and Ozcan, 2013) suggesting dual- career couples experience a lot of job stress, which is associated with marital quality (Buck and Neff, 2012; Sandberg et al., 2012).

\section{Moderating Effects of Perceived Dyadic Coping}

We hypothesized that supportive and common dyadic coping would moderate the association between job stress and their own marital quality (actor effect) and partner's reports of marital quality (partner effect). Below we expand upon the results from these models.

\section{Perceived Partner Supportive Dyadic Coping}

Data from this study supported our hypothesis, suggesting that perceived partner supportive dyadic coping moderated the negative association of job stress and marital quality for husbands and wives. Said differently, when individuals reported greater partner's supportive dyadic coping, they also experienced higher level of marital quality. These results are consistent with previous studies (e.g., Wunderer and Schneewind, 2008; Papp and Witt, 2010; Falconier et al., 2013; Herzberg, 2013; Nicholls and Perry, 2016), which have found supportive dyadic coping to have a beneficial effect on marital quality (Bodenmann et al., 2006b, 2016; Falconier et al., 2013; Breitenstein et al., 2018).

\section{Common Dyadic Coping}

Data from this study supported our hypothesis, suggesting that common dyadic coping moderated the negative association of job stress (actor and partner effect) for both husbands and wives. These results are in line with previous studies that have found that common dyadic coping may play a moderating role in association between stress and marital outcomes (i.e., relationship satisfaction, marital quality) (e.g., Bodenmann, 2005; 
Bodenmann et al., 2010; Falconier et al., 2013). Given that common dyadic coping refer to partners' perception of handling stressful situations, these results support understanding stress and coping as a dyadic context (Randall and Bodenmann, 2009, 2017). In situations where one of the partners, or both, faces a significant stressor, viewing stress as a dyadic stress (i.e., "our stress") and engaging in common dyadic coping can help partners cope with the stress, by fostering a sense of "we-ness" within the couple (Vedes et al., unpublished). Therefore, this strong association between positive dyadic coping techniques and ability to cope with stress suggests that the way in which couples manage and interact with stress and conflict in their marital life is considered as the most important determinants of marital satisfaction (Vedes et al., 2013), and the satisfaction of the relationship may dependent on positive dyadic coping during times of distress (Falconier et al., 2015).

\section{Role of Gender}

Another goal of the present study was to examine possible gender differences between job stress and marital quality. Results from this study revealed that when wives reported greater job stress they also reported lower marital quality, however, this effect was not found for husbands. One's is a very important culture is a very important factor for predicting gender differences in the coping process between couples (Hilpert et al., 2016). In Iran Khojasteh mehr et al. (2013) in their research with 150 couples, found that dyadic coping in women had a greater effect on their marital satisfaction than men, because the support that women under stress receive from their husbands has a great influence on the quality of their marital life.

Additionally, women may engage in greater dyadic coping behaviors due to their greater attentiveness to their partner's needs (Bodenmann et al., 2006a), as women are thought to be more sensitive to changes in their marital relationships (Bodenmann et al., 2004). This greater engagement in dyadic coping behaviors may be particularly true for couples who come from a society wherein men and women carry different roles and responsibilities in the relationship (see Hilpert et al., 2016).

\section{Limitations and Future Directions}

Although this study is one of the few studies that has used a dyadic sample of dual-career couples from Iran to examine associations between job stress and marital quality, it is notwithstanding limitations. First, data for this study was based on cross-sectional data, which limits our ability to make causal inferences and further test associations between stress spillover and crossover. To better address for stress spillover (i.e., external stress to internal stress; see Randall and Bodenmann, 2009) and crossover future research should utilize longitudinal data (e.g., Bodenmann and Cina, 2006; Bodenmann et al., 2006a). Second, this study relied on the use of self-report assessments, which may contain bias (Spector, 1994). As such, future research is encouraged to use a multi-method approach that includes more objective measures, such as observational measures and interview methods, which may provide a better understanding of the nature of how and when supportive dyadic coping is utilized especially given the cultural context. Third, it is important to examine other variables that may further moderate the association between job stress and marital quality. One such variable is the presence of children in the home. Having a child affects the workfamily conflict (Mennino et al., 2005), and negatively affects the individual's job performance (Patel et al., 2006), as such the presence of children may. Lastly, this study chose to focus on supportive and common dyadic coping due to its robust positive associations with relationship well-being (see Falconier et al., 2015). To further understand the role of dyadic coping in the context of dual-career couples, future research should also examine other types of dyadic coping (e.g., delegated and negative dyadic coping), which may help relationship researchers and clinicians working with couples identify other forms of effective coping on the relationship between job stress and marital quality. Also, considering the cultural differences between Iran and Western countries regarding gender roles and its possible effects on family-work conflict of the couples, it is suggested that future research would measure specific gender roles.

\section{CONCLUSION}

The number of dual- career couples is increasing in Iran (Ghodrati, 2015). In addition to stress common to all couples (Jackson et al., 2016), some couples may experience higher levels of stress due to work-family conflicts (Nohe et al., 2015), which can have negative implications on their relational wellbeing (Randall and Bodenmann, 2009, 2017). Recent research cross-culturally has shown that supportive and common dyadic coping have buffering effect on reducing the impact of stress and can enhance marital quality (Bodenmann et al., 2010; Falconier et al., 2016).

Perhaps not surprisingly, the results of this study found that job stress was negatively associated with marital quality; however, perceived partner supportive and common dyadic coping moderated the association. The findings of this study have improved our understanding of stress processes in marital quality of dual- career couples. The findings of this study improve our understanding of stress and coping processes for dual-career couples in Iran, and the importance of engaging in supportive and common dyadic coping. These findings suggest that dyadic coping plays a very important role both in reducing stress and improving the quality of relationships in dual-career couples in Iran. The findings of this study are important implications for relationship researchers and clinical experts in understanding the effects of work-family stress on marital quality in dual-career couples and their gender differences in their rate and effect of job stress. Moreover, teaching coping skills can be effective both in reducing stress and improving the quality.

\section{AUTHOR CONTRIBUTIONS}

MF organized the database and performed the statistical analysis. RF wrote the first draft of the manuscript and performed the statistical analysis. MF, RF, and AR wrote the sections of the manuscript. All authors contributed to the manuscript revision, read, and approved the submitted version. 


\section{REFERENCES}

Abbasi-Shavazi, M. J., and McDonald, R. (2008). "Family change in Iran: religion, revolution, and the state," in Proceedings of the International Family Change: Ideational Perspectives, eds R. Jayakody, A. Thornton, and W. Axinn (New York, NY: Taylor \& Francis Group).

Allen, T. D., and Finkelstein, L. M. (2014). Work-family conflict among members of full-time dual-earner couples: an examination of family life stage, gender, and age. J. Occup. Health Psychol. 19, 376-384. doi: 10.1037/a0036941

Anafarta, N. (2011). The relationship between work-family conflict and job satisfaction: a structural equation modeling (SEM) approach. Int. J. Bus. Manage. 6:168. doi: 10.5539/ijbm.v6n4p168

Arbuckle, J. L. (2006). Amos 7.0 User's Guide. Chicago: SPSS.

Askari-Nodoushan, A., Abbasi-Shavazi, M. J., and Sadeghi, R. (2009). Mothers, daughters and marriage: intergenerational differences in ideas and attitudes of marriage in Yazd. Womens Strateg. Stud. 11, 7-36.

Atta, M., Adil, A., Shujja, S., and Shakir, S. (2013). Role of trust in marital satisfaction among single and dual-career couples. Int. J. Res. Stud. Psychol. 2, 53-62.

Azadarmaki, T., Sharifi Saei, M., Isari, M., and Talebi, S. (2012). The typology of premarital sex patterns in Iran. Sociol. Cult. Stud. 2, 1-34.

Bachem, R., Levin, Y., Zhou, X., Zerach, G., and Solomon, Z. (2018). The role of parental posttraumatic stress, marital adjustment, and dyadic self-disclosure in intergenerational transmission of trauma: a family system approach. J. Marit. Fam. Ther. 44, 543-555. doi: 10.1111/jmft.12266

Barling, J., Kelloway, E. K., and Frone, M. R. (eds) (2004). Handbook of Work Stress. Thousand Oaks, CA: Sage Publications. doi: 10.1111/jmft.12266

Barnett, R. C., and Gareis, K. C. (2006). "Role theory perspectives on work and family," in The Work and Family Handbook: Multi-Disciplinary Perspectives, Methods, And Approaches, eds M. Pitt-Catsouphes, E. E. Kossek, and S. Sweet (Mahwah, NJ: Lawrence Erlbaum Associates Publishers), 209-221.

Bodenmann, G. (2005). "Dyadic coping and its significance for marital functioning," in Couples Coping with Stress: Emerging Perspectives on Dyadic Coping, eds T. A. Revenson, K. Kayser, and G. Bodenmann (Washington, DC: American Psychological Association), 33-50.

Bodenmann, G., Charvoz, L., Widmer, K., and Bradbury, T. (2004). Differences in individual and dyadic coping among low and high depressed, partially remitted, and non-depressed persons. J. Psychopathol. Behav. Assess. 26, 75-85. doi: 10.1023/B:JOBA.0000013655.45146.47

Bodenmann, G., and Cina, A. (2006). Stress and coping among stable satisfied, stable-dissatisfied and separated/divorced Swiss couples: a 5- year prospective longitudinal study. J. Divorce Remarriage 44, 71-89. doi: 10.1300/j087v44 n01_04

Bodenmann, G., Ledermann, T., Blattner, D., and Galluzzo, C. (2006a). Associations among everyday stress, critical life events, and sexual problems. J. Nervous Ment. Dis. 194, 494-501. doi: 10.1097/01.nmd.0000228504.15569.b6

Bodenmann, G., Ledermann, T., and Bradbury, T. N. (2007). Stress, sex, and satisfaction in marriage. Pers. Relat. 14, 551-569. doi: 10.1111/j.1475-6811. 2007.00171.x

Bodenmann, G., Meuwly, N., Bradbury, T. N., Gmelch, S., and Ledermann, T. (2010). Stress, anger, and verbal aggression in intimate relationships: moderating effects of individual and dyadic coping. J. Soc. Pers. Relat. 27, 408-424. doi: 10.1177/0265407510361616

Bodenmann, G., Meuwly, N., and Kayser, K. (2011). Two conceptualizations of dyadic coping and their potential for predicting relationship quality and individual well-being. Eur. Psychol. 16, 255-266. doi: 10.1027/1016-9040/ a000068

Bodenmann, G., Pihet, S., and Kayser, K. (2006b). The relationship between dyadic coping and marital quality: a 2-year longitudinal study. J. Fam. Psychol. 20, 485-493. doi: 10.1037/0893-3200.20.3.485

Bodenmann, G., Randall, A. K., and Falconier, M. K. (2016). Coping in Couples: The Systemic Transactional Model (STM). Couples Coping with Stress: A Cross Cultural Perspective. New York, NY: Routledge, 5-22.

Breitenstein, C. J., Milek, A., Nussbeck, F. W., Davila, J., and Bodenmann, G. (2018). Stress, dyadic coping, and relationship satisfaction in late adolescent couples. J. Soc. Pers. Relat. 35, 770-790. doi: 10.1177/0265407517698049

Buck, A. A., and Neff, L. A. (2012). Stress spillover in early marriage: the role of self-regulatory depletion. J. Fam. Psychol. 26, 698-708. doi: 10.1037/a0029260
Chiara, G., Eva, G., Elisa, M., Luca, T., and Piera, B. (2014). Psychometrical properties of the dyadic adjustment scale for measurement of marital quality with Italian couples. Proc. Soc. Behav. Sci. 127, 499-503. doi: 10.1016/j.sbspro. 2014.03.298

Cook, W. L., and Kenny, D. A. (2005). the actor-partner interdependence model: a model of bidirectional effects in developmental studies. Int. J. Behav. Dev. 29, 101-109. doi: 10.1080/01650250444000405

Cousins, R., MacKay, C. J., Clarke, S. D., Kelly, C., Kelly, P. J., and McCaig, R. H. (2004). Management standards' and work related stress in the UK: practical development. Work Stress 18, 113-136. doi: 10.1080/02678370410001734322

Edwards, J. R., and Rothbard, N. P. (2000). Mechanisms linking work and family: clarifying the relationships between work and family constructs. Acad. Manag. Rev. 25, 178-199. doi: 10.5465/amr.2000.2791609

Efeoğlu, I. E., and Ozcan, S. (2013). Work-family conflict and its association with job performance and family satisfaction among physicians. Aust. J. Basic Appl. Sci. 7, 43-48.

Falconier, M., Randall, A., and Bodenmann, G. (2016). Couples Coping with Stress A Cultural Perspective. Abingdon: Routledge, doi: 10.4324/9781315644394

Falconier, M. K., Jackson, J. B., Hilpert, P., and Bodenmann, G. (2015). Dyadic coping and relationship satisfaction: a meta-analysis. Clin. Psychol. Rev. 42, 28-46. doi: 10.1016/j.cpr.2015.07.002

Falconier, M. K., Nussbeck, F., and Bodenmann, G. (2013). Immigration stress and relationship satisfaction in Latino couples: the role of dyadic coping. J. Soc. Clin. Psychol. 32, 813-843. doi: 10.1521/jscp.2013.32.8.813

Fallahchai, R., Fallahi, M., Charhartangi, S. H., and Bodenmann, G. (2017). Psychometric properties and factorial validity of the dyadic coping inventory the persian version. Curr. Psychol.

Fallahchai, R., and Khaluee, S. (2016). Comparison of quality of life and psychological well-being among dual career couples and single career couples. J. Modern Ind. 7, 19-27.

Fellows, K. J., Chiu, H.-Y., Hill, E. J., and Hawkins, A. J. (2016). Work-family conflict and couple relationship quality: a meta-analytic study. J. Fam. Econ. Issues 37, 509-518. doi: 10.1007/s10834-015-9450-7

Fișiloğlu, H., and Demir, A. (2000). Applicability of the dyadic adjustment scale for measurement of marital quality with Turkish couples. Eur. J. Psychol. Assess. 16, 214-218. doi: 10.1027//1015-5759.16.3.214

Gasbarrini, M. F., Snyder, D. K., Iafrate, R., Bertoni, A., Margola, D., Gasbarrini, M. F., et al. (2015). Investigating the relation between shared stressors and marital satisfaction: the moderating effects of dyadic coping and communication. Fam. Sci. 6, 143-149. doi: 10.1080/19424620.2015.1082044

Geurts, S. A. E., and Demerouti, E. (2003). "Work/non-work interface: a review of theories and findings," in The Handbook of Work and Health Psychology, eds M. J. Schabracq, J. A. M. Winnubst, and C. L. Cooper (Chichester: Wiley), 279-312. doi: 10.1002/0470013400.ch14

Ghodrati, H. (2015). Women's employment fluctuations in Iran and its deviations from the world trends. J. Soc. Dev. 9, 29-52.

Haddock, S. A., Zimmerman, T. S., Lyness, K. P., and Ziemba, S. J. (2006). Practices of dual earner couples successfully balancing work and family. J. Fam. Econ. Issues 27, 207-234. doi: 10.1007/s10834-006-9014-y

Haines, V. Y., Marchand, A., and Harvey, S. (2006). Crossover of workplace aggression experiences in dual-earner couples. J. Occup. Health Psychol. 11, 305-314. doi: 10.1037/1076-8998.11.4.305

Hassan, Z., Dollard, M. F., and Winefield, A. H. (2010). Work-family conflict in east vs western countries. Cross Cult. Manage. 17, 30-49. doi: 10.1108/ 13527601011016899

Herzberg, P. (2013). Coping in relationships: the interplay between individual and dyadic coping and their effects on relationship satisfaction. Anxiety Stress Coping 26, 136-153. doi: 10.1080/10615806.2012. 655726

Herzberg, P. Y., and Sierau, S. (2016). "Dyadic coping in German in couples," in Couples Coping with Stress, eds M. Falconier, A. Randall, and G. Bodenmann (New York, NY: Routledge), 122-126.

Hilpert, P., Randall, A. K., Sorokowski, P., Atkins, D. C., Sorokowska, A., Ahmadi, K., et al. (2016). The associations of dyadic coping and relationship satisfaction vary between and within nations: a 35-nation study. Front. Psychol. 7:1106. doi: 10.3389/fpsyg.2016.01106

Hilpert, P., Xu, F., Milek, A., Atkins, D. C., Bodenmann, G., and Bradbury, T. N. (2018). Couples coping with stress: between-person differences and 
within-person processes. J. Fam. Psychol. 32, 366-374. doi: 10.1037/fam00 00380

Hu, L.-T., and Bentler, P. M. (1999). Cutoff criteria for fit indexes in covariance structure analysis: conventional criteria versus new alternatives. Struct. Equat. Model. 6, 1-55. doi: 10.1080/10705519909540118

Iafrate, R., Bertoni, A., Margola, D., Cigoli, V., and Acitelli, L. (2012). The link between perceptual congruence and couple relationship satisfaction in dyadic coping. Eur. Psychol. 17, 73-82. doi: 10.1027/1016-9040/a000069

Jackson, G. L., Trail, T. E., Kennedy, D. P., Williamson, H. C., Bradbury, T. N., and Karney, B. R. (2016). The salience and severity of relationship problems among low-income couples. J. Fam. Psychol. 30, 2-11. doi: 10.1037/fam0000158

Kenny, D. A., Kashy, D. A., and Cook, W. L. (2006). Dyadic Data Analysis. New York, NY: The Guilford Press. doi: 10.1037/fam0000158

Khojasteh mehr, R., Naderi, F., and Sodani, M. (2013). The mediating role of dyadic coping on the relationship between marital standards and marital satisfaction. J. Manage. Syst. 3, 47-67.

Khosravi, M., Azami, S., Elahifar, A., and Dekani, M. (2010). Comparison of couples' marital satisfaction with both single-staffed couples with respect to their general health. Woman Fam. Stud. 3, 27-37.

Lapierre, L. M., and Allen, T. D. (2006). Work-supportive family, family-supportive supervision, use of organizational benefits, and problem-focused coping: implications for work-family conflict and employee well-being. J. Occup. Health Psychol. 11, 169-181. doi: 10.1037/1076-8998.11.2.169

Ledermann, T., and Kenny, D. A. (2017). Analyzing dyadic data with multilevel modeling versus structural equation modeling: a tale of two methods. J. Fam. Psychol. 31, 442-452. doi: 10.1037/fam0000290

Levesque, C., Lafontaine, M. F., Caron, A., Flesch, J. L., and Bjornson, S. (2014). Dyadic empathy, dyadic coping, and relationship satisfaction: a dyadic model. Eur. J. Psychol. 10, 118-134. doi: 10.1080/0092623X.2016.1208698

MacCallum, R. C., Browne, M. W., and Sugawara, H. M. (1996). Power analysis and determination of sample size for covariance structure modeling. Psychol. Methods 1, 130-149. doi: 10.1037/1082-989X.1.2.130

Mache, S., Bernburg, M., Vitzthum, K., Groneberg, D. A., Klapp, B. F., and Danzer, G. (2015). Managing work-family conflict in the medical profession: working conditions and individual resources as related factors. BMJ Open 5:e006871. doi: 10.1136/bmjopen-2014-006871

Marcatto, F., Colautti, L., Filon, F. L., Luís, O. D., and Ferrante, D. (2014). The HSE management standards indicator tool: concurrent and construct validity. Occup. Med. 64, 365-371. doi: 10.1093/occmed/kqu038

Martinengo, G., Jacob, J. I., and Jeffrey Hill, E. (2010). Gender and the work-family interface: exploring differences across the family life course. J. Fam. Issues 31, 1363-1390. doi: 10.1177/0192513X10361709

Mazhari, M., Zahrakar, K., Shakarami, M., Davarniya, R., and Abdollah Zadeh, A. (2016). The effect of Relationship Enhancement Program (REP) on reducing marital conflicts of dual- career couples. Iran J. Nurs. 29, 32-44. doi: 10.29252/ ijn.29.102.32

Mennino, S. F., Rubin, B. A., and Brayfield, A. (2005). Home-to-job and job-to home spillover: the impact of company policies and workplace culture. Sociol. Q. 46, 107-135. doi: 10.1111/j.1533-8525.2005.00006.x

Merz, C. A., Meuwly, N., Randall, A. K., and Bodenmann, G. (2014). Engaging in dyadic coping: buffering the impact of everyday stress on prospective relationship satisfaction. Fam. Sci. 5, 30-37. doi: 10.1080/19424620.2014. 927385

Michel, J. S., Mitchelson, J. K., Kotrba, L. M., LeBreton, J. L., and Baltes, B. B. (2009). A comparative test of work-family conflict models and critical examination of work family linkages. J. Vocat. Behav. 74, 199-218. doi: 10.1016/j.jvb.2008. 12.005

Motahari, Z., Kh, A., Behzadpoor, S., and Azmoodeh, F. (2012). Effectiveness of mindfulness on reducing couple burnout in mothers with ADHD children. J. Fam. Couns. Psychother. 3, 592-612.

National Organization for Civil Registration (2017). The Rate of Marriage and Divorce in Iran. Available at: http://www.sabteahval.ir/Default.aspx?tabid $=4773$

Nazari, A., and Goli, M. (2008). The effects of solution- focused psychotherapy on the marital satisfaction of dual career couples. J. Knowl. Health 2, 35-40.

Neff, L. A., and Karney, B. R. (2005). To know you is to love you: the implications of global adoration and specific accuracy for marital relationships. J. Pers. Soc. Psychol. 88, 480-497. doi: 10.1037/0022-3514.88.3.480
Neff, L. A., and Karney, B. R. (2007). Stress crossover in newlywed marriage: a longitudinal and dyadic perspective. J. Marriage Fam. 69, 594-607. doi: 10.1111/j.1741-3737.2007.00394.x

Nicholls, A. R., and Perry, J. L. (2016). Perceptions of coach-athlete relationship are more important to coaches than athletes in predicting dyadic coping and stress appraisals: an actor-partner independence mediation model. Front. Psychol. 7:447. doi: 10.3389/fpsyg.2016.00447

Nohe, C., Meier, L. L., Sonntag, K., and Michel, A. (2015). The chicken or the egg? A meta-analysis of panel studies of the relationship between work-family conflict and strain. J. Appl. Psychol. 100, 522-536. doi: 10.1037/a0038012

Obradovic, J., and Cudina-Obradovic, M. (2009). Work-related stressors of workfamily conflict and stress crossover on marriage quality. Društvena Istraživanja $18,437-460$.

Oreizi, H., Dibaji, M., and Sadeghi, M. (2011). Investigation the relationship of work-family conflict with perceived organizational support, job stress and self-mastery in expatriate workers. Res. Clin. Psychol. Couns. 2, 151-170.

Papp, L. M., and Witt, N. L. (2010). Romantic partners' individual coping strategies and dyadic coping: implications for relationship functioning. J. Fam. Psychol. 24, 551-559. doi: 10.1037/a0020836

Patel, C. J., Govender, V., Paruk, Z., and Ramgoon, S. (2006). Working mothers: family-work conflict, job performance and family/work variables. SA J. Ind. Psychol. 32, 39-45. doi: 10.4102/sajip.v32i2.238

Quek, K. M. T., Knudson-Martin, C., Orpen, S., and Victor, J. (2011). Gender equality during the transition to parenthood: a longitudinal study of dual career couples in Singapore. J. Soc. Pers. Relat. 28, 943-962. doi: 10.1177/ 0265407510397989

Rafatjah, M. (2011). Changing gender stereotypes in Iran. Int. J. Womens Res. 1, $155-168$.

Randall, A. K., and Bodenmann, G. (2009). The role of stress on close relationships and marital satisfaction. Clin. Psychol. Rev. 29, 105-115. doi: 10.1016/j.cpr.2008. 10.004

Randall, A. K., and Bodenmann, G. (2017). Stress and its associations with relationship satisfaction. Curr. Opin. Psychol. 13, 96-106. doi: 10.1016/j.copsyc. 2016.05.010

Randall, A. K., Totenhagen, C. J., Walsh, K. J., Adams, C., and Tao, C. (2017). Coping with workplace minority stress: associations between dyadic coping and anxiety among women in same-sex relationships. J. Lesbian Stud. 21, 70-87. doi: 10.1080/10894160.2016.1142353

Richter, J., Rostami, A., and Ghazinour, M. (2014). Marital satisfaction, coping, and social support in female medical staff members in Tehran University Hospitals. Interpersona 8, 115-127. doi: 10.5964/ijpr.v8i1.139

Rottmann, N., Hansen, D. G., Larsen, P. V., Nicolaisen, A., Flyger, H., Johansen, C., et al. (2015). Dyadic coping within couples dealing with breast cancer: a longitudinal, population-based study. Health Psychol. 34, 486-495. doi: 10.1037/ hea0000218

Ruffieux, M., Nussbeck, F. W., and Bodenmann, G. (2014). Long-term prediction of relationship satisfaction and stability by stress, coping, communication, and well-being. J. Divorce Remarriage 55, 485-501. doi: 10.1080/10502556.2014. 931767

Saginak, K. A., and Saginak, M. A. (2005). Balancing work and family: equity, gender, and marital satisfaction. Fam. J. 13, 162-166. doi: 10.1177/ 1066480704273230

Sanai Zakir, B. (2000). Marriage and Family Assessment Scales. Tehran: Besat Publications. doi: 10.1177/1066480704273230

Sandberg, J. G., Yorgason, J. B., Miller, R. B., and Hill, E. J. (2012). Family to-work spillover in Singapore: marital distress, physical and mental health, and work satisfaction. Fam. Relat. 61, 1-15. doi: 10.1111/j.1741-3729.2011. 00682.x

Saraie, H., and Tajdari, A. (2011). Left discourse in Iran: global ideas in Iranian context. Sociol. Cult. Stud. 1, 7-23.

Schaer, M., Bodenmann, G., and Klink, T. (2008). Balancing work and relationship: couples coping enhancement training (CCET) in the workplace. Appl. Psychol. 57, 71-89. doi: 10.1111/j.1464-0597.2008.00355.x

Schulz, M. S., Cowan, P. A., Pape Cowan, C., and Brennan, R. T. (2004). Coming home upset: gender, marital satisfaction, and the daily spillover of workday experience into couple interactions. J. Fam. Psychol. 18, 250-263. doi: 10.1037/ 0893-3200.18.1.250 
Shojaei, J., and Yazdkhasti, B. (2017). A systematic review of studies of fertility decline in the last two decades. Womens Strateg. Stud. 19, 137-159.

Šimunic, A., and Gregov, L. (2012). Conflict between work and family roles and satisfaction among nurses in different shift systems in Croatia: a questionnaire survey. Arch. Ind. Hygiene Toxicol. 63, 189-197. doi: 10.2478/10004-1254-632012-2159

Soleimanian, A., and Nazari, A. M. (2007). Investigation and comparison of marital satisfaction of dual- career couples and one couple employed. Couns. Res. 6, 103-122. doi: 10.2478/10004-1254-63-2012-2159

Spanier, G. B. (2001). Dyadic Adjustment Scale (DAS): User's manual MHS. Available at: https://books.google.com/books?id=eaM9mwEACAAJ

Spector, P. E. (1994). Using self-report questionnaires in OB research: a comment on the use of a controversial method. J. Organ. Behav. 15, 385-392. doi: 10.1002/job.4030150503

Spell, C. S., Haar, J., and O'Driscoll, M. (2009). Managing work-family conflict: exploring individual and organizational options. N. Z. J. Hum. Resour. Manag. 9, 200-215. doi: 10.1002/job.4030150503

Statistics Center of Iran (2018). Marriage and Divorce in the First and Second Years of Marriage in Iran. Available at: https://www.amar.org.ir

Sultana, N., Tabassum, A., and Abdullah, A. M. (2014). Dual-career couples in Bangladesh: exploring the challenges. Can. J. Fam. Youth 6, 29-57.

Tatman, A. W., Hovestadt, A. J., Yelsma, P., Fenell, D. L., and Canfield, B. S. (2006). Work and family conflict: an often overlooked issue in couple and family therapy. Contemp. Fam. Ther. 28, 39-51. doi: 10.1007/s10591-0069693-4

van Steenbergen, E. F., Kluwer, E. S., and Karney, B. R. (2011). Workload and the trajectory of marital satisfaction in newlyweds: job satisfaction, gender, and parental status as moderators. J. Fam. Psychol. 25, 345-355. doi: 10.1037/ a0023653

Vedes, A., Nussbeck, F., Bodenmann, G., Lind, W., and Ferreira, A. (2013). Psychometric properties and validity of the dyadic coping inventory in portuguese. Swiss J. Psychol. 72, 149-157. doi: 10.1024/1421-0185/a000108

Wang, P., Lawler, J. J., and Shi, K. (2010). Work-family conflict, self-efficacy, job satisfaction, and gender: evidences from Asia. J. Leadersh. Organ. Stud. 17, 298-308. doi: 10.1177/1548051810368546

Wierda-Boer, H. H., Gerris, J. R., and Vermulst, A. A. (2009). Personality, stress, and work-family interferences in dual earner couples. J. Individ. Differ. 30, 6-19. doi: 10.1027/1614-0001.30.1.6

Wunderer, E., and Schneewind, K. (2008). The relationship between marital standards, dyadic coping and marital satisfaction. Eur. J. Soc. Psychol. 38, 462-476. doi: 10.1002/ejsp.405

Yucel, D. (2017). Work-family balance and marital satisfaction: the mediating effects of mental and physical health. Soc. Ment. Health 7, 175-195. doi: 10. $1177 / 2156869317713069$

Conflict of Interest Statement: The authors declare that the research was conducted in the absence of any commercial or financial relationships that could be construed as a potential conflict of interest.

Copyright (c) 2019 Fallahchai, Fallahi and Randall. This is an open-access article distributed under the terms of the Creative Commons Attribution License (CC BY). The use, distribution or reproduction in other forums is permitted, provided the original author(s) and the copyright owner(s) are credited and that the original publication in this journal is cited, in accordance with accepted academic practice. No use, distribution or reproduction is permitted which does not comply with these terms. 


\section{OPEN ACCESS}

Edited by:

Guy Bodenmann,

University of Zurich, Switzerland

Reviewed by:

Johan Karremans,

Radboud University Nijmegen,

Netherlands

Dayna Lee-Baggley,

Dalhousie University, Canada

*Correspondence:

Tamás Martos

tamas.martos@psy.u-szeged.hu

Specialty section:

This article was submitted to Clinical and Health Psychology, a section of the journal

Frontiers in Psychology

Received: 19 June 2018 Accepted: 11 February 2019

Published: 28 February 2019

Citation:

Martos T, Sallay V, Nagy $M$, Gregus H and Filep O (2019) Stress and Dyadic Coping in Personal Projects of Couples -

A Pattern-Oriented Analysis.

Front. Psychol. 10:400. doi: 10.3389/fpsyg.2019.00400

\section{Stress and Dyadic Coping in Personal Projects of Couples - A Pattern-Oriented Analysis}

\author{
Tamás Martos ${ }^{1 *}$, Viola Sallay ${ }^{1}$, Marianna Nagy ${ }^{1}$, Henrietta Gregus ${ }^{1}$ and Orsolya Filep ${ }^{2}$ \\ ${ }^{1}$ Institute of Psychology, University of Szeged, Szeged, Hungary, ${ }^{2}$ Doctoral School, Semmelweis University, Budapest, \\ Hungary
}

Relational accounts of goal striving have barely considered dyadic coping as an element of the process, nor has dyadic coping research utilized the unique advantages of the goal construct (e.g., in form of personal project assessment) so far. Therefore, the primary aim of the present study was to explore stress and dyadic coping experiences associated with the personal projects of partners in a close relationship. Moreover, we approached data analysis in a pattern-oriented way, instead of using variable-centered linear models. We used cross-sectional data from 270 married and cohabiting Hungarian heterosexual couples (mean age 40.1 \pm 11.2 and $37.8 \pm 10.9$ years for male and female partners, respectively). Partners individually completed an adapted version of the Personal Project Assessment procedure. First, they named an important but stressful personal project. Respondents appraised their experiences with the chosen personal project along several predefined aspects. These included: (1) stress experiences; (2) dyadic coping, using the adapted Dyadic Coping Inventory; (3) positive emotions; and (4) sense of community. The Relationship Assessment Scale was also assessed. Cluster analysis of both partners' stress experiences, positive and negative dyadic coping strategies in their own personal projects revealed six relationship-level clusters. Cluster solutions represented typical variations of the stress and dyadic coping patterns of the couples, and could be arranged in a three- (lower, medium, and higher stress) by-two (positively vs. negatively balanced dyadic coping pattern) array. Further analyses indicated the general trend that couples with lower (vs. higher) stress together with more positively (vs. negatively) balanced dyadic coping may have experienced better functioning in projects (more positive emotions and higher sense of community) and higher relationship satisfaction. Results confirm that the partners' pursuit of their personal projects is embedded in their relationship, and their functioning in these projects may partly depend on dyadic coping with the stress that arises during the accomplishment of the project. By using a pattern-oriented approach to dyadic data, we were able to distill stress and coping patterns that capture the specific types of couples' relationships and indicate the non-linear and multidimensional nature of stress and dyadic coping processes.

Keywords: stress, dyadic coping, self-regulation, Dyadic Coping Inventory, personal project assessment, dyadic data, cluster analysis, relationship satisfaction 


\section{INTRODUCTION}

People often pursue important personal goals in their lives that are related to the goals of important others. For example, when a husband seeks to arrange a vacation together with his spouse, but the wife has to take an important exam, they will have to cooperate in their personal pursuits in order to maintain a well-functioning relationship. Moreover, the accomplishment of these goals is often accompanied by the experience of stress (c.f., Carver et al., 2008) that requires joint stress management efforts in the relationship. Building on this, in the present study, we aim to connect two domains of relationship functioning in close relationships. First, we focus on systemic accounts of self-regulation: on everyday personal projects of partners as these are embedded in their close relationship; and second, on dyadic coping (and more specifically, on the Systemic Transactional Model) to coping with stress. Then, we link these domains, and describe how a personal-project-based approach may add to our present knowledge about the processes of dyadic coping with stress.

\section{Personal Goals, Personal Projects, and Close Relationships}

The pursuit and successful accomplishment of personal goals are vital parts of individual self-regulation and well-being (e.g., Brunstein, 1993; Baumeister et al., 2007; Klug and Maier, 2015). Recent theoretical approaches to self-regulation have emphasized the fundamentally relational nature of the goal-striving processes. Individual self-regulation is closely interwoven with the selfregulation efforts of important others in the sense that, from a systemic point of view, close relationships themselves may be treated as units of analysis (Fitzsimons and Finkel, 2015; Fitzsimons et al., 2015), whereby individual and relational regulations are circularly related to each other (Finkel and Fitzsimons, 2011; Fitzsimons and Finkel, 2011). While working on their personal goals, individuals are constantly faced with challenges that result from the goals and actions of others. In fact, according to Transactive Goal Dynamics Theory (Fitzsimons and Finkel, 2015; Fitzsimons et al., 2015), personal goals of individuals in a committed relationship may also be regarded as interrelated and linked goals that can be best understood in the context of the close relationship.

Goal constructs have successfully been applied in studies of relationship functioning in general (Kaplan and Maddux, 2002), of relational-level adjustment to life transitions (Salmela-Aro et al., 2010) and of the management of relationship conflicts (Gere and Schimmack, 2013). It was also found that mutual support for partners' personal goals and strivings significantly contributes toward relationship satisfaction; in return, the experience of a higher relationship quality fosters further support and goal coherence (Molden et al., 2009; Overall et al., 2010; Hofmann et al., 2015).

In these studies, similarly to the individual level research tradition of goal-directed self-regulation, personal goals have often been conceptualized in more concrete terms such as the pursuit of personal strivings, personal projects or actual concerns (c.f., Emmons, 1997). For the present study, we will apply personal projects as the core theoretical and methodological construct. Personal projects are sets of personally important pursuits of individuals that are embedded in their everyday ecological contexts (Little, 1983, 2006). While overarching goals like 'performing well' may represent general rules of selfregulation, the project 'I will pass my next professional exam' is deeply embedded in the actual context of the person. Moreover, it refers to desired future states as well (e.g., professional achievement). This way, an investigation of personal projects is capable of capturing both the actual social ecological context of an individual's life and its future-oriented component (Little, 2015). Since close relationships are among the most important contexts of individual goal striving (c.f., Fitzsimons and Finkel, 2015), it may be inferred that personal projects are ideal units for psychological assessment and analysis of relationship related experiences.

\section{Stress and Coping in Personal Projects}

Processes of coping with stress and goal-directed behavior are often treated as distinct concepts. In the stress literature, people often (and implicitly) are depicted as if they were required to face difficulties and stressful circumstances independently of their previous actions. Their agency only appears in their coping efforts, and the quality of coping with these situations influences their quality of life, as well as their health and well-being (Thoits, 2010; Carnes, 2017; Praharso et al., 2017). However, important theoretical work connects stress and coping with self-regulation. For example, Carver et al. (2008) argue that stress and coping can be better understood as linked to goal-directed action. Stress is viewed as goal-related frustration, whereas coping is regarded as the self-regulation process that helps the individual find a new way to accomplish the goal or to disengage from it. When pursuing an 'exam' project, for example, one can easily run into difficulties with time management or a complicated learning task. Personal projects themselves may evoke stress in the individual, and coping efforts to regulate this stress become part of project accomplishment. In a similar way, we may assume that partners also have to regulate their personal projects with regard to their relationships, and thus, project-related coping processes may also have relational implications.

\section{Dyadic Coping With Stress in Close Relationships}

Recent theoretical developments concerning stress and coping acknowledge that individual stress often becomes dyadic stress that impacts both members of a couple. According to Bodenmann (1997), dyadic coping involves the joint process of a couple responding to an individual stressor of one of the partners'. The reason for that is to restore the homeostasis of the relationship. In dyadic coping both partners' stress management skills are activated. The importance of effective dyadic coping is emphasized by the fact that chronic stress can negatively affect communication among partners, as well as the quality and the development of a relationship (Papp and Witt, 2010). In addition, unmitigated stress can increase the chance of divorce in the 
long term (Bodenmann, 2000, 2005; Story and Bradbury, 2004). Consequently, the recognition, that coping processes have dyadic aspects as well has led to the concept of dyadic coping.

Dyadic stress, dyadic coping and the connection between the two concepts have become an area for extensive research in the last few decades (Bodenmann, 1997; Falconier et al., 2016; Sim et al., 2017; Staff et al., 2017). One of the most often used dyadic coping models is the Systemic Transactional Model (STM, Bodenmann, 1995). STM identifies the circular processes of dyadic coping that involve both partners' coordinated actions of stress communication, partner's reactions, and the appraisal of these reactions by the stressed partner. STM also considers common coping: when partners are involved in joint action to handle stress. According to the model, the mutual dyadic coping efforts of partners can be classified as positive (supportive and delegated acts of dyadic coping) or negative (hostile, ambivalent and superficial ways of dyadic coping). Several studies have used STM to investigate the significance of dyadic coping in couples' lives and its interrelation with relationship satisfaction (c.f., Falconier et al., 2015a, 2016). Positive dyadic coping with stress is associated with well-being and better relationship quality, while negative dyadic coping more often occurs between couples who experience distress in their closeness (Bodenmann et al., 2004; Herzberg, 2013; Falconier et al., 2015a).

By now, the stress-buffering potential of positive dyadic coping skill has been well documented (Merz et al., 2014; Falconier et al., 2015b; Breitenstein et al., 2018; Hilpert et al., 2018), showing that extra-dyadic or external stress (emerging outside of the relationship) may have a spillover effect on intradyadic or internal stress (conflict and tension between partners) only when partners' dyadic coping behaviors tend more toward negative strategies. The adverse effects of unmitigated dyadic stress reinforce STM's claims about the importance of adequate dyadic coping in long-term relationship functioning. However, further research is needed to understand how processes of stress and dyadic coping are nested in the everyday life context of couples (c.f., Bodenmann et al., 2016) as they construct their life circumstances through their active pursuit of goals, desires and accomplishments. For example, a couple's stress stemming from one partner's project (e.g., the wife's professional exam) can be co-regulated by the couple's dyadic coping strategies; which, in turn, will impact the accomplishment of the project. Thus, we turn to examining the interrelations of dyadic coping and the relationship-level regulation of personal projects.

\section{Dyadic Coping, Goals, and Personal Projects}

To the best of our knowledge, relational accounts of goal striving have barely considered dyadic coping as an element of the process. Moreover, while in STM goals play an important role in the dyadic coping process on a theoretical level (c.f., Bodenmann, 1995; Bodenmann et al., 2016), dyadic coping research rarely addressed the specific role of goals so far, except for a few but notable examples. For example, Kuster et al. (2017) tested approach and avoidance orientations in relationship goals in relation to dyadic coping strategies. Their results showed that approach-oriented goal striving in romantic relationships was associated with better relational outcomes, including more effective stress communication and better dyadic coping, whereas using avoidance goals in the relationship produced more negative consequences. In another study (Koranyi et al., 2017) researchers found that experiencing partner's stress might increase implicit preferences for communal goals that, in turn, predicted stronger inclinations to provide support in form of supportive dyadic coping. However, these associations were primarily true for participants with high relationship satisfaction. These studies provide support for the theoretical notion of STM where goals are results of primary and secondary appraisals as well as antecedents of the actual coping behavior (Bodenmann et al., 2016). This way, dyadic coping behaviors may serve specific individual and relationship oriented goals in a stressful situation. For example, supportive dyadic coping helps to fulfill relationship goals by reducing partner's emotional stress arousal and bad mood while assisting the partner's own efforts (c.f., Bodenmann et al., 2016, p. 13).

Using a personalized approach to goal striving, Martos et al. (2019) recently extended the personal project assessment methodology to the study of dyadic coping strategies, and demonstrated the reliability and validity of this approach in a pilot study. They argued that the construct of dyadic coping, as formulated in STM, might be included in models of relationshiplevel regulation of personal projects. Moreover, the assessment procedure might provide a more contextualized methodological approach to dyadic coping as well. With a sample of couples, the authors used the Dyadic Coping Inventory (DCI) to assess dyadic coping strategies. Partners in a dyad were asked to separately choose an actual stressful personal project of their own and to rate this project along the slightly modified items of the DCI. Thus, the procedure tapped into the everyday relationship experiences of the partners, and also assessed dyadic coping behaviors in this context. However, personal projects represent a distinct aspect of the motivational system that is complementary to the goals described in STM. Personal projects are larger scale pursuits in time that may include several forms of relationship regulation processes, among them, dyadic coping; while during the process of dyadic coping partners activate more proximal, actual goals that drive their coping with stress. Thus, personal projects and goals in dyadic coping represent different levels in the complex organization of individual and relational self-regulation.

\section{The Present Study}

Building on and extending the work of Martos et al. (2019), in the present research we connect the following aspects of individual and relationship functioning. We examine individually pursued personal projects of each member of a couple while we assume that their projects and, consequently, their project related experiences are embedded in the broader context of the couple's relationship. Therefore, we seek to study the partners' experiences of stress and dyadic coping in the context of their personal projects. The stress experienced by either partner during the accomplishment of their personal project may be approached with dyadic coping strategies jointly applied by the partners. Furthermore, the quality of this stress and dyadic coping process 
TABLE 1 | Conceptual and methodological network of the study.

\begin{tabular}{|c|c|c|c|c|c|}
\hline General context & Studied context & Measur & d experience & Primary reference of the experience & Analytic approach \\
\hline \multirow{6}{*}{$\begin{array}{l}\text { Couple's relationship } \\
\text { functioning }\end{array}$} & \multirow{5}{*}{$\begin{array}{l}\text { Partners' personal } \\
\text { projects }\end{array}$} & & tress & Individual & \multirow{3}{*}{ Pattern-oriented/explorative (Step 1) } \\
\hline & & Positive & \multirow{2}{*}{ Dyadic coping } & Relational & \\
\hline & & Negative & & Relational & \\
\hline & & \multicolumn{2}{|c|}{ Positive emotions } & Individual & \multirow{3}{*}{ Outcome-oriented/deductive (Step 2) } \\
\hline & & \multicolumn{2}{|c|}{ Sense of community } & Relational & \\
\hline & \multicolumn{3}{|c|}{ Relationship satisfaction in general } & Relational & \\
\hline
\end{tabular}

may have an impact on other personal project related experiences and may be associated with relationship functioning in general as well. Successful dyadic coping with project related stress may help to maintain positive emotions in the partners during the accomplishment of the personal project; it may also enhance their sense of community concerning the project (c.f., Fowers and Owenz, 2010; Randall et al., 2013). Consequently, in the context of the personal projects of the partners, we examined the association of stress and dyadic coping with two kinds of experience: positive emotions associated with the personal project and sense of community with the partner in the project. Hereby, positive emotions refer to primarily individual experiences of the partners during the accomplishment of the projects, whereas project related sense of community refers to primarily relational experiences of the partners. Moreover, in the broader context of the relationship, personal project related stress and dyadic coping experiences may be connected to relationship functioning in general as well. Accordingly, we assessed relationship satisfaction, a commonly used indicator of the general functionality of a romantic relationship in relation to stress (Randall and Bodenmann, 2017).

In order to address the holistic, and potentially non-linear nature of this systemic stress and dyadic coping functioning in dyads, we firstly apply a pattern-oriented analytic approach (Bergman and Trost, 2006) to data analysis and explore the relationship level patterns (c.f., Seiffge-Krenke and Burk, 2013; Czikmantori et al., 2018) of stress and dyadic coping experiences in the context of the personal projects of the partners. In the next step, we relate these emergent patterns to a series of outcomes that represent individual and relational functioning, both in the context of the personal projects themselves and in the broader context of the couple's relationship. Table 1 presents an overview on the conceptual and methodological network of our study. Moreover, we summarize the core aspects and assumptions of our study in detail in the following sections.

\section{Assessment of the Personal Projects}

Treating personal projects as conceptual units (c.f., Little, 2006) provides a powerful way to study personal and interpersonal processes in their everyday context. Moreover, as we have argued previously, dyadic coping with stress may be an important component of the effective relationship-level regulation of personal project attainment. The corresponding methodology of personal project assessment is a flexible and complex measurement tool that is suitable for assessing ecologically valid, contextually embedded experiences of respondents. The typical assessment procedure includes an individual elicitation of personal projects (e.g., "arranging a vacation trip for my parents"), followed by the measurement of several projectrelated characteristics (Little and Gee, 2007). In our study, we have adapted this personal project based procedure to capture stress experiences and dyadic coping strategies, as well as experiences of positive emotions and sense of community with the partner.

\section{Stress and Dyadic Coping in Personal Projects}

We used personal project related stress appraisals and dyadic coping strategies as the core units for the pattern-oriented analytic approach. In a previous piece of work (c.f., Martos et al., 2019) we measured only dyadic coping strategies via the personal project assessment procedure, that is, relational experiences in the project. However, since previous research has confirmed that the extent of perceived stress in a relationship may vary considerably, and this can have an adverse effect on relationship functioning (e.g., Merz et al., 2014; Hilpert et al., 2018), the inclusion of personal project related stress appears necessary for understanding the complex relationship between stress and dyadic coping in the partners' personal project pursuits. Therefore, in addition to a personal project based assessment of dyadic coping strategies, we also aimed to measure the amount of stress that was experienced by the partners during the accomplishment of their individual projects.

\section{Pattern-Oriented Analysis}

Scientific attention is being increasingly focused on the theoretical and practical differences between two branches of data-analysis strategies, namely the variable- and the pattern-oriented approaches, the latter of which is also called the person-oriented or person-centered approach (for a recent review, see Howard and Hoffman, 2017). Traditional variable-oriented approaches focus on separate individual characteristics (variables) and the linear associations between them. This way, results from a variable-oriented approach inform central tendencies, general rules and grand means. In contrast, a pattern-oriented approach seeks to maintain a holistic view of the individual (Bergman and Trost, 2006). It utilizes a complex set of multiple interdependent characteristics simultaneously and investigates how these characteristics relate to each other in specific ways (i.e., how they form types) and how they interact with each other as parts of a complex integrated system (Bergman et al., 2003; Bergman and Lundh, 2015). Pattern-oriented approach focuses on the whole system as the unit of analysis; in our case, on the couple, and more specifically, on the partners' interrelated, 
systemic functioning in their personal project pursuits. This way, the pattern-oriented approach can be viewed as complementary to the variable-oriented approach (Asendorpf, 2003; Czikmantori et al., 2018).

While research into dyadic relationships has been dominated by variable-oriented studies - for example, with the extensive use of the Actor-Partner Interdependence Model (APIM; Kenny and Ledermann, 2010) -, studies with a pattern-oriented approach to dyadic data are also attracting scholarly attention (Gagliardi et al., 2013; Seiffge-Krenke and Burk, 2013; Cao et al., 2015; Wood et al., 2015; Czikmantori et al., 2018). Accordingly, we assumed that a pattern-oriented approach may be an appropriate way to approach the personal projectrelated stress and dyadic coping experiences of partners in a close relationship. Since both stress and dyadic coping were assessed in relation to the same concrete personal projects, these experiences describe elements of the integrated, systemic functioning of the couple. Moreover, pattern-oriented data analysis is suitable for revealing complex interaction patterns in multiple characteristics, without the limitations of traditional (variable-centered) two- or three-way interaction analyses. As a result, we expected to explore and identify meaningfully different patterns of stress and dyadic coping in the personal-projectrelated experiences of couples. We also assumed that these emergent patterns would represent characteristic variations in couples' relationship functioning.

\section{General Assumptions on Outcomes}

In addition to the previous explorative analytic step, we planned a deductive, outcome-oriented analysis where the emergent stress and dyadic coping patterns would be compared to a series of potential outcomes of the stress and dyadic coping process. Although pattern-oriented approaches serve for exploratory purposes rather than hypothesis testing (Bergman and ElKhouri, 2003), we may still formulate a couple of general assumptions regarding the associations with outcomes. As a general pattern, we expected that higher stress together with less positive strategies of dyadic coping in personal projects would be associated with a lower level of beneficial experiences in the personal projects themselves (positive emotions and sense of community) as well as lower relationship satisfaction in general. However, the exact configurations of these associations required detailed exploration.

\section{MATERIALS AND METHODS}

\section{Procedure}

We conducted a cross-sectional study with voluntary participants who filled in an internet-based questionnaire. The respondents were recruited by trained students via an online advertisement on personal forums and social media. The participants were informed about the general aims of the study (i.e., the study of personal goals in a relationship context) and about the anonymity and confidentiality of data handling. Participants explicitly gave their informed consent through responding to the first question. Acceptance of this informed consent question was a prerequisite for subsequently filling in the questionnaire. Participants received no credit or monetary compensation for their participation. The research design was approved by the Unified Ethical Committee for Psychological Research of Hungarian Universities (EPKEB).

TABLE 2 | Sociodemographic characteristics of the sample.

\begin{tabular}{|c|c|c|c|c|c|c|c|c|c|c|c|}
\hline & & \multicolumn{5}{|c|}{ Cohabiting } & \multicolumn{5}{|c|}{ Married } \\
\hline & & $N$ & $m$ & $S D$ & Min & Max & $N$ & $m$ & $S D$ & Min & Max \\
\hline \multirow[t]{2}{*}{ Age } & $M$ & 103 & 34.07 & 9.72 & 23 & 66 & 167 & 43.85 & 10.45 & 26 & 76 \\
\hline & $F$ & 103 & 32.54 & 9.29 & 21 & 63 & 167 & 40.96 & 10.65 & 25 & 73 \\
\hline \multirow[t]{2}{*}{ Subjective financial status } & $M$ & 102 & 6.14 & 1.77 & 1 & 10 & 165 & 6.07 & 1.89 & 1 & 10 \\
\hline & $F$ & 103 & 5.81 & 1.69 & 1 & 9 & 166 & 6.09 & 1.86 & 1 & 10 \\
\hline \multirow[t]{2}{*}{ Length of relationship } & $M$ & 94 & 7.26 & 7.53 & 1 & 45 & 118 & 18.33 & 11.80 & 1 & 50 \\
\hline & $F$ & 93 & 6.70 & 5.88 & 1 & 35 & 114 & 18.14 & 11.70 & 1 & 50 \\
\hline \multirow[t]{2}{*}{ Number of children } & $M$ & 53 & 0.13 & 0.52 & 0 & 3 & 87 & 0.86 & 1.02 & 0 & 4 \\
\hline & $F$ & 53 & 0.02 & 0.14 & 0 & 1 & 87 & 0.99 & 1.04 & 0 & 4 \\
\hline
\end{tabular}

TABLE 3 | Examples of couples' personal projects.

\begin{tabular}{|c|c|c|c|}
\hline Couple no. & Male partner's project & Female partner's project & Coded as \\
\hline 7 & Insulate the house & Install insulation in the rooms in the attic & 'Same project' \\
\hline 63 & Quit smoking because we will have a baby & Stop smoking due to pending birth of child & 'Same project' \\
\hline 128 & Learn photography properly & Improve my English & 'Different projects' \\
\hline 140 & Take a nice vacation together & Pass professional exam & 'Different projects' \\
\hline 161 & Take less medication & Settle our debts & 'Different projects' \\
\hline 188 & Buy a new car & Finish the paintwork in the flat & 'Different projects' \\
\hline
\end{tabular}

Coding process referred whether the partners named essentially the same vs. different personal project. 


\section{Sample}

Table 2 presents descriptive statistics for the participants. In sum, a community sample of 270 married and cohabiting Hungarian heterosexual couples (mean age $40.1 \pm 11.2$ and $37.8 \pm 10.9$ years for male and female partners, respectively) were assessed. The average length of relationship was 13.42 years $(S D=11.51)$. Data was missing for 129 couples concerning their number of children; from the remaining 141 couples 49 were raising at least one underage child, while 92 had no children. Respondents rated their subjective financial status as slightly above average (on a 110 scale $M=6.04$ ). Approximately $28.3 \%$ of the participants ( 92 men and 61 women) had a primary, $58.0 \%$ a secondary (147 men and 166 women) and $13.7 \%$ (31 men and 43 women) a tertiary level of education.

\section{Measures}

\section{Personal Project Assessment}

\section{Overview}

Partners individually completed an adapted version of the Personal Project Assessment procedure. We assessed the experiences of participants related to stressful personal projects via an adapted version of the personal project assessment procedure (see Little and Gee, 2007). As a first step (project elicitation), participants were asked to write a short list of their current personal projects. We defined personal projects as "the goals and strivings that you are currently working on in your everyday life" (see Table 3 for examples of the personal projects in the study). In the second step, respondents selected the "most stressful" personal project from the list, leaving the respondents to define how they interpreted stress in their projects. Finally, participants were instructed to assess their personal experiences with their stressful personal project along several predefined aspects. These included (1) stressful and negative feelings as experienced during working on the projects; (2) project-related dyadic coping actions and evaluations - using the slightly modified items of the Dyadic Coping Inventory; and (3) further project-relevant experiences (i.e., positive emotions and sense of community).

\section{Stress in personal projects}

In order to estimate the extent of aggregated stress perceived in the chosen personal project we asked four questions. Two questions concerned the respondent's own experiences with the project ("How difficult is it for you to work on this project?" and "How much negative emotion/how much tension do you feel while working on this project?"). Two other questions asked about the same issues but from the point of view of the partner; that is, how the respondent perceives the experiences of their partner concerning the project ("How difficult is it for your partner to cooperate in this project?" and "How much negative emotion/how much tension does your partner feel concerning this project?"). Responses were scored on a 7-point, Likert-type scale ( 1 = low agreement, 7 = high agreement). Averaged scores of own and partner's stress appraisals were calculated for further analysis for each respondent.

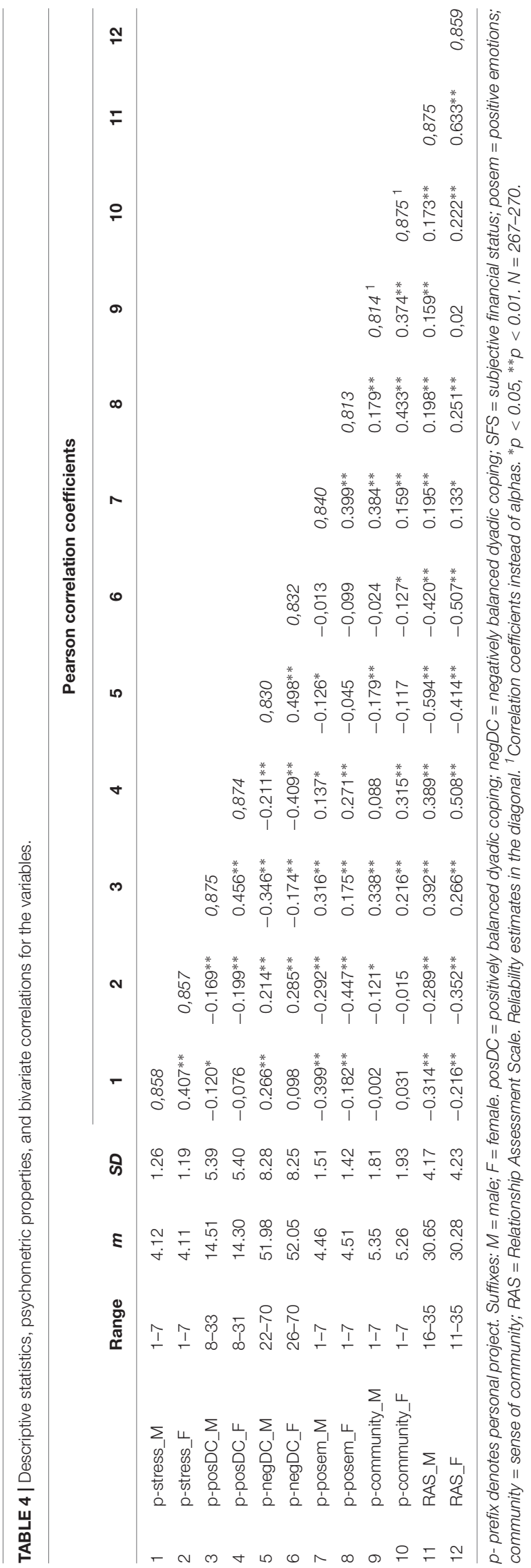




\section{Dyadic coping in personal projects}

We assessed dyadic coping experiences as they were related to the selected stressful personal projects. For the purposes of this study, we adapted the items of the Dyadic Coping Inventory (DCI, Bodenmann, 2008; Hungarian version: Martos et al., 2012). The same procedure was used as in research by Martos et al. (2019). The 37 items of the DCI assessed the couples' dyadic coping strategies. Subscales included stress communication (for example "When I feel stressed, I tell my partner openly how I feel, and that I would appreciate his/her support"), supportive, delegated and negative coping (for example "My partner blames me for not coping well enough with stress."). Respondents were also asked to indicate the stress communication of their partners and the way they react to their partners' stress in a supportive, delegated, or negative way. Finally, items assessed the frequency of common coping (for example, "We try to cope with problems together, and search for ascertained solutions."). Two additional items referred to the evaluation of the dyadic coping process (for example, "I am satisfied with the support I receive from my partner, and the way we deal with stress together."). We used a reworded version of the DCI in which phrases concerning the items had been modified from general present tense to past tense (e.g., "When I felt stressed I told my partner..." instead of "When I feel stressed I tell my partner..."). Moreover, in the instructions we referred explicitly to the chosen personal project, asking the respondents to indicate how often they experienced the presented coping behaviors in relation to their project in the past few weeks $(1=$ very rarely, $5=$ very often). For further analysis, we calculated summed scores of positive (stress communication, supportive, delegated and common) as well as negative dyadic coping strategies for each respondent.

\section{Positive emotions in personal project}

In addition to the stress and dyadic coping assessment, respondents were also asked to rate their personal project along further dimensions. Firstly, they rated the amount of positive emotions experienced throughout the project. In addition, participants also rated this aspect from their partner's point of view; that is, the partner's positive emotions in the personal project of the respondent. Finally, respondents rated the amount of "joyful experiences together" related to the personal project. Responses were scored on a 7point, Likert-type scale $(1=$ low agreement, $7=$ high agreement). Ratings of positive emotional experiences (self and attributed to the partner) as well as joyful experiences together were averaged into the "positive emotions in the personal project" variable (henceforth "positive emotions") for each respondent.

\section{Sense of community in personal project}

In a similar way, respondents were asked to indicate the extent they regarded the selected project as a "joint project," (i.e., how much they felt community with their partner concerning the project). Respondents also indicated the extent of agreement to which their partner regarded the project of the respondent as a “joint project." Responses were scored on a 7-point, Likert-type scale ( $1=$ low agreement, $7=$ high agreement). Self and attributed ratings of "joint project" of the personal project were averaged into the "sense of community in the personal project" variable (henceforth "sense of community").

\section{Relationship Assessment Scale}

Participants indicated their relationship satisfaction by scoring items using the Relationship Assessment Scale (RAS; Hendrick, 1988; Martos et al., 2014). The seven RAS items assessed the respondent's satisfaction with their own relationship (sample item: "How well does your partner meet your needs?"). Responses indicate the degree of agreement on a 5-point, Likert-type scale ( $1=$ little agreement, $5=$ high agreement). The alpha coefficient indicated excellent internal consistency in the sample (alphas $=0.875$ and 0.859 for male and female partners, respectively).

\section{RESULTS}

\section{Overview of the Analytical Process}

After initial data screening, the variables for the study were identified and their basic psychometric properties and bivariate associations were computed (see Table 4 for an overview). As a first analytic step, explorative cluster analysis using the pattern recognition module of the ROPstat software (Vargha, 2016) was

TABLE 5 | Adequacy indexes of cluster solutions 3-10.

\begin{tabular}{|c|c|c|c|c|c|c|}
\hline Step & Cluster N & EESS \% & Point biserial & XieBeni (mod) & Silhouette coefficient & HC mean \\
\hline$i=260$ & 10 & 58.43 & 0.291 & 0.127 & 0.430 & 0.861 \\
\hline$i=261$ & 9 & 56.01 & 0.309 & 0.349 & 0.419 & 0.907 \\
\hline$i=262$ & 8 & 53.31 & 0.309 & -0.030 & 0.411 & 0.959 \\
\hline$i=263$ & 7 & 50.39 & 0.333 & 0.278 & 0.432 & 1.015 \\
\hline$i=264$ & 6 & 46.99 & 0.34 & 0.361 & 0.418 & 1.081 \\
\hline$i=265$ & 5 & 43.00 & 0.331 & 0.281 & 0.405 & 1.157 \\
\hline$i=266$ & 4 & 37.97 & 0.32 & 0.088 & 0.389 & 1.254 \\
\hline$i=267$ & 3 & 30.04 & 0.312 & -0.135 & 0.364 & 1.409 \\
\hline After relocation & 6 & 50.52 & 0.358 & 0.333 & 0.473 & 1.009 \\
\hline
\end{tabular}

EESS = Explained Error Sum of Squares; Point biserial = point biserial correlation coefficient; XieBeni $($ mod $)=$ modified Xie-Beni index; HC = Homogeneity of Cluster index . 


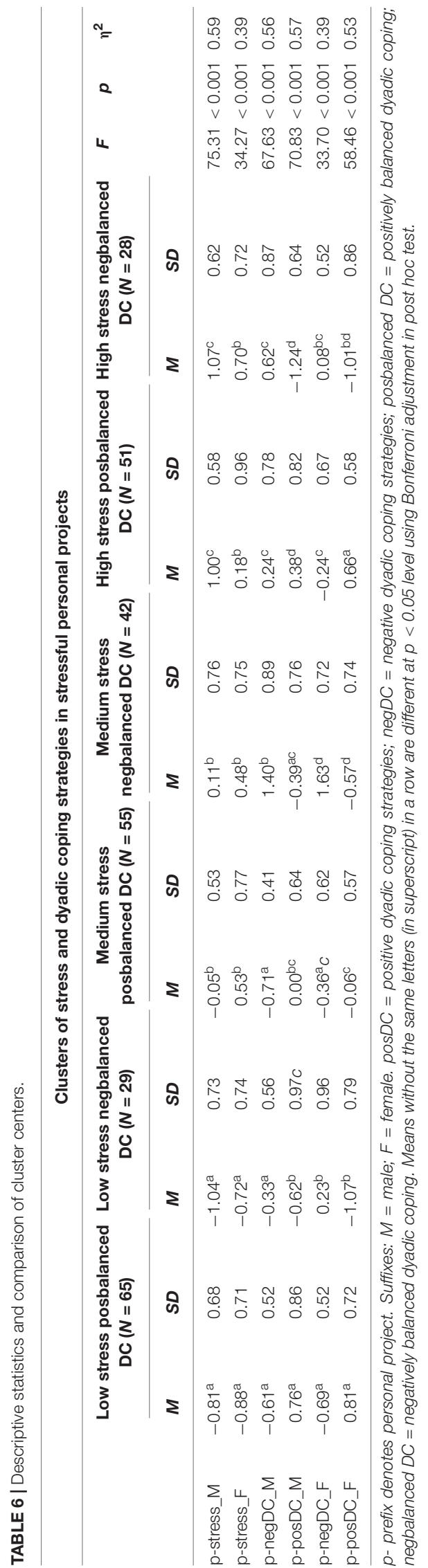

run on the aggregated stress and dyadic coping scores of the partners. Since we treated the couple as the unit of the analysis, corresponding data from both partners of a couple were included in this analysis. Through an iterative process (c.f., Takács et al., 2015; Vargha et al., 2016) we identified the appropriate number of clusters. In the next analytic step, we deductively compared the clusters - representing subsamples of couples with specific stress and dyadic coping patterns in their personal projects - with respect to basic demographic characteristics. It was also tested whether the clusters differed in the outcome variables; partners' positive emotions and sense of community as experienced in their stressful personal projects, as well as in relationship satisfaction of the partners. Cluster comparisons were performed using ANCOVA with Bonferroni post hoc test.

\section{Preliminary Analyses}

First we tested whether aggregated ratings of personal project experiences formed reliable subscales. Alpha coefficients (Table 4, diagonal) were appropriate in magnitude for all of the subscales; ranging from 0.813 (positive emotions in female respondents) to 0.875 (DCI, Negative Dyadic Coping in male respondents). For sense of community items we calculated inter item correlations as estimates of reliability ( 0.814 and 0.875 for male and female respondents, respectively). After initial data processing we ran descriptive statistics and bivariate correlations for the variables in the study (see Table 4). Inspection of bivariate correlations revealed that the association between the two genders on the same scores were of only medium to low effect size for all of the coping scales $(r=0.374, p<0.001$ to $r=0.498 ; p<0.001)$ while RAS was strongly associated $(r=0.633, p<0.001)$. The conceptually similar subscales of Positive Dyadic Coping and Project Positivity indicated medium associations $(r=0.316$, $p<0.001$ and $r=0.271, p<0.001$ for male and female partners, respectively) while there were low effect sizes between Negative Dyadic Coping and Project Stress $(r=0.266, p<0.001$ and $r=0.285, p<0.001$ for male and female partners, respectively).

The presence of partners with the same (vs. different) projects in the sample was considered to have a potentially confounding effect on results. Therefore, we also checked personal projects to identify partners with same projects; that is, we looked for cases where both partners named essentially identical projects (see Table 3 for examples). Two independent raters content-analyzed the individual project descriptions and decided whether descriptions referred to the same (vs. different) personal project between partners. The reliability of inter-rater decisions was assessed using intraclass correlation (two-way, consistency, average-measures ICC; Hallgren, 2012), yielding an ICC coefficient of $0.922(95 \%$ CI $=0.902-0.939)$. Discrepancies were resolved by team discussion. In sum, 66 couples (24.6\%) referenced the same projects. There were no statistically significant differences between couples with and without the same personal projects in terms of demographic variables (age, relationship status, relationship duration) nor several psychological characteristics (project stress, positive and negative dyadic coping, positive emotions in projects, as well as relationship satisfaction) after correcting the alpha level for multiple comparisons. We found only one significant difference; 


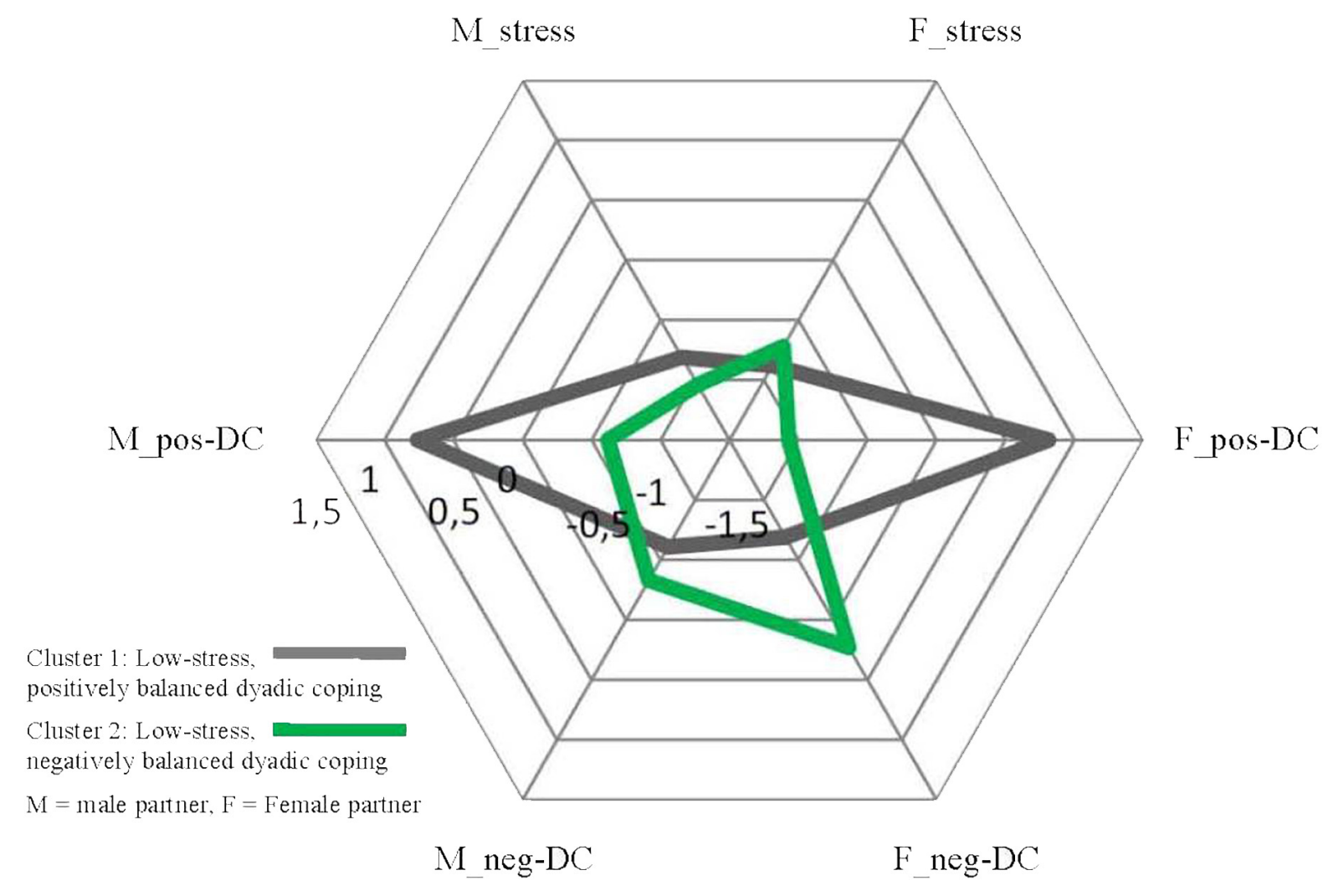

FIGURE 1 | Low-stress couples: Cluster centers of Cluster 1 and 2, z-scores.

on average, partners with the same project had higher sense of community in their projects. ${ }^{1}$

\section{Cluster Analysis of the Stress and Dyadic Coping Variables}

We conducted a series of hierarchical cluster analyses to determine the best-fitting cluster solution (c.f., Vargha et al., 2015) Cluster analysis was run on standardized scores of the initial variables (stress, positive and negative dyadic coping of both partners in their projects; in sum, six variables) via Ward method with squared Euclidean distances, which maximizes the difference between the groups and minimizes it between the clusters. Following the procedure described by Vargha et al. (2015), we compared 3-10 cluster solutions with regard to their adequacy. For each of these actual cluster solutions we examined the most important adequacy measures (explained variance, Point-bi-serial Correlation, Silhouette Coefficient, average cluster homogeneity; c.f., Vargha et al., 2016). Adequacy measures are presented in Table 5 for the cluster solutions with 3-10 clusters. We determined the final number of clusters based on inspection of the adequacy measures as well as preliminary interpretation of the potentially well fitting cluster solutions. As a result, we retained the six-cluster solution for further analysis because this solution maximized goodness of fit, explanatory power

\footnotetext{
${ }^{1}$ Results are available on request from the corresponding author.
}

and interpretability. Comparison of the adequacy measures (see Table 5) shows that the $N=6$ clusters solution is appropriate in several ways. First, EESS $=46.99 \%$, which is adequate considering that we may expect it to increase after relocation (Vargha et al., 2016). Moreover, inspection of the change diagram of added EESS\% showed that there is an elbow around solutions 6 and 7. This means that the addition of the seventh cluster adds proportionally less to this value than the previous solutions in terms of EESS\%. Second, the point-bi-serial coefficient is well above the 0.3 threshold, while the Homogeneity Coefficients of the clusters are around 1.0. More importantly, the modified XieBeni index indicated a local maximum for this solution. This was interpreted to mean that the six-cluster solution was locally the most homogeneous compared to its neighbors. Preliminary interpretation of this solution also confirmed its viability. The next solution with a similar modified Xie-Beni index was the nine-cluster solution. However, retaining nine clusters would have led to challenges both in interpretation and because of the resulting low sample size of cluster memberships which may have produced non-significant associations in group comparison. Therefore, to maintain the robustness of results, we decided to apply the six-cluster solution for the analysis. Following the confirmation of the appropriate number of clusters, a relocation process was performed so that the individual cases matched their final cluster better. As a result, EESS\% increased to $50.52 \%$, and the modified Xie-Beni index remained above 0.3. Upon 


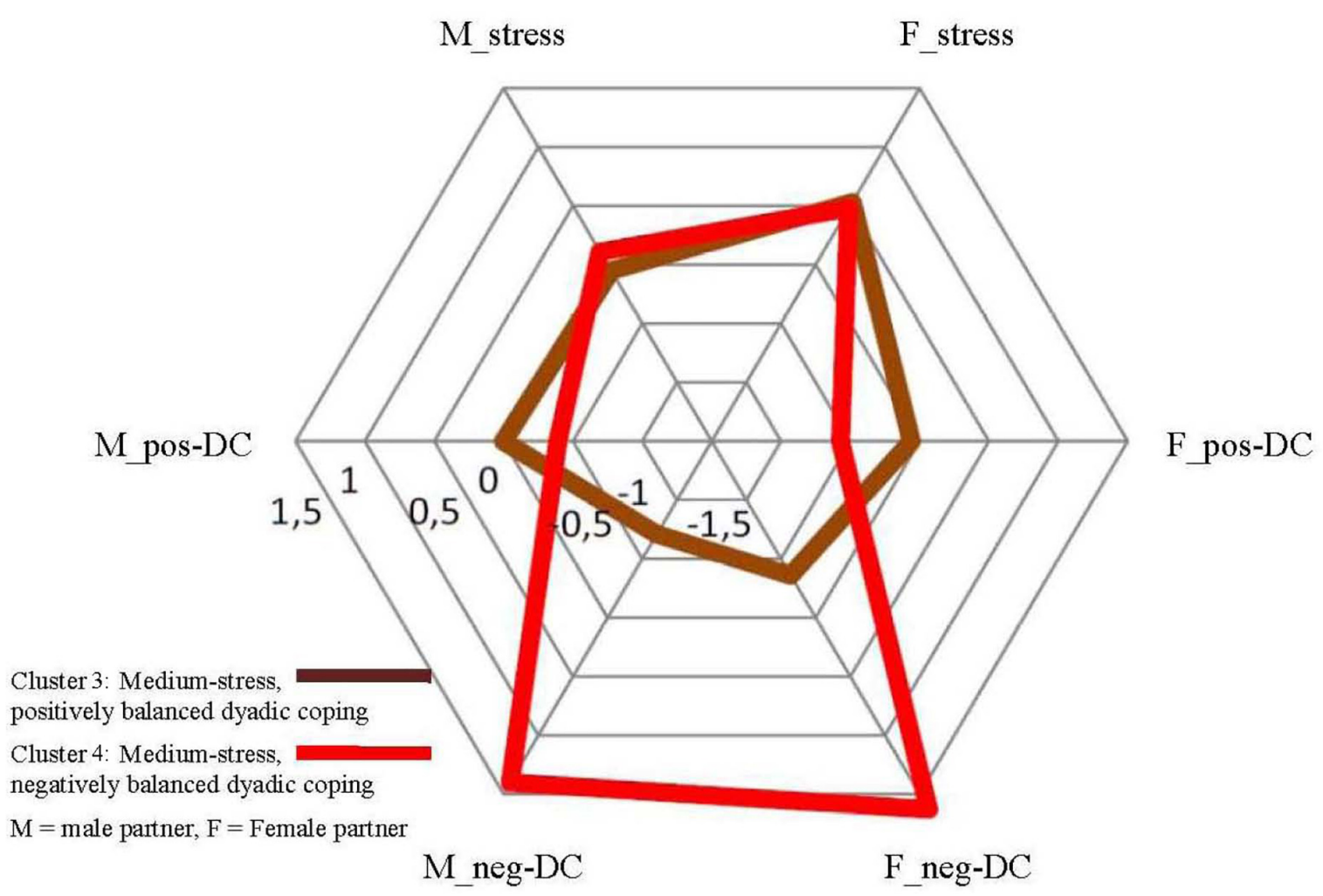

FIGURE 2 | Medium-stress couples: Cluster centers of Cluster 3 and 4, z-scores.

relocation, the individual cases were assigned to the clusters for further analysis. The proportion of each of the clusters in the examined sample can be regarded as fairly balanced, ranging from 10.37 to $24.07 \%$.

We ran a series of ANOVAs to determine how the clusters as groups of couples were different in terms of the initial dimensions of stress and dyadic coping of the partners (see Table 6). Closer inspection of the cluster centers and their graphic representation (see Figures 1-3) indicated that cluster solutions could be grouped in a three- (lower, medium, and higher stress) -by-two (positively vs. negatively balanced dyadic coping pattern) array. Clusters 1 and 2 are characterized by a low level of experienced stress in the personal projects of the partners ( $z$-scores between -1.04 and -0.72 ), Clusters 3 and 4 with a medium level of experienced stress ( $\mathrm{z}$-scores between -0.05 and $0.53)$ and Clusters 5 and 6 with a medium level of experienced stress (z-scores between 0.18 and 1.07). It is important to note that Clusters 3-4 and 5-6 differ primarily and significantly in terms of the scores of male partners while there are no significant differences between the female scores in these four clusters. Still, we propose the interpretation of 'medium' vs. 'high' level stress because partners in Clusters 5 and 6 struggle with significantly more total stress in their lives than couples in Clusters 3 and 4. However, in later interpretations it is important to keep in mind that the main difference can be traced back to the stress level of the male partners. Concerning the latter analytical element it was clear that in Clusters 1, 3, and 5 positive dyadic coping experiences outweigh negative dyadic coping experiences in the projects of both partners, while the opposite is true for the couples in Clusters 2, 4, and 6. In these clusters negative ways of dyadic coping are relatively more frequent than positive ways in both partners' experiences. While this description is true for the balance between positive and negative dyadic coping, the actual levels of these strategies vary across patterns. The frequencies of positive and negative dyadic coping are not equal in the clusters; however, they do not follow a linear pattern either. For example, negative dyadic coping is extremely high in both partners in Cluster 4 together with only medium-level couple stress. In a similar way, a high level of positive dyadic coping is indicated in partners, especially in women, in Clusters 1 and 5; that is, in couples with low and high stress, while in Cluster 3 medium-level stress was accompanied with only a medium level of positive dyadic coping. With all these nuances in mind, in the subsequent analyses we refer to the clusters using the abovementioned characteristics of low, medium, and high stress, as well as positively and negatively balanced dyadic coping experiences.

\section{Comparison of the Clusters}

Furthermore, one-way ANOVAs were conducted to detect differences between the six clusters regarding the sociodemographic characteristics of the partners. There was no significant difference in the partners' age $[F(5,264)=0.827$, $p=0.532 ; F(5,264)=0.906, p=0.478$ for male and female partners, respectively), length of relationship $[F(5,206)=0.317$, 


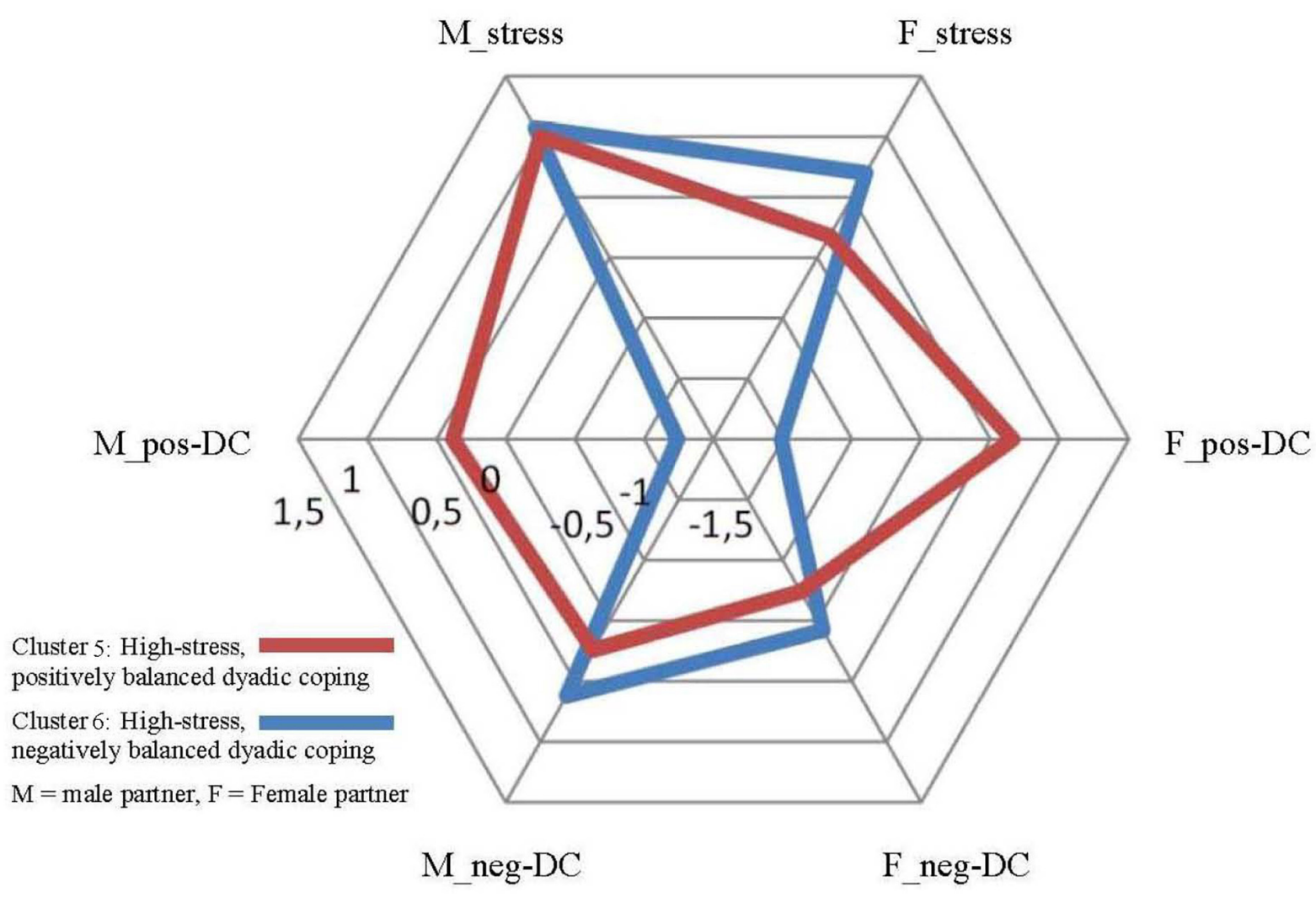

FIGURE 3 | High-stress couples: Cluster centers of Cluster 5 and 6, z-scores.

$p=0.902 ; F(5,201)=0.368, p=0.870]$ and number of underage children $[F(5,134)=1.170, p=0.327 ; F(5,134)=0.679$, $p=0.640]$. In contrast, the analysis showed significant differences for subjective financial status regarding both genders $[F(5$, $261)=2.864, p=0.015 ; F(5,263)=3.526, p=0.004]$. Bonferroni post hoc analysis showed that in couples with a low level of experienced stress and positive coping both spouses reported the highest subjective financial status (Cluster 1) while, unexpectedly, in couples with a medium level of experienced stress and negative coping (Cluster 4 ) the lowest subjective financial status level was reported $(p=0.019$ and $p=0.004)$. There was no significant association between cluster membership and relationship status (marriage vs. cohabitation) [chi-square $=1.29(5), p=0.936$ ] and between cluster membership and education either [chisquare $=14.49(10), p=0.152$ and chi-square $=14.35(10)$, $p=0.158$, for male and female partners, respectively].

In the next step, we tested whether couples in the six clusters were different across a series of outcome measures, such as the partners' positive emotions and sense of community experienced in their own projects, as well as their relationship satisfaction (see Figures 4-6). Since the preliminary analyses partly showed the significant associations of the clusters with sociodemographic characteristics, we performed subsequent group comparisons using a series of ANCOVAs, where the main effects were controlled for the subjective financial status scores of both partners. We assessed group differences using post hoc tests with
Bonferroni adjustment. There were significant differences for both spouses for project positivity $[F(5,258)=8.608, p<0.001$, $\left.\eta^{2}=0.143 ; F(5,258)=2.815, p=0.017, \eta^{2}=0.052\right]$, project community $\left[F(5,258)=3.911, p=0.002, \eta^{2}=0.070 ; F(5\right.$, 258) $\left.=2.861, p=0.016, \eta^{2}=0.053\right]$ and relationship satisfaction $\left[F(5,256)=25.467, p<0.001, \eta^{2}=0.332 ; F(5,257)=15.192\right.$, $\left.p<0.001, \eta^{2}=0.228\right]$. Means and standard deviations for the clusters with regard to the partners' project positivity, project community and RAS scores are presented in Table 7. Further investigation of the clusters as subgroups indicates the general trend that couples with lower (vs. higher) stress along with more positively (vs. negatively) balanced dyadic coping appear to experience better functioning in projects and higher relationship satisfaction. However, there are notable exceptions - for example, couples with lower stress but rather negatively balanced dyadic coping exhibit fairly high satisfaction but low positive emotions and sense of community in their projects.

\section{Additional Computations}

In a subsequent series of analyses, we tested whether the results with the clusters were dependent on the proportion of couples with the same (vs. different) projects. First, we compared the frequency of couples with the same project in the six clusters. Chi-square statistics indicated that the differences in distribution were statistically not significant $\left[\chi^{2}=5.15(5), p=0.398\right]$. We also ran the above-described ANCOVAs with an additional covariate 


\section{Project positivity}

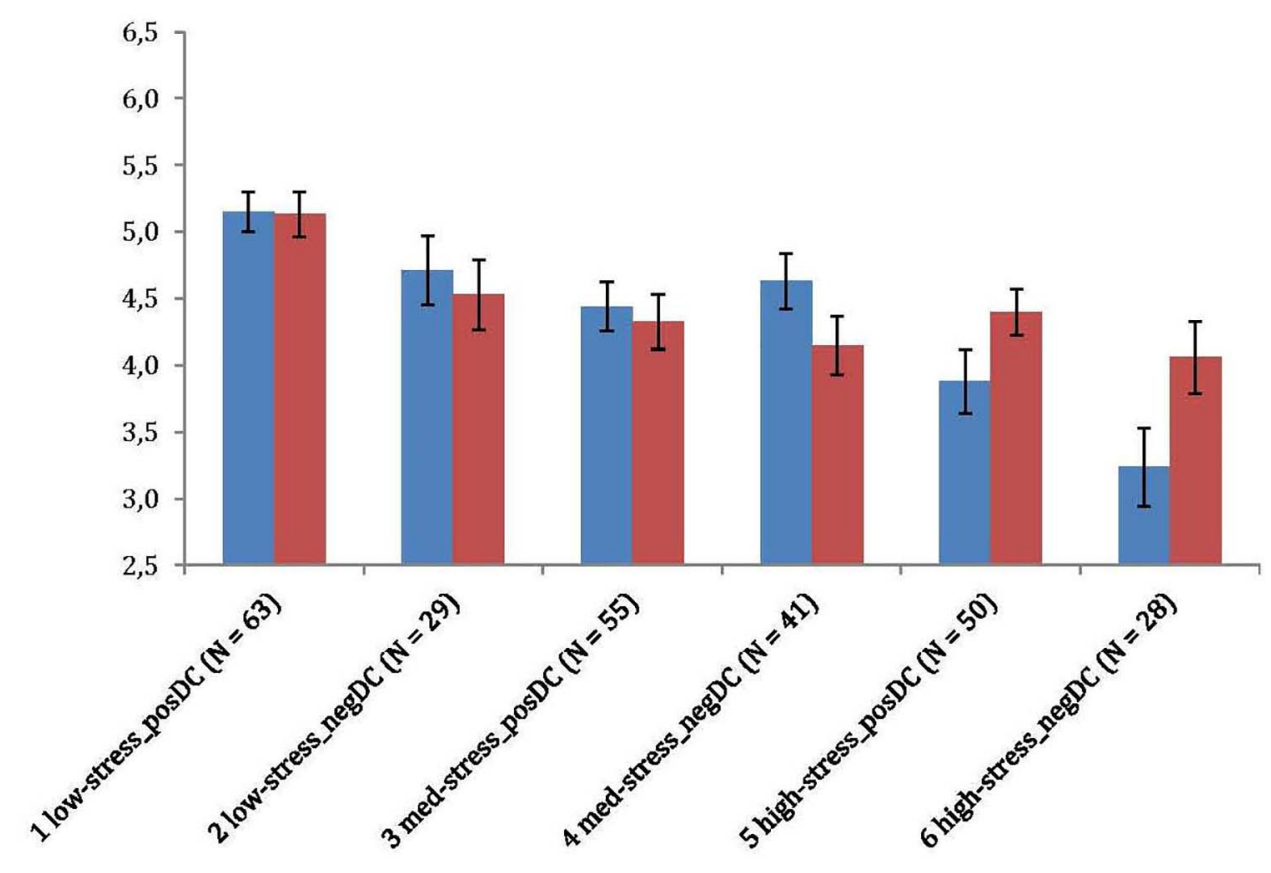

- Male Partners

- Female Partners

FIGURE 4 | Differences of clusters: positive emotions in personal project.

that coded shared (vs. not shared) projects in couples. Results indicated only minor differences in comparison to the abovepresented data. ${ }^{1}$

\section{DISCUSSION}

In the present study, we examined the personal projectrelated stress and dyadic coping experiences of adult Hungarian couples who were living together in a committed long-term relationship. To the best of our knowledge, this is the first attempt to study these relationship processes via personal project assessment. Using a pattern-oriented approach to dyadic data analysis, we were able to extract six meaningfully different stress and dyadic coping clusters that captured distinct patterns of couples' relationship processes connected to the personal project pursuit of the partners. Furthermore, we demonstrated that these patterns represented the non-linear and multidimensional nature of stress and dyadic coping in couples. We have argued that, through assessing dyadic coping processes in personal projects, the dyadic regulation of everyday pursuits can be studied in an ecologically valid way. Moreover, this approach may extend and add to the concept of dyadic coping as formulated in STM. Consequently, clusters of stress and dyadic coping represent different types of dyadic regulation processes as they are embedded in the everyday lives of couples. In the discussion below, we focus primarily on the potential implications and consequences of these relationship-level regulatory patterns.

\section{Relationship-Level Patterns of Stress and Dyadic Coping}

Through cluster analysis we identified six clusters with specific patterns of stress and dyadic coping experiences in the personal projects of the partners, where clusters were subsamples of couples. The clusters could be roughly characterized by two features: the level of stress experienced by the partners (lower, medium and higher stress) coupled with a positively vs. negatively balanced dyadic coping style. These patterns were mainly independent of sociodemographic characteristics, such as age, education, and length of relationship; this points to their potential generalizability as everyday regulatory strategies. However, we found significant associations with the subjective appraisals of financial status of the respondents. Closer inspection of these patterns generates a series of conclusions about the relationship-level regulation of everyday goals.

First, clusters represent non-linear, relationship-level associations between stress and dyadic coping experiences of couples. In bivariate associations, experienced stress was clearly directly correlated to negative (and in inverse correlation with positive) dyadic strategies within individual responses and partly also between partners (although effect sizes were typically in the low-to-medium range). Moreover, 


\section{Project community}

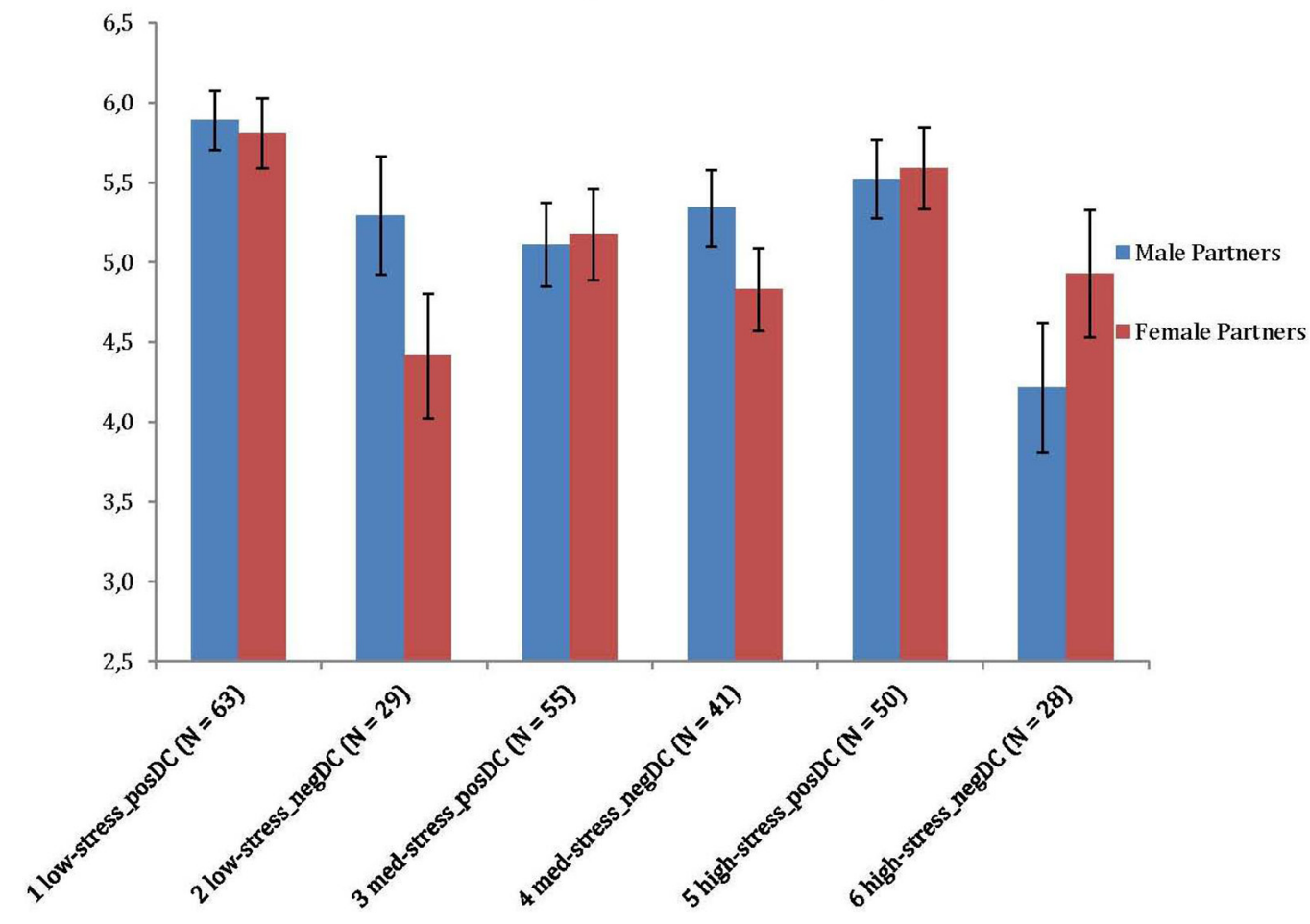

FIGURE 5 | Differences of clusters: sense of community in personal project.

correlations between negative and positive dyadic coping strategies were also inverse and in the medium range. However, clusters represented systematic exceptions to these findings; at every level of stress, dyadic coping strategies showed both combinations of positively and negatively balanced coping patterns. Accordingly, we were able to identify "out of the rule" subgroups of couples. For example, in Cluster 5 , a high level of stress appeared together with the relative dominance of positive dyadic coping in partners. Furthermore, in Cluster 4, negative dyadic coping was at its highest in both partners from among all clusters, in spite of only a medium level of stress.

Second, the detected patterns showed considerable symmetry; that is, relatively high "agreement" between partners with respect to the measured characteristics. Since the projects that were assessed in this procedure were individually chosen and personally important, this high agreement may represent deeper interdependence between the partners' self-regulation processes. Interdependence can appear in at least two ways; the projects may be the same or closely interconnected (e.g., quit smoking because of the birth of a baby; couple No. 63 in Table 3) and the way the partners pursue them may be interconnected too (e.g., when partners mutually support each other's important but different - pursuits; Kaplan and Maddux, 2002; Kuster et al., 2017). Both possibilities are in line with previous theorizing about goal interdependence in close relationships (Fitzsimons and Finkel, 2015).
Finally, our results confirm that a pattern oriented approach to dyadic data is an appropriate way to explore complex interaction patterns in a series of characteristics, in our case, stress and dyadic coping appraisals of partners. Studies on pattern-oriented approach assert that the strength of a pattern-oriented approach lies in its potential to detect non-linear, "outside the general rule" variations in the complex associations of relationship constructs (e.g., Gagliardi et al., 2013; Cao et al., 2015; Czikmantori et al., 2018), while preserving the holistic view of a couple's relationship. Non-linearity was not only present in the emergent clusters of stress and dyadic coping but also in their associations with outcome characteristics.

\section{Associations Between Stress and Dyadic Coping Patterns With Outcomes}

We assumed that specific patterns of stress and dyadic coping experiences might be associated with the partners' functioning in their personal projects (positive emotions and sense of community) and with their relationship satisfaction. Confirming this assumption in the deductive analytic step of the study, we found significant differences between the clusters with regard to these outcomes. On the whole, higher experienced stress in projects was only associated with adverse outcomes when it was also associated with the higher prevalence of negative and lower prevalence of positive dyadic coping strategies. This was primarily true for 


\section{Relationship Satisfaction}

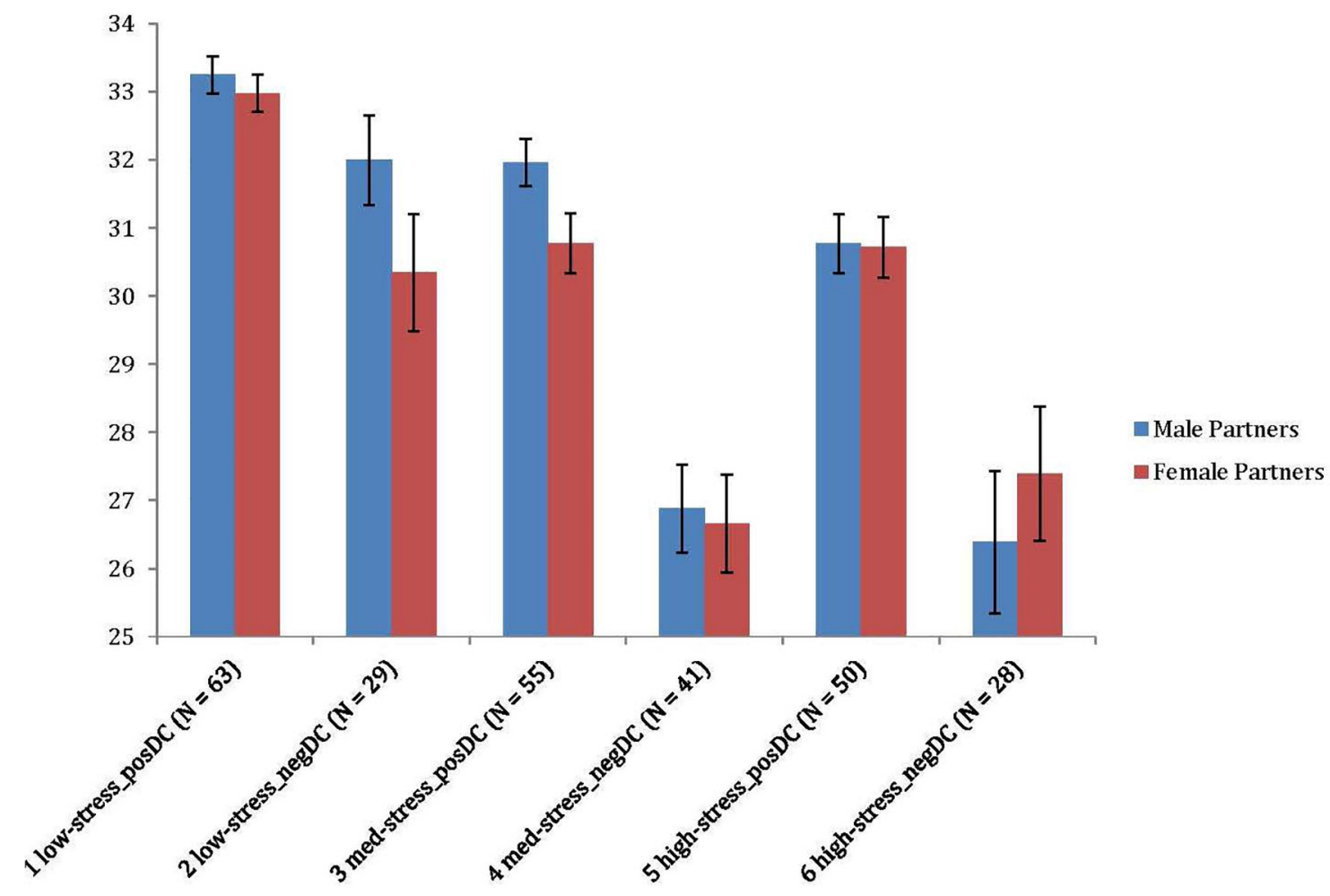

FIGURE 6 | Differences of clusters: relationship satisfaction.

lower sense of community in the personal project and lower general relationship satisfaction. In contrast, medium and high experienced stress was buffered against adverse effects by a positively balanced coping pattern in the projects. Associations of positive emotional experiences deviated from this trend. Couples that experienced lower stress in their projects felt generally more positive emotions in their projects than higher stressed couples, independent of the quality of their dyadic coping experiences. Below we give detailed implications of these results. We also provide further considerations on additional findings in Appendix 1.

\section{Positive Emotions in Personal Project}

In our study, positive emotions referred to primarily individual experiences of the partners in their personal projects. This way, the direct link of higher perceived stress to diminishing positive emotions may indicate a specific risk to health and well-being of the partners. According to an earlier longitudinal study, greater decreases in positive affect in response to daily stressors represent a risk of higher mortality (Mroczek et al., 2013). However, recent research has also found that the adaptive regulation of emotional states in the individual is connected to more positive dyadic coping of the partners (Rusu et al., 2018) and that positive emotions play a crucial role in successful coping processes (c.f., Folkman, 2008). Therefore, later research may address how and to what extent the appropriate use of positive dyadic strategies can still help couples to preserve positive emotional experiences even during the accomplishment of stressful personal projects.

\section{Sense of Community in Personal Project}

For both genders, complex patterns of stress and dyadic coping strategies were in a significant non-linear association with sense of community, a primarily relational experience of the partners in their personal projects. Male partners experienced the strongest sense of community both when dealing with low- and high-stress projects, paired with a positive dyadic coping pattern in their relationships. Female partners' sense of community was highest when low-stress projects were paired with positive coping patterns in the couple. Similarly, with negatively balanced dyadic coping strategies in relationship-level regulation of personal projects, male and female partners showed divergent vulnerabilities to diminished sense of community. Women were especially sensitive to low-level stress and negative dyadic coping (Cluster 2) in their sense of community, while men felt similarly when the partners were highly stressed in their projects, paired with negative dyadic coping (Cluster 6).

The protection of sense of community in personal projects even in the face of high stress and lower actual positive emotions may be beneficial for the short- and long-term functioning of relationships in many ways. The mutual pursuit of goals has been found to be associated with better relationship functioning, higher support and better goal progress (Kaplan and Maddux, 


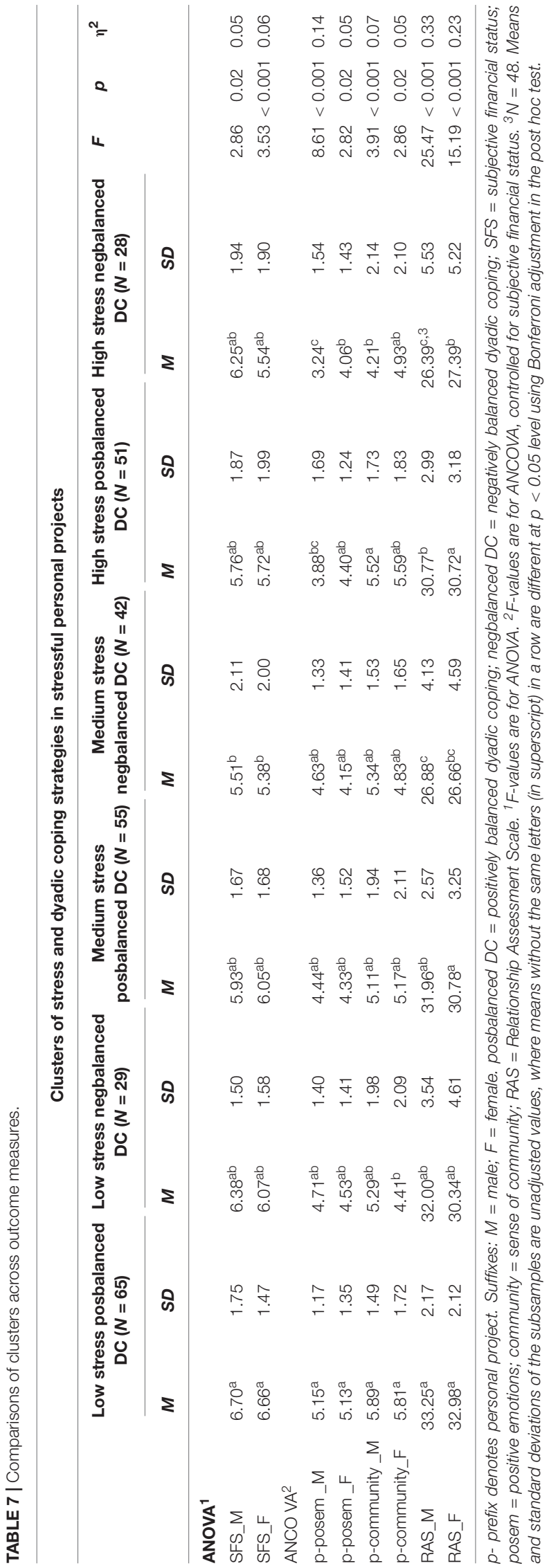

2002; Avivi et al., 2009) and the mutual sharing of future selves may support enjoyment in cooperation and personal well-being (Schindler et al., 2010). On the other hand, lack of goal similarity and joint goal planning may be indicators of the long-term risk of relationship dissolution (Arránz Becker, 2013; Gere et al., 2016), presumably because joint goal pursuits help partners to better align themselves with each other, and to involve each other in important strivings and the related decisions. Finally, emotional and practical coordination of important personal pursuits may extend and reinforce the partners' communal orientation which in turn may support further dyadic coping efforts (Randall et al., 2013; Koranyi et al., 2017).

\section{Relationship Satisfaction}

The interaction between stress level and the quality of dyadic coping strategies applied by the couple to cope with this stress was especially evident when we focused on outcomes in the context of the relationship in general, that is, in the case of relationship satisfaction. Clusters with a positive dyadic coping style in personal projects - even when paired with medium or high stress - were associated with higher relationship satisfaction than clusters with negative balance in dyadic coping. These associations had considerable effect sizes, and clearly showed that positively tuned dyadic coping strategies in personal projects may mitigate the adverse effect of heightened stress. Moreover, such findings are comparable to those of previous research about the stress-buffering effect of positive dyadic coping (Merz et al., 2014; Falconier et al., 2015b; Breitenstein et al., 2018; Hilpert et al., 2018). It is important to note, however, that project-related stress cannot be identified as purely external or internal; therefore dyadic coping in personal projects can be conceptualized as much as managing stress as just buffering against stress spillover. Consequently, higher relationship satisfaction may be regarded as a resource for stress management of the couple. The significance of high relationship satisfaction for relational self-regulation is highlighted by the fact that it may promote the effective goal pursuit of partners (Hofmann et al., 2015; Cappuzzello and Gere, 2018). Moreover, couples who were more satisfied with their relationship were more likely to choose to resolve stressful situations in a cooperative way (Bodenmann and Cina, 2000; Koranyi et al., 2017). These studies point to the possibility of a bidirectional association between stress, dyadic coping and relationship satisfaction. Research on relationship-level processes of personal project pursuit may add further insights into these phenomena.

\section{Limitations}

In interpreting the results of the current paper, one should bear in mind certain limitations. First, the cross-sectional research design does not allow for causal explanations of the results. Second, due to our reliance on self-reported responses solely, the validity of answers might be affected. Third, since the examined sample consisted of Hungarian respondents exclusively, future research can aim at investigating the cross-cultural generalizability of stress and dyadic coping patterns. Fourth, we assessed only one personal project for each respondent - later studies may use a more extended measurement of personal projects and the 
experiences associated with them. Fifth, we did not assess the other partner's real experiences concerning the personal project of one partner, but relied only on his or her perceptions. This leaves open the possibility that perceptions on the other partner's stress, positive emotions, or even dyadic coping behaviors were biased to a certain extent. Sixth, our emergent clusters represent complex interactions of multiple characteristics in an explorative, heuristic way. Later research should test these associations with more theoretically driven, targeted moderation analyses, for example in form of two- or three-way interaction analyses between gender, stress and dyadic coping. Finally, in order to make the cluster solutions easier to interpret, we focused on aggregated positive and negative dyadic coping scores only. A finer-grained analysis that differentiates between self and partner aspects and several forms of dyadic coping may strengthen the scientific power of the approach presented here.

\section{CONCLUSION AND FUTURE DIRECTIONS}

Our research documents the protective role of positively balanced dyadic coping strategies in the pursuit of everyday personal projects of people living in committed relationships. Dyadic coping processes proved to be important elements of the systemic, relationship-level regulation of personal projects in couples and when positively balanced, they predicted higher quality of relationship functioning, even when projects were rather stressful. These results have many implications for methodology, theory and the potential applications of dyadic coping research.

First, the results confirm previous notions that dyadic coping can be studied in a psychometrically reliable and ecologically valid way using personal projects as units (c.f., Martos et al., 2019). Through the dyadic coping-focused assessment of personal projects we can tackle everyday relationship-level experiences and the related regulatory strategies of couples. Later studies could use personal project assessment in the study of dyadic coping with specific life circumstances, like chronic illness or financial strain (c.f., Meier et al., 2011; Traa et al., 2015).

Second, on a more theoretical level the results also demonstrate that the pursuit of personally relevant projects is deeply connected to the interpersonal reality of a couple's relationship (c.f., Fitzsimons et al., 2015) while, by definition, they reflect personal and contextual features at the same time (Little, 2006). More or less consciously, people may face a considerable amount of stress when striving toward important personal goals. In other instances, life challenges arise, and projects are used to handle the resulting stress. Important relationships may play a role in both processes. Moreover, later

\section{REFERENCES}

Archuleta, K. L., Britt, S. L., Tonn, T. J., and Grable, J. E. (2011). Financial satisfaction and financial stressors in marital satisfaction. Psychol. Rep. 108, 563-576. doi: 10.2466/07.21.PR0.108.2.563-576 research may address how overarching pursuits, like personal projects are modified through the actual dyadic coping processes: the activated coping goals (c.f., Bodenmann, 1995; Bodenmann et al., 2016) and the resulting experiences of positive emotions and sense of community. In sum, we may assume that a series of complex intra- and interpersonal transactions exist involving relational features - such as dyadic coping strategies and contextual-environmental conditions and challenges. The dynamics and details of these transactional processes should be a target of later studies: understanding the former may deepen our understanding of relationship functioning as they are embedded in everyday life situations.

Finally, the results of the pattern-oriented approach presented here have implications for praxis. Identification of high-risk relationship patterns in couples may increase the sensitivity of practitioners to special configurations of vulnerability. Our results also confirm the importance of specific situations, such as financial strain, a frequent latent stressor for many couples (c.f., Martos et al., 2016). In these situations, application of STM-based training programs like Couples Coping Enhancement Training (CCET, Bodenmann and Shantinath, 2004) and TOGETHER (Falconier, 2015) may help couples to improve their individual and dyadic coping strategies. In our sample, partners who had highly stressful personal projects but applied positive dyadic coping strategies demonstrated the opportunity of fulfilling bonds and shared sense of community, even when they faced challenges in their life pursuits.

\section{DATA AVAILABILITY}

The raw database for this study that supports the conclusions of this manuscript will be made available by the authors, without undue reservation, to any qualified researcher.

\section{AUTHOR CONTRIBUTIONS}

TM and VS designed the study and carried out the research. TM and OF conducted data analysis. TM and HG conducted content analysis of personal projects. TM, VS, and OF wrote sections of the manuscript. HG and MN did the literature review and revised the manuscript. All authors contributed to the final interpretation of findings and read and approved this final version.

\section{FUNDING}

The research described herein was partly supported by the Hungarian Scientific Research Fund (OTKA) under grant number PD 105685. 
Avivi, Y. E., Laurenceau, J. P., and Carver, C. S. (2009). Linking relationship quality to perceived mutuality of relationship goals and perceived goal progress. J. Soc. Clin. Psychol. 28, 137-164. doi: 10.1521/jscp.2009.28.2.137

Barton, A. W., Futris, T. G., and Nielsen, R. B. (2015). Linking financial distress to marital quality: the intermediary roles of demand/withdraw and spousal gratitude expressions: demand/withdraw and spousal gratitude expressions. Pers. Relationsh. 22, 536-549. doi: 10.1111/pere.12094

Baumeister, R. F., Schmeichel, B. J., and Vohs, K. D. (2007). "Self-regulation and the executive function: the self as controlling agent," in Social Psychology: Handbook of Basic Principles, 2nd Edn, eds A. W. Kruglanski and E. T. Higgins (New York, NY: Guilford Press), 516-539.

Bergman, L., Magnusson, D., and El-Khouri, B. (2003). Studying Individual Development in an Inter-Individual Context: A Person-Oriented Approach. Mahwah, NJ: Lawrence Erlbaum. doi: 10.4324/9781410606822

Bergman, L. R., and El-Khouri, B. M. (2003). A person-oriented approach: methods for today and methods for tomorrow. New Dir. Child Adolesc. Dev. 2003, 25-38. doi: $10.1002 / \mathrm{cd} .80$

Bergman, L. R., and Lundh, L.-G. (2015). The person-oriented approach: roots and roads to the future. J. Pers. Oriented Res. 1, 1-6. doi: 10.17505/jpor.2015.01

Bergman, L. R., and Trost, K. (2006). The person-oriented versus the variable-oriented approach: Are they complementary, opposites, or exploring different worlds? Merrill Palmer Q. 52, 601-632. doi: 10.1353/mpq.2006. 0023

Bodenmann, G. (1995). A systemic-transactional conceptualization of stress and coping in couples. Swiss J. Psychol. 54, 34-49. doi: 10.1016/j.cpr.2008. 10.004

Bodenmann, G. (1997). Dyadic coping: a systemic-transactional view of stress and coping among couples: theory and empirical findings. Eur. Rev. Appl. Psychol. $47,137-140$.

Bodenmann, G. (2000). Stress und Coping bei Paaren. Göttingen: Hogrefe.

Bodenmann, G. (2005). "Dyadic coping and its significance for marital functioning," in Couples Coping with Stress: Emerging Perspectives on Dyadic Coping, eds T. A. Revenson, K. Kayser, and G. Bodenmann (Washington, DC: American Psychological Association), 33-50. doi: 10.1037/11031-002

Bodenmann, G. (2008). Dyadisches Coping Inventar. Test Manual [Dyadic Coping Inventory. Test Manual]. Bern: Huber.

Bodenmann, G., and Cina, A. (2000). Stress und coping als prädiktoren für scheidung: eine prospektive Fünf-Jahre-Längsschnittstudie. [Stress and coping as predictors of divorce: a prospecive five years longitudinal study.]. Z. Familienforsch. 12, 5-20.

Bodenmann, G., Randall, A. K., and Falconier, M. K. (2016). “Coping in couples the systemic transactional model (STM)," in Couples Coping with Stress: A CrossCultural Perspective, eds M. K. Falconier, A. K. Randall, and G. Bodenmann (New York, NY: Routledge), 5-22.

Bodenmann, G., and Shantinath, S. D. (2004). The couples coping enhancement training (CCET): a new approach to prevention of marital distress based upon stress and coping. Fam. Relat. 53, 477-484. doi: 10.1111/j.0197-6664.2004. 00056.x

Bodenmann, G., Widmer, K., Charvoz, L., and Brandbury, T. (2004). Differences in individual and dyadic coping among low and high depressed, partially remitted, and nondepressed persons. J. Psychopathol. Behav. Assess. 26, 75-85. doi: 10.1023/B:JOBA.0000013655.45146.47

Breitenstein, C. J., Milek, A., Nussbeck, F. W., Davila, J., and Bodenmann, G. (2018). Stress, dyadic coping, and relationship satisfaction in late adolescent couples. J. Soc. Pers. Relationsh. 35, 770-790. doi: 10.1177/0265407517698049

Brunstein, J. C. (1993). Personal goals and subjective well-being: a longitudinal study. J. Personal. Soc. Psychol. 65, 1061-1070. doi: 10.1037/0022-3514.65.5. 1061

Cao, H., Fang, X., Fine, M. A., Ju, X., Lan, J., and Liu, X. (2015). Beyond the average marital communication: latent profiles of the observed interactions among Chinese newlywed couples. J. Fam. Psychol. 29, 850-862. doi: 10.1037/ fam0000126

Cappuzzello, A. C., and Gere, J. (2018). Can you make my goals easier to achieve? Effects of partner instrumentality on goal pursuit and relationship satisfaction. Pers. Relationsh. 45, 549-559. doi: 10.1111/pere.12238

Carnes, A. M. (2017). Bringing work stress home: the impact of role conflict and role overload on spousal marital satisfaction. J. Occup. Organ. Psychol. 90, 153-176. doi: 10.1111/joop.12163
Carver, C. S., Scheier, M. F., and Fulford, D. (2008). "Self-regulatory processes, stress, and coping," in Handbook of Personality. Theory and Research, 3rd Edn, eds O. P. John, R. W. Robins, and L. A. Pervin (New York, NY: Guilford), 725-742.

Czikmantori, T., Hagemeyer, B., and Engeser, S. (2018). A dyadic typology of social desires in couples. J. Personal. doi: 10.1111/jopy.12338 [Epub ahead of print].

Dew, J., Britt, S., and Huston, S. (2012). Examining the relationship between financial issues and divorce. Fam. Relat. 61, 615-628. doi: 10.1111/j.1741-3729. 2012.00715.x

Dew, J., and Dakin, J. (2011). Financial disagreements and marital conflict tactics. J. Financ. Ther. 2:7. doi: 10.4148/jft.v2i1.1414

Emmons, R. A. (1997). "Motives and life goals," in Handbook of Personality Psychology, eds S. Briggs, R. Hogan, and J. A. Johnson (San Diego, CA: Academic Press), 485-512. doi: 10.1016/B978-012134645-4/50021-4

Falconier, M. K. (2015). TOGETHER - a couples' program to improve communication, coping, and financial management skills: development and initial pilot-testing. J. Marital Fam. Ther. 41, 236-250. doi: 10.1111/jmft.12052

Falconier, M. K., Jackson, J. B., Hilpert, P., and Bodenmann, G. (2015a). Dyadic coping and relationship satisfaction: a meta-analysis. Clin. Psychol. Rev. 42, 28-46. doi: 10.1016/j.cpr.2015.07.002

Falconier, M. K., Nussbeck, F., Bodenmann, G., Schneider, H., and Bradbury, T. (2015b). Stress from daily hassles in couples: its effects on intradyadic stress, relationship satisfaction, and physical and psychological well-being. J. Marital Fam. Ther. 41, 221-235. doi: 10.1111/jmft.12073

Falconier, M. K., Randall, A. K., and Bodenmann, G. (eds) (2016). Couples Coping with Stress: A Cross-Cultural Perspective. New York, NY: Routledge. doi: 10. 4324/9781315644394

Finkel, E. J., and Fitzsimons, G. M. (2011). "The effects of social relationships on self-regulation," in Handbook of Self-Regulation: Research, Theory, and Applications, eds K. D. Vohs and R. F. Baumeister (New York, NY: Guilford), 390-406.

Fitzsimons, G. M., and Finkel, E. J. (2011). "The effects of self-regulation on social relationships," in Handbook of Self-Regulation: Research, Theory, and Applications, eds K. D. Vohs and R. F. Baumeister (New York, NY: Guilford), 407-421.

Fitzsimons, G. M., and Finkel, E. J. (2015). Goal interdependence. Curr. Opin. Psychol. 1, 10-13. doi: 10.1016/j.copsyc.2014.11.015

Fitzsimons, G. M., Finkel, E. J., and vanDellen, M. R. (2015). Transactive goal dynamics. Psychol. Rev. 122, 648-673. doi: 10.1037/a0039654

Folkman, S. (2008). The case for positive emotions in the stress process. Anxiety Stress Coping 21, 3-14. doi: 10.1080/10615800701740457

Fowers, B. J., and Owenz, M. B. (2010). A eudaimonic theory of marital quality. J. Fam. Theory Rev. 2, 334-352. doi: 10.1111/j.1756-2589.2010. 00065.x

Gagliardi, S., Bodenmann, G., Heinrichs, N., Bertoni, A. M., Iafrate, R., and Donato, S. (2013). Unterschiede in der Partnerschaftsqualität und im dyadischen Coping bei verschiedenen bindungsbezogenen Paartypen. Psychother. Psychosom. Med. Psychol. 63, 185-192. doi: 10.1055/s-00321333291

Gere, J., Almeida, D. M., and Martire, L. M. (2016). The effects of lack of joint goal planning on divorce over 10 years. PLoS One 11:e0163543. doi: 10.1371/journal. pone. 0163543

Gere, J., and Schimmack, U. (2013). When romantic partners' goals conflict: effects on relationship quality and subjective well-being. J. Happiness Stud. 14, 37-49. doi: 10.1007/s10902-011-9314-2

Hallgren, K. A. (2012). Computing inter-rater reliability for observational data: an overview and tutorial. Tutor. Quant. Methods Psychol. 8, 23-34. doi: 10.20982/ tqmp.08.1.p023

Hendrick, S. S. (1988). A generic measure of relationship satisfaction. J. Marriage Fam. 50, 93-98. doi: 10.2307/352430

Herzberg, P. Y. (2013). Coping in relationships: the interplay between individual and dyadic coping and their effects on relationship satisfaction. Anxiety Stress Coping 26, 136-153. doi: 10.1080/10615806.2012.655726

Hilpert, P., Xu, F., Milek, A., Atkins, D. C., Bodenmann, G., and Bradbury, T. N. (2018). Couples coping with stress: between-person differences and within-person processes. J. Fam. Psychol. 32, 366-374. doi: 10.1037/fam000 0380 
Hofmann, W., Finkel, E. J., and Fitzsimons, G. M. (2015). Close relationships and self-regulation: how relationship satisfaction facilitates momentary goal pursuit. J. Personal. Soc. Psychol. 109, 434-452. doi: 10.1037/pspi0000020

Howard, M. C., and Hoffman, M. E. (2017). Variable-centered, person-centered, and person-specific approaches: where theory meets the method. Organ. Res. Methods 21, 846-876. doi: 10.1177/1094428117744021

Kaplan, M., and Maddux, J. E. (2002). Goals and marital satisfaction: perceived support for personal goals and collective efficacy for collective goals. J. Soc. Clin. Psychol. 21, 157-164. doi: 10.1521/jscp.21.2.157.22513

Kenny, D. A., and Ledermann, T. (2010). Detecting, measuring, and testing dyadic patterns in the actor-partner interdependence model. J. Fam. Psychol. 24, 359-366. doi: 10.1037/a0019651

Klug, H. J., and Maier, G. W. (2015). Linking goal progress and subjective wellbeing: a meta-analysis. J. Happiness Stud. 16, 37-65. doi: 10.1007/s10902-0139493-0

Koranyi, N., Hilpert, P., Job, V., and Bodenmann, G. (2017). Automatic affectivemotivational regulation processes underlying supportive dyadic coping: the role of increased implicit positive attitudes toward communal goals in response to a stressed relationship partner. Anxiety Stress Coping 30, 521-535. doi: 10.1080/ 10615806.2017.1292421

Kuster, M., Backes, S., Brandstätter, V., Nussbeck, F. W., Bradbury, T. N., SutterStickel, D., et al. (2017). Approach-avoidance goals and relationship problems, communication of stress, and dyadic coping in couples. Motiv. Emot. 41, 576-590. doi: 10.1007/s11031-017-9629-3

Little, B. R. (1983). Personal projects: a rationale and method for investigation. Environ. Behav. 15, 273-309. doi: 10.1177/0013916583153002

Little, B. R. (2006). Personality science and self-regulation: personal projects as integrative units. Appl. Psychol. 55, 419-427. doi: 10.1111/j.1464-0597.2006. 00262.x

Little, B. R. (2015). The integrative challenge in personality science: personal projects as units of analysis. J. Res. Personal. 56, 93-101. doi: 10.1016/j.jrp.2014. 10.008

Little, B. R., and Gee, T. L. (2007). "The methodology of personal projects analysis: four modules and a funnel," in Personal Project Pursuit: Goals, Action and Human Flourishing, eds B. R. Little, K. Salmela-Aro, and S. D. Phillips (Mahwah, NJ: Lawrence Erlbaum Associates), 51-93.

Martos, T., Sallay, V., Nistor, M., and Józsa, P. (2012). Párkapcsolati megküzdés és jóllét - a Páros megküzdés Kérdőív magyar változata [Dyadic coping and wellbeing - Hungarian version of the Dyadic Coping Inventory]. Psychiatr. Hung. $27,446-458$

Martos, T., Sallay, V., Szabó, T., Lakatos, C. S., and Tóth-Vajna, R. (2014). A Kapcsolati Elégedettség Skála Magyar változatának (RAS-H) pszichometriai jellemzõi. Mentálhig. Pszichoszom. 15, 245-258. doi: 10.1556/Mental.15. 2014.3.6

Martos, T., Sallay, V., and Tóth-Vajna, R. (2016). "Dyadic coping in hungarian couples," in Couples Coping with Stress: A Cross-Cultural Perspective, eds M. K. Falconier, A. K. Randall, and G. Bodenmann (New York, NY: Routledge), $170-186$.

Martos, T., Szabó, E., Koren, R., and Sallay, V. (2019). Dyadic coping in personal projects of romantic partners: assessment and associations with relationship satisfaction. Curr. Psychol. (accepted).

Meier, C., Bodenmann, G., Mörgeli, H., and Jenewein, J. (2011). Dyadic coping, quality of life, and psychological distress among chronic obstructive pulmonary disease patients and their partners. Int. J. Chron. Obstruct. Pulmon. Dis. 6, 583-595. doi: 10.2147/COPD.S24508

Merz, C. A., Meuwly, N., Randall, A. K., and Bodenmann, G. (2014). Engaging in dyadic coping: buffering the impact of everyday stress on prospective relationship satisfaction. Fam. Sci. 5, 30-37. doi: 10.1080/19424620.2014.927385

Molden, D. C., Lucas, G. M., Finkel, E. J., Kumashiro, M., and Rusbult, C. (2009). Perceived support for promotion-focused and prevention-focused goals: associations with well-being in unmarried and married couples. Psychol. Sci. 7, 787-793. doi: 10.1111/j.1467-9280.2009.02362

Mroczek, D. K., Stawski, R. S., Turiano, N. A., Chan, W., Almeida, D. M., Neupert, S. D., et al. (2013). Emotional reactivity and mortality: longitudinal findings from the VA Normative Aging Study. J Gerontol. B Psychol. Sci. Soc. Sci. 70, 398-406. doi: 10.1093/geronb/gbt107

Overall, N. C., Fletcher, G. J. O., and Simpson, J. A. (2010). Helping each other grow: romantic partner support, self-improvement, and relationship quality. Personal. Soc. Psychol. Bull. 36, 1496-1513. doi: 10.1177/0146167210383045
Papp, L. M., and Witt, N. L. (2010). Romantic partners' individual coping strategies and dyadic doping: implications for relationship functioning. J. Fam. Psychol. 24, 551-559. doi: 10.1037/a0020836

Praharso, N. F., Tear, M. J., and Cruwys, T. (2017). Stressful life transitions and wellbeing: a comparison of the stress buffering hypothesis and the social identity model of identity change. Psychiatry Res. 247, 264-275. doi: 10.1016/j.psychres. 2016.11.039

Randall, A. K., and Bodenmann, G. (2017). Stress and its associations with relationship satisfaction. Curr. Opin. Psychol. 13, 96-106. doi: 10.1016/j.copsyc. 2016.05.010

Randall, A. K., Post, J. H., Reed, R. G., and Butler, E. A. (2013). Cooperating with your romantic partner: associations with interpersonal emotion coordination. J. Soc. Pers. Relationsh. 30, 1072-1095. doi: 10.1177/0265407513481864

Rusu, P. P., Bodenmann, G., and Kayser, K. (2018). Cognitive emotion regulation and positive dyadic outcomes in married couples. J. Soc. Pers. Relationsh. 36, 359-376. doi: 10.1177/0265407517751664

Salmela-Aro, K., Nurmi, J., Saisto, T., and Halmesmäki, E. (2010). Spousal support for personal goals and relationship satisfaction among women during the transition to parenthood. Int. J. Behav. Dev. 34, 229-237. doi: 10.1177/ 0165025409350956

Schindler, I., Berg, C. A., Butler, J. M., Fortenberry, K. T., and Wiebe, D. J. (2010). Late-midlife and older couples' shared possible selves and psychological well-being during times of illness: the role of collaborative problem solving. J. Gerontol. B Psychol. Sci. Soc. Sci. 65, 416-424.

Seiffge-Krenke, I., and Burk, W. J. (2013). Friends or lovers? Person-and variableoriented perspectives on dyadic similarity in adolescent romantic relationships. J. Soc. Pers. Relationsh. 30, 711-733. doi: 10.1177/0265407512467562

Sim, A., Cordier, R., Vaz, S., Parsons, R., and Falkmer, T. (2017). Relationship satisfaction and dyadic coping in couples with a child with autism spectrum disorder. J. Autism Dev. Disord. 47, 3562-3573. doi: 10.1007/s10803-0173275-1

Staff, H. R., Didymus, F. F., and Backhouse, S. H. (2017). The antecedents and outcomes of dyadic coping in close personal relationships: a systematic review and narrative synthesis. J. Anxiety Stress Coping 30, 498-520. doi: 10.1080/ 10615806.2017.1329931

Story, L. B., and Bradbury, T. N. (2004). Understanding marriage and stress: essential questions and challenges. Clin. Psychol. Rev. 23, 1139-1162. doi: 10.1016/j.cpr.2003.10.002

Takács, S., Makrai, B., and Vargha, A. (2015). Klasszifikációs módszerek mutatói. [Indexes of classification methods.]. Psychol. Hung. 3, 67-88.

Thoits, P. A. (2010). Stress and health: major findings and policy implications. J. Health Soc. Behav. 51, 41-53. doi: 10.1177/0022146510383499

Traa, M. J., De Vries, J., Bodenmann, G., and Den Oudsten, B. L. (2015). Dyadic coping and relationship functioning in couples coping with cancer: a systematic review. Br. J. Health Psychol. 20, 85-114. doi: 10.1111/bjhp.12094

Vargha, A. (2016). A ROPstat statisztikai programcsomag. [The ROPstat statistical package.]. Stat. Szle. 94, 1165-1192. doi: 10.20311/stat2016.11-12.hu 1165

Vargha, A., Bergman, L. R., and Takács, S. (2016). Performing cluster analysis within a person-oriented context: some methods for evaluating the quality of cluster solutions. J. Pers. Oriented Res. 2, 78-86. doi: 10.17505/jpor.2016.08

Vargha, A., Torma, B., and Bergman, L. R. (2015). ROPstat: a general statistical package useful for conducting person-oriented analyses. J. Pers. Oriented Res. 1, 87-98. doi: 10.17505/jpor.2015.09

Wood, C. A., Helms, H. M., Supple, A. J., and Perlman, D. (2015). Gendertyped attributes and marital satisfaction among Mexican immigrant couples: a latent profile approach. J. Fam. Psychol. 29, 321-330. doi: 10.1037/fam000 0077

Conflict of Interest Statement: The authors declare that the research was conducted in the absence of any commercial or financial relationships that could be construed as a potential conflict of interest.

Copyright (c) 2019 Martos, Sallay, Nagy, Gregus and Filep. This is an open-access article distributed under the terms of the Creative Commons Attribution License (CC BY). The use, distribution or reproduction in other forums is permitted, provided the original author(s) and the copyright owner(s) are credited and that the original publication in this journal is cited, in accordance with accepted academic practice. No use, distribution or reproduction is permitted which does not comply with these terms. 


\section{APPENDIX 1: DISCUSSION OF ADDITIONAL FINDINGS}

\section{The Role of Perceived Financial Status in Stress and Coping Patterns}

One contextual feature that has received attention in recent studies related to relationship functioning and dyadic coping is the financial situation of couples. Research findings have confirmed that financial distress significantly increases the risk of negative impacts on relationship satisfaction and that this risk is modulated by the way couples manage financial challenges in their daily interactions (Archuleta et al., 2011; Barton et al., 2015). In fact, financial strain may lead to relationship dissatisfaction and dissolution when coupled with severe disagreement, while financial disagreements are among the most severe types of disagreement in relationships (Dew et al., 2012; Dew and Dakin, 2011).

As a corollary finding that fits this trend, we have documented how the stress and dyadic coping clusters were significantly different with regard to the level of subjective financial status of both partners. Interestingly, the lowest levels of perceived financial status were found in Cluster 4 in which stress was only at a medium level but negative dyadic coping was highest in both partners. Although general patterns of associations remained significant after controlling for financial status variables, later studies may include and scrutinize measures of financial status as well.

\section{The Role of Shared Projects}

We also tested whether our results were affected by the presence of certain couples' shared projects in the sample. Roughly a quarter of the couples specified the same project as most stressful. However, inclusion of this distinction in the analyses did not change the results considerably; the proportion of couples with shared projects was quite stable across the clusters, while associations of clusters with outcomes remained largely unaffected by this variable as well. While null findings have to be interpreted cautiously, we conclude that our results show the relative importance of general regulation processes over the specific content of personal projects. Regardless of their shared vs. non-shared content, individual personal projects are jointly regulated by the couples, and the associated stress and dyadic coping experiences seem to represent important aspects of relationship functioning. Later investigations may strengthen these notions or confirm the potentially distinctive role of shared personal project pursuits in relationship functioning. 


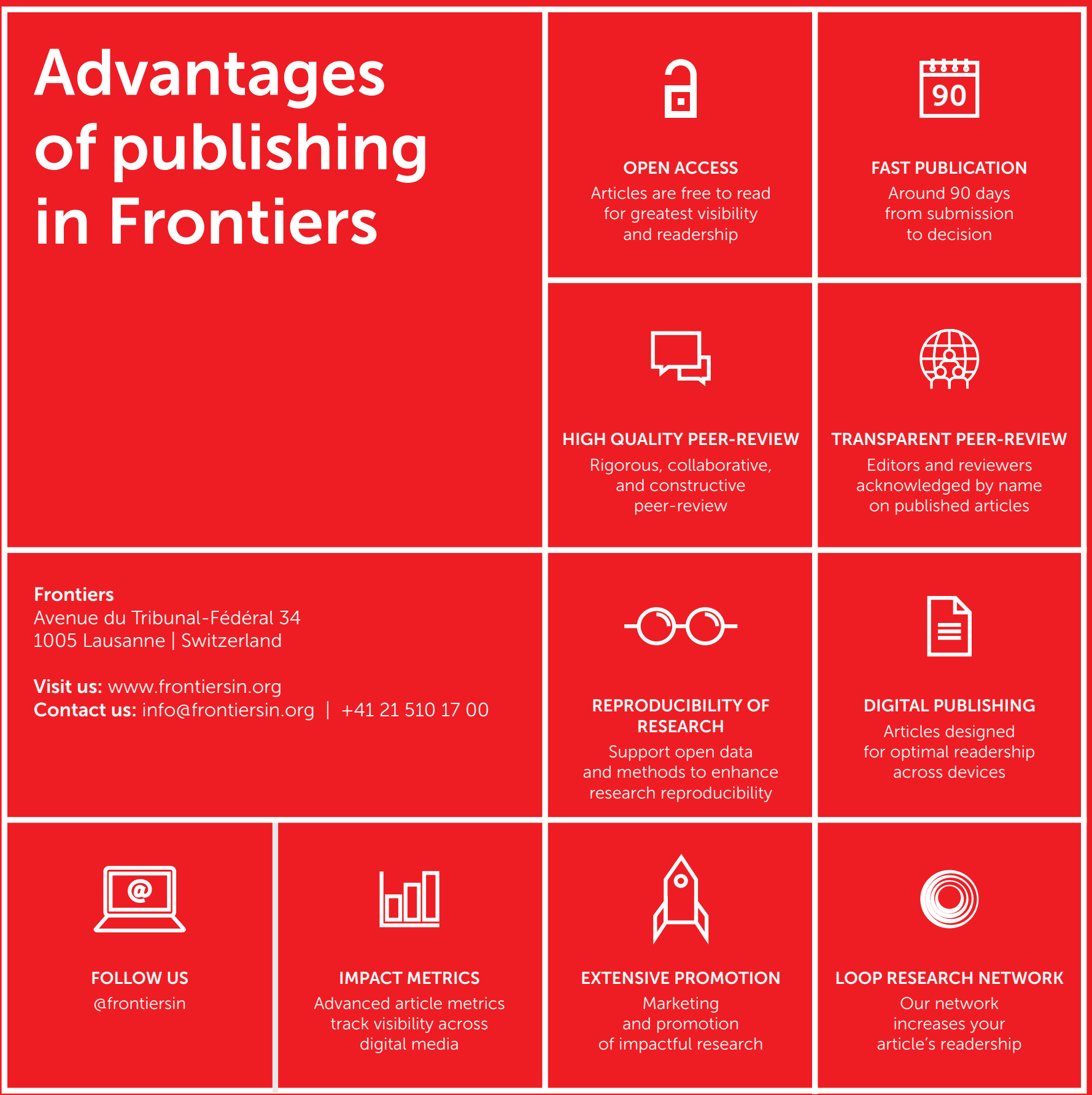

